\title{
Intramolecular Sakurai Allylation of Geminal Bis(silyl) Enamide with Indolenine. A Diastereoselective Cyclization to Form Functionalized Hexahydropyrido[3,4-b]Indole
}

\author{
Yi Chen, Xuanyi Song, Lu Gao* and Zhenlei Song* \\ Key Laboratory of Drug-Targeting and Drug Delivery System of the Education Ministry and \\ Sichuan Province, Sichuan Engineering Laboratory for Plant-Sourced Drug and Sichuan \\ Research Center for Drug Precision Industrial Technology, West China School of Pharmacy, \\ Sichuan University, Chengdu 610041, China. \\ lugao@scu.edu.cn; zhenleisong@scu.edu.cn
}

Table of Contents

1. General Methods S2

2. Experimental Procedures and Spectral Data of Products. S3-S59

2.1. Preparation of allylic carbonates S1-S26 ...S3-S10

2.2. Preparation of $7 a-7 d$ S11- S20

2.3. Screening of racemic reaction conditions form 2 S21

2.4. Preparation of indolenine $2 \mathrm{a}-2 \mathrm{ad}$. .S21-S38

2.5. Screening of asymmetric reaction conditions to form $(S)-2 t$ using diphosphine ligands and phosphoramidite ligands. S39-S40

2.6. General procedure of asymmetric reaction conditions with $\mathrm{Pd}(\mathrm{dba})_{2}$ .S40-S42

2.7. Preparation of 3a-3v. .S43-S55

2.8. Preparation of 8 . .556

2.9. Preparation of 4 . .S57-S58

2.10. Crystal preparation of 4 . S58

2.11. X-ray crystallographic structure of 4 . . .558

2.12. X-ray crystallographic data of 4 . .S59

3. ${ }^{1} \mathrm{H}$ and ${ }^{13} \mathrm{C}$ NMR Spectral Copies ..S60-S239 


\section{General Methods}

Unless otherwise noted, all commercial reagents which were purchased from Energy chemical company, J\&K Scientific Ltd, Aladdin, and so forth were used without any purification. Unless otherwise noted, all reactions were performed using common anhydrous, inert atmosphere techniques under ambient atmosphere; when the reactions required heating, the heat source was oil bath. Reactions were monitored by TLC which was performed on glass-backed silica plates and visualized using $\mathrm{UV}$ light at $254 \mathrm{~nm}, \mathrm{KMnO}_{4}$ stains or anisaldehyde stains. Column chromatography was performed using silica gel (200-300 mesh) eluting with EtOAc/petroleum ether. ${ }^{1} \mathrm{H}$ NMR spectra were recorded at $400 \mathrm{MHz}$ (Varian and Bruker) and $600 \mathrm{MHz}$ (Agilent), ${ }^{13} \mathrm{C}$ NMR spectra were recorded at $100 \mathrm{MHz}$ (Bruker) and $150 \mathrm{MHz}$ (Agilent) using $\mathrm{CDCl}_{3}$ or DMSO-d $\mathrm{d}_{6}$ with TMS as standard. The chemical shift $(\delta)$ are reported in parts per million (ppm) on the scale relative to $\mathrm{CDCl}_{3}\left(\delta=7.26\right.$ for ${ }^{1} \mathrm{H}$ NMR, $\delta=77.00$ for ${ }^{13} \mathrm{C}$ NMR $)$ or DMSO-d $\mathrm{d}_{6}\left(\delta=2.50\right.$ for ${ }^{1} \mathrm{H}$ NMR, $\delta=39.50$ for ${ }^{13} \mathrm{C}$ NMR), and the coupling constants $(J)$ are given in Hz. The abbreviations used are as follows: s, singlet; d, doublet; t, triplet; q, quartet; dd, doublet of doublet; ddd, doublet of doublet of doublet; dt, doublet of triplets; td, triplet of doublet; m, multiplet. Infrared spectra were obtained using $\mathrm{KCl}$ plates on a VECTOR22. High-resolution mass spectral analyses performed on Waters Q-TOF. X-ray diffraction experiment was carried out on Xcalibur E and the data obtained was deposited at the Cambridge Crystallographic Data Centre. Diastereomeric ratio was determined by ${ }^{1} \mathrm{H}$ NMR analysis and enantiomeric excess was determined by HPLC (Agilent Technologies: 1260 Infinity II) analysis on a Daicel Chiralpak ${ }^{\circledR}$ IC column. $\mathrm{CH}_{2} \mathrm{Cl}_{2}$ were distilled from $\mathrm{CaH}_{2}$. THF, $\mathrm{Et}_{2} \mathrm{O}$ and toluene were distilled from Na. All spectral data obtained for new compounds are reported here. 


\section{Experimental Procedures and Spectral Data of Products}

\subsection{Preparation of allylic carbonates S1-S26.}

Allylic carbonates S1-S26 were synthesized by the following general procedure. ${ }^{1} \mathbf{S 1 1}$, S14, S16-S23, S25-S26 are known compounds and their ${ }^{1} \mathrm{H}$ NMR spectra data are consistent with those reported. ${ }^{2}$ S12-S13, S15, S24 are new compounds and were fully characterized.

\section{Representative Procedure: preparation of S7}
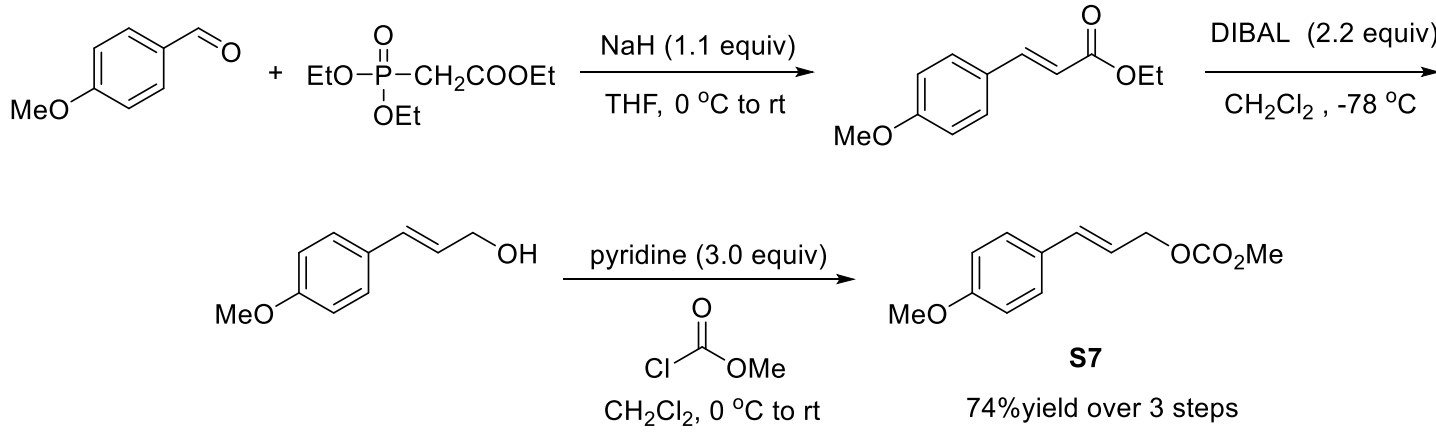

To a suspension of $\mathrm{NaH}$ (440 $\mathrm{mg}, 11 \mathrm{mmol}, 60 \%$ in mineral oil) in THF (40 mL) at $0{ }^{\circ} \mathrm{C}$ under argon was added triethyl phosphonoacetate $(2.2 \mathrm{~mL}, 11 \mathrm{mmol})$ dropwise. The reaction mixture was stirred for 15 minutes before adding a solution of the $p$-anisaldehyde $(1.36 \mathrm{~g}, 10 \mathrm{mmol})$ in THF $(10 \mathrm{~mL})$. The resulting mixture was stirred for $4 \mathrm{~h}$ at room temperature before quenching with sat. aq. $\mathrm{NH}_{4} \mathrm{Cl}(25 \mathrm{~mL})$. The aqueous layer was extracted with EtOAc $(3 \times 30 \mathrm{~mL})$. The combined organic layers were dried over anhydrous $\mathrm{Na}_{2} \mathrm{SO}_{4}$, concentrated by rotary evaporation. The crude $\alpha$, $\beta$-unsaturated ester was used in the next step without purification.

To a solution of the above $\alpha, \beta$-unsaturated ester $(10 \mathrm{mmol})$ in $\mathrm{CH}_{2} \mathrm{Cl}_{2}(30 \mathrm{~mL})$ at $-78{ }^{\circ} \mathrm{C}$ under argon was added dropwise DIBAL $(14.7 \mathrm{~mL}, 22 \mathrm{mmol}, 1.5 \mathrm{~mol} / \mathrm{L}$ in

\footnotetext{
1. Qiu, G. Y. S.; Mamboury, M.; Wang, Q.; Zhu, J. P. Angew. Chem., Int. Ed. 2016, 55, 15377-15381.

2. (a) Trost, B. M.; Miller, J. R.; Hoffman, C. M. J. Am. Chem. Soc. 2011, 133, 8165-8167. (b) Hatano, M.; Kamiya, S.; Ishihara, K. Chem. Commun. 2012, 48, 9465-9467. (c) Dai, Y. J.; Wu, F.; Zang, Z. H.; You, H. Z.; Gong, H. G. Chem. Eur. J. 2012, 18, 808-812. (d) Grange, R. L.; Clizbe, E. A.; Counsell, E. J.; Evans, P. A. Chem. Sci. 2015, 6, 777-781. (e) Nakatake, D.; Yazaki, R.; Matsushima, Y.; Ohshima, T. Adv. Synth. Catal. 2016, 358, 2569-2574. (f) Lundgren, R. J.; Thomas, B. N. Chem. Commun. 2016, 52, 958-961. (g) Bai, X. D.; Wang, J.; He, Y. Adv. Synth. Catal. 2018, 361, 496-501. (h) Murakami, R.; Sano, K.; Iwai, T.; Taniguchi, T.; Monde, K.; Sawamura, M. Angew. Chem., Int. Ed. 2018, 57, 9465-9469. (i) Jiang, X.Y.; Boehm, P.; Hartwig, J. F. J. Am. Chem. Soc. 2018, 140, 1239-1242. (j) Takizawa, K.; Sekino, T.; Sato, S.; Yoshino, T.; Kojima, M.; Matsunaga, S., Angew. Chem., Int. Ed.2019, 58, 9199-9203.
} 
toluene). The reaction was stirred for $2 \mathrm{~h}$ at room temperature before quenching with sat. aq. potassium sodium tartrate $(25 \mathrm{~mL})$. The aqueous layer was extracted with $\mathrm{CH}_{2} \mathrm{Cl}_{2}(3 \times 20 \mathrm{~mL})$ and the combined organic layers were dried over anhydrous $\mathrm{Na}_{2} \mathrm{SO}_{4}$, concentrated by rotary evaporation. The crude allylic alcohol was used in the next step without purification.

To a solution of the allylic alcohol $(10 \mathrm{mmol})$ in $\mathrm{CH}_{2} \mathrm{Cl}_{2}(50 \mathrm{~mL})$ at $0{ }^{\circ} \mathrm{C}$ under argon was added pyridine $(2.4 \mathrm{~mL}, 30 \mathrm{mmol})$. The reaction mixture was stirred for $15 \mathrm{~min}$ before adding methyl chloroformate $(1.6 \mathrm{~mL}, 20 \mathrm{mmol})$. The resulting mixture was warmed to room temperature and stirred for $12 \mathrm{~h}$. The reaction was washed with sat. aq. $\mathrm{NaCl}(30 \mathrm{~mL})$ and extracted with $\mathrm{Et}_{2} \mathrm{O}(3 \times 20 \mathrm{~mL})$. The combined organic layers were washed with aq. $1 \mathrm{~N} \mathrm{HCl}$ solution $(30 \mathrm{~mL})$, dried over anhydrous $\mathrm{Na}_{2} \mathrm{SO}_{4}$ and concentrated by rotary evaporation. The crude product was purified by silica gel column chromatography (gradient eluent: petroleum ether/EtOAc $=500: 1 \rightarrow 50: 1$ ) to afford the desired allylic carbonate $\mathbf{S 7}$ as a light yellow solid, $1.65 \mathrm{~g}, 74 \%$ yield over 3 steps. ${ }^{1} \mathrm{H}$ NMR $\left(400 \mathrm{MHz}, \mathrm{CDCl}_{3}\right) \delta 7.34(\mathrm{~d}, J=8.8 \mathrm{~Hz}, 2 \mathrm{H}), 6.87(\mathrm{~d}, J=8.8 \mathrm{~Hz}$, 2H), $6.65(\mathrm{~d}, J=16.0 \mathrm{~Hz}, 1 \mathrm{H}), 6.16\left(\mathrm{dt}, J_{1}=16.0 \mathrm{~Hz}, J_{2}=6.8 \mathrm{~Hz}, 1 \mathrm{H}\right), 4.77(\mathrm{~d}, J=$ $6.8 \mathrm{~Hz}, 2 \mathrm{H}), 3.80(\mathrm{~s}, 3 \mathrm{H}), 3.79$ (s, 3H).

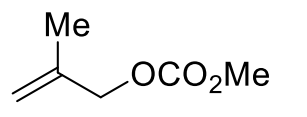

s1

S1: The title compound (starting from $20 \mathrm{mmol}$ 2-methylprop-2-en-1-ol) was isolated by silica gel column chromatography (gradient eluent: petroleum ether/EtOAc $=500: 1$ $\rightarrow 50: 1)$ as a light yellow oil, $1.9 \mathrm{~g}, 73 \%$ yield. ${ }^{1} \mathrm{H} \mathrm{NMR}\left(400 \mathrm{MHz}, \mathrm{CDCl}_{3}\right) \delta 5.00(\mathrm{~s}$, 1H), 4.93 (s, 1H), 4.53 (s, 2H), 3.78 (s, 3H), 1.75 (s, 3H).

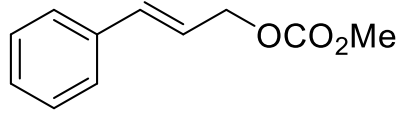

S2

S2: The title compound (starting from $40 \mathrm{mmol}$ 3-phenyl-2-propen-1-ol) was isolated by silica gel column chromatography (gradient eluent: petroleum ether/EtOAc $=$ $500: 1 \rightarrow 50: 1)$ as a white solid, $7.46 \mathrm{~g}, 97 \%$ yield. ${ }^{1} \mathrm{H}$ NMR $\left(400 \mathrm{MHz}, \mathrm{CDCl}_{3}\right) \delta$ 7.40-7.37 (m, 2H), 7.34-7.30 (m, 2H), 7.28-7.23 (m, 1H), $6.70(\mathrm{~d}, J=16.0 \mathrm{~Hz}, 1 \mathrm{H})$, $6.31\left(\mathrm{dt}, J_{1}=16.0 \mathrm{~Hz}, J_{2}=6.4 \mathrm{~Hz}, 1 \mathrm{H}\right), 4.79\left(\mathrm{dd}, J_{1}=6.4 \mathrm{~Hz}, J_{2}=1.6 \mathrm{~Hz}, 2 \mathrm{H}\right), 3.80$ 
$(\mathrm{s}, 3 \mathrm{H})$.

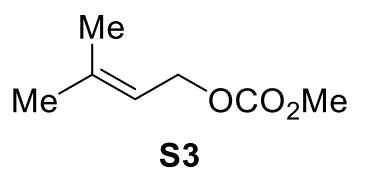

S3: The title compound (starting from $20 \mathrm{mmol}$ 3-methyl-2-buten-1-ol) was isolated by silica gel column chromatography (gradient eluent: petroleum ether/EtOAc $=$ 500:1 $\rightarrow 50: 1)$ as a light yellow oil, $2.58 \mathrm{~g}, 90 \%$ yield. ${ }^{1} \mathrm{H}$ NMR $\left(400 \mathrm{MHz}, \mathrm{CDCl}_{3}\right) \delta$ $5.36(\mathrm{t}, J=7.2 \mathrm{~Hz}, 1 \mathrm{H}), 4.62(\mathrm{~d}, J=7.2 \mathrm{~Hz}, 1 \mathrm{H}), 3.75(\mathrm{~s}, 3 \mathrm{H}), 1.74(\mathrm{~s}, 3 \mathrm{H}), 1.71(\mathrm{~s}$, $3 \mathrm{H})$.

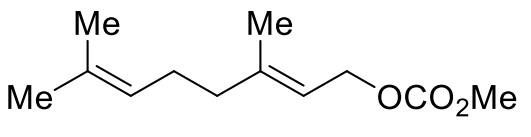

S4

S4: The title compound (starting from $20 \mathrm{mmol}$ geraniol) was isolated by silica gel column chromatography (gradient eluent: petroleum ether/EtOAc $=500: 1 \rightarrow 50: 1)$ as a colorless oil, $4.16 \mathrm{~g}, 98 \%$ yield. ${ }^{1} \mathrm{H}$ NMR $(400 \mathrm{MHz}$, $\left.\mathrm{CDCl}_{3}\right) \delta 5.39-5.34(\mathrm{~m}, 1 \mathrm{H}), 5.09-5.05(\mathrm{~m}, 1 \mathrm{H}), 4.65(\mathrm{~d}, J=7.2 \mathrm{~Hz}, 2 \mathrm{H}), 3.76(\mathrm{~s}, 3 \mathrm{H})$, 2.12-2.01 (m, 4H), $1.71(\mathrm{~s}, 3 \mathrm{H}), 1.70(\mathrm{~s}, 3 \mathrm{H}), 1.59(\mathrm{~s}, 3 \mathrm{H})$<smiles>COC(C)OC1C=CCCC1</smiles>

S5

S5: The title compound (starting from $10 \mathrm{mmol}$ 2-cyclohexen-1-ol) was isolated by silica gel column chromatography (gradient eluent: petroleum ether/EtOAc $=500: 1 \rightarrow 50: 1)$ as a light yellow oil, $1.51 \mathrm{~g}, 95 \%$ yield. ${ }^{1} \mathrm{H}$ NMR $\left(400 \mathrm{MHz}, \mathrm{CDCl}_{3}\right) \delta 5.98\left(\mathrm{dt}, J_{1}=10.0 \mathrm{~Hz}, J_{2}=4.0 \mathrm{~Hz}\right.$, 1H), 5.77-5.73 (m, 1H), 5.12-5.08 (m, 1H), $3.75(\mathrm{~s}, 3 \mathrm{H}), 2.07-1.58(\mathrm{~m}, 6 \mathrm{H})$.<smiles>CC=CCOC(C)=O</smiles>

S6

S6: The title compound (starting from $20 \mathrm{mmol}$ crotonyl alcohol) was isolated by silica gel column chromatography (gradient eluent: petroleum ether/EtOAc $=500: 1 \rightarrow 50: 1)$ as a light yellow oil, cis:trans $=1: 19,2.42 \mathrm{~g}, 93 \%$ yield. ${ }^{1} \mathrm{H}$ NMR $\left(400 \mathrm{MHz}, \mathrm{CDCl}_{3}\right) \delta 5.86-5.77(\mathrm{~m}, 1 \mathrm{H})$, 5.62-5.55 (m, 1H), $4.54(\mathrm{~d}, J=6.4 \mathrm{~Hz}, 2 \mathrm{H}), 3.75(\mathrm{~s}, 3 \mathrm{H}), 1.71(\mathrm{~d}, J=6.4 \mathrm{~Hz}, 3 \mathrm{H})$.<smiles>CC(=O)OC/C=C/c1ccc(C(F)(F)F)cc1</smiles>

58
S8: The title compound (starting from $10 \mathrm{mmol}$ 4-(trifluoromethyl)benzaldehyde) was isolated by silica gel column chromatography (gradient eluent: petroleum ether/EtOAc $=500: 1 \rightarrow 50: 1)$ as a yellow solid, $1.90 \mathrm{~g}, 73 \%$ yield over 3 steps. ${ }^{1} \mathrm{H}$ NMR $\left(400 \mathrm{MHz}, \mathrm{CDCl}_{3}\right) \delta 7.58(\mathrm{~d}, J=8.0 \mathrm{~Hz}, 2 \mathrm{H}), 7.48(\mathrm{~d}, J=8.0 \mathrm{~Hz}, 2 \mathrm{H})$, 
$6.73(\mathrm{~d}, J=16.0 \mathrm{~Hz}, 1 \mathrm{H}), 6.16\left(\mathrm{dt}, J_{1}=16.0 \mathrm{~Hz}, J_{2}=6.4 \mathrm{~Hz}, 1 \mathrm{H}\right), 4.82(\mathrm{~d}, J=6.4 \mathrm{~Hz}$, $2 \mathrm{H}), 3.81(\mathrm{~s}, 3 \mathrm{H})$.<smiles>COCC/C=C/c1ccc(F)cc1</smiles>

S9

S9: The title compound (starting from $10 \mathrm{mmol}$ 4-fluorobenzaldehyde) was isolated by silica gel column chromatography (gradient eluent: petroleum ether/EtOAc $=500: 1 \rightarrow 50: 1)$ as a white solid, $1.64 \mathrm{~g}, 78 \%$ yield over 3 steps. ${ }^{1} \mathrm{H}$ NMR $\left(400 \mathrm{MHz}, \mathrm{CDCl}_{3}\right) \delta 7.37\left(\mathrm{dd}, J_{1}=8.8 \mathrm{~Hz}, J_{2}=5.6 \mathrm{~Hz}, 2 \mathrm{H}\right), 7.00(\mathrm{t}, J=8.8 \mathrm{~Hz}, 2 \mathrm{H})$, $6.66(\mathrm{~d}, J=16.0 \mathrm{~Hz}, 1 \mathrm{H}), 6.21\left(\mathrm{dt}, J_{1}=16.0 \mathrm{~Hz}, J_{2}=6.4 \mathrm{~Hz}, 1 \mathrm{H}\right), 4.78(\mathrm{~d}, J=6.4 \mathrm{~Hz}$, $2 \mathrm{H}), 3.80(\mathrm{~s}, 3 \mathrm{H})$.<smiles>COc1ccccc1/C=C/COC(C)=O</smiles>

S10: The title compound (starting from $10 \mathrm{mmol}$ $o$-anisaldehyde) was isolated by silica gel column chromatography (gradient eluent: petroleum ether/EtOAc $=$ 500:1 $\rightarrow 50: 1)$ as a light yellow oil, $1.79 \mathrm{~g}, 81 \%$ yield over 3 steps. ${ }^{1} \mathrm{H}$ NMR (400 MHz, $\left.\mathrm{CDCl}_{3}\right) \delta 7.43(\mathrm{~d}, J=7.6 \mathrm{~Hz}, 1 \mathrm{H}), 7.24(\mathrm{t}, J=8.4 \mathrm{~Hz}, 1 \mathrm{H}), 7.03(\mathrm{~d}, J=16.0 \mathrm{~Hz}, 1 \mathrm{H})$, $6.92(\mathrm{t}, J=7.6 \mathrm{~Hz}, 1 \mathrm{H}), 6.88(\mathrm{~d}, J=8.4 \mathrm{~Hz}, 1 \mathrm{H}), 6.33\left(\mathrm{dt}, J_{1}=16.0 \mathrm{~Hz}, J_{2}=6.4 \mathrm{~Hz}\right.$, $1 \mathrm{H}), 4.81(\mathrm{~d}, J=6.4 \mathrm{~Hz}, 2 \mathrm{H}), 3.84(\mathrm{~s}, 3 \mathrm{H}), 3.80(\mathrm{~s}, 3 \mathrm{H})$.<smiles></smiles>

S11: The title compound (starting from $10 \mathrm{mmol}$ 3-methoxybenzaldehyde) was isolated by silica gel column chromatography (gradient eluent: petroleum ether/EtOAc $=500: 1 \rightarrow 50: 1)$ as a light yellow oil, $1.51 \mathrm{~g}, 68 \%$ yield over 3 steps. ${ }^{1} \mathrm{H}$ NMR (400 MHz, $\left.\mathrm{CDCl}_{3}\right) \delta 7.26(\mathrm{t}, J=8.0 \mathrm{~Hz}, 1 \mathrm{H}), 7.02(\mathrm{~d}, J=7.6 \mathrm{~Hz}, 1 \mathrm{H}), 6.95(\mathrm{~s}$, $1 \mathrm{H}), 6.86\left(\mathrm{dd}, J_{1}=8.0 \mathrm{~Hz}, J_{2}=2.4 \mathrm{~Hz}, 1 \mathrm{H}\right), 6.70(\mathrm{~d}, J=16.0 \mathrm{~Hz}, 1 \mathrm{H}), 6.33\left(\mathrm{dt}, J_{1}=\right.$ $\left.16.0 \mathrm{~Hz}, J_{2}=6.4 \mathrm{~Hz}, 1 \mathrm{H}\right), 4.82(\mathrm{~d}, J=6.4 \mathrm{~Hz}, 2 \mathrm{H}), 3.83(\mathrm{~s}, 6 \mathrm{H})$.<smiles>CC(=O)OC/C=C/c1ccc2c(c1)OCCO2</smiles>

ether/EtOAc $=500: 1 \rightarrow 30: 1)$ as a white solid, $2.0 \mathrm{~g}, 80 \%$ yield over 3 steps, m. p. $=$ 54.5-55.9 ${ }^{\circ} \mathrm{C} .{ }^{1} \mathrm{H}$ NMR $\left(400 \mathrm{MHz}, \mathrm{CDCl}_{3}\right) \delta 6.89\left(\mathrm{td}, J_{1}=8.4 \mathrm{~Hz}, J_{2}=2.0 \mathrm{~Hz}, 2 \mathrm{H}\right)$, $6.81(\mathrm{~d}, J=8.0 \mathrm{~Hz}, 1 \mathrm{H}), 6.58(\mathrm{~d}, J=15.6 \mathrm{~Hz}, 1 \mathrm{H}), 6.13\left(\mathrm{dt}, J_{1}=16.0 \mathrm{~Hz}, J_{2}=6.4 \mathrm{~Hz}\right.$, 
1H), $4.75(\mathrm{~d}, J=6.4 \mathrm{~Hz}, 2 \mathrm{H}), 4.23(\mathrm{~s}, 4 \mathrm{H}), 3.79(\mathrm{~s}, 3 \mathrm{H}),{ }^{13} \mathrm{C} \mathrm{NMR}\left(100 \mathrm{MHz}, \mathrm{CDCl}_{3}\right)$ $\delta 155.6,143.7,143.4,134.4,129.7,120.6,120.1,117.3,115.2,68.5,64.3,64.2,54.7$; IR (neat) $\mathrm{cm}^{-1} 2955,1743,1656,1583,1507,1440,1378,1254,1066,964,886$; HRMS (ESI-TOF) m/z: $\left[\mathrm{M}+\mathrm{Na}^{+} \mathrm{Calcd}\right.$ for $\mathrm{C}_{13} \mathrm{H}_{14} \mathrm{O}_{5} \mathrm{Na}^{+}:$273.0733, found 273.0734 .<smiles></smiles>

$\mathbf{S 1 3}$

S13: The title compound (starting from $10 \mathrm{mmol}$ 2-methylbenzaldehyde) was isolated by silica gel column chromatography (gradient eluent: petroleum ether/EtOAc $=$ $500: 1 \rightarrow 50: 1)$ as a light yellow oil, $1.33 \mathrm{~g}, 64 \%$ yield over 3 steps. ${ }^{1} \mathrm{H}$ NMR $\left(400 \mathrm{MHz}, \mathrm{CDCl}_{3}\right) \delta$ 7.46-7.44 (m, 1H), 7.20-7.14 (m, 3H), 6.95 (d, $J$ $=15.6 \mathrm{~Hz}, 1 \mathrm{H}), 6.20\left(\mathrm{dt}, J_{1}=15.6 \mathrm{~Hz}, J_{2}=6.4 \mathrm{~Hz}, 1 \mathrm{H}\right), 4.83(\mathrm{~d}, J=6.4 \mathrm{~Hz}, 2 \mathrm{H}), 3.82$ (s, 3H), $2.36(\mathrm{~s}, 3 \mathrm{H}) ;{ }^{13} \mathrm{C} \mathrm{NMR}\left(100 \mathrm{MHz}, \mathrm{CDCl}_{3}\right) \delta 155.6,135.7,135.1,132.7,130.2$, 128.0, 126.1, 125.8, 123.7,68.6, 54.7, 19.6; IR (neat) $\mathrm{cm}^{-1} 2955,1743,1484,1440$, 1397, 1251, 1122, 943, 790; HRMS (ESI-TOF) m/z: $[\mathrm{M}+\mathrm{K}]^{+}$Calcd for $\mathrm{C}_{12} \mathrm{H}_{14} \mathrm{O}_{3} \mathrm{~K}^{+}$: 245.0575, found 245.0571 .<smiles>CC(=O)OC/C=C/c1ccc(Cl)cc1</smiles>

S14
S14: The title compound (starting from $10 \mathrm{mmol}$ 4-chlorobenzaldehyde) was isolated by silica gel column chromatography (gradient eluent: petroleum ether/EtOAc $=500: 1 \rightarrow 50: 1)$ as a white solid, $1.67 \mathrm{~g}, 74 \%$ yield over 3 steps. ${ }^{1} \mathrm{H}$ NMR $\left(400 \mathrm{MHz}, \mathrm{CDCl}_{3}\right) \delta$ 7.32-7.27 (m, 4H), $6.65(\mathrm{~d}, J=16.0 \mathrm{~Hz}, 1 \mathrm{H}), 6.26\left(\mathrm{dt}, J_{1}=16.0\right.$ $\left.\mathrm{Hz}, J_{2}=6.4 \mathrm{~Hz}, 1 \mathrm{H}\right), 4.78(\mathrm{~d}, J=6.4 \mathrm{~Hz}, 2 \mathrm{H}), 3.80(\mathrm{~s}, 3 \mathrm{H})$.<smiles>CC(=O)OC/C=C/c1ccc(Cl)c(Cl)c1</smiles>
ether/EtOAc $=500: 1 \rightarrow 50: 1)$ as a white solid, $1.98 \mathrm{~g}, 76 \%$ yield over 3 steps, m. p. = 42.5-43. ${ }^{\circ} \mathrm{C} .{ }^{1} \mathrm{H}$ NMR $\left(400 \mathrm{MHz}, \mathrm{CDCl}_{3}\right) \delta 7.45(\mathrm{~d}, J=2.0 \mathrm{~Hz}, 1 \mathrm{H}), 7.39(\mathrm{~d}, J=8.4$ $\mathrm{Hz}, 1 \mathrm{H}), 7.20\left(\mathrm{dd}, J_{1}=8.4 \mathrm{~Hz}, J_{2}=2.0 \mathrm{~Hz}, 1 \mathrm{H}\right), 6.60(\mathrm{~d}, J=16.0 \mathrm{~Hz}, 1 \mathrm{H}), 6.28\left(\mathrm{dt}, J_{1}\right.$ $\left.=16.0 \mathrm{~Hz}, J_{2}=6.4 \mathrm{~Hz}, 1 \mathrm{H}\right), 4.78(\mathrm{~d}, J=6.4 \mathrm{~Hz}, 2 \mathrm{H}), 3.81(\mathrm{~s}, 3 \mathrm{H}) ;{ }^{13} \mathrm{C}$ NMR $(100$ $\left.\mathrm{MHz}, \mathrm{CDCl}_{3}\right) \delta 155.5,136.1,132.7,131.9,131.8,130.5,128.3,125.7,124.6,67.7$, 
54.9; IR (neat) $\mathrm{cm}^{-1} 2956,1743,1554,1472,1441,1253,1132,1027,950,886$; HRMS (ESI-TOF) m/z: $[\mathrm{M}+\mathrm{Na}]^{+}$Calcd for $\mathrm{C}_{11} \mathrm{H}_{10} \mathrm{Cl}_{2} \mathrm{O}_{3} \mathrm{Na}^{+}: 282.9899$, found 282.9901 .<smiles>CC(=O)OC/C=C/c1ccc(Br)cc1</smiles>

S16

S16: The title compound (starting from $10 \mathrm{mmol}$ 4-bromobenzaldehyde) was isolated by silica gel column chromatography (gradient eluent: petroleum ether/EtOAc $=500: 1 \rightarrow 50: 1)$ as a white solid, $2.41 \mathrm{~g}, 89 \%$ yield over 3 steps. ${ }^{1} \mathrm{H}$ NMR $\left(400 \mathrm{MHz}, \mathrm{CDCl}_{3}\right) \delta 7.46(\mathrm{~d}, J=8.4 \mathrm{~Hz}, 2 \mathrm{H}), 7.26(\mathrm{~d}, J=8.4 \mathrm{~Hz}, 2 \mathrm{H}), 6.64(\mathrm{~d}, J=$ $15.6 \mathrm{~Hz}, 1 \mathrm{H}), 6.30\left(\mathrm{dt}, J_{1}=16.0 \mathrm{~Hz}, J_{2}=6.4 \mathrm{~Hz}, 1 \mathrm{H}\right), 4.78(\mathrm{~d}, J=6.4 \mathrm{~Hz}, 2 \mathrm{H}), 3.81(\mathrm{~s}$, $3 \mathrm{H})$.<smiles>CC(=O)OC/C=C/c1ccc(-c2ccccc2)cc1</smiles>

petroleum ether/EtOAc $=500: 1 \rightarrow 50: 1)$ as a white solid, $1.88 \mathrm{~g}, 70 \%$ yield over 3 steps. ${ }^{1} \mathrm{H}$ NMR $\left(400 \mathrm{MHz}, \mathrm{CDCl}_{3}\right) \delta$ 7.63-7.57 (m, 4H), 7.49-7.44 (m, 4H), 7.38-7.34 $(\mathrm{m}, 1 \mathrm{H}), 6.76(\mathrm{~d}, J=16.0 \mathrm{~Hz}, 1 \mathrm{H}), 6.36\left(\mathrm{dt}, J_{1}=16.0 \mathrm{~Hz}, J_{2}=6.4 \mathrm{~Hz}, 1 \mathrm{H}\right), 4.84(\mathrm{dd}$, $\left.J_{1}=6.4 \mathrm{~Hz}, J_{2}=1.2 \mathrm{~Hz}, 2 \mathrm{H}\right), 3.83(\mathrm{~s}, 3 \mathrm{H})$.

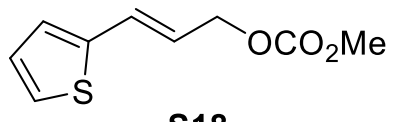

S18: The title compound (starting from $10 \mathrm{mmol}$ 2-thenaldehyde) was isolated by silica gel column chromatography (gradient eluent: petroleum ether/EtOAc $=500: 1 \rightarrow 50: 1$ ) as a yellow oil, 1.5 g, 76\% yield over 3 steps. ${ }^{1} \mathrm{H}$ NMR $\left(400 \mathrm{MHz}, \mathrm{CDCl}_{3}\right) \delta 7.20(\mathrm{~d}, J=5.2 \mathrm{~Hz}$, 1H), 7.00-6.95 (m, 2H), $6.83(\mathrm{~d}, J=16.0 \mathrm{~Hz}, 1 \mathrm{H}), 6.12\left(\mathrm{dt}, J_{1}=15.6 \mathrm{~Hz}, J_{2}=6.4 \mathrm{~Hz}\right.$, $1 \mathrm{H}), 4.75(\mathrm{~d}, J=6.4 \mathrm{~Hz}, 2 \mathrm{H}), 3.80(\mathrm{~s}, 3 \mathrm{H})$.<smiles>CC(=O)OC/C=C/c1ccc2ccccc2c1</smiles>

S19
S19: The title compound (starting from $10 \mathrm{mmol}$ 2-naphthaldehyde) was isolated by silica gel column chromatography (gradient eluent: petroleum ether/EtOAc $=500: 1 \rightarrow 50: 1)$ as a white solid, $1.82 \mathrm{~g}, 75 \%$ yield over 3 steps. ${ }^{1} \mathrm{H}$ NMR $\left(400 \mathrm{MHz}, \mathrm{CDCl}_{3}\right) \delta$ 7.82-7.76 (m, 4H), 7.61-7.59 (d, $\left.J=8.0 \mathrm{~Hz}, 1 \mathrm{H}\right), 7.50-7.45(\mathrm{~m}$, 
2H), $6.88(\mathrm{~d}, J=15.6 \mathrm{~Hz}, 1 \mathrm{H}), 6.43\left(\mathrm{dt}, J_{1}=16.0 \mathrm{~Hz}, J_{2}=6.4 \mathrm{~Hz}, 1 \mathrm{H}\right), 4.87(\mathrm{~d}, J=$ $6.4 \mathrm{~Hz}, 2 \mathrm{H}), 3.84$ (s, 3H).

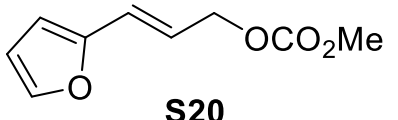

$\mathbf{S 2 0}$

S20: The title compound (starting from $10 \mathrm{mmol}$ furfural) was isolated by silica gel column chromatography (gradient eluent: petroleum ether/EtOAc $=500: 1 \rightarrow 50: 1)$ as a reddish brown oil, $1.36 \mathrm{~g}, 75 \%$ yield over 3 steps. ${ }^{1} \mathrm{H}$ NMR $\left(400 \mathrm{MHz}, \mathrm{CDCl}_{3}\right) \delta 7.35(\mathrm{~d}, J=1.6 \mathrm{~Hz}, 1 \mathrm{H}), 6.51(\mathrm{~d}, J=$ $16.0 \mathrm{~Hz}, 1 \mathrm{H}), 6.37\left(\mathrm{dd}, J_{1}=3.2 \mathrm{~Hz}, J_{2}=1.6 \mathrm{~Hz}, 1 \mathrm{H}\right), 6.29(\mathrm{~d}, J=3.2 \mathrm{~Hz}, 1 \mathrm{H}), 6.21$ $\left(\mathrm{dt}, J_{1}=15.6 \mathrm{~Hz}, J_{2}=6.4 \mathrm{~Hz}, 1 \mathrm{H}\right), 4.76(\mathrm{~d}, J=6.4 \mathrm{~Hz}, 2 \mathrm{H}), 3.79(\mathrm{~s}, 3 \mathrm{H})$.<smiles>CCOCC=Cc1ccc([N+](=O)[O-])cc1</smiles>

S21: The title compound (starting from $3.28 \mathrm{mmol}$ 4-nitrociamyl alcohol) was isolated by silica gel column chromatography (gradient eluent: petroleum ether/EtOAc $=500: 1 \rightarrow 30: 1$ ) as a yellow solid, $600 \mathrm{mg}, 77 \%$ yield. ${ }^{1} \mathrm{H}$ NMR $\left(400 \mathrm{MHz}, \mathrm{CDCl}_{3}\right) \delta 8.19(\mathrm{~d}, J=8.8 \mathrm{~Hz}, 2 \mathrm{H})$, $7.53(\mathrm{~d}, J=8.8 \mathrm{~Hz}, 2 \mathrm{H}), 6.76(\mathrm{~d}, J=16.0 \mathrm{~Hz}, 1 \mathrm{H}), 6.47\left(\mathrm{dt}, J_{1}=16.0 \mathrm{~Hz}, J_{2}=6.0 \mathrm{~Hz}\right.$, $1 \mathrm{H}), 4.84(\mathrm{~d}, J=6.0 \mathrm{~Hz}, 2 \mathrm{H}), 3.82(\mathrm{~s}, 3 \mathrm{H})$.<smiles></smiles>
phenylacetaldehyde) was isolated by silica gel column

chromatography (gradient eluent: petroleum ether/EtOAc $=500: 1 \rightarrow 50: 1$ ) as a light yellow solid, $1.85 \mathrm{~g}, 85 \%$ yield over 3 steps. ${ }^{1} \mathrm{H}$ NMR (400 MHz, $\left.\mathrm{CDCl}_{3}\right) \delta 7.41(\mathrm{~d}, J$ $=7.2 \mathrm{~Hz}, 2 \mathrm{H}), 7.32(\mathrm{t}, J=7.2 \mathrm{~Hz}, 2 \mathrm{H}), 7.24(\mathrm{t}, J=7.2 \mathrm{~Hz}, 1 \mathrm{H}), 6.80\left(\mathrm{dd}, J_{1}=15.6 \mathrm{~Hz}\right.$, $\left.J_{2}=10.4 \mathrm{~Hz}, 1 \mathrm{H}\right), 6.62(\mathrm{~d}, J=15.6 \mathrm{~Hz}, 1 \mathrm{H}), 6.49\left(\mathrm{dd}, J_{1}=14.8 \mathrm{~Hz}, J_{2}=10.4 \mathrm{~Hz}, 1 \mathrm{H}\right)$, $5.90\left(\mathrm{dt}, J_{1}=14.8 \mathrm{~Hz}, J_{2}=6.4 \mathrm{~Hz}, 1 \mathrm{H}\right), 4.73(\mathrm{~d}, J=6.4 \mathrm{~Hz}, 2 \mathrm{H}), 3.80(\mathrm{~s}, 3 \mathrm{H})$.

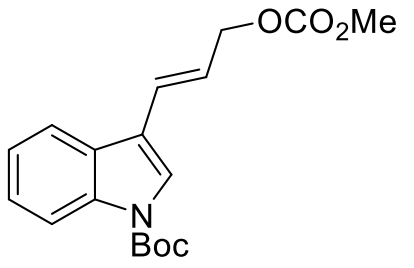

S23

S23: The title compound (starting from $10 \mathrm{mmol}$ 1-Boc-3-formylindole) was isolated by silica gel column chromatography (gradient eluent: petroleum ether/EtOAc $=$ $500: 1 \rightarrow 30: 1)$ as a light yellow oil, $875 \mathrm{mg}, 42 \%$ yield over 3 steps. ${ }^{1} \mathrm{H}$ NMR $\left(400 \mathrm{MHz}, \mathrm{CDCl}_{3}\right) \delta 8.18(\mathrm{~d}, J=8.4 \mathrm{~Hz}, 1 \mathrm{H}), 7.78(\mathrm{~d}, J=8.4 \mathrm{~Hz}$, 1H), $7.65(\mathrm{~s}, 1 \mathrm{H}), 7.34$ (t, $J=8.4 \mathrm{~Hz}, 1 \mathrm{H}), 7.28(\mathrm{t}, J=8.4 \mathrm{~Hz}, 1 \mathrm{H}), 6.82(\mathrm{~d}, J=16.0$ $\mathrm{Hz}, 1 \mathrm{H}), 6.39\left(\mathrm{dt}, J_{1}=16.0 \mathrm{~Hz}, J_{2}=6.4 \mathrm{~Hz}, 1 \mathrm{H}\right), 4.83(\mathrm{~d}, J=6.4 \mathrm{~Hz}, 2 \mathrm{H}), 3.82(\mathrm{~s}$, 
3H), 1.67 (s, 9H).<smiles>CC(=O)OC/C=C/c1ccc(C(C)=O)cc1</smiles>

S24: The title compound (starting from $1.7 \mathrm{mmol}$ 1-[4-[(1E)-3-Hydroxy-1-propen-1-yl]phenyl]ethenone ${ }^{3}$ ) was isolated by silica gel column chromatography (gradient eluent: petroleum ether/EtOAc $=500: 1 \rightarrow 30: 1$ ) as a white solid, $300 \mathrm{mg}, 75 \%$ yield, m. p. = 89.0-90.0 ${ }^{\circ} \mathrm{C} .{ }^{1} \mathrm{H} \mathrm{NMR}\left(400 \mathrm{MHz}, \mathrm{CDCl}_{3}\right) \delta 7.92(\mathrm{~d}$, $J=8.4 \mathrm{~Hz}, 2 \mathrm{H}), 7.47(\mathrm{~d}, J=8.4 \mathrm{~Hz}, 2 \mathrm{H}), 6.73(\mathrm{~d}, J=16.0 \mathrm{~Hz}, 1 \mathrm{H}), 6.40\left(\mathrm{dt}, J_{1}=16.0\right.$ $\left.\mathrm{Hz}, J_{2}=6.0 \mathrm{~Hz}, 1 \mathrm{H}\right), 4.81\left(\mathrm{dd}, J_{1}=6.0 \mathrm{~Hz}, J_{2}=1.6 \mathrm{~Hz}, 2 \mathrm{H}\right), 3.81(\mathrm{~s}, 3 \mathrm{H}), 2.58(\mathrm{~s}, 3 \mathrm{H})$; ${ }^{13} \mathrm{C}$ NMR $\left(100 \mathrm{MHz}, \mathrm{CDCl}_{3}\right) \delta 197.4,155.5,140.6,136.4,133.1,128.7,126.7,125.4$, 67.9, 54.9, 26.5; IR (neat) $\mathrm{cm}^{-1} 2965,1752,1676,1604,1444,1277,955,838$; HRMS (ESI-TOF) $\mathrm{m} / \mathrm{z}:[\mathrm{M}+\mathrm{Na}]^{+}$Calcd for $\mathrm{C}_{13} \mathrm{H}_{14} \mathrm{O}_{4} \mathrm{Na}^{+}:$257.0784, found 257.0787.<smiles>C=C(COC(C)=O)c1ccccc1</smiles>

S25: The title compound (starting from $10 \mathrm{mmol}$ beta-methylenephenethyl alcohol ${ }^{4}$ ) was isolated by silica gel column chromatography (gradient eluent: petroleum ether/EtOAc $=$ 500:1 $\rightarrow 50: 1)$ as a yellow oil, $1.91 \mathrm{~g}, 99 \%$ yield. ${ }^{1} \mathrm{H}$ NMR $\left(400 \mathrm{MHz}, \mathrm{CDCl}_{3}\right) \delta$ 7.46-7.43 (m, 2H), 7.38-7.26 (m, 3H), $5.58(\mathrm{~s}, 1 \mathrm{H}), 5.42(\mathrm{~s}, 1 \mathrm{H}), 5.05(\mathrm{~s}, 2 \mathrm{H}), 3.80(\mathrm{~s}$, $3 \mathrm{H})$.<smiles>CC(=O)OC/C=C/c1ccc(C(=O)O[Na])cc1</smiles>

S26: The title compound starting from $4.83 \mathrm{mmol}$ benzoic acid, 4-[(1E)-3-hydroxy-1-propen-1-yl]-, methyl ester ${ }^{5}$ was isolated by silica gel column chromatography (gradient eluent: petroleum ether/EtOAc $=500: 1 \rightarrow 30: 1$ ) as a white solid, $1.11 \mathrm{~g}, 92 \%$ yield. ${ }^{1} \mathrm{H}$ NMR $\left(400 \mathrm{MHz}, \mathrm{CDCl}_{3}\right) \delta 7.99(\mathrm{~d}, J=8.0 \mathrm{~Hz}, 2 \mathrm{H}), 7.44$ $(\mathrm{d}, J=8.0 \mathrm{~Hz}, 2 \mathrm{H}), 6.72(\mathrm{~d}, J=16.0 \mathrm{~Hz}, 1 \mathrm{H}), 6.40\left(\mathrm{dt}, J_{1}=16.0 \mathrm{~Hz}, J_{2}=6.0 \mathrm{~Hz}, 1 \mathrm{H}\right)$, $4.81(\mathrm{~d}, J=6.0 \mathrm{~Hz}, 2 \mathrm{H}), 3.90(\mathrm{~s}, 3 \mathrm{H}), 3.80(\mathrm{~s}, 3 \mathrm{H})$.

\footnotetext{
3 Shi, Y.; Hoveyda, A. H. Angew. Chem., Int. Ed. 2016, 128, 3516-3519.

4 Duan, Z. C.; Hu, X. P.; Zhang, C.; Wang, D. Y.; Yu, S. B.; Zheng, Z. J. Org. Chem. 2009, 74, 9191-9194.

5 Yang, B.; Wang, Z. X. Org. Lett. 2019, 21, 7965-7969.
} 


\subsection{Preparation of $7 a-7 d .{ }^{6,7}$}

\section{Preparation of $7 a$}
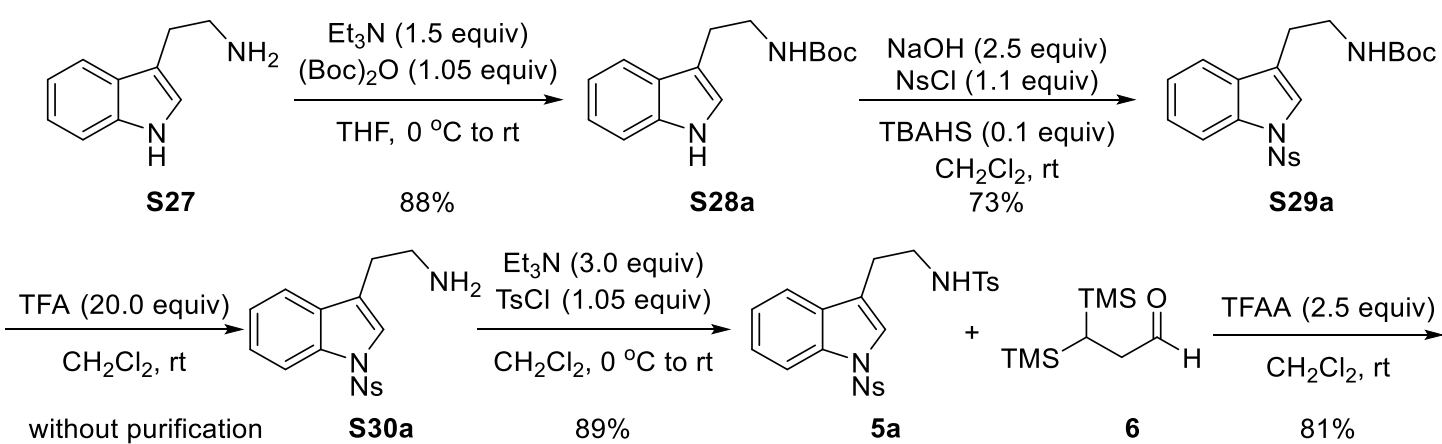

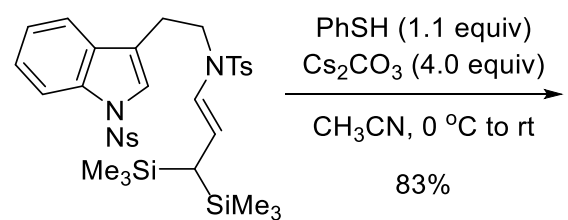

S31a

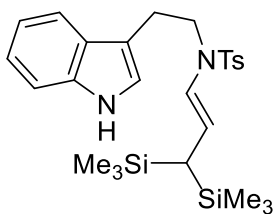

$7 \mathbf{a}$

To a solution of tryptamine $\mathbf{S 2 7}(32.04 \mathrm{~g}, 200 \mathrm{mmol})$ and triethylamine (41.7 mL, 300 mmol $)$ in THF $(270 \mathrm{~mL})$ at $0{ }^{\circ} \mathrm{C}$ under argon was added dropwise a solution of di-tert-butyl dicarbonate (45.83 g, $210 \mathrm{mmol})$ in THF (30 mL) via syringe. The reaction mixture was stirred at $0{ }^{\circ} \mathrm{C}$ for 10 minutes, and then warmed to room temperature with additional stirring for $12 \mathrm{~h}$. The reaction was quenched with sat. aq. $\mathrm{NH}_{4} \mathrm{Cl}(3 \times 100 \mathrm{~mL})$. The organic layers were dried over anhydrous $\mathrm{Na}_{2} \mathrm{SO}_{4}$ and concentrated by rotary evaporation. The crude product was recrystallized through $\mathrm{CH}_{2} \mathrm{Cl}_{2}$ and $n$-hexane to obtain the desired product $\mathbf{S 2 8 a}$ as a white solid, $45.59 \mathrm{~g}, 88 \%$ yield. ${ }^{1} \mathrm{H}$ NMR $\left(400 \mathrm{MHz}, \mathrm{CDCl}_{3}\right) \delta 8.21(\mathrm{~s}, 1 \mathrm{H}), 7.63(\mathrm{~d}, J=8.0 \mathrm{~Hz}, 1 \mathrm{H}), 7.38(\mathrm{~d}, J$ $=8.0 \mathrm{~Hz}, 1 \mathrm{H}), 7.21\left(\mathrm{dd}, J_{1}=8.0 \mathrm{~Hz}, J_{2}=7.2 \mathrm{~Hz}, 1 \mathrm{H}\right), 7.13\left(\mathrm{dd}, J_{1}=8.0 \mathrm{~Hz}, J_{2}=7.2\right.$ $\mathrm{Hz}, 1 \mathrm{H}), 7.02(\mathrm{~s}, 1 \mathrm{H}), 4.65(\mathrm{~s}, 1 \mathrm{H}), 3.47(\mathrm{q}, J=6.8 \mathrm{~Hz}, 2 \mathrm{H}), 2.96(\mathrm{t}, J=6.8 \mathrm{~Hz}, 2 \mathrm{H})$, $1.45(\mathrm{~s}, 9 \mathrm{H})$.

To a solution of $\mathbf{S 2 8 a}(10.41 \mathrm{~g}, 40 \mathrm{mmol})$ in $\mathrm{CH}_{2} \mathrm{Cl}_{2}(200 \mathrm{~mL})$ at room temperature 
under argon were added powdered $\mathrm{NaOH}(4 \mathrm{~g}, 100 \mathrm{mmol})$ and TBAHS (1.36 g, 4 mmol). The resulting suspension was stirred for 10 min before adding a solution of $\mathrm{NsCl}(9.75 \mathrm{~g}, 44 \mathrm{mmol})$ in $\mathrm{CH}_{2} \mathrm{Cl}_{2}(30 \mathrm{~mL})$. The reaction was stirred at room temperature for $16 \mathrm{~h}$ before quenching with sat. aq. $\mathrm{NH}_{4} \mathrm{Cl}(100 \mathrm{~mL})$. The organic layer was extracted with $\mathrm{CH}_{2} \mathrm{Cl}_{2}(3 \times 100 \mathrm{~mL})$, dried over anhydrous $\mathrm{Na}_{2} \mathrm{SO}_{4}$ and concentrated by rotary evaporation. The crude product was purified by silica gel column chromatography (eluent: $\mathrm{CH}_{2} \mathrm{Cl}_{2}$ ) to afford the desired product $\mathbf{S 2 9}$ a as a light yellow solid, 12.94 g, 73\% yield. m. p. = 119.6-120.8 ${ }^{\circ} \mathrm{C} .{ }^{1} \mathrm{H}$ NMR (400 MHz, $\mathrm{CDCl}_{3}$ ) $\delta 7.81(\mathrm{t}, J=8.4 \mathrm{~Hz}, 2 \mathrm{H}), 7.70(\mathrm{~d}, J=4.4 \mathrm{~Hz}, 2 \mathrm{H}), 7.64\left(\mathrm{dt}, J_{1}=8.4 \mathrm{~Hz}, J_{2}=4.4 \mathrm{~Hz}\right.$, 1H), $7.59(\mathrm{~d}, J=8.0 \mathrm{~Hz}, 1 \mathrm{H}), 7.41(\mathrm{~s}, 1 \mathrm{H}), 7.34-7.27(\mathrm{~m}, 2 \mathrm{H}), 4.78(\mathrm{~s}, 1 \mathrm{H}), 3.47$ (q, $J$ $=6.8 \mathrm{~Hz}, 2 \mathrm{H}), 2.92(\mathrm{t}, J=6.8 \mathrm{~Hz}, 2 \mathrm{H}), 1.42(\mathrm{~s}, 9 \mathrm{H}) \cdot{ }^{13} \mathrm{C} \mathrm{NMR}\left(100 \mathrm{MHz}, \mathrm{CDCl}_{3}\right) \delta$ $155.8,147.8,135.0,134.7,132.3,131.8,130.8,129.8,125.1,124.8,124.2,123.7$, 119.8, 113.4, 79.3, 40.0, 28.3, 25.6; IR (neat) $\mathrm{cm}^{-1} 3432,2978,1695,1543,1512$, 1447, 1365, 1275, 1122, 977, 763; HRMS (ESI-TOF) m/z: $[\mathrm{M}+\mathrm{Na}]^{+}$Calcd for $\mathrm{C}_{21} \mathrm{H}_{23} \mathrm{~N}_{3} \mathrm{O}_{6} \mathrm{SNa}^{+}$: 468.1200, found 468.1192.

To a solution of $\mathbf{S 2 9 a}(12.94 \mathrm{~g}, 29.05 \mathrm{mmol})$ in $\mathrm{CH}_{2} \mathrm{Cl}_{2}(200 \mathrm{~mL})$ at room temperature was added TFA $(43.2 \mathrm{~mL}, 581 \mathrm{mmol})$. The reaction was stirred for $2 \mathrm{~h}$ before cooling to $0{ }^{\circ} \mathrm{C}$ and quenching slowly with sat. aq. $\mathrm{NaHCO}_{3}(100 \mathrm{~mL})$. The aqueous layer was extracted with $\mathrm{CH}_{2} \mathrm{Cl}_{2}(3 \times 50 \mathrm{~mL})$. The combined organic layers were dried over anhydrous $\mathrm{Na}_{2} \mathrm{SO}_{4}$ and concentrated by rotary evaporation. To a solution of the crude product $\mathbf{S 3 0 a}$ in $\mathrm{CH}_{2} \mathrm{Cl}_{2}(120 \mathrm{~mL})$ at $0{ }^{\circ} \mathrm{C}$ under argon were added triethylamine (12.1 $\mathrm{mL}, 87.15 \mathrm{mmol})$, and a solution of $\mathrm{TsCl}(5.82 \mathrm{~g}, 30.5 \mathrm{mmol})$ in $\mathrm{CH}_{2} \mathrm{Cl}_{2}(30 \mathrm{~mL})$. The reaction was warmed to room temperature and stirred for $12 \mathrm{~h}$. The reaction was quenched with aq. $1 \mathrm{~N} \mathrm{HCl}$ solution $(30 \mathrm{~mL})$. The aqueous layer was extracted with $\mathrm{CH}_{2} \mathrm{Cl}_{2}(3 \times 50 \mathrm{~mL})$. The combined organic layers were dried over anhydrous $\mathrm{Na}_{2} \mathrm{SO}_{4}$ and concentrated by rotary evaporation. The crude product was purified by silica gel column chromatography (gradient eluent: petroleum ether/EtOAc $=20: 1 \rightarrow 2: 1$ ) to afford the desired product 5a as a yellow solid, 9.73 g, 89\% yield. m. p. = 152.2-154.8 ${ }^{\circ} \mathrm{C} .{ }^{1} \mathrm{H}$ NMR (600 MHz, DMSO) $\delta 8.10(\mathrm{~d}, J=7.8 \mathrm{~Hz}, 1 \mathrm{H}), 7.93(\mathrm{t}, J=$ 
$7.8 \mathrm{~Hz}, 1 \mathrm{H}), 7.79$ (t, $J=8.4 \mathrm{~Hz}, 1 \mathrm{H}), 7.76(\mathrm{~d}, J=8.4 \mathrm{~Hz}, 1 \mathrm{H}), 7.72(\mathrm{~d}, J=7.8 \mathrm{~Hz}$, 2H), $7.63(\mathrm{~d}, J=7.8 \mathrm{~Hz}, 2 \mathrm{H}), 7.57(\mathrm{~d}, J=7.8 \mathrm{~Hz}, 1 \mathrm{H}), 7.53(\mathrm{~s}, 1 \mathrm{H}), 7.34(\mathrm{t}, J=8.4$ $\mathrm{Hz}, 1 \mathrm{H}), 7.31-7.28$ (m, 3H), 3.10 (q, J=7.2 Hz, 2H), 2.83 (t, $J=7.2 \mathrm{~Hz}, 2 \mathrm{H}), 2.34$ (s, 3H). ${ }^{13} \mathrm{C}$ NMR (100 MHz, DMSO) $\delta 147.2$, 142.6, 137.6, 136.3, 134.4, 133.4, 130.3, 129.9, 129.6, 129.2, 126.4, 125.3, 125.1, 124.5, 123.8, 119.9, 119.7, 113.1, 42.0, 24.6, 21.0; IR (neat) $\mathrm{cm}^{-1} 3283,2920,1540,1446,1373,1317,1175,1155,1121,1091,852$; HRMS (ESI-TOF) m/z: $[\mathrm{M}+\mathrm{Na}]^{+}$Calcd for $\mathrm{C}_{23} \mathrm{H}_{21} \mathrm{~N}_{3} \mathrm{O}_{6} \mathrm{~S}_{2} \mathrm{Na}^{+}: 522.0764$, found 522.0768 .

To a solution of $5 \mathbf{a}(3.0 \mathrm{~g}, 6 \mathrm{mmol})$ and aldehyde $\mathbf{6}^{8}(2.43 \mathrm{~g}, 12 \mathrm{mmol})$ in $\mathrm{CH}_{2} \mathrm{Cl}_{2}(100$ $\mathrm{mL})$ at room temperature under argon was added TFAA $(2.1 \mathrm{~mL}, 15 \mathrm{mmol})$ and $\mathrm{MgSO}_{4}(10 \mathrm{~g})$. The reaction mixture was stirred for $12 \mathrm{~h}$ before filtrating through a pad of celite. The resulting mixture was washed with $\mathrm{CH}_{2} \mathrm{Cl}_{2}$ and concentrated by rotary evaporation. The residues were purified by silica gel column chromatography (gradient eluent: petroleum ether/EtOAc $=20: 1 \rightarrow 5: 1$ ) to afford the desired product S31a as a light yellow solid, $3.31 \mathrm{~g}, 81 \%$ yield. m. p. $=64.9-66.6{ }^{\circ} \mathrm{C} .{ }^{1} \mathrm{H}$ NMR $(600$ $\left.\mathrm{MHz} \mathrm{CDCl}_{3}\right) \delta 7.84(\mathrm{~d}, J=7.8 \mathrm{~Hz}, 1 \mathrm{H}), 7.72(\mathrm{t}, J=7.8 \mathrm{~Hz}, 1 \mathrm{H}), 7.67(\mathrm{t}, J=7.2 \mathrm{~Hz}$, 1H), $7.62(\mathrm{t}, J=7.8 \mathrm{~Hz}, 4 \mathrm{H}), 7.58(\mathrm{~d}, J=7.2 \mathrm{~Hz}, 1 \mathrm{H}), 7.41(\mathrm{~s}, 1 \mathrm{H}), 7.32(\mathrm{~d}, J=7.2$ $\mathrm{Hz}, 2 \mathrm{H}), 7.26(\mathrm{~d}, J=7.2 \mathrm{~Hz}, 2 \mathrm{H}), 6.00(\mathrm{~d}, J=13.2 \mathrm{~Hz}, 1 \mathrm{H}), 5.13\left(\mathrm{dd}, J_{1}=13.2 \mathrm{~Hz}, J_{2}\right.$ $=12.6 \mathrm{~Hz}, 1 \mathrm{H}), 3.55(\mathrm{t}, J=7.2 \mathrm{~Hz}, 2 \mathrm{H}), 3.01(\mathrm{t}, J=7.2 \mathrm{~Hz}, 2 \mathrm{H}), 2.40(\mathrm{~s}, 3 \mathrm{H}), 1.05(\mathrm{~d}$, $J=12.6 \mathrm{~Hz}, 1 \mathrm{H}), 0.03(\mathrm{~s}, 18 \mathrm{H}) .{ }^{13} \mathrm{C} \mathrm{NMR}\left(150 \mathrm{MHz}, \mathrm{CDCl}_{3}\right) \delta 147.8,143.5,135.7$, $135.1,134.5,132.8,132.0,130.6,129.7,129.5,127.0,125.2,124.8,124.3,123.8$, $122.4,119.6,119.5,119.2,113.7,46.4,23.5,22.1,21.5,-0.4$; IR (neat) $\mathrm{cm}^{-1} 2952$, 1545, 1449, 1382, 1352, 1249, 1161, 1123, 979, 838; HRMS (ESI-TOF) m/z: [M + $\mathrm{Na}]^{+}$Calcd for $\mathrm{C}_{32} \mathrm{H}_{41} \mathrm{~N}_{3} \mathrm{O}_{6} \mathrm{~S}_{2} \mathrm{Si}_{2} \mathrm{Na}^{+}$: 706.1868, found 706.1869 .

To a solution of S31a $(4.45 \mathrm{~g}, 6.5 \mathrm{mmol})$ in $\mathrm{CH}_{3} \mathrm{CN}(50 \mathrm{~mL})$ at $0{ }^{\circ} \mathrm{C}$ under argon were added $\mathrm{Cs}_{2} \mathrm{CO}_{3}(8.47 \mathrm{~g}, 26 \mathrm{mmol})$ and $\mathrm{PhSH}(0.73 \mathrm{~mL}, 7.15 \mathrm{mmol})$. The reaction mixture was warmed to room temperature and stirred for $0.5 \mathrm{~h}$. The reaction was quenched with sat. aq. $\mathrm{NH}_{4} \mathrm{Cl}(30 \mathrm{~mL})$. The aqueous layer was extracted with EtOAc

8. Song, Z. L.; Lei, Z.; Gao, L.; Wu, X.; Li, L. J. Org. Lett. 2010, 12, 5298-5301. 
$(3 \times 30 \mathrm{~mL})$. The combined organic layers were dried over $\mathrm{Na}_{2} \mathrm{SO}_{4}$, concentrated by rotary evaporation. The crude products were purified by silica gel column chromatography (gradient eluent: petroleum ether/EtOAc $=50: 1 \rightarrow 15: 1$ ) to afford the desired product 7a as a light yellow solid, $1.5 \mathrm{~g}, 83 \%$ yield. m. p. $=112.8-115.9^{\circ} \mathrm{C}$. ${ }^{1} \mathrm{H}$ NMR (600 MHz, $\left.\mathrm{CDCl}_{3}\right) \delta 8.14(\mathrm{~s}, 1 \mathrm{H}), 7.67(\mathrm{~d}, J=8.4 \mathrm{~Hz}, 2 \mathrm{H}), 7.62(\mathrm{~d}, J=8.4$ $\mathrm{Hz}, 1 \mathrm{H}), 7.38(\mathrm{~d}, J=8.4 \mathrm{~Hz}, 1 \mathrm{H}), 7.26(\mathrm{~d}, J=7.8 \mathrm{~Hz}, 2 \mathrm{H}), 7.21(\mathrm{t}, J=8.4 \mathrm{~Hz}, 1 \mathrm{H})$, $7.14(\mathrm{t}, J=7.8 \mathrm{~Hz}, 1 \mathrm{H}), 7.05(\mathrm{~s}, 1 \mathrm{H}), 6.11(\mathrm{~d}, J=13.8 \mathrm{~Hz}, 1 \mathrm{H}), 5.22\left(\mathrm{dd}, J_{1}=13.8 \mathrm{~Hz}\right.$ $\left.J_{2}=12.0 \mathrm{~Hz}, 1 \mathrm{H}\right), 3.54(\mathrm{t}, J=7.8 \mathrm{~Hz}, 2 \mathrm{H}), 3.08(\mathrm{t}, J=7.8 \mathrm{~Hz}, 2 \mathrm{H}), 2.40(\mathrm{~s}, 3 \mathrm{H}), 1.07$ $(\mathrm{d}, J=12.0 \mathrm{~Hz}, 1 \mathrm{H}), 0.07(\mathrm{~s}, 18 \mathrm{H}) .{ }^{13} \mathrm{C} \mathrm{NMR}\left(150 \mathrm{MHz}, \mathrm{CDCl}_{3}\right) \delta 143.2,136.2$, $135.9,129.5,127.2,126.9,122.8,122.1,122.0,119.3,118.5,118.3,112.4,111.3$, 47.6, 24.0, 22.0, 21.4, -0.4; IR (neat) $\mathrm{cm}^{-1} 3409,2951,1597,1493,1457,1339,1248$, 1159, 1092, 956, 835; HRMS (ESI-TOF) m/z: $\left[\mathrm{M}+\mathrm{Na}^{+}\right.$Calcd for $\mathrm{C}_{26} \mathrm{H}_{38} \mathrm{~N}_{2} \mathrm{O}_{2} \mathrm{SSi}_{2} \mathrm{Na}^{+}:$521.2085, found 521.2079.

\section{$\underline{\text { Preparation of } 7 b}$}

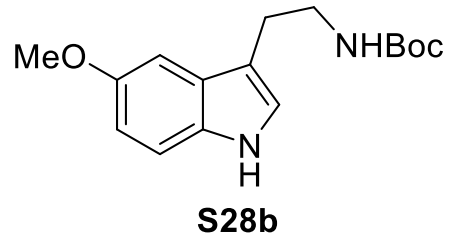

S28b: The title compound (starting from $87.38 \mathrm{mmol}$ 5-methoxytryptamine) was recrystallized through $\mathrm{CH}_{2} \mathrm{Cl}_{2}$ and $n$-hexane to obtain the desired product $\mathbf{S 2 8 b}$ as a white solid, 23.6 g, 93\% yield. ${ }^{1} \mathrm{H}$ NMR (400 MHz, $\left.\mathrm{CDCl}_{3}\right) \delta 8.20(\mathrm{~s}, 1 \mathrm{H}), 7.24(\mathrm{~d}, J=8.8 \mathrm{~Hz}, 1 \mathrm{H}), 7.02(\mathrm{~s}, 1 \mathrm{H}), 6.96(\mathrm{~s}, 1 \mathrm{H}), 6.87\left(\mathrm{dd}, J_{1}\right.$ $\left.=8.8 \mathrm{~Hz}, J_{2}=2.4 \mathrm{~Hz}, 1 \mathrm{H}\right), 4.68(\mathrm{~s}, 1 \mathrm{H}), 3.85(\mathrm{~s}, 3 \mathrm{H}), 3.45(\mathrm{q}, J=6.8 \mathrm{~Hz}, 2 \mathrm{H}), 2.91(\mathrm{t}$, $J=6.8 \mathrm{~Hz}, 2 \mathrm{H}), 1.44(\mathrm{~s}, 9 \mathrm{H})$.

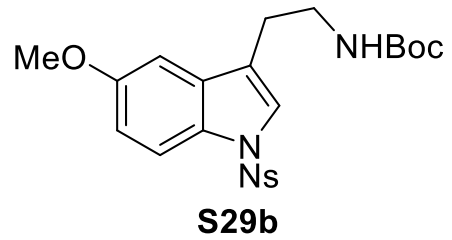

S29b: The title compound (starting from $25.12 \mathrm{mmol}$ S28b) was isolated by silica gel column chromatography (eluent: $\mathrm{CH}_{2} \mathrm{Cl}_{2}$ ) to afford the desired product $\mathbf{S 2 9} \mathbf{b}$ as a tan solid, $6.13 \mathrm{~g}, 51 \%$ yield. m. p. $=139.2-140.9{ }^{\circ} \mathrm{C} .{ }^{1} \mathrm{H}$ NMR (400 MHz, $\left.\mathrm{CDCl}_{3}\right) \delta$ 7.73-7.68 (m, 4H), 7.63-7.59 (m, 1H), $7.37(\mathrm{~s}, 1 \mathrm{H}), 6.99$ (s, 1H), $7.93\left(\mathrm{dd}, J_{1}=8.8 \mathrm{~Hz}, J_{2}=2.4 \mathrm{~Hz}, 1 \mathrm{H}\right), 4.77(\mathrm{~s}, 1 \mathrm{H}), 3.84(\mathrm{~s}, 3 \mathrm{H}), 3.47(\mathrm{q}, J=$ $6.8 \mathrm{~Hz}, 2 \mathrm{H}), 2.88(\mathrm{t}, J=6.8 \mathrm{~Hz}, 2 \mathrm{H}), 1.42(\mathrm{~s}, 9 \mathrm{H}) .{ }^{13} \mathrm{C} \mathrm{NMR}\left(100 \mathrm{MHz}, \mathrm{CDCl}_{3}\right) \delta$ $156.8,155.8,147.8,134.6,132.3,131.9,129.6,129.6,124.9,124.8,120.0,114.4$, 
113.9, 102.5, 79.3, 55.7, 39.8, 28.3, 25.6; IR (neat) $\mathrm{cm}^{-1} 3355,2932,1679,1539,1519$, 1364, 1278, 1231, 1123, 982, 889; HRMS (ESI-TOF) m/z: $[\mathrm{M}+\mathrm{Na}]^{+}$Calcd for $\mathrm{C}_{22} \mathrm{H}_{25} \mathrm{~N}_{3} \mathrm{O}_{7} \mathrm{SNa}^{+}$: 498.1305, found 498.1301.

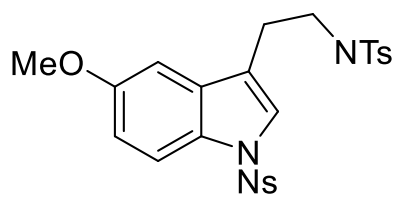

$5 b$

5b: The title compound (starting from 10 mmol S29b) was isolated by silica gel column chromatography (gradient eluent: petroleum ether/EtOAc $=20: 1 \rightarrow 2: 1)$ to afford the desired product $\mathbf{5 b}$ as a yellow solid, $4.40 \mathrm{~g}, 83 \%$ yield. $\mathrm{m}$. p. $=157.9-159.8{ }^{\circ} \mathrm{C} .{ }^{1} \mathrm{H}$ NMR $(400 \mathrm{MHz}, \mathrm{DMSO}) \delta 8.09(\mathrm{~d}, J=8.4 \mathrm{~Hz}, 1 \mathrm{H}), 7.91(\mathrm{t}$, $J=7.6 \mathrm{~Hz}, 1 \mathrm{H}), 7.78(\mathrm{t}, J=7.6 \mathrm{~Hz}, 1 \mathrm{H}), 7.72(\mathrm{t}, J=5.6 \mathrm{~Hz}, 1 \mathrm{H}), 7.68(\mathrm{~d}, J=8.0 \mathrm{~Hz}$, 1H), 7.64-7.59 (m, 3H), 7.49 (s, 1H), $7.31(\mathrm{~d}, J=8.0 \mathrm{~Hz}, 2 \mathrm{H}), 7.07$ (d, $J=2.8 \mathrm{~Hz}$, $1 \mathrm{H}), 6.95\left(\mathrm{dd}, J_{1}=8.8 \mathrm{~Hz}, J_{2}=2.8 \mathrm{~Hz}, 1 \mathrm{H}\right), 3.77(\mathrm{~s}, 3 \mathrm{H}), 3.11(\mathrm{q}, J=6.8 \mathrm{~Hz}, 2 \mathrm{H})$, $2.81(\mathrm{t}, J=6.8 \mathrm{~Hz}, 2 \mathrm{H}), 2.34(\mathrm{~s}, 3 \mathrm{H}) .{ }^{13} \mathrm{C} \mathrm{NMR}(100 \mathrm{MHz}, \mathrm{DMSO}) \delta 156.5,147.2$, $142.6,137.6,136.1,133.4,131.6,130.0,129.6,128.9,126.5,125.3,125.2,120.0$, 114.0, 113.8, 102.7, 55.6, 41.9, 24.7, 21.0. IR (neat) $\mathrm{cm}^{-1} 3295,2925,1549,1472$, 1376, 1216, 1179, 1147, 1038, 930; HRMS (ESI-TOF) m/z: $[\mathrm{M}+\mathrm{Na}]^{+}$Calcd for $\mathrm{C}_{24} \mathrm{H}_{23} \mathrm{~N}_{3} \mathrm{O}_{7} \mathrm{~S}_{2} \mathrm{Na}^{+}:$552.0870, found 552.0876.

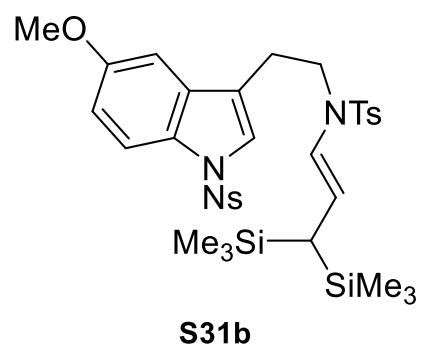

S31b: The title compound (starting from $3.78 \mathrm{mmol} \mathbf{5 b}$ ) was isolated by silica gel column chromatography (gradient eluent: petroleum ether/EtOAc $=20: 1 \rightarrow 5: 1)$ to afford the desired product $\mathbf{S 3 1 b}$ as a light yellow solid, 618 mg, $23 \%$ yield. m. p. $=53.8-55.3{ }^{\circ} \mathrm{C} .{ }^{1} \mathrm{H}$ NMR $(400 \mathrm{MHz}$, $\left.\mathrm{CDCl}_{3}\right) \delta 7.74-7.57(\mathrm{~m}, 7 \mathrm{H}), 7.36(\mathrm{~s}, 1 \mathrm{H}), 7.27(\mathrm{~d}, J=8.0 \mathrm{~Hz}, 2 \mathrm{H}), 7.04(\mathrm{~d}, J=2.4 \mathrm{~Hz}$, $1 \mathrm{H}), 6.94\left(\mathrm{dd}, J_{1}=9.2 \mathrm{~Hz}, J_{2}=2.4 \mathrm{~Hz}, 1 \mathrm{H}\right), 5.99(\mathrm{~d}, J=13.6 \mathrm{~Hz}, 1 \mathrm{H}), 5.15\left(\mathrm{dd}, J_{1}=\right.$ $\left.13.6 \mathrm{~Hz}, J_{2}=12.0 \mathrm{~Hz}, 1 \mathrm{H}\right), 3.87(\mathrm{~s}, 3 \mathrm{H}), 3.53(\mathrm{t}, J=7.6 \mathrm{~Hz}, 2 \mathrm{H}), 2.98(\mathrm{t}, J=7.6 \mathrm{~Hz}$, 2H), $2.40(\mathrm{~s}, 3 \mathrm{H}), 1.04(\mathrm{~d}, J=12.4 \mathrm{~Hz}, 1 \mathrm{H}), 0.02(\mathrm{~s}, 18 \mathrm{H}) .{ }^{13} \mathrm{C}$ NMR (100 MHz, $\left.\mathrm{CDCl}_{3}\right) \delta 156.9,147.8,143.5,135.7,134.4,132.7,132.1,131.7,129.7,129.6,129.4$, 127.0, 124.9, 124.8, 122.4, 119.8, 119.3, 114.6, 114.1, 102.2, 55.7, 46.3, 23.8, 22.1, 21.5, -0.4. IR (neat) $\mathrm{cm}^{-1} 2952,1543,1474,1378,1345,1247,1159,1119,1031,836$; HRMS (ESI-TOF) m/z: $[\mathrm{M}+\mathrm{Na}]^{+}$Calcd for $\mathrm{C}_{33} \mathrm{H}_{43} \mathrm{~N}_{3} \mathrm{O}_{7} \mathrm{~S}_{2} \mathrm{Si}_{2} \mathrm{Na}^{+}: 736.1973$, found 
736.1974.

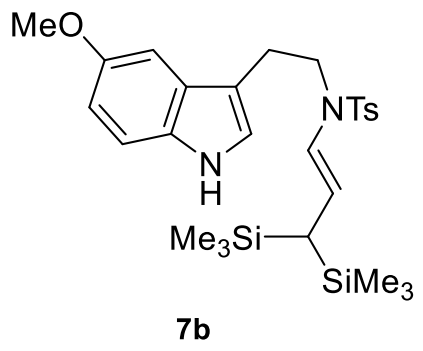

7b: The title compound (starting from $0.87 \mathrm{mmol} \mathrm{S31b}$ ) was isolated by silica gel column chromatography (gradient eluent: petroleum ether/EtOAc $=50: 1 \rightarrow 15: 1)$ to afford the desired product $\mathbf{7 b}$ as a light yellow solid, 403 mg, $88 \%$ yield. m. p. $=80.7-83.0{ }^{\circ} \mathrm{C} .{ }^{1} \mathrm{H}$ NMR $(400 \mathrm{MHz}$, $\left.\mathrm{CDCl}_{3}\right) \delta 8.08(\mathrm{~s}, 1 \mathrm{H}), 7.67(\mathrm{~d}, J=8.0 \mathrm{~Hz}, 2 \mathrm{H}), 7.26-7.24(\mathrm{~m}, 3 \mathrm{H}), 7.08(\mathrm{~d}, J=2.4 \mathrm{~Hz}$, $1 \mathrm{H}), 7.02(\mathrm{~d}, J=2.4 \mathrm{~Hz}, 1 \mathrm{H}), 6.88\left(\mathrm{dd}, J_{1}=8.8 \mathrm{~Hz}, J_{2}=2.4 \mathrm{~Hz}, 1 \mathrm{H}\right), 6.06(\mathrm{~d}, J=13.6$ $\mathrm{Hz}, 1 \mathrm{H}), 5.21\left(\mathrm{dd}, J_{1}=13.6 \mathrm{~Hz}, J_{2}=12.4 \mathrm{~Hz}, 1 \mathrm{H}\right), 3.89(\mathrm{~s}, 3 \mathrm{H}), 3.54(\mathrm{t}, J=7.6 \mathrm{~Hz}$, 2H), $3.54(\mathrm{t}, J=7.6 \mathrm{~Hz}, 2 \mathrm{H}), 2.40(\mathrm{~s}, 3 \mathrm{H}), 1.06(\mathrm{~d}, J=12.4 \mathrm{~Hz}, 1 \mathrm{H}), 0.05(\mathrm{~s}, 18 \mathrm{H})$. ${ }^{13} \mathrm{C}$ NMR $\left(100 \mathrm{MHz}, \mathrm{CDCl}_{3}\right) \delta 153.9,143.2,135.9,131.3,129.5,127.6,126.9,122.8$, $122.7,118.9,112.3,112.1,112.0,100.4,55.9,47.6,24.2,22.0,21.4,-0.5$. IR (neat) $\mathrm{cm}^{-1} 3385,2954,1586,1486,1439,1337,1247,1162,1080,944,862$; HRMS (ESI-TOF) m/z: [M + Na $]^{+}$Calcd for $\mathrm{C}_{27} \mathrm{H}_{40} \mathrm{~N}_{2} \mathrm{O}_{3} \mathrm{SSi}_{2} \mathrm{Na}^{+}:$551.2190, found 551.2190.

\section{Preparation of $7 c$}

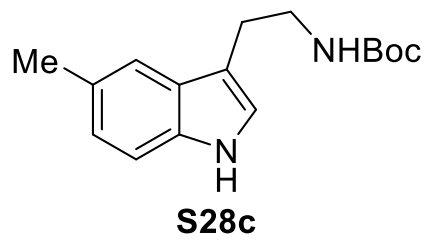

S28c: The title compound (starting from $87.38 \mathrm{mmol}$ 5-methyltryptamine hydrochloride) was recrystallized through $\mathrm{CH}_{2} \mathrm{Cl}_{2}$ and $n$-hexane to obtain the desired product S28c as a white solid, $22.77 \mathrm{~g}, 95 \%$ yield. ${ }^{1} \mathrm{H}$ NMR (400 MHz, $\left.\mathrm{CDCl}_{3}\right) \delta 8.17(\mathrm{~s}, 1 \mathrm{H}), 7.40(\mathrm{~s}, 1 \mathrm{H}), 7.27(\mathrm{~d}, J=8.0 \mathrm{~Hz}, 1 \mathrm{H}), 7.06(\mathrm{~d}$, $J=8.0 \mathrm{~Hz}, 1 \mathrm{H}), 6.97(\mathrm{~s}, 1 \mathrm{H}), 4.68(\mathrm{~s}, 1 \mathrm{H}), 3.47(\mathrm{q}, J=6.8 \mathrm{~Hz}, 2 \mathrm{H}), 2.94(\mathrm{t}, J=6.8$ $\mathrm{Hz}, 2 \mathrm{H}), 2.48$ (s, 3H), 1.47 (s, 9H).

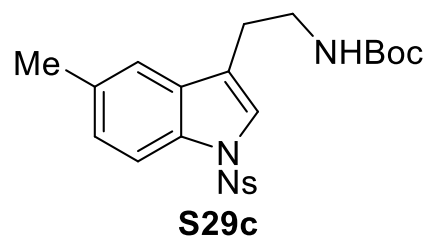

S29c: The title compound (starting from $25.12 \mathrm{mmol} \mathrm{S28c}$ ) was isolated by silica gel column chromatography (eluent: $\mathrm{CH}_{2} \mathrm{Cl}_{2}$ ) to afford the desired product $\mathbf{S 2 9 c}$ as a dark brown solid, $5.54 \mathrm{~g}, 48 \%$ yield. m. p. $=78.1-80.2{ }^{\circ} \mathrm{C} .{ }^{1} \mathrm{H}$ NMR (400 MHz, $\left.\mathrm{CDCl}_{3}\right) \delta 7.76(\mathrm{~d}, J=7.6 \mathrm{~Hz}, 1 \mathrm{H}), 7.71-7.67(\mathrm{~m}, 3 \mathrm{H}), 7.64-7.58(\mathrm{~m}$, $1 \mathrm{H}), 7.36(\mathrm{~d}, J=8.0 \mathrm{~Hz}, 2 \mathrm{H}), 7.14(\mathrm{~d}, J=8.4 \mathrm{~Hz}, 1 \mathrm{H}), 4.76(\mathrm{~s}, 1 \mathrm{H}), 3.46(\mathrm{q}, J=6.8$ 
$\mathrm{Hz}, 2 \mathrm{H}), 2.89$ (t, $J=6.8 \mathrm{~Hz}, 2 \mathrm{H}), 2.43$ (s, 3H), 1.43 (s, 9H). ${ }^{13} \mathrm{C}$ NMR (100 MHz, $\left.\mathrm{CDCl}_{3}\right) \delta 155.8,147.8,134.6,133.5,133.2,132.3,131.9,131.0,129.7,126.4,124.7$, 124.3, 119.7, 113.2, 79.3, 40.0, 28.3, 25.5, 21.3. IR (neat) $\mathrm{cm}^{-1} 3425,2976,1693$, 1542, 1511, 1364, 1271, 1172, 1121, 979, 851; HRMS (ESI-TOF) m/z: $[\mathrm{M}+\mathrm{Na}]^{+}$ Calcd for $\mathrm{C}_{22} \mathrm{H}_{25} \mathrm{~N}_{3} \mathrm{O}_{6} \mathrm{SNa}^{+}$: 482.1356, found 482.1359 .

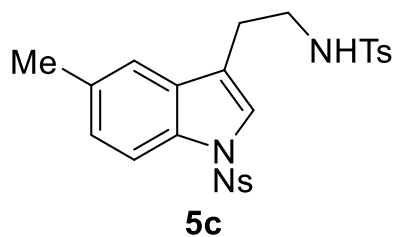

5c: The title compound (starting from $10 \mathrm{mmol} \mathrm{S29c)}$ was isolated by silica gel column chromatography (gradient eluent: petroleum ether/EtOAc $=20: 1 \rightarrow 2: 1)$ to afford the desired product $\mathbf{5 c}$ as a white solid, $4.10 \mathrm{~g}, 80 \%$ yield. m. p. $=168.9-172.3{ }^{\circ} \mathrm{C} .{ }^{1} \mathrm{H}$ NMR $(400 \mathrm{MHz}, \mathrm{DMSO}) \delta 8.09(\mathrm{~d}, J=8.0 \mathrm{~Hz}, 1 \mathrm{H}), 7.91(\mathrm{t}, J=$ $7.6 \mathrm{~Hz}, 1 \mathrm{H}), 7.77(\mathrm{t}, J=7.6 \mathrm{~Hz}, 1 \mathrm{H}), 7.73(\mathrm{t}, J=5.6 \mathrm{~Hz}, 1 \mathrm{H}), 7.69(\mathrm{~d}, J=8.4 \mathrm{~Hz}, 1 \mathrm{H})$, $7.65(\mathrm{~d}, J=8.0 \mathrm{~Hz}, 2 \mathrm{H}), 7.60(\mathrm{~d}, J=8.4 \mathrm{~Hz}, 1 \mathrm{H}), 7.48(\mathrm{~s}, 1 \mathrm{H}), 7.32(\mathrm{~s}, 1 \mathrm{H}), 7.30(\mathrm{~d}, J$ $=8.4 \mathrm{~Hz}, 2 \mathrm{H}), 7.16(\mathrm{~d}, J=8.4 \mathrm{~Hz}, 1 \mathrm{H}), 3.11(\mathrm{q}, J=6.8 \mathrm{~Hz}, 2 \mathrm{H}), 2.80(\mathrm{t}, J=6.8 \mathrm{~Hz}$, 2H), 2.35 (s, 3H), 2.34 (s, 3H). ${ }^{13} \mathrm{C}$ NMR (100 MHz, DMSO) $\delta$ 147.2, 142.6, 137.6, $136.1,133.3,133.2,132.7,130.6,130.0,129.6,129.0,126.5,126.4,125.2,124.7$, 119.7, 119.6, 112.9, 41.9, 24.6, 21.0, 20.9. IR (neat) $\mathrm{cm}^{-1} 3270,2922,1543,1468$, 1374, 1295, 1175, 1153, 998, 852; HRMS (ESI-TOF) m/z: $[\mathrm{M}+\mathrm{Na}]^{+}$Calcd for $\mathrm{C}_{24} \mathrm{H}_{23} \mathrm{~N}_{3} \mathrm{O}_{6} \mathrm{~S}_{2} \mathrm{Na}^{+}$: 536.0920, found 536.0917.
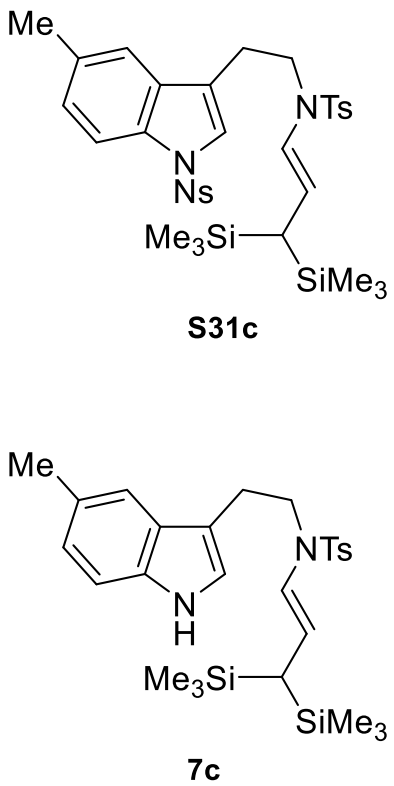

S31c: After the reaction was completed, the crude product was purified by silica gel column chromatography (gradient eluent: petroleum ether/EtOAc $=20: 1 \rightarrow 5: 1)$ to obtain 360 mg of an inseparable mixture. The inseparable mixture was used in the next step.

7c: The title compound (starting from $0.52 \mathrm{mmol} \mathrm{S31c)}$ was isolated by silica gel column chromatography (gradient eluent: petroleum ether/EtOAc $=50: 1 \rightarrow 15: 1)$ to afford the desired product 7c as a light yellow solid, $169 \mathrm{mg}, 64 \%$ yield. m. p. $=79.6-81.5^{\circ} \mathrm{C} .{ }^{1} \mathrm{H}$ NMR $\left(600 \mathrm{MHz}, \mathrm{CDCl}_{3}\right) \delta$ $7.96(\mathrm{~s}, 1 \mathrm{H}), 7.67(\mathrm{~d}, J=8.4 \mathrm{~Hz}, 2 \mathrm{H}), 7.37(\mathrm{~s}, 1 \mathrm{H}), 7.28-7.25(\mathrm{~m}, 3 \mathrm{H}), 7.04(\mathrm{~d}, J=8.4$ 
$\mathrm{Hz}, 1 \mathrm{H}), 7.03(\mathrm{~s}, 1 \mathrm{H}), 6.13(\mathrm{~d}, J=13.8 \mathrm{~Hz}, 1 \mathrm{H}), 5.19$ (dd, $J_{1}=13.8 \mathrm{~Hz}, J_{2}=12.0 \mathrm{~Hz}$, 1H), $3.52(\mathrm{t}, J=7.8 \mathrm{~Hz}, 2 \mathrm{H}), 3.05(\mathrm{t}, J=7.8 \mathrm{~Hz}, 2 \mathrm{H}), 2.47(\mathrm{~s}, 3 \mathrm{H}), 2.40$ (s, 3H), 1.06 $(\mathrm{d}, J=12.6 \mathrm{~Hz}, 1 \mathrm{H}), 0.07(\mathrm{~s}, 18 \mathrm{H}) .{ }^{13} \mathrm{C} \mathrm{NMR}\left(150 \mathrm{MHz}, \mathrm{CDCl}_{3}\right) \delta$ 143.2, 136.1, $134.5,129.5,128.7,127.5,127.0,123.7,123.0,122.3,118.2,117.6,112.1,110.9$, 47.7, 24.0, 22.0, 21.4, -0.4. IR (neat) $\mathrm{cm}^{-1} 3389,2953,1598,1434,1337,1247,1160$, 1092, 1036, 947, 861; HRMS (ESI-TOF) m/z: $\left[\mathrm{M}+\mathrm{Na}^{+}\right.$Calcd for $\mathrm{C}_{27} \mathrm{H}_{40} \mathrm{~N}_{2} \mathrm{O}_{2} \mathrm{SSi}_{2} \mathrm{Na}^{+}$: 535.2241, found 535.2249.

\section{Preparation of $7 d$}<smiles>CC(C)(C)NCCc1c[nH]c2ccc(Cl)cc12</smiles>

S28d

S28d: The title compound (starting from $87.38 \mathrm{mmol}$ 2-(5-chloro-1H-indol-3-yl)ethanamine hydrochloride) was recrystallized through $\mathrm{CH}_{2} \mathrm{Cl}_{2}$ and $n$-hexane to obtain the desired product S28d as a white solid, $23.44 \mathrm{~g}, 91 \%$ yield.

${ }^{1} \mathrm{H}$ NMR $\left(400 \mathrm{MHz}, \mathrm{CDCl}_{3}\right) \delta 8.45(\mathrm{~s}, 1 \mathrm{H}), 7.55(\mathrm{~s}, 1 \mathrm{H}), 7.27(\mathrm{~d}, J=8.4 \mathrm{~Hz}, 1 \mathrm{H})$, $7.45\left(\mathrm{dd}, J_{1}=8.8 \mathrm{~Hz}, J_{2}=2.0 \mathrm{~Hz}, 1 \mathrm{H}\right), 7.00(\mathrm{~s}, 1 \mathrm{H}), 4.67(\mathrm{~s}, 1 \mathrm{H}), 3.43(\mathrm{q}, J=6.8 \mathrm{~Hz}$, 2H), $2.89(\mathrm{t}, J=6.8 \mathrm{~Hz}, 2 \mathrm{H}), 1.46(\mathrm{~s}, 9 \mathrm{H})$.

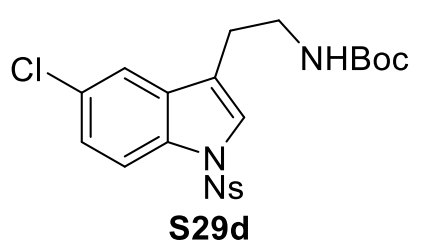

$\left.\mathrm{CDCl}_{3}\right) \delta 7.78(\mathrm{~d}, J=8.0 \mathrm{~Hz}, 1 \mathrm{H}), 7.73-7.70(\mathrm{~m}, 3 \mathrm{H}), 7.66-7.62(\mathrm{~m}, 1 \mathrm{H}), 7.51(\mathrm{~s}, 1 \mathrm{H})$, $7.41(\mathrm{~s}, 1 \mathrm{H}), 7.27(\mathrm{~d}, J=8.0 \mathrm{~Hz}, 1 \mathrm{H}), 4.75(\mathrm{~s}, 1 \mathrm{H}), 3.43(\mathrm{q}, J=6.8 \mathrm{~Hz}, 2 \mathrm{H}), 2.86(\mathrm{t}, J$ $=6.8 \mathrm{~Hz}, 2 \mathrm{H}), 1.41(\mathrm{~s}, 9 \mathrm{H}) .{ }^{13} \mathrm{C} \mathrm{NMR}\left(100 \mathrm{MHz}, \mathrm{CDCl}_{3}\right) \delta 155.8,147.8,135.0,133.3$, 132.5, 132.2, 131.6, 129.8, 129.7, 125.6, 125.3, 125.0, 122.3, 119.6, 114.5, 79.4, 40.1, 28.3, 25.5. IR (neat) $\mathrm{cm}^{-1} 3440,2977,1689,1542,1442,1364,1249,1174,1121,976$, 851; HRMS (ESI-TOF) m/z: $[\mathrm{M}+\mathrm{Na}]^{+}$Calcd for $\mathrm{C}_{21} \mathrm{H}_{22} \mathrm{ClN}_{3} \mathrm{O}_{6} \mathrm{SNa}^{+}$: 502.0810 , found 502.0815 .<smiles>[13CH3]n1cc(CC[SnH3])c2cc(Cl)ccc21</smiles>

5d: The title compound (starting from $10 \mathrm{mmol}$ S29d) was isolated by silica gel column chromatography (gradient

S29d: The title compound (starting from $25.12 \mathrm{mmol}$ S28d) was isolated by silica gel column chromatography (eluent: $\mathrm{CH}_{2} \mathrm{Cl}_{2}$ ) to afford the desired product $\mathbf{S 2 9 d}$ as a yellow viscous semi-solid, $6.38 \mathrm{~g}$, 53\% yield. ${ }^{1} \mathrm{H}$ NMR (400 MHz, 
eluent: petroleum ether/EtOAc $=20: 1 \rightarrow 2: 1)$ to afford the desired product $\mathbf{5 d}$ as a white solid, 3.89 g, $73 \%$ yield. m. p. = 156.7-162.1 ${ }^{\circ} \mathrm{C} .{ }^{1} \mathrm{H}$ NMR (400 MHz, DMSO) $\delta$ $8.13(\mathrm{~d}, J=8.0 \mathrm{~Hz}, 1 \mathrm{H}), 7.95(\mathrm{t}, J=8.0 \mathrm{~Hz}, 1 \mathrm{H}), 7.84-7.76(\mathrm{~m}, 2 \mathrm{H}), 7.73-7.65(\mathrm{~m}$, 3H), 7.60 (s, 1H), 7.59 (d, $J=8.0 \mathrm{~Hz}, 2 \mathrm{H}), 7.38(\mathrm{~d}, J=8.8 \mathrm{~Hz}, 1 \mathrm{H}), 7.26(\mathrm{~d}, J=8.0$ $\mathrm{Hz}, 2 \mathrm{H}), 3.10(\mathrm{q}, J=6.4 \mathrm{~Hz}, 2 \mathrm{H}), 2.80(\mathrm{t}, J=6.8 \mathrm{~Hz}, 2 \mathrm{H}), 2.33(\mathrm{~s}, 3 \mathrm{H}) .{ }^{13} \mathrm{C} \mathrm{NMR}$ (100 MHz, DMSO) $\delta$ 147.2, 142.6, 137.6, 136.5, 133.6, 133.0, 131.9, 129.7, 129.6, $129.3,128.7,126.4,126.3,125.5,125.1,119.7,119.3,114.7,42.0,24.4,21.0$. IR (neat) $\mathrm{cm}^{-1} 3267,2923,1544,1443,1375,1292,1193,1149,1126,862$; HRMS (ESI-TOF) m/z: $[\mathrm{M}+\mathrm{Na}]^{+}$Calcd for $\mathrm{C}_{23} \mathrm{H}_{20} \mathrm{ClN}_{3} \mathrm{O}_{6} \mathrm{~S}_{2} \mathrm{Na}^{+}:$556.0374, found 556.0378 .

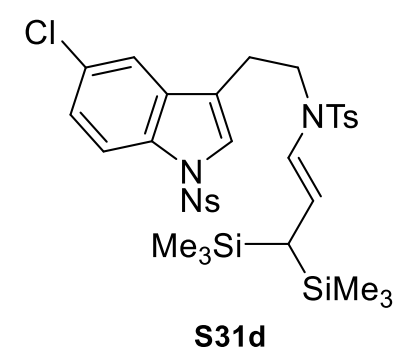

S31d: The title compound (starting from $2.81 \mathrm{mmol} 5 \mathbf{d}$ ) was isolated by silica gel column chromatography (gradient eluent: petroleum ether/EtOAc $=20: 1 \rightarrow 5: 1)$ to afford the desired product S31d as a light yellow solid, $474 \mathrm{mg}, 24 \%$ yield. m. p. $=56.8-58.2{ }^{\circ} \mathrm{C} .{ }^{1} \mathrm{H}$ NMR $\left(400 \mathrm{MHz}, \mathrm{CDCl}_{3}\right) \delta$ $7.77(\mathrm{~d}, J=8.8 \mathrm{~Hz}, 1 \mathrm{H}), 7.76\left(\mathrm{dd}, J_{1}=7.6 \mathrm{~Hz}, J_{2}=1.6 \mathrm{~Hz}, 1 \mathrm{H}\right), 7.72\left(\mathrm{dd}, J_{1}=6.8 \mathrm{~Hz}\right.$, $\left.J_{2}=2.0 \mathrm{~Hz}, 1 \mathrm{H}\right), 7.68-7.65(\mathrm{~m}, 1 \mathrm{H}), 7.64-7.60(\mathrm{~m}, 3 \mathrm{H}), 7.51(\mathrm{~d}, J=2.0 \mathrm{~Hz}, 1 \mathrm{H}), 7.46$ (s, 1H), 7.29-7.26 (m, 3H), $5.97(\mathrm{~d}, J=13.6 \mathrm{~Hz}, 1 \mathrm{H}), 5.16\left(\mathrm{dd}, J_{1}=14.0 \mathrm{~Hz}, J_{2}=12.4\right.$ $\mathrm{Hz}, 1 \mathrm{H}), 3.53(\mathrm{t}, J=7.2 \mathrm{~Hz}, 2 \mathrm{H}), 2.96(\mathrm{t}, J=7.2 \mathrm{~Hz}, 2 \mathrm{H}), 2.41(\mathrm{~s}, 3 \mathrm{H}), 1.05$ (d, $J=$ $12.4 \mathrm{~Hz}, 1 \mathrm{H}), 0.03(\mathrm{~s}, 18 \mathrm{H}) .{ }^{13} \mathrm{C} \mathrm{NMR}\left(100 \mathrm{MHz}, \mathrm{CDCl}_{3}\right) \delta \delta 147.7,143.5,135.6$, $134.7,133.5,133.0,131.9,129.8,129.7,129.5,127.0,125.8,125.4,124.9,122.3$, 120.1, 119.3, 118.7, 114.8, 46.3, 23.2, 22.2, 21.5, -0.4. IR (neat) $\mathrm{cm}^{-1} 2953,1544$, 1444, 1383, 1248, 1160, 1122, 1090, 966, 838; HRMS (ESI-TOF) m/z: $[\mathrm{M}+\mathrm{Na}]^{+}$ Calcd for $\mathrm{C}_{32} \mathrm{H}_{40} \mathrm{ClN}_{3} \mathrm{O}_{6} \mathrm{~S}_{2} \mathrm{Si}_{2} \mathrm{Na}^{+}:$740.1478, found 740.1483 .

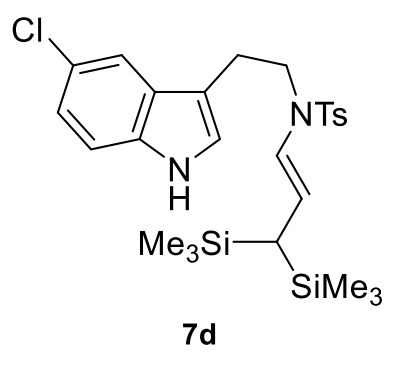

7d: The title compound (starting from 0.65 mmol S31d) was isolated by silica gel column chromatography (gradient eluent: petroleum ether/EtOAc $=50: 1 \rightarrow 15: 1)$ to afford the desired product $\mathbf{7 d}$ as a light yellow solid, $309 \mathrm{mg}, 90 \%$ yield. m. p. $=118.9-122.1^{\circ} \mathrm{C} .{ }^{1} \mathrm{H}$ NMR $\left(400 \mathrm{MHz}, \mathrm{CDCl}_{3}\right) \delta$ 
$8.19(\mathrm{~s}, 1 \mathrm{H}), 7.65(\mathrm{~d}, J=8.0 \mathrm{~Hz}, 2 \mathrm{H}), 7.49(\mathrm{~s}, 1 \mathrm{H}), 7.27-7.24(\mathrm{~m}, 3 \mathrm{H}), 7.13(\mathrm{~d}, J=8.4$ Hz, 1H), 7.09 (s, 1H), 6.03 (d, $J=13.6 \mathrm{~Hz}, 1 \mathrm{H}), 5.16$ (t, $J=12.8 \mathrm{~Hz}, 1 \mathrm{H}), 3.51-3.48$ $(\mathrm{m}, 2 \mathrm{H}), 3.02-2.98(\mathrm{~m}, 2 \mathrm{H}), 2.39(\mathrm{~s}, 3 \mathrm{H}), 1.05(\mathrm{~d}, J=12.4 \mathrm{~Hz}, 1 \mathrm{H}), 0.04(\mathrm{~s}, 18 \mathrm{H}) .{ }^{13} \mathrm{C}$ NMR $\left(150 \mathrm{MHz}, \mathrm{CDCl}_{3}\right) \delta 143.3,135.8,134.5,129.6,128.4,127.0,125.1,123.6$, $122.7,122.3,119.2,118.0,112.3,112.2,47.5,23.8,22.1,21.5,-0.4$. IR (neat) $\mathrm{cm}^{-1}$ 3365, 2953, 1598, 1456, 1347, 1248, 1159, 1072, 1031, 957, 856; HRMS (ESI-TOF) $\mathrm{m} / \mathrm{z}:[\mathrm{M}+\mathrm{Na}]^{+}$Calcd for $\mathrm{C}_{26} \mathrm{H}_{37} \mathrm{ClN}_{2} \mathrm{O}_{2} \mathrm{SSi}_{2} \mathrm{Na}^{+}$: 555.1695, found 555.1691. 


\subsection{Screening of racemic reaction conditions to form $2^{[a]}$.}

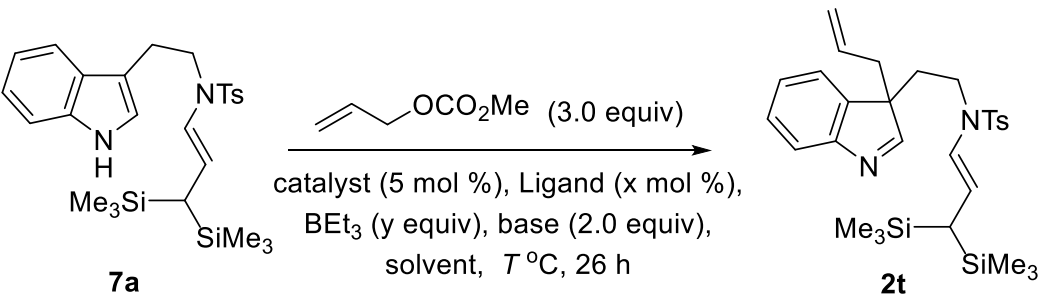

\begin{tabular}{|c|c|c|c|c|c|c|c|c|}
\hline entry & Catalyst & Ligand & $x$ & solvent & $\mathrm{y}$ & base & $T\left({ }^{\circ} \mathrm{C}\right)$ & yield $^{[b]}$ \\
\hline 1 & {$[\operatorname{lr}(\operatorname{cod}) \mathrm{Cl}]_{2}$} & - & - & THF & - & $\mathrm{Cs}_{2} \mathrm{CO}_{3}$ & 50 & NR \\
\hline 2 & {$[\operatorname{lr}(\operatorname{cod}) \mathrm{Cl}]_{2}$} & $\mathrm{PPh}_{3}$ & $10 \%$ & THF & - & $\mathrm{Cs}_{2} \mathrm{CO}_{3}$ & 50 & NR \\
\hline 3 & {$[\operatorname{lr}(\operatorname{cod}) \mathrm{Cl}]_{2}$} & $(R)$-MonoPhos & $10 \%$ & THF & - & $\mathrm{Cs}_{2} \mathrm{CO}_{3}$ & 50 & NR \\
\hline 4 & {$[\operatorname{lr}(\operatorname{cod}) \mathrm{Cl}]_{2}$} & $(R)$-MonoPhos & $10 \%$ & $\mathrm{CH}_{2} \mathrm{Cl}_{2}$ & - & $\mathrm{Cs}_{2} \mathrm{CO}_{3}$ & 50 & NR \\
\hline 5 & {$[\operatorname{lr}(\operatorname{cod}) \mathrm{Cl}]_{2}$} & $(R)$-MonoPhos & $10 \%$ & 1,4-dioxane & - & $\mathrm{Cs}_{2} \mathrm{CO}_{3}$ & 50 & NR \\
\hline 6 & $\mathrm{Pd}_{2}(\mathrm{dba})_{3}$ & $\mathrm{P}(2 \text {-furyl })_{3}$ & $10 \%$ & $\mathrm{CH}_{2} \mathrm{Cl}_{2}$ & - & - & $\mathrm{rt}$ & $18 \%$ \\
\hline 7 & $\mathrm{Pd}_{2}(\mathrm{dba})_{3}$ & $P(2-\text { furyl })_{3}$ & $10 \%$ & $\mathrm{CH}_{2} \mathrm{Cl}_{2}$ & 1.0 & - & $\mathrm{rt}$ & $61 \%$ \\
\hline 8 & $\mathrm{Pd}_{2}(\mathrm{dba})_{3}$ & $\mathrm{PPh}_{3}$ & $10 \%$ & $\mathrm{CH}_{2} \mathrm{Cl}_{2}$ & 1.0 & - & $\mathrm{rt}$ & $79 \%$ \\
\hline 9 & $\mathrm{Pd}_{2}(\mathrm{dba})_{3}\left(\mathrm{CHCl}_{3}\right)$ & $\mathrm{PPh}_{3}$ & $10 \%$ & $\mathrm{CH}_{2} \mathrm{Cl}_{2}$ & 1.0 & - & $\mathrm{rt}$ & $72 \%$ \\
\hline 10 & $\mathrm{Pd}\left(\mathrm{PPh}_{3}\right)_{4}$ & - & - & $\mathrm{CH}_{2} \mathrm{Cl}_{2}$ & 1.0 & - & rt & $85 \%$ \\
\hline 11 & $\mathrm{Pd}(\mathrm{dba})_{2}$ & $\mathrm{PPh}_{3}$ & $10 \%$ & $\mathrm{CH}_{2} \mathrm{Cl}_{2}$ & 1.0 & - & $\mathrm{rt}$ & $84 \%$ \\
\hline 12 & $\mathrm{Pd}(\mathrm{acac})_{2}$ & $\mathrm{PPh}_{3}$ & $10 \%$ & $\mathrm{CH}_{2} \mathrm{Cl}_{2}$ & 1.0 & - & $\mathrm{rt}$ & $13 \%$ \\
\hline 13 & $\mathrm{Pd}(\mathrm{OAc})_{2}$ & $\mathrm{PPh}_{3}$ & $10 \%$ & $\mathrm{CH}_{2} \mathrm{Cl}_{2}$ & 1.0 & - & $\mathrm{rt}$ & NR \\
\hline 14 & $\mathrm{Pd}\left(\mathrm{CF}_{3} \mathrm{COO}\right)_{2}$ & $\mathrm{PPh}_{3}$ & $10 \%$ & $\mathrm{CH}_{2} \mathrm{Cl}_{2}$ & 1.0 & - & $\mathrm{rt}$ & NR \\
\hline 15 & $\mathrm{Pd}\left(\mathrm{PPh}_{3}\right)_{2} \mathrm{Cl}_{2}$ & $\mathrm{PPh}_{3}$ & $10 \%$ & $\mathrm{CH}_{2} \mathrm{Cl}_{2}$ & 1.0 & - & $\mathrm{rt}$ & NR \\
\hline 16 & $\mathrm{Pd}(\mathrm{dppf}) \mathrm{Cl}_{2}$ & $\mathrm{PPh}_{3}$ & $10 \%$ & $\mathrm{CH}_{2} \mathrm{Cl}_{2}$ & 1.0 & - & $\mathrm{rt}$ & $12 \%$ \\
\hline 17 & {$\left[\mathrm{PdCl}\left(\mathrm{C}_{3} \mathrm{H}_{5}\right)\right]_{2}$} & $\mathrm{PPh}_{3}$ & $10 \%$ & $\mathrm{CH}_{2} \mathrm{Cl}_{2}$ & 1.0 & - & $\mathrm{rt}$ & NR \\
\hline 18 & $\mathrm{Pd}\left(\mathrm{t}-\mathrm{Bu}_{3} \mathrm{P}\right)_{2}$ & - & - & $\mathrm{CH}_{2} \mathrm{Cl}_{2}$ & 1.0 & - & $\mathrm{rt}$ & NR \\
\hline 19 & $\mathrm{Pd}\left(\mathrm{PPh}_{3}\right)_{4}$ & - & - & $\mathrm{Et}_{2} \mathrm{O}$ & 1.0 & - & $\mathrm{rt}$ & $43 \%$ \\
\hline 20 & $\mathrm{Pd}\left(\mathrm{PPh}_{3}\right)_{4}$ & - & - & DCE & 1.0 & - & $\mathrm{rt}$ & $48 \%$ \\
\hline
\end{tabular}

${ }^{a}$ Reaction conditions: 7a $(0.1 \mathrm{mmol})$, allylic methyl carbonate $(0.3 \mathrm{mmol})$, catalyst $(5 \mathrm{~mol} \%), \mathrm{rt}, 26 \mathrm{~h}$. ${ }^{b}$ Isolated yields.

\subsection{Preparation of indolenine 2a-2ad.}

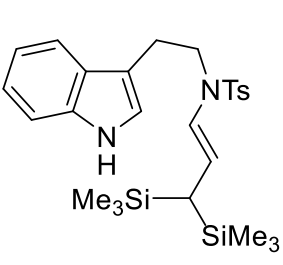

$7 a$

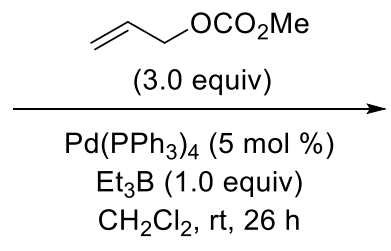

$85 \%$

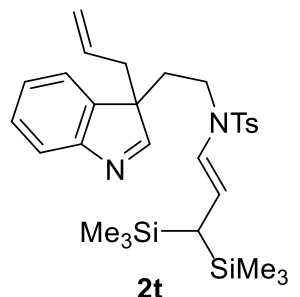

$2 \mathrm{t}$ 
To a solution of 7a $(50 \mathrm{mg}, 0.1 \mathrm{mmol})$ and $\mathrm{Pd}\left(\mathrm{PPh}_{3}\right)_{4}(5.8 \mathrm{mg}, 5 \mathrm{~mol} \%)$ in $\mathrm{CH}_{2} \mathrm{Cl}_{2}$ $(2.0 \mathrm{~mL})$ at room temperature under argon was added dropwise $\mathrm{Et}_{3} \mathrm{~B}(0.1 \mathrm{~mL}, 0.1$ $\mathrm{mmol}, 1.0 \mathrm{~mol} / \mathrm{L}$ in hexane) via syringe. The reaction mixture was stirred for $5 \mathrm{~min}$ before adding allylic methyl carbonate $(34 \mu \mathrm{L}, 0.3 \mathrm{mmol})$. The reaction mixture was stirred at room temperature for $26 \mathrm{~h}$. The reaction was concentrated by rotary evaporation. The crude products were purified by silica gel column chromatography (gradient eluent: petroleum ether/EtOAc $=50: 1 \rightarrow 10: 1)$ to afford the desired product $\mathbf{2 t}$ as a light yellow oil, $46 \mathrm{mg}, 85 \%$ yield.

To a solution of 7a $(500 \mathrm{mg}, 1 \mathrm{mmol})$ and $\mathrm{Pd}\left(\mathrm{PPh}_{3}\right)_{4}(58 \mathrm{mg}, 5 \mathrm{~mol} \%)$ in $\mathrm{CH}_{2} \mathrm{Cl}_{2}(20$ $\mathrm{mL})$ at room temperature under argon was added dropwise $\mathrm{Et}_{3} \mathrm{~B}(1 \mathrm{~mL}, 1 \mathrm{mmol}, 1.0$ $\mathrm{mol} / \mathrm{L}$ in hexane) via syringe. The reaction mixture was stirred for $5 \mathrm{~min}$ before adding allylic methyl carbonate $(0.34 \mathrm{~mL}, 3 \mathrm{mmol})$. The reaction mixture was stirred at room temperature for $26 \mathrm{~h}$. The reaction was concentrated by rotary evaporation. The crude products were purified by silica gel column chromatography (gradient eluent: petroleum ether/EtOAc $=50: 1 \rightarrow 10: 1)$ to afford the desired product $2 \mathbf{t}$ as a light yellow oil, $420 \mathrm{mg}$, $78 \%$ yield.

${ }^{1} \mathrm{H}$ NMR (400 MHz, $\left.\mathrm{CDCl}_{3}\right) \delta 8.06(\mathrm{~s}, 1 \mathrm{H}), 7.63(\mathrm{~d}, J=7.6 \mathrm{~Hz}, 1 \mathrm{H}), 7.47$ (d, $J=8.0$ $\mathrm{Hz}, 2 \mathrm{H}), 7.35$ (t, $J=7.6 \mathrm{~Hz}, 1 \mathrm{H}), 7.31-7.27$ (m, 2H), 7.19 (d, $J=8.0 \mathrm{~Hz}, 2 \mathrm{H}), 5.93$ (d, $J=13.6 \mathrm{~Hz}, 1 \mathrm{H}), 5.51-5.41(\mathrm{~m}, 1 \mathrm{H}), 5.04-5.00(\mathrm{~m}, 2 \mathrm{H}), 4.68\left(\mathrm{dd}, J_{1}=13.6 \mathrm{~Hz}, J_{2}=\right.$ $12.0 \mathrm{~Hz}, 1 \mathrm{H}), 2.83\left(\mathrm{ddd}, J_{1}=14.0 \mathrm{~Hz}, J_{2}=11.2 \mathrm{~Hz}, J_{3}=5.6 \mathrm{~Hz}, 1 \mathrm{H}\right), 2.57$ (ddd, $J_{1}=$ $\left.14.0 \mathrm{~Hz}, J_{2}=10.8 \mathrm{~Hz}, J_{3}=5.2 \mathrm{~Hz}, 1 \mathrm{H}\right), 2.50(\mathrm{~d}, J=7.2 \mathrm{~Hz}, 2 \mathrm{H}), 2.36(\mathrm{~s}, 3 \mathrm{H})$, 2.31-2.18 (m, 2H), $0.91(\mathrm{~d}, J=12.4 \mathrm{~Hz}, 1 \mathrm{H}), 0.01(\mathrm{~s}, 9 \mathrm{H}),-0.06(\mathrm{~s}, 9 \mathrm{H}),{ }^{13} \mathrm{C} \mathrm{NMR}$ $\left(100 \mathrm{MHz}, \mathrm{CDCl}_{3}\right) \delta 177.1,155.3,143.3,140.3,135.6,131.8,129.6,128.2,126.8$, $126.4,122.5,121.8,121.5,119.3,116.2,59.9,41.9,39.6,32.1,21.8,21.4,-0.4,-0.5$; IR (neat) $\mathrm{cm}^{-1} 2952,1597,1555,1455,1354,1250,1163,1091,1035,953,860,838$; HRMS (ESI-TOF) m/z: $[\mathrm{M}+\mathrm{H}]^{+}$Calcd for $\mathrm{C}_{29} \mathrm{H}_{43} \mathrm{~N}_{2} \mathrm{O}_{2} \mathrm{SSi}_{2}{ }^{+}: 539.2578$, found 539.2573 . 


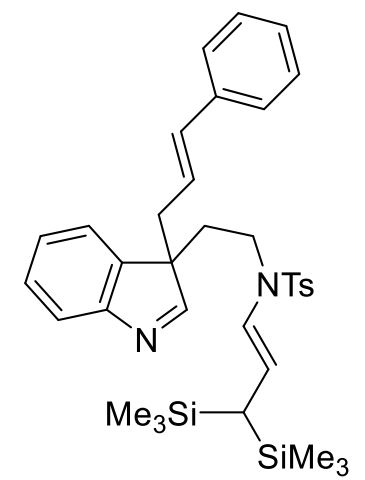

$2 a$

2a: The title compound were purified by silica gel column chromatography (gradient eluent: petroleum ether/EtOAc $=$ $50: 1 \rightarrow 10: 1)$ to afford the desired product $\mathbf{2 a}$ as a light yellow oil, $59 \mathrm{mg}, 95 \%$ yield. ${ }^{1} \mathrm{H}$ NMR (400 MHz, $\left.\mathrm{CDCl}_{3}\right) \delta 8.20$ (s, $1 \mathrm{H}), 7.72(\mathrm{~d}, J=7.6 \mathrm{~Hz}, 1 \mathrm{H}), 7.55(\mathrm{~d}, J=8.0 \mathrm{~Hz}, 2 \mathrm{H}), 7.46(\mathrm{~d}$, $J=7.2 \mathrm{~Hz}, 1 \mathrm{H}), 7.43(\mathrm{~d}, J=7.2 \mathrm{~Hz}, 1 \mathrm{H}), 7.38(\mathrm{~d}, J=7.6 \mathrm{~Hz}$, 1H), 7.34-7.28 (m, 5H), $7.26(\mathrm{~d}, J=8.0 \mathrm{~Hz}, 2 \mathrm{H}), 6.47(\mathrm{~d}, J=$ $15.6 \mathrm{~Hz}, 1 \mathrm{H}), 6.02(\mathrm{~d}, J=14.0 \mathrm{~Hz}, 1 \mathrm{H}), 5.98\left(\mathrm{dt}, J_{1}=15.6 \mathrm{~Hz}\right.$, $\left.J_{2}=7.6 \mathrm{~Hz}, 1 \mathrm{H}\right), 4.75\left(\mathrm{dd}, J_{1}=13.6 \mathrm{~Hz}, J_{2}=12.0 \mathrm{~Hz}, 1 \mathrm{H}\right), 2.94\left(\mathrm{ddd}, J_{1}=14.0 \mathrm{~Hz}, J_{2}\right.$ $\left.=11.2 \mathrm{~Hz}, J_{3}=5.6 \mathrm{~Hz}, 1 \mathrm{H}\right), 2.76-2.62(\mathrm{~m}, 3 \mathrm{H}), 2.43(\mathrm{~s}, 3 \mathrm{H}), 2.42-2.32(\mathrm{~m}, 2 \mathrm{H}), 0.98$ $(\mathrm{d}, J=12.0 \mathrm{~Hz}, 1 \mathrm{H}), 0.06(\mathrm{~s}, 9 \mathrm{H}),-0.01(\mathrm{~s}, 9 \mathrm{H}) ;{ }^{13} \mathrm{C} \mathrm{NMR}\left(100 \mathrm{MHz}, \mathrm{CDCl}_{3}\right) \delta 177.1$, $155.3,143.3,140.3,136.7,135.6,134.3,129.6,128.4,128.3,127.5,126.8,126.5$, $126.2,123.3,122.5,121.9,121.6,116.3,60.3,42.0,38.9,32.1,21.8,21.4,-0.4,-0.6$; IR (neat) $\mathrm{cm}^{-1} 2952,1597,1555,1495,1454,1352,1248,1161,1090,1033,965,859$, 836; HRMS (ESI-TOF) m/z: $[\mathrm{M}+\mathrm{Na}]^{+}$Calcd for $\mathrm{C}_{35} \mathrm{H}_{46} \mathrm{~N}_{2} \mathrm{O}_{2} \mathrm{SSi}_{2} \mathrm{Na}^{+}$: 637.2711, found 637.2712 .

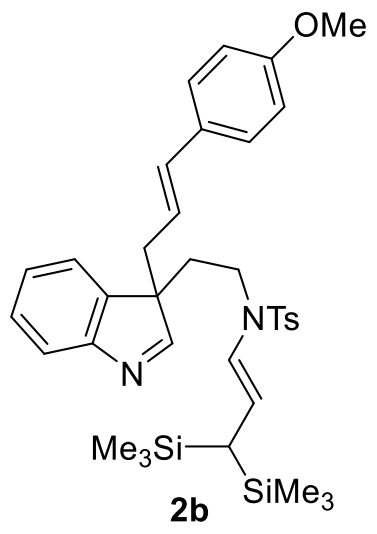

2b: The title compound were purified by silica gel column chromatography (gradient eluent: petroleum ether/EtOAc $=$ $50: 1 \rightarrow 10: 1)$ to afford the desired product $\mathbf{2 b}$ as a light yellow oil, $62 \mathrm{mg}$, 96\% yield. ${ }^{1} \mathrm{H} \mathrm{NMR}\left(400 \mathrm{MHz}, \mathrm{CDCl}_{3}\right) \delta 8.11(\mathrm{~s}$, 1H), $7.63(\mathrm{~d}, J=7.6 \mathrm{~Hz}, 1 \mathrm{H}), 7.47(\mathrm{~d}, J=8.4 \mathrm{~Hz}, 2 \mathrm{H}), 7.38$ $(\mathrm{d}, J=7.2 \mathrm{~Hz}, 1 \mathrm{H}), 7.34(\mathrm{~d}, J=7.6 \mathrm{~Hz}, 1 \mathrm{H}), 7.28(\mathrm{t}, J=7.2$ $\mathrm{Hz}, 1 \mathrm{H}), 7.18-7.15(\mathrm{~m}, 4 \mathrm{H}), 6.81(\mathrm{~d}, J=8.8 \mathrm{~Hz}, 2 \mathrm{H}), 6.33(\mathrm{~d}$, $J=15.6 \mathrm{~Hz}, 1 \mathrm{H}), 5.94(\mathrm{~d}, J=13.6 \mathrm{~Hz}, 1 \mathrm{H}), 5.77\left(\mathrm{dt}, J_{1}=15.6 \mathrm{~Hz}, J_{2}=7.6 \mathrm{~Hz}, 1 \mathrm{H}\right)$, $4.66\left(\mathrm{dd}, J_{1}=13.6 \mathrm{~Hz}, J_{2}=12.4 \mathrm{~Hz}, 1 \mathrm{H}\right), 3.79(\mathrm{~s}, 3 \mathrm{H}), 2.87\left(\mathrm{ddd}, J_{1}=14.0 \mathrm{~Hz}, J_{2}=\right.$ $\left.10.8 \mathrm{~Hz}, J_{3}=5.6 \mathrm{~Hz}, 1 \mathrm{H}\right), 2.64-2.51(\mathrm{~m}, 3 \mathrm{H}), 2.35(\mathrm{~s}, 3 \mathrm{H}), 2.33-2.23(\mathrm{~m}, 2 \mathrm{H}), 0.90(\mathrm{~d}$, $J=12.0 \mathrm{~Hz}, 1 \mathrm{H}),-0.02(\mathrm{~s}, 9 \mathrm{H}),-0.09(\mathrm{~s}, 9 \mathrm{H}) ;{ }^{13} \mathrm{C} \mathrm{NMR}\left(100 \mathrm{MHz}, \mathrm{CDCl}_{3}\right) \delta 177.3$, $159.1,155.3,143.3,140.4,135.7,133.7,129.6,129.6,128.3,127.4,126.8,126.4$, $122.5,121.9,121.6,121.0,116.2,113.9,60.3,55.3,42.0,38.9,32.1,21.8,21.4,-0.4$, 
-0.6; IR (neat) $\mathrm{cm}^{-1} 2953,1607,1555,1510,1455,1351,1247,1161,1090,1034,966$, 859, 828; HRMS (ESI-TOF) m/z: $[\mathrm{M}+\mathrm{H}]^{+}$Calcd for $\mathrm{C}_{36} \mathrm{H}_{49} \mathrm{~N}_{2} \mathrm{O}_{3} \mathrm{SSi}_{2}{ }^{+}$: 645.2997, found 645.2999.

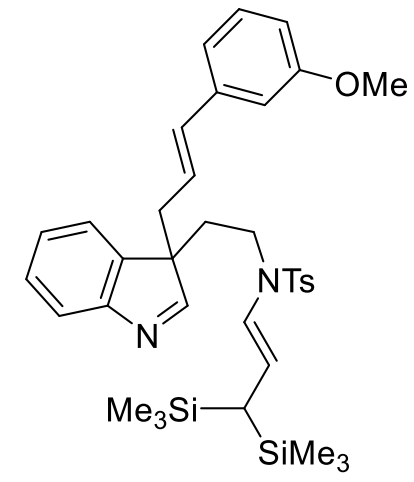

2c

2c: The title compound were purified by silica gel column chromatography (gradient eluent: petroleum ether/EtOAc $=$ $50: 1 \rightarrow 10: 1)$ to afford the desired product $\mathbf{2 c}$ as a light yellow oil, $55 \mathrm{mg}, 85 \%$ yield. ${ }^{1} \mathrm{H} \mathrm{NMR}\left(400 \mathrm{MHz}, \mathrm{CDCl}_{3}\right) \delta$ $8.11(\mathrm{~s}, 1 \mathrm{H}), 7.64(\mathrm{~d}, J=7.6 \mathrm{~Hz}, 1 \mathrm{H}), 7.47(\mathrm{~d}, J=8.4 \mathrm{~Hz}$, 2H), 7.38-7.28 (m, 3H), 7.20-7.16 (m, 3H), $6.83(\mathrm{~d}, J=7.6$ $\mathrm{Hz}, 1 \mathrm{H}), 6.77-6.75(\mathrm{~m}, 2 \mathrm{H}), 6.36(\mathrm{~d}, J=15.6 \mathrm{~Hz}, 1 \mathrm{H}), 5.93$ $(\mathrm{d}, J=13.6 \mathrm{~Hz}, 1 \mathrm{H}), 5.89\left(\mathrm{dt}, J_{1}=15.6 \mathrm{~Hz}, J_{2}=7.6 \mathrm{~Hz}, 1 \mathrm{H}\right)$, $4.67\left(\mathrm{dd}, J_{1}=13.6 \mathrm{~Hz}, J_{2}=12.4 \mathrm{~Hz}, 1 \mathrm{H}\right), 3.79(\mathrm{~s}, 3 \mathrm{H}), 2.87\left(\mathrm{ddd}, J_{1}=14.0 \mathrm{~Hz}, J_{2}=\right.$ $\left.10.8 \mathrm{~Hz}, J_{3}=5.2 \mathrm{~Hz}, 1 \mathrm{H}\right), 2.67-2.54(\mathrm{~m}, 3 \mathrm{H}), 2.35(\mathrm{~s}, 3 \mathrm{H}), 2.33-2.23(\mathrm{~m}, 2 \mathrm{H}), 0.90(\mathrm{~d}$, $J=12.4 \mathrm{~Hz}, 1 \mathrm{H}),-0.02(\mathrm{~s}, 9 \mathrm{H}),-0.09(\mathrm{~s}, 9 \mathrm{H}) ;{ }^{13} \mathrm{C} \mathrm{NMR}\left(100 \mathrm{MHz}, \mathrm{CDCl}_{3}\right) \delta 177.1$, $159.7,155.3,143.4,140.3,138.2,135.6,134.2$, 129.6, 129.4, 128.3, 126.8, 126.5, 123.6, 122.5, 121.9, 121.6, 118.9, 116.3, 113.0, 111.7, 60.2, 55.2, 42.0, 38.8, 32.2, 21.8, 21.4, -0.4, -0.6; IR (neat) $\mathrm{cm}^{-1} 2951,1597,1579,1555,1491,1352,1249,1161$, 1090, 1039, 959, 859, 837; HRMS (ESI-TOF) m/z: $[\mathrm{M}+\mathrm{H}]^{+}$Calcd for $\mathrm{C}_{36} \mathrm{H}_{49} \mathrm{~N}_{2} \mathrm{O}_{3} \mathrm{SSi}_{2}{ }^{+}:$645.2997, found 645.2996.

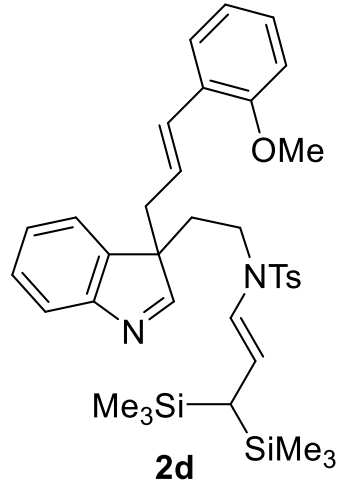

2d: The title compound were purified by silica gel column chromatography (gradient eluent: petroleum ether/EtOAc $=$ $50: 1 \rightarrow 10: 1)$ to afford the desired product $\mathbf{2 d}$ as a light yellow oil, $62 \mathrm{mg}$, 96\% yield. ${ }^{1} \mathrm{H}$ NMR (400 MHz, $\left.\mathrm{CDCl}_{3}\right) \delta 8.21$ (s, 1H), $7.72(\mathrm{~d}, J=7.6 \mathrm{~Hz}, 1 \mathrm{H}), 7.55(\mathrm{~d}, J=8.0 \mathrm{~Hz}, 2 \mathrm{H}), 7.44(\mathrm{t}$, $J=7.6 \mathrm{~Hz}, 2 \mathrm{H}), 7.36(\mathrm{t}, J=7.2 \mathrm{~Hz}, 1 \mathrm{H}), 7.30(\mathrm{~d}, J=7.6 \mathrm{~Hz}$, 1H), $7.26(\mathrm{t}, J=8.4 \mathrm{~Hz}, 3 \mathrm{H}), 7.00(\mathrm{~d}, J=7.2 \mathrm{~Hz}, 1 \mathrm{H}), 6.92(\mathrm{~d}$, $J=8.8 \mathrm{~Hz}, 1 \mathrm{H}), \quad 6.79(\mathrm{~d}, J=16.0 \mathrm{~Hz}, 1 \mathrm{H}), 6.02(\mathrm{~d}, J=13.6 \mathrm{~Hz}, 1 \mathrm{H}), 5.98\left(\mathrm{dt}, J_{1}=\right.$ $\left.16.0 \mathrm{~Hz}, J_{2}=7.6 \mathrm{~Hz}, 1 \mathrm{H}\right), 4.75\left(\mathrm{dd}, J_{1}=13.6 \mathrm{~Hz}, J_{2}=12.4 \mathrm{~Hz}, 1 \mathrm{H}\right), 3.88(\mathrm{~s}, 3 \mathrm{H}), 2.94$ $\left(\mathrm{ddd}, J_{1}=14.0 \mathrm{~Hz}, J_{2}=11.2 \mathrm{~Hz}, J_{3}=5.6 \mathrm{~Hz}, 1 \mathrm{H}\right), 2.77-2.61(\mathrm{~m}, 3 \mathrm{H}), 2.43(\mathrm{~s}, 3 \mathrm{H})$, 
2.41-2.33 (m, 2H), $0.98(\mathrm{~d}, J=12.0 \mathrm{~Hz}, 1 \mathrm{H}), 0.06(\mathrm{~s}, 9 \mathrm{H}),-0.01(\mathrm{~s}, 9 \mathrm{H}) ;{ }^{13} \mathrm{C} \mathrm{NMR}$ $\left(100 \mathrm{MHz}, \mathrm{CDCl}_{3}\right) \delta 177.3,156.5,155.3,143.3,140.5,135.8,129.6,129.1,128.5$, 128.2, 126.8, 126.4, 125.9, 124.0, 122.6, 122.0, 121.5, 120.5, 116.3, 110.8, 60.3, 55.4, 42.1, 39.3, 32.1, 21.8, 21.4, -0.4, -0.6; IR (neat) $\mathrm{cm}^{-1} 2951,1597,1554,1488,1463$, 1352, 1244, 1161, 1090, 1029, 974, 859, 836; HRMS (ESI-TOF) m/z: $[\mathrm{M}+\mathrm{H}]^{+} \mathrm{Calcd}$ for $\mathrm{C}_{36} \mathrm{H}_{49} \mathrm{~N}_{2} \mathrm{O}_{3} \mathrm{SSi}_{2}{ }^{+}$: 645.2997, found 645.2998.

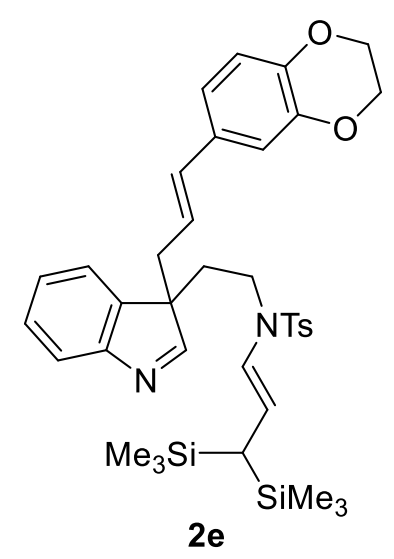

2e: The title compound were purified by silica gel column chromatography (gradient eluent: petroleum ether/EtOAc $=$ $50: 1 \rightarrow 10: 1)$ to afford the desired product $2 \mathbf{e}$ as a light yellow oil, $45 \mathrm{mg}, 66 \%$ yield. ${ }^{1} \mathrm{H}$ NMR (400 MHz, $\mathrm{CDCl}_{3}$ ) $\delta 8.11$ (s, 1H), $7.64(\mathrm{~d}, J=7.6 \mathrm{~Hz}, 1 \mathrm{H}), 7.47(\mathrm{~d}, J=8.4 \mathrm{~Hz}, 2 \mathrm{H})$, 7.38-7.27 (m, 3H), $7.19(\mathrm{~d}, J=8.0 \mathrm{~Hz}, 2 \mathrm{H}), 6.77-6.71(\mathrm{~m}$, $3 \mathrm{H}), 6.26(\mathrm{~d}, J=15.6 \mathrm{~Hz}, 1 \mathrm{H}), 5.94(\mathrm{~d}, J=13.6 \mathrm{~Hz}, 1 \mathrm{H})$, $5.74\left(\mathrm{dt}, J_{1}=16.0 \mathrm{~Hz}, J_{2}=8.0 \mathrm{~Hz}, 1 \mathrm{H}\right), 4.67\left(\mathrm{dd}, J_{1}=13.2\right.$ $\left.\mathrm{Hz}, J_{2}=11.6 \mathrm{~Hz}, 1 \mathrm{H}\right), 4.24(\mathrm{~s}, 4 \mathrm{H}), 2.85\left(\mathrm{ddd}, J_{1}=14.0 \mathrm{~Hz}, J_{2}=10.8 \mathrm{~Hz}, J_{3}=5.6 \mathrm{~Hz}\right.$, $1 \mathrm{H}), 2.65-2.52(\mathrm{~m}, 3 \mathrm{H}), 2.36(\mathrm{~s}, 3 \mathrm{H}), 2.32-2.22(\mathrm{~m}, 2 \mathrm{H}), 0.91(\mathrm{~d}, J=12.0 \mathrm{~Hz}, 1 \mathrm{H})$, $-0.01(\mathrm{~s}, 9 \mathrm{H}),-0.08(\mathrm{~s}, 9 \mathrm{H}) ;{ }^{13} \mathrm{C} \mathrm{NMR}\left(100 \mathrm{MHz}, \mathrm{CDCl}_{3}\right) \delta 177.2,155.3,143.4,143.3$, 143.1 , 140.3, 135.7, 133.5, 130.6, 129.6, 128.3, 126.8, 126.4, 122.5, 121.9, 121.6, $119.7,117.1,116.3,114.7,64.4,64.3,60.3,42.0,38.8,32.1,21.8,21.4,-0.4,-0.5$; IR (neat) $\mathrm{cm}^{-1} 2951,1736,1582,1506,1456,1351,1248,1161,1090,1067,965,860$, 837; HRMS (ESI-TOF) m/z: [M + H] $]^{+}$Calcd for $\mathrm{C}_{37} \mathrm{H}_{49} \mathrm{~N}_{2} \mathrm{O}_{4} \mathrm{SSi}_{2}{ }^{+}$: 673.2946, found 673.2945 .

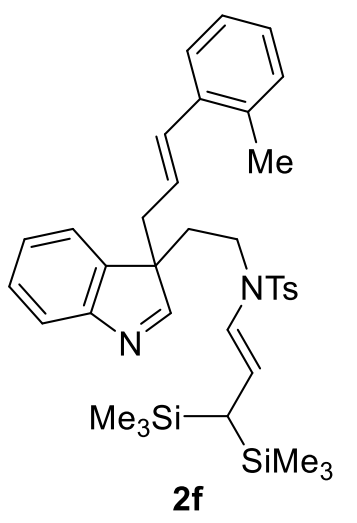

2f: The title compound were purified by silica gel column chromatography (gradient eluent: petroleum ether/EtOAc $=$ $50: 1 \rightarrow 10: 1)$ to afford the desired product $\mathbf{2 f}$ as a light yellow oil, $53 \mathrm{mg}, 83 \%$ yield. ${ }^{1} \mathrm{H}$ NMR (400 MHz, $\left.\mathrm{CDCl}_{3}\right) \delta 8.13$ (s, $1 \mathrm{H}), 7.63(\mathrm{~d}, J=7.6 \mathrm{~Hz}, 1 \mathrm{H}), 7.47(\mathrm{~d}, J=8.4 \mathrm{~Hz}, 2 \mathrm{H})$, 7.38-7.33 (m, 2H), 7.28 (t, $J=7.6 \mathrm{~Hz}, 1 \mathrm{H}), 7.19$ (d, $J=7.6 \mathrm{~Hz}$, 3H), 7.13-7.06 (m, 3H), $6.53(\mathrm{~d}, J=15.6 \mathrm{~Hz}, 1 \mathrm{H}), 5.94(\mathrm{~d}, J=$ 
$13.6 \mathrm{~Hz}, 1 \mathrm{H}), 5.72\left(\mathrm{dt}, J_{1}=15.2 \mathrm{~Hz}, J_{2}=7.6 \mathrm{~Hz}, 1 \mathrm{H}\right), 4.67\left(\mathrm{dd}, J_{1}=13.6 \mathrm{~Hz}, J_{2}=\right.$ $12.0 \mathrm{~Hz}, 1 \mathrm{H}), 2.87\left(\mathrm{ddd}, J_{1}=14.0 \mathrm{~Hz}, J_{2}=11.2 \mathrm{~Hz}, J_{3}=5.2 \mathrm{~Hz}, 1 \mathrm{H}\right), 2.73-2.51(\mathrm{~m}$, $3 \mathrm{H}), 2.35(\mathrm{~s}, 3 \mathrm{H}), 2.34-2.28(\mathrm{~m}, 2 \mathrm{H}), 2.20(\mathrm{~s}, 3 \mathrm{H}), 0.90(\mathrm{~d}, J=12.0 \mathrm{~Hz}, 1 \mathrm{H}),-0.02(\mathrm{~s}$, 9H), -0.09 (s, 9H); ${ }^{13} \mathrm{C}$ NMR (100 MHz, $\left.\mathrm{CDCl}_{3}\right) \delta 177.1,155.3,143.4,140.3,136.0$, 135.6, 135.1, 132.3, 130.1, 129.6, 128.3, 127.4, 126.8, 126.5, 126.0, 125.8, 124.6, $122.6,121.9,121.6,116.3,60.4,42.0,39.1,32.3,21.8,21.4,19.7,-0.4,-0.6$; IR (neat) $\mathrm{cm}^{-1} 2951,1597,1554,1455,1353,1248,1162,1090,1034,950,859,837$; HRMS (ESI-TOF) m/z: $[\mathrm{M}+\mathrm{H}]^{+}$Calcd for $\mathrm{C}_{36} \mathrm{H}_{49} \mathrm{~N}_{2} \mathrm{O}_{2} \mathrm{SSi}_{2}{ }^{+}$: 629.3048, found 629.3047.

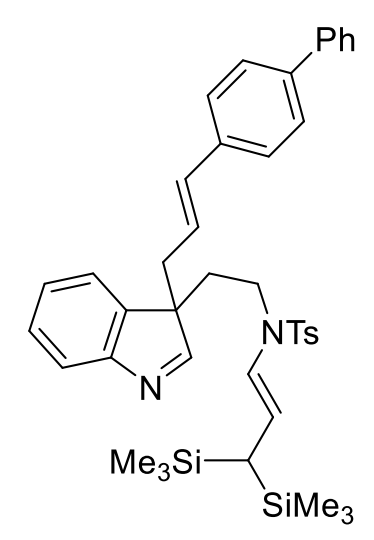

2g

2g: The title compound were purified by silica gel column chromatography (gradient eluent: petroleum ether/EtOAc $=$ $50: 1 \rightarrow 10: 1)$ to afford the desired product $\mathbf{2} \mathbf{g}$ as a light yellow oil, $50 \mathrm{mg}, 72 \%$ yield. ${ }^{1} \mathrm{H} \mathrm{NMR}\left(400 \mathrm{MHz}, \mathrm{CDCl}_{3}\right) \delta 8.14(\mathrm{~s}$, $1 \mathrm{H}), 7.65(\mathrm{~d}, J=7.2 \mathrm{~Hz}, 1 \mathrm{H}), 7.58(\mathrm{~d}, J=8.0 \mathrm{~Hz}, 2 \mathrm{H}), 7.52(\mathrm{~d}$, $J=8.0 \mathrm{~Hz}, 2 \mathrm{H}), 7.48(\mathrm{~d}, J=8.0 \mathrm{~Hz}, 2 \mathrm{H}), 7.43-7.34(\mathrm{~m}, 5 \mathrm{H})$, $7.31(\mathrm{~d}, J=7.6 \mathrm{~Hz}, 2 \mathrm{H}), 7.19(\mathrm{~d}, J=8.0 \mathrm{~Hz}, 2 \mathrm{H}), 6.43(\mathrm{~d}, J=$ $16.0 \mathrm{~Hz}, 1 \mathrm{H}), 5.95\left(\mathrm{dt}, J_{1}=16.0 \mathrm{~Hz}, J_{2}=7.2 \mathrm{~Hz}, 1 \mathrm{H}\right), 5.91(\mathrm{~d}, J$ $=14.0 \mathrm{~Hz}, 1 \mathrm{H}), 4.68\left(\mathrm{dd}, J_{1}=13.6 \mathrm{~Hz}, J_{2}=12.0 \mathrm{~Hz}, 1 \mathrm{H}\right), 2.86\left(\mathrm{ddd}, J_{1}=14.0 \mathrm{~Hz}, J_{2}\right.$ $\left.=10.8 \mathrm{~Hz}, J_{3}=5.6 \mathrm{~Hz}, 1 \mathrm{H}\right), 2.71-2.53(\mathrm{~m}, 3 \mathrm{H}), 2.39-2.27(\mathrm{~m}, 2 \mathrm{H}), 2.36(\mathrm{~s}, 3 \mathrm{H}), 0.90$ $(\mathrm{d}, J=12.0 \mathrm{~Hz}, 1 \mathrm{H}),-0.02(\mathrm{~s}, 9 \mathrm{H}),-0.09(\mathrm{~s}, 9 \mathrm{H}) ;{ }^{13} \mathrm{C} \mathrm{NMR}\left(100 \mathrm{MHz}, \mathrm{CDCl}_{3}\right) \delta$ 177.1, 155.3, 143.4, 140.7, 140.4, 140.3, 135.8, 133.8, 129.6, 128.8, 128.4, 127.3, 127.2, 126.9, 126.8, 126.7, 126.5, 123.4, 122.6, 121.9, 121.7, 116.4, 60.3, 42.1, 38.9, 32.2, 21.9, 21.4, -0.4, -0.5; IR (neat) $\mathrm{cm}^{-1} 2951,1736,1598,1554,1486,1454,1352$,

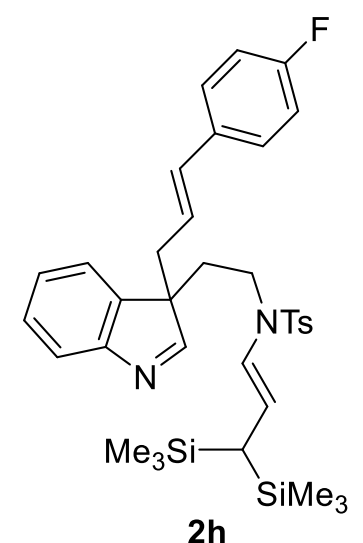

2h
1248, 1162, 1090, 1036, 948, 859, 836, 756; HRMS (ESI-TOF) $\mathrm{m} / \mathrm{z}:[\mathrm{M}+\mathrm{H}]^{+}$Calcd for $\mathrm{C}_{41} \mathrm{H}_{51} \mathrm{~N}_{2} \mathrm{O}_{2} \mathrm{SSi}_{2}{ }^{+}:$691.3204, found 691.3209.

2h: The title compound were purified by silica gel column chromatography (gradient eluent: petroleum ether/EtOAc $=$ $50: 1 \rightarrow 10: 1)$ to afford the desired product $\mathbf{2 h}$ as a light yellow oil, $44 \mathrm{mg}, 68 \%$ yield. ${ }^{1} \mathrm{H} \mathrm{NMR}\left(400 \mathrm{MHz}, \mathrm{CDCl}_{3}\right) \delta 8.11$ (s, 
1H), $7.64(\mathrm{~d}, J=7.6 \mathrm{~Hz}, 1 \mathrm{H}), 7.47(\mathrm{~d}, J=8.0 \mathrm{~Hz}, 2 \mathrm{H}), 7.39(\mathrm{~d}, J=7.6 \mathrm{~Hz}, 1 \mathrm{H})$, 7.35-7.28 (m, 2H), 7.19-7.16 (m, 4H), $6.94(\mathrm{t}, J=8.4 \mathrm{~Hz}, 2 \mathrm{H}), 6.35(\mathrm{~d}, J=15.6 \mathrm{~Hz}$, $1 \mathrm{H}), 5.94(\mathrm{~d}, J=12.4 \mathrm{~Hz}, 1 \mathrm{H}), 5.79\left(\mathrm{dt}, J_{1}=15.6 \mathrm{~Hz}, J_{2}=7.6 \mathrm{~Hz}, 1 \mathrm{H}\right), 4.66\left(\mathrm{dd}, J_{1}=\right.$ $\left.13.6 \mathrm{~Hz}, J_{2}=12.0 \mathrm{~Hz}, 1 \mathrm{H}\right), 2.85\left(\mathrm{ddd}, J_{1}=14.0 \mathrm{~Hz}, J_{2}=11.2 \mathrm{~Hz}, J_{3}=5.6 \mathrm{~Hz}, 1 \mathrm{H}\right)$, 2.67-2.52 (m, 3H), $2.35(\mathrm{~s}, 3 \mathrm{H}), 2.33-2.24(\mathrm{~m}, 2 \mathrm{H}), 0.90(\mathrm{~d}, J=12.0 \mathrm{~Hz}, 1 \mathrm{H}),-0.03(\mathrm{~s}$, 9H), -0.10 (s, 9H); $\left.{ }^{13} \mathrm{C} \mathrm{NMR} \mathrm{(100} \mathrm{MHz,} \mathrm{CDCl}_{3}\right) \delta 177.1,163.4(\mathrm{~d}, J=245.2 \mathrm{~Hz})$, $155.3,143.4,140.3,135.6,133.1,132.9$ (d, $J=3.2 \mathrm{~Hz}), 129.6,128.4,127.7$ (d, $J=$ $7.8 \mathrm{~Hz}), 126.8,126.5,123.0(\mathrm{~d}, J=2.2 \mathrm{~Hz}), 122.5,121.8,121.6,116.2,115.4(\mathrm{~d}, J=$ 21.4 Hz), 60.3, 41.9, 38.8, 32.2, 21.8, 21.4, -0.4, -0.6; IR (neat) $\mathrm{cm}^{-1} 2952,1599,1555$, 1508, 1455, 1352, 1248, 1160, 1090, 1034, 938, 858, 836; HRMS (ESI-TOF) m/z: [M $+\mathrm{H}]^{+}$Calcd for $\mathrm{C}_{35} \mathrm{H}_{46} \mathrm{FN}_{2} \mathrm{O}_{2} \mathrm{SSi}_{2}{ }^{+}:$633.2797, found 633.2797.

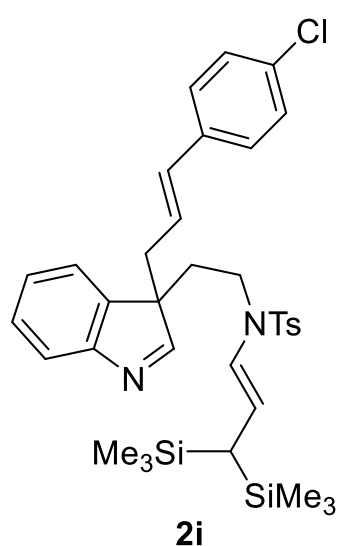

2i 2i: The title compound were purified by silica gel column chromatography (gradient eluent: petroleum ether/EtOAc $=$ $50: 1 \rightarrow 10: 1)$ to afford the desired product $2 \mathbf{i}$ as a light yellow oil, $38 \mathrm{mg}, 58 \%$ yield. ${ }^{1} \mathrm{H}$ NMR $\left(400 \mathrm{MHz}, \mathrm{CDCl}_{3}\right) \delta 8.16(\mathrm{~s}$, 1H), $7.69(\mathrm{~d}, J=7.6 \mathrm{~Hz}, 1 \mathrm{H}), 7.52(\mathrm{~d}, J=8.0 \mathrm{~Hz}, 2 \mathrm{H})$, 7.44-7.31 (m, 4H), 7.28-7.17 (m, 5H), $6.38(\mathrm{~d}, J=15.6 \mathrm{~Hz}, 1 \mathrm{H})$, $5.98(\mathrm{~d}, J=5.2 \mathrm{~Hz}, 1 \mathrm{H}), 5.89\left(\mathrm{dt}, J_{1}=15.6 \mathrm{~Hz}, J_{2}=7.6 \mathrm{~Hz}\right.$, $1 \mathrm{H}), 4.73\left(\mathrm{dd}, J_{1}=13.6 \mathrm{~Hz}, J_{2}=12.0 \mathrm{~Hz}, 1 \mathrm{H}\right), 2.91\left(\mathrm{ddd}, J_{1}=\right.$ $\left.14.4 \mathrm{~Hz}, J_{2}=11.2 \mathrm{~Hz}, J_{3}=5.6 \mathrm{~Hz}, 1 \mathrm{H}\right), 2.70(\mathrm{~d}, J=7.6 \mathrm{~Hz}, 2 \mathrm{H}), 2.62\left(\mathrm{ddd}, J_{1}=14.0\right.$ $\left.\mathrm{Hz}, J_{2}=10.4 \mathrm{~Hz}, J_{3}=5.2 \mathrm{~Hz}, 1 \mathrm{H}\right), 2.41(\mathrm{~s}, 3 \mathrm{H}), 2.38-2.28(\mathrm{~m}, 2 \mathrm{H}), 0.95(\mathrm{~d}, J=12.0$ $\mathrm{Hz}, 1 \mathrm{H}), 0.03$ (s, 9H), -0.04 (s, 9H); $\left.{ }^{13} \mathrm{C} \mathrm{NMR} \mathrm{(100} \mathrm{MHz,} \mathrm{CDCl}_{3}\right) \delta$ 177.0, 155.3, 143.4, 140.2, 135.6, 135.2, 133.1, 133.0, 129.6, 128.6, 128.4, 127.4, 126.8, 126.5, $124.0,122.5,121.8,121.6,116.4,60.2,42.0,38.8,32.3,21.8,21.4,-0.4,-0.6$; IR (neat) $\mathrm{cm}^{-1} 2952,1596,1555,1490,1455,1351,1248,1161,1090,1035,940,858$, 827; HRMS (ESI-TOF) m/z: [M + Na ${ }^{+}$Calcd for $\mathrm{C}_{35} \mathrm{H}_{45} \mathrm{ClN}_{2} \mathrm{O}_{2} \mathrm{SSi}_{2} \mathrm{Na}^{+}: 671.2321$, found 671.2321 . 


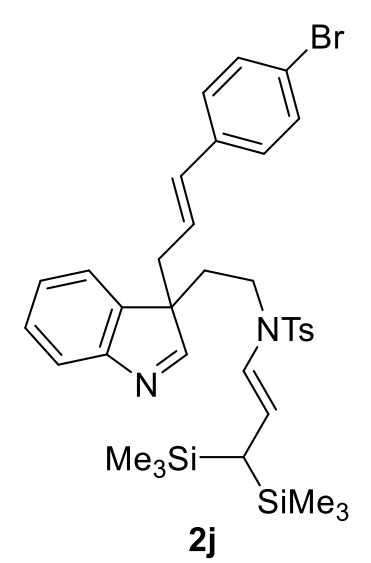

2j: The title compound were purified by silica gel column chromatography (gradient eluent: petroleum ether/EtOAc $=$ $50: 1 \rightarrow 10: 1)$ to afford the desired product $\mathbf{2} \mathbf{j}$ as a light yellow oil, $44 \mathrm{mg}, 62 \%$ yield. ${ }^{1} \mathrm{H}$ NMR (400 MHz, $\left.\mathrm{CDCl}_{3}\right) \delta 8.11(\mathrm{~s}$, 1H), $7.64(\mathrm{~d}, J=7.6 \mathrm{~Hz}, 1 \mathrm{H}), 7.47(\mathrm{~d}, J=8.0 \mathrm{~Hz}, 2 \mathrm{H})$, 7.38-7.28 (m, 5H), $7.18(\mathrm{~d}, J=8.0 \mathrm{~Hz}, 2 \mathrm{H}), 7.08(\mathrm{~d}, J=8.0 \mathrm{~Hz}$, 2H), $6.31(\mathrm{~d}, J=15.6 \mathrm{~Hz}, 1 \mathrm{H}), 5.93(\mathrm{~d}, J=13.6 \mathrm{~Hz}, 1 \mathrm{H}), 5.87$ $\left(\mathrm{dd}, J_{1}=15.6 \mathrm{~Hz}, J_{2}=7.6 \mathrm{~Hz}, 1 \mathrm{H}\right), 4.67\left(\mathrm{t}, J_{1}=13.2 \mathrm{~Hz}, 1 \mathrm{H}\right)$, $2.85\left(\mathrm{ddd}, J_{1}=14.4 \mathrm{~Hz}, J_{2}=11.2 \mathrm{~Hz}, J_{3}=5.6 \mathrm{~Hz}, 1 \mathrm{H}\right), 2.64(\mathrm{~d}, J=7.6 \mathrm{~Hz}, 2 \mathrm{H}), 2.57$ $\left(\mathrm{ddd}, J_{1}=19.2 \mathrm{~Hz}, J_{2}=10.8 \mathrm{~Hz}, J_{3}=5.2 \mathrm{~Hz}, 1 \mathrm{H}\right), 2.36(\mathrm{~s}, 3 \mathrm{H}), 2.33-2.24(\mathrm{~m}, 2 \mathrm{H})$, $0.90(\mathrm{~d}, J=12.0 \mathrm{~Hz}, 1 \mathrm{H}),-0.02(\mathrm{~s}, 9 \mathrm{H}),-0.09(\mathrm{~s}, 9 \mathrm{H}) ;{ }^{13} \mathrm{C} \mathrm{NMR}\left(100 \mathrm{MHz}, \mathrm{CDCl}_{3}\right) \delta$ 177.0, 155.3, 143.4, 140.2, 135.6, 133.1, 131.5, 129.6, 128.4, 127.7, 126.8, 126.5, $124.2,122.5,121.8,121.7,121.2,116.4,60.2,42.0,38.8,32.3,21.8,21.4,-0.4,-0.5$; IR (neat) $\mathrm{cm}^{-1} 2952,1607,1555,1487,1455,1351,1248,1161,1090,1034,966,858$, 826; HRMS (ESI-TOF) m/z: $[\mathrm{M}+\mathrm{H}]^{+}$Calcd for $\mathrm{C}_{35} \mathrm{H}_{46} \mathrm{BrN}_{2} \mathrm{O}_{2} \mathrm{SSi}_{2}{ }^{+}$: 693.1996, found 693.2001.

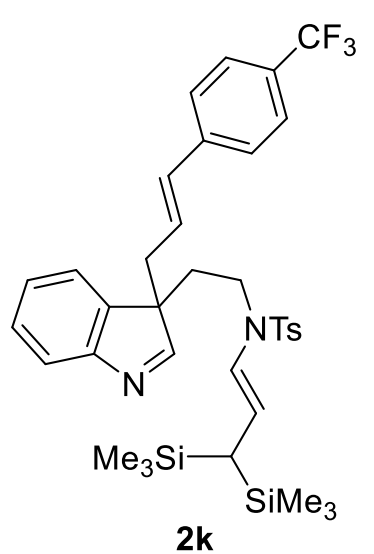

2k: The title compound were purified by silica gel column chromatography (gradient eluent: petroleum ether/EtOAc $=$ $50: 1 \rightarrow 10: 1)$ to afford the desired product $\mathbf{2 k}$ as a light yellow oil, $41 \mathrm{mg}, 59 \%$ yield. ${ }^{1} \mathrm{H} \mathrm{NMR}\left(400 \mathrm{MHz}, \mathrm{CDCl}_{3}\right) \delta 8.12$ (s, $1 \mathrm{H}), 7.64(\mathrm{~d}, J=8.0 \mathrm{~Hz}, 1 \mathrm{H}), 7.51(\mathrm{~d}, J=8.0 \mathrm{~Hz}, 2 \mathrm{H}), 7.47(\mathrm{~d}$, $J=8.4 \mathrm{~Hz}, 2 \mathrm{H}), 7.40-7.28(\mathrm{~m}, 5 \mathrm{H}), 7.19(\mathrm{~d}, J=8.0 \mathrm{~Hz}, 2 \mathrm{H})$, $6.41(\mathrm{~d}, J=15.6 \mathrm{~Hz}, 1 \mathrm{H}), 5.94\left(\mathrm{dt}, J_{1}=15.6 \mathrm{~Hz}, J_{2}=7.6 \mathrm{~Hz}\right.$, $1 \mathrm{H}), 5.93(\mathrm{~d}, J=13.6 \mathrm{~Hz}, 1 \mathrm{H}), 4.68\left(\mathrm{dd}, J_{1}=13.6 \mathrm{~Hz}, J_{2}=\right.$ $12.0 \mathrm{~Hz}, 1 \mathrm{H}), 2.86\left(\mathrm{ddd}, J_{1}=14.0 \mathrm{~Hz}, J_{2}=11.2 \mathrm{~Hz}, J_{3}=5.6 \mathrm{~Hz}, 1 \mathrm{H}\right), 2.70(\mathrm{~d}, J=7.6$ $\mathrm{Hz}, 2 \mathrm{H}), 2.58\left(\mathrm{ddd}, J_{1}=14.0 \mathrm{~Hz}, J_{2}=10.8 \mathrm{~Hz}, J_{3}=5.6 \mathrm{~Hz}, 1 \mathrm{H}\right), 2.36(\mathrm{~s}, 3 \mathrm{H})$, 2.34-2.24 (m, 2H), $0.90(\mathrm{~d}, J=12.0 \mathrm{~Hz}, 1 \mathrm{H}),-0.02(\mathrm{~s}, 9 \mathrm{H}),-0.09(\mathrm{~s}, 9 \mathrm{H}) ;{ }^{13} \mathrm{C} \mathrm{NMR}$ $\left(100 \mathrm{MHz}, \mathrm{CDCl}_{3}\right) \delta 176.8,155.3,143.4,140.2,140.1,135.7,132.9,129.6,128.5$, $126.8,126.6,126.4,126.2,125.4(\mathrm{q}, J=3.9 \mathrm{~Hz}), 122.6,121.8,121.7,116.7,60.2$, 
42.1, 38.7, 32.4, 21.9, 21.4, -0.4, -0.5; IR (neat) $\mathrm{cm}^{-1} 2952,1614,1555,1455,1351$, 1323, 1248, 1160, 1121, 1090, 1066, 950, 859, 828; HRMS (ESI-TOF) m/z: $[\mathrm{M}+\mathrm{H}]^{+}$ Calcd for $\mathrm{C}_{36} \mathrm{H}_{46} \mathrm{~F}_{3} \mathrm{~N}_{2} \mathrm{O}_{2} \mathrm{SSi}_{2}{ }^{+}$: 683.2765, found 683.2767.

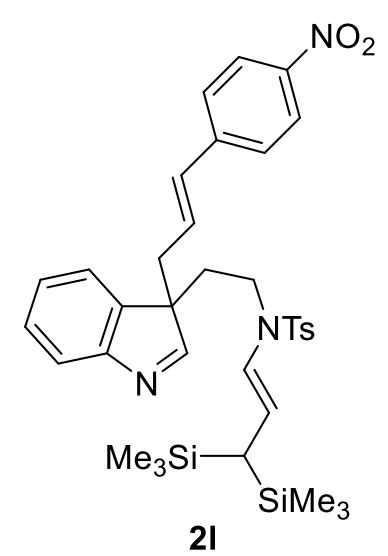

21: The title compound were purified by silica gel column chromatography (gradient eluent: petroleum ether/EtOAc $=$ $50: 1 \rightarrow 8: 1)$ to afford the desired product $\mathbf{2 l}$ as a light yellow oil, $21 \mathrm{mg}, 31 \%$ yield. ${ }^{1} \mathrm{H} \mathrm{NMR}\left(400 \mathrm{MHz}, \mathrm{CDCl}_{3}\right) \delta 8.08(\mathrm{~d}$, $J=7.2 \mathrm{~Hz}, 2 \mathrm{H}), 8.07(\mathrm{~s}, 1 \mathrm{H}), 7.60(\mathrm{~d}, J=7.6 \mathrm{~Hz}, 1 \mathrm{H}), 7.44(\mathrm{~d}$, $J=8.0 \mathrm{~Hz}, 2 \mathrm{H}), 7.36-7.25(\mathrm{~m}, 4 \mathrm{H}), 7.22(\mathrm{~s}, 1 \mathrm{H}), 7.15(\mathrm{~d}, J=$ $8.0 \mathrm{~Hz}, 2 \mathrm{H}), 6.39(\mathrm{~d}, J=15.6 \mathrm{~Hz}, 1 \mathrm{H}), 5.94\left(\mathrm{dt}, J_{1}=15.6 \mathrm{~Hz}\right.$, $\left.J_{2}=7.6 \mathrm{~Hz}, 1 \mathrm{H}\right), 5.88(\mathrm{~d}, J=13.6 \mathrm{~Hz}, 1 \mathrm{H}), 4.65\left(\mathrm{t}, J_{1}=12.8\right.$

$\mathrm{Hz}, 1 \mathrm{H}), 2.83\left(\mathrm{ddd}, J_{1}=16.0 \mathrm{~Hz}, J_{2}=11.6 \mathrm{~Hz}, J_{3}=5.2 \mathrm{~Hz}, 1 \mathrm{H}\right), 2.71(\mathrm{~d}, J=7.6 \mathrm{~Hz}$, $2 \mathrm{H}), 2.56\left(\mathrm{ddd}, J_{1}=16.0 \mathrm{~Hz}, J_{2}=10.8 \mathrm{~Hz}, J_{3}=5.6 \mathrm{~Hz}, 1 \mathrm{H}\right), 2.32(\mathrm{~s}, 3 \mathrm{H}), 2.28-2.17$ $(\mathrm{m}, 2 \mathrm{H}), 0.87(\mathrm{~d}, J=12.4 \mathrm{~Hz}, 1 \mathrm{H}),-0.06(\mathrm{~s}, 9 \mathrm{H}),-0.13(\mathrm{~s}, 9 \mathrm{H}) ;{ }^{13} \mathrm{C} \mathrm{NMR}(100 \mathrm{MHz}$, $\left.\mathrm{CDCl}_{3}\right) \delta 176.6,155.2,146.8,143.5,143.0,139.9,135.5,132.2,129.6,128.6,128.5$, $126.8,126.7,126.7,123.9,122.5,121.8,121.7,116.8,60.2,42.0,38.8,32.6,21.8$, 21.4, -0.4, -0.5; IR (neat) $\mathrm{cm}^{-1} 2952,1596,1555,1515,1455,1341,1248,1161,1090$, 1034, 953, 858, 837; HRMS (ESI-TOF) m/z: $\left[\mathrm{M}+\mathrm{Na}^{+}\right.$Calcd for $\mathrm{C}_{35} \mathrm{H}_{45} \mathrm{~N}_{3} \mathrm{O}_{4} \mathrm{SSi}_{2} \mathrm{Na}^{+}:$682.2562, found 682.2564 .

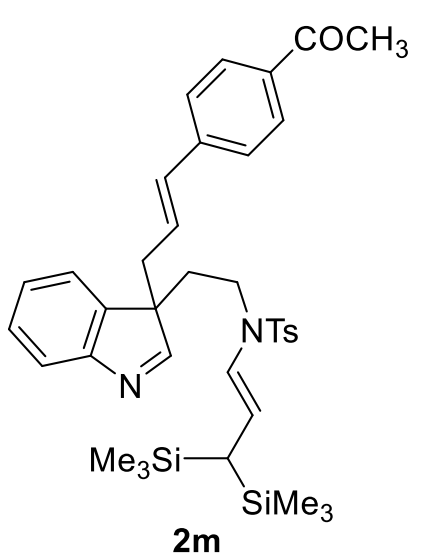

2m: The title compound were purified by silica gel column chromatography (gradient eluent: petroleum ether/EtOAc $=$ $50: 1 \rightarrow 8: 1)$ to afford the desired product $\mathbf{2} \mathbf{m}$ as a light yellow oil, $36 \mathrm{mg}, 54 \%$ yield. ${ }^{1} \mathrm{H}$ NMR (400 $\left.\mathrm{MHz}, \mathrm{CDCl}_{3}\right)$ $\delta 8.12(\mathrm{~s}, 1 \mathrm{H}), 7.86(\mathrm{~d}, J=7.6 \mathrm{~Hz}, 2 \mathrm{H}), 7.64(\mathrm{~d}, J=7.6 \mathrm{~Hz}$, $1 \mathrm{H}), 7.47(\mathrm{~d}, J=8.0 \mathrm{~Hz}, 2 \mathrm{H}), 7.39-7.26(\mathrm{~m}, 5 \mathrm{H}), 7.19(\mathrm{~d}, J$ $=8.0 \mathrm{~Hz}, 2 \mathrm{H}), 6.42(\mathrm{~d}, J=15.6 \mathrm{~Hz}, 1 \mathrm{H}), 5.98\left(\mathrm{dt}, J_{1}=15.6\right.$ $\left.\mathrm{Hz}, J_{2}=7.6 \mathrm{~Hz}, 1 \mathrm{H}\right), 5.92(\mathrm{~d}, J=13.6 \mathrm{~Hz}, 1 \mathrm{H}), 4.68(\mathrm{dd}$, $\left.J_{1}=13.6 \mathrm{~Hz}, J_{2}=12.0 \mathrm{~Hz}, 1 \mathrm{H}\right), 2.86\left(\mathrm{ddd}, J_{1}=14.0 \mathrm{~Hz}, J_{2}=10.8 \mathrm{~Hz}, J_{3}=5.2 \mathrm{~Hz}\right.$, $1 \mathrm{H}), 2.70(\mathrm{~d}, J=7.6 \mathrm{~Hz}, 2 \mathrm{H}), 2.62-2.52(\mathrm{~m}, 1 \mathrm{H}), 2.57(\mathrm{~s}, 3 \mathrm{H}), 2.35(\mathrm{~s}, 3 \mathrm{H}), 2.33-2.24$ 
(m, 2H), $0.90(\mathrm{~d}, J=12.0 \mathrm{~Hz}, 1 \mathrm{H}),-0.02$ (s, 9H), -0.09 (s, 9H); ${ }^{13} \mathrm{C}$ NMR $(100 \mathrm{MHz}$, $\left.\mathrm{CDCl}_{3}\right) \delta 197.5,176.8,155.2,143.4,141.3,140.1,135.9,135.6,133.3,129.6,128.7$, 128.5, 126.8, 126.6, 126.4, 126.3, 122.5, 121.8, 121.7, 116.5, 60.2, 42.0, 38.9, 32.4, 26.6, 21.8, 21.4, -0.4, -0.6; IR (neat) $\mathrm{cm}^{-1}$ 2952, 1680, 1602, 1356, 1265, 1162, 1090, 956, 860, 839; HRMS (ESI-TOF) $\mathrm{m} / \mathrm{z}:[\mathrm{M}+\mathrm{Na}]^{+}$Calcd for $\mathrm{C}_{37} \mathrm{H}_{48} \mathrm{~N}_{2} \mathrm{O}_{3} \mathrm{SSi}_{2} \mathrm{Na}^{+}: 679.2816$, found 679.2811.

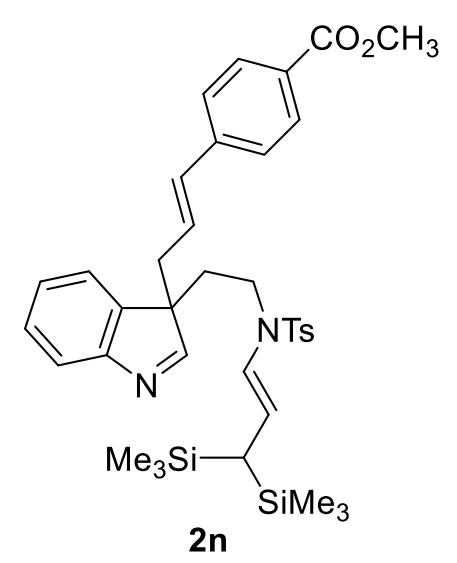

2n: The title compound were purified by silica gel column chromatography (gradient eluent: petroleum ether/EtOAc $=50: 1 \rightarrow 8: 1)$ to afford the desired product $\mathbf{2 n}$ as a light yellow oil, $43 \mathrm{mg}, 64 \%$ yield. ${ }^{1} \mathrm{H} \mathrm{NMR}\left(400 \mathrm{MHz}, \mathrm{CDCl}_{3}\right)$ $\delta 8.12(\mathrm{~s}, 1 \mathrm{H}), 7.93(\mathrm{~d}, J=8.0 \mathrm{~Hz}, 2 \mathrm{H}), 7.64(\mathrm{~d}, J=7.6$ $\mathrm{Hz}, 1 \mathrm{H}), 7.47$ (d, J=8.0 Hz, 2H), 7.39-7.29 (m, 3H), 7.26 $(\mathrm{d}, J=8.4 \mathrm{~Hz}, 2 \mathrm{H}), 7.18(\mathrm{~d}, J=8.0 \mathrm{~Hz}, 2 \mathrm{H}), 6.41(\mathrm{~d}, J=$ $15.6 \mathrm{~Hz}, 1 \mathrm{H}), 5.98\left(\mathrm{dt}, J_{1}=15.6 \mathrm{~Hz}, J_{2}=7.6 \mathrm{~Hz}, 1 \mathrm{H}\right)$, $5.93(\mathrm{~d}, J=13.6 \mathrm{~Hz}, 1 \mathrm{H}), 4.68\left(\mathrm{dd}, J_{1}=13.6 \mathrm{~Hz}, J_{2}=12.0 \mathrm{~Hz}, 1 \mathrm{H}\right), 3.89(\mathrm{~s}, 3 \mathrm{H}), 2.86$ $\left(\mathrm{ddd}, J_{1}=14.4 \mathrm{~Hz}, J_{2}=11.2 \mathrm{~Hz}, J_{3}=5.6 \mathrm{~Hz}, 1 \mathrm{H}\right), 2.68(\mathrm{~d}, J=7.2 \mathrm{~Hz}, 2 \mathrm{H}), 2.62-2.54$ (m, 1H), $2.35(\mathrm{~s}, 3 \mathrm{H}), 2.33-2.25(\mathrm{~m}, 2 \mathrm{H}), 0.90(\mathrm{~d}, J=12.0 \mathrm{~Hz}, 1 \mathrm{H}),-0.03(\mathrm{~s}, 9 \mathrm{H})$, -0.09 (s, 9H); ${ }^{13} \mathrm{C}$ NMR (100 MHz, $\left.\mathrm{CDCl}_{3}\right) \delta 176.8,166.8,155.3,143.4,141.1,140.1$, $135.7,133.4$, 129.8, 129.6, 129.0, 128.5, 126.8, 126.6, 126.2, 126.1, 122.6, 121.8, 121.7, 116.6, 60.2, 52.0, 42.0, 38.9, 32.3, 21.8, 21.4, -0.4, -0.5; IR (neat) $\mathrm{cm}^{-1} 2952$, 1717, 1606, 1436, 1351, 1265, 1162, 1110, 1035, 957, 861, 839; HRMS (ESI-TOF) $\mathrm{m} / \mathrm{z}:[\mathrm{M}+\mathrm{Na}]^{+}$Calcd for $\mathrm{C}_{37} \mathrm{H}_{48} \mathrm{~N}_{2} \mathrm{O}_{4} \mathrm{SSi}_{2} \mathrm{Na}^{+}:$695.2766, found 695.2767.

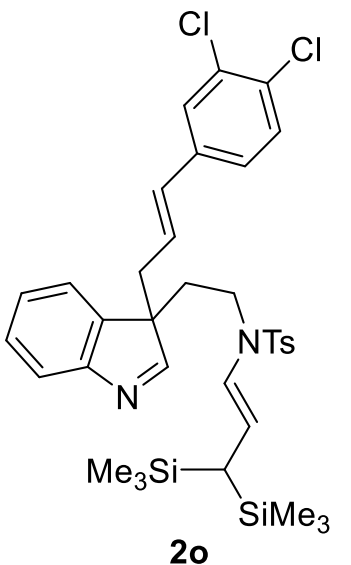

20

20: The title compound were purified by silica gel column chromatography (gradient eluent: petroleum ether/EtOAc $=$ $50: 1 \rightarrow 10: 1)$ to afford the desired product 20 as a light yellow oil, $41 \mathrm{mg}, 60 \%$ yield. ${ }^{1} \mathrm{H} \mathrm{NMR}\left(400 \mathrm{MHz}, \mathrm{CDCl}_{3}\right) \delta 8.04$ (s, 1H), $7.58(\mathrm{~d}, J=7.6 \mathrm{~Hz}, 1 \mathrm{H}), 7.40(\mathrm{~d}, J=8.4 \mathrm{~Hz}, 2 \mathrm{H}), 7.31$ $\left(\mathrm{ddd}, J_{1}=8.0 \mathrm{~Hz}, J_{2}=6.8 \mathrm{~Hz}, J_{3}=2.4 \mathrm{~Hz}, 1 \mathrm{H}\right), 7.26-7.22(\mathrm{~m}$, $3 \mathrm{H}), 7.21(\mathrm{~d}, J=2.0 \mathrm{~Hz}, 1 \mathrm{H}), 7.19(\mathrm{~s}, 1 \mathrm{H}), 7.12(\mathrm{~d}, J=8.0 \mathrm{~Hz}$, 
2H), $6.95\left(\mathrm{dd}, J_{1}=8.4 \mathrm{~Hz}, J_{2}=2.4 \mathrm{~Hz}, 1 \mathrm{H}\right), 6.20(\mathrm{~d}, J=15.6 \mathrm{~Hz}, 1 \mathrm{H}), 5.86(\mathrm{~d}, J=$ $13.6 \mathrm{~Hz}, 1 \mathrm{H}), 5.78\left(\mathrm{dt}, J_{1}=15.6 \mathrm{~Hz}, J_{2}=7.6 \mathrm{~Hz}, 1 \mathrm{H}\right), 4.61\left(\mathrm{dd}, J_{1}=13.6 \mathrm{~Hz}, J_{2}=\right.$ $12.4 \mathrm{~Hz}, 1 \mathrm{H}), 2.79\left(\mathrm{ddd}, J_{1}=14.0 \mathrm{~Hz}, J_{2}=11.2 \mathrm{~Hz}, J_{3}=5.6 \mathrm{~Hz}, 1 \mathrm{H}\right), 2.59(\mathrm{~d}, J=7.6$ $\mathrm{Hz}, 2 \mathrm{H}), 2.51\left(\mathrm{ddd}, J_{1}=14.0 \mathrm{~Hz}, J_{2}=10.8 \mathrm{~Hz}, J_{3}=5.6 \mathrm{~Hz}, 1 \mathrm{H}\right), 2.29(\mathrm{~s}, 3 \mathrm{H})$, 2.26-2.16 (m, 2H), $0.84(\mathrm{~d}, J=12.0 \mathrm{~Hz}, 1 \mathrm{H}),-0.08(\mathrm{~s}, 9 \mathrm{H}),-0.15(\mathrm{~s}, 9 \mathrm{H}),{ }^{13} \mathrm{C} \mathrm{NMR}$ $\left(100 \mathrm{MHz}, \mathrm{CDCl}_{3}\right) \delta 176.8,155.2,143.4,140.0,136.7,135.6,132.6,131.9,131.1$, $130.4,129.6,128.5,127.9,126.8,126.6,125.5,125.4,122.5,121.8,121.7,116.5$, 60.2, 42.0, 38.7, 32.3, 21.8, 21.4, -0.4, -0.5; IR (neat) $\mathrm{cm}^{-1} 2952,1597,1554,1472$, 1351, 1249, 1161, 1090, 1028, 950, 859, 836; HRMS (ESI-TOF) m/z: $[\mathrm{M}+\mathrm{Na}]^{+}$ Calcd for $\mathrm{C}_{35} \mathrm{H}_{44} \mathrm{Cl}_{2} \mathrm{~N}_{2} \mathrm{O}_{2} \mathrm{SSi}_{2} \mathrm{Na}^{+}$: 705.1931, found 705.1930.

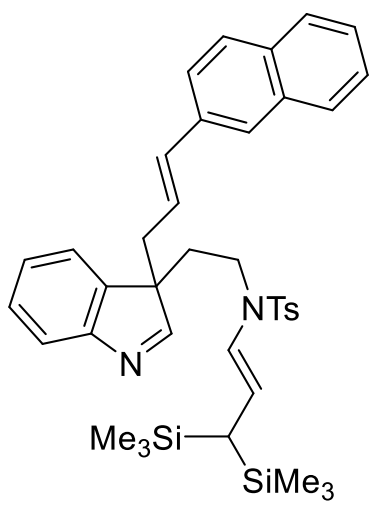

$2 p$

2p: The title compound were purified by silica gel column chromatography (gradient eluent: petroleum ether/EtOAc $=$ $50: 1 \rightarrow 10: 1)$ to afford the desired product $\mathbf{2 p}$ as a light yellow oil, $56 \mathrm{mg}, 84 \%$ yield. ${ }^{1} \mathrm{H}$ NMR (400 MHz, $\left.\mathrm{CDCl}_{3}\right) \delta 8.15$ (s, 1H), $7.78(\mathrm{~d}, J=6.8 \mathrm{~Hz}, 2 \mathrm{H}), 7.74(\mathrm{~d}, J=8.8 \mathrm{~Hz}, 1 \mathrm{H}), 7.65$ (d, $J=7.6 \mathrm{~Hz}, 1 \mathrm{H}), 7.59$ (s, 1H), $7.48(\mathrm{~d}, J=8.0 \mathrm{~Hz}, 2 \mathrm{H})$, 7.45-7.39 (m, 3H), $7.37(\mathrm{~d}, J=6.8 \mathrm{~Hz}, 2 \mathrm{H}), 7.31-7.28(\mathrm{~m}$, $1 \mathrm{H}), 7.18(\mathrm{~d}, J=8.0 \mathrm{~Hz}, 2 \mathrm{H}), 6.55(\mathrm{~d}, J=16.0 \mathrm{~Hz}, 1 \mathrm{H}), 6.03$ $\left(\mathrm{dt}, J_{1}=15.6 \mathrm{~Hz}, J_{2}=7.6 \mathrm{~Hz}, 1 \mathrm{H}\right), 5.94(\mathrm{~d}, J=13.6 \mathrm{~Hz}, 1 \mathrm{H}), 4.69\left(\mathrm{dd}, J_{1}=13.6 \mathrm{~Hz}\right.$, $\left.J_{2}=12.0 \mathrm{~Hz}, 1 \mathrm{H}\right), 2.88\left(\mathrm{ddd}, J_{1}=14.0 \mathrm{~Hz}, J_{2}=11.2 \mathrm{~Hz}, J_{3}=5.6 \mathrm{~Hz}, 1 \mathrm{H}\right), 2.74-2.57$ (m, 3H), 2.41-2.28 (m, 2H), 2.35 (s, 3H), $0.90(\mathrm{~d}, J=12.0 \mathrm{~Hz}, 1 \mathrm{H}),-0.02(\mathrm{~s}, 9 \mathrm{H})$, -0.09 (s, 9H); ${ }^{13} \mathrm{C}$ NMR (100 MHz, $\left.\mathrm{CDCl}_{3}\right) \delta 177.1,155.3,143.4,140.3,135.7,134.4$, 134.1, 133.5, 132.9, 129.6, 128.4, 128.1, 127.9, 127.6, 126.8, 126.5, 126.2, 126.1, 125.8, 123.7, 123.4, 122.5, 121.9, 121.6, 116.3, 60.3, 42.0, 39.0, 32.2, 21.8, 21.4, -0.4, -0.6; IR (neat) $\mathrm{cm}^{-1} 2951,1597,1555,1454,1352,1249,1162,1090,1035,960,860$, 838; HRMS (ESI-TOF) m/z: [M + H] $]^{+}$Calcd for $\mathrm{C}_{39} \mathrm{H}_{49} \mathrm{~N}_{2} \mathrm{O}_{2} \mathrm{SSi}_{2}{ }^{+}: 665.3048$, found 665.3052 . 


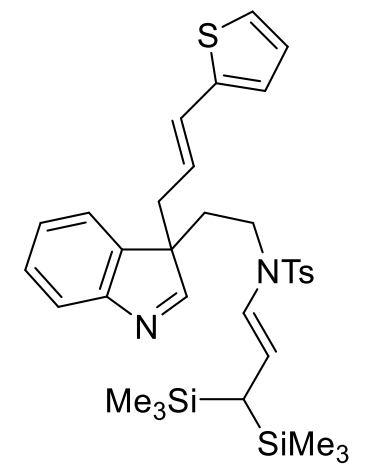

$2 q$

2q: The title compound were purified by silica gel column chromatography (gradient eluent: petroleum ether/EtOAc $=$ $50: 1 \rightarrow 10: 1)$ to afford the desired product $\mathbf{2 q}$ as a brown oil, 58 mg, 92\% yield. ${ }^{1} \mathrm{H}$ NMR (400 $\left.\mathrm{MHz}, \mathrm{CDCl}_{3}\right) \delta 8.11(\mathrm{~s}, 1 \mathrm{H})$, $7.64(\mathrm{~d}, J=7.6 \mathrm{~Hz}, 1 \mathrm{H}), 7.47(\mathrm{~d}, J=8.4 \mathrm{~Hz}, 2 \mathrm{H}), 7.38-7.28(\mathrm{~m}$, $3 \mathrm{H}), 7.18(\mathrm{~d}, J=8.0 \mathrm{~Hz}, 2 \mathrm{H}), 7.10(\mathrm{~d}, J=4.8 \mathrm{~Hz}, 1 \mathrm{H}), 6.91(\mathrm{dd}$, $\left.J_{1}=5.2 \mathrm{~Hz}, J_{2}=3.6 \mathrm{~Hz}, 1 \mathrm{H}\right), 6.85(\mathrm{~d}, J=3.6 \mathrm{~Hz}, 1 \mathrm{H}), 6.51(\mathrm{~d}$, $J=15.2 \mathrm{~Hz}, 1 \mathrm{H}), 5.93(\mathrm{~d}, J=14.0 \mathrm{~Hz}, 1 \mathrm{H}), 5.76\left(\mathrm{dt}, J_{1}=15.2 \mathrm{~Hz}, J_{2}=7.6 \mathrm{~Hz}, 1 \mathrm{H}\right)$, $4.68\left(\mathrm{dd}, J_{1}=13.6 \mathrm{~Hz}, J_{2}=12.4 \mathrm{~Hz}, 1 \mathrm{H}\right), 2.84\left(\mathrm{ddd}, J_{1}=14.0 \mathrm{~Hz}, J_{2}=11.2 \mathrm{~Hz}, J_{3}=\right.$ $5.6 \mathrm{~Hz}, 1 \mathrm{H}), 2.63-2.51(\mathrm{~m}, 3 \mathrm{H}), 2.35(\mathrm{~s}, 3 \mathrm{H}), 2.32-2.22(\mathrm{~m}, 2 \mathrm{H}), 0.90(\mathrm{~d}, J=12.0 \mathrm{~Hz}$, 1H), -0.02 (s, 9H), -0.08 (s, 9H); ${ }^{13} \mathrm{C}$ NMR $\left(100 \mathrm{MHz}, \mathrm{CDCl}_{3}\right) \delta 177.0,155.2,143.3$, $141.7,140.2$, 135.6, 129.6, 128.4, 127.4, 127.2, 126.8, 126.5, 125.4, 124.0, 123.0, 122.5, 121.9, 121.6, 116.4, 60.2, 41.9, 38.7, 32.0, 21.8, 21.4, -0.4, -0.6; IR (neat) $\mathrm{cm}^{-1}$ 2952, 1597, 1555, 1455, 1352, 1249, 1163, 1090, 1036, 959, 860, 838; HRMS (ESI-TOF) $\mathrm{m} / \mathrm{z}:[\mathrm{M}+\mathrm{H}]^{+}$Calcd for $\mathrm{C}_{33} \mathrm{H}_{45} \mathrm{~N}_{2} \mathrm{O}_{2} \mathrm{~S}_{2} \mathrm{Si}_{2}{ }^{+}$: 621.2455, found 621.2456.

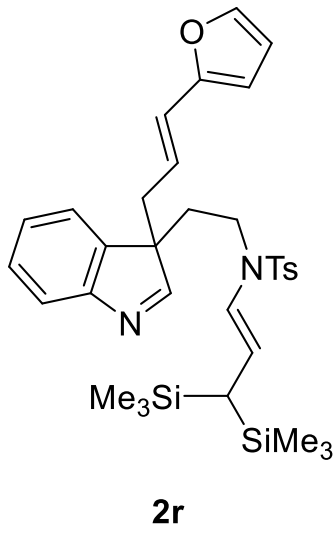

2r: The title compound were purified by silica gel column chromatography (gradient eluent: petroleum ether/EtOAc $=$ $50: 1 \rightarrow 10: 1)$ to afford the desired product $\mathbf{2 r}$ as a brownish red oil, $54 \mathrm{mg}, 88 \%$ yield. ${ }^{1} \mathrm{H}$ NMR (400 MHz, $\left.\mathrm{CDCl}_{3}\right) \delta 8.11(\mathrm{~s}$, $1 \mathrm{H}), 7.64(\mathrm{~d}, J=7.6 \mathrm{~Hz}, 1 \mathrm{H}), 7.46(\mathrm{~d}, J=8.4 \mathrm{~Hz}, 2 \mathrm{H})$, $7.38-7.26(\mathrm{~m}, 4 \mathrm{H}), 7.18(\mathrm{~d}, J=8.0 \mathrm{~Hz}, 2 \mathrm{H}), 6.33\left(\mathrm{dd}, J_{1}=3.2\right.$ $\left.\mathrm{Hz}, J_{2}=2.0 \mathrm{~Hz}, 1 \mathrm{H}\right), 6.21(\mathrm{~d}, J=15.6 \mathrm{~Hz}, 1 \mathrm{H}), 6.14(\mathrm{~d}, J=3.6$

$\mathrm{Hz}, 1 \mathrm{H}), 5.90\left(\mathrm{dt}, J_{1}=15.6 \mathrm{~Hz}, J_{2}=7.6 \mathrm{~Hz}, 1 \mathrm{H}\right), 5.94(\mathrm{~d}, J=14.0 \mathrm{~Hz}, 1 \mathrm{H}), 4.66(\mathrm{dd}$, $\left.J_{1}=13.6 \mathrm{~Hz}, J_{2}=12.0 \mathrm{~Hz}, 1 \mathrm{H}\right), 2.82\left(\mathrm{ddd}, J_{1}=13.6 \mathrm{~Hz}, J_{2}=10.8 \mathrm{~Hz}, J_{3}=5.2 \mathrm{~Hz}\right.$, 1H), 2.62-2.49 (m, 3H), $2.35(\mathrm{~s}, 3 \mathrm{H}), 2.32-2.22(\mathrm{~m}, 2 \mathrm{H}), 0.90(\mathrm{~d}, J=12.0 \mathrm{~Hz}, 1 \mathrm{H})$, -0.02 (s, 9H), -0.09 (s, 9H); ${ }^{13} \mathrm{C}$ NMR $\left(100 \mathrm{MHz}, \mathrm{CDCl}_{3}\right) \delta 177.1,155.2,152.1,143.3$, $141.7,140.3,135.7,129.6,128.3,126.8,126.5,122.6,122.5,122.1,121.9,121.6$, 116.2, 111.2, 107.6, 60.2, 41.9, 38.8, 31.9, 21.8, 21.4, -0.4, -0.6; IR (neat) $\mathrm{cm}^{-1} 2952$, 1597, 1555, 1456, 1352, 1248, 1162, 1090, 1034, 957, 860, 837; HRMS (ESI-TOF) 
$\mathrm{m} / \mathrm{z}:[\mathrm{M}+\mathrm{H}]^{+}$Calcd for $\mathrm{C}_{33} \mathrm{H}_{45} \mathrm{~N}_{2} \mathrm{O}_{3} \mathrm{SSi}_{2}{ }^{+}:$605.2684, found 605.2682.

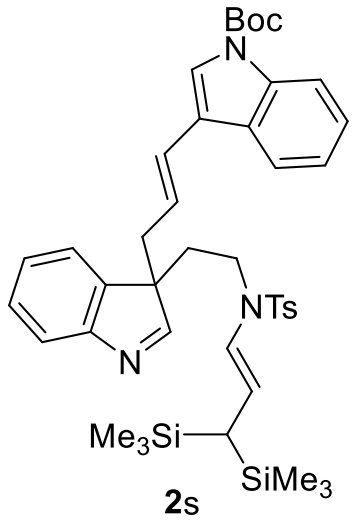

2s: The title compound were purified by silica gel column chromatography (gradient eluent: petroleum ether/EtOAc $=$ $50: 1 \rightarrow 8: 1)$ to afford the desired product $2 \mathbf{s}$ as a light yellow oil, $37 \mathrm{mg}, 49 \%$ yield. ${ }^{1} \mathrm{H}$ NMR (400 MHz, $\left.\mathrm{CDCl}_{3}\right) \delta 8.14(\mathrm{~s}$, $1 \mathrm{H}), 8.12(\mathrm{~s}, 1 \mathrm{H}), 7.64(\mathrm{~d}, J=7.6 \mathrm{~Hz}, 1 \mathrm{H}), 7.49-7.46(\mathrm{~m}, 4 \mathrm{H})$, 7.39-7.35 (m, 2H), 7.32-7.27 (m, 2H), $7.21(\mathrm{t}, J=7.6 \mathrm{~Hz}, 1 \mathrm{H})$, $7.18(\mathrm{~d}, J=8.0 \mathrm{~Hz}, 2 \mathrm{H}), 7.45(\mathrm{~d}, J=16.0 \mathrm{~Hz}, 1 \mathrm{H}), 5.94(\mathrm{~d}, J=$ $13.6 \mathrm{~Hz}, 1 \mathrm{H}), 5.92\left(\mathrm{dt}, J_{1}=15.6 \mathrm{~Hz}, J_{2}=7.6 \mathrm{~Hz}, 1 \mathrm{H}\right), 4.69\left(\mathrm{dd}, J_{1}=13.6 \mathrm{~Hz}, J_{2}=\right.$ $12.0 \mathrm{~Hz}, 1 \mathrm{H}), 2.89\left(\mathrm{ddd}, J_{1}=14.4 \mathrm{~Hz}, J_{2}=11.2 \mathrm{~Hz}, J_{3}=5.6 \mathrm{~Hz}, 1 \mathrm{H}\right), 2.73-2.57(\mathrm{~m}$, 3H), 2.40-2.27 (m, 2H), 2.35 (s, 3H), $1.66(\mathrm{~s}, 9 \mathrm{H}), 0.90$ (d, $J=12.4 \mathrm{~Hz}, 1 \mathrm{H}),-0.03$ (s, 9H), $-0.10(\mathrm{~s}, 9 \mathrm{H}) ;{ }^{13} \mathrm{C} \mathrm{NMR}\left(100 \mathrm{MHz}, \mathrm{CDCl}_{3}\right) \delta 177.1,155.4,149.5,143.4,140.4$, $135.8,129.6,128.6,128.3,126.8,126.5,125.3,124.6,123.8,123.5,122.9,122.6$, $121.9,121.6,119.8,118.2,116.5,115.3,83.8,60.4,42.1,39.4,32.3,28.2,21.9,21.4$, $-0.4,-0.5$; IR (neat) $\mathrm{cm}^{-1} 2952,1732,1597,1555,1451,1368,1249,1158,1090,1023$, 960, 858, 837; HRMS (ESI-TOF) $\mathrm{m} / \mathrm{z}:[\mathrm{M}+\mathrm{H}]^{+}$Calcd for $\mathrm{C}_{42} \mathrm{H}_{56} \mathrm{~N}_{3} \mathrm{O}_{4} \mathrm{SSi}_{2}{ }^{+}$: 754.3525, found 754.3526 .

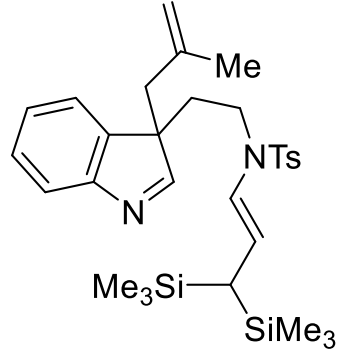

$2 u$

2u: The title compound were purified by silica gel column chromatography (gradient eluent: petroleum ether/EtOAc $=$ $50: 1 \rightarrow 10: 1)$ to afford the desired product $\mathbf{2 u}$ as a colorless oil, $49 \mathrm{mg}, 87 \%$ yield. ${ }^{1} \mathrm{H}$ NMR $\left(400 \mathrm{MHz}, \mathrm{CDCl}_{3}\right) \delta 8.11(\mathrm{~s}, 1 \mathrm{H})$, $7.61(\mathrm{~d}, J=7.6 \mathrm{~Hz}, 1 \mathrm{H}), 7.46(\mathrm{~d}, J=8.0 \mathrm{~Hz}, 2 \mathrm{H}), 7.37-7.25(\mathrm{~m}$, $3 \mathrm{H}), 7.19(\mathrm{~d}, J=8.0 \mathrm{~Hz}, 2 \mathrm{H}), 5.95(\mathrm{~d}, J=14.0 \mathrm{~Hz}, 1 \mathrm{H})$, 4.71-4.70 (m, 1H), $4.64(\mathrm{t}, J=13.6 \mathrm{~Hz}, 1 \mathrm{H}), 4.56(\mathrm{~s}, 1 \mathrm{H}), 2.78\left(\mathrm{ddd}, J_{1}=14.0 \mathrm{~Hz}, J_{2}\right.$ $\left.=11.6 \mathrm{~Hz}, J_{3}=4.8 \mathrm{~Hz}, 1 \mathrm{H}\right), 2.70(\mathrm{~d}, J=13.2 \mathrm{~Hz}, 1 \mathrm{H}), 2.53-2.48(\mathrm{~m}, 1 \mathrm{H}), 2.46(\mathrm{~d}, J=$ $13.2 \mathrm{~Hz}, 1 \mathrm{H}), 2.36$ (s, 3H), 2.32-2.18 (m, 2H), 1.43 (s, 3H), 0.91 (d, J = $12.0 \mathrm{~Hz}, 1 \mathrm{H})$, 0.01 (s, 9H), -0.07 (s, 9H); ${ }^{13} \mathrm{C}$ NMR (100 MHz, $\left.\mathrm{CDCl}_{3}\right) \delta 177.4,155.2,143.3,140.6$, $140.2,135.7,129.6,128.2,126.8,126.4,122.6,121.8,121.5,115.9,115.5,60.2,44.0$, 41.8, 33.2, 24.0, 21.8, 21.4, -0.4, -0.5; IR (neat) $\mathrm{cm}^{-1} 2951,1597,1554,1465,1354$, 
1249, 1163, 1090, 1034, 950, 860, 838; HRMS (ESI-TOF) m/z: $[\mathrm{M}+\mathrm{H}]^{+}$Calcd for $\mathrm{C}_{30} \mathrm{H}_{45} \mathrm{~N}_{2} \mathrm{O}_{2} \mathrm{SSi}_{2}{ }^{+}:$553.2735, found 553.2730.

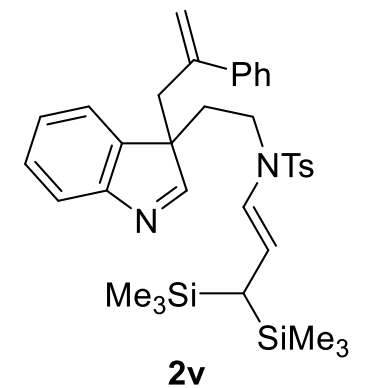

2v: The title compound were purified by silica gel column chromatography (gradient eluent: petroleum ether/EtOAc $=$ $50: 1 \rightarrow 10: 1)$ to afford the desired product $2 \mathbf{v}$ as a light yellow oil, $43 \mathrm{mg}$, 69\% yield. ${ }^{1} \mathrm{H}$ NMR (400 MHz, $\left.\mathrm{CDCl}_{3}\right) \delta 7.58(\mathrm{~s}$, 1H), $7.51(\mathrm{~d}, J=7.6 \mathrm{~Hz}, 1 \mathrm{H}), 7.40(\mathrm{~d}, J=8.4 \mathrm{~Hz}, 2 \mathrm{H})$, 7.27-7.20 (m, 4H), 7.16-7.08 (m, 6H), $5.90(\mathrm{~d} J=13.6 \mathrm{~Hz}, 1 \mathrm{H})$, $\mathrm{Hz}, 1 \mathrm{H}), 2.89(\mathrm{~d}, J=13.6 \mathrm{~Hz}, 1 \mathrm{H}), 2.66\left(\mathrm{ddd}, J_{1}=14.0 \mathrm{~Hz}, J_{2}=11.6 \mathrm{~Hz}, J_{3}=5.2 \mathrm{~Hz}\right.$, $1 \mathrm{H}), 2.43\left(\mathrm{ddd}, J_{1}=16.4 \mathrm{~Hz}, J_{2}=12.0 \mathrm{~Hz}, J_{3}=5.2 \mathrm{~Hz}, 1 \mathrm{H}\right), 2.32(\mathrm{~s}, 3 \mathrm{H}), 2.27-2.12$ $(\mathrm{m}, 2 \mathrm{H}), 0.86(\mathrm{~d}, J=12.0 \mathrm{~Hz}, 1 \mathrm{H}),-0.03(\mathrm{~s}, 9 \mathrm{H}),-0.11(\mathrm{~s}, 9 \mathrm{H}) ;{ }^{13} \mathrm{C} \mathrm{NMR}(100 \mathrm{MHz}$, $\left.\mathrm{CDCl}_{3}\right) \delta 176.7,155.1,143.7,143.3,141.2,140.2,135.7,129.6,128.4,128.1,127.7$, 126.8, 126.3, 126.2, 122.6, 122.0, 121.3, 117.6, 115.8, 60.2, 42.1, 41.9, 32.7, 21.8, 21.4, -0.4, -0.5; IR (neat) $\mathrm{cm}^{-1} 2951,1597,1553,1493,1465,1353,1248,1162,1090$, 1032, 957, 859, 837, 758; HRMS (ESI-TOF) m/z: $[\mathrm{M}+\mathrm{H}]^{+}$Calcd for $\mathrm{C}_{35} \mathrm{H}_{47} \mathrm{~N}_{2} \mathrm{O}_{2} \mathrm{SSi}_{2}{ }^{+}:$615.2891, found 615.2889.

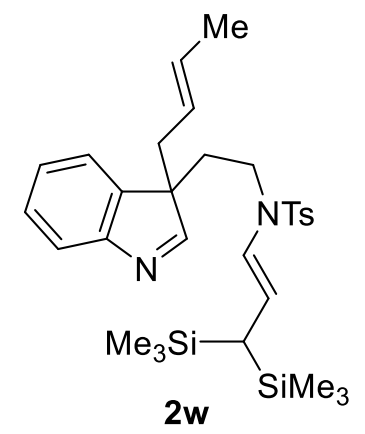

$(\mathrm{d}, J=14.0 \mathrm{~Hz}, 1 \mathrm{H}), 5.52-5.43(\mathrm{~m}, 1 \mathrm{H}), 5.22-5.15(\mathrm{~m}, 1 \mathrm{H}), 4.66\left(\mathrm{dd}, J_{1}=13.6 \mathrm{~Hz}, J_{2}\right.$ $=12.0 \mathrm{~Hz}, 1 \mathrm{H}), 2.81\left(\mathrm{ddd}, J_{1}=14.0 \mathrm{~Hz}, J_{2}=11.2 \mathrm{~Hz}, J_{3}=5.2 \mathrm{~Hz}, 1 \mathrm{H}\right), 2.51\left(\mathrm{ddd}, J_{1}\right.$ $\left.=14.0 \mathrm{~Hz}, J_{2}=11.2 \mathrm{~Hz}, J_{3}=5.6 \mathrm{~Hz}, 1 \mathrm{H}\right), 2.40-2.37(\mathrm{~m}, 5 \mathrm{H}), 2.32-2.18(\mathrm{~m}, 2 \mathrm{H}), 1.60$ $(\mathrm{d}, J=5.6 \mathrm{~Hz}, 3 \mathrm{H}), 0.93(\mathrm{~d}, J=12.4 \mathrm{~Hz}, 1 \mathrm{H}), 0.03(\mathrm{~s}, 9 \mathrm{H}),-0.05(\mathrm{~s}, 9 \mathrm{H}) ;{ }^{13} \mathrm{C} \mathrm{NMR}$ $\left(100 \mathrm{MHz}, \mathrm{CDCl}_{3}\right) \delta 177.5,155.3,143.3,140.6,135.7,130.1,129.6,128.1,126.8$, 126.3, 124.4, 122.6, 121.8, 121.4, 115.8, 60.1, 41.9, 38.6, 31.9, 21.8, 21.4, 17.9, -0.4, 
-0.5; IR (neat) $\mathrm{cm}^{-1} 2952,1597,1555,1454,1353,1248,1161,1090,1033,953,858$, 826; HRMS (ESI-TOF) m/z: $[\mathrm{M}+\mathrm{Na}]^{+}$Calcd for $\mathrm{C}_{30} \mathrm{H}_{44} \mathrm{~N}_{2} \mathrm{O}_{2} \mathrm{SSi}_{2} \mathrm{Na}^{+}:$575.2554, found 575.2556 .

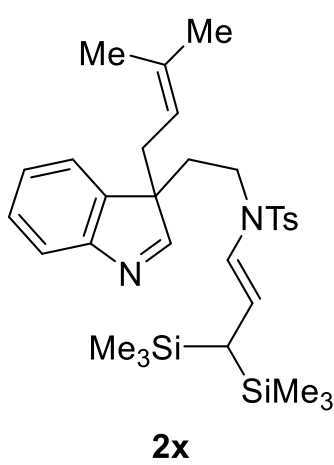

2x: The title compound were purified by silica gel column chromatography (gradient eluent: petroleum ether/EtOAc $=$ $50: 1 \rightarrow 10: 1)$ to afford the desired product $\mathbf{2} \mathbf{x}$ as a light yellow oil, $17 \mathrm{mg}, 29 \%$ yield. ${ }^{1} \mathrm{H} \mathrm{NMR}\left(400 \mathrm{MHz}, \mathrm{CDCl}_{3}\right) \delta 8.04(\mathrm{~s}$, $1 \mathrm{H}), 7.62(\mathrm{~d}, J=7.6 \mathrm{~Hz}, 1 \mathrm{H}), 7.46(\mathrm{~d}, J=8.0 \mathrm{~Hz}, 2 \mathrm{H})$, 7.36-7.27 (m, 3H), 7.19 (d, $J=8.0 \mathrm{~Hz}, 2 \mathrm{H}), 5.96(\mathrm{~d}, J=13.6$ $\mathrm{Hz}, 1 \mathrm{H}), 4.19(\mathrm{t}, J=7.6 \mathrm{~Hz}, 1 \mathrm{H}), 4.66\left(\mathrm{dd}, J_{1}=13.6 \mathrm{~Hz}, J_{2}=12.0 \mathrm{~Hz}, 1 \mathrm{H}\right), 2.79$ (ddd, $\left.J_{1}=14.0 \mathrm{~Hz}, J_{2}=11.2 \mathrm{~Hz}, J_{3}=5.2 \mathrm{~Hz}, 1 \mathrm{H}\right), 2.54-2.37(\mathrm{~m}, 3 \mathrm{H}), 2.36(\mathrm{~s}, 3 \mathrm{H})$, 2.29-2.22 (m, 2H), $1.62(\mathrm{~s}, 3 \mathrm{H}), 1.52(\mathrm{~s}, 3 \mathrm{H}), 0.91$ (d, J=12.0 Hz, 1H), 0.02 (s, 9H), -0.06 (s, 9H); ${ }^{13} \mathrm{C}$ NMR $\left(100 \mathrm{MHz}, \mathrm{CDCl}_{3}\right) \delta 177.7,155.3,143.3,140.8,135.8,135.7$, 129.6, 128.1, 126.8, 126.3, 122.6, 121.7, 121.4, 117.8, 115.6, 60.7, 41.9, 34.0, 31.8, 25.9, 21.8, 21.4, 17.9, -0.4, -0.5; IR (neat) $\mathrm{cm}^{-1} 2952,1597,1454,1354,1249,1163$, 1090, 1033, 948, 860, 838; HRMS (ESI-TOF) m/z: $[\mathrm{M}+\mathrm{H}]^{+}$Calcd for $\mathrm{C}_{31} \mathrm{H}_{47} \mathrm{~N}_{2} \mathrm{O}_{2} \mathrm{SSi}_{2}{ }^{+}:$567.2891, found 567.2893.

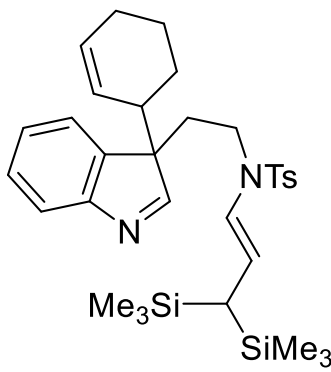

2y

2y: The title compound were purified by silica gel column chromatography (gradient eluent: petroleum ether/EtOAc $=$ $50: 1 \rightarrow 10: 1)$ to afford the desired product $\mathbf{2 y}$ as a light yellow oil, $46 \mathrm{mg}$, 79\% yield, 2:1 dr. ${ }^{1} \mathrm{H}$ NMR (400 $\left.\mathrm{MHz}, \mathrm{CDCl}_{3}\right) \delta$ $8.07(\mathrm{~s}, 1 \mathrm{H}), 7.62(\mathrm{~d}, J=7.6 \mathrm{~Hz}, 1 \mathrm{H}), 7.45(\mathrm{~d}, J=8.4 \mathrm{~Hz}, 2 \mathrm{H})$, $7.35(\mathrm{t}, J=7.2 \mathrm{~Hz}, 1 \mathrm{H}), 7.31-7.23(\mathrm{~m}, 2 \mathrm{H}), 7.18(\mathrm{~d}, J=8.0 \mathrm{~Hz}$, $2 \mathrm{H}), 5.95\left(\mathrm{dd}, J_{1}=13.6 \mathrm{~Hz}, J_{2}=7.2 \mathrm{~Hz}, 1 \mathrm{H}\right), 5.79-5.66(\mathrm{~m}, 1 \mathrm{H}), 5.18(\mathrm{~d}, J=10.4 \mathrm{~Hz}$, $1 \mathrm{H}), 4.64\left(\mathrm{dd}, J_{1}=13.6 \mathrm{~Hz}, J_{2}=12.0 \mathrm{~Hz}, 1 \mathrm{H}\right), 2.78\left(\mathrm{ddd}, J_{1}=13.6 \mathrm{~Hz}, J_{2}=10.0 \mathrm{~Hz}\right.$, $\left.J_{3}=6.4 \mathrm{~Hz}, 1 \mathrm{H}\right), 2.75-2.68(\mathrm{~m}, 1 \mathrm{H}), 2.54-2.40(\mathrm{~m}, 2 \mathrm{H}), 2.36(\mathrm{~s}, 3 \mathrm{H}), 2.33-2.19(\mathrm{~m}$, 2H), 1.92-1.84 (m, 3H), 1.50-1.42 (m, 2H), 0.90 (d, J = $12.0 \mathrm{~Hz}, 1 \mathrm{H}), 0.01(\mathrm{~s}, 9 \mathrm{H})$, -0.07 (s, 9H); ${ }^{13} \mathrm{C}$ NMR (100 MHz, $\left.\mathrm{CDCl}_{3}\right) \delta 177.1,155.7,143.3,139.9,135.7,130.6$, $129.6,128.2,126.8,126.4,125.4,122.6,122.4,121.3,115.7,63.4,41.9,40.6,30.4$, 
25.3, 24.8, 22.0, 21.8, 21.4, -0.4, -0.5; IR (neat) $\mathrm{cm}^{-1} 3025,2950,1634,1597,1555$, 1494, 1454, 1354, 1248, 1162, 1090, 1033, 946, 859, 830; HRMS (ESI-TOF) m/z: [M $+\mathrm{Na}]^{+}$Calcd for $\mathrm{C}_{32} \mathrm{H}_{46} \mathrm{~N}_{2} \mathrm{O}_{2} \mathrm{SSi}_{2} \mathrm{Na}^{+}:$601.2711, found 601.2716.

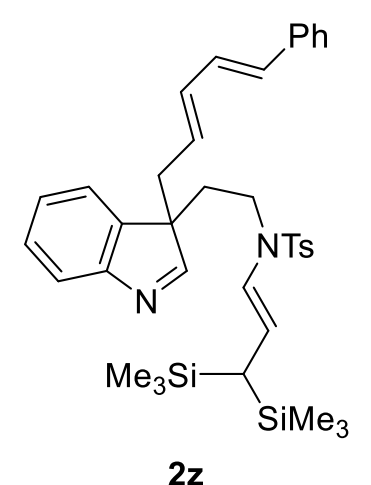

2z: The title compound were purified by silica gel column chromatography (gradient eluent: petroleum ether/EtOAc $=$ $50: 1 \rightarrow 10: 1)$ to afford the desired product $\mathbf{2 z}$ as a colorless oil, $54 \mathrm{mg}, 83 \%$ yield. ${ }^{1} \mathrm{H}$ NMR $\left(400 \mathrm{MHz}, \mathrm{CDCl}_{3}\right) \delta 8.09(\mathrm{~s}, 1 \mathrm{H})$, $7.64(\mathrm{~d}, J=8.0 \mathrm{~Hz}, 1 \mathrm{H}), 7.48(\mathrm{~d}, J=7.6 \mathrm{~Hz}, 2 \mathrm{H}), 7.39-7.27(\mathrm{~m}$, $7 \mathrm{H}), 7.22(\mathrm{~d}, J=7.2 \mathrm{~Hz}, 1 \mathrm{H}), 7.19(\mathrm{~d}, J=8.0 \mathrm{~Hz}, 2 \mathrm{H}), 6.61(\mathrm{dd}$, $\left.J_{1}=15.6 \mathrm{~Hz}, J_{2}=10.4 \mathrm{~Hz}, 1 \mathrm{H}\right), 6.45(\mathrm{~d}, J=15.6 \mathrm{~Hz}, 1 \mathrm{H}), 6.18$ $\left(\mathrm{dd}, J_{1}=15.2 \mathrm{~Hz}, J_{2}=10.4 \mathrm{~Hz}, 1 \mathrm{H}\right), 5.94(\mathrm{~d}, J=13.6 \mathrm{~Hz}, 1 \mathrm{H}), 5.48\left(\mathrm{dt}, J_{1}=15.2 \mathrm{~Hz}\right.$, $\left.J_{2}=7.6 \mathrm{~Hz}, 1 \mathrm{H}\right), 4.69\left(\mathrm{dd}, J_{1}=13.6 \mathrm{~Hz}, J_{2}=12.0 \mathrm{~Hz}, 1 \mathrm{H}\right), 2.84\left(\mathrm{ddd}, J_{1}=14.0 \mathrm{~Hz}, J_{2}\right.$ $\left.=11.2 \mathrm{~Hz}, J_{3}=5.2 \mathrm{~Hz}, 1 \mathrm{H}\right), 2.62-2.52(\mathrm{~m}, 3 \mathrm{H}), 2.36(\mathrm{~s}, 3 \mathrm{H}), 2.32-2.21(\mathrm{~m}, 2 \mathrm{H})$, $0.91(\mathrm{~d}, J=12.0 \mathrm{~Hz}, 1 \mathrm{H}), 0.01(\mathrm{~s}, 9 \mathrm{H}),-0.07(\mathrm{~s}, 9 \mathrm{H}) ;{ }^{13} \mathrm{C} \mathrm{NMR}\left(100 \mathrm{MHz}, \mathrm{CDCl}_{3}\right) \delta$ $177.1,155.3,143.3,140.4,137.1,135.7,134.7,132.0,129.6,128.6,128.3,128.2$, $127.5,127.4,126.8,126.5,126.3,122.6,121.8,121.6,116.4,60.3,42.0,38.7,32.2$, 21.9, 21.4, -0.4, -0.5; IR (neat) $\mathrm{cm}^{-1} 2951,1596,1554,1484,1454,1352,1248,1161$, 1090, 1033, 946, 859, 827, 753; HRMS (ESI-TOF) m/z: $[\mathrm{M}+\mathrm{H}]^{+}$Calcd for $\mathrm{C}_{37} \mathrm{H}_{49} \mathrm{~N}_{2} \mathrm{O}_{2} \mathrm{SSi}_{2}{ }^{+}$: 641.3048, found 641.3043.

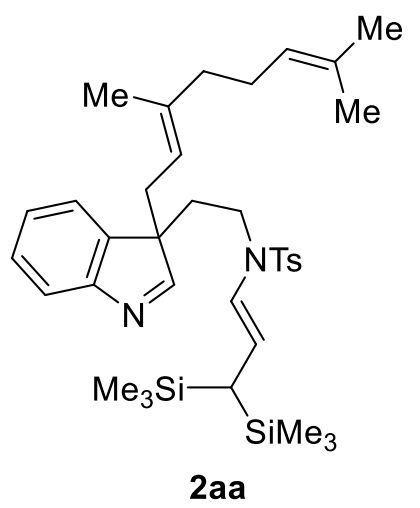

2aa: The title compound were purified by silica gel column chromatography (gradient eluent: petroleum ether/EtOAc $=$ $50: 1 \rightarrow 10: 1)$ to afford the desired product $2 \mathbf{a a}$ as a light yellow oil, $44 \mathrm{mg}, 68 \%$ yield. ${ }^{1} \mathrm{H}$ NMR $\left(400 \mathrm{MHz}, \mathrm{CDCl}_{3}\right)$ $\delta 8.04(\mathrm{~s}, 1 \mathrm{H}), 7.61(\mathrm{~d}, J=7.6 \mathrm{~Hz}, 1 \mathrm{H}), 7.46(\mathrm{~d}, J=8.0 \mathrm{~Hz}$, 2H), 7.36-7.29 (m, 2H), 7.25 (t, $J=7.2 \mathrm{~Hz}, 1 \mathrm{H}), 7.19$ (d, $J$ $=8.0 \mathrm{~Hz}, 2 \mathrm{H}), 5.95(\mathrm{~d}, J=13.6 \mathrm{~Hz}, 1 \mathrm{H}), 4.97(\mathrm{t}, J=7.2 \mathrm{~Hz}$,

$1 \mathrm{H}), 4.87(\mathrm{t}, J=7.6 \mathrm{~Hz}, 1 \mathrm{H}), 4.67\left(\mathrm{dd}, J_{1}=13.6 \mathrm{~Hz}, J_{2}=12.0 \mathrm{~Hz}, 1 \mathrm{H}\right), 2.81\left(\mathrm{ddd}, J_{1}\right.$ $\left.=14.0 \mathrm{~Hz}, J_{2}=11.2 \mathrm{~Hz}, J_{3}=5.6 \mathrm{~Hz}, 1 \mathrm{H}\right), 2.53\left(\mathrm{ddd}, J_{1}=14.0 \mathrm{~Hz}, J_{2}=11.2 \mathrm{~Hz}, J_{3}=\right.$ $5.6 \mathrm{~Hz}, 1 \mathrm{H}), 2.44(\mathrm{t}, J=7.2 \mathrm{~Hz}, 2 \mathrm{H}), 2.36(\mathrm{~s}, 3 \mathrm{H}), 2.32-2.19(\mathrm{~m}, 2 \mathrm{H}), 1.96-1.86(\mathrm{~m}$, 
4H), $1.66(\mathrm{~s}, 3 \mathrm{H}), 1.55(\mathrm{~s}, 3 \mathrm{H}), 1.51(\mathrm{~s}, 3 \mathrm{H}), 0.91(\mathrm{~d}, J=12.0 \mathrm{~Hz}, 1 \mathrm{H}), 0.01(\mathrm{~s}, 9 \mathrm{H})$, -0.07 (s, 9H); ${ }^{13} \mathrm{C}$ NMR (100 MHz, $\left.\mathrm{CDCl}_{3}\right) \delta 177.6,155.3,143.3,140.7,139.3,135.7$, $131.5,129.6,128.1,126.8,126.3,123.9,122.6,121.7,121.4,117.5,115.9,60.7,42.0$, $39.7,33.8,32.0,26.5,25.7,21.8,21.4,17.7,16.2,-0.4,-0.5$; IR (neat) $\mathrm{cm}^{-1} 2952$, 1597, 1555, 1454, 1355, 1249, 1163, 1090, 1034, 950, 860, 837; HRMS (ESI-TOF) $\mathrm{m} / \mathrm{z}:[\mathrm{M}+\mathrm{H}]^{+}$Calcd for $\mathrm{C}_{36} \mathrm{H}_{55} \mathrm{~N}_{2} \mathrm{O}_{2} \mathrm{SSi}_{2}{ }^{+}: 635.3517$, found 635.3520.

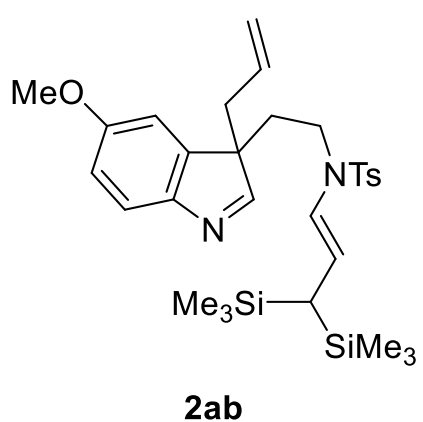

$2 a b$

2ab: The title compound were purified by silica gel column chromatography (gradient eluent: petroleum ether/EtOAc $=50: 1 \rightarrow 10: 1)$ to afford the desired product 2ab as a light yellow oil, $50 \mathrm{mg}, 87 \%$ yield. ${ }^{1} \mathrm{H}$ NMR (400 $\left.\mathrm{MHz}, \mathrm{CDCl}_{3}\right) \delta 7.91(\mathrm{~s}, 1 \mathrm{H}), 7.52(\mathrm{~d}, J=9.2 \mathrm{~Hz}, 1 \mathrm{H}), 7.47$ $(\mathrm{d}, J=8.0 \mathrm{~Hz}, 2 \mathrm{H}), 7.19(\mathrm{~d}, J=8.0 \mathrm{~Hz}, 2 \mathrm{H}), 6.87-6.84(\mathrm{~m}$, $2 \mathrm{H}), 5.91(\mathrm{~d}, J=13.6 \mathrm{~Hz}, 1 \mathrm{H}), 5.51-5.41(\mathrm{~m}, 1 \mathrm{H}), 5.04-4.98(\mathrm{~m}, 2 \mathrm{H}), 4.78\left(\mathrm{dd}, J_{1}=\right.$ $\left.13.6 \mathrm{~Hz}, J_{2}=12.0 \mathrm{~Hz}, 1 \mathrm{H}\right), 3.82(\mathrm{~s}, 3 \mathrm{H}), 2.85\left(\mathrm{ddd}, J_{1}=14.0 \mathrm{~Hz}, J_{2}=11.6 \mathrm{~Hz}, J_{3}=\right.$ $5.2 \mathrm{~Hz}, 1 \mathrm{H}), 2.66\left(\mathrm{ddd}, J_{1}=14.0 \mathrm{~Hz}, J_{2}=11.2 \mathrm{~Hz}, J_{3}=5.2 \mathrm{~Hz}, 1 \mathrm{H}\right), 2.51-2.41(\mathrm{~m}$, 2H), $2.36(\mathrm{~s}, 3 \mathrm{H}), 2.28-2.11(\mathrm{~m}, 2 \mathrm{H}), 0.92(\mathrm{~d}, J=12.4 \mathrm{~Hz}, 1 \mathrm{H}), 0.00(\mathrm{~s}, 9 \mathrm{H}),-0.05(\mathrm{~s}$, $9 \mathrm{H}) ;{ }^{13} \mathrm{C} \mathrm{NMR}\left(100 \mathrm{MHz}, \mathrm{CDCl}_{3}\right) \delta 174.8,158.8,149.0,143.3,142.1,135.6,131.8$, $129.6,126.8,122.5,121.7,119.2,117.0,112.5,108.7,59.9,55.6,42.1,39.8,32.3$, 21.8, 21.4, -0.4, -0.6. IR (neat) $\mathrm{cm}^{-1} 2952,1592,1469,1353,1248,1161,1090,1063$, 1030, 952, 859; HRMS (ESI-TOF) m/z: $[\mathrm{M}+\mathrm{Na}]^{+}$Calcd for $\mathrm{C}_{30} \mathrm{H}_{44} \mathrm{~N}_{2} \mathrm{O}_{3} \mathrm{SSi}_{2} \mathrm{Na}^{+}$: 591.2503, found 591.2495.

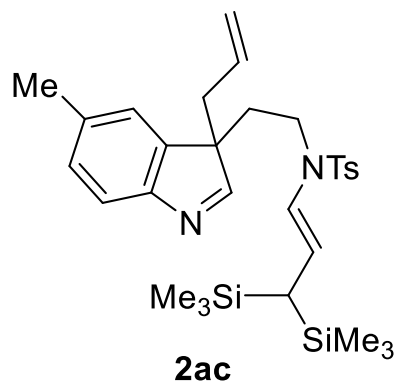

2ac: The title compound were purified by silica gel column chromatography (gradient eluent: petroleum ether/EtOAc $=$ $50: 1 \rightarrow 10: 1)$ to afford the desired product $\mathbf{2 a c}$ as a light yellow oil, $40 \mathrm{mg}, 75 \%$ yield. ${ }^{1} \mathrm{H}$ NMR $\left(400 \mathrm{MHz}, \mathrm{CDCl}_{3}\right) \delta$ $7.98(\mathrm{~s}, 1 \mathrm{H}), 7.49$ (d, $J=8.4 \mathrm{~Hz}, 1 \mathrm{H}), 7.47(\mathrm{~d}, J=8.0 \mathrm{~Hz}$, 2H), $7.19(\mathrm{~d}, J=8.0 \mathrm{~Hz}, 2 \mathrm{H}), 7.15(\mathrm{~d}, J=8.0 \mathrm{~Hz}, 1 \mathrm{H}), 7.10$ (s, 1H), $5.92(\mathrm{~d}, J=13.6 \mathrm{~Hz}, 1 \mathrm{H}), 5.51-5.41(\mathrm{~m}, 1 \mathrm{H}), 5.04-4.97(\mathrm{~m}, 2 \mathrm{H}), 4.76\left(\mathrm{dd}, J_{1}\right.$ $\left.=13.6 \mathrm{~Hz}, J_{2}=12.0 \mathrm{~Hz}, 1 \mathrm{H}\right), 2.85\left(\mathrm{ddd}, J_{1}=14.0 \mathrm{~Hz}, J_{2}=10.8 \mathrm{~Hz}, J_{3}=5.6 \mathrm{~Hz}, 1 \mathrm{H}\right)$, 
$2.63\left(\mathrm{ddd}, J_{1}=14.0 \mathrm{~Hz}, J_{2}=10.8 \mathrm{~Hz}, J_{3}=5.6 \mathrm{~Hz}, 1 \mathrm{H}\right), 2.51-2.43(\mathrm{~m}, 2 \mathrm{H}), 2.40(\mathrm{~s}$, $3 \mathrm{H}), 2.36(\mathrm{~s}, 3 \mathrm{H}), 2.28-2.15(\mathrm{~m}, 2 \mathrm{H}), 0.92(\mathrm{~d}, J=12.0 \mathrm{~Hz}, 1 \mathrm{H}), 0.01(\mathrm{~s}, 9 \mathrm{H}),-0.05(\mathrm{~s}$, $9 \mathrm{H}) ;{ }^{13} \mathrm{C} \mathrm{NMR}\left(100 \mathrm{MHz}, \mathrm{CDCl}_{3}\right) \delta 176.2,153.2,143.3,140.5,136.3,135.7,132.0$, 129.6, 128.9, 126.8, 122.6, 122.4, 121.0, 119.1, 116.7, 59.6, 42.1, 39.8, 32.2, 21.8, 21.6, 21.4, -0.4, -0.5. IR (neat) $\mathrm{cm}^{-1} 2952,1737,1597,1463,1354,1245,1161,1091$, 1044, 951, 859; HRMS (ESI-TOF) m/z: $[\mathrm{M}+\mathrm{Na}]^{+}$Calcd for $\mathrm{C}_{30} \mathrm{H}_{44} \mathrm{~N}_{2} \mathrm{O}_{2} \mathrm{SSi}_{2} \mathrm{Na}^{+}$: 575.2554, found 575.2546.

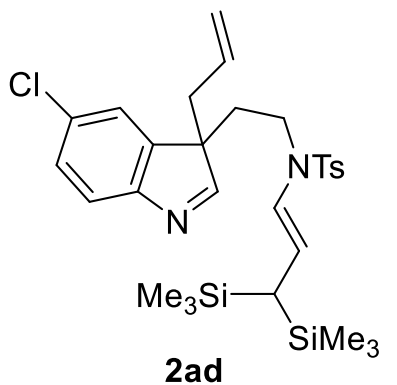

2ad: The title compound were purified by silica gel column chromatography (gradient eluent: petroleum ether/EtOAc $=$ $50: 1 \rightarrow 10: 1)$ to afford the desired product $\mathbf{2 a d}$ as a light yellow oil, $32 \mathrm{mg}, 55 \%$ yield. ${ }^{1} \mathrm{H}$ NMR $\left(400 \mathrm{MHz} \mathrm{CDCl}_{3}\right) \delta$ $8.07(\mathrm{~s}, 1 \mathrm{H}), 7.54(\mathrm{~d}, J=8.4 \mathrm{~Hz}, 1 \mathrm{H}), 7.50(\mathrm{~d}, J=8.4 \mathrm{~Hz}$, $2 \mathrm{H}), 7.34\left(\mathrm{dd}, J_{1}=8.0 \mathrm{~Hz}, J_{2}=2.0 \mathrm{~Hz}, 1 \mathrm{H}\right), 7.26(\mathrm{~d}, J=2.0$

$\mathrm{Hz}, 1 \mathrm{H}), 7.21(\mathrm{~d}, J=8.0 \mathrm{~Hz}, 2 \mathrm{H}), 5.87(\mathrm{~d}, J=13.2 \mathrm{~Hz}, 1 \mathrm{H}), 5.49-5.38(\mathrm{~m}, 1 \mathrm{H})$, 5.06-5.01 (m, 2H), $4.81\left(\mathrm{dd}, J_{1}=13.6 \mathrm{~Hz}, J_{2}=12.4 \mathrm{~Hz}, 1 \mathrm{H}\right), 2.89$ (ddd, $J_{1}=14.0 \mathrm{~Hz}$, $\left.J_{2}=11.2 \mathrm{~Hz}, J_{3}=5.2 \mathrm{~Hz}, 1 \mathrm{H}\right), 2.65\left(\mathrm{ddd}, J_{1}=14.0 \mathrm{~Hz}, J_{2}=10.8 \mathrm{~Hz}, J_{3}=5.2 \mathrm{~Hz}, 1 \mathrm{H}\right)$, $2.49(\mathrm{~d}, J=6.8 \mathrm{~Hz}, 2 \mathrm{H}), 2.37(\mathrm{~s}, 3 \mathrm{H}), 2.29-2.13(\mathrm{~m}, 2 \mathrm{H}), 0.93(\mathrm{~d}, J=12.4 \mathrm{~Hz}, 1 \mathrm{H})$, 0.00 (s, 9H), -0.05 (s, 9H); ${ }^{13} \mathrm{C}$ NMR (100 MHz, $\left.\mathrm{CDCl}_{3}\right) \delta 177.4,153.8,143.4,142.3$, $135.5,132.5,131.3,129.6,128.5,126.9,122.4,122.3,119.7,118.1,60.4,42.2,39.4$, 32.1, 21.9, 21.5, -0.4, -0.5. IR (neat) $\mathrm{cm}^{-1} 2953,1737,1598,1448,1352,1247,1161$, 1090, 1044, 952, 859; HRMS (ESI-TOF) m/z: $[\mathrm{M}+\mathrm{Na}]^{+}$Calcd for $\mathrm{C}_{29} \mathrm{H}_{41} \mathrm{ClN}_{2} \mathrm{O}_{2} \mathrm{SSi}_{2} \mathrm{Na}^{+}:$595.2008, found 595.2005. 
2.5. Screening of asymmetric reaction conditions to form (S)-2t using diphosphine ligands and phosphoramidite ligands ${ }^{[a]}$.

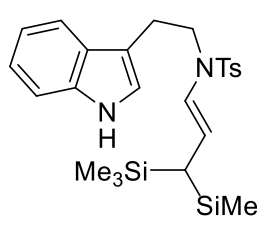

$7 \mathbf{a}$

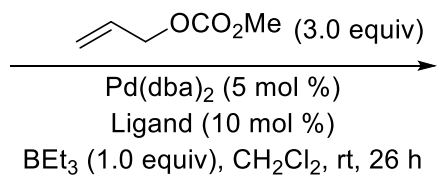

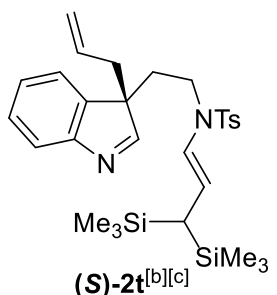

(S) $-2 \mathrm{t}^{[\mathrm{b}][\mathrm{c}]}$

A: biphosphine ligand

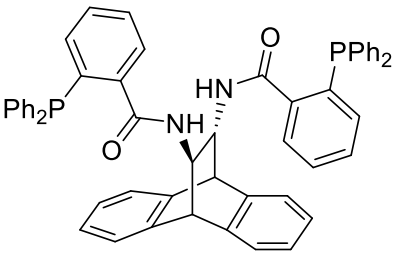

L1: $57 \%$ yield, $37 \%$ ee<smiles>CC1(C)O[C@H](CP)[C@@H](CP)O1</smiles>

L8: $92 \%$ yield, racemic<smiles>CC(P)P(P)c1ccccc1</smiles>

L11: NR

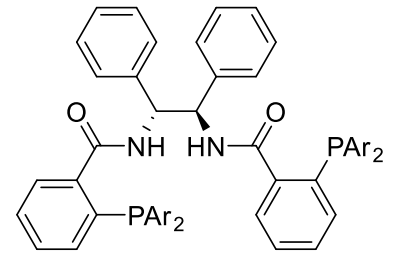

L2: $\mathrm{Ar}=\mathrm{Ph}, 31 \%$ yield, $43 \%$ ee L3: $\mathrm{Ar}=2-\mathrm{Me}-\mathrm{Ph}, \mathrm{NR}$ L4: $\mathrm{Ar}=4-\mathrm{Me}-\mathrm{Ph}, \mathrm{NR}$<smiles></smiles>

L9: NR

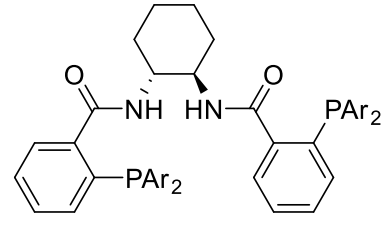

L5: $\mathrm{Ar}=\mathrm{Ph}, 60 \%$ yield, $8 \%$ ee

L6: $\mathrm{Ar}=3-\mathrm{Me}-\mathrm{Ph}, \mathrm{NR}$ L7: $\mathrm{Ar}=4-\mathrm{Me}-\mathrm{Ph}, \mathrm{NR}$

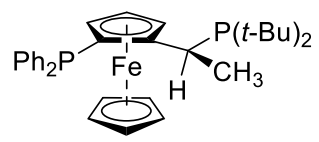

L10: NR

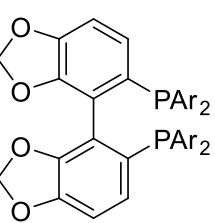

L12: $\mathrm{Ar}=3,5-\mathrm{Me}-\mathrm{C}_{6} \mathrm{H}_{3}, 64 \%$ yield, $15 \%$ ee

L13: $\mathrm{Ar}=3,5$-di-tert-butyl-4-methoxyphenyl $11 \%$ yield, $2 \%$ ee<smiles></smiles>

L14: NR<smiles>COc1ccccc1P(CCP(c1ccccc1)c1ccccc1OC)c1ccccc1</smiles>

L15: NR<smiles>Pc1ccccc1</smiles>

L16: NR 
B: monophosphine ligand

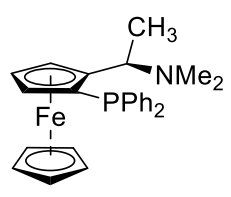

L17: $(S)-(R)-P P F A: ~ N R$

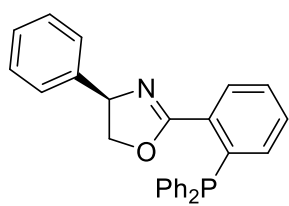

L18: Stoltz type ligand: NR

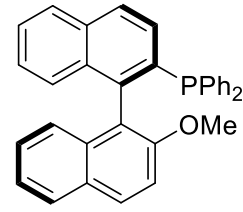

L19: (R)-MOP: NR

C: phosphoramidite ligand<smiles>[R]c1cc2ccccc2c(-c2c([R])cc3ccccc3c2OP(O)N(C)C)c1[R]</smiles>
L20: $R^{1}=H, 85 \%$ yield, $9.5 \%$ ee
L21: $\mathrm{R}^{1}=\mathrm{Cl}, 41 \%$ yield, $2 \%$ ee
L22: $\mathrm{R}^{1}=\mathrm{Me}, 51 \%$ yield, $12.5 \%$ ee L23: $\mathrm{R}^{1}=n-\mathrm{Bu}, \mathrm{NR}$
L24: $\mathrm{R}^{1}=\mathrm{Ph}, 35 \%$ yield, $3 \%$ ee
L25: $\mathrm{R}^{1}=4-\mathrm{OMeC}_{6} \mathrm{H}_{4}, \mathrm{NR}$

L26: $\mathrm{R}^{1}=2-$ Naphth, NR<smiles>[R]N([R])P(Oc1cc2ccccc2cc1C)Oc1c(C)cc2ccccc2c1-c1ccccc1</smiles>

L27: $\mathrm{R}^{2}=i-\mathrm{Pr}, 12 \%$ yield, racemic

L28: $R^{2}=B n, 27 \%$ yield, racemic

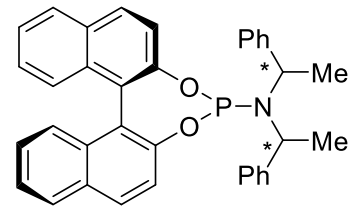

L29: $\mathrm{R}_{\mathrm{a}} \mathrm{S}_{\mathrm{c}} \mathrm{S}_{\mathrm{c}}, 77 \%$ yield, racemic<smiles></smiles>

L30: NR

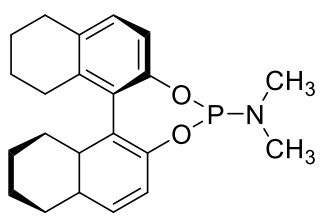

L31: NR

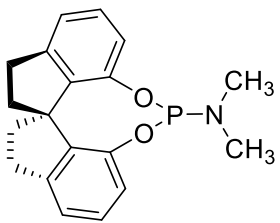

L32: NR

${ }^{a}$ Reaction conditions: 7a $(0.1 \mathrm{mmol})$, allylic methyl carbonate $(0.3 \mathrm{mmol}), \mathrm{Pd}(\mathrm{dba})_{2}(5 \mathrm{~mol} \%)$, ligand (10 mol \%), $\mathrm{BEt}_{3}(0.1 \mathrm{mmol}), 2.0 \mathrm{~mL} \mathrm{CH}_{2} \mathrm{Cl}_{2}, \mathrm{rt}, 26 \mathrm{~h} .{ }^{b}$ Isolated yields. ${ }^{\mathrm{c}}$ Determined by HPLC.

\subsection{General procedure of asymmetric reaction conditions with $\mathrm{Pd}(\mathrm{dba})_{2}$.}

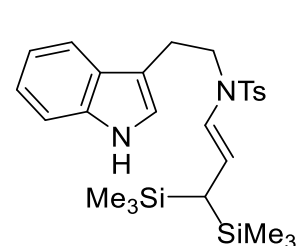

$7 a$

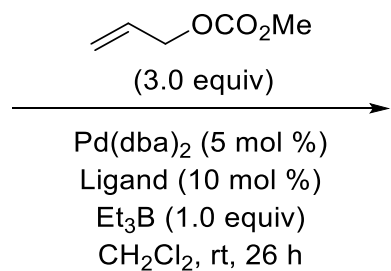

$\mathrm{CH}_{2} \mathrm{Cl}_{2}$, rt, $26 \mathrm{~h}$

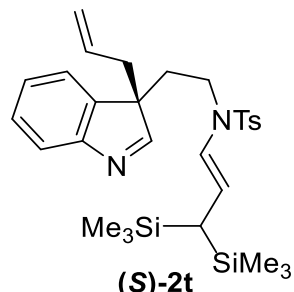

(S)-2t

A solution of $\mathrm{Pd}(\mathrm{dba})_{2}(5.8 \mathrm{mg}, 5 \mathrm{~mol} \%)$ and Ligand (10 mol \%) in $\mathrm{CH}_{2} \mathrm{Cl}_{2}(2.0 \mathrm{~mL})$ was stirred for $10 \mathrm{~min}$ at room temperature under argon. To the above solution were 
added $7 \mathbf{a}(50 \mathrm{mg}, 0.1 \mathrm{mmol})$ and $\mathrm{Et}_{3} \mathrm{~B}(0.1 \mathrm{~mL}, 0.1 \mathrm{mmol}, 1.0 \mathrm{~mol} / \mathrm{L}$ in hexane $)$. The reaction was stirred for $5 \mathrm{~min}$ before adding allylic methyl carbonate ( $34 \mu \mathrm{L}, 0.3$ $\mathrm{mmol})$. The reaction was stirred at room temperature for $26 \mathrm{~h}$ before concentration by rotary evaporation. The crude products were purified by silica gel column chromatography (gradient eluent: petroleum ether/EtOAc $=50: 1 \rightarrow 10: 1$ ) to afford (S)-2t. The ee was determined on a Daicel Chiralpak ${ }^{\circledR}$ IC column with hexane $/ 2$-propanol $=20 / 1$, flow $=1 \mathrm{~mL} / \mathrm{min}$.

(S)-2t: $31 \mathrm{mg}, 57 \%$ yield, 37\% ee (using $\mathbf{L 1}$ as ligand in the reaction). The ee was determined on a Daicel Chiralpak ${ }^{\circledR}$ IC column with hexane/2-propanol $=20 / 1$, flow $=$ $1 \mathrm{~mL} / \mathrm{min}$. Retention times: $21.1 \mathrm{~min}$ [major enantiomer], $28.4 \mathrm{~min}$ [minor enantiomer].

(S)-2t: $17 \mathrm{mg}, 31 \%$ yield, $43 \%$ ee (using $\mathbf{L 2}$ as ligand in the reaction). The ee was determined on a Daicel Chiralpak ${ }^{\circledR}$ IC column with hexane/2-propanol $=20 / 1$, flow $=$ $1 \mathrm{~mL} / \mathrm{min}$. Retention times: $21.8 \mathrm{~min}$ [major enantiomer], $30.0 \mathrm{~min}$ [minor enantiomer].
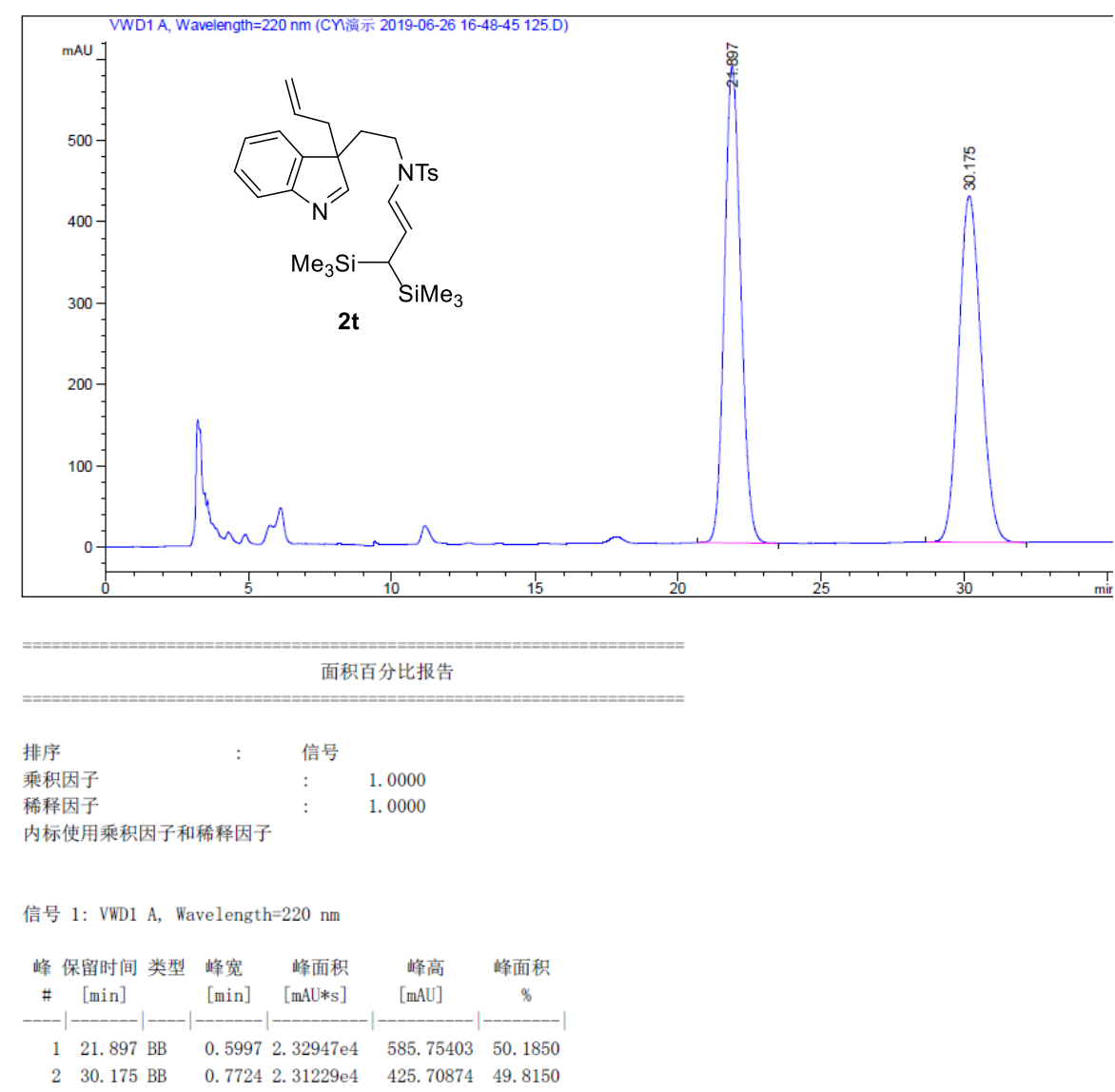


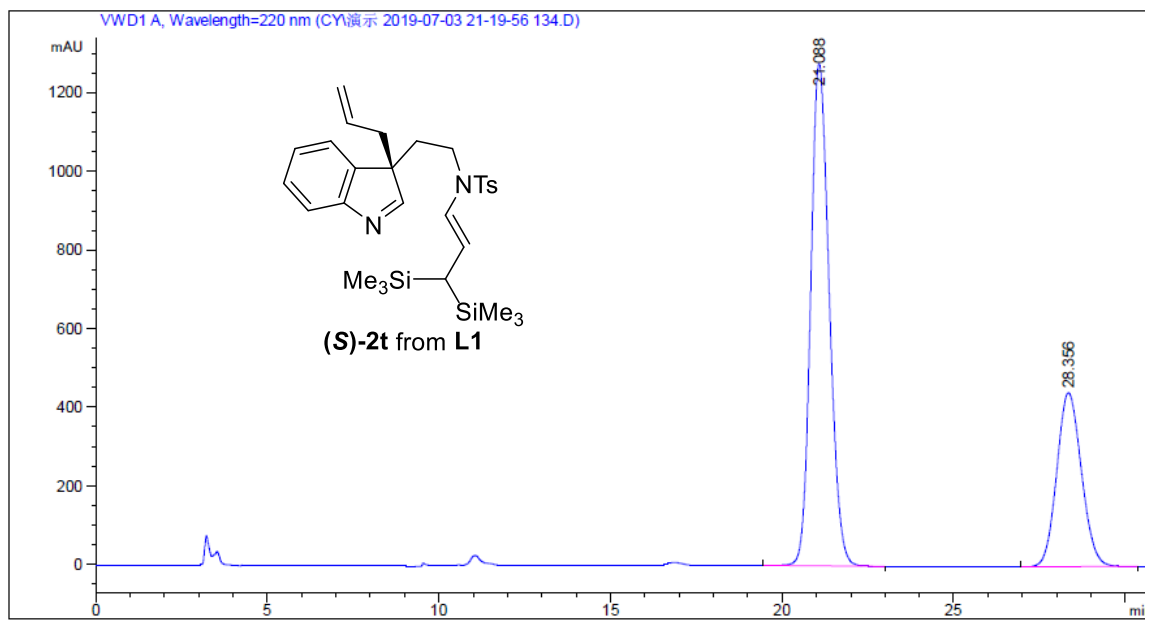

面积百分比报告

排序

信号

乘积因子 : $\quad 1.0000$

1.0000

内标使用乘积因子和稀释因子

信号 1: VWD1 A, Wavelength=220 nm

峰 保留时间 类型峰宽峰面积峰高峰面积

[min] [mAU*s]

[mAU] $\%$

$\begin{array}{llllll}21.088 \mathrm{BB} & 0.5487 & 4.79637 \mathrm{e} 4 & 1278.54968 & 68.4558\end{array}$

$\begin{array}{llllll}2 & 28.356 \mathrm{BB} & 0.6978 & 2.21015 \mathrm{e} 4 & 442.47772 & 31.5442\end{array}$

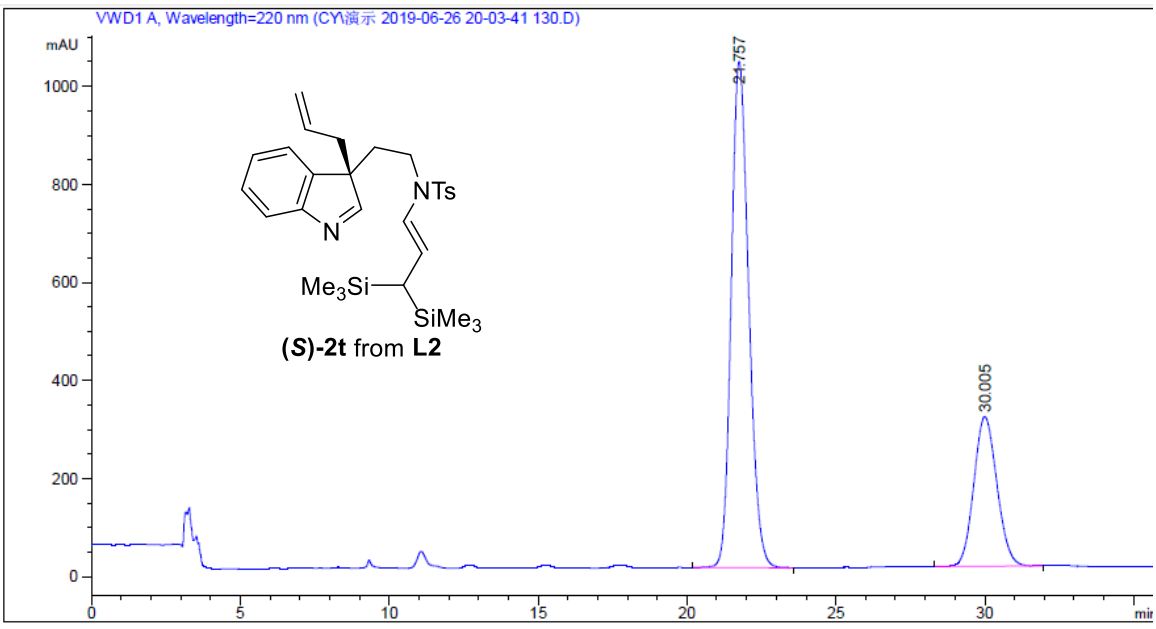

面积百分比报告

排序

信号

乘积因子

: $\quad 1.0000$

稀释因子

1.0000

内标使用乘积因子和稀释因子

信号 1: VWD1 A, Wavelength=220 nm

峰 保留时间 类型峰宽峰面积峰高峰面积

\# [min] [min] [mAU*s] [mAU] \%

$\begin{array}{llllll}1 & 21.757 \mathrm{BB} & 0.5896 & 4.20709 \mathrm{e} 4 & 1031.98071 & 71.8312\end{array}$

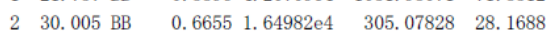




\subsection{Preparation of $3 a-3 v$.}
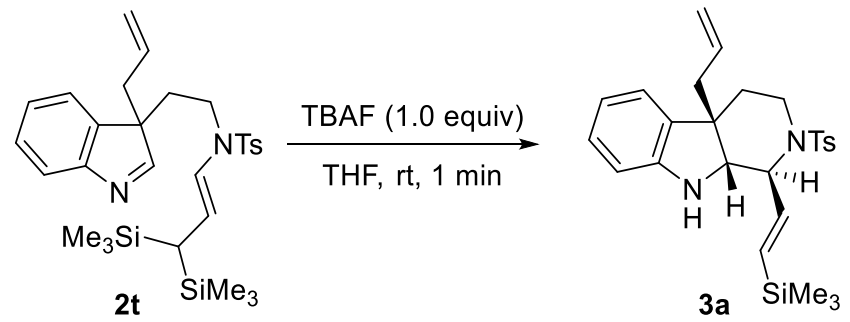

To a solution of $\mathbf{2 t}(54 \mathrm{mg}, 0.1 \mathrm{mmol})$ in THF $(1 \mathrm{~mL})$ at room temperature under argon was added $4 \AA$ molecular sieve $(50 \mathrm{mg})$ and TBAF $(0.1 \mathrm{~mL}, 0.1 \mathrm{mmol}, 1.0$ $\mathrm{mol} / \mathrm{L}$ in THF). The reaction was stirred for $1 \mathrm{~min}$ before quickly quenching with sat. aq. $\mathrm{NH}_{4} \mathrm{Cl}$ until $\mathrm{pH} \sim 7$. The aqueous layer was extracted with $\mathrm{CH}_{2} \mathrm{Cl}_{2}(3 \times 5 \mathrm{~mL})$ and $\mathrm{Et}_{2} \mathrm{O}(2 \times 5 \mathrm{~mL})$. The combined organic layers were dried over anhydrous $\mathrm{Na}_{2} \mathrm{SO}_{4}$, concentrated by rotary evaporation. The crude products were purified by silica gel column chromatography (gradient eluent: petroleum ether/EtOAc $=100: 1 \rightarrow 20: 1$ ) to afford the desired product 3a as a colorless oil, $40 \mathrm{mg}$, 86\% yield, 9:1 $d r$.

To a solution of $\mathbf{2 t}(540 \mathrm{mg}, 1 \mathrm{mmol})$ in $\mathrm{THF}(10 \mathrm{~mL})$ at room temperature under argon was added $4 \AA$ molecular sieve $(500 \mathrm{mg})$ and TBAF $(1 \mathrm{~mL}, 1 \mathrm{mmol}, 1.0 \mathrm{~mol} / \mathrm{L}$ in THF). The reaction was stirred for 1 min before quickly quenching with sat. aq. $\mathrm{NH}_{4} \mathrm{Cl}$ until $\mathrm{pH} \sim 7$. The aqueous layer was extracted with $\mathrm{CH}_{2} \mathrm{Cl}_{2}(3 \times 50 \mathrm{~mL})$ and $\mathrm{Et}_{2} \mathrm{O}(2 \times 50 \mathrm{~mL})$. The combined organic layers were dried over anhydrous $\mathrm{Na}_{2} \mathrm{SO}_{4}$, concentrated by rotary evaporation. The crude products were purified by silica gel column chromatography (gradient eluent: petroleum ether/EtOAc $=100: 1 \rightarrow 20: 1$ ) to afford the desired product 3a as a colorless oil, $335 \mathrm{mg}, 76 \%$ yield, 9:1 $d r$.

${ }^{1} \mathrm{H}$ NMR (400 MHz, $\left.\mathrm{CDCl}_{3}\right) \delta 7.63(\mathrm{~d}, J=8.4 \mathrm{~Hz}, 2 \mathrm{H}), 7.22(\mathrm{~d}, J=8.0 \mathrm{~Hz}, 2 \mathrm{H}), 6.95$ $\left(\mathrm{td}, J_{1}=7.6 \mathrm{~Hz}, J_{2}=1.2 \mathrm{~Hz}, 1 \mathrm{H}\right), 6.89(\mathrm{~d}, J=7.2 \mathrm{~Hz}, 1 \mathrm{H}), 6.65(\mathrm{t}, J=7.6 \mathrm{~Hz}, 1 \mathrm{H})$, $6.22(\mathrm{~d}, J=7.6 \mathrm{~Hz}, 1 \mathrm{H}), 6.07\left(\mathrm{dd}, J_{1}=18.4 \mathrm{~Hz}, J_{2}=3.2 \mathrm{~Hz}, 1 \mathrm{H}\right), 6.01\left(\mathrm{dd}, J_{1}=18.4\right.$ $\left.\mathrm{Hz}, J_{2}=1.2 \mathrm{~Hz}, 1 \mathrm{H}\right), 5.59-5.48(\mathrm{~m}, 1 \mathrm{H}), 5.04-4.98(\mathrm{~m}, 2 \mathrm{H}), 4.25-4.24(\mathrm{~m}, 1 \mathrm{H}), 3.86$ $(\mathrm{d}, J=3.2 \mathrm{~Hz}, 1 \mathrm{H}), 3.55\left(\mathrm{dt}, J_{1}=11.2 \mathrm{~Hz}, J_{2}=4.4 \mathrm{~Hz}, 1 \mathrm{H}\right), 3.31(\mathrm{~s}, 1 \mathrm{H}), 2.87\left(\mathrm{td}, J_{1}\right.$ $\left.=11.2 \mathrm{~Hz}, J_{2}=3.6 \mathrm{~Hz}, 1 \mathrm{H}\right), 2.49-2.35(\mathrm{~m}, 1 \mathrm{H}), 2.42(\mathrm{~s}, 3 \mathrm{H}), 2.23\left(\mathrm{dd}, J_{1}=14.0 \mathrm{~Hz}\right.$, $\left.J_{2}=8.8 \mathrm{~Hz}, 1 \mathrm{H}\right), 1.92\left(\mathrm{ddd}, J_{1}=14.4 \mathrm{~Hz}, J_{2}=12.0 \mathrm{~Hz}, J_{3}=4.4 \mathrm{~Hz}, 1 \mathrm{H}\right), 1.72\left(\mathrm{dt}, J_{1}=\right.$ 
$\left.13.6 \mathrm{~Hz}, J_{2}=4.0 \mathrm{~Hz}, 1 \mathrm{H}\right), 0.09(\mathrm{~s}, 9 \mathrm{H}) ;{ }^{13} \mathrm{C} \mathrm{NMR}\left(100 \mathrm{MHz}, \mathrm{CDCl}_{3}\right) \delta 149.5,144.2$, $142.9,136.3,134.2,132.8,132.1,129.3,127.9,127.4,122.6,118.5,118.3,108.8$, 64.2, 61.0, 45.9, 44.4, 40.2, 33.2, 21.5, -1.4; IR (neat) $\mathrm{cm}^{-1} 3381,2952,1608,1486$, 1467, 1344, 1246, 1158, 1093, 998, 866, 837; HRMS (ESI-TOF) m/z: [M + H] Calcd for $\mathrm{C}_{26} \mathrm{H}_{35} \mathrm{~N}_{2} \mathrm{O}_{2} \mathrm{SSi}^{+}$: 467.2183, found 467.2176.

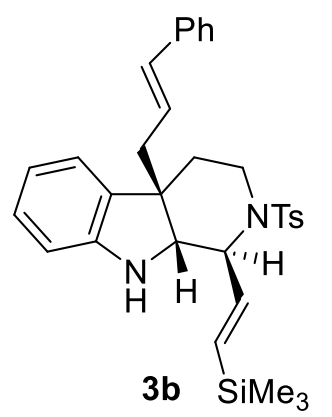

3b: The title compound were purified by silica gel column chromatography (gradient eluent: petroleum ether/EtOAc $=100: 1$ $\rightarrow 20: 1)$ to afford the desired product $\mathbf{3 b}$ as a colorless oil, $51 \mathrm{mg}$, 82\% yield, 7:1 dr. ${ }^{1} \mathrm{H}$ NMR (400 $\left.\mathrm{MHz}, \mathrm{CDCl}_{3}\right) \delta 7.65(\mathrm{~d}, J=8.4$ $\mathrm{Hz}, 2 \mathrm{H}), 7.32-7.28(\mathrm{~m}, 2 \mathrm{H}), 7.24-7.19(\mathrm{~m}, 4 \mathrm{H}), 6.99$ (t, $J=7.6$ $\mathrm{Hz}, 1 \mathrm{H}), 6.96(\mathrm{~d}, J=7.6 \mathrm{~Hz}, 1 \mathrm{H}), 6.70(\mathrm{t}, J=7.6 \mathrm{~Hz}, 1 \mathrm{H}), 6.38$ $(\mathrm{d}, J=16.0 \mathrm{~Hz}, 1 \mathrm{H}), 6.28(\mathrm{~d}, J=7.6 \mathrm{~Hz}, 1 \mathrm{H}), 6.13\left(\mathrm{dd}, J_{1}=18.8 \mathrm{~Hz}, J_{2}=3.6 \mathrm{~Hz}, 1 \mathrm{H}\right)$, $6.04\left(\mathrm{dd}, J_{1}=18.8 \mathrm{~Hz}, J_{2}=1.6 \mathrm{~Hz}, 1 \mathrm{H}\right), 6.00-5.93(\mathrm{~m}, 1 \mathrm{H}), 4.26-4.24(\mathrm{~m}, 1 \mathrm{H}), 3.91$ $(\mathrm{d}, J=2.8 \mathrm{~Hz}, 1 \mathrm{H}), 3.58\left(\mathrm{dt}, J_{1}=11.2 \mathrm{~Hz}, J_{2}=4.4 \mathrm{~Hz}, 1 \mathrm{H}\right), 3.38(\mathrm{~s}, 1 \mathrm{H}), 2.93\left(\mathrm{td}, J_{1}\right.$ $\left.=11.6 \mathrm{~Hz}, J_{2}=3.6 \mathrm{~Hz}, 1 \mathrm{H}\right), 2.65\left(\mathrm{dd}, J_{1}=15.2 \mathrm{~Hz}, J_{2}=6.4 \mathrm{~Hz}, 1 \mathrm{H}\right), 2.44(\mathrm{~s}, 3 \mathrm{H})$, 2.42-2.38 (m, 1H), $1.99\left(\mathrm{ddd}, J_{1}=14.4 \mathrm{~Hz}, J_{2}=11.2 \mathrm{~Hz}, J_{3}=4.0 \mathrm{~Hz}, 1 \mathrm{H}\right), 1.81(\mathrm{dt}$, $\left.J_{1}=14.0 \mathrm{~Hz}, J_{2}=4.0 \mathrm{~Hz}, 1 \mathrm{H}\right), 0.13(\mathrm{~s}, 9 \mathrm{H}) ;{ }^{13} \mathrm{C} \mathrm{NMR}\left(100 \mathrm{MHz}, \mathrm{CDCl}_{3}\right) \delta 149.4$, 144.0, 142.9, 137.0, 136.1, 133.3, 132.7, 132.3, 129.3, 128.5, 127.9, 127.4, 127.3, $126.1,125.8,122.6,118.6,108.9,64.3,61.1,46.3,43.6,40.3,33.3,21.5,-1.3$; IR (neat) $\mathrm{cm}^{-1} 3384,2921,1607,1485,1246,1159,1092,966,866,837$; HRMS (ESI-TOF) m/z: [M + H] $]^{+}$Calcd for $\mathrm{C}_{32} \mathrm{H}_{39} \mathrm{~N}_{2} \mathrm{O}_{2} \mathrm{SSi}^{+}$: 543.2496, found 543.2494.

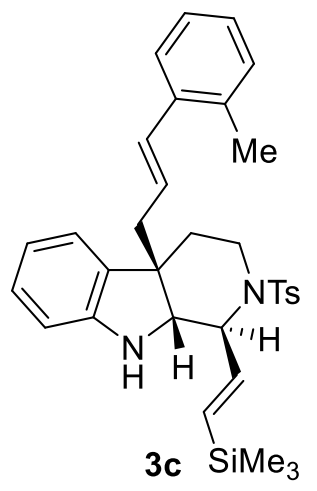

3c: The title compound were purified by silica gel column chromatography (gradient eluent: petroleum ether/EtOAc $=100: 1$ $\rightarrow 20: 1)$ to afford the desired product $\mathbf{3 c}$ as a colorless oil, $40 \mathrm{mg}$, $72 \%$ yield, 6:1 $d r .{ }^{1} \mathrm{H}$ NMR (400 MHz, $\left.\mathrm{CDCl}_{3}\right) \delta 7.63(\mathrm{~d}, J=8.4$ $\mathrm{Hz}, 2 \mathrm{H}), 7.24-7.19$ (m, 3H), 7.13-7.07 (m, 3H), 6.97 (dd, $J_{1}=7.6$ $\left.\mathrm{Hz}, J_{2}=1.2 \mathrm{~Hz}, 1 \mathrm{H}\right), 6.93\left(\mathrm{dd}, J_{1}=7.6 \mathrm{~Hz}, J_{2}=1.2 \mathrm{~Hz}, 1 \mathrm{H}\right), 6.66$ $(\mathrm{t}, J=7.6 \mathrm{~Hz}, 1 \mathrm{H}), 6.55(\mathrm{~d}, J=15.6 \mathrm{~Hz}, 1 \mathrm{H}), 6.22(\mathrm{~d}, J=7.6 \mathrm{~Hz}$, $1 \mathrm{H}), 6.13\left(\mathrm{dd}, J_{1}=18.8 \mathrm{~Hz}, J_{2}=3.6 \mathrm{~Hz}, 1 \mathrm{H}\right), 6.05\left(\mathrm{dd}, J_{1}=18.8 \mathrm{~Hz}, J_{2}=1.6 \mathrm{~Hz}, 1 \mathrm{H}\right)$, 
5.85-5.76 (m, 1H), 4.22-4.20 (m, 1H), $3.93(\mathrm{t}, J=2.8 \mathrm{~Hz}, 1 \mathrm{H}), 3.61\left(\mathrm{dt}, J_{1}=11.2 \mathrm{~Hz}\right.$, $\left.J_{2}=4.0 \mathrm{~Hz}, 1 \mathrm{H}\right), 3.31(\mathrm{~s}, 1 \mathrm{H}), 2.87\left(\mathrm{td}, J_{1}=11.6 \mathrm{~Hz}, J_{2}=3.6 \mathrm{~Hz}, 1 \mathrm{H}\right), 2.64\left(\mathrm{dd}, J_{1}=\right.$ $\left.14.0 \mathrm{~Hz}, J_{2}=6.0 \mathrm{~Hz}, 1 \mathrm{H}\right), 2.42(\mathrm{~s}, 3 \mathrm{H}), 2.41-2.37(\mathrm{~m}, 1 \mathrm{H}), 2.26(\mathrm{~s}, 3 \mathrm{H}), 2.06-1.97(\mathrm{~m}$, $1 \mathrm{H}), 1.80\left(\mathrm{dt}, J_{1}=14.0 \mathrm{~Hz}, J_{2}=3.6 \mathrm{~Hz}, 1 \mathrm{H}\right), 0.10(\mathrm{~s}, 9 \mathrm{H}) ;{ }^{13} \mathrm{C} \mathrm{NMR}(100 \mathrm{MHz}$, $\left.\mathrm{CDCl}_{3}\right) \delta 149.5,144.2,142.9,136.4,136.0,134.9,132.5,132.3,131.5,130.1,129.3$, 127.9, 127.4, 127.2, 127.2, 126.0, 125.6, 122.8, 118.5, 108.8, 64.4, 61.2, 46.4, 44.2, 40.5, 33.4, 21.5, 19.8, -1.3. IR (neat) $\mathrm{cm}^{-1} 3379,2923,1607,1486,1345,1259,1160$, 1089, 1016, 839, 812; HRMS (ESI-TOF) m/z: $[\mathrm{M}+\mathrm{Na}]^{+}$Calcd for $\mathrm{C}_{33} \mathrm{H}_{40} \mathrm{~N}_{2} \mathrm{O}_{2} \mathrm{SSiNa}^{+}:$579.2472, found 579.2468.

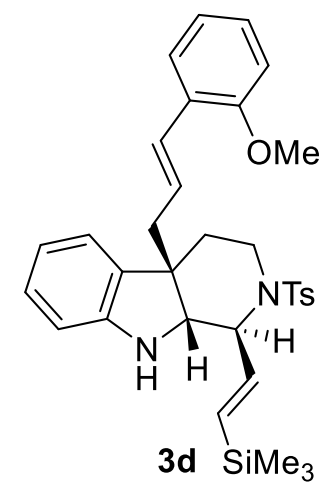

3d: The title compound were purified by silica gel column chromatography (gradient eluent: petroleum ether/EtOAc $=100: 1$ $\rightarrow 20: 1)$ to afford the desired product $\mathbf{3 d}$ as a colorless oil, $47 \mathrm{mg}$, $82 \%$ yield, 4:1 dr. ${ }^{1} \mathrm{H}$ NMR $\left(400 \mathrm{MHz}, \mathrm{CDCl}_{3}\right) \delta 7.63(\mathrm{~d}, J=8.4$ $\mathrm{Hz}, 2 \mathrm{H}), 7.25-7.14(\mathrm{~m}, 4 \mathrm{H}), 6.97\left(\mathrm{dd}, J_{1}=7.6 \mathrm{~Hz}, J_{2}=1.2 \mathrm{~Hz}\right.$, $1 \mathrm{H})$, 6.94-6.93 (m, 1H), 6.87-6.81 (m, 2H), 6.72-6.65 (m, 2H), $6.23(\mathrm{~d}, J=7.6 \mathrm{~Hz}, 1 \mathrm{H}), 6.10\left(\mathrm{dd}, J_{1}=18.8 \mathrm{~Hz}, J_{2}=3.2 \mathrm{~Hz}, 1 \mathrm{H}\right)$, $6.02\left(\mathrm{dd}, J_{1}=18.4 \mathrm{~Hz}, J_{2}=1.6 \mathrm{~Hz}, 2 \mathrm{H}\right), 5.94-5.85(\mathrm{~m}, 1 \mathrm{H}), 4.27-4.25(\mathrm{~m}, 1 \mathrm{H}), 3.94$ $(\mathrm{d}, J=3.2 \mathrm{~Hz}, 1 \mathrm{H}), 3.79(\mathrm{~s}, 3 \mathrm{H}), 3.55\left(\mathrm{dt}, J_{1}=11.2 \mathrm{~Hz}, J_{2}=4.0 \mathrm{~Hz}, 1 \mathrm{H}\right), 3.31(\mathrm{~s}, 1 \mathrm{H})$, $2.90\left(\mathrm{td}, J_{1}=11.6 \mathrm{~Hz}, J_{2}=3.6 \mathrm{~Hz}, 1 \mathrm{H}\right), 2.64\left(\mathrm{ddd}, J_{1}=14.0 \mathrm{~Hz}, J_{2}=5.6 \mathrm{~Hz}, J_{3}=1.6\right.$ $\mathrm{Hz}, 1 \mathrm{H}), 2.42(\mathrm{~s}, 3 \mathrm{H}), 2.41-2.37(\mathrm{~m}, 1 \mathrm{H}), 1.95\left(\mathrm{ddd}, J_{1}=14.0 \mathrm{~Hz}, J_{2}=11.2 \mathrm{~Hz}, J_{3}=\right.$ $4.0 \mathrm{~Hz}, 1 \mathrm{H}), 1.76\left(\mathrm{dt}, J_{1}=14.0 \mathrm{~Hz}, J_{2}=4.0 \mathrm{~Hz}, 1 \mathrm{H}\right), 0.11(\mathrm{~s}, 9 \mathrm{H}) ;{ }^{13} \mathrm{C} \mathrm{NMR}(100$ $\left.\mathrm{MHz}, \mathrm{CDCl}_{3}\right) \delta 156.3,149.5,144.1,142.8,136.2,132.9,132.1,129.3,128.3,128.0$, $127.8,127.4,126.4,126.3,126.2,122.7,120.5$, 118.5, 110.7, 108.8, 64.1, 60.9, 55.3, 46.3, 43.9, 40.2, 33.5, 21.5, -1.4. IR (neat) $\mathrm{cm}^{-1} 3367,2923,1600,1487,1344,1261$, 1160, 1090, 1019, 839, 800; HRMS (ESI-TOF) m/z: $[\mathrm{M}+\mathrm{Na}]^{+}$Calcd for $\mathrm{C}_{33} \mathrm{H}_{40} \mathrm{~N}_{2} \mathrm{O}_{3} \mathrm{SSiNa}^{+}$: 595.2421, found 595.2435. 


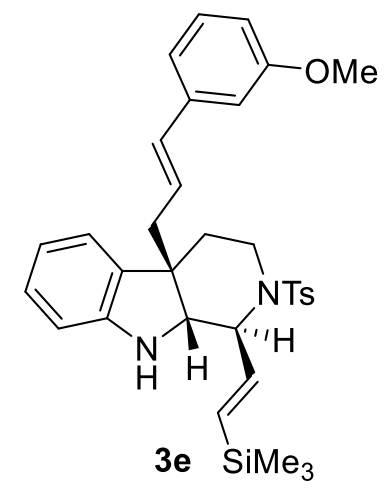

3e: The title compound were purified by silica gel column chromatography (gradient eluent: petroleum ether/EtOAc $=$ $100: 1 \rightarrow 20: 1)$ to afford the desired product $\mathbf{3 e}$ as a colorless oil, $43 \mathrm{mg}, 75 \%$ yield, 5:1 $d r .{ }^{1} \mathrm{H}$ NMR (400 $\left.\mathrm{MHz} \mathrm{CDCl}_{3}\right) \delta$ $7.63(\mathrm{~d}, J=8.4 \mathrm{~Hz}, 2 \mathrm{H}), 7.22-7.15(\mathrm{~m}, 3 \mathrm{H}), 6.98-6.90(\mathrm{~m}$, 2H), $6.84(\mathrm{~d}, J=8.0 \mathrm{~Hz}, 1 \mathrm{H}), 6.76-6.65(\mathrm{~m}, 3 \mathrm{H}), 6.32(\mathrm{~d}, J=$ $16.0 \mathrm{~Hz}, 1 \mathrm{H}), 6.25(\mathrm{~d}, J=7.6 \mathrm{~Hz}, 1 \mathrm{H}), 6.11\left(\mathrm{dd}, J_{1}=18.4 \mathrm{~Hz}\right.$, $\left.J_{2}=3.6 \mathrm{~Hz} 1 \mathrm{H}\right), 6.01\left(\mathrm{dd}, J_{1}=18.4 \mathrm{~Hz}, J_{2}=1.6 \mathrm{~Hz} 1 \mathrm{H}\right), 5.97-5.89(\mathrm{~m}, 1 \mathrm{H}), 4.22-4.20$ $(\mathrm{m}, 1 \mathrm{H}), 3.87(\mathrm{~d}, J=3.2 \mathrm{~Hz}, 1 \mathrm{H}), 3.77(\mathrm{~s}, 3 \mathrm{H}), 3.55\left(\mathrm{dt}, J_{1}=11.2 \mathrm{~Hz}, J_{2}=4.0 \mathrm{~Hz}\right.$, $1 \mathrm{H}), 3.54(\mathrm{~s}, 1 \mathrm{H}), 2.89\left(\mathrm{td}, J_{1}=11.2 \mathrm{~Hz}, J_{2}=3.2 \mathrm{~Hz}, 1 \mathrm{H}\right), 2.60\left(\mathrm{ddd}, J_{1}=14.0 \mathrm{~Hz}, J_{2}\right.$ $\left.=5.6 \mathrm{~Hz}, J_{3}=1.6 \mathrm{~Hz}, 1 \mathrm{H}\right), 2.41(\mathrm{~s}, 3 \mathrm{H}), 2.38(\mathrm{~d}, J=4.0 \mathrm{~Hz}, 1 \mathrm{H}), 1.95\left(\mathrm{ddd}, J_{1}=14.4\right.$ $\left.\mathrm{Hz}, J_{2}=11.2 \mathrm{~Hz}, J_{3}=4.0 \mathrm{~Hz}, 1 \mathrm{H}\right), 1.77\left(\mathrm{dt}, J_{1}=14.0 \mathrm{~Hz}, J_{2}=4.0 \mathrm{~Hz}, 1 \mathrm{H}\right), 0.10(\mathrm{~s}$, $9 \mathrm{H}) ;{ }^{13} \mathrm{C} \mathrm{NMR}\left(100 \mathrm{MHz}, \mathrm{CDCl}_{3}\right) \delta 159.7,149.4,143.9,142.9,138.5,136.1,133.2$, $132.6,132.3,129.5,129.3,127.9,127.4,126.2,122.6,118.7,118.6,112.5,111.8$, 108.9, 64.4, 61.2, 55.2, 46.3, 43.5, 40.3, 33.3, 21.5, -1.3. IR (neat) $\mathrm{cm}^{-1} 3368,2922$, 1603, 1464, 1343, 1261, 1159, 1090, 988, 839, 813; HRMS (ESI-TOF) m/z: [M + $\mathrm{Na}]^{+}$Calcd for $\mathrm{C}_{33} \mathrm{H}_{40} \mathrm{~N}_{2} \mathrm{O}_{3} \mathrm{SSiNa}^{+}$: 595.2421, found 595.2431.

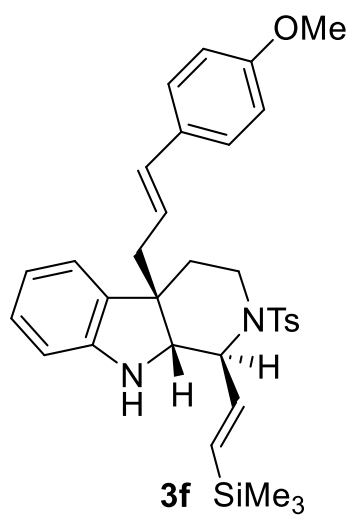

3f: The title compound were purified by silica gel column chromatography (gradient eluent: petroleum ether/EtOAc $=$ $100: 1 \rightarrow 20: 1)$ to afford the desired product $\mathbf{3 f}$ as a colorless oil, $51 \mathrm{mg}, 79 \%$ yield, 7:1 $d r .{ }^{1} \mathrm{H}$ NMR (400 MHz, $\left.\mathrm{CDCl}_{3}\right) \delta 7.63$ $(\mathrm{d}, J=8.4 \mathrm{~Hz}, 2 \mathrm{H}), 7.22-7.16(\mathrm{~m}, 4 \mathrm{H}), 6.97(\mathrm{t}, J=7.6 \mathrm{~Hz}, 1 \mathrm{H})$, $6.93(\mathrm{~d}, J=7.6 \mathrm{~Hz}, 1 \mathrm{H}), 6.80(\mathrm{~d}, J=8.4 \mathrm{~Hz}, 2 \mathrm{H}), 6.67(\mathrm{t}, J=$ $7.6 \mathrm{~Hz}, 1 \mathrm{H}), 6.30(\mathrm{~d}, J=16.0 \mathrm{~Hz}, 1 \mathrm{H}), 6.24(\mathrm{~d}, J=7.6 \mathrm{~Hz}, 1 \mathrm{H})$, $6.10\left(\mathrm{dd}, J_{1}=18.8 \mathrm{~Hz}, J_{2}=3.6 \mathrm{~Hz}, 1 \mathrm{H}\right), 6.02\left(\mathrm{dd}, J_{1}=18.8 \mathrm{~Hz}, J_{2}=1.6 \mathrm{~Hz}, 1 \mathrm{H}\right)$, 5.82-5.74 (m, 1H), 4.23-4.20 (m, 1H), $3.88(\mathrm{~d}, J=2.4 \mathrm{~Hz}, 1 \mathrm{H}), 3.78(\mathrm{~s}, 3 \mathrm{H}), 3.55(\mathrm{dt}$, $\left.J_{1}=11.2 \mathrm{~Hz}, J_{2}=4.4 \mathrm{~Hz}, 1 \mathrm{H}\right), 3.33(\mathrm{~s}, 1 \mathrm{H}), 2.88\left(\mathrm{td}, J_{1}=11.2 \mathrm{~Hz}, J_{2}=3.6 \mathrm{~Hz}, 1 \mathrm{H}\right)$, $2.61\left(\mathrm{dd}, J_{1}=14.0 \mathrm{~Hz}, J_{2}=4.0 \mathrm{~Hz}, 1 \mathrm{H}\right), 2.42(\mathrm{~s}, 3 \mathrm{H}), 2.38-2.32(\mathrm{~m}, 1 \mathrm{H}), 1.94\left(\mathrm{ddd}, J_{1}\right.$ $\left.=14.4 \mathrm{~Hz}, J_{2}=11.6 \mathrm{~Hz}, J_{3}=4.0 \mathrm{~Hz}, 1 \mathrm{H}\right), 1.77\left(\mathrm{dt}, J_{1}=14.0 \mathrm{~Hz}, J_{2}=4.0 \mathrm{~Hz}, 1 \mathrm{H}\right)$, 
$0.11(\mathrm{~s}, 9 \mathrm{H}) ;{ }^{13} \mathrm{C} \mathrm{NMR}\left(100 \mathrm{MHz}, \mathrm{CDCl}_{3}\right) \delta$ 159.0, 149.5, 144.0, 142.9, 136.2, 132.8, $132.7,132.2,129.9,129.3,127.9,127.4,127.2,123.5,122.6,118.5,113.9$, 108.9, 64.3, 61.1, 55.3, 46.3, 43.5, 40.3, 33.3, 21.5, -1.3; IR (neat) $\mathrm{cm}^{-1} 3372,2926,1607$, 1510, 1486, 1264, 1247, 1161, 1092, 968, 839; HRMS (ESI-TOF) m/z: $[\mathrm{M}+\mathrm{H}]^{+}$ Calcd for $\mathrm{C}_{33} \mathrm{H}_{41} \mathrm{~N}_{2} \mathrm{O}_{3} \mathrm{SSi}^{+}$: 573.2602, found 573.2600.

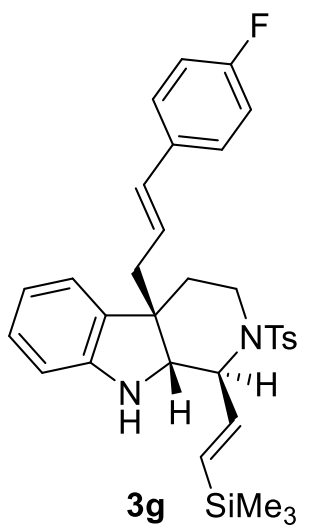

3g: The title compound were purified by silica gel column chromatography (gradient eluent: petroleum ether/EtOAc $=100: 1$ $\rightarrow 20: 1)$ to afford the desired product $\mathbf{3 g}$ as a colorless oil, $35 \mathrm{mg}$, $63 \%$ yield, 3:1 $d r .{ }^{1} \mathrm{H}$ NMR $\left(400 \mathrm{MHz}, \mathrm{CDCl}_{3}\right) \delta 7.62(\mathrm{~d}, J=8.4$ $\mathrm{Hz}, 2 \mathrm{H}), 7.22-7.17(\mathrm{~m}, 4 \mathrm{H}), 6.99-6.90(\mathrm{~m}, 4 \mathrm{H}), 6.68(\mathrm{t}, J=7.6$ $\mathrm{Hz}, 1 \mathrm{H}), 6.31(\mathrm{~d}, J=16.0 \mathrm{~Hz}, 1 \mathrm{H}), 6.26(\mathrm{~d}, J=8.0 \mathrm{~Hz}, 1 \mathrm{H}), 6.11$ $\left(\mathrm{dd}, J_{1}=18.8 \mathrm{~Hz}, J_{2}=3.6 \mathrm{~Hz}, 1 \mathrm{H}\right), 5.96\left(\mathrm{dd}, J_{1}=18.8 \mathrm{~Hz}, J_{2}=\right.$ $1.6 \mathrm{~Hz}, 1 \mathrm{H}), 5.88-5.81(\mathrm{~m}, 1 \mathrm{H}), 4.22-4.20(\mathrm{~m}, 1 \mathrm{H}), 3.86(\mathrm{~d}, J=3.2 \mathrm{~Hz}, 1 \mathrm{H}), 3.55$ (dt, $\left.J_{1}=11.2 \mathrm{~Hz}, J_{2}=4.4 \mathrm{~Hz}, 1 \mathrm{H}\right), 3.36(\mathrm{~s}, 1 \mathrm{H}), 2.89\left(\mathrm{td}, J_{1}=11.2 \mathrm{~Hz}, J_{2}=3.2 \mathrm{~Hz}, 1 \mathrm{H}\right)$, 2.62-2.57 (m, 1H), $2.41(\mathrm{~s}, 3 \mathrm{H}), 2.38-2.34(\mathrm{~m}, 1 \mathrm{H}), 1.95\left(\mathrm{ddd}, J_{1}=14.0 \mathrm{~Hz}, J_{2}=11.2\right.$ $\left.\mathrm{Hz}, J_{3}=4.0 \mathrm{~Hz}, 1 \mathrm{H}\right), 1.77\left(\mathrm{dt}, J_{1}=14.0 \mathrm{~Hz}, J_{2}=4.0 \mathrm{~Hz}, 1 \mathrm{H}\right), 0.10(\mathrm{~s}, 9 \mathrm{H}) ;{ }^{13} \mathrm{C} \mathrm{NMR}$ $\left(100 \mathrm{MHz}, \mathrm{CDCl}_{3}\right) \delta 163.4(\mathrm{~d}, J=245.0 \mathrm{~Hz}), 149.5,144.0,142.9,136.3,133.3(\mathrm{~d}, J=$ $3.4 \mathrm{~Hz}), 132.6,132.4,132.2,129.3,128.0,127.6$ (d, $J=7.9 \mathrm{~Hz}), 127.4,125.6$ (d, $J=$ $2.3 \mathrm{~Hz}), 122.6,118.6,115.5(\mathrm{~d}, J=21.4 \mathrm{~Hz}), 109.0,64.5,61.2,46.3,43.6,40.3,33.2$, 21.5, -1.3. IR (neat) $\mathrm{cm}^{-1} 3379,2922,1604,1486,1345,1261,1159,1091,1017,839$, 812; HRMS (ESI-TOF) m/z: [M + Na ${ }^{+}$Calcd for $\mathrm{C}_{32} \mathrm{H}_{37} \mathrm{FN}_{2} \mathrm{O}_{2} \mathrm{SSiNa}^{+}:$583.2221, found 583.2210.

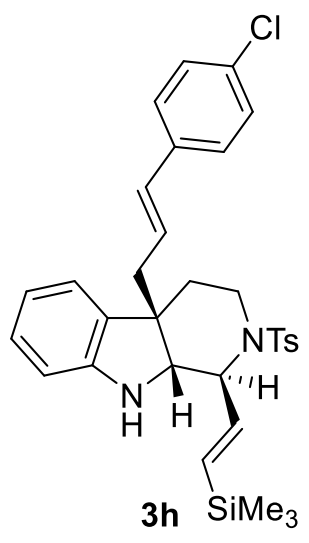

3h: The title compound were purified by silica gel column chromatography (gradient eluent: petroleum ether/EtOAc $=100: 1$ $\rightarrow 20: 1)$ to afford the desired product $\mathbf{3 h}$ as a colorless oil, $37 \mathrm{mg}$, 64\% yield, 8:1 dr. ${ }^{1} \mathrm{H}$ NMR (400 MHz, $\left.\mathrm{CDCl}_{3}\right) \delta 7.60(\mathrm{~d}, J=8.0$ $\mathrm{Hz}, 2 \mathrm{H}), 7.24-7.19(\mathrm{~m}, 5 \mathrm{H}), 7.14(\mathrm{~d}, J=8.8 \mathrm{~Hz}, 2 \mathrm{H}), 7.02-6.95$ $(\mathrm{m}, 1 \mathrm{H}), 6.92-6.85(\mathrm{~m}, 1 \mathrm{H}), 6.71-6.66(\mathrm{~m}, 1 \mathrm{H}), 6.26(\mathrm{~d}, J=7.6$ $\mathrm{Hz}, 1 \mathrm{H}), 6.10\left(\mathrm{dd}, J_{1}=18.8 \mathrm{~Hz}, J_{2}=3.6 \mathrm{~Hz}, 1 \mathrm{H}\right), 6.00-5.87(\mathrm{~m}$, 
$2 \mathrm{H}), 4.21-4.19(\mathrm{~m}, 1 \mathrm{H}), 3.84(\mathrm{~d}, J=3.2 \mathrm{~Hz}, 1 \mathrm{H}), 3.55\left(\mathrm{dt}, J_{1}=11.2 \mathrm{~Hz}, J_{2}=4.0 \mathrm{~Hz}\right.$, $1 \mathrm{H}), 3.37(\mathrm{~s}, 1 \mathrm{H}), 2.89\left(\mathrm{td}, J_{1}=11.6 \mathrm{~Hz}, J_{2}=3.6 \mathrm{~Hz}, 1 \mathrm{H}\right), 2.60\left(\mathrm{ddd}, J_{1}=14.0 \mathrm{~Hz}, J_{2}\right.$ $\left.=6.0 \mathrm{~Hz}, J_{3}=1.6 \mathrm{~Hz}, 1 \mathrm{H}\right), 2.41(\mathrm{~s}, 3 \mathrm{H}), 2.38-2.35(\mathrm{~m}, 1 \mathrm{H}), 1.95\left(\mathrm{ddd}, J_{1}=14.0 \mathrm{~Hz}, J_{2}\right.$ $\left.=11.2 \mathrm{~Hz}, J_{3}=4.0 \mathrm{~Hz}, 1 \mathrm{H}\right), 1.78\left(\mathrm{dt}, J_{1}=14.0 \mathrm{~Hz}, J_{2}=4.0 \mathrm{~Hz}, 1 \mathrm{H}\right), 0.10(\mathrm{~s}, 9 \mathrm{H}) ;{ }^{13} \mathrm{C}$ NMR $\left(100 \mathrm{MHz}, \mathrm{CDCl}_{3}\right) \delta 149.4,143.9,142.9,140.3,136.1,135.5,132.9,132.5$, 132.4, 132.1, 129.3, 128.6, 128.0, 127.4, 127.3, 126.6, 122.6, 118.6, 109.0, 64.4, 61.2, 46.3, 43.6, 40.3, 33.2, 21.5, -1.3. IR (neat) $\mathrm{cm}^{-1} 3375,2921,1607,1488,1344,1248$, 1160, 1092, 839, 816; HRMS (ESI-TOF) m/z: $[\mathrm{M}+\mathrm{Na}]^{+}$Calcd for $\mathrm{C}_{32} \mathrm{H}_{37} \mathrm{ClN}_{2} \mathrm{O}_{2} \mathrm{SSiNa}^{+}$: 599.1926, found 599.1919.

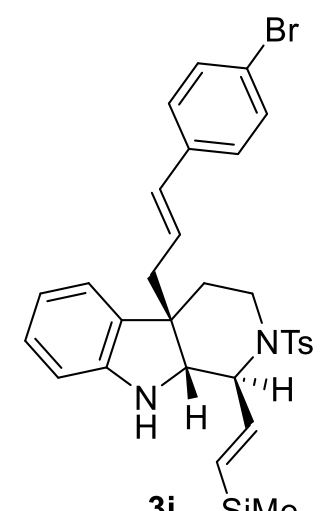

3i: The title compound were purified by silica gel column chromatography (gradient eluent: petroleum ether/EtOAc $=100: 1$ $\rightarrow 20: 1)$ to afford the desired product $3 \mathbf{i}$ as a yellow oil, $37 \mathrm{mg}, 60 \%$ yield, 4:1 $d r .{ }^{1} \mathrm{H}$ NMR $\left(400 \mathrm{MHz}, \mathrm{CDCl}_{3}\right) \delta 7.62(\mathrm{~d}, J=8.0 \mathrm{~Hz}$, 2H), $7.37(\mathrm{~d}, J=8.4 \mathrm{~Hz}, 2 \mathrm{H}), 7.22(\mathrm{~d}, J=8.0 \mathrm{~Hz}, 2 \mathrm{H}), 7.09(\mathrm{~d}, J$ $=8.4 \mathrm{~Hz}, 2 \mathrm{H}), 6.97\left(\mathrm{td}, J_{1}=7.6 \mathrm{~Hz}, J_{2}=1.2 \mathrm{~Hz}, 1 \mathrm{H}\right), 6.91(\mathrm{~d}, J=$ $7.2 \mathrm{~Hz}, 1 \mathrm{H}), 6.69\left(\mathrm{dd}, J_{1}=7.6 \mathrm{~Hz}, J_{2}=2.4 \mathrm{~Hz}, 1 \mathrm{H}\right), 6.28-6.24(\mathrm{~m}$, $2 \mathrm{H}), 6.10\left(\mathrm{dd}, J_{1}=18.8 \mathrm{~Hz}, J_{2}=3.6 \mathrm{~Hz}, 1 \mathrm{H}\right), 6.01-5.89(\mathrm{~m}, 2 \mathrm{H}), 4.21-4.18(\mathrm{~m}, 1 \mathrm{H})$, $3.84(\mathrm{~d}, J=2.8 \mathrm{~Hz}, 1 \mathrm{H}), 3.54\left(\mathrm{dt}, J_{1}=11.6 \mathrm{~Hz}, J_{2}=4.4 \mathrm{~Hz}, 1 \mathrm{H}\right), 3.37(\mathrm{~s}, 1 \mathrm{H}), 2.89$ $\left(\mathrm{td}, J_{1}=11.6 \mathrm{~Hz}, J_{2}=3.6 \mathrm{~Hz}, 1 \mathrm{H}\right), 2.60\left(\mathrm{ddd}, J_{1}=14.0 \mathrm{~Hz}, J_{2}=6.0 \mathrm{~Hz}, J_{3}=1.2 \mathrm{~Hz}\right.$, $1 \mathrm{H}), 2.41(\mathrm{~s}, 3 \mathrm{H}), 2.38-2.34(\mathrm{~m}, 1 \mathrm{H}), 1.95\left(\mathrm{ddd}, J_{1}=14.0 \mathrm{~Hz}, J_{2}=11.2 \mathrm{~Hz}, J_{3}=4.0\right.$ $\mathrm{Hz}, 1 \mathrm{H}), 1.78\left(\mathrm{dt}, J_{1}=14.0 \mathrm{~Hz}, J_{2}=4.0 \mathrm{~Hz}, 1 \mathrm{H}\right), 0.10(\mathrm{~s}, 9 \mathrm{H}) ;{ }^{13} \mathrm{C} \mathrm{NMR}(100 \mathrm{MHz}$, $\left.\mathrm{CDCl}_{3}\right) \delta 149.4,143.9,142.9,136.1,135.9,132.5,132.4,132.2,131.6,129.3,128.0$, 127.6, 127.4, 126.7, 122.6, 121.0, 118.6, 109.0, 64.4, 61.2, 46.3, 43.6, 40.3, 33.2, 21.5, -1.3. IR (neat) $\mathrm{cm}^{-1} 3377,2922,1736,1605,1463,1261,1160,1089,1010,841$; HRMS (ESI-TOF) m/z: [M + Na $]^{+}$Calcd for $\mathrm{C}_{32} \mathrm{H}_{37} \mathrm{BrN}_{2} \mathrm{O}_{2} \mathrm{SSiNa}^{+}: 643.1421$, found 643.1425 . 


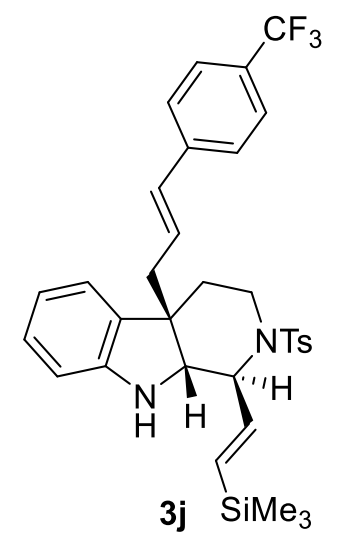

3j: The title compound were purified by silica gel column chromatography (gradient eluent: petroleum ether/EtOAc $=$ $100: 1 \rightarrow 20: 1)$ to afford the desired product $\mathbf{3} \mathbf{j}$ as a colorless oil, $39 \mathrm{mg}, 64 \%$ yield, 7:1 dr. ${ }^{1} \mathrm{H}$ NMR $\left(400 \mathrm{MHz} \mathrm{CDCl}_{3}\right) \delta 7.62(\mathrm{~d}$, $J=8.4 \mathrm{~Hz}, 2 \mathrm{H}), 7.50(\mathrm{~d}, J=8.4 \mathrm{~Hz}, 2 \mathrm{H}), 7.32(\mathrm{~d}, J=8.4 \mathrm{~Hz}$, $2 \mathrm{H}), 7.22(\mathrm{~d}, J=8.0 \mathrm{~Hz}, 2 \mathrm{H}), 6.98\left(\mathrm{td}, J_{1}=7.2 \mathrm{~Hz}, J_{2}=1.2 \mathrm{~Hz}\right.$, $1 \mathrm{H}), 6.92\left(\mathrm{dd}, J_{1}=7.6 \mathrm{~Hz}, J_{2}=1.2 \mathrm{~Hz}, 1 \mathrm{H}\right), 6.69\left(\mathrm{td}, J_{1}=7.2 \mathrm{~Hz}\right.$,

$\left.J_{2}=1.2 \mathrm{~Hz}, 1 \mathrm{H}\right), 6.38(\mathrm{~d}, J=16.0 \mathrm{~Hz}, 1 \mathrm{H}), 6.28(\mathrm{~d}, J=8.0 \mathrm{~Hz}, 1 \mathrm{H}), 6.10-5.95(\mathrm{~m}$, $3 \mathrm{H}), 4.19-4.17(\mathrm{~m}, 1 \mathrm{H}), 3.83(\mathrm{~d}, J=3.2 \mathrm{~Hz}, 1 \mathrm{H}), 3.56\left(\mathrm{dt}, J_{1}=11.2 \mathrm{~Hz}, J_{2}=4.0 \mathrm{~Hz}\right.$, $1 \mathrm{H}), 3.39(\mathrm{~s}, 1 \mathrm{H}), 2.89\left(\mathrm{td}, J_{1}=11.6 \mathrm{~Hz}, J_{2}=3.6 \mathrm{~Hz}, 1 \mathrm{H}\right), 2.62\left(\mathrm{ddd}, J_{1}=14.0 \mathrm{~Hz}, J_{2}\right.$ $\left.=6.0 \mathrm{~Hz}, J_{3}=1.6 \mathrm{~Hz}, 1 \mathrm{H}\right), 2.45-2.37(\mathrm{~m}, 1 \mathrm{H}), 2.41(\mathrm{~s}, 3 \mathrm{H}), 1.97\left(\mathrm{ddd}, J_{1}=14.0 \mathrm{~Hz}\right.$, $\left.J_{2}=11.6 \mathrm{~Hz}, J_{3}=4.0 \mathrm{~Hz}, 1 \mathrm{H}\right), 1.80\left(\mathrm{dt}, J_{1}=14.0 \mathrm{~Hz}, J_{2}=4.0 \mathrm{~Hz}, 1 \mathrm{H}\right), 0.10(\mathrm{~s}, 9 \mathrm{H}) ;$ ${ }^{13} \mathrm{C}$ NMR $\left(100 \mathrm{MHz}, \mathrm{CDCl}_{3}\right) \delta 149.4,143.8,143.0,140.4,136.1,132.5,132.3,132.1$, 129.3, 128.7, 128.1, 127.4, 126.2, 125.5 (q, $J=3.9 \mathrm{~Hz}), 122.6,118.6,109.0,64.5$, 61.3, 46.3, 43.6, 40.4, 33.1, 21.5, -1.3. IR (neat) $\mathrm{cm}^{-1} 3365,2922,1610,1464,1325$, 1260, 1161, 1067, 1016, 839; HRMS (ESI-TOF) m/z: $[\mathrm{M}+\mathrm{Na}]^{+}$Calcd for $\mathrm{C}_{33} \mathrm{H}_{37} \mathrm{~F}_{3} \mathrm{~N}_{2} \mathrm{O}_{2} \mathrm{SSiNa}^{+}$: 633.2189, found 633.2189.

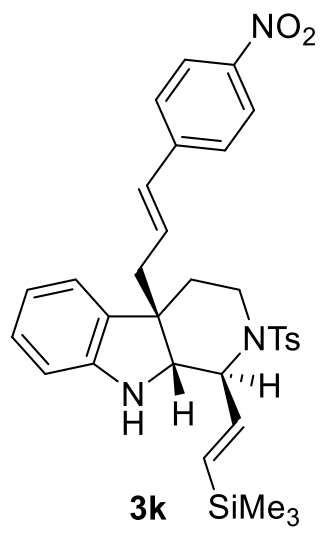

3k: The title compound were purified by silica gel column chromatography (gradient eluent: petroleum ether/EtOAc $=$ $100: 1 \rightarrow 20: 1)$ to afford the desired product $\mathbf{3 k}$ as a yellow oil, 40 mg, 68\% yield, 7:1 $d r .{ }^{1} \mathrm{H}$ NMR $\left(400 \mathrm{MHz}, \mathrm{CDCl}_{3}\right) \delta 8.12(\mathrm{~d}, J$ $=8.8 \mathrm{~Hz}, 2 \mathrm{H}), 7.61(\mathrm{~d}, J=8.4 \mathrm{~Hz}, 2 \mathrm{H}), 7.35(\mathrm{~d}, J=9.2 \mathrm{~Hz}, 2 \mathrm{H})$, $7.22(\mathrm{~d}, J=8.0 \mathrm{~Hz}, 2 \mathrm{H}), 7.01\left(\mathrm{td}, J_{1}=7.6 \mathrm{~Hz}, J_{2}=1.2 \mathrm{~Hz}, 1 \mathrm{H}\right)$, $6.92\left(\mathrm{dd}, J_{1}=7.6 \mathrm{~Hz}, J_{2}=1.2 \mathrm{~Hz}, 1 \mathrm{H}\right), 6.69\left(\mathrm{td}, J_{1}=8.0 \mathrm{~Hz}, J_{2}=\right.$ $1.2 \mathrm{~Hz}, 1 \mathrm{H}), 6.37(\mathrm{~d}, J=16.0 \mathrm{~Hz}, 1 \mathrm{H}), 6.29(\mathrm{~d}, J=8.0 \mathrm{~Hz}, 1 \mathrm{H}), 6.18-6.10(\mathrm{~m}, 1 \mathrm{H})$, $6.06(\mathrm{~d}, J=4.0 \mathrm{~Hz}, 1 \mathrm{H}), 5.99\left(\mathrm{dd}, J_{1}=18.8 \mathrm{~Hz}, J_{2}=2.0 \mathrm{~Hz}, 1 \mathrm{H}\right), 4.16-4.14(\mathrm{~m}, 1 \mathrm{H})$, $3.81(\mathrm{~d}, J=3.2 \mathrm{~Hz}, 1 \mathrm{H}), 3.57\left(\mathrm{dt}, J_{1}=11.6 \mathrm{~Hz}, J_{2}=4.4 \mathrm{~Hz}, 1 \mathrm{H}\right), 3.41(\mathrm{~s}, 1 \mathrm{H}), 2.89$ $\left(\mathrm{td}, J_{1}=11.2 \mathrm{~Hz}, J_{2}=3.6 \mathrm{~Hz}, 1 \mathrm{H}\right), 2.64\left(\mathrm{ddd}, J_{1}=14.0 \mathrm{~Hz}, J_{2}=6.0 \mathrm{~Hz}, J_{3}=1.6 \mathrm{~Hz}\right.$, $1 \mathrm{H}), 2.47-2.34(\mathrm{~m}, 1 \mathrm{H}), 2.41(\mathrm{~s}, 3 \mathrm{H}), 1.97\left(\mathrm{ddd}, J_{1}=14.0 \mathrm{~Hz}, J_{2}=11.2 \mathrm{~Hz}, J_{3}=4.0\right.$ 
$\mathrm{Hz}, 1 \mathrm{H}), 1.82\left(\mathrm{dt}, J_{1}=14.0 \mathrm{~Hz}, J_{2}=4.0 \mathrm{~Hz}, 1 \mathrm{H}\right), 0.10(\mathrm{~s}, 9 \mathrm{H}) ;{ }^{13} \mathrm{C} \mathrm{NMR}(100 \mathrm{MHz}$, $\left.\mathrm{CDCl}_{3}\right) \delta 149.4,146.7,143.8,143.4,143.0,136.0,132.7,132.0,131.4,131.3,129.3$, 128.2, 127.4, 126.6, 124.0, 122.5, 118.7, 109.1, 64.6, 61.4, 46.4, 43.8, 40.5, 33.1, 21.5, -1.3. IR (neat) $\mathrm{cm}^{-1} 3376,2922,1597,1517,1462,1341,1262,1060,1090,1018,840$; HRMS (ESI-TOF) m/z: $[\mathrm{M}+\mathrm{Na}]^{+}$Calcd for $\mathrm{C}_{32} \mathrm{H}_{37} \mathrm{~N}_{3} \mathrm{O}_{4} \mathrm{SSiNa}^{+}:$610.2166, found 610.2166.

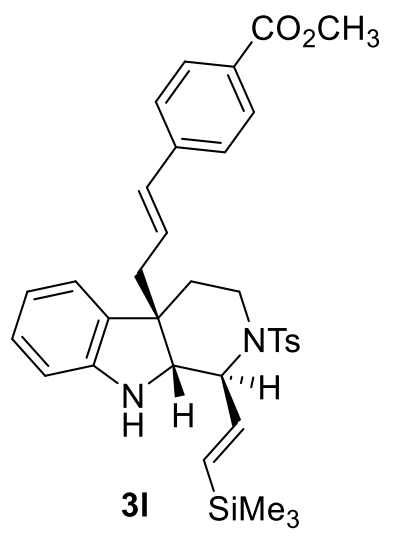

31: The title compound were purified by silica gel column chromatography (gradient eluent: petroleum ether/EtOAc $=$ $100: 1 \rightarrow 20: 1)$ to afford the desired product $\mathbf{3 1}$ as a colorless oil, $42 \mathrm{mg}, 70 \%$ yield, 4:1 $d r .{ }^{1} \mathrm{H} \mathrm{NMR}\left(400 \mathrm{MHz}, \mathrm{CDCl}_{3}\right) \delta$ $7.92(\mathrm{~d}, J=8.4 \mathrm{~Hz}, 2 \mathrm{H}), 7.62(\mathrm{~d}, J=8.4 \mathrm{~Hz}, 2 \mathrm{H}), 7.31-7.28$ $(\mathrm{m}, 2 \mathrm{H}), 7.22(\mathrm{~d}, J=8.0 \mathrm{~Hz}, 2 \mathrm{H}), 6.98\left(\mathrm{td}, J_{1}=7.6 \mathrm{~Hz}, J_{2}=\right.$ $1.2 \mathrm{~Hz}, 1 \mathrm{H}), 6.92\left(\mathrm{dd}, J_{1}=7.6 \mathrm{~Hz}, J_{2}=1.2 \mathrm{~Hz}, 1 \mathrm{H}\right), 6.69(\mathrm{td}$, $\left.J_{1}=7.2 \mathrm{~Hz}, J_{2}=0.8 \mathrm{~Hz}, 1 \mathrm{H}\right), 6.38(\mathrm{~d}, J=15.6 \mathrm{~Hz}, 1 \mathrm{H}), 6.27(\mathrm{~d}, J=7.6 \mathrm{~Hz}, 1 \mathrm{H})$, 6.11-6.03 (m, 2H), $6.01\left(\mathrm{dd}, J_{1}=18.8 \mathrm{~Hz}, J_{2}=1.6 \mathrm{~Hz}, 1 \mathrm{H}\right), 4.21-4.18(\mathrm{~m}, 1 \mathrm{H}), 3.89$ (s, 3H), $3.85(\mathrm{~d}, J=3.2 \mathrm{~Hz}, 1 \mathrm{H}), 3.56\left(\mathrm{dt}, J_{1}=11.2 \mathrm{~Hz}, J_{2}=4.4 \mathrm{~Hz}, 1 \mathrm{H}\right), 3.38(\mathrm{~s}, 1 \mathrm{H})$, $2.90\left(\mathrm{td}, J_{1}=11.2 \mathrm{~Hz}, J_{2}=3.6 \mathrm{~Hz}, 1 \mathrm{H}\right), 2.63\left(\mathrm{ddd}, J_{1}=14.0 \mathrm{~Hz}, J_{2}=5.6 \mathrm{~Hz}, J_{3}=1.6\right.$ $\mathrm{Hz}, 1 \mathrm{H}), 2.41(\mathrm{~s}, 3 \mathrm{H}), 2.39-2.31(\mathrm{~m}, 1 \mathrm{H}), 1.96\left(\mathrm{ddd}, J_{1}=14.4 \mathrm{~Hz}, J_{2}=11.2 \mathrm{~Hz}, J_{3}=\right.$ $3.6 \mathrm{~Hz}, 1 \mathrm{H}), 1.78\left(\mathrm{dt}, J_{1}=14.0 \mathrm{~Hz}, J_{2}=4.0 \mathrm{~Hz}, 1 \mathrm{H}\right), 0.10(\mathrm{~s}, 9 \mathrm{H}) ;{ }^{13} \mathrm{C} \mathrm{NMR}(100$ $\left.\mathrm{MHz}, \mathrm{CDCl}_{3}\right) \delta 166.8,149.4,143.8,142.9,141.4,136.1,132.5,132.5,132.4,129.9$, 129.3, 128.8, 128.7, 128.1, 127.4, 126.0, 122.6, 118.6, 109.0, 64.5, 61.2, 52.1, 46.3, 43.7, 40.3, 33.2, 21.5, -1.3. IR (neat) $\mathrm{cm}^{-1} 3376,2923,1716,1606,1463,1262,1160$,

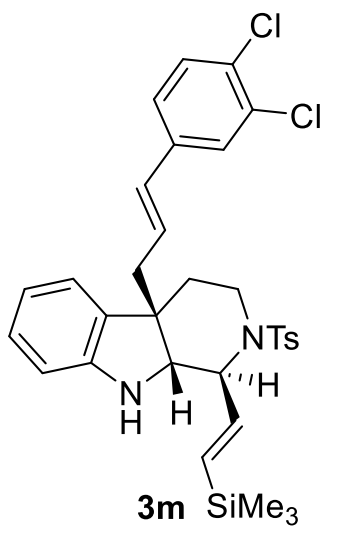
1109, 1016, 840; HRMS (ESI-TOF) m/z: $[\mathrm{M}+\mathrm{Na}]^{+}$Calcd for $\mathrm{C}_{34} \mathrm{H}_{40} \mathrm{~N}_{2} \mathrm{O}_{4} \mathrm{SSiNa}^{+}:$623.2370, found 623.2355.

3m: The title compound were purified by silica gel column chromatography (gradient eluent: petroleum ether/EtOAc $=$ $100: 1 \rightarrow 20: 1)$ to afford the desired product $\mathbf{3 m}$ as a colorless oil, $33 \mathrm{mg}, 54 \%$ yield, 4:1 $d r .{ }^{1} \mathrm{H}$ NMR (400 $\left.\mathrm{MHz}, \mathrm{CDCl}_{3}\right) \delta$ $7.62(\mathrm{~d}, J=8.4 \mathrm{~Hz}, 2 \mathrm{H}), 7.31-7.29(\mathrm{~m}, 2 \mathrm{H}), 7.22(\mathrm{~d}, J=8.0 \mathrm{~Hz}$ 
2H), $7.05\left(\mathrm{dd}, J_{1}=8.4 \mathrm{~Hz}, J_{2}=2.0 \mathrm{~Hz}, 1 \mathrm{H}\right), 6.98\left(\mathrm{td}, J_{1}=7.6 \mathrm{~Hz}, J_{2}=1.2 \mathrm{~Hz}, 1 \mathrm{H}\right)$, $6.91\left(\mathrm{dd}, J_{1}=7.6 \mathrm{~Hz}, J_{2}=1.2 \mathrm{~Hz}, 1 \mathrm{H}\right), 6.69\left(\mathrm{td}, J_{1}=7.2 \mathrm{~Hz}, J_{2}=0.8 \mathrm{~Hz}, 1 \mathrm{H}\right), 6.27(\mathrm{~d}$, $J=7.6 \mathrm{~Hz}, 1 \mathrm{H}), 6.24(\mathrm{~d}, J=16.0 \mathrm{~Hz}, 1 \mathrm{H}), 6.08\left(\mathrm{dd}, J_{1}=18.4 \mathrm{~Hz}, J_{2}=4.0 \mathrm{~Hz}, 1 \mathrm{H}\right)$, 6.00-5.90 (m, 2H), 4.19-4.16 (m, 1H), $3.81(\mathrm{~d}, J=3.2 \mathrm{~Hz}, 1 \mathrm{H}), 3.55\left(\mathrm{dt}, J_{1}=11.6 \mathrm{~Hz}\right.$, $\left.J_{2}=4.4 \mathrm{~Hz}, 1 \mathrm{H}\right), 3.38(\mathrm{~s}, 1 \mathrm{H}), 2.90\left(\mathrm{td}, J_{1}=11.6 \mathrm{~Hz}, J_{2}=3.6 \mathrm{~Hz}, 1 \mathrm{H}\right), 2.60\left(\mathrm{ddd}, J_{1}\right.$ $\left.=14.0 \mathrm{~Hz}, J_{2}=6.0 \mathrm{~Hz}, J_{3}=1.6 \mathrm{~Hz}, 1 \mathrm{H}\right), 2.42(\mathrm{~s}, 3 \mathrm{H}), 2.39-2.35(\mathrm{~m}, 1 \mathrm{H}), 1.95\left(\mathrm{ddd}, J_{1}\right.$ $\left.=14.0 \mathrm{~Hz}, J_{2}=11.6 \mathrm{~Hz}, J_{3}=4.0 \mathrm{~Hz}, 1 \mathrm{H}\right), 1.78\left(\mathrm{dt}, J_{1}=14.0 \mathrm{~Hz}, J_{2}=4.0 \mathrm{~Hz}, 1 \mathrm{H}\right)$, $0.10(\mathrm{~s}, 9 \mathrm{H}) ;{ }^{13} \mathrm{C} \mathrm{NMR}\left(100 \mathrm{MHz}, \mathrm{CDCl}_{3}\right) \delta 149.4,143.8,143.0,137.1,136.1,132.6$, $132.5,132.3,131.1,130.9,130.4,129.3,128.1,127.7,127.4,125.3,122.6,118.7$, 109.0, 64.5, 61.3, 46.3, 43.6, 40.4, 33.1, 21.5, -1.3. IR (neat) $\mathrm{cm}^{-1} 3375,2922,1605$, 1465, 1345, 1261, 1160, 1090, 865, 804; HRMS (ESI-TOF) m/z: [M + Na $]^{+}$Calcd for $\mathrm{C}_{32} \mathrm{H}_{36} \mathrm{Cl}_{2} \mathrm{~N}_{2} \mathrm{O}_{2} \mathrm{SSiNa}^{+}$: 633.1536, found 633.1524.

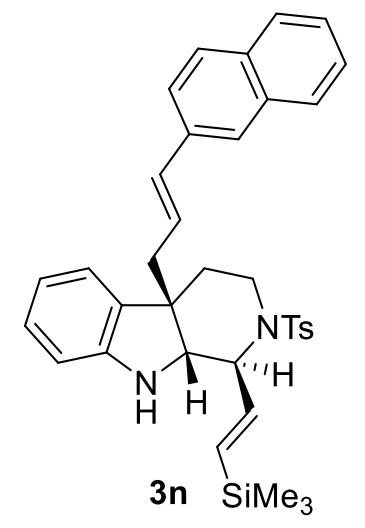

3n: The title compound were purified by silica gel column chromatography (gradient eluent: petroleum ether/EtOAc $=$ $100: 1 \rightarrow 20: 1)$ to afford the desired product $\mathbf{3 n}$ as a yellow oil, $38 \mathrm{mg}, 64 \%$ yield, 4:1 $d r .{ }^{1} \mathrm{H} \mathrm{NMR}\left(400 \mathrm{MHz}, \mathrm{CDCl}_{3}\right) \delta 7.76$ $\left(\mathrm{dd}, J_{1}=6.8 \mathrm{~Hz}, J_{2}=2.4 \mathrm{~Hz}, 2 \mathrm{H}\right), 7.71(\mathrm{~d}, J=8.8 \mathrm{~Hz}, 1 \mathrm{H})$, 7.63-7.59 (m, 3H), 7.44-7.39 (m, 3H), 7.22 (d, $J=8.0 \mathrm{~Hz}, 2 \mathrm{H})$, 7.04-6.94 (m, 2H), $6.71\left(\mathrm{td}, J_{1}=7.6 \mathrm{~Hz}, J_{2}=1.2 \mathrm{~Hz}, 1 \mathrm{H}\right)$, 6.53-6.48 (m, 1H), 6.27 (d, $J=7.6 \mathrm{~Hz}, 1 \mathrm{H}), 6.14-5.99(\mathrm{~m}, 3 \mathrm{H}), 4.24-4.22(\mathrm{~m}, 1 \mathrm{H})$, $3.91(\mathrm{~d}, J=2.8 \mathrm{~Hz}, 1 \mathrm{H}), 3.57\left(\mathrm{dt}, J_{1}=11.6 \mathrm{~Hz}, J_{2}=4.0 \mathrm{~Hz}, 1 \mathrm{H}\right), 3.38(\mathrm{~s}, 1 \mathrm{H}), 2.91$ $\left(\operatorname{td}, J_{1}=11.2 \mathrm{~Hz}, J_{2}=3.2 \mathrm{~Hz}, 1 \mathrm{H}\right), 2.67\left(\mathrm{ddd}, J_{1}=14.0 \mathrm{~Hz}, J_{2}=5.6 \mathrm{~Hz}, J_{3}=1.6 \mathrm{~Hz}\right.$, $1 \mathrm{H}), 2.48-2.44(\mathrm{~m}, 1 \mathrm{H}), 2.42(\mathrm{~s}, 3 \mathrm{H}), 1.95\left(\mathrm{ddd}, J_{1}=14.0 \mathrm{~Hz}, J_{2}=11.2 \mathrm{~Hz}, J_{3}=4.0\right.$ $\mathrm{Hz}, 1 \mathrm{H}), 1.81\left(\mathrm{dt}, J_{1}=14.0 \mathrm{~Hz}, J_{2}=4.0 \mathrm{~Hz}, 1 \mathrm{H}\right), 0.12(\mathrm{~s}, 9 \mathrm{H}) ;{ }^{13} \mathrm{C} \mathrm{NMR}(100 \mathrm{MHz}$, $\left.\mathrm{CDCl}_{3}\right) \delta 149.5,143.9,142.9,136.1,134.5,133.5,133.5,132.8,132.7,132.4,129.3$, $128.1,128.0,127.8,127.6,127.4,127.4,126.2$, 126.2, 125.7, 123.5, 122.7, 118.6, 109.0, 64.4, 61.2, 46.4, 43.7, 40.3, 33.3, 29.7, 22.7, 21.5, 14.1, -1.3. IR (neat) $\mathrm{cm}^{-1}$ 3406, 2923, 1602, 1486, 1330, 1250, 1159, 1087, 932, 840, 813; HRMS (ESI-TOF) $\mathrm{m} / \mathrm{z}:[\mathrm{M}+\mathrm{Na}]^{+}$Calcd for $\mathrm{C}_{36} \mathrm{H}_{40} \mathrm{~N}_{2} \mathrm{O}_{2} \mathrm{SSiNa}^{+}$: 615.2472, found 615.2468. 


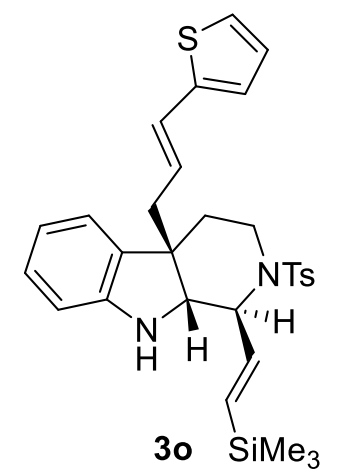

3o: The title compound were purified by silica gel column chromatography (gradient eluent: petroleum ether/EtOAc $=100: 1$ $\rightarrow 20: 1)$ to afford the desired product $\mathbf{3 0}$ as alight yellow oil, 46 mg, 73\% yield, 7:1 $d r .{ }^{1} \mathrm{H}$ NMR $\left(400 \mathrm{MHz}, \mathrm{CDCl}_{3}\right) \delta 7.62(\mathrm{~d}, J=$ $8.0 \mathrm{~Hz}, 2 \mathrm{H}), 7.21(\mathrm{~d}, J=8.0 \mathrm{~Hz}, 2 \mathrm{H}), 7.07(\mathrm{~d}, J=4.8 \mathrm{~Hz}, 1 \mathrm{H})$, $6.97(\mathrm{t}, J=7.6 \mathrm{~Hz}, 1 \mathrm{H}), 6.93-6.90(\mathrm{~m}, 2 \mathrm{H}), 6.84(\mathrm{~d}, J=2.8 \mathrm{~Hz}$, 1H), $6.67(\mathrm{t}, J=7.6 \mathrm{~Hz}, 1 \mathrm{H}), 6.47(\mathrm{~d}, J=15.6 \mathrm{~Hz}, 1 \mathrm{H}), 6.27(\mathrm{~d}, J=7.6 \mathrm{~Hz}, 1 \mathrm{H}), 6.10$ $\left(\mathrm{dd}, J_{1}=18.4 \mathrm{~Hz}, J_{2}=3.6 \mathrm{~Hz}, 1 \mathrm{H}\right), 6.00\left(\mathrm{dd}, J_{1}=18.8 \mathrm{~Hz}, J_{2}=1.6 \mathrm{~Hz}, 1 \mathrm{H}\right)$, 5.82-5.75 (m, 1H), 4.21-4.19 (m, 1H), $3.84(\mathrm{~d}, J=2.4 \mathrm{~Hz}, 1 \mathrm{H}), 3.57\left(\mathrm{dt}, J_{1}=11.2 \mathrm{~Hz}\right.$, $\left.J_{2}=4.4 \mathrm{~Hz}, 1 \mathrm{H}\right), 3.40(\mathrm{~s}, 1 \mathrm{H}), 2.93\left(\mathrm{td}, J_{1}=11.2 \mathrm{~Hz}, J_{2}=3.6 \mathrm{~Hz}, 1 \mathrm{H}\right), 2.59\left(\mathrm{dd}, J_{1}=\right.$ $\left.14.0 \mathrm{~Hz}, J_{2}=6.0 \mathrm{~Hz}, 1 \mathrm{H}\right), 2.41(\mathrm{~s}, 3 \mathrm{H}), 2.38-2.33(\mathrm{~m}, 1 \mathrm{H}), 1.94\left(\mathrm{ddd}, J_{1}=14.4 \mathrm{~Hz}, J_{2}\right.$ $\left.=11.2 \mathrm{~Hz}, J_{3}=4.0 \mathrm{~Hz}, 1 \mathrm{H}\right), 1.79\left(\mathrm{dt}, J_{1}=14.0 \mathrm{~Hz}, J_{2}=4.4 \mathrm{~Hz}, 1 \mathrm{H}\right), 0.11(\mathrm{~s}, 9 \mathrm{H}) ;{ }^{13} \mathrm{C}$ NMR (100 MHz, $\left.\mathrm{CDCl}_{3}\right) \delta 149.4,143.9,142.9,142.1,136.1,132.5,132.4,129.3$, 127.9, 127.4, 127.2, 126.4, 125.5, 124.8, 123.7, 122.6, 118.6, 109.0, 64.5, 61.2, 46.3, 43.4, 40.3, 33.0, 21.5, -1.3. IR (neat) $\mathrm{cm}^{-1} 3380,2923,1607,1485,1345,1247,1159$, 1091, 957, 866, 837; HRMS (ESI-TOF) m/z: $\left[\mathrm{M}+\mathrm{Na}^{+}\right.$Calcd for $\mathrm{C}_{30} \mathrm{H}_{36} \mathrm{~N}_{2} \mathrm{O}_{2} \mathrm{~S}_{2} \mathrm{SiNa}^{+}:$571.1880, found 571.1880.

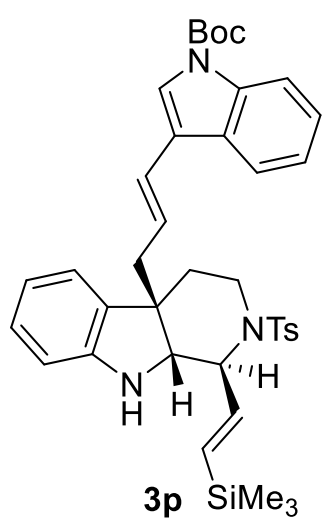

3p: The title compound were purified by silica gel column chromatography (gradient eluent: petroleum ether/EtOAc $=$ 100:1 $\rightarrow 20: 1)$ to afford the desired product $\mathbf{3 p}$ as a colorless oil, $32 \mathrm{mg}, 47 \%$ yield, $7: 1 d r .{ }^{1} \mathrm{H}$ NMR $\left(400 \mathrm{MHz}, \mathrm{CDCl}_{3}\right) \delta 8.12(\mathrm{~d}$, $J=8.4 \mathrm{~Hz}, 1 \mathrm{H}), 7.62(\mathrm{~d}, J=8.4,2 \mathrm{H}), 7.47(\mathrm{~d}, J=7.2 \mathrm{~Hz}, 2 \mathrm{H})$, 7.33-7.27 (m, 1H), 7.22-7.17 (m, 3H), 7.00-6.94 (m, 2H), $6.69(\mathrm{t}$, $J=7.6 \mathrm{~Hz}, 1 \mathrm{H}), 6.44(\mathrm{~d}, J=16.0 \mathrm{~Hz}, 1 \mathrm{H}), 6.26(\mathrm{~d}, J=7.6 \mathrm{~Hz}$, $1 \mathrm{H}), 6.13\left(\mathrm{dd}, J_{1}=18.8 \mathrm{~Hz}, J_{2}=4.0 \mathrm{~Hz}, 1 \mathrm{H}\right), 6.04-5.94(\mathrm{~m}, 2 \mathrm{H}), 4.21-4.19(\mathrm{~m}, 1 \mathrm{H})$, $3.92(\mathrm{~d}, J=3.2 \mathrm{~Hz}, 1 \mathrm{H}), 3.58\left(\mathrm{dt}, J_{1}=11.2 \mathrm{~Hz}, J_{2}=4.0 \mathrm{~Hz}, 1 \mathrm{H}\right), 3.37(\mathrm{~s}, 1 \mathrm{H}), 2.90$ $\left(\mathrm{td}, J_{1}=11.2 \mathrm{~Hz}, J_{2}=3.2 \mathrm{~Hz}, 1 \mathrm{H}\right), 2.65\left(\mathrm{ddd}, J_{1}=14.0 \mathrm{~Hz}, J_{2}=6.0 \mathrm{~Hz}, J_{3}=1.6 \mathrm{~Hz}\right.$, $1 \mathrm{H}), 2.41(\mathrm{~s}, 3 \mathrm{H}), 2.40-2.32(\mathrm{~m}, 1 \mathrm{H}), 2.05-1.97(\mathrm{~m}, 1 \mathrm{H}), 1.82\left(\mathrm{dt}, J_{1}=14.4 \mathrm{~Hz}, J_{2}=\right.$ $4.0 \mathrm{~Hz}, 1 \mathrm{H}), 1.65$ (s, 9H), 0.10 (s, 9H); ${ }^{13} \mathrm{C}$ NMR $\left(150 \mathrm{MHz}, \mathrm{CDCl}_{3}\right) \delta$ 149.6, 149.5, 
$144.1,142.9,136.1,135.8,132.5,132.4,129.4,129.3,128.7,128.0,127.4,126.5$, 124.6, 124.3, 123.1, 122.8, 122.7, 119.7, 118.6, 115.3, 108.9, 83.8, 64.5, 61.3, 46.4, 44.4, 40.5, 33.2, 28.2, 21.5, -1.3. IR (neat) $\mathrm{cm}^{-1} 3375,2922,1734,1604,1454,1370$, 1258, 1157, 1088, 1018, 799; HRMS (ESI-TOF) m/z: $[\mathrm{M}+\mathrm{Na}]^{+}$Calcd for $\mathrm{C}_{39} \mathrm{H}_{47} \mathrm{~N}_{3} \mathrm{O}_{4} \mathrm{SSiNa}^{+}$: 704.2949, found 704.2946.

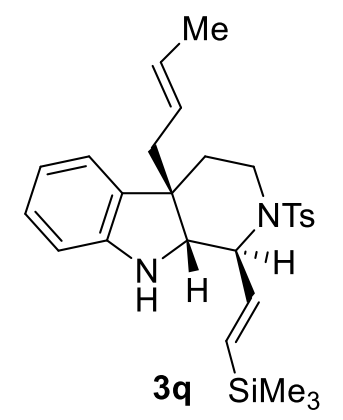

3q: The title compound were purified by silica gel column chromatography (gradient eluent: petroleum ether/EtOAc $=100: 1$ $\rightarrow 20: 1)$ to afford the desired product $\mathbf{3 q}$ as a light yellow oil, 37 mg, 77\% yield, 4:1 dr. ${ }^{1} \mathrm{H}$ NMR (400 MHz, $\left.\mathrm{CDCl}_{3}\right) \delta 7.63$ (d, $J=$ $8.0 \mathrm{~Hz}, 2 \mathrm{H}), 7.22(\mathrm{~d}, J=8.0 \mathrm{~Hz}, 2 \mathrm{H}), 6.94\left(\mathrm{td}, J_{1}=7.6 \mathrm{~Hz}, J_{2}=\right.$ $1.2 \mathrm{~Hz}, 1 \mathrm{H}), 6.87(\mathrm{~d}, J=7.2 \mathrm{~Hz}, 1 \mathrm{H}), 6.65(\mathrm{t}, J=7.2 \mathrm{~Hz}, 1 \mathrm{H})$, $6.23(\mathrm{~d}, J=7.6 \mathrm{~Hz}, 1 \mathrm{H}), 6.07\left(\mathrm{dd}, J_{1}=18.4 \mathrm{~Hz}, J_{2}=2.8 \mathrm{~Hz}, 1 \mathrm{H}\right), 6.01-5.96(\mathrm{~m}, 1 \mathrm{H})$, 5.45-5.36 (m, 1H), 5.20-5.12 (m, 1H), $4.26(\mathrm{t}, J=2.8 \mathrm{~Hz}, 1 \mathrm{H}), 3.85(\mathrm{~d}, J=3.2 \mathrm{~Hz}$, $1 \mathrm{H}), 3.53\left(\mathrm{dt}, J_{1}=11.2 \mathrm{~Hz}, J_{2}=4.4 \mathrm{~Hz}, 1 \mathrm{H}\right), 3.34(\mathrm{~s}, 1 \mathrm{H}), 2.87\left(\mathrm{td}, J_{1}=11.2 \mathrm{~Hz}, J_{2}=\right.$ $3.6 \mathrm{~Hz}, 1 \mathrm{H}), 2.41(\mathrm{~s}, 3 \mathrm{H}), 2.39-2.31(\mathrm{~m}, 1 \mathrm{H}), 2.18\left(\mathrm{dd}, J_{1}=14.0 \mathrm{~Hz}, J_{2}=8.8 \mathrm{~Hz}, 1 \mathrm{H}\right)$, $1.92\left(\mathrm{ddd}, J_{1}=14.4 \mathrm{~Hz}, J_{2}=11.6 \mathrm{~Hz}, J_{3}=4.4 \mathrm{~Hz}, 1 \mathrm{H}\right), 1.69\left(\mathrm{dt}, J_{1}=14.0 \mathrm{~Hz}, J_{2}=4.0\right.$ $\mathrm{Hz}, 1 \mathrm{H}), 1.60(\mathrm{~d}, J=6.4 \mathrm{~Hz}, 1 \mathrm{H}), 0.10(\mathrm{~s}, 9 \mathrm{H}) ;{ }^{13} \mathrm{C} \mathrm{NMR}\left(150 \mathrm{MHz}, \mathrm{CDCl}_{3}\right) \delta 149.4$, 144.0, 142.8, 136.2, 133.2, 131.9, 129.2, 128.9, 127.7, 127.4, 126.5, 122.5, 118.4, 108.7, 64.0, 61.0, 45.9, 42.9, 40.1, 33.1, 21.4, 18.0, -1.4. IR (neat) $\mathrm{cm}^{-1} 3380,2923$, 1607, 1486, 1346, 1247, 1159, 1094, 968, 866, 837; HRMS (ESI-TOF) m/z: [M + H] ${ }^{+}$ Calcd for $\mathrm{C}_{27} \mathrm{H}_{37} \mathrm{~N}_{2} \mathrm{O}_{2} \mathrm{SSi}^{+}$: 481.2340 , found 481.2334 .

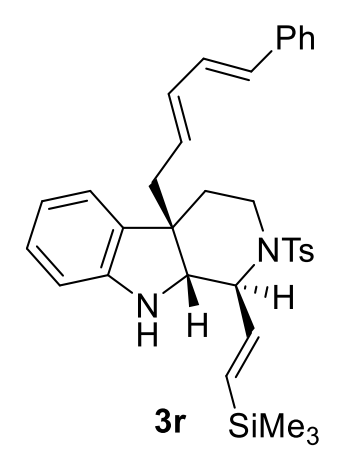

3r: The title compound were purified by silica gel column chromatography (gradient eluent: petroleum ether/EtOAc $=100: 1$ $\rightarrow 20: 1)$ to afford the desired product $\mathbf{3 r}$ as a light yellow oil, 46 mg, 81\% yield, 5:1 dr. ${ }^{1} \mathrm{H}$ NMR (400 MHz, $\left.\mathrm{CDCl}_{3}\right) \delta 7.58(\mathrm{~d}, J=$ $8.0 \mathrm{~Hz}, 2 \mathrm{H}), 7.28(\mathrm{~s}, 1 \mathrm{H}), 7.24(\mathrm{~d}, J=7.6 \mathrm{~Hz}, 2 \mathrm{H}), 7.20(\mathrm{~s}, 1 \mathrm{H})$, 1H), 6.63-6.56 (m, 2H), $6.38(\mathrm{~d}, J=15.6 \mathrm{~Hz}, 1 \mathrm{H}), 6.19(\mathrm{~d}, J=7.6 \mathrm{~Hz}, 1 \mathrm{H}), 6.13(\mathrm{dd}$, $\left.J_{1}=15.2 \mathrm{~Hz}, J_{2}=10.4 \mathrm{~Hz}, 1 \mathrm{H}\right), 6.05\left(\mathrm{dd}, J_{1}=18.8 \mathrm{~Hz}, J_{2}=3.6 \mathrm{~Hz}, 1 \mathrm{H}\right), 5.97\left(\mathrm{dd}, J_{1}\right.$ 
$\left.=18.8 \mathrm{~Hz}, J_{2}=1.6 \mathrm{~Hz}, 1 \mathrm{H}\right), 5.51-5.44(\mathrm{~m}, 1 \mathrm{H}), 4.21-4.19(\mathrm{~m}, 1 \mathrm{H}), 3.81(\mathrm{~d}, J=2.8 \mathrm{~Hz}$, $1 \mathrm{H}), 3.50\left(\mathrm{dt}, J_{1}=11.2 \mathrm{~Hz}, J_{2}=4.4 \mathrm{~Hz}, 1 \mathrm{H}\right), 3.32(\mathrm{~s}, 1 \mathrm{H}), 2.87\left(\mathrm{td}, J_{1}=11.2 \mathrm{~Hz}, J_{2}=\right.$ $3.6 \mathrm{~Hz}, 1 \mathrm{H}), 2.53\left(\mathrm{dd}, J_{1}=14.4 \mathrm{~Hz}, J_{2}=6.0 \mathrm{~Hz}, 1 \mathrm{H}\right), 2.36(\mathrm{~s}, 3 \mathrm{H}), 2.32-2.23(\mathrm{~m}, 1 \mathrm{H})$, $1.88\left(\mathrm{ddd}, J_{1}=14.0 \mathrm{~Hz}, J_{2}=11.2 \mathrm{~Hz}, J_{3}=4.0 \mathrm{~Hz}, 1 \mathrm{H}\right), 1.69\left(\mathrm{dt}, J_{1}=14.0 \mathrm{~Hz}, J_{2}=4.0\right.$ $\mathrm{Hz}, 1 \mathrm{H}), 0.07$ (s, 9H); ${ }^{13} \mathrm{C} \mathrm{NMR}\left(100 \mathrm{MHz}, \mathrm{CDCl}_{3}\right) \delta 149.4,144.0,142.9,137.2$, $136.1,133.9,132.7,132.2,131.2,130.2,129.3,128.6,128.5,127.9,127.4,127.4$, 126.2, 122.6, 118.5, 108.8, 64.3, 61.0, 46.3, 43.3, 40.1, 33.2, 21.5, -1.3. IR (neat) $\mathrm{cm}^{-1}$ 3391, 2952, 1608, 1485,1247, 1159, 1092, 988, 864, 837; HRMS (ESI-TOF) m/z: [M $+\mathrm{Na}]^{+}$Calcd for $\mathrm{C}_{34} \mathrm{H}_{40} \mathrm{~N}_{2} \mathrm{O}_{2} \mathrm{SSiNa}^{+}:$591.2472, found 591.2479.

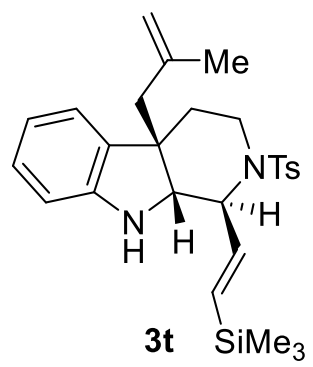

3t: The title compound were purified by silica gel column chromatography (gradient eluent: petroleum ether/EtOAc $=100: 1$ $\rightarrow 20: 1)$ to afford the desired product $\mathbf{3 t}$ as alight yellow oil, 40 mg, 83\% yield, 9:1 $d r .{ }^{1} \mathrm{H}$ NMR (400 $\left.\mathrm{MHz}, \mathrm{CDCl}_{3}\right) \delta 7.62(\mathrm{~d}, J=$ $8.4 \mathrm{~Hz}, 2 \mathrm{H}), 7.23(\mathrm{~d}, J=8.0 \mathrm{~Hz}, 2 \mathrm{H}), 6.94\left(\mathrm{td}, J_{1}=7.2 \mathrm{~Hz}, J_{2}=\right.$ $1.2 \mathrm{~Hz}, 1 \mathrm{H}), 6.89$ (d, $J=7.2 \mathrm{~Hz}, 1 \mathrm{H}), 6.62(\mathrm{t}, J=7.2 \mathrm{~Hz}, 1 \mathrm{H}), 6.14$ (d, $J=8.0 \mathrm{~Hz}$, 1H), 6.11-6.01 (m, 2H), $4.79(\mathrm{t}, J=2.0 \mathrm{~Hz}, 1 \mathrm{H}), 4.57(\mathrm{~s}, 1 \mathrm{H}), 4.25(\mathrm{t}, J=2.4 \mathrm{~Hz}, 1 \mathrm{H})$, $4.04(\mathrm{~d}, J=2.8 \mathrm{~Hz}, 1 \mathrm{H}), 3.56\left(\mathrm{dt}, J_{1}=10.8 \mathrm{~Hz}, J_{2}=4.0 \mathrm{~Hz}, 1 \mathrm{H}\right), 3.23(\mathrm{~s}, 1 \mathrm{H}), 2.83$ $\left(\mathrm{td}, J_{1}=12.4 \mathrm{~Hz}, J_{2}=3.2 \mathrm{~Hz}, 1 \mathrm{H}\right), 2.51-2.39(\mathrm{~m}, 1 \mathrm{H}), 2.43(\mathrm{~s}, 3 \mathrm{H}), 2.19$ (d, $J=14.0$ $\mathrm{Hz}, 1 \mathrm{H}), 1.97\left(\mathrm{ddd}, J_{1}=14.0 \mathrm{~Hz}, J_{2}=12.8 \mathrm{~Hz}, J_{3}=4.0 \mathrm{~Hz}, 1 \mathrm{H}\right), 1.73\left(\mathrm{dt}, J_{1}=14.0\right.$ $\left.\mathrm{Hz}, J_{2}=3.6 \mathrm{~Hz}, 1 \mathrm{H}\right), 1.33(\mathrm{~s}, 3 \mathrm{H}), 0.09(\mathrm{~s}, 9 \mathrm{H}) ;{ }^{13} \mathrm{C} \mathrm{NMR}\left(150 \mathrm{MHz}, \mathrm{CDCl}_{3}\right) \delta 149.2$, $144.4,142.8,142.5,136.0,132.9,132.0,129.3,127.8,127.3,122.9,118.2,115.4$, 108.6, 63.2, 61.0, 48.3, 45.8, 40.2, 34.8, 24.3, 21.4, -1.3. IR (neat) $\mathrm{cm}^{-1} 3390,2953$, 1608, 1486, 1344, 1247, 1158, 1099, 1002, 867, 837; HRMS (ESI-TOF) m/z: [M + $\mathrm{H}]^{+}$Calcd for $\mathrm{C}_{27} \mathrm{H}_{37} \mathrm{~N}_{2} \mathrm{O}_{2} \mathrm{SSi}^{+}: 481.2340$, found 481.2343 .

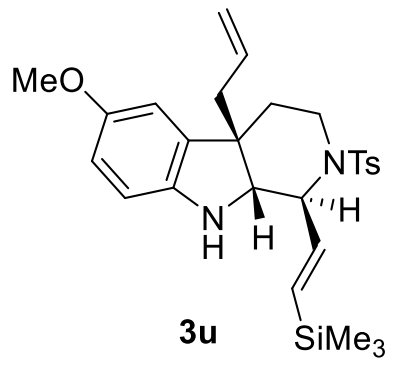

3u: The title compound were purified by silica gel column chromatography (gradient eluent: petroleum ether/EtOAc $=$ $100: 1 \rightarrow 20: 1)$ to afford the desired product $\mathbf{3 u}$ as a light yellow oil, $44 \mathrm{mg}$, 88\% yield, 3:1 $d r .{ }^{1} \mathrm{H}$ NMR (400 MHz, $\left.\mathrm{CDCl}_{3}\right) \delta 7.64(\mathrm{~d}, J=8.0 \mathrm{~Hz}, 2 \mathrm{H}), 7.22(\mathrm{~d}, J=8.0 \mathrm{~Hz}, 2 \mathrm{H})$, 
$6.54\left(\mathrm{dd}, J_{1}=8.4 \mathrm{~Hz}, J_{2}=2.4 \mathrm{~Hz}, 1 \mathrm{H}\right), 6.50(\mathrm{~d}, J=2.4 \mathrm{~Hz}, 1 \mathrm{H}), 6.22(\mathrm{~d}, J=8.4 \mathrm{~Hz}$, $1 \mathrm{H}), 6.05\left(\mathrm{dd}, J_{1}=18.8 \mathrm{~Hz}, J_{2}=3.6 \mathrm{~Hz}, 1 \mathrm{H}\right), 5.97\left(\mathrm{dd}, J_{1}=18.8 \mathrm{~Hz}, J_{2}=1.6 \mathrm{~Hz}, 1 \mathrm{H}\right)$, 5.61-5.50 (m, 1H), 5.04-4.91 (m, 2H), 4.24-4.22 (m, 1H), $3.83(\mathrm{~d}, J=3.2 \mathrm{~Hz}, 1 \mathrm{H})$, $3.71(\mathrm{~s}, 3 \mathrm{H}), 3.53\left(\mathrm{dt}, J_{1}=11.6 \mathrm{~Hz}, J_{2}=4.4 \mathrm{~Hz}, 1 \mathrm{H}\right), 3.18(\mathrm{~s}, 1 \mathrm{H}), 2.90\left(\mathrm{td}, J_{1}=11.2\right.$ $\left.\mathrm{Hz}, J_{2}=3.6 \mathrm{~Hz}, 1 \mathrm{H}\right), 2.46-2.39(\mathrm{~m}, 1 \mathrm{H}), 2.41(\mathrm{~s}, 3 \mathrm{H}), 2.25\left(\mathrm{dd}, J_{1}=14.0 \mathrm{~Hz}, J_{2}=8.8\right.$ $\mathrm{Hz}, 1 \mathrm{H}), 1.92\left(\mathrm{ddd}, J_{1}=14.4 \mathrm{~Hz}, J_{2}=11.2 \mathrm{~Hz}, J_{3}=4.0 \mathrm{~Hz}, 1 \mathrm{H}\right), 1.72\left(\mathrm{dt}, J_{1}=14.0\right.$ $\left.\mathrm{Hz}, J_{2}=4.0 \mathrm{~Hz}, 1 \mathrm{H}\right), 0.08(\mathrm{~s}, 9 \mathrm{H}) ;{ }^{13} \mathrm{C} \mathrm{NMR}\left(100 \mathrm{MHz}, \mathrm{CDCl}_{3}\right) \delta 153.4,144.0,143.4$, $142.9,136.2$, 134.8, 134.1, 132.1, 129.3, 127.4, 127.4, 118.4, 112.6, 109.7, 109.5, 64.7, 61.1, 55.9, 46.3, 44.0, 40.1, 33.1, 21.5, -1.4. IR (neat) $\mathrm{cm}^{-1} 3366,2952,1598$, 1493, 1345, 1247, 1214, 1159, 1094, 998, 866, 839; HRMS (ESI-TOF) m/z: [M + H] ${ }^{+}$ Calcd for $\mathrm{C}_{27} \mathrm{H}_{37} \mathrm{~N}_{2} \mathrm{O}_{3} \mathrm{SSi}^{+}$: 497.2289, found 497.2285.

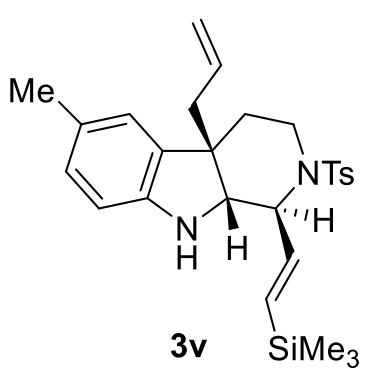

3v: The title compound were purified by silica gel column chromatography (gradient eluent: petroleum ether/EtOAc $=$ $100: 1 \rightarrow 20: 1)$ to afford the desired product $\mathbf{3 v}$ as a light yellow oil, $43 \mathrm{mg}$, 89\% yield, 10:1 dr. ${ }^{1} \mathrm{H}$ NMR (400 MHz, $\left.\mathrm{CDCl}_{3}\right) \delta 7.64(\mathrm{~d}, J=8.0 \mathrm{~Hz}, 2 \mathrm{H}), 7.22(\mathrm{~d}, J=8.0 \mathrm{~Hz}, 2 \mathrm{H})$, $6.77(\mathrm{~d}, J=8.0 \mathrm{~Hz}, 1 \mathrm{H}), 6.68(\mathrm{~s}, 1 \mathrm{H}), 6.16(\mathrm{~d}, J=7.6 \mathrm{~Hz}, 1 \mathrm{H}), 6.07\left(\mathrm{dd}, J_{1}=18.8 \mathrm{~Hz}\right.$, $\left.J_{2}=3.2 \mathrm{~Hz}, 1 \mathrm{H}\right), 5.95\left(\mathrm{dd}, J_{1}=18.8 \mathrm{~Hz}, J_{2}=1.2 \mathrm{~Hz}, 1 \mathrm{H}\right), 5.59-5.49(\mathrm{~m}, 1 \mathrm{H})$, 5.04-4.91 (m, 2H), 4.23-4.22 (m, 1H), $3.83(\mathrm{~d}, J=3.2 \mathrm{~Hz}, 1 \mathrm{H}), 3.55\left(\mathrm{dt}, J_{1}=11.6 \mathrm{~Hz}\right.$, $\left.J_{2}=4.4 \mathrm{~Hz}, 1 \mathrm{H}\right), 3.21(\mathrm{~s}, 3 \mathrm{H}), 2.90\left(\mathrm{td}, J_{1}=11.2 \mathrm{~Hz}, J_{2}=3.6 \mathrm{~Hz}, 1 \mathrm{H}\right), 2.48-2.39(\mathrm{~m}$, $1 \mathrm{H}), 2.42(\mathrm{~s}, 3 \mathrm{H}), 2.24-2.15(\mathrm{~m}, 1 \mathrm{H}), 2.21(\mathrm{~s}, 3 \mathrm{H}), 1.93\left(\mathrm{ddd}, J_{1}=14.4 \mathrm{~Hz}, J_{2}=11.6\right.$ $\left.\mathrm{Hz}, J_{3}=4.0 \mathrm{~Hz}, 1 \mathrm{H}\right), 1.71\left(\mathrm{dt}, J_{1}=14.0 \mathrm{~Hz}, J_{2}=4.0 \mathrm{~Hz}, 1 \mathrm{H}\right), 0.09(\mathrm{~s}, 9 \mathrm{H}) ;{ }^{13} \mathrm{C} \mathrm{NMR}$ $\left(100 \mathrm{MHz} \mathrm{CDCl}_{3}\right) \delta 147.1,144.1,142.8,136.2,134.4,133.2,132.0,129.3,128.3$, 127.9, 127.4, 123.2, 118.2, 108.9, 64.3, 61.0, 45.8, 44.2, 40.2, 33.3, 21.5, 20.9, -1.4. IR (neat) $\mathrm{cm}^{-1} 3375,2952,1615,1496,1344,1246,1158,1094,996,910,865,837$; HRMS (ESI-TOF) m/z: $[\mathrm{M}+\mathrm{H}]^{+}$Calcd for $\mathrm{C}_{27} \mathrm{H}_{37} \mathrm{~N}_{2} \mathrm{O}_{2} \mathrm{SSi}^{+}$: 481.2340 , found 481.2335 . 


\subsection{Preparation of 8 .}

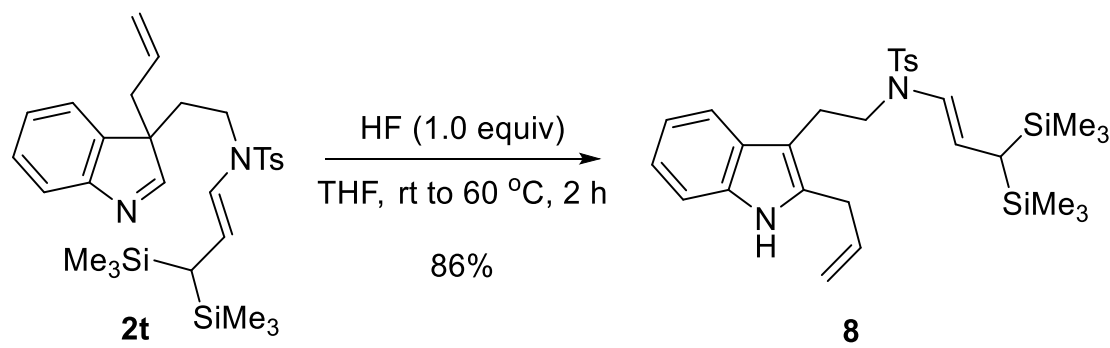

To a solution of $2 \mathrm{t}(54 \mathrm{mg}, 0.1 \mathrm{mmol})$ in THF $(1.0 \mathrm{~mL})$ at room temperature under argon was added dropwise HF ( $5 \mu \mathrm{L}, 0.1 \mathrm{mmol}, 40 \%$ solution in water) via syringe. The reaction mixture was warmed to $60{ }^{\circ} \mathrm{C}$ by oil bath and stirred for $2 \mathrm{~h}$. The reaction was quenched with sat. aq. $\mathrm{NaHCO}_{3}(2 \mathrm{~mL})$. The aqueous layer was extracted with EtOAc $(3 \times 5 \mathrm{~mL})$. The combined organic layers were dried over anhydrous $\mathrm{Na}_{2} \mathrm{SO}_{4}$, concentrated by rotary evaporation. The crude products were purified by silica gel column chromatography (gradient eluent: petroleum ether/EtOAc $=100: 1 \rightarrow 20: 1)$ to afford product $8(46 \mathrm{mg}, 86 \%$ yield $)$ as a light yellow oil. ${ }^{1} \mathrm{H}$ NMR (400 MHz, $\left.\mathrm{CDCl}_{3}\right) \delta 7.84(\mathrm{~s}, 1 \mathrm{H}), 7.64(\mathrm{~d}, J=8.0 \mathrm{~Hz}, 2 \mathrm{H}), 7.55(\mathrm{~d}, J=$ $7.6 \mathrm{~Hz}, 1 \mathrm{H}), 7.29$ (d, $J=8.0 \mathrm{~Hz}, 1 \mathrm{H}), 7.23(\mathrm{~d}, J=8.0 \mathrm{~Hz}, 2 \mathrm{H}), 7.16-7.08(\mathrm{~m}, 2 \mathrm{H})$, $6.19(\mathrm{~d}, J=13.6 \mathrm{~Hz}, 1 \mathrm{H}), 6.01-5.91(\mathrm{~m}, 1 \mathrm{H}), 5.20-5.09(\mathrm{~m}, 3 \mathrm{H}), 3.53(\mathrm{~d}, J=6.4 \mathrm{~Hz}$, 2H), 3.40-3.36 (m, 2H), 3.04-3.00 (m, 2H), $2.37(\mathrm{~s}, 3 \mathrm{H}), 1.04(\mathrm{~d}, J=12.4 \mathrm{~Hz}, 1 \mathrm{H})$, $0.05(\mathrm{~s}, 18 \mathrm{H}) ;{ }^{13} \mathrm{C} \mathrm{NMR}\left(100 \mathrm{MHz}, \mathrm{CDCl}_{3}\right) \delta 143.2,136.1,135.4,134.9,133.2,129.5$, $128.3,126.9,123.3,121.4,119.5,118.0,117.3,116.0,110.5,108.3,47.4,30.5,23.3$, 21.9, 21.4, -0.4. IR (neat) $\mathrm{cm}^{-1} 3397,2952,1637,1460,1352,1315,1249,1161,1092$, 970, 860, 838; HRMS (ESI-TOF) m/z: $[\mathrm{M}+\mathrm{Na}]^{+}$Calcd for $\mathrm{C}_{29} \mathrm{H}_{42} \mathrm{~N}_{2} \mathrm{O}_{2} \mathrm{SSi}_{2} \mathrm{Na}^{+}$: 561.2398, found 561.2409. 


\subsection{Preparation of 4 .}

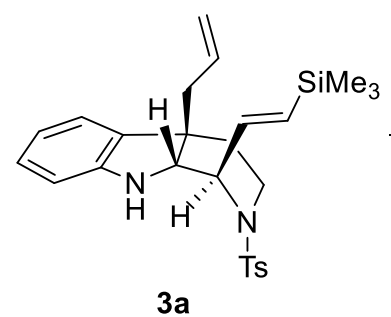

1) $\mathrm{ClCO}_{2} \mathrm{Me}$, pyridine

$\mathrm{CH}_{2} \mathrm{Cl}_{2}, 0^{\circ} \mathrm{C}$ to $\mathrm{rt}, 1 \mathrm{~h}$

2) cat. 9 (30 mol \%)

toluene, $120^{\circ} \mathrm{C}, 48 \mathrm{~h}$

$52 \%$
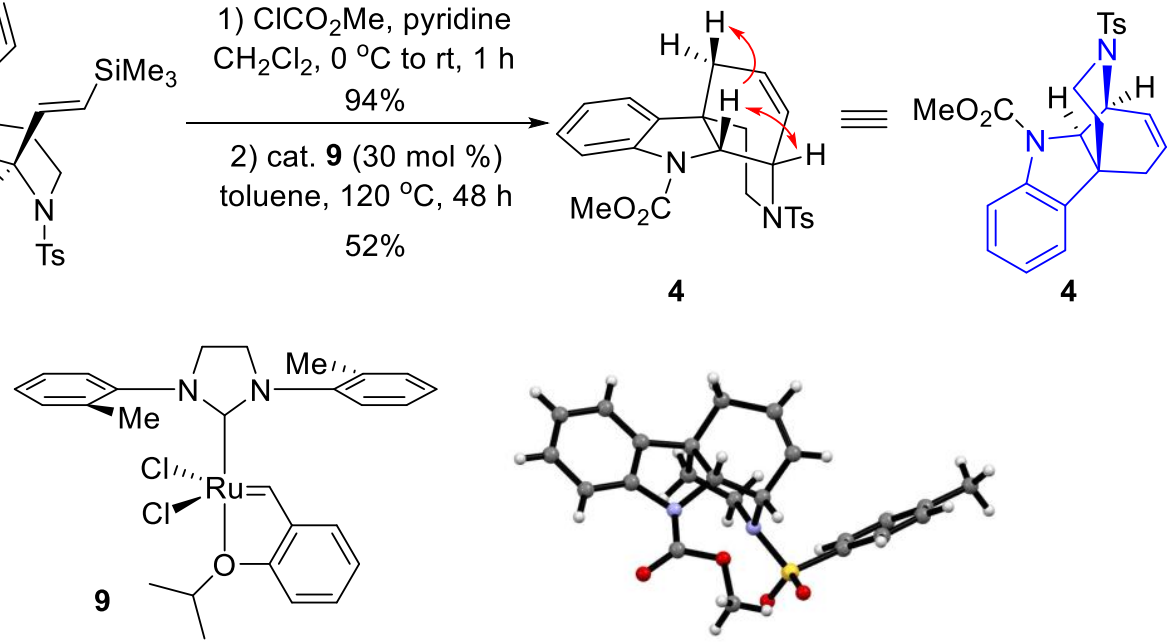

To a solution of $3 \mathbf{a}(52 \mathrm{mg}, 0.11 \mathrm{mmol})$ in $\mathrm{CH}_{2} \mathrm{Cl}_{2}(2 \mathrm{~mL})$ at $0{ }^{\circ} \mathrm{C}$ under argon was added pyridine $(27 \mu \mathrm{L}, 0.33 \mathrm{mmol}$ ). The reaction mixture was stirred for $10 \mathrm{~min}$ before adding methyl chloroformate $(18 \mu \mathrm{L}, 0.22 \mathrm{mmol})$. The reaction was warmed to room temperature and stirred for $1 \mathrm{~h}$. The reaction was quenched with sat. aq. $\mathrm{NaCl}(5$ $\mathrm{mL})$. The aqueous layer was extracted with $\mathrm{CH}_{2} \mathrm{Cl}_{2}(3 \times 5 \mathrm{~mL})$. The combined organic layers were washed with aq. $1 \mathrm{~N} \mathrm{HCl}$ solution $(10 \mathrm{~mL})$, dried over anhydrous $\mathrm{Na}_{2} \mathrm{SO}_{4}$ and concentrated by rotary evaporation. The crude product was purified by silica gel column chromatography (gradient eluent: petroleum ether/EtOAc $=100: 1 \rightarrow$ 20:1) to afford carbamate intermediate (55 $\mathrm{mg}, 94 \%$ yield) as a colorless oil.

To a solution of the carbamate intermediate $(55 \mathrm{mg}, 0.105 \mathrm{mmol})$ in toluene $(2 \mathrm{~mL})$ at room temperature under argon was added cat. 9 (18 $\mathrm{mg}, 30 \mathrm{~mol} \%)$. The reaction mixture was warmed to $120{ }^{\circ} \mathrm{C}$ by oil bath and stirred for $48 \mathrm{~h}$. The reaction was concentrated by rotary evaporation to give the crude products, which were purified by silica gel column chromatography (gradient eluent: petroleum ether/EtOAc $=50: 1 \rightarrow$ 10:1) to afford $4(23 \mathrm{mg}, 52 \%)$ as a white solid. m. p. $=161.2-163.7{ }^{\circ} \mathrm{C} .{ }^{1} \mathrm{H}$ NMR $\left(400 \mathrm{MHz}, \mathrm{CDCl}_{3}\right) \delta 7.86(\mathrm{~d}, J=7.6 \mathrm{~Hz}, 1 \mathrm{H}), 7.71(\mathrm{~d}, J=8.0 \mathrm{~Hz}, 2 \mathrm{H}), 7.30(\mathrm{~d}, J=$ $8.0 \mathrm{~Hz}, 2 \mathrm{H}), 7.23\left(\mathrm{td}, J_{1}=7.6 \mathrm{~Hz}, J_{2}=1.6 \mathrm{~Hz}, 1 \mathrm{H}\right), 7.06(\mathrm{~d}, J=7.6 \mathrm{~Hz}, 1 \mathrm{H}), 6.99(\mathrm{t}, J$ $=7.6 \mathrm{~Hz}, 1 \mathrm{H}), 6.02\left(\mathrm{dt}, J_{1}=10.0 \mathrm{~Hz}, J_{2}=3.6 \mathrm{~Hz}, 1 \mathrm{H}\right), 5.59\left(\mathrm{dd}, J_{1}=6.0 \mathrm{~Hz}, J_{2}=3.2\right.$ $\mathrm{Hz}, 1 \mathrm{H}), 5.32-5.27(\mathrm{~m}, 1 \mathrm{H}), 3.87(\mathrm{~s}, 3 \mathrm{H}), 3.71(\mathrm{~d}, J=2.8 \mathrm{~Hz}, 1 \mathrm{H}), 3.44$ (ddd, $J_{1}=$ 
$\left.12.0 \mathrm{~Hz}, J_{2}=6.0 \mathrm{~Hz}, J_{3}=2.0 \mathrm{~Hz}, 1 \mathrm{H}\right), 2.98\left(\mathrm{td}, J_{1}=12.4 \mathrm{~Hz}, J_{2}=4.0 \mathrm{~Hz}, 1 \mathrm{H}\right), 2.57(\mathrm{t}$, $J=3.2 \mathrm{~Hz}, 1 \mathrm{H}), 2.43(\mathrm{~s}, 3 \mathrm{H}), 1.67-1.59(\mathrm{~m}, 1 \mathrm{H}), 1.56-1.51(\mathrm{~m}, 1 \mathrm{H}) ;{ }^{13} \mathrm{C}$ NMR $(100$ $\left.\mathrm{MHz}, \mathrm{CDCl}_{3}\right) \delta 155.0,143.3,141.9,138.6,136.9,132.0,129.6,128.0,127.3,123.0$, 122.4, 120.6, 115.6, 65.1, 52.7, 49.6, 39.3, 38.6, 36.2, 35.4, 21.5. IR (neat) $\mathrm{cm}^{-1} 2922$, 1713, 1474, 1443, 1355, 1271, 1159, 1063, 919; HRMS (ESI-TOF) m/z: $[\mathrm{M}+\mathrm{Na}]^{+}$ Calcd for $\mathrm{C}_{23} \mathrm{H}_{24} \mathrm{~N}_{2} \mathrm{O}_{4} \mathrm{SNa}^{+}$: 447.1349, found 447.1358 .

\subsection{Crystal preparation of 4.}

Method for single crystals cultivation: compound $4(5 \mathrm{mg})$ was dissolved in $0.2 \mathrm{~mL}$ of ethyl acetate/n-hexane $(\mathrm{v} 1 / \mathrm{v} 2=1: 1)$ in a vial at room temperature. The vial was sealed, and it was crystallized to give crystal as colorless prisms after the solvent was slowly volatilized in 7 days at room temperature $\left(\sim 25^{\circ} \mathrm{C}\right)$. All diffraction data were collected by Xcalibur E equipped with a Mo radiation source $(\mathrm{K} \alpha=0.71073 \AA$ A $)$ at 293.15 K. CCDC 2017152 [4] contains the supplementary crystallographic data for this paper. These data can be obtained free of charge via www.ccdc.cam.ac.uk/data_request/cif, or by emailing data_request@ccdc.cam.ac.uk, or by contacting The Cambridge Crystallographic Data Centre, 12 Union Road, Cambridge CB2 1EZ, UK; fax: +44 1223336033.

\subsection{X-ray crystallographic structure of 4.}

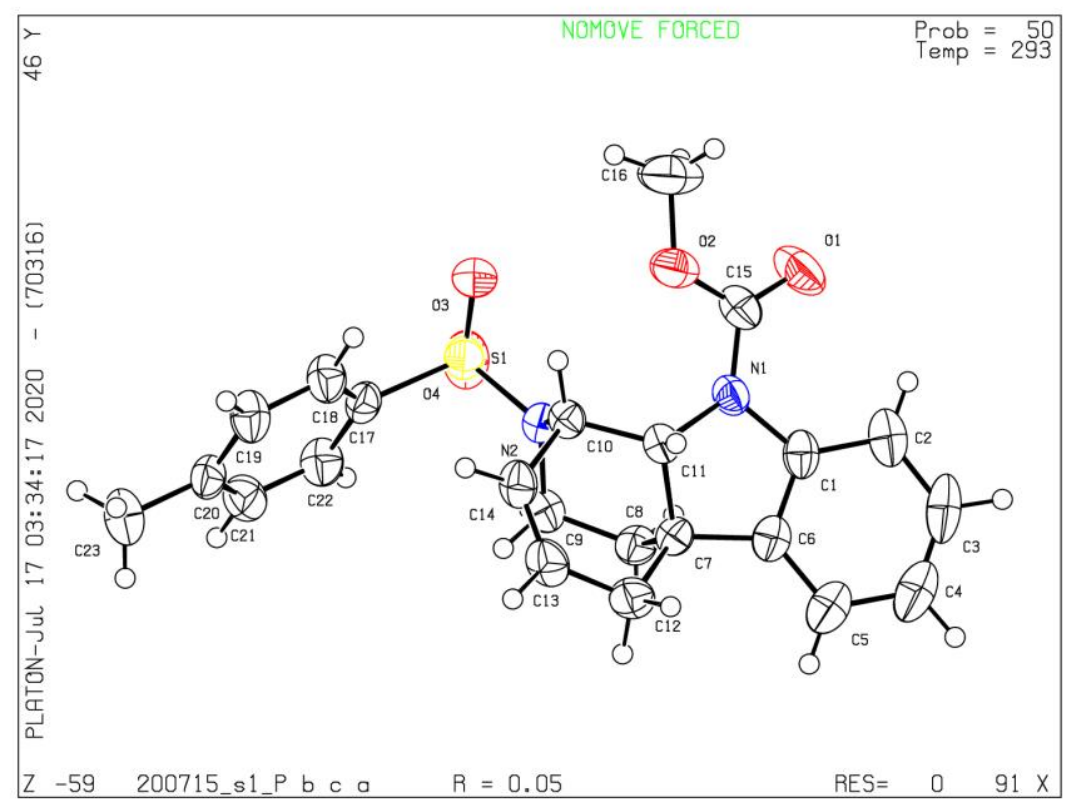


Figure 1. X-ray crystallographic structure of 4 (50\% thermal ellipsoid contour probability, CCDC 2017152)

\subsection{X-ray crystallographic data of 4 .}

Identification code

Empirical formula

Formula weight

Temperature/K

Crystal system

Space group

$\mathrm{a} / \AA$

$\mathrm{b} / \AA$

$\mathrm{c} / \AA$

$\alpha /^{\circ}$

$\beta /^{\circ}$

$\gamma /{ }^{\circ}$

Volume $/ \AA^{3}$

$\mathrm{Z}$

$\rho_{\text {calc }} \mathrm{g} / \mathrm{cm}^{3}$

$\mu / \mathrm{mm}^{-1}$

$\mathrm{F}(000)$

Crystal size $/ \mathrm{mm}^{3}$

Radiation

$2 \Theta$ range for data collection $/^{\circ}$

Index ranges

Reflections collected

Independent reflections

Data/restraints/parameters

Goodness-of-fit on $\mathrm{F}^{2}$

Final R indexes $[\mathrm{I}>=2 \sigma(\mathrm{I})]$

Final $\mathrm{R}$ indexes [all data]

Largest diff. peak/hole / e $\AA^{-3}$
4 (CCDC 2017152)

$\mathrm{C}_{23} \mathrm{H}_{24} \mathrm{~N}_{2} \mathrm{O}_{4} \mathrm{~S}$

424.50

293.15

orthorhombic

Pbca

12.5994(6)

$13.1510(4)$

25.1729(13)

90

90

90

4171.0(3)

8

1.352

0.188

1792.0

$0.35 \times 0.3 \times 0.25$

$\operatorname{MoK} \alpha(\lambda=0.71073)$

6.196 to 52.744

$-15 \leq \mathrm{h} \leq 7,-16 \leq \mathrm{k} \leq 13,-26 \leq 1 \leq 30$

11373

$4212\left[\mathrm{R}_{\text {int }}=0.0245, \mathrm{R}_{\text {sigma }}=0.0345\right]$

$4212 / 0 / 273$

1.030

$\mathrm{R}_{1}=0.0500, \mathrm{wR}_{2}=0.1116$

$\mathrm{R}_{1}=0.0768, \mathrm{wR}_{2}=0.1270$

$0.24 /-0.42$ 
CY-5-113_H1_CDCl3_2019-10-8.10.fid - $400 \mathrm{MHz}$

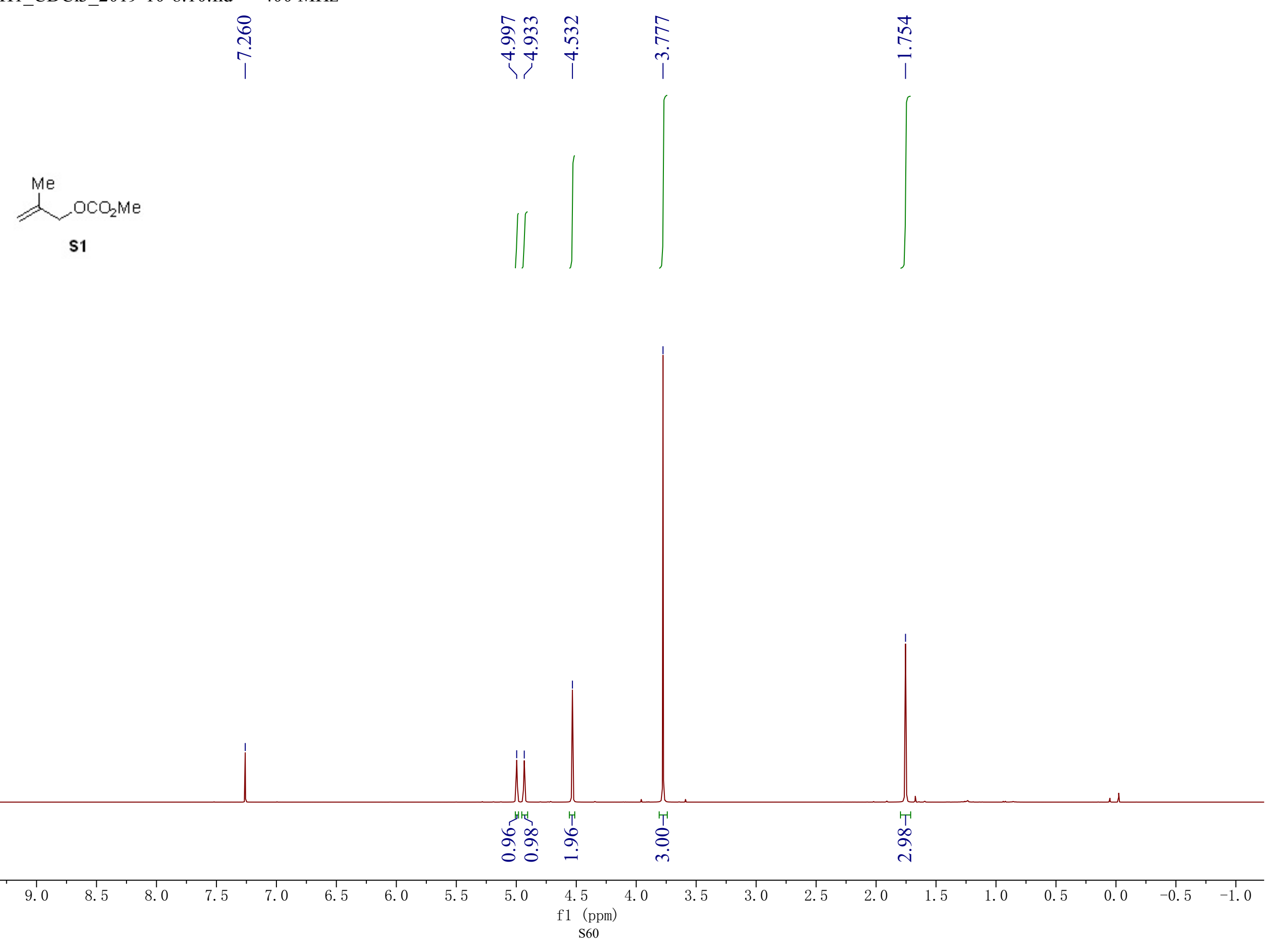




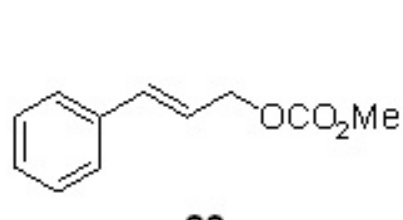

S2
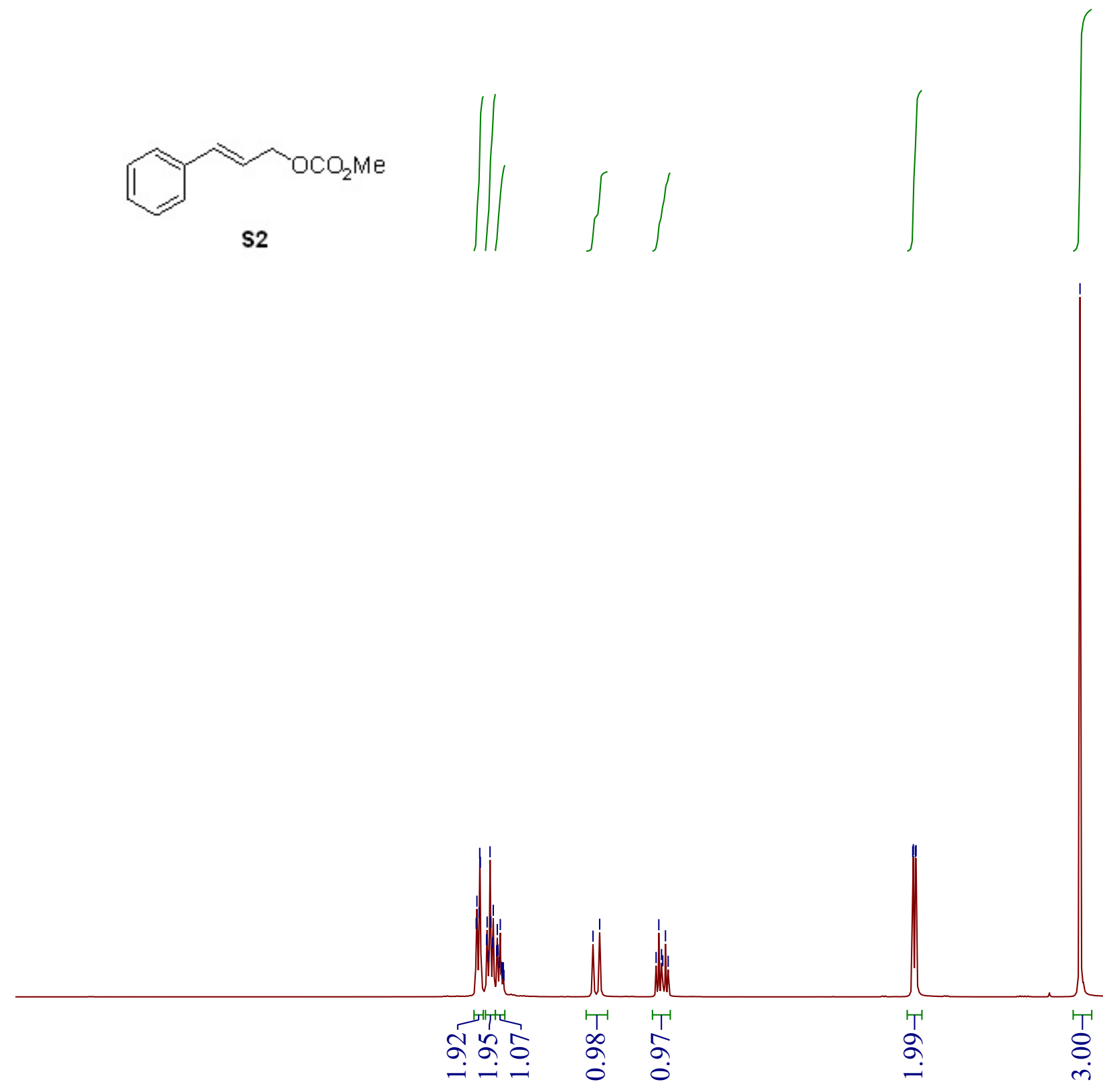

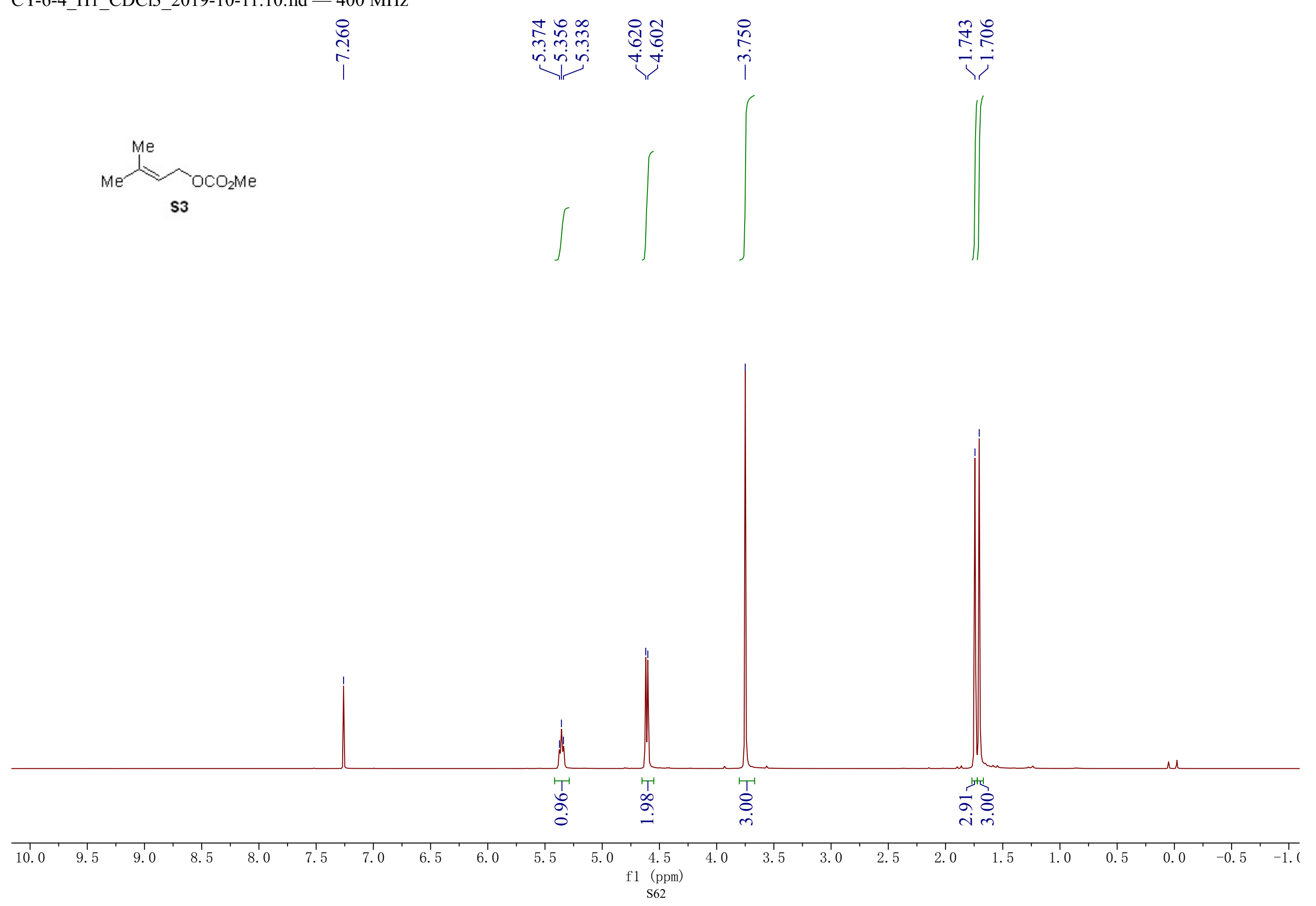

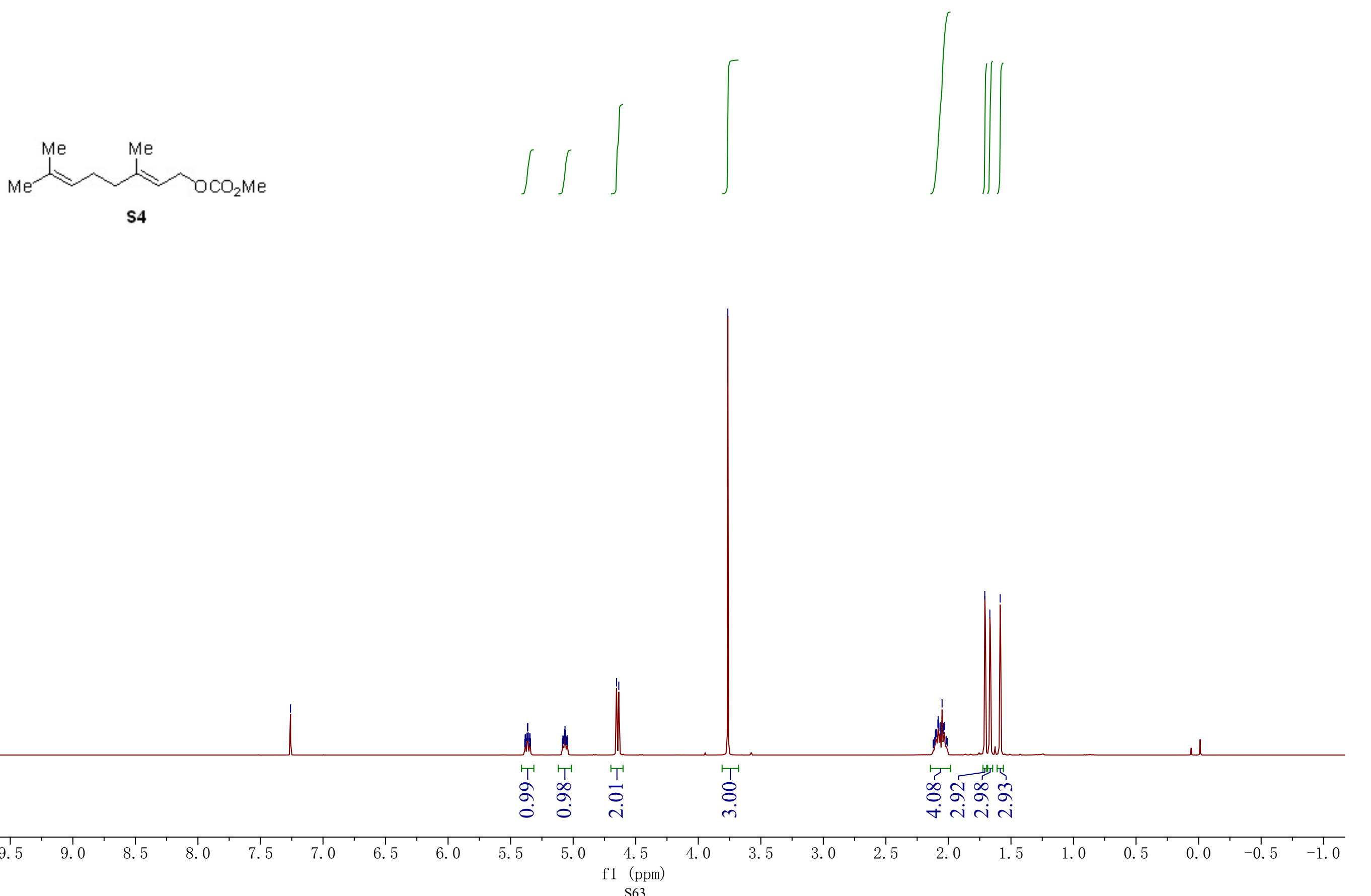

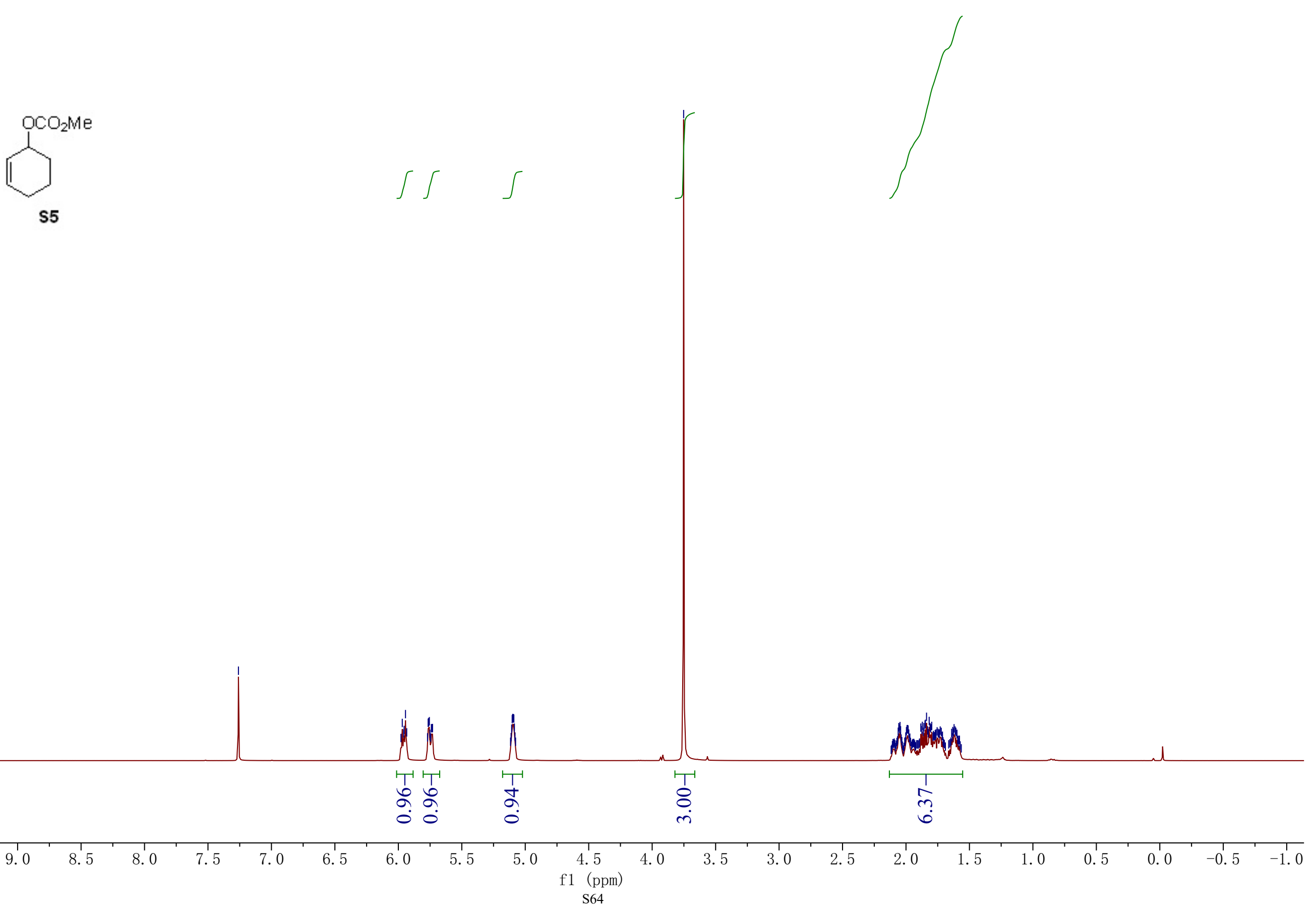

S5 

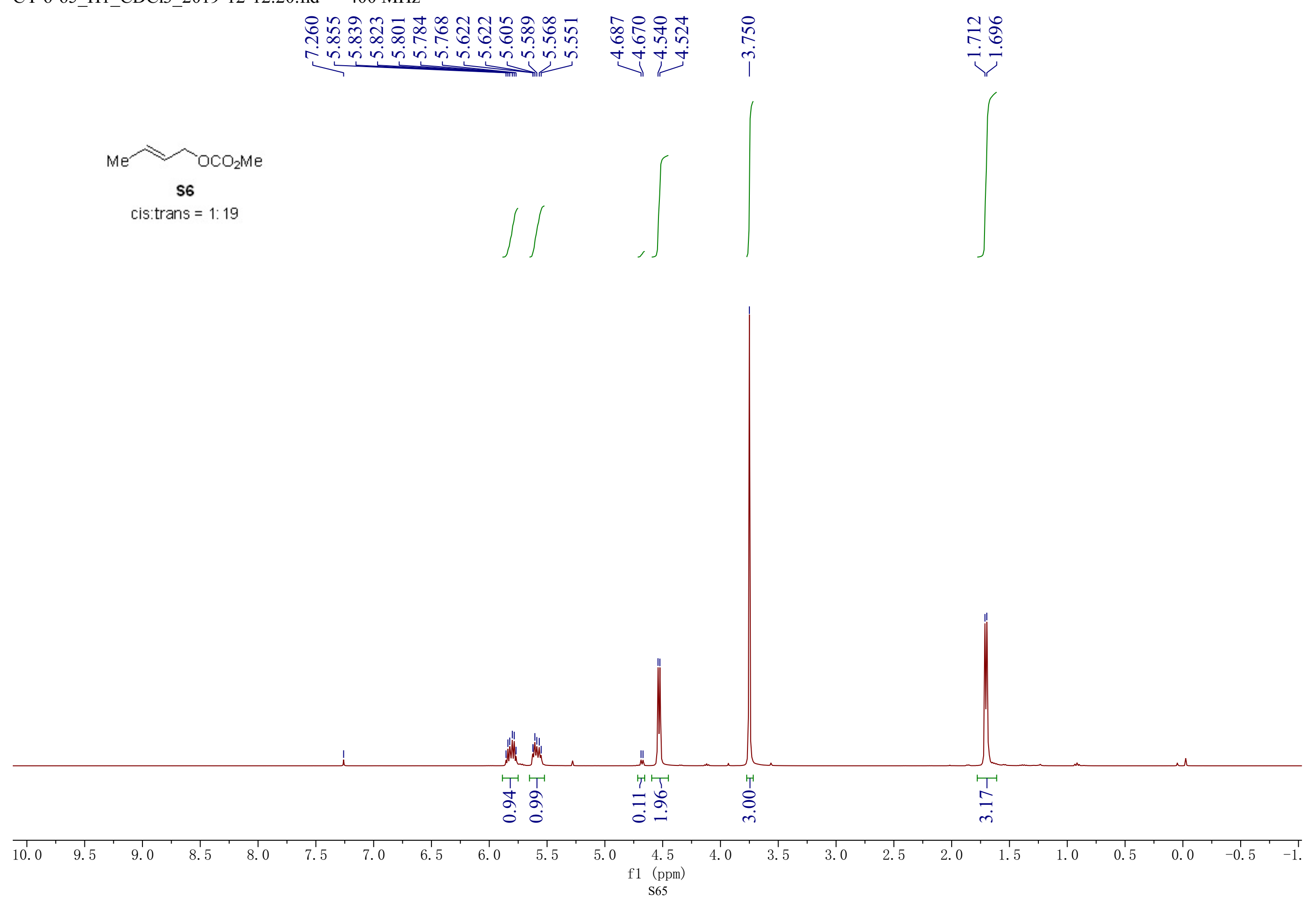

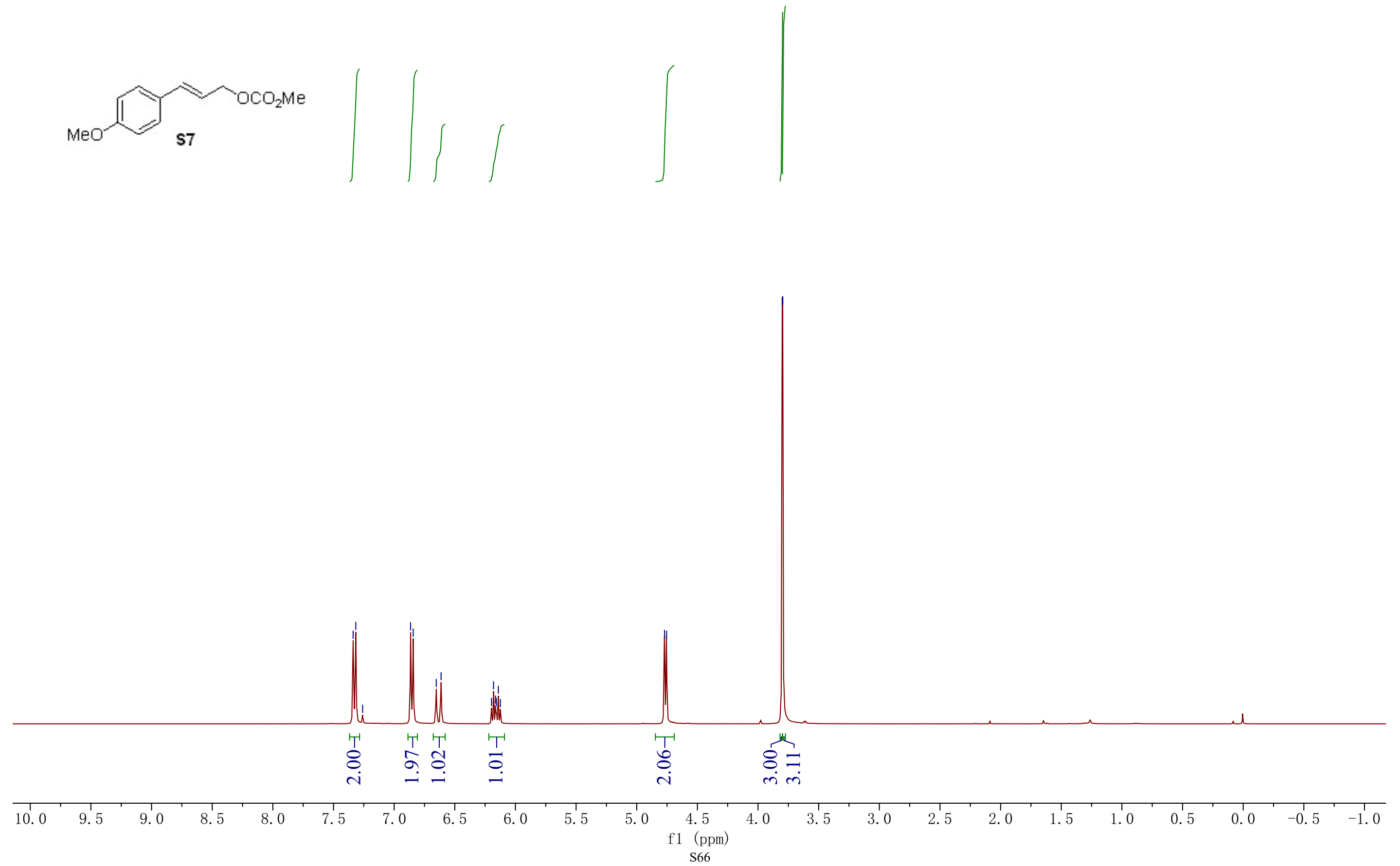

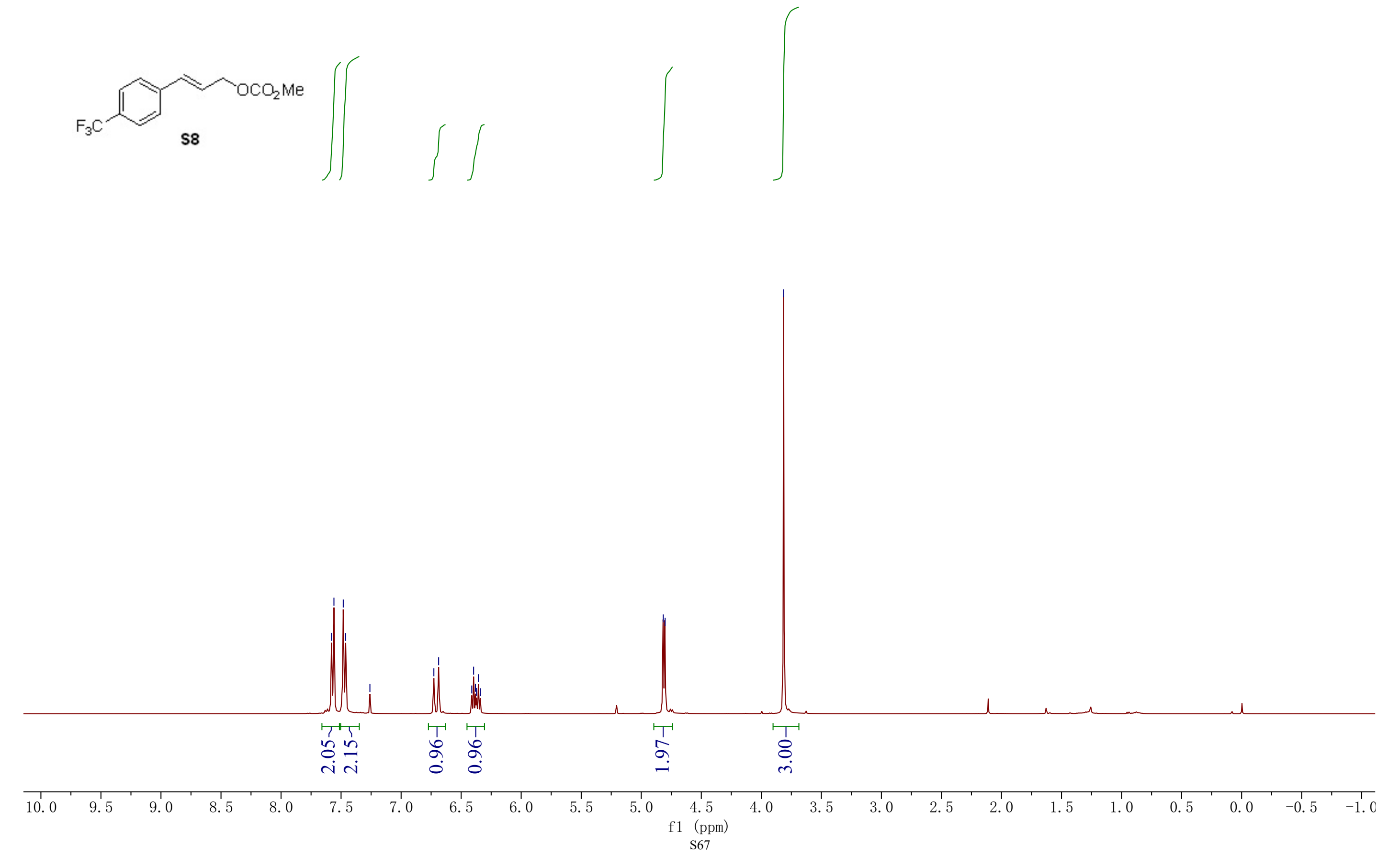


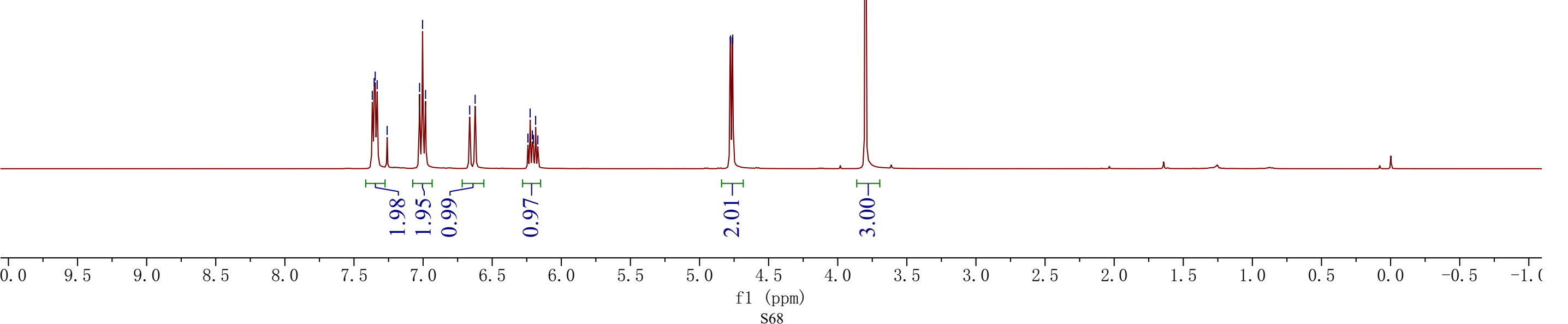



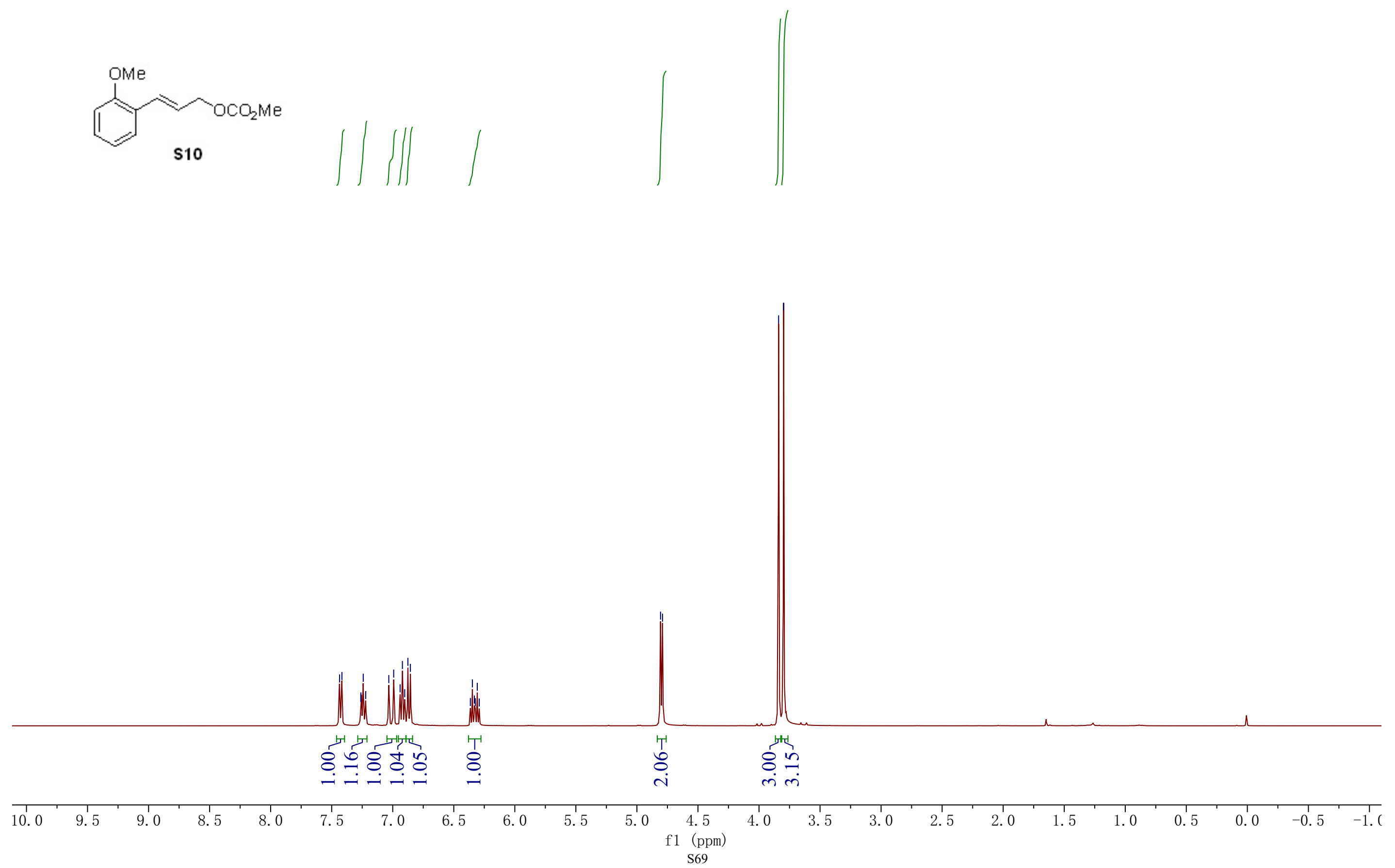


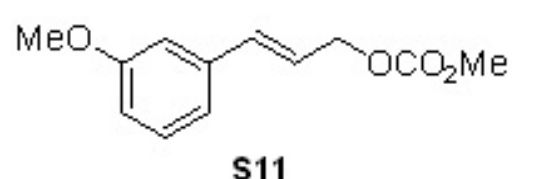

s11 

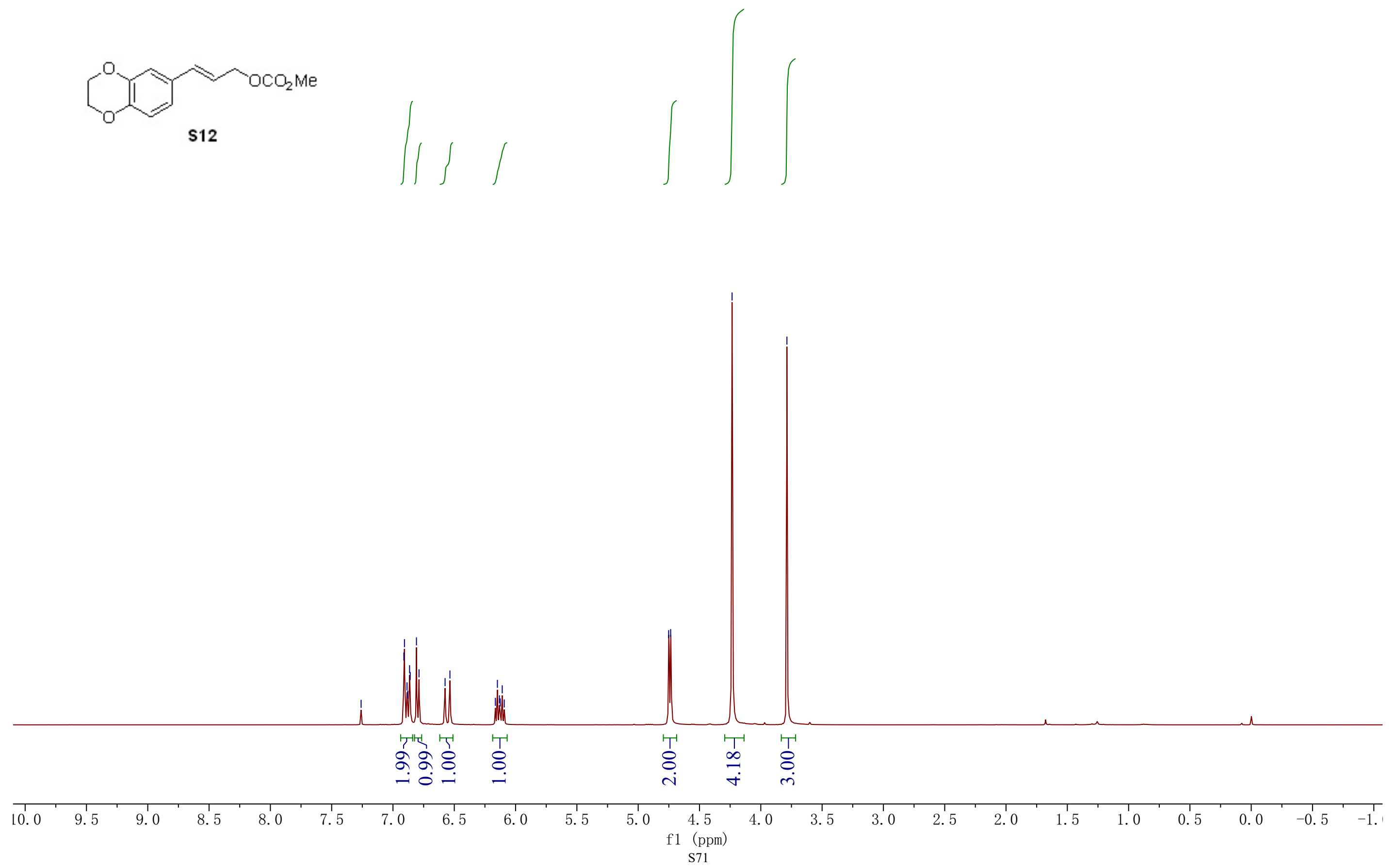


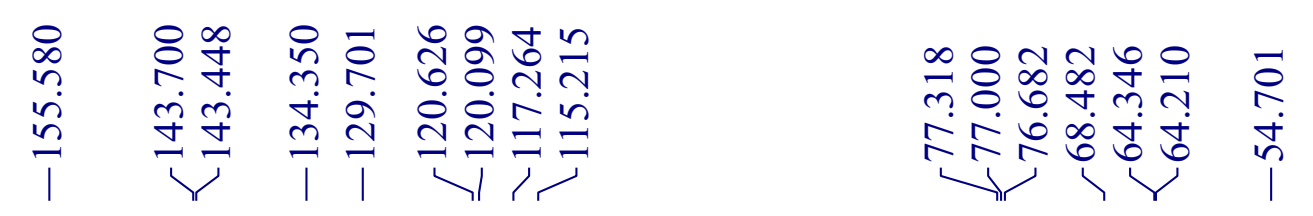

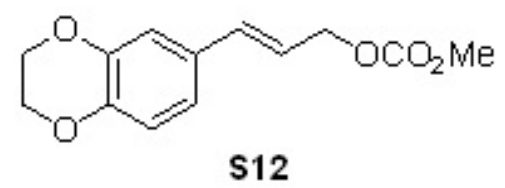

$\mathrm{S12}$

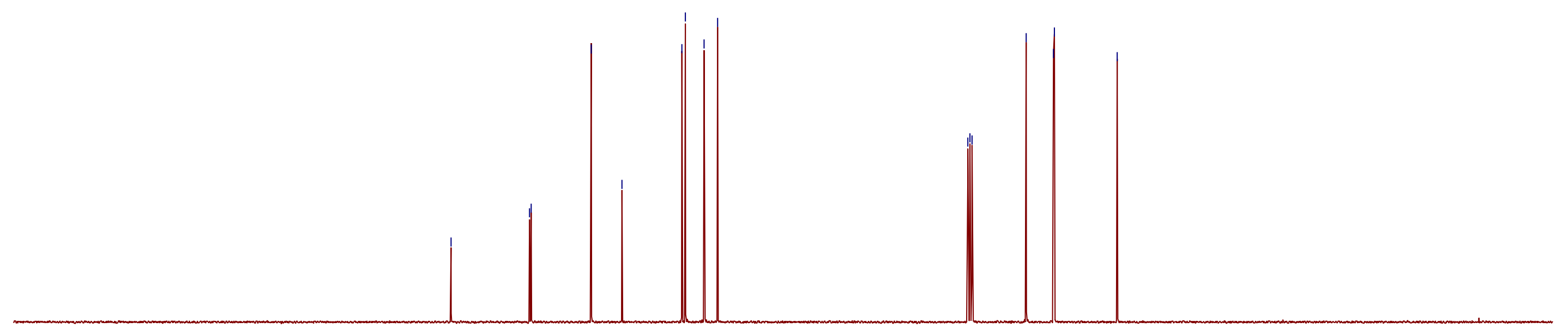


CY-6-42-A4-CDCL3-H1-2019-11-14.80.fid - $400 \mathrm{MHz}$

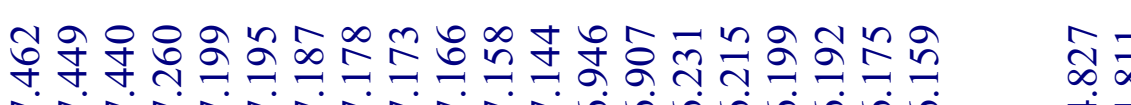

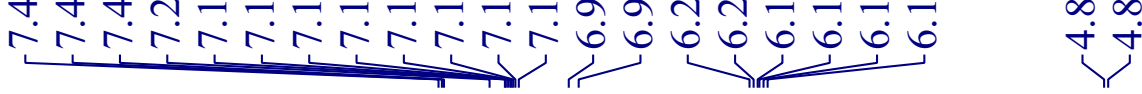

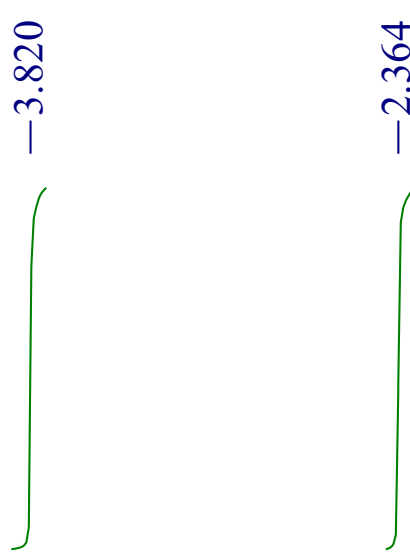

513
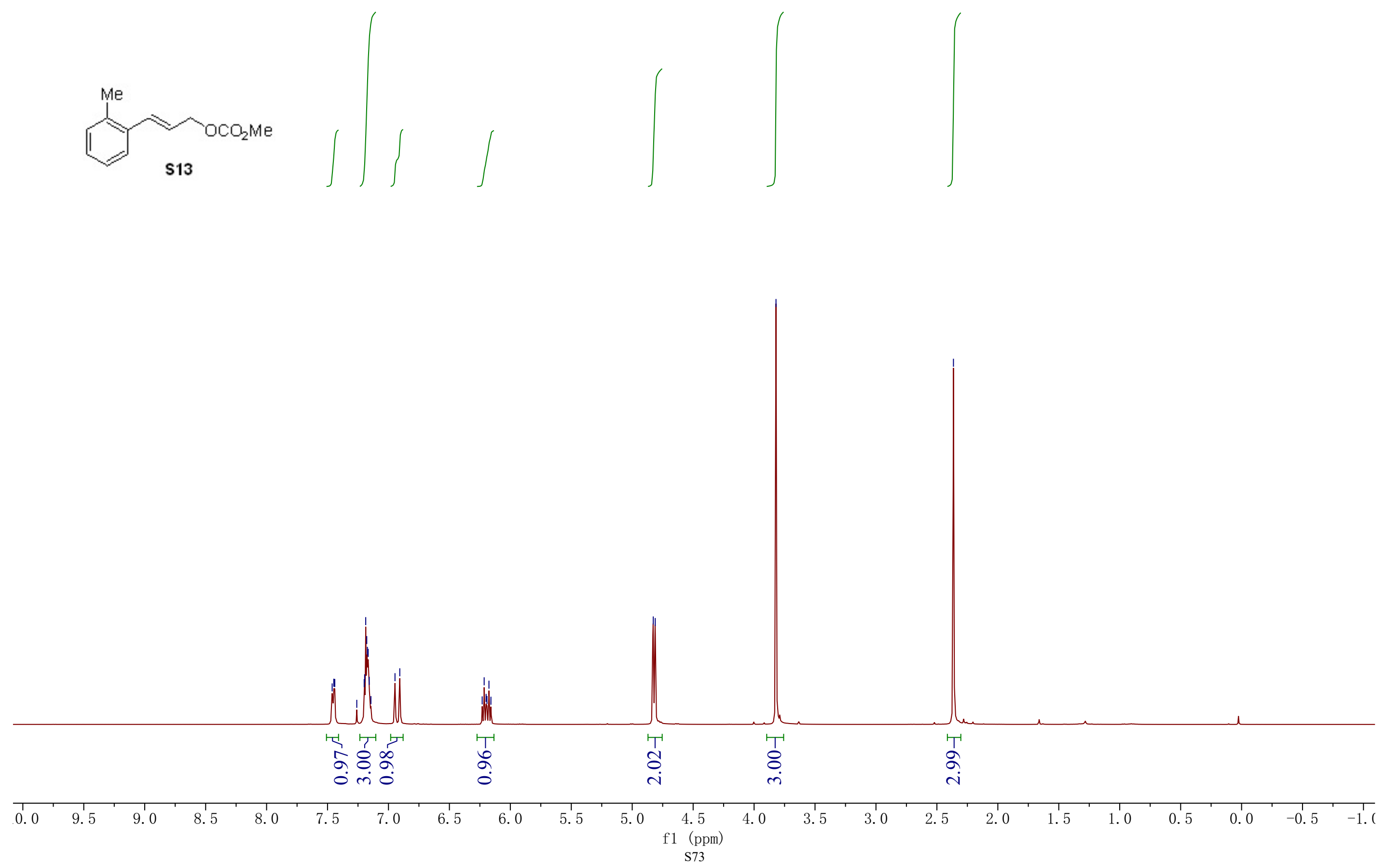

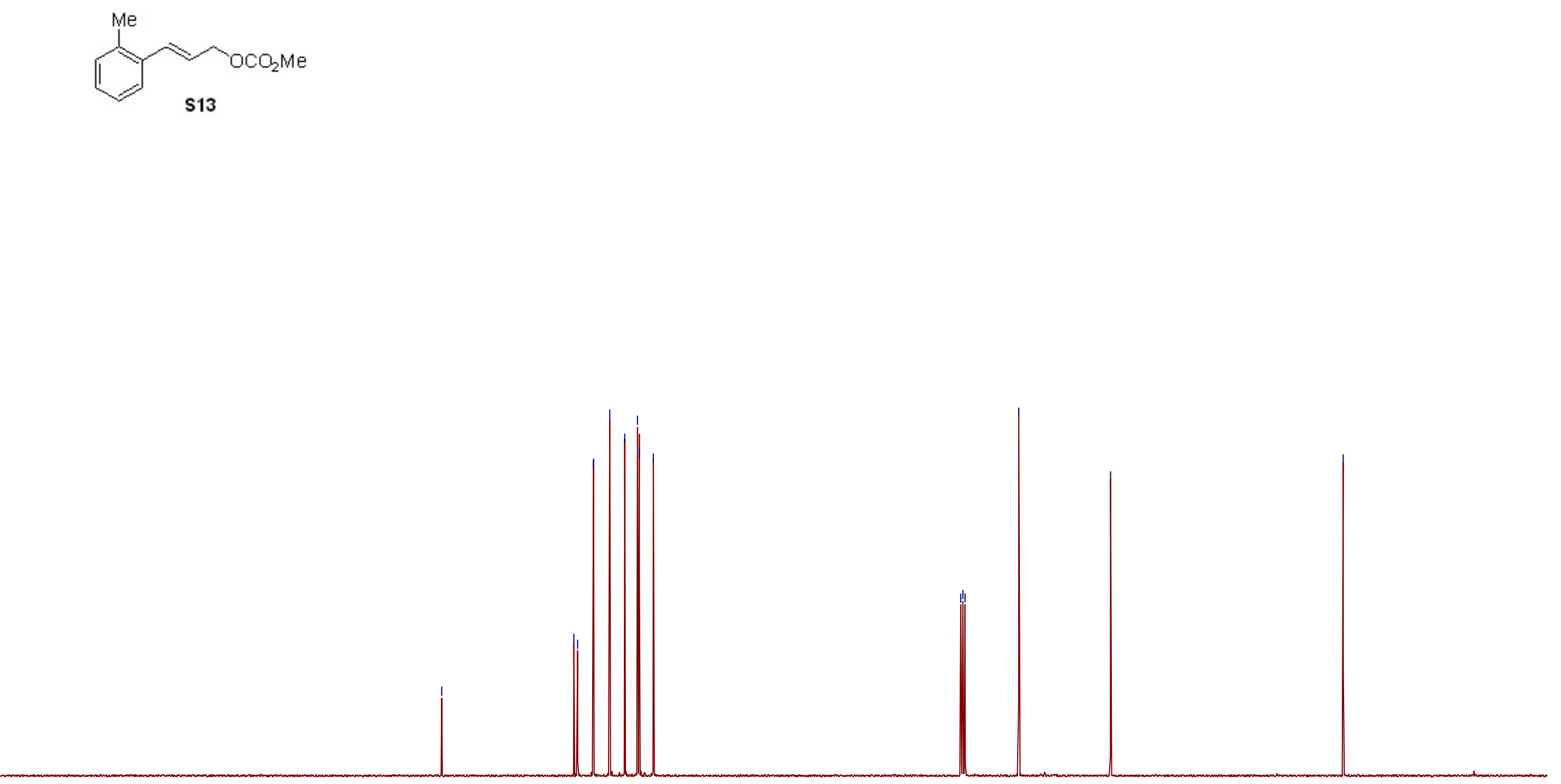

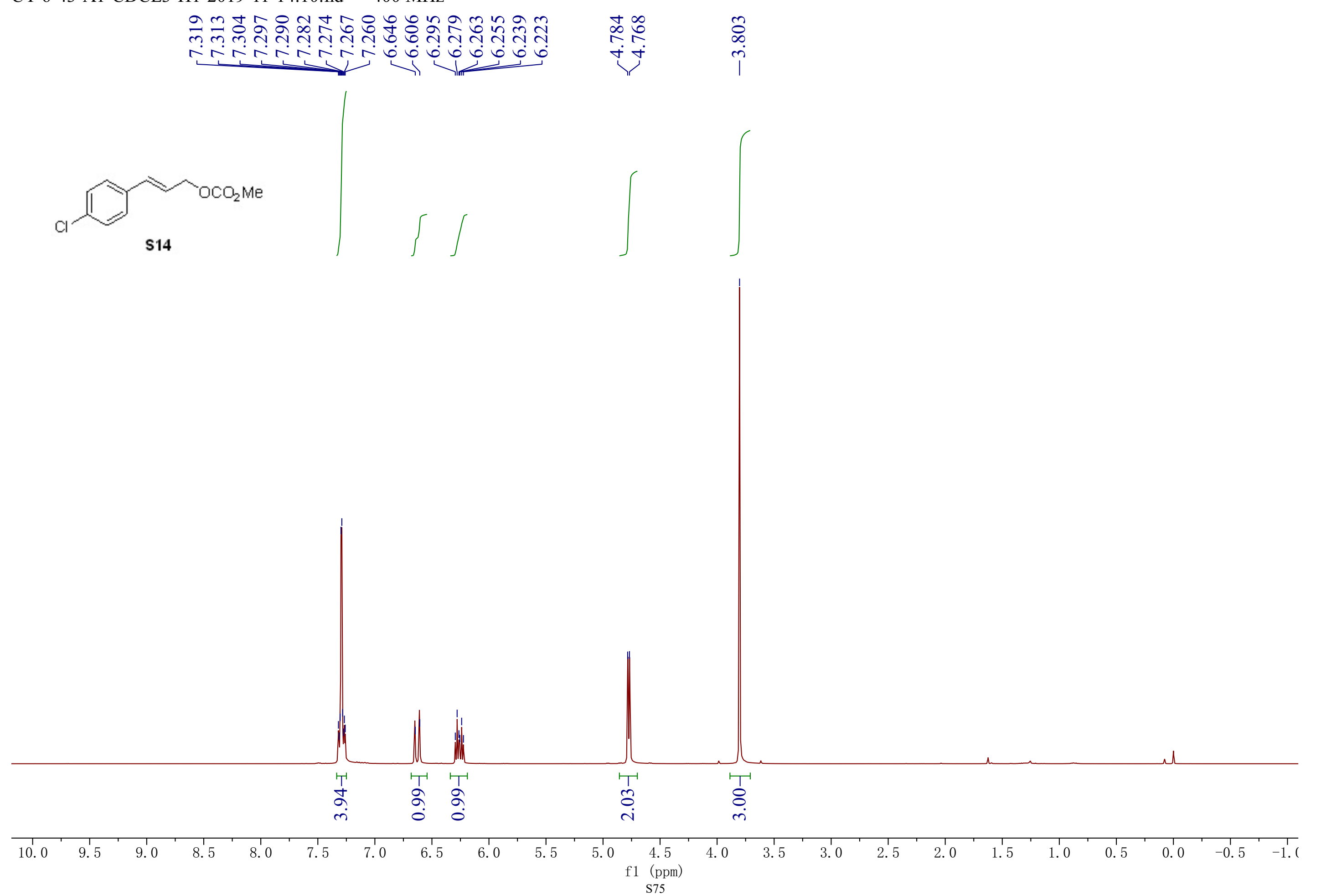

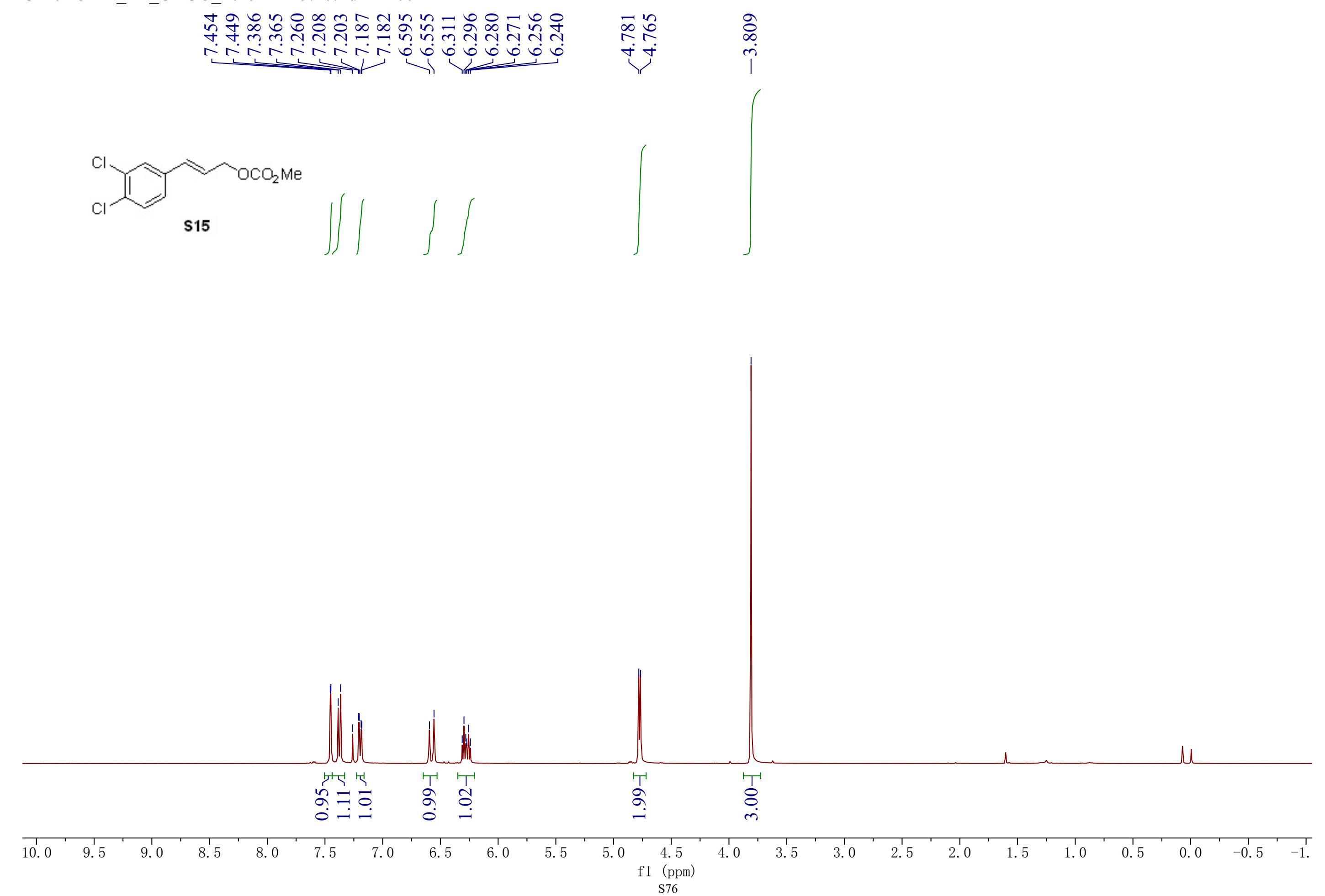

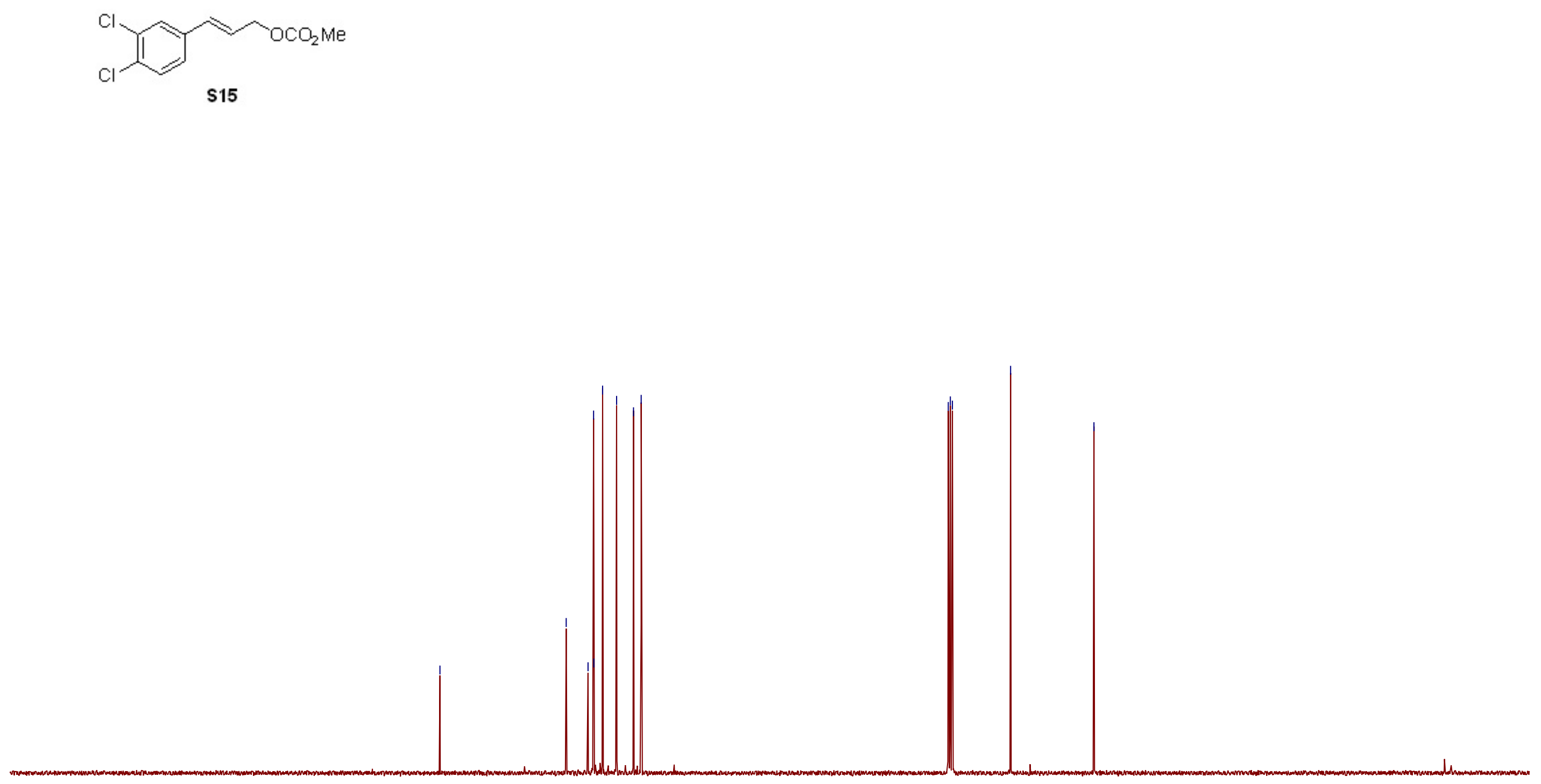
CY-6-43-A3_H1_CDCl3_2019-11-15.50.fid - $400 \mathrm{MHz}$

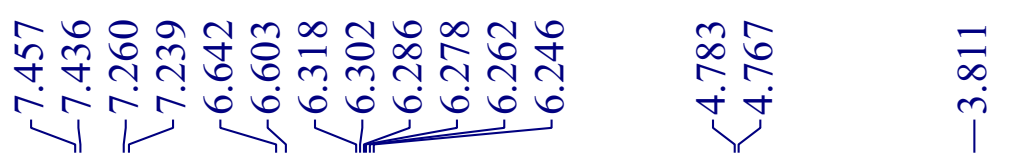

$\overbrace{\mathrm{S} 16} \mathrm{OCO}_{2} \mathrm{Me}$
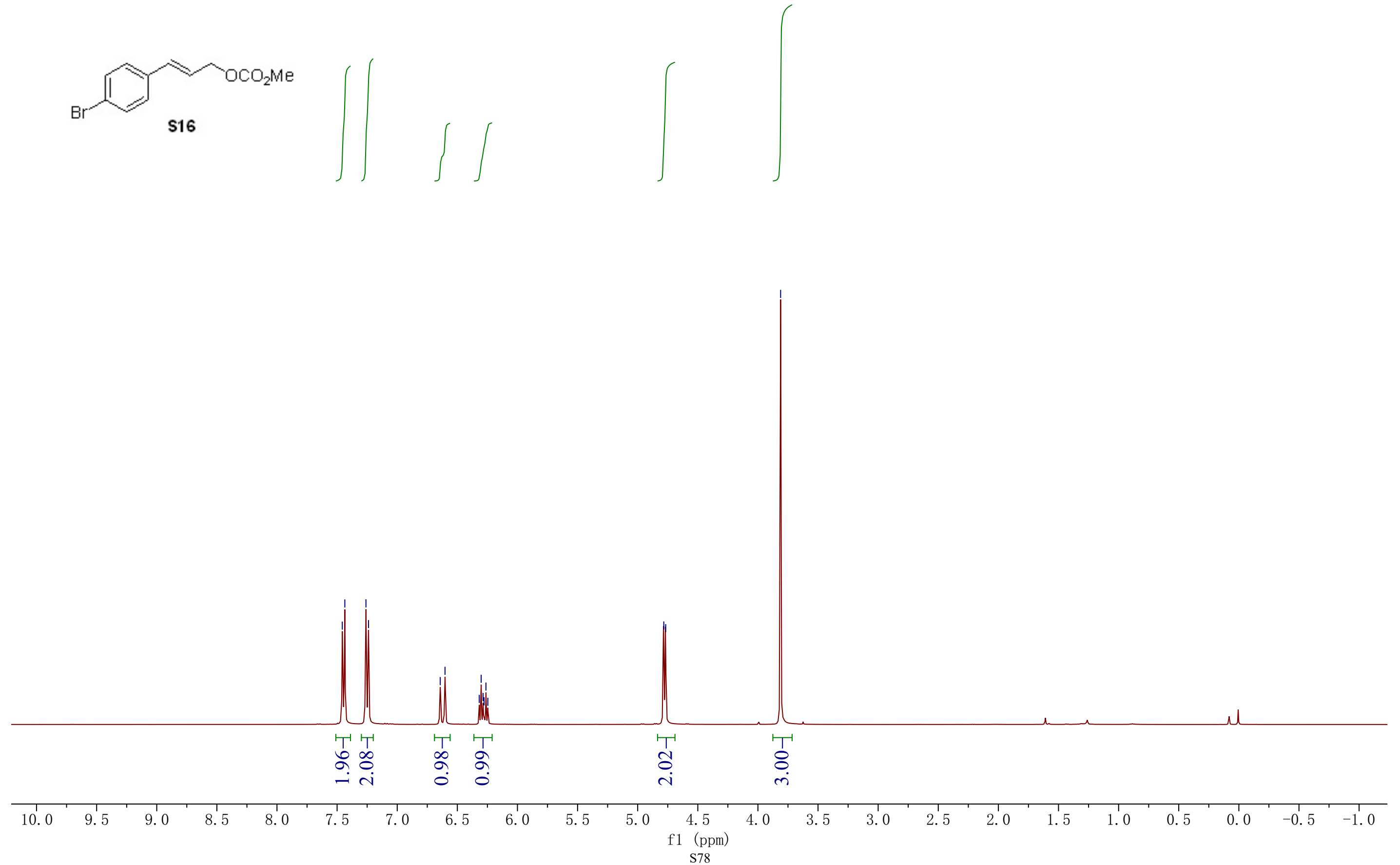

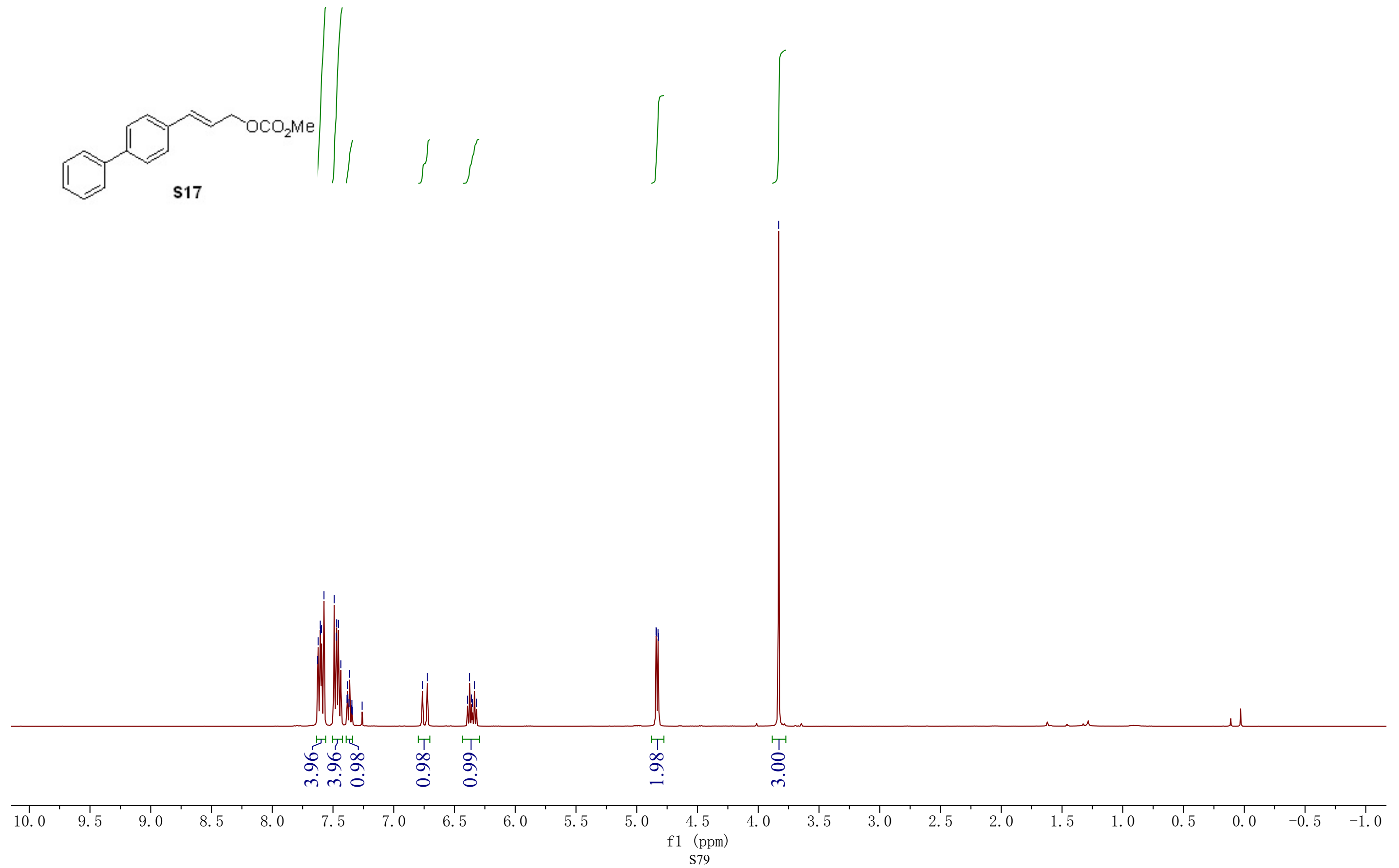

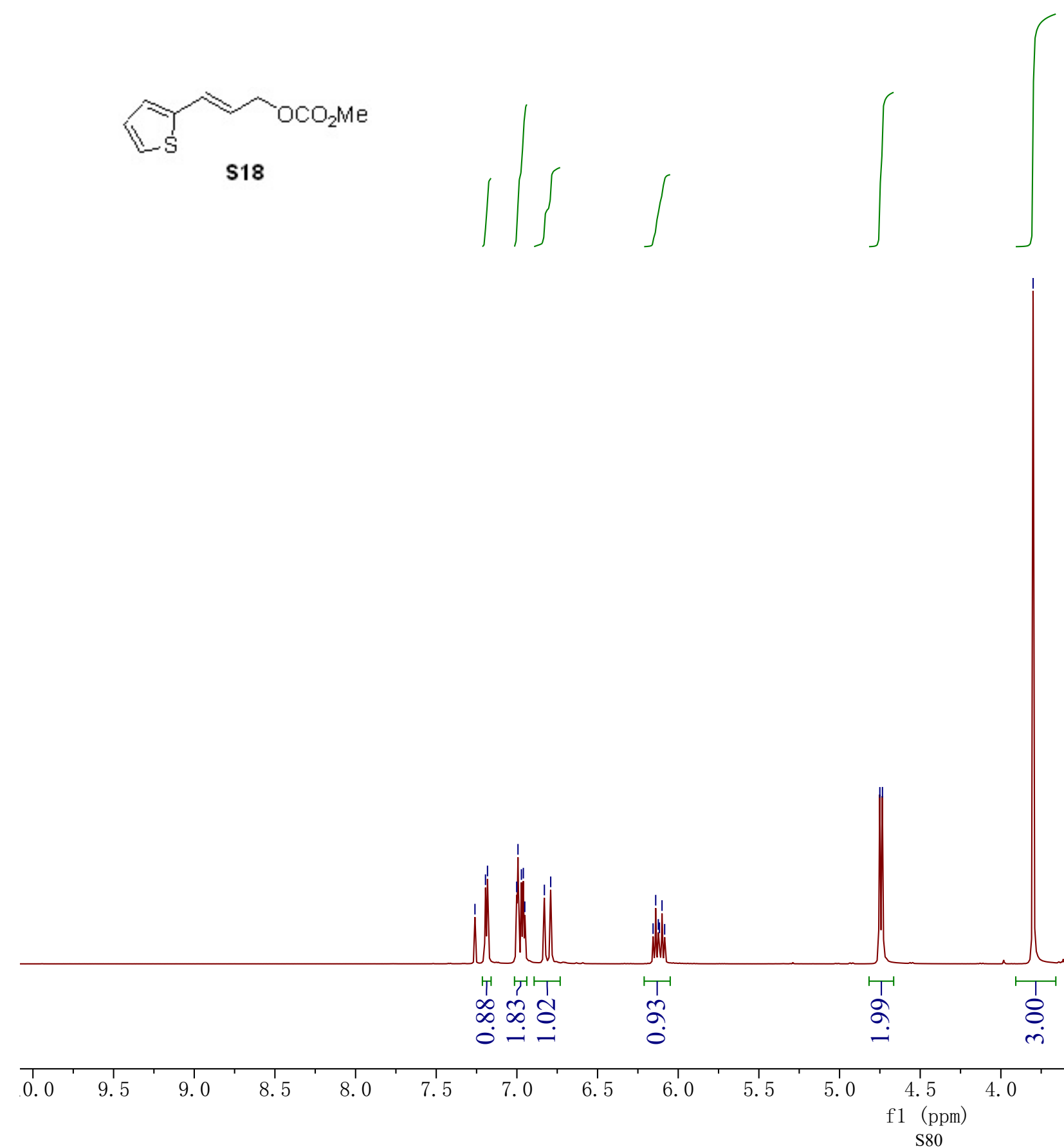

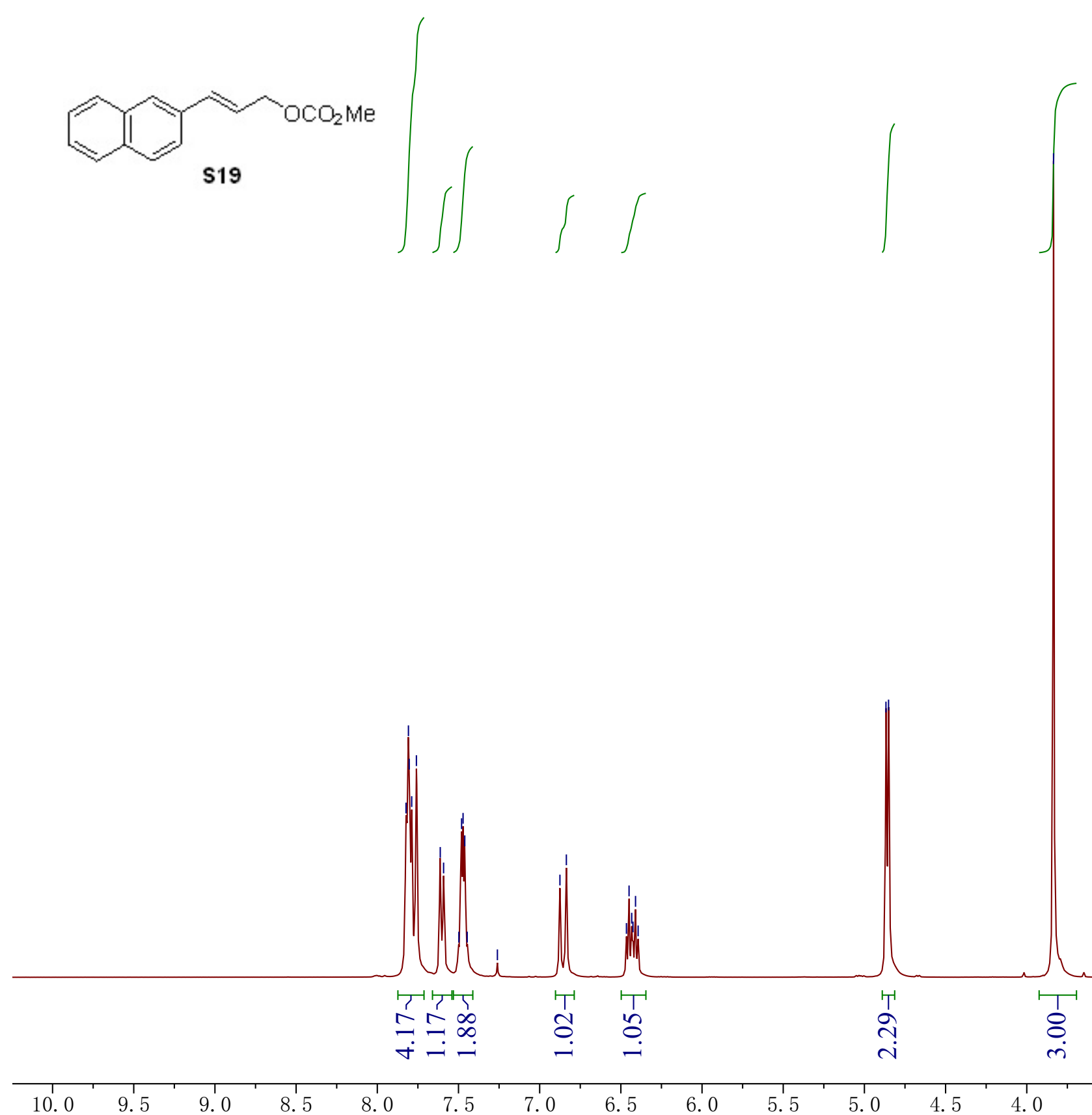

7. 5

$0 \quad 6.5$




\section{CY-6-49-A2-CDCL3-2019-11-19-400 MHz}

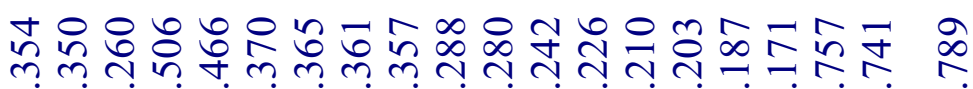

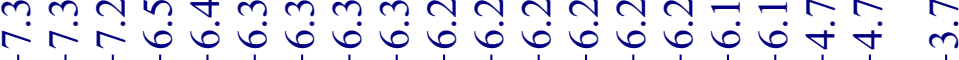

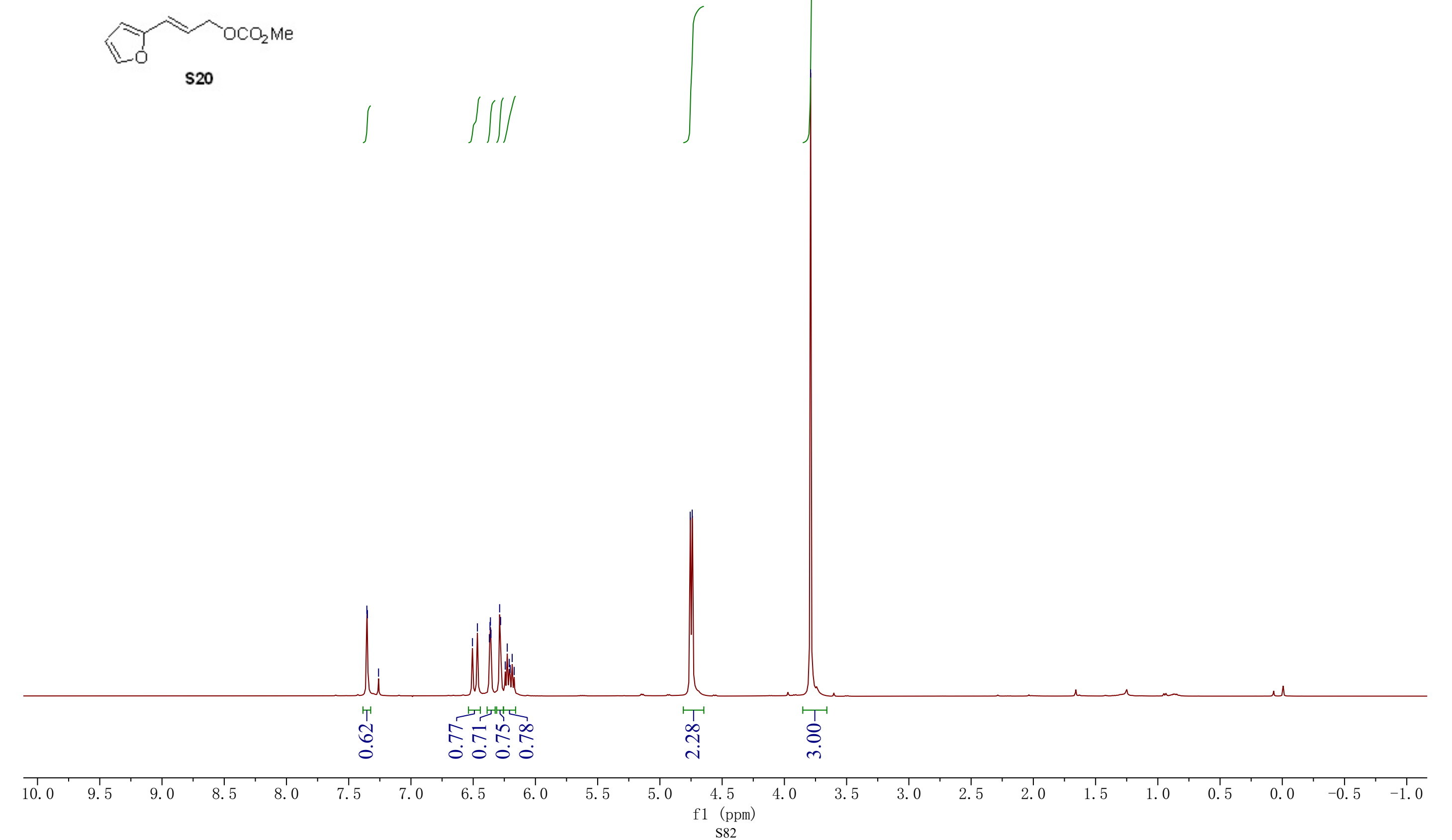

$\mathbf{s} 20$

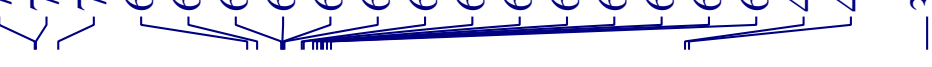



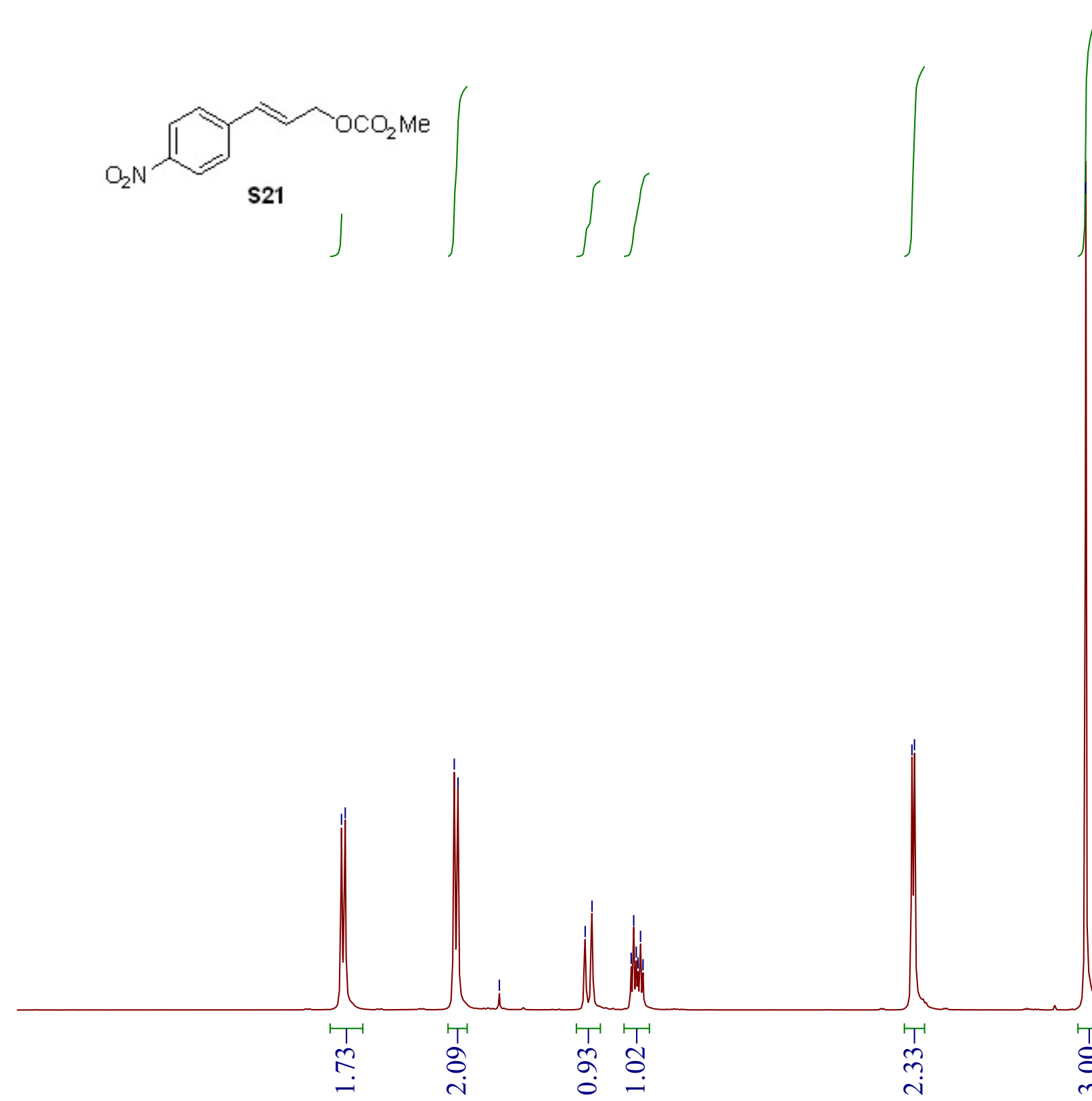

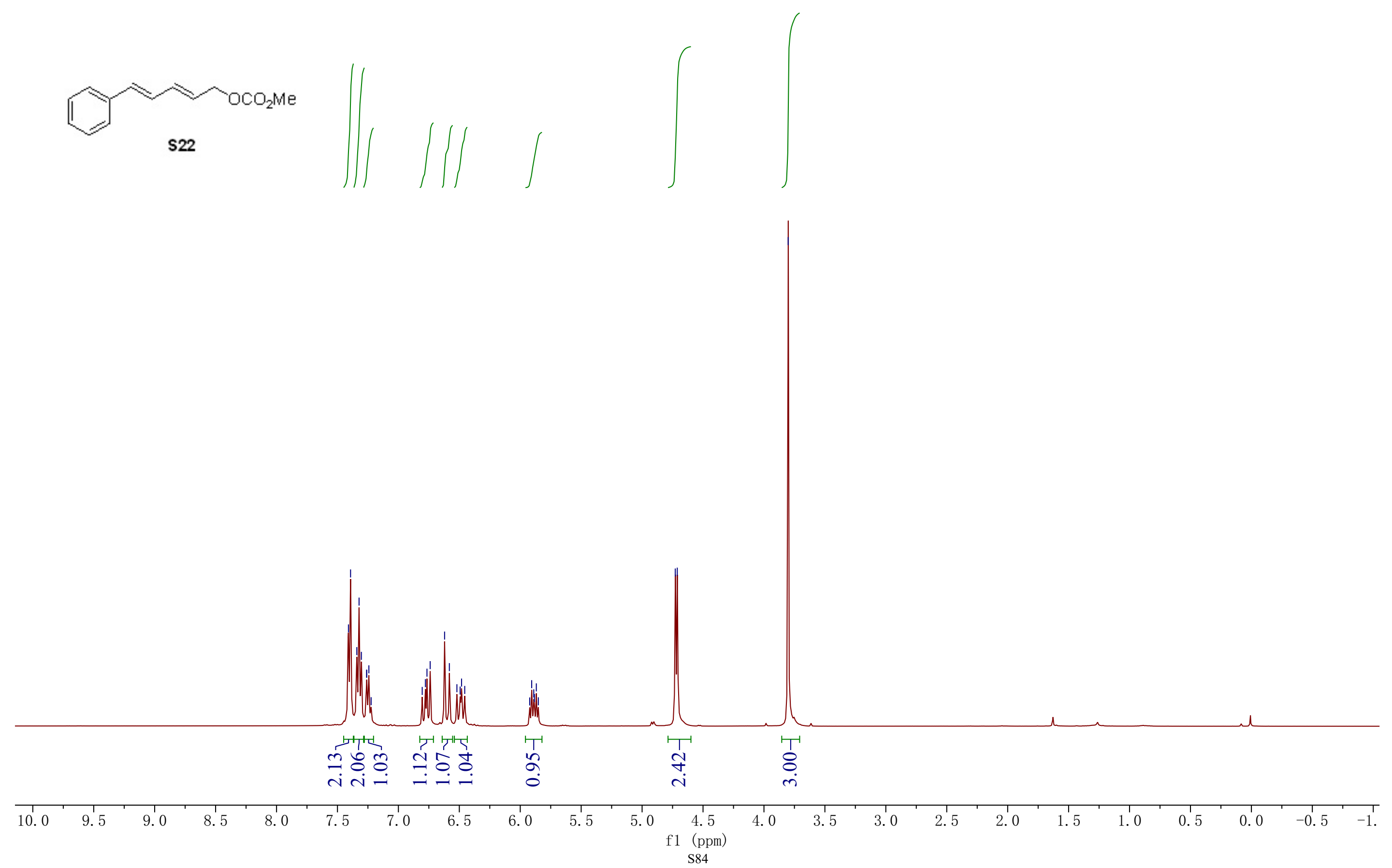

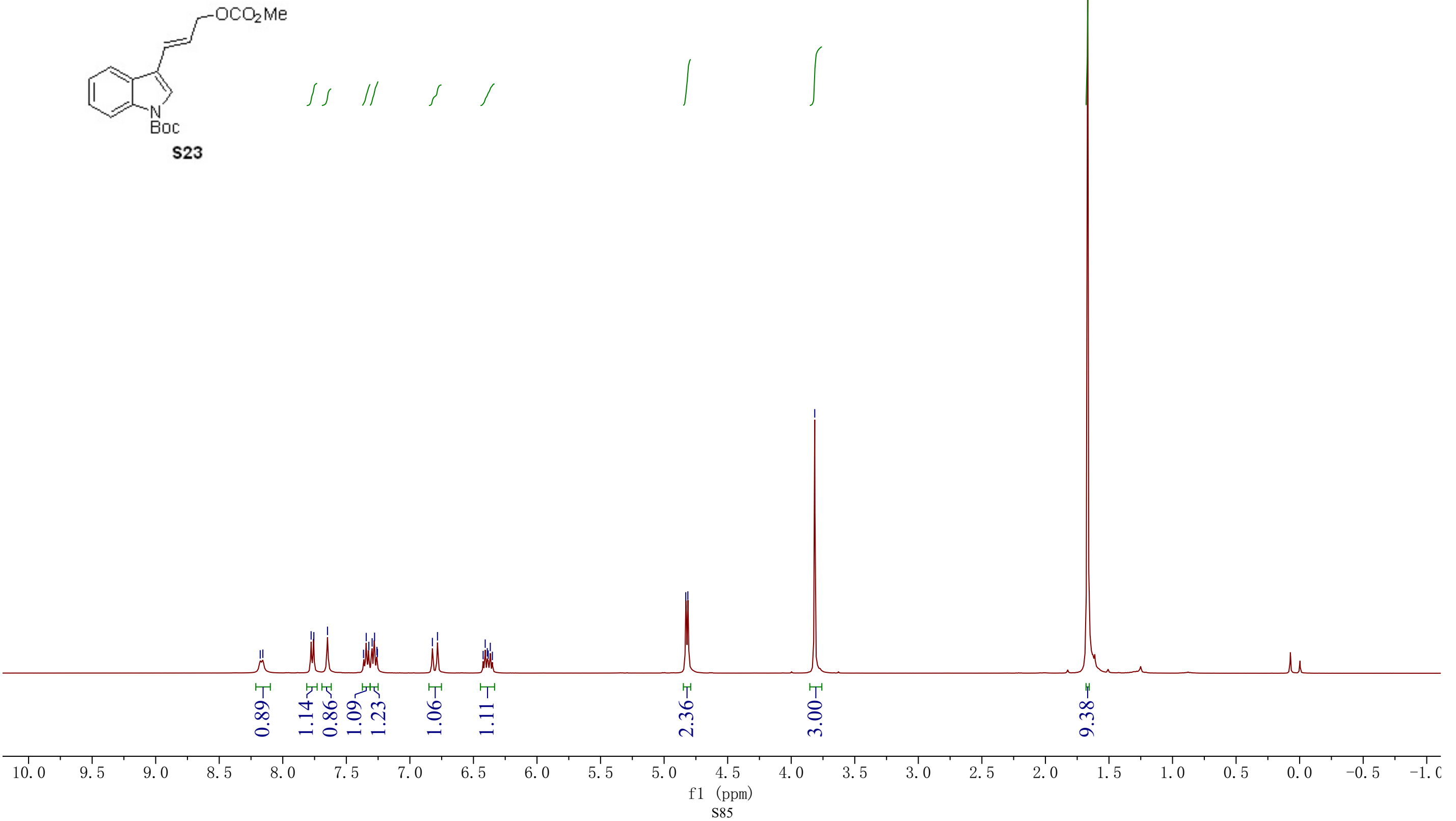

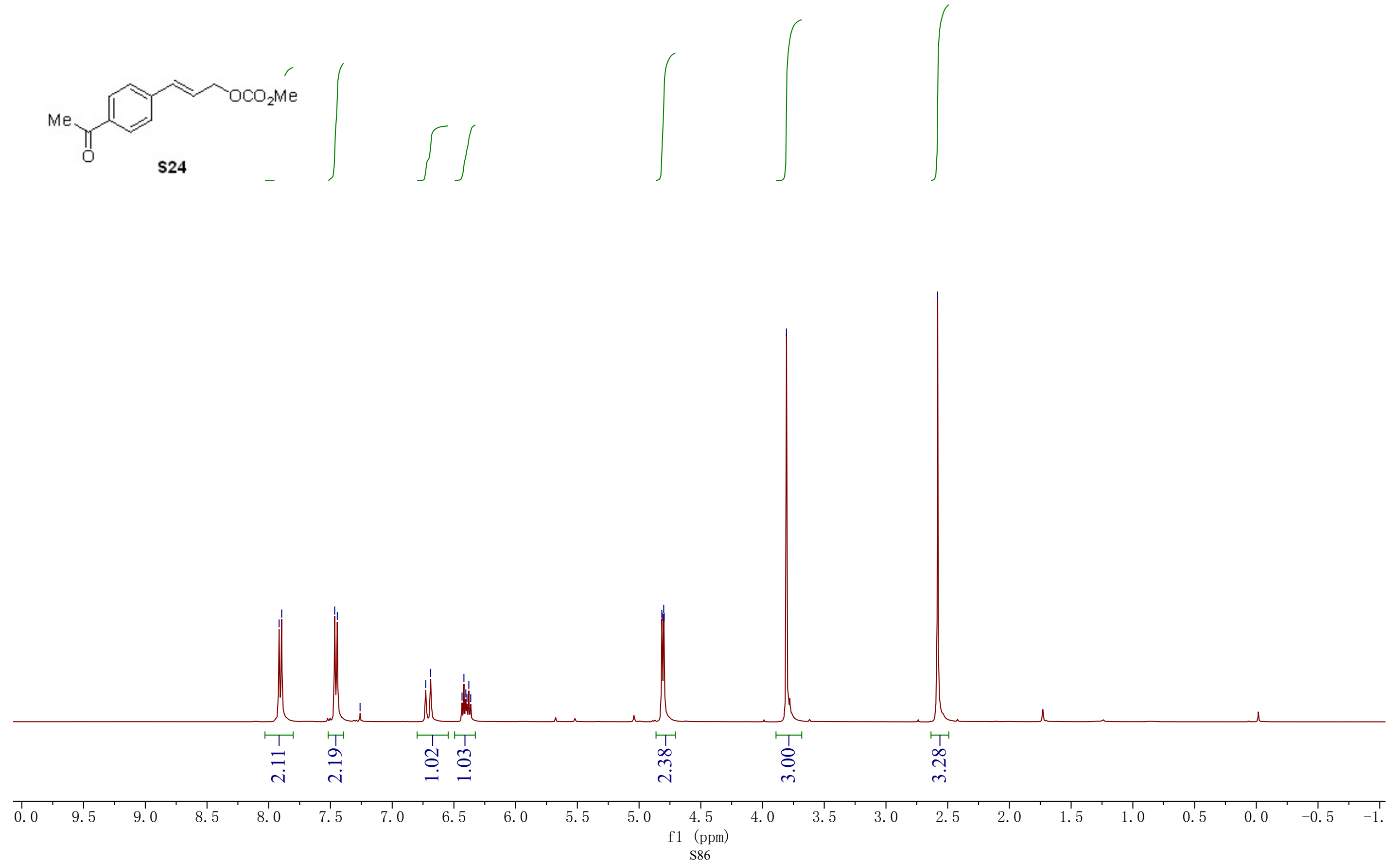
CY-6-66_C13_CDCl3_2019-12-13.10.fid - 100 MHz

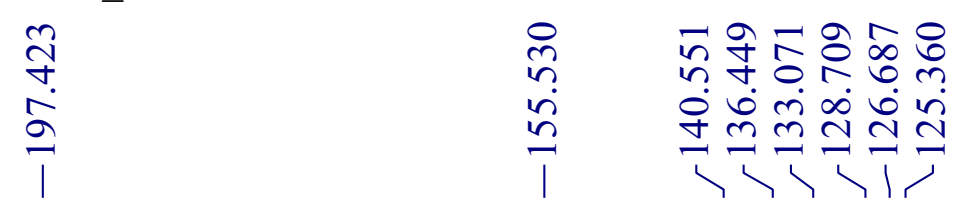

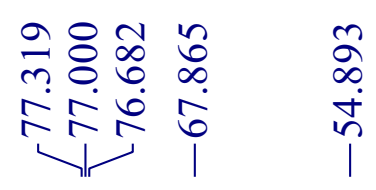
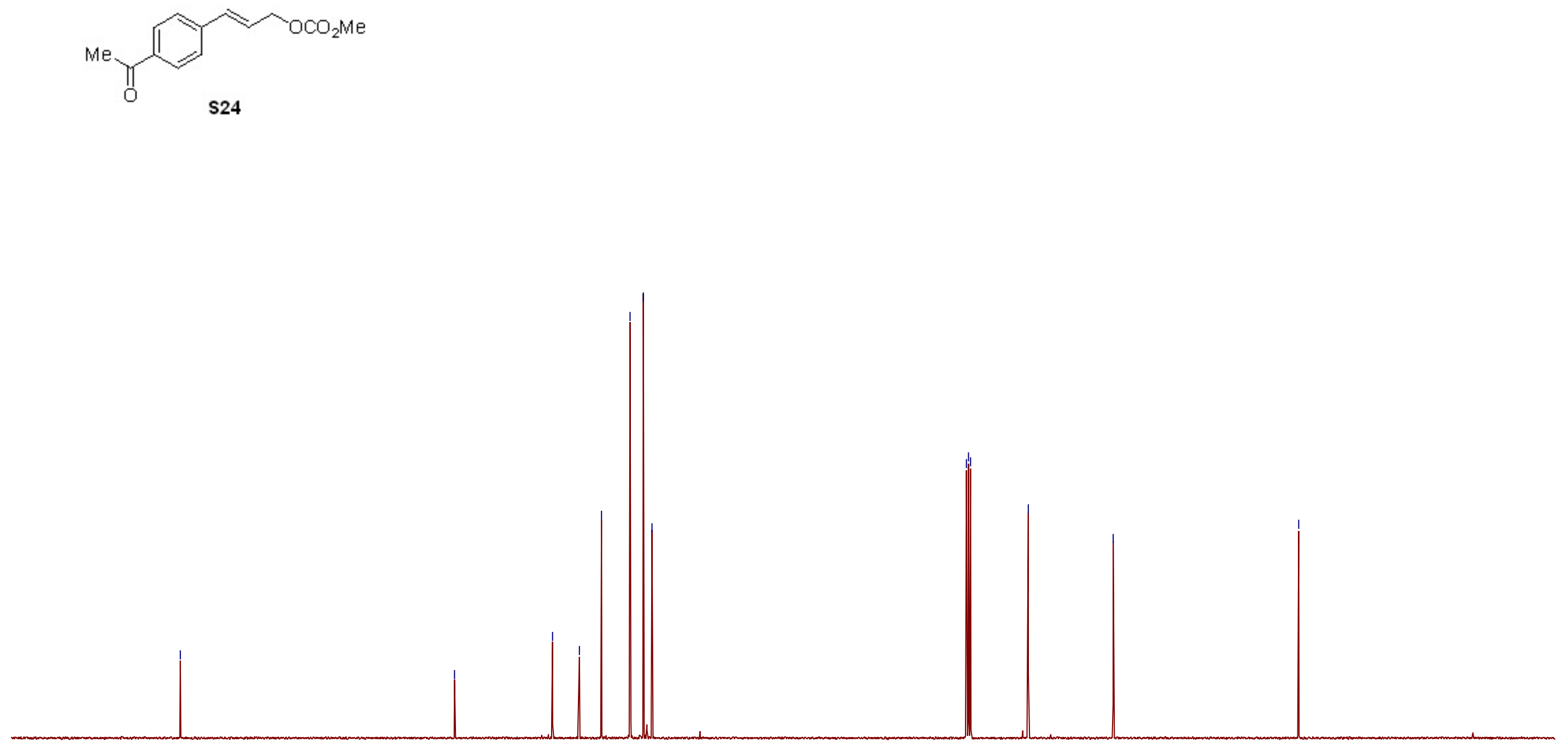


\section{CY-6-69-CDCL3-H1-2019-12-16- $400 \mathrm{MHz}$}

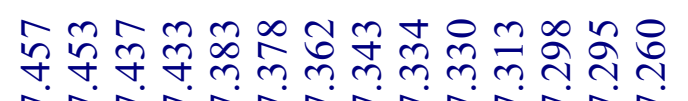

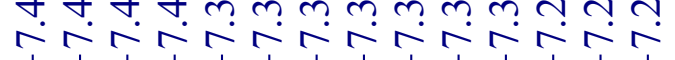

๙

in is

$\stackrel{\mathrm{C}}{\mathrm{PCO}} \mathrm{Me}$

S25
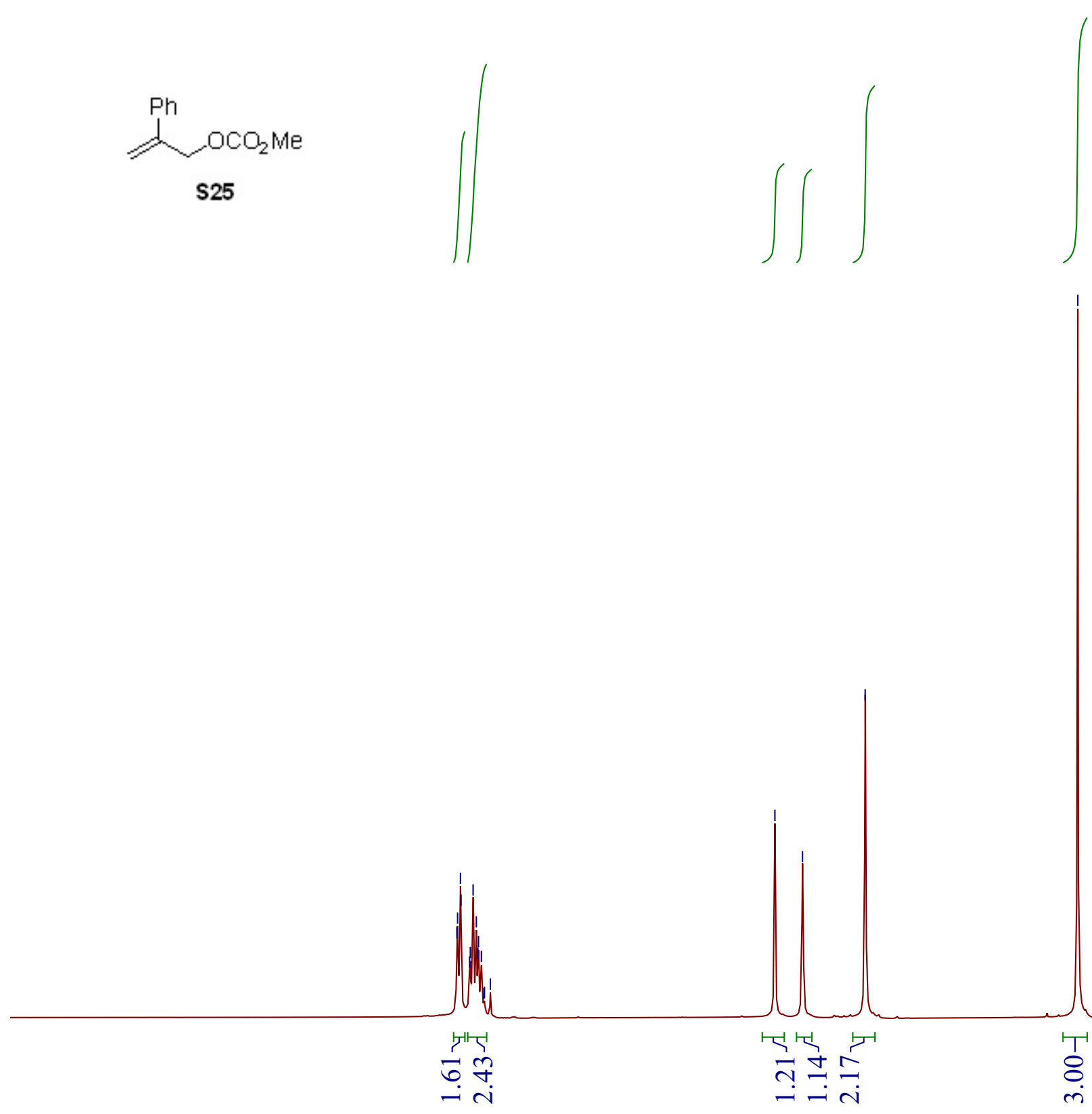

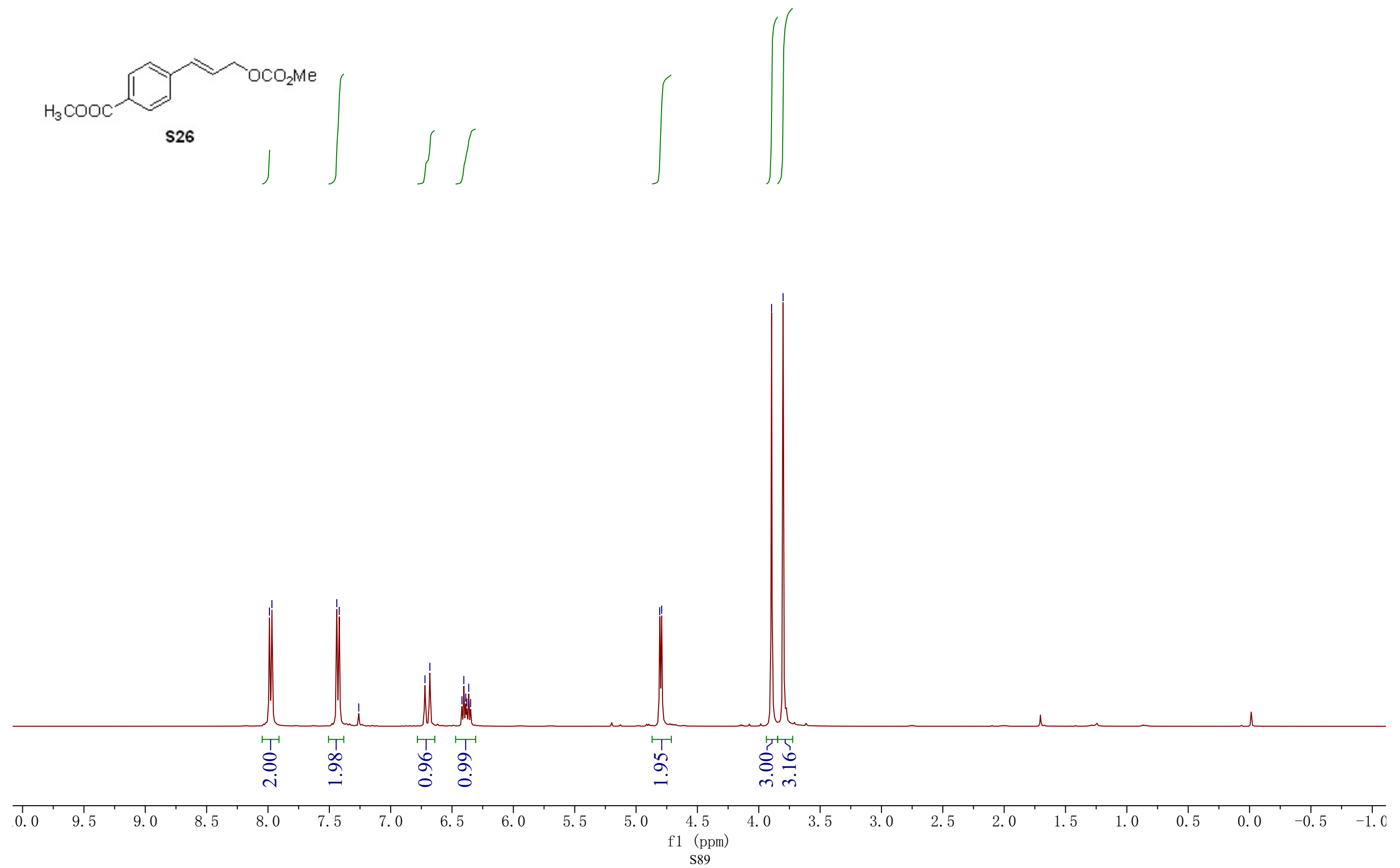

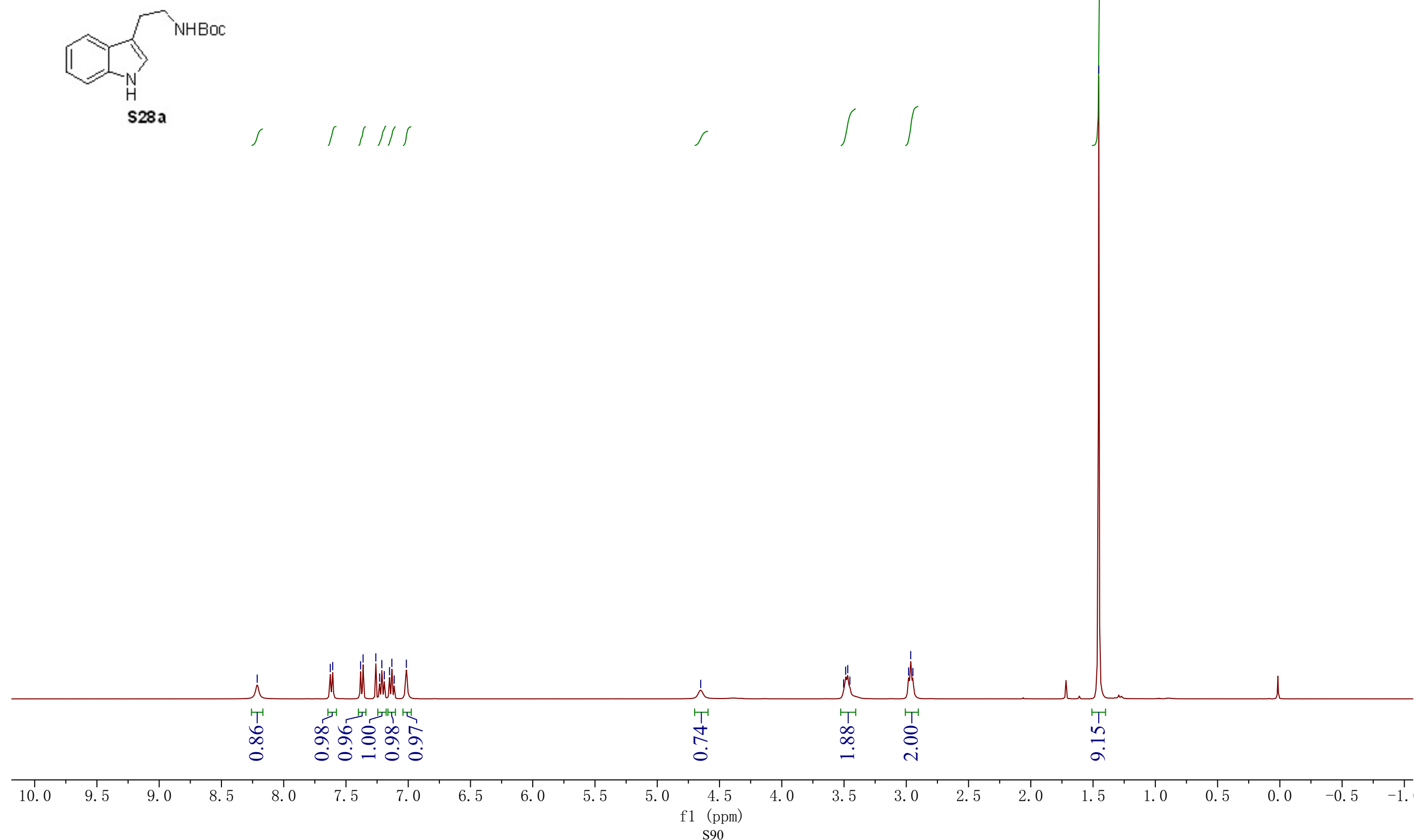
<smiles>COc1ccc2[nH]cc(CCNC(=O)OCc3ccccc3)c2c1</smiles>

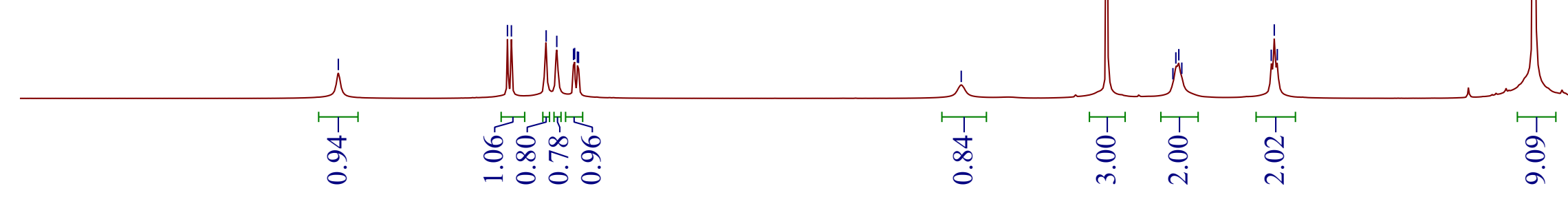


CY-6-61-A2-2_H1_CDCl3_2019-12-11.200.fid - $400 \mathrm{MHz}$

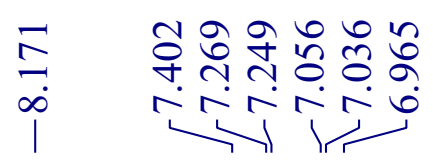<smiles>Cc1ccc2[nH]cc(CCNC(C)(C)C)c2c1</smiles>

$\mathrm{S} 28 \mathrm{c}$

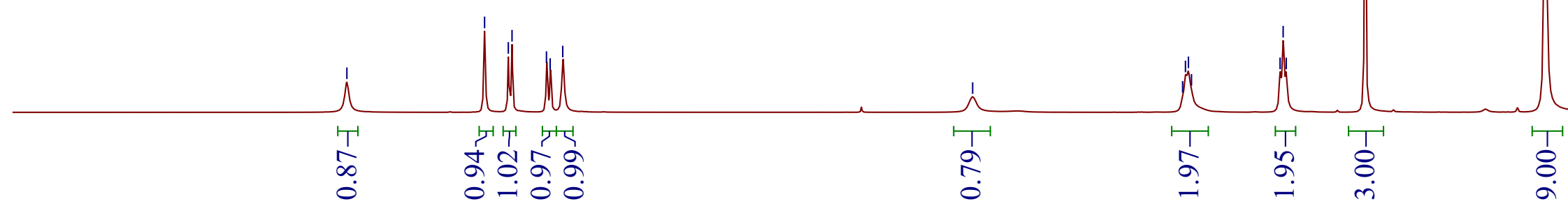




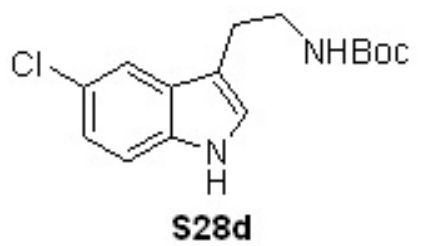



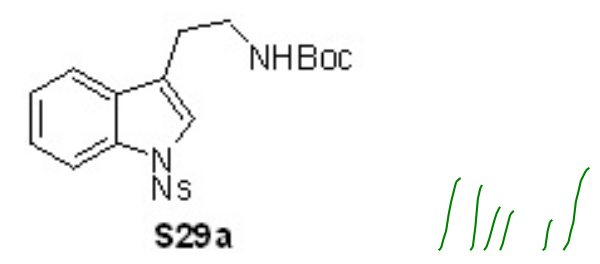

1
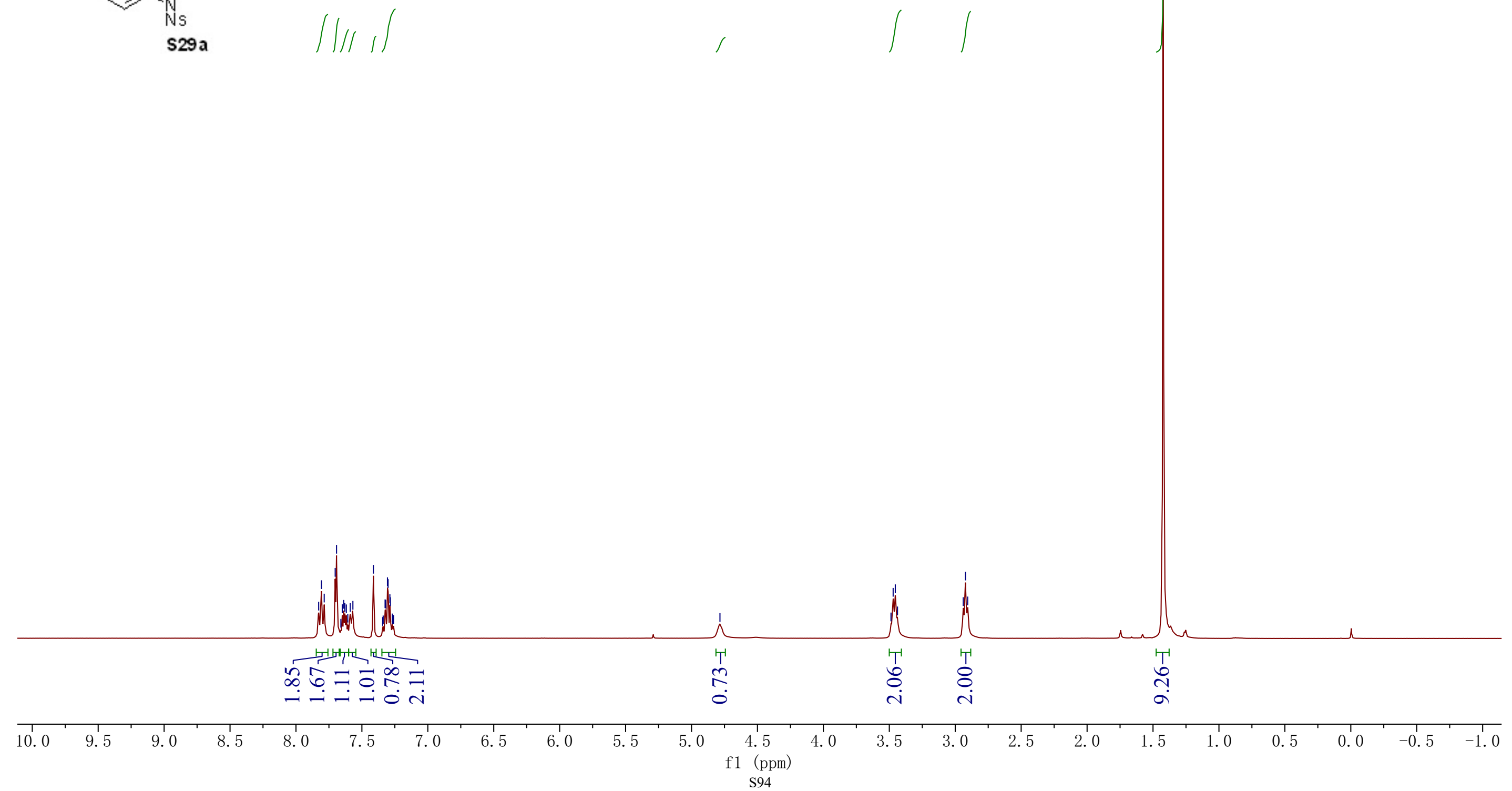

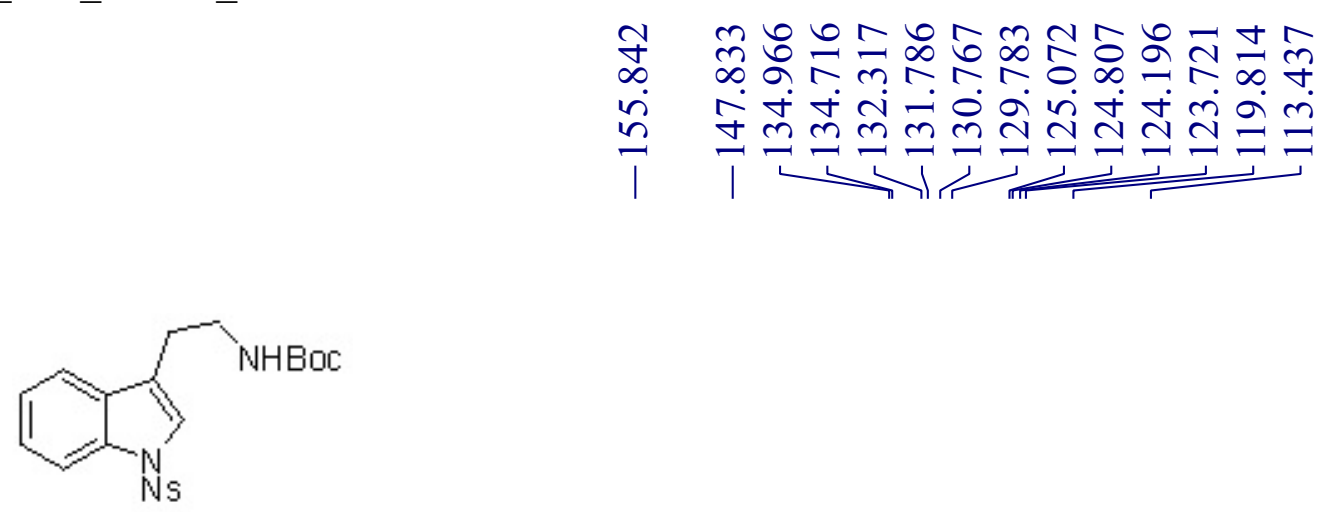

S29a

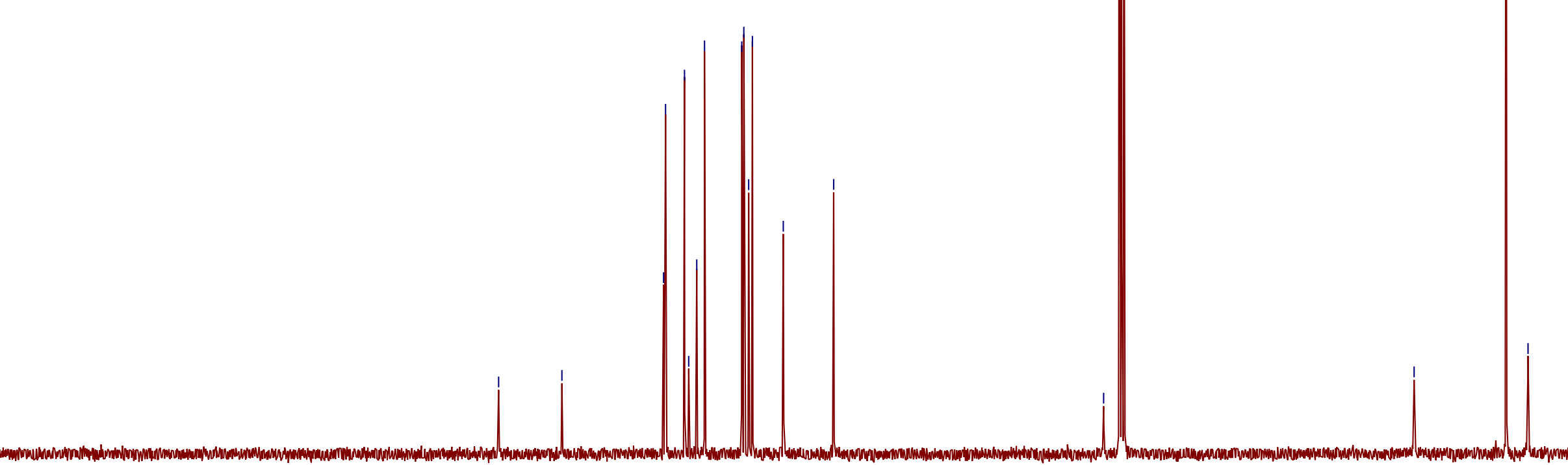


<smiles>COc1ccc2c(c1)c(CCNC(=O)OCc1ccccc1)cn2S</smiles>
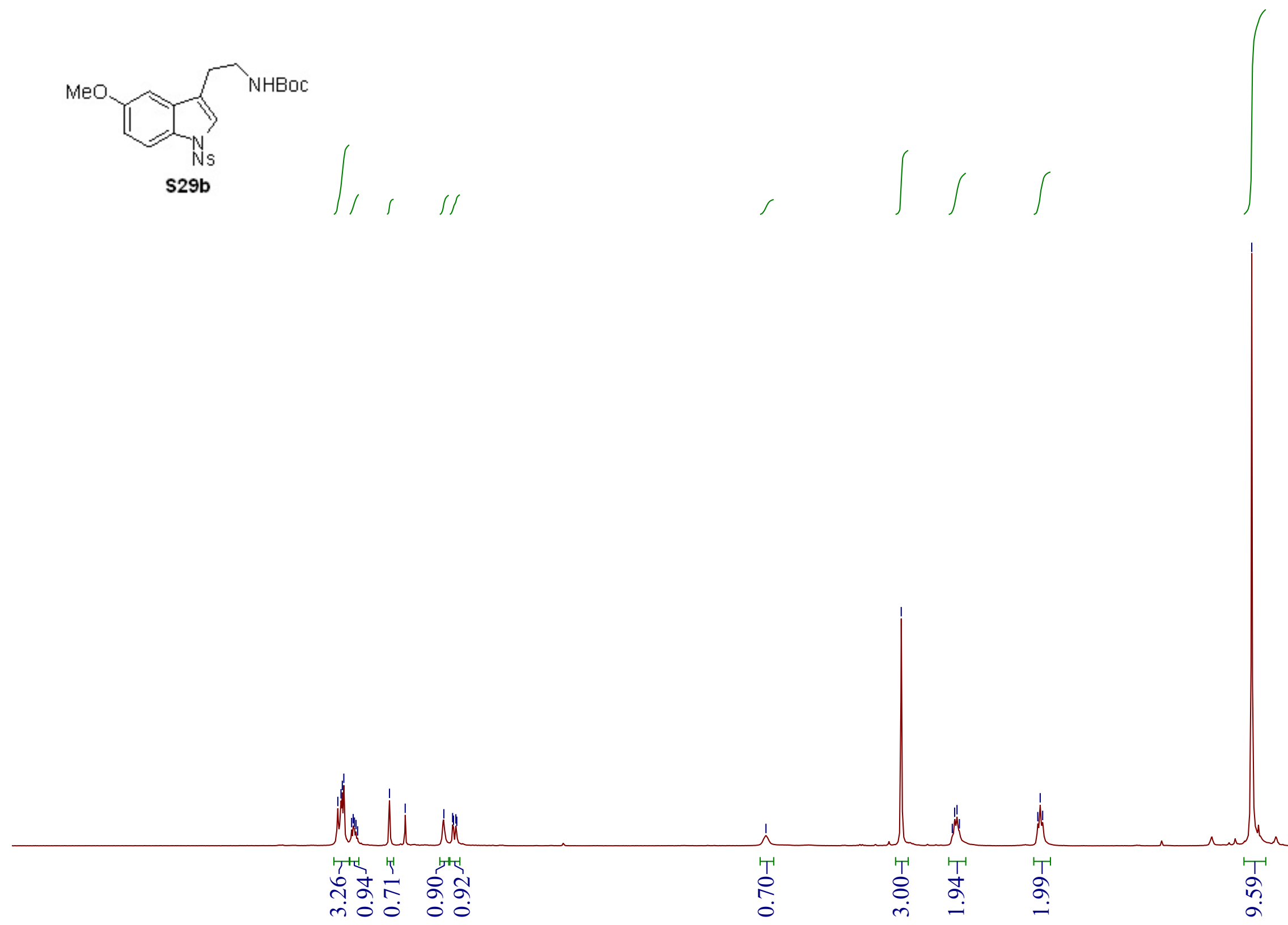


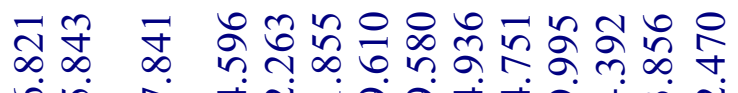

$$
\begin{aligned}
& \text { 品守 }
\end{aligned}
$$

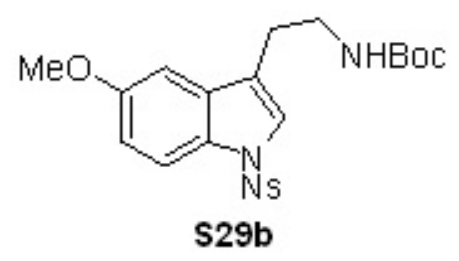

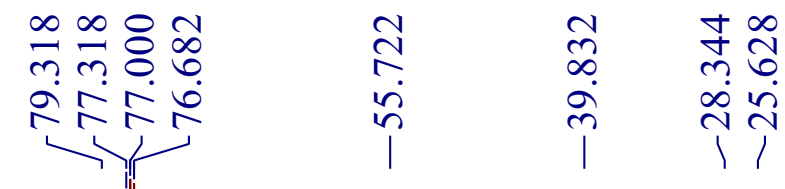

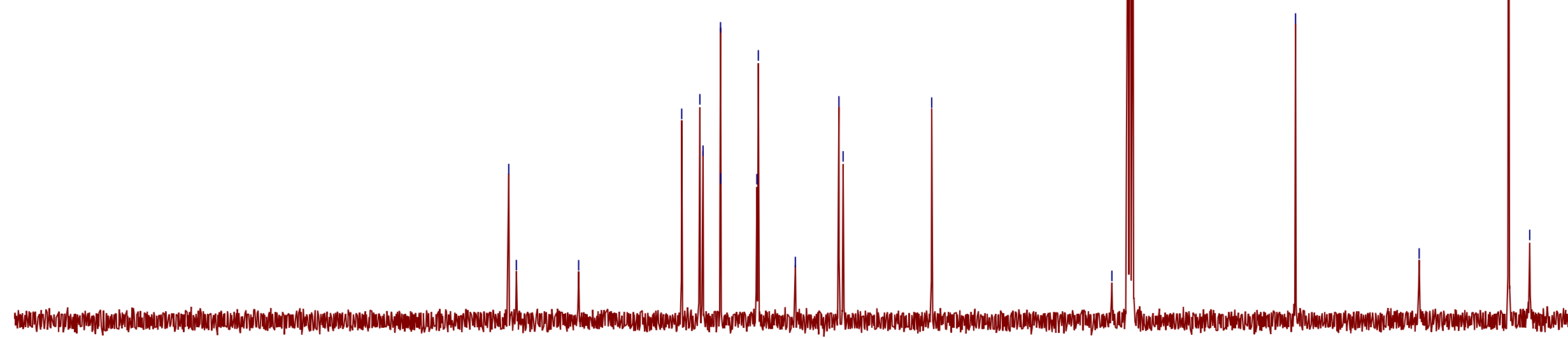



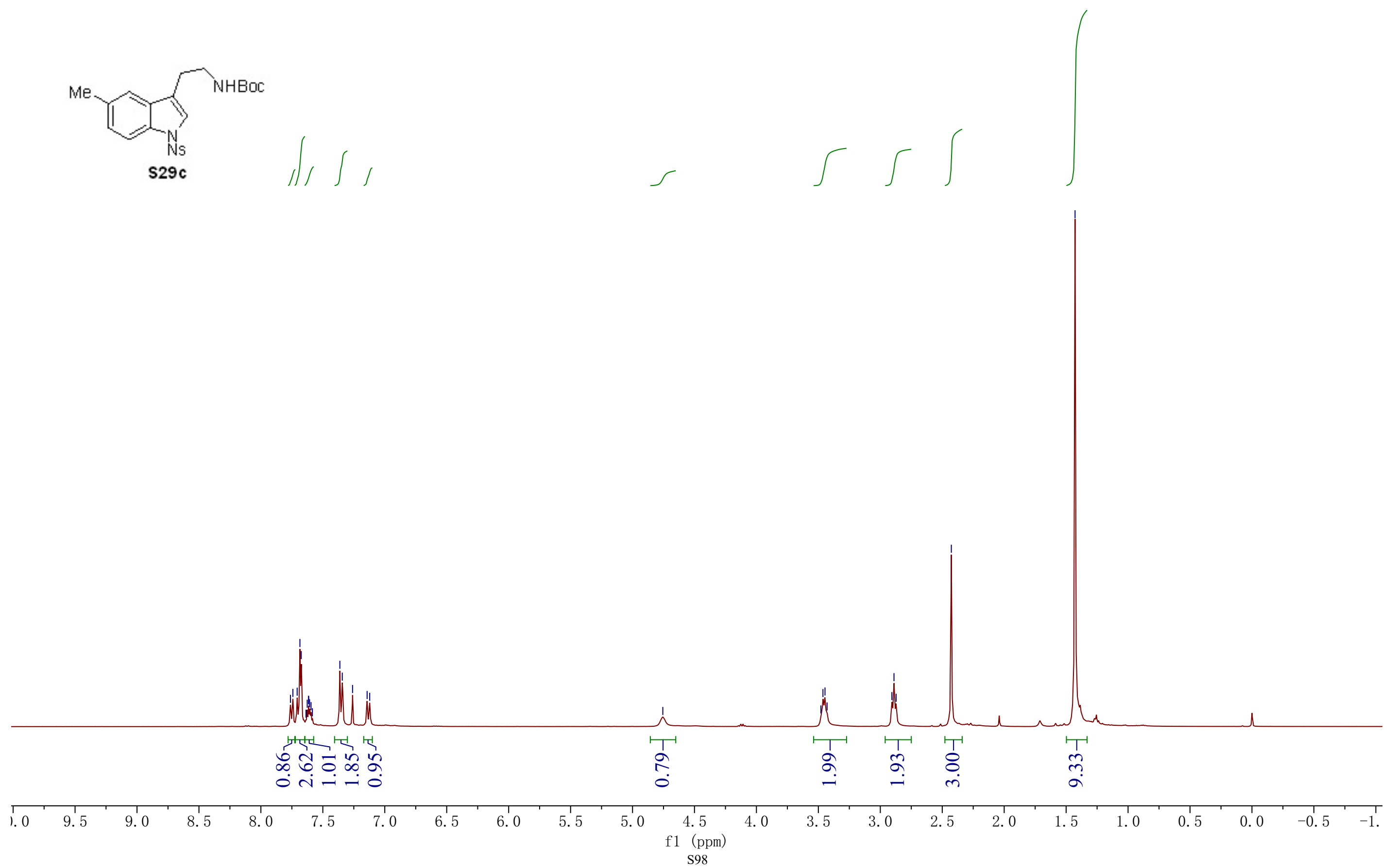

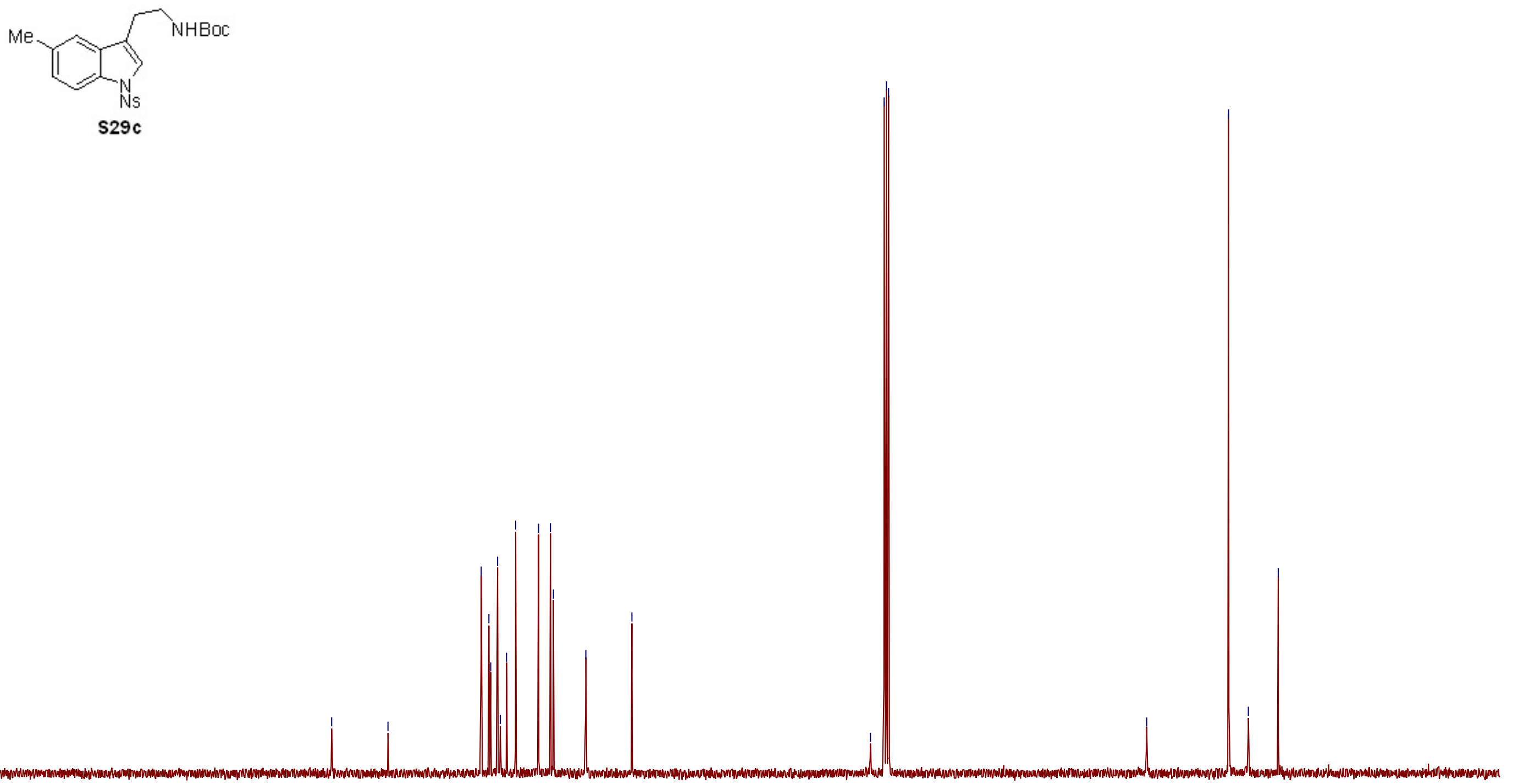


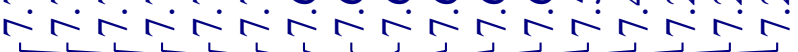
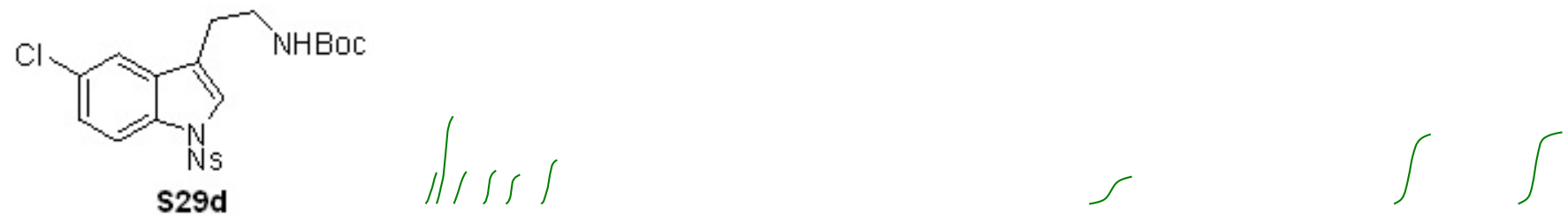<smiles>C1CCCC1</smiles>

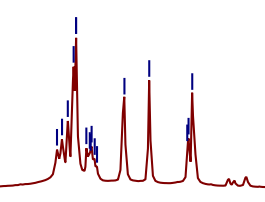
क्ष

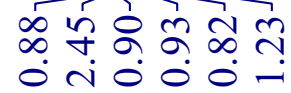




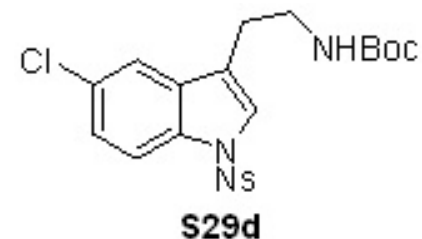

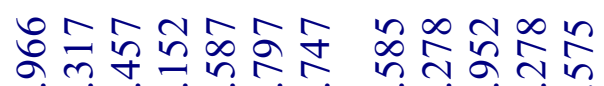

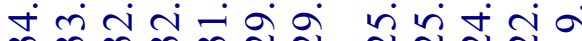
mmmmㄷำ 드는

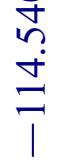
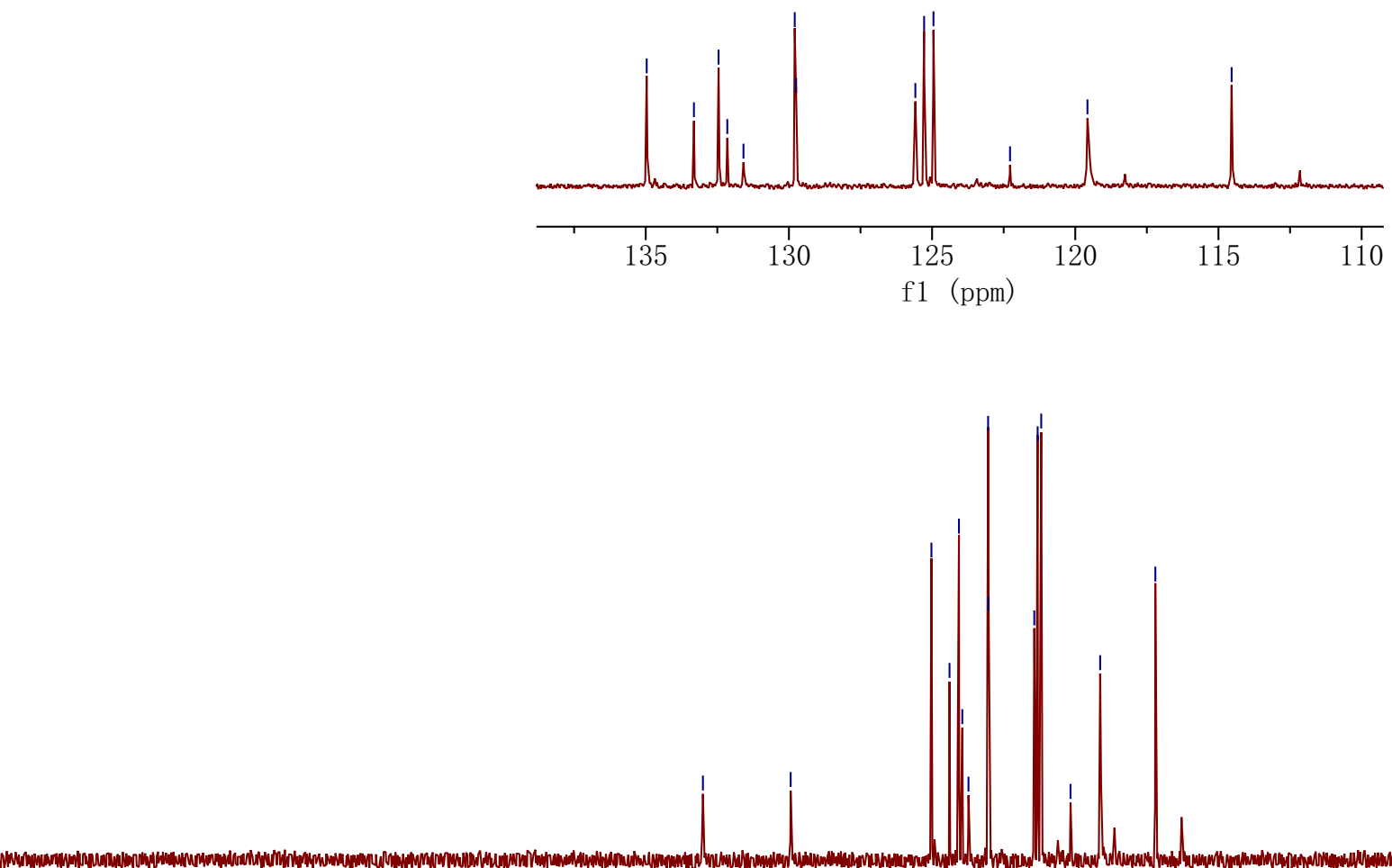

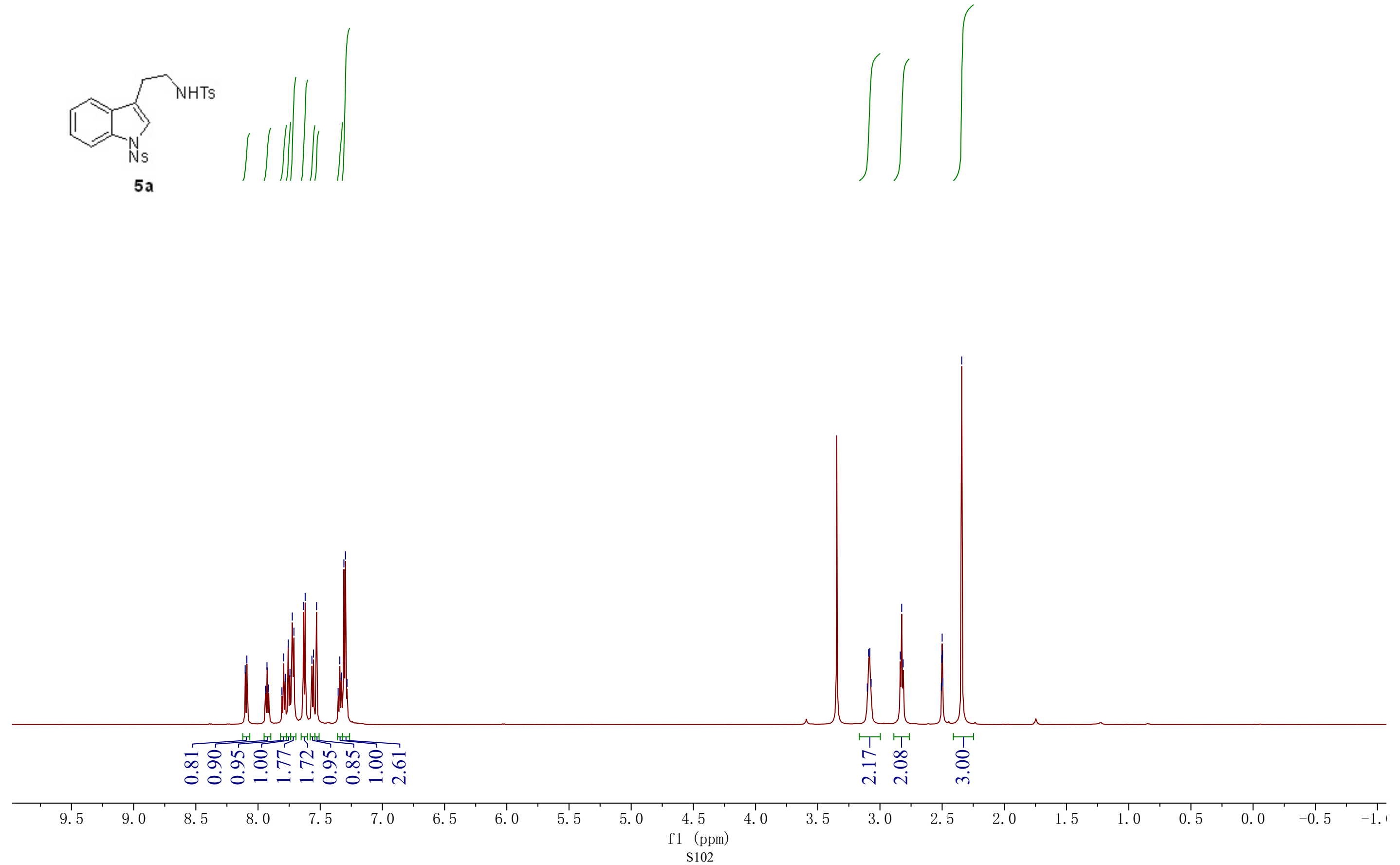


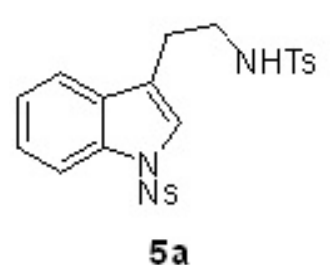

$$
\begin{aligned}
& \text { ஸै? } \\
& \text { ํํํำ } \\
& \text { 군근 }
\end{aligned}
$$
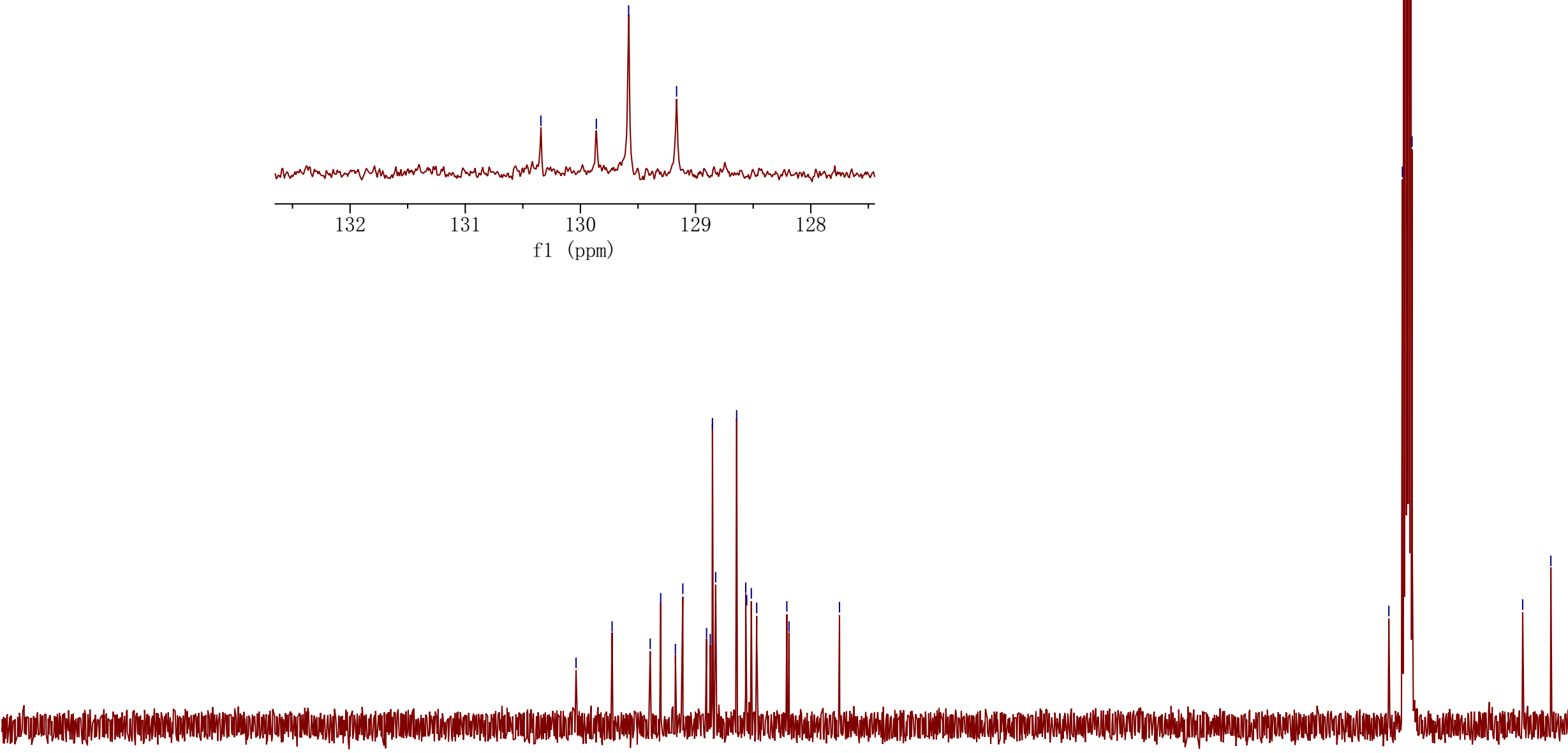


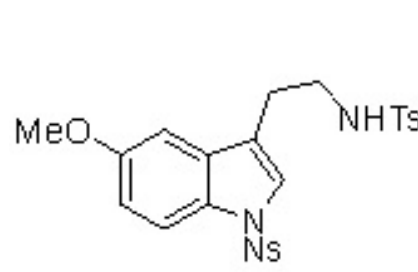

$5 \mathbf{b}$
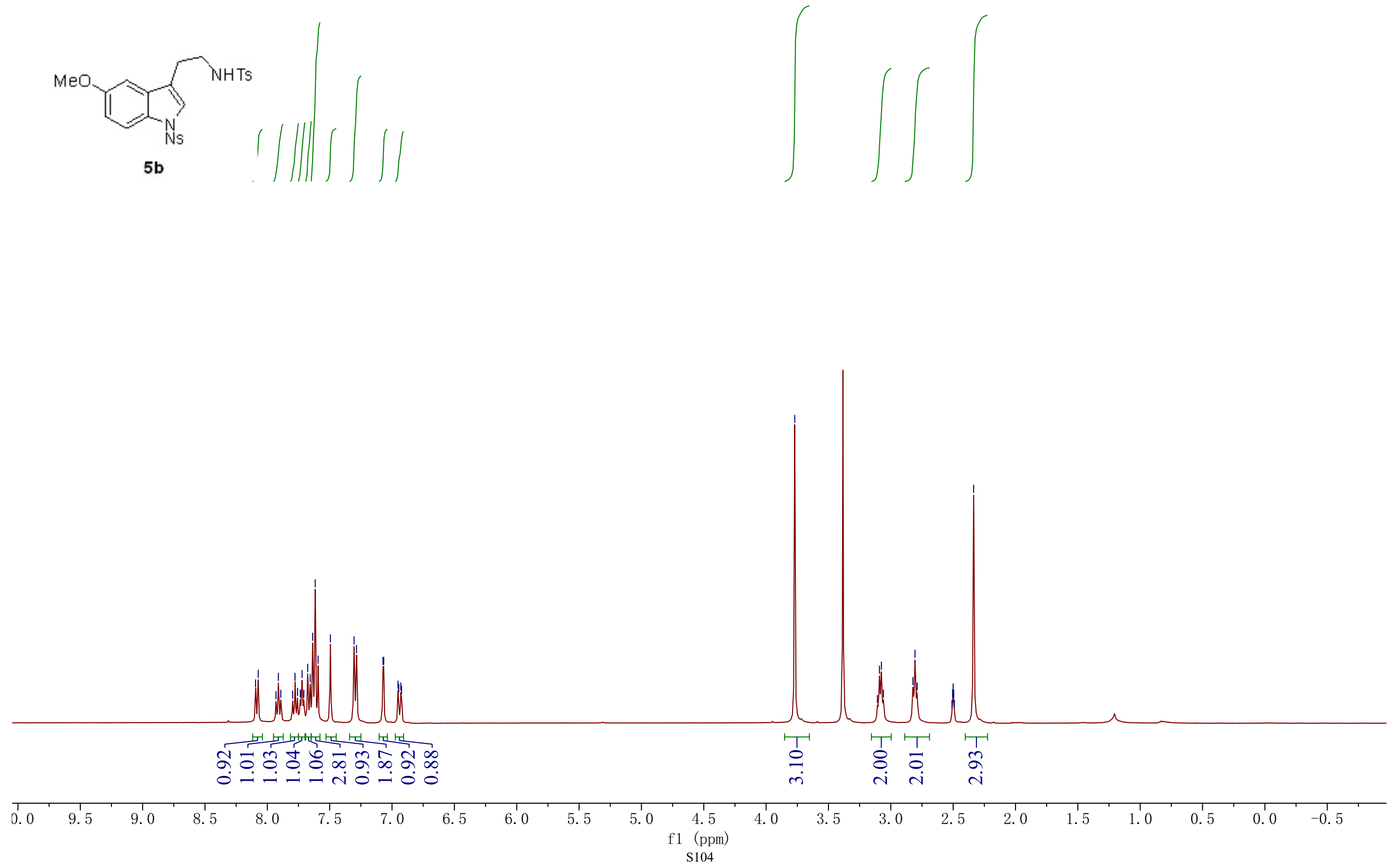


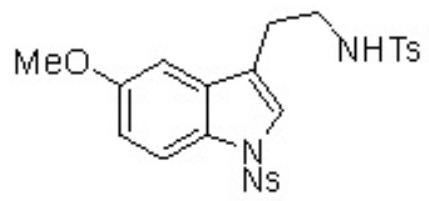

$5 \mathrm{~b}$

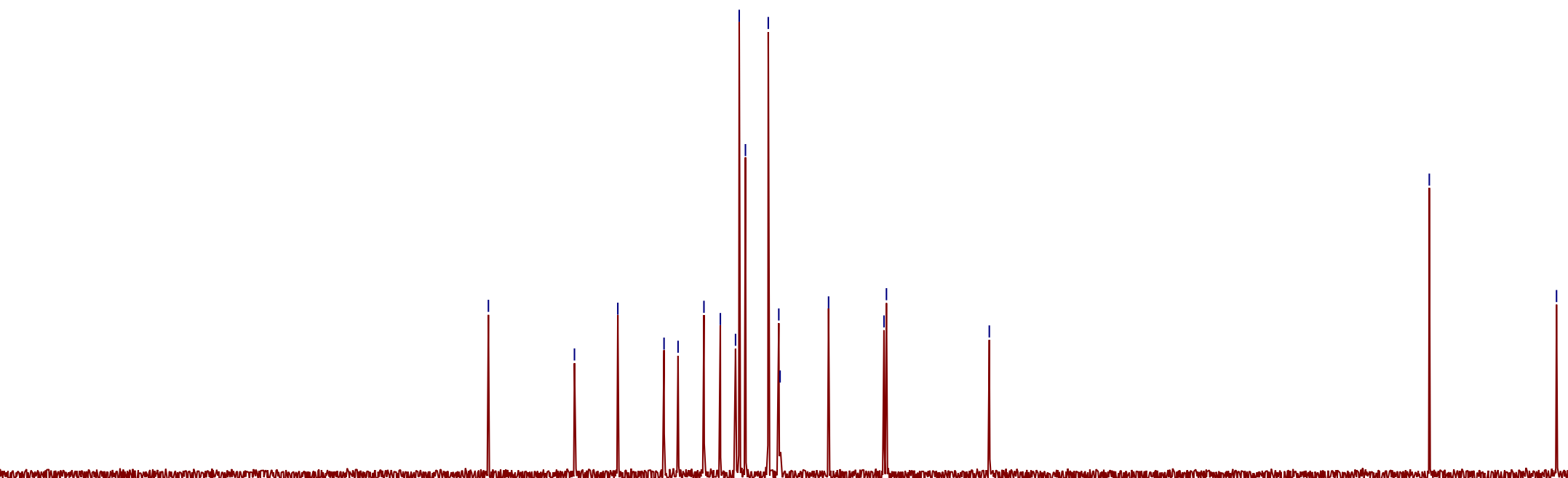



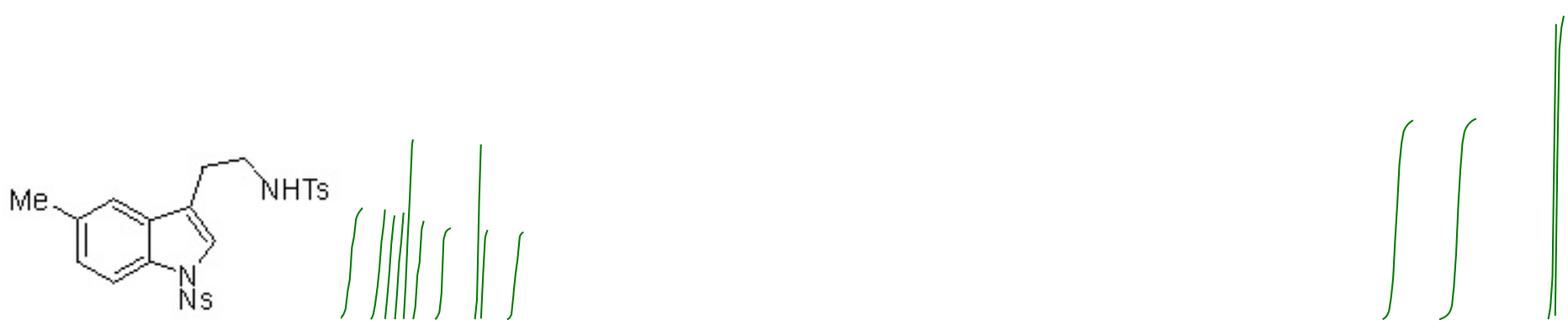

$5 \mathrm{c}$

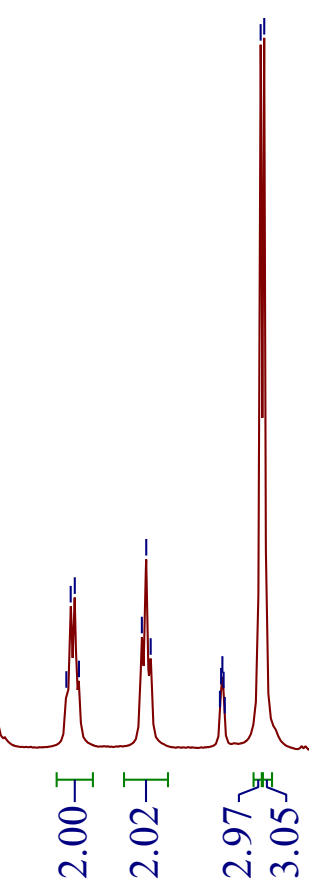



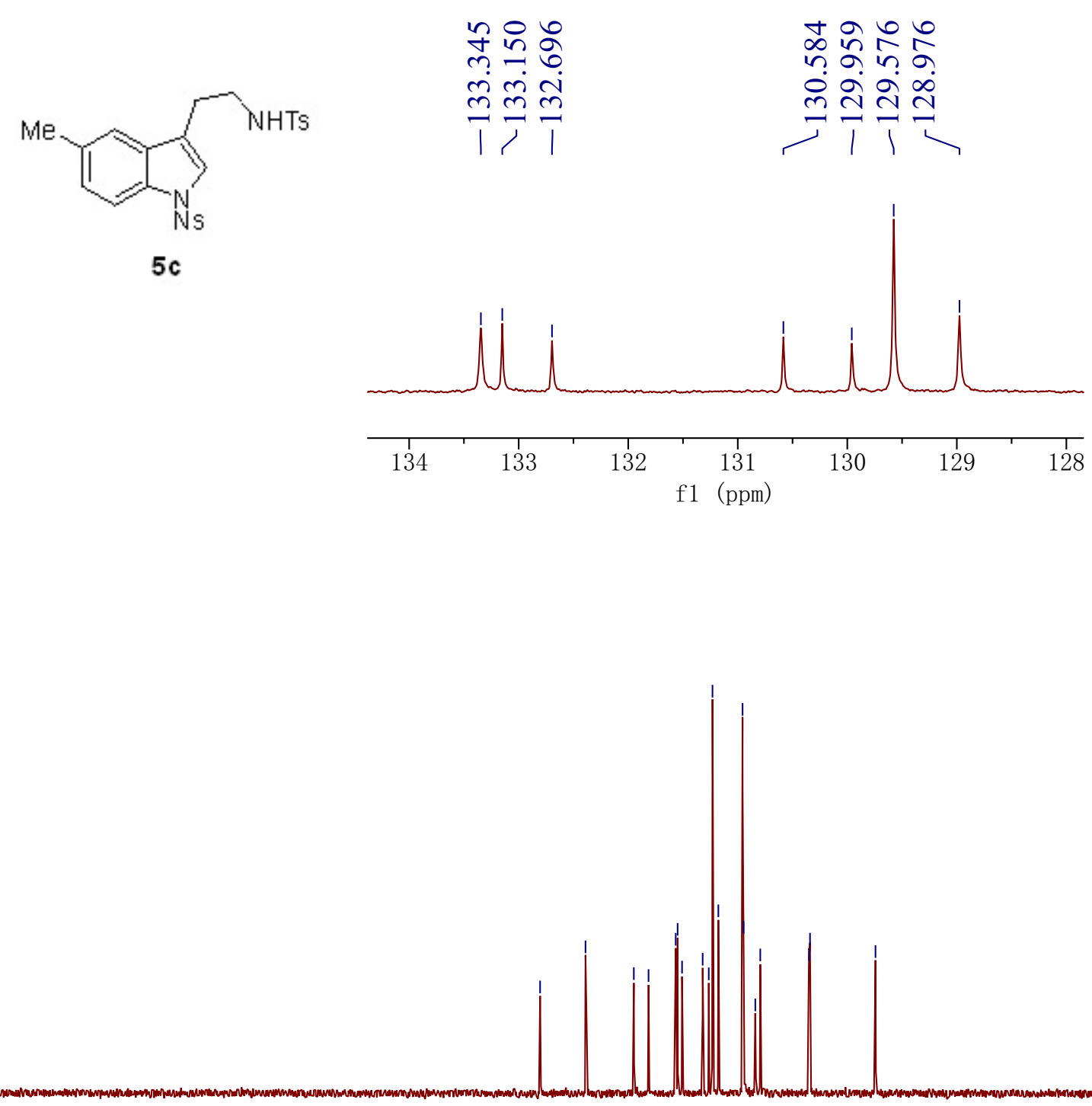

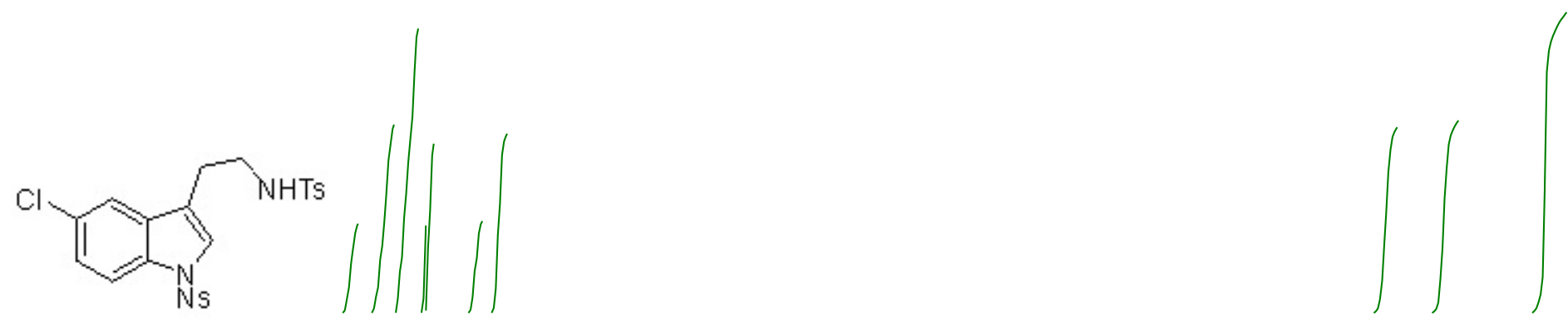

$5 d$
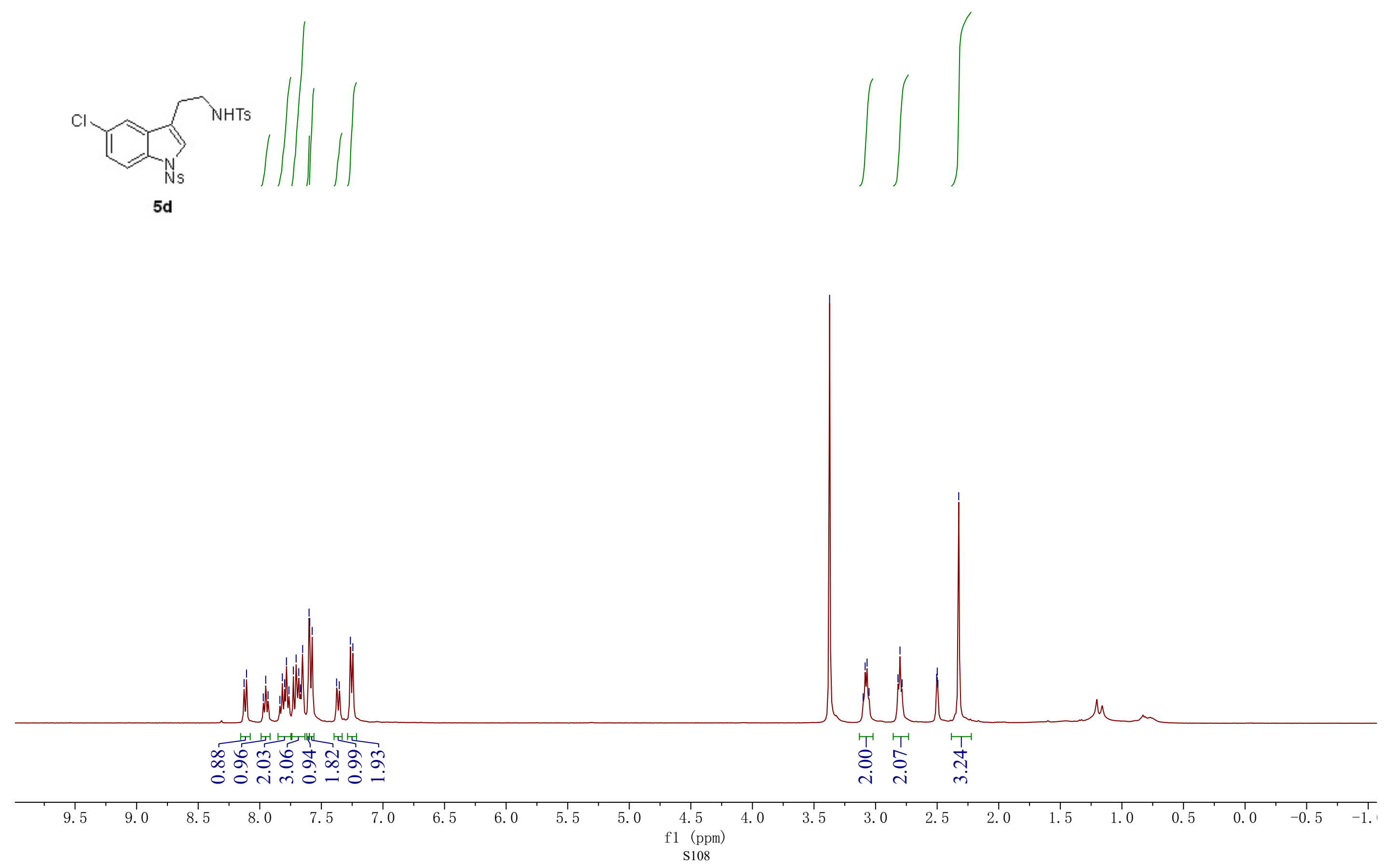

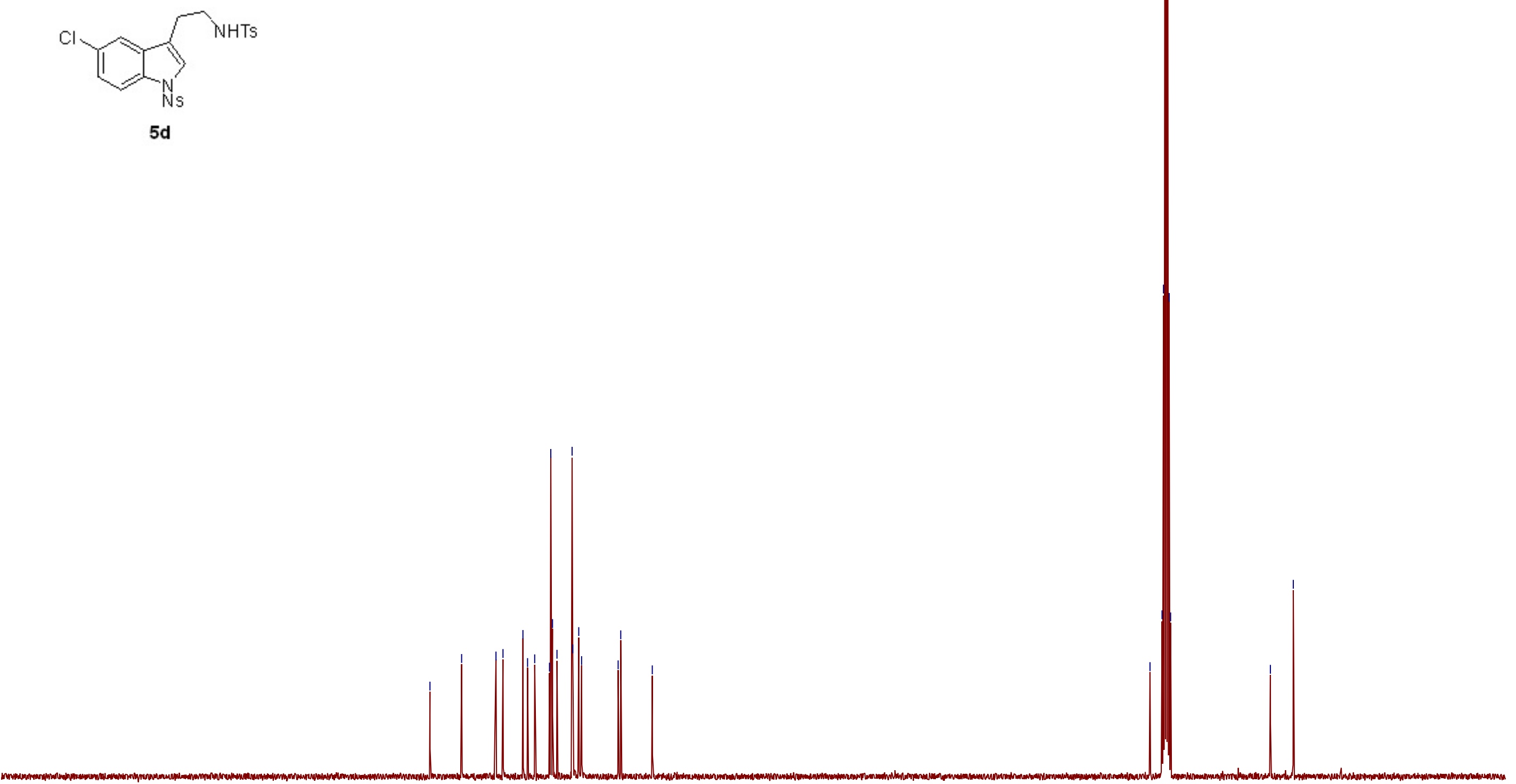

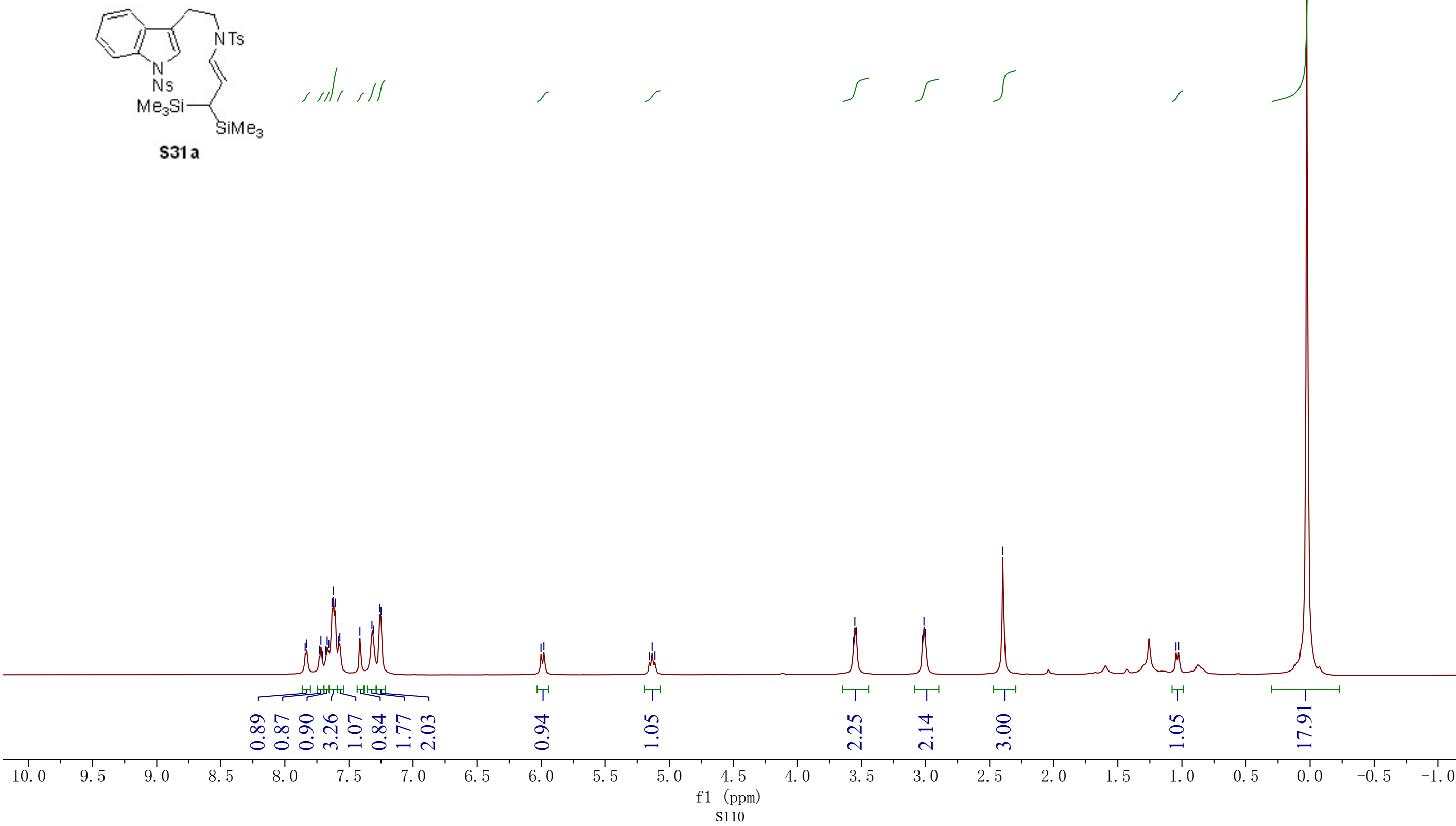


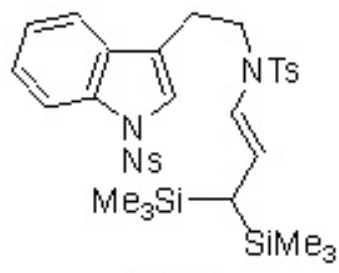

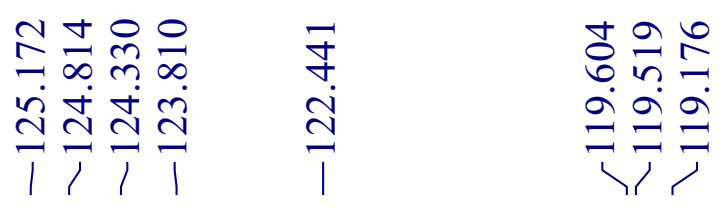

S31a
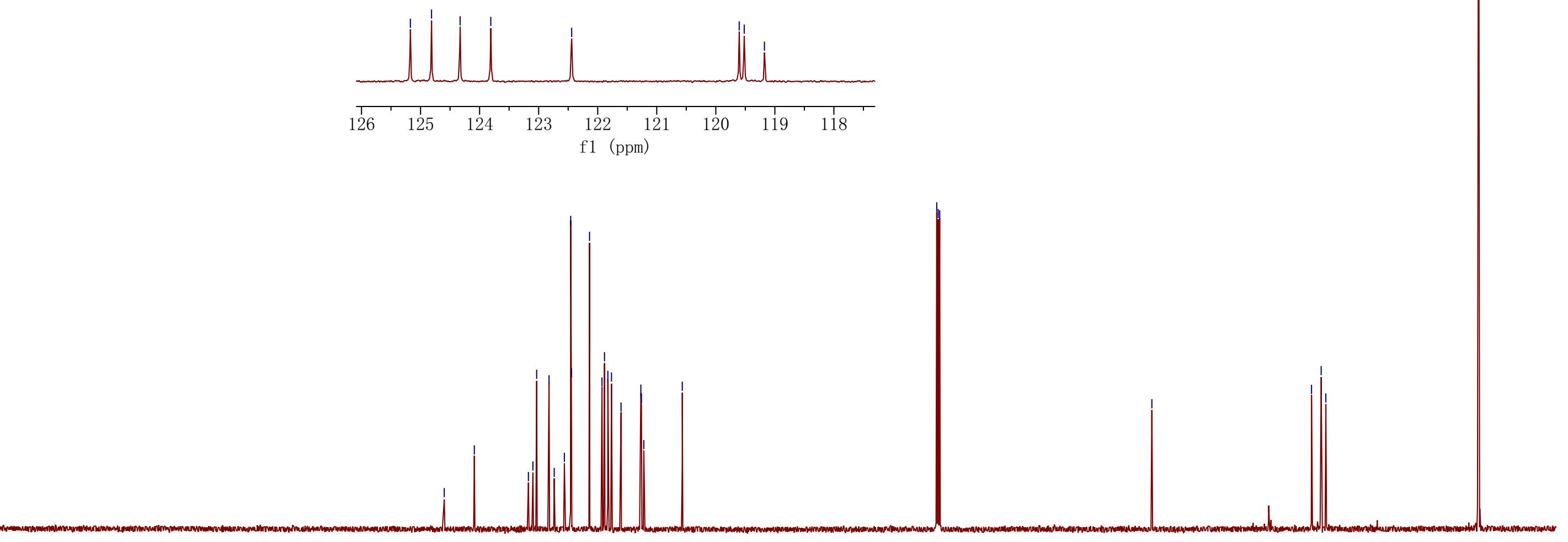
$\mathrm{MeO}$
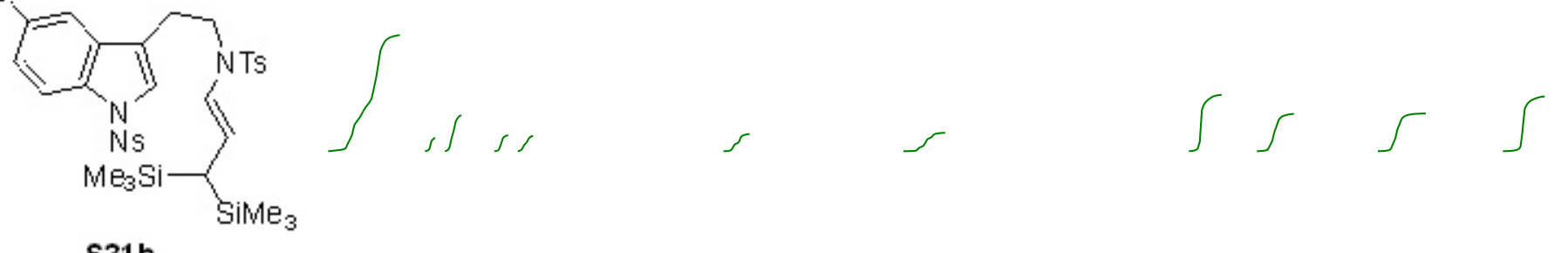

S31b

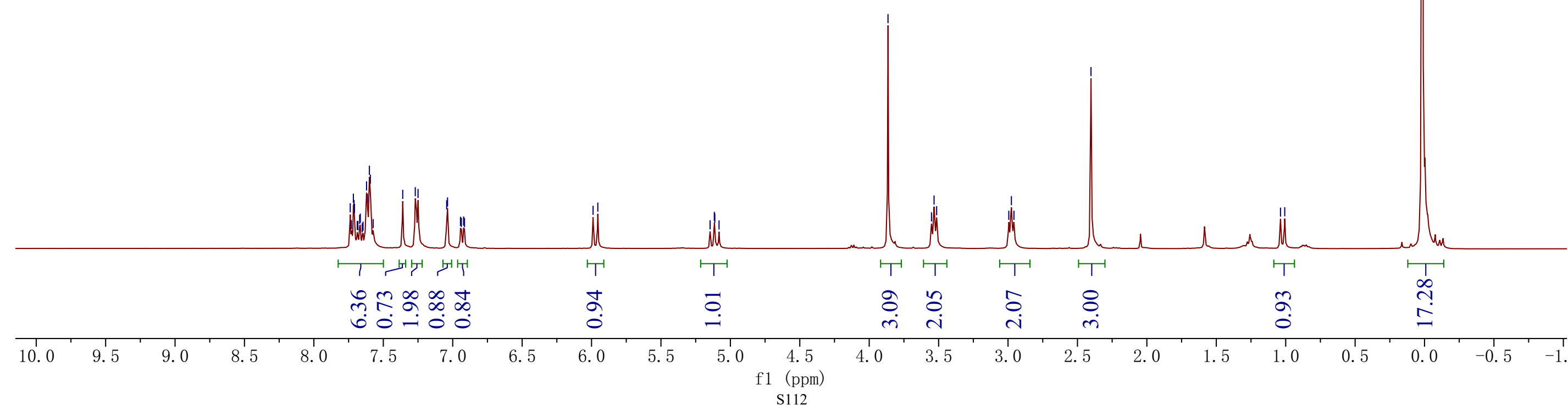


MeO

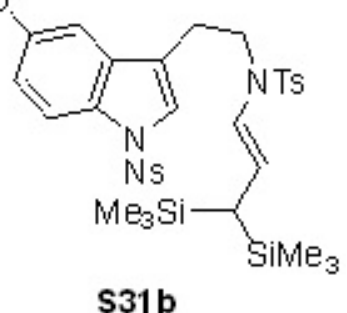

S31b

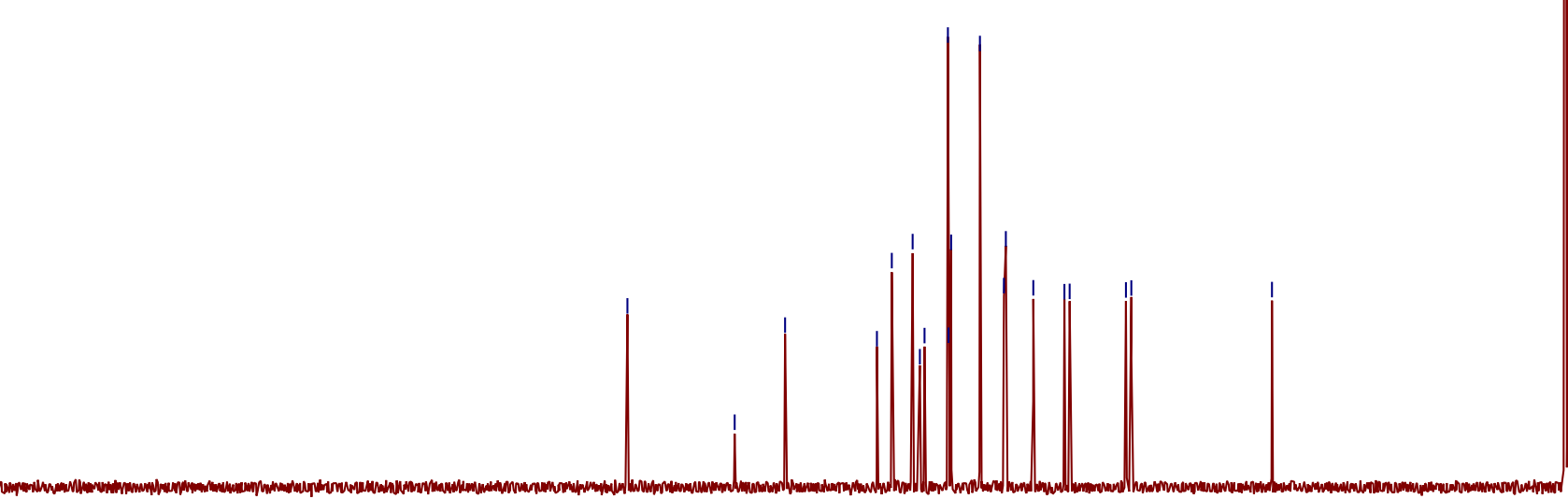



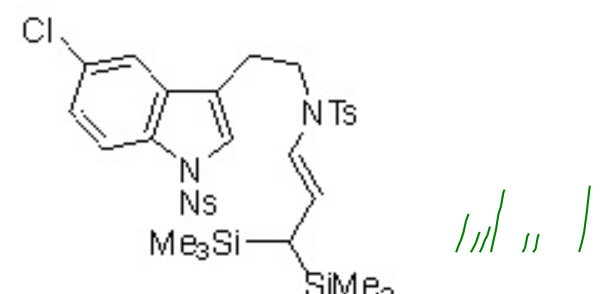

S31d

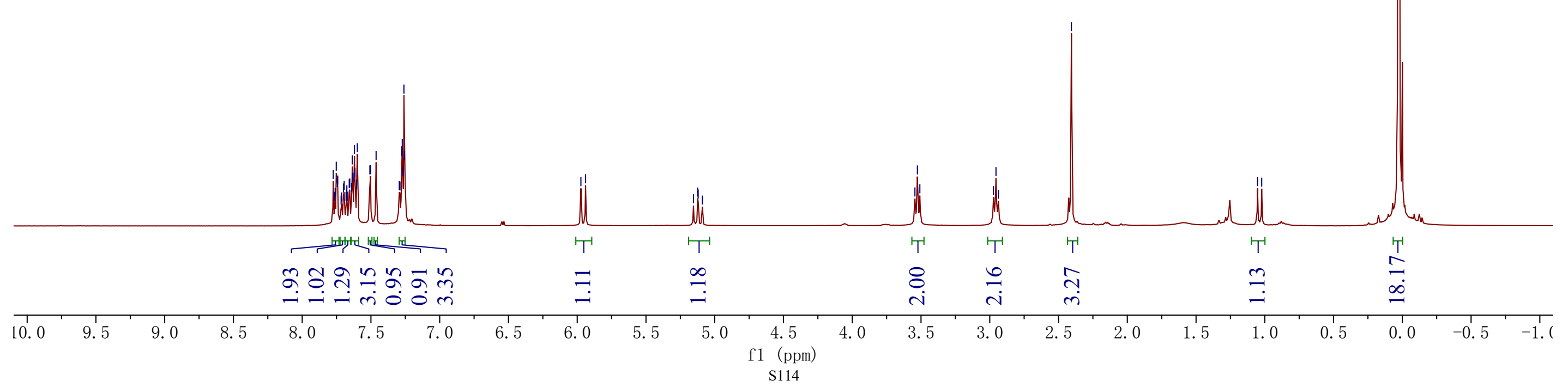



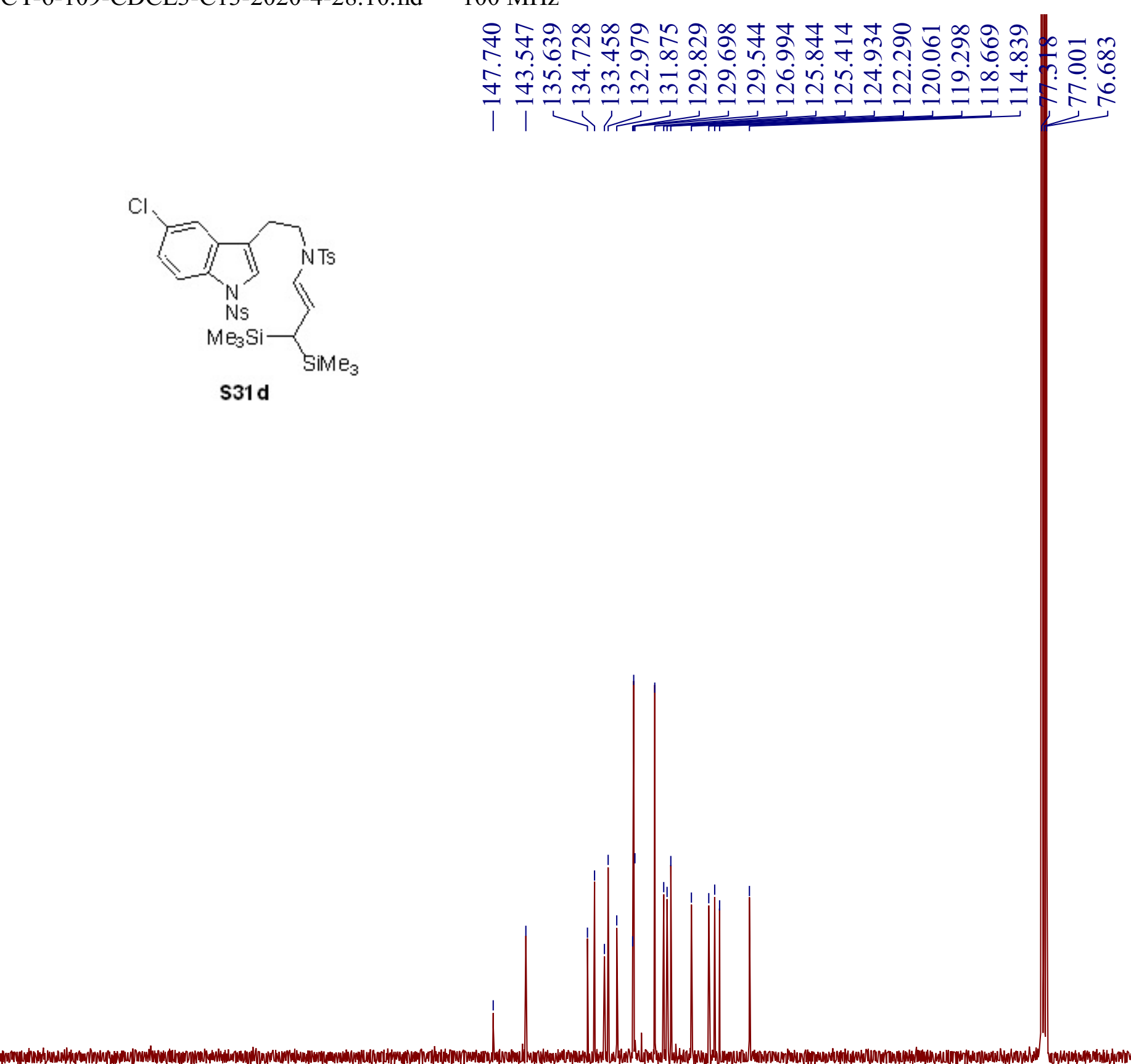


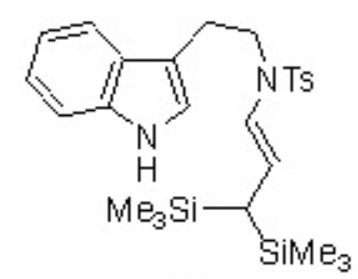

$7 a$

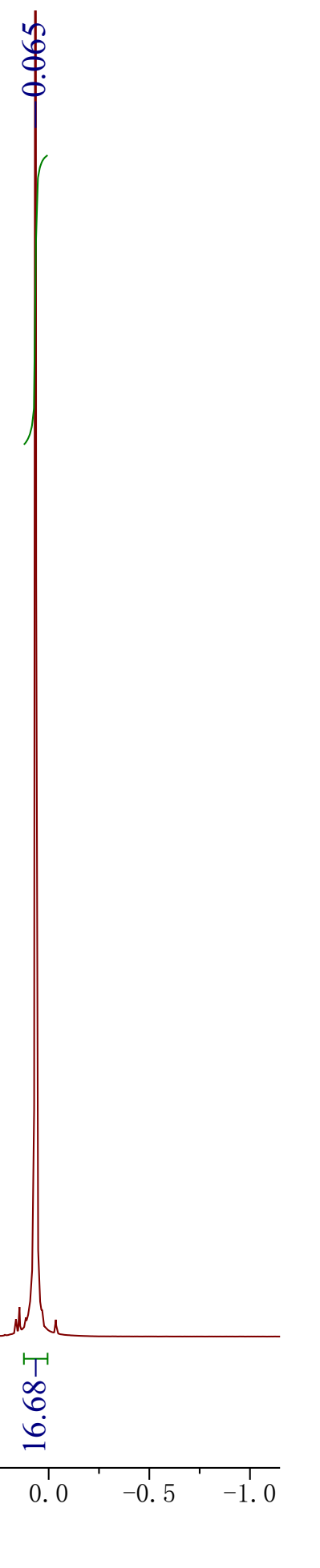



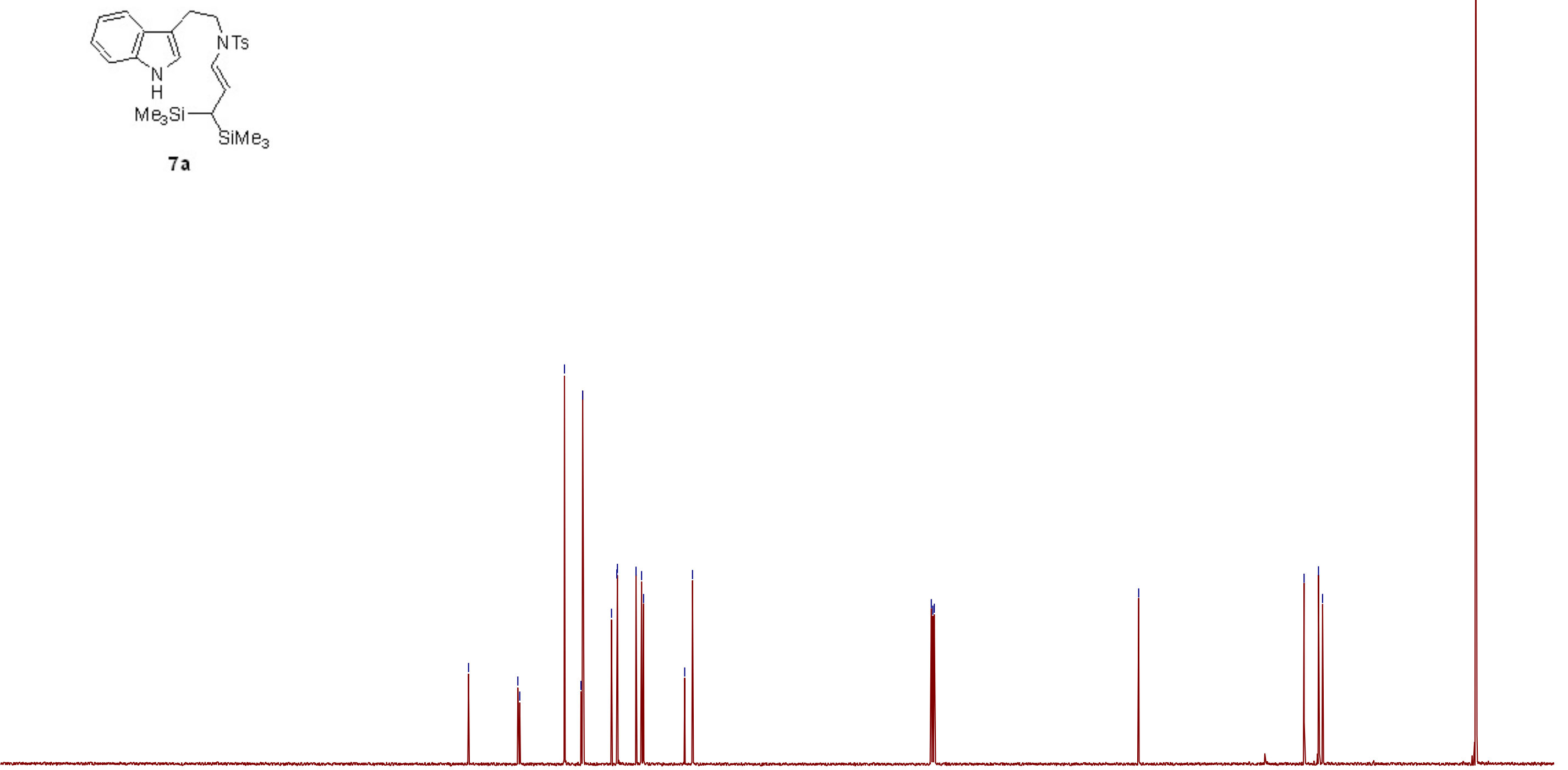
$\mathrm{MeO}$

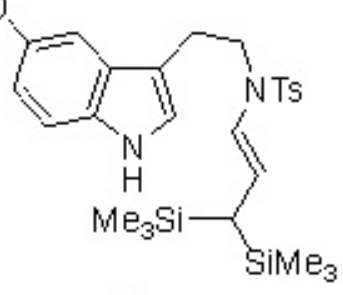

$7 \mathrm{~b}$

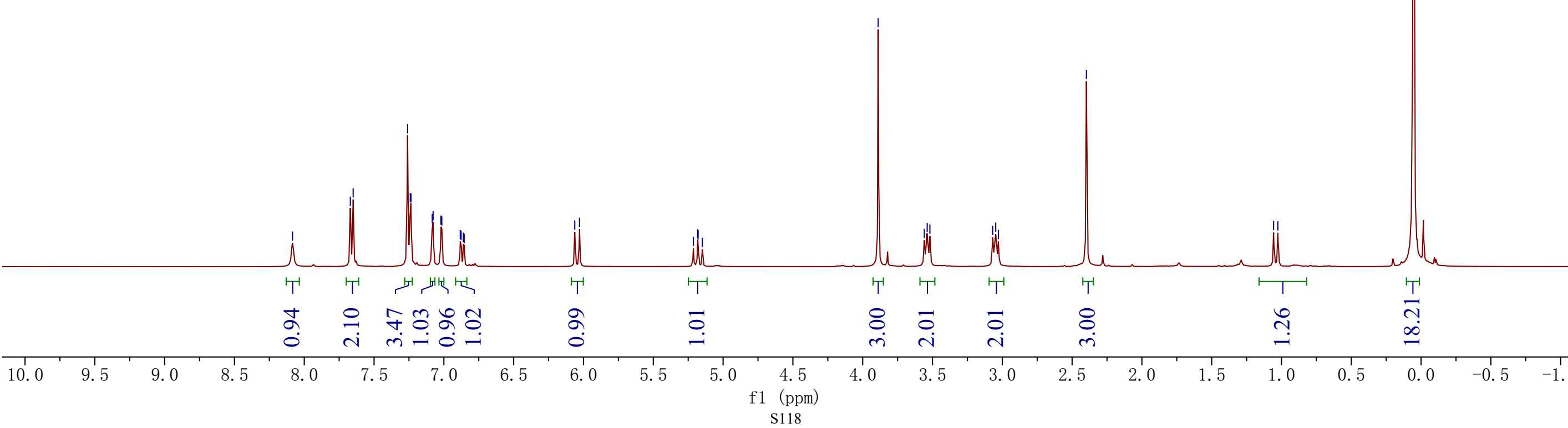




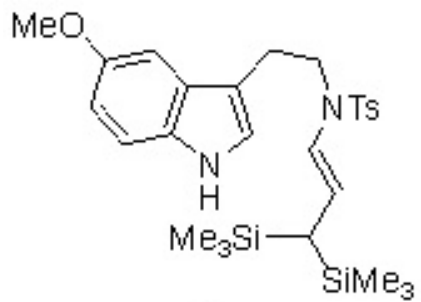

$7 \mathrm{~b}$

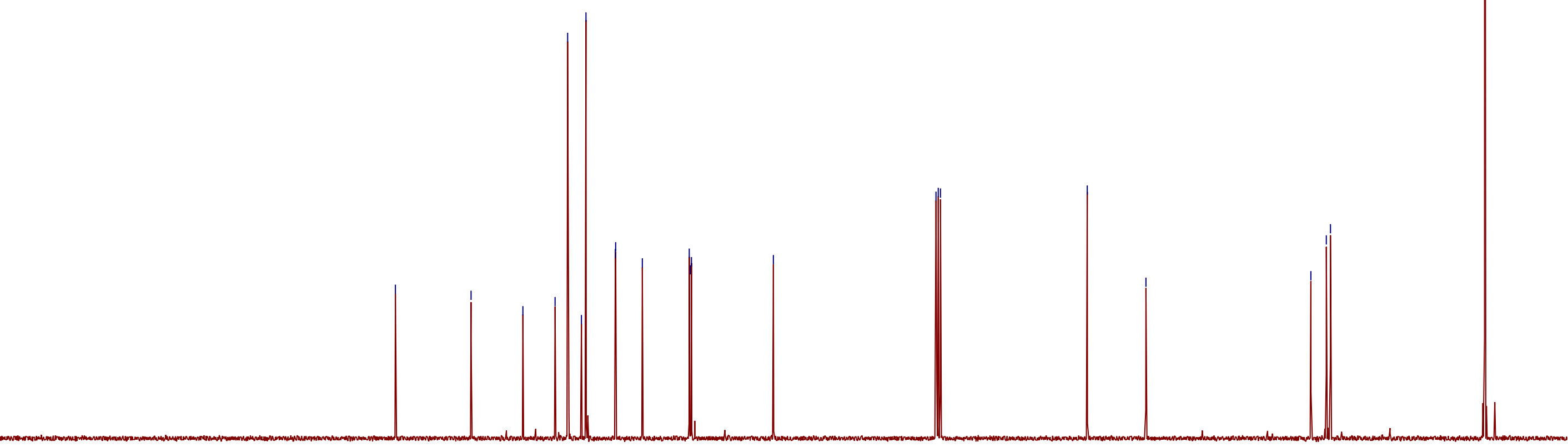


Me,

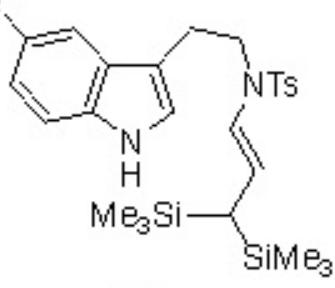

$7 \mathrm{c}$

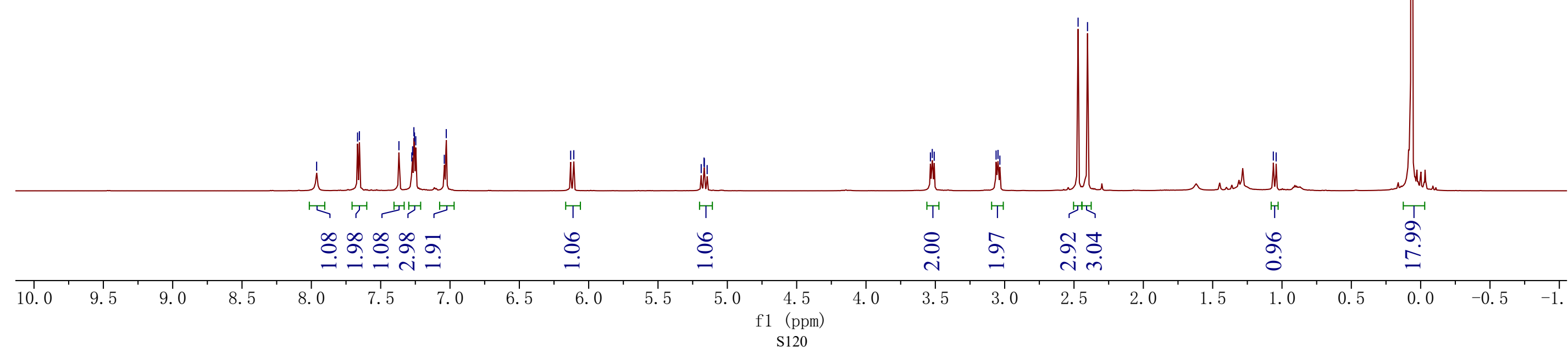




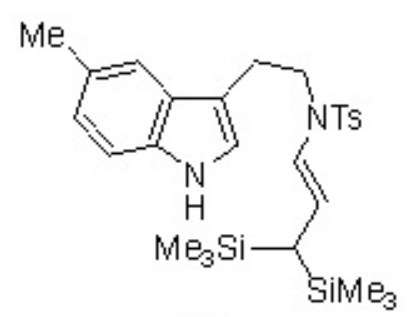

$7 c$

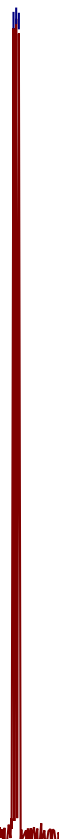



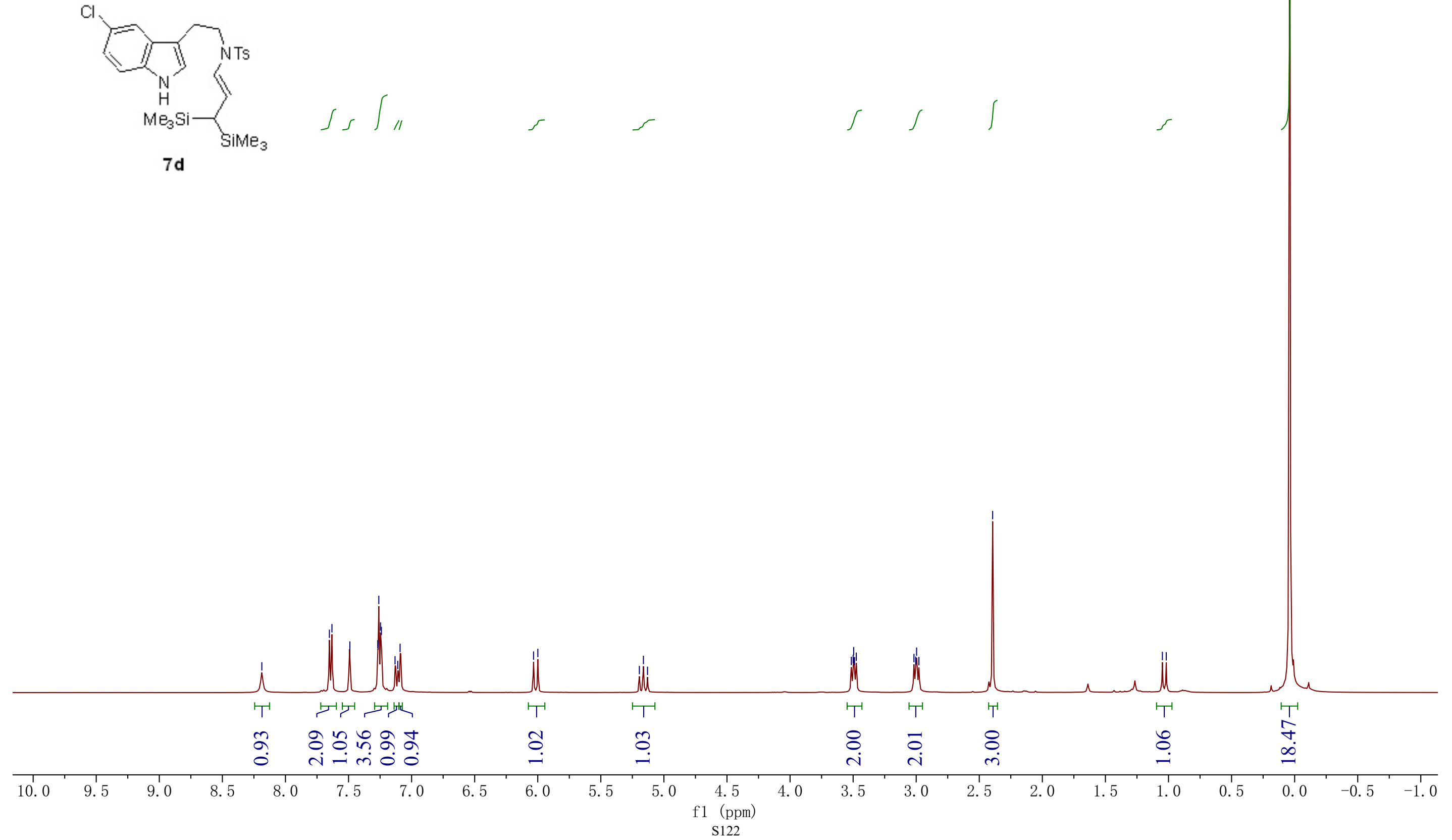


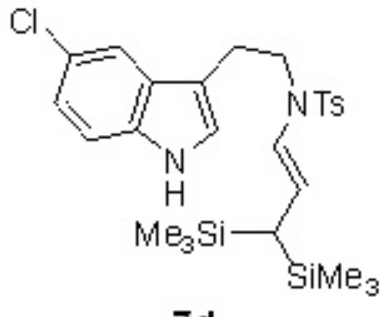

7d

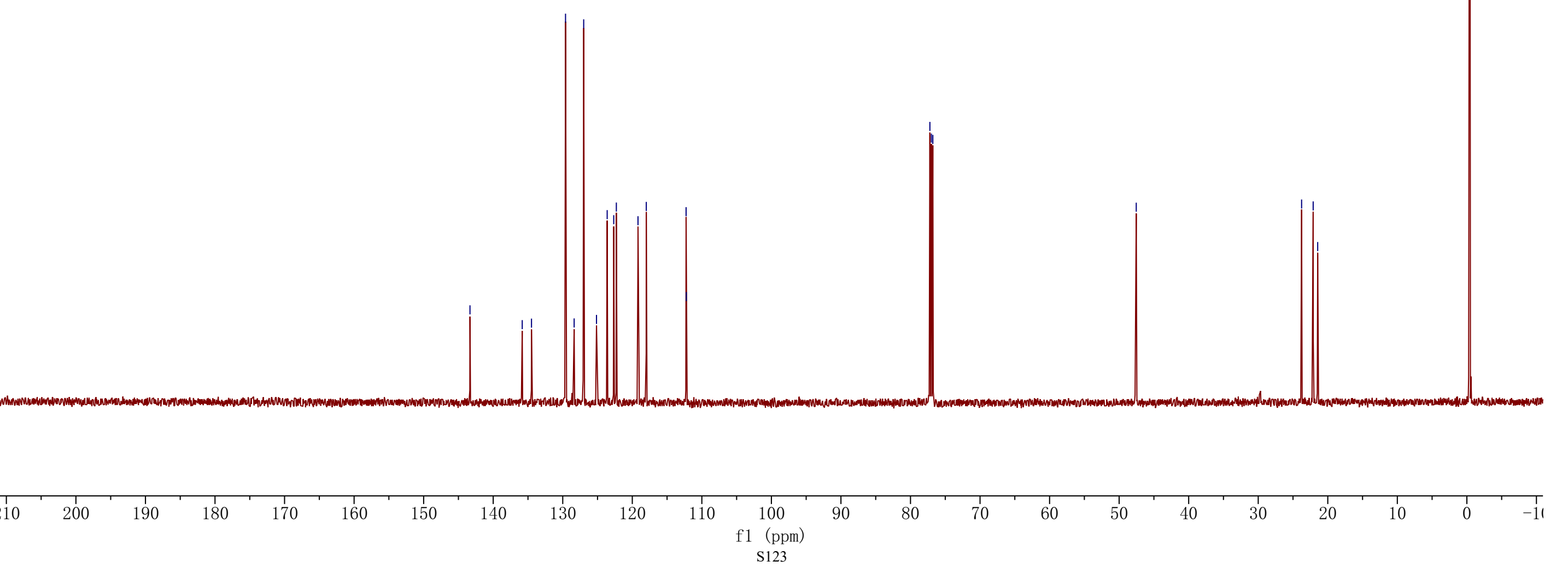




\section{CY-6-59-B1-CDCL3-H1-2019-12-9.10.fid - $400 \mathrm{MHz}$}

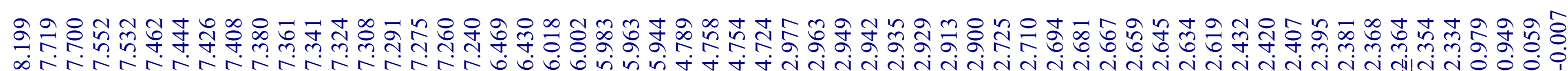

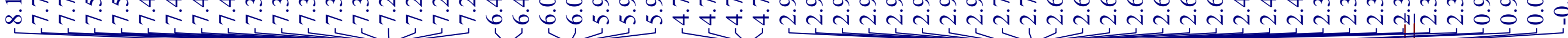
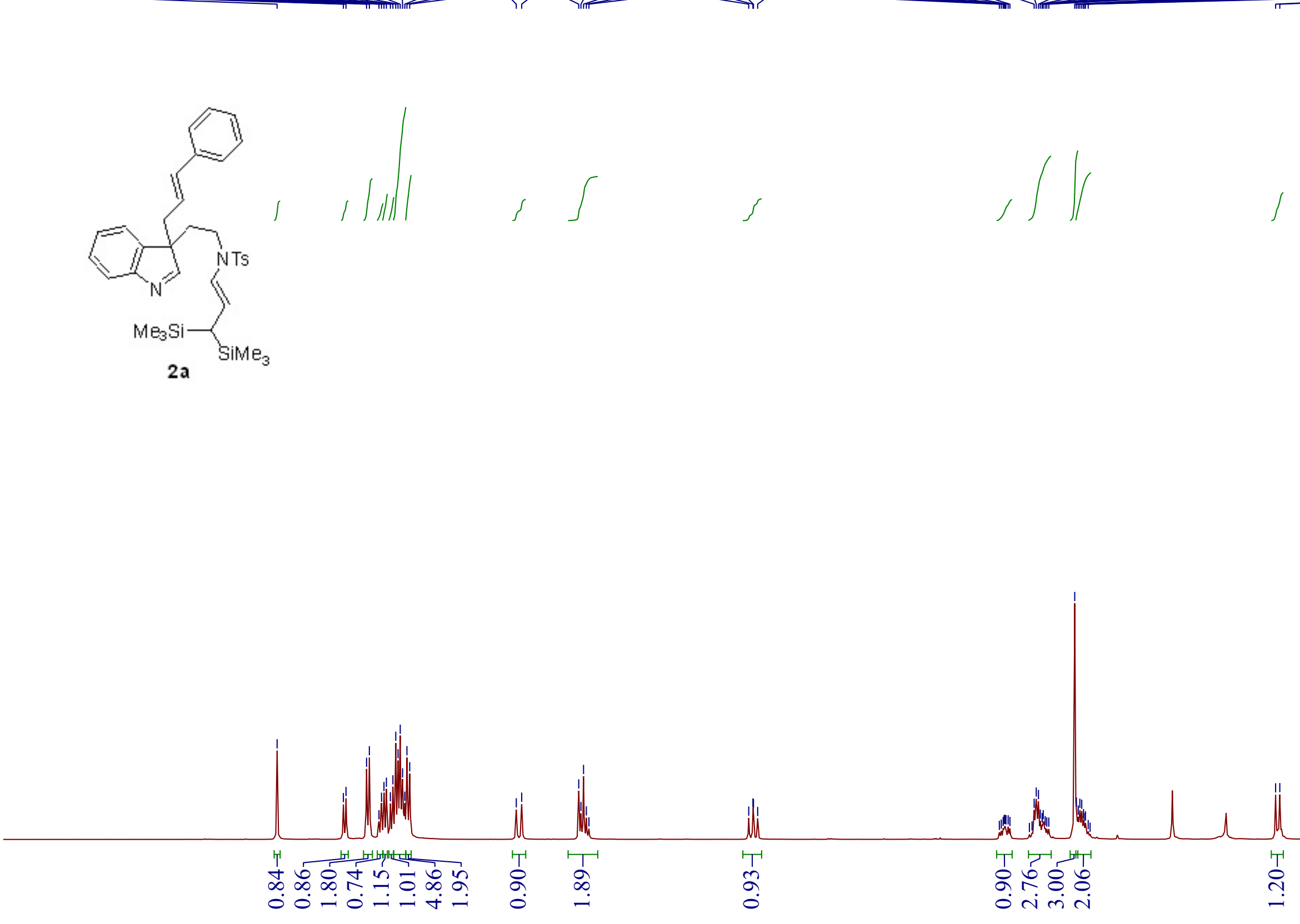


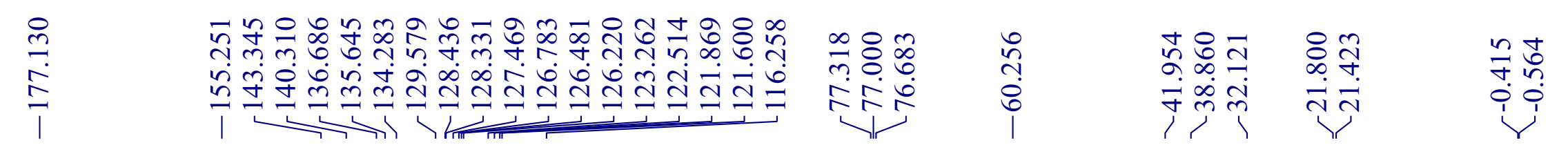
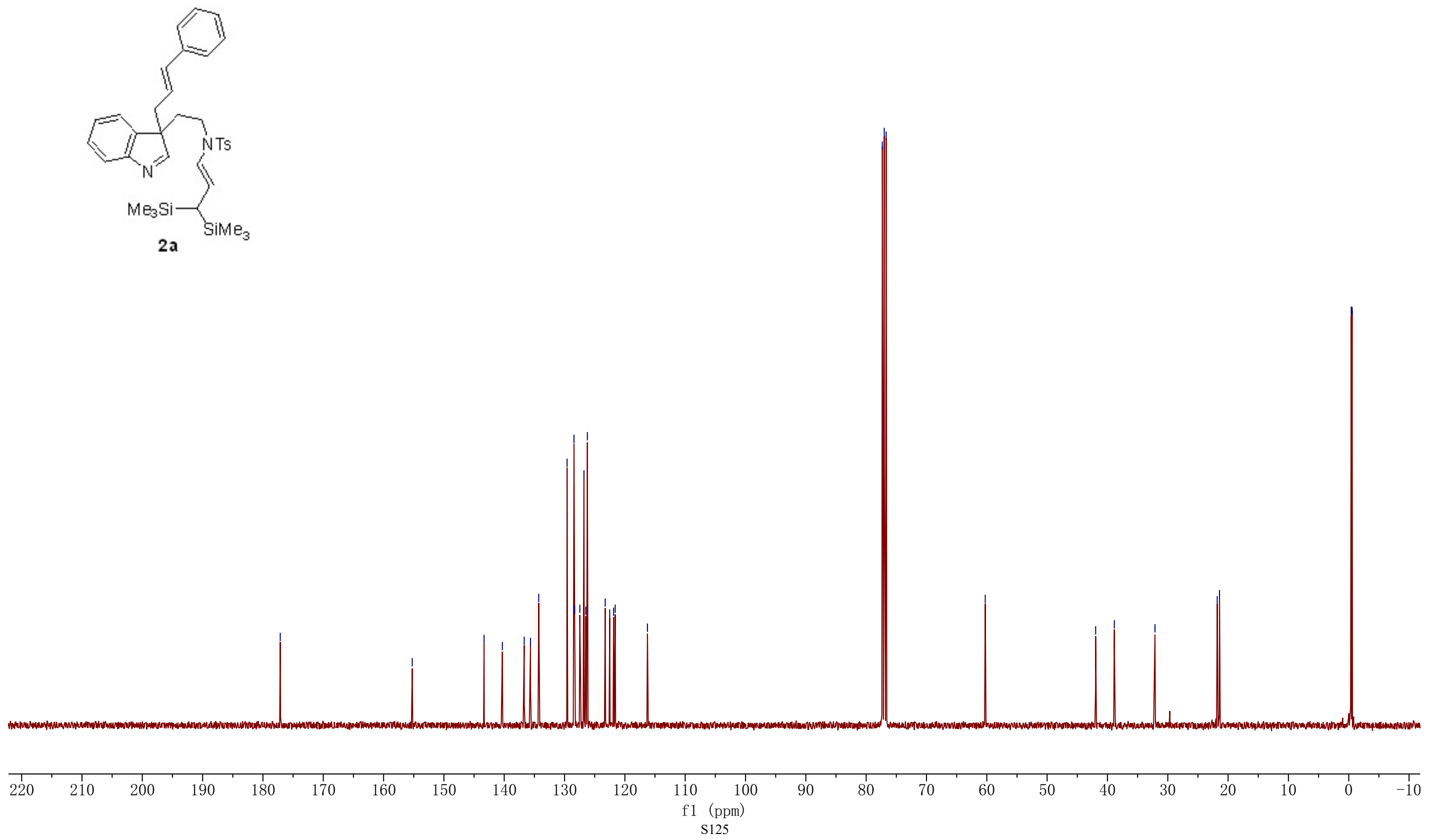

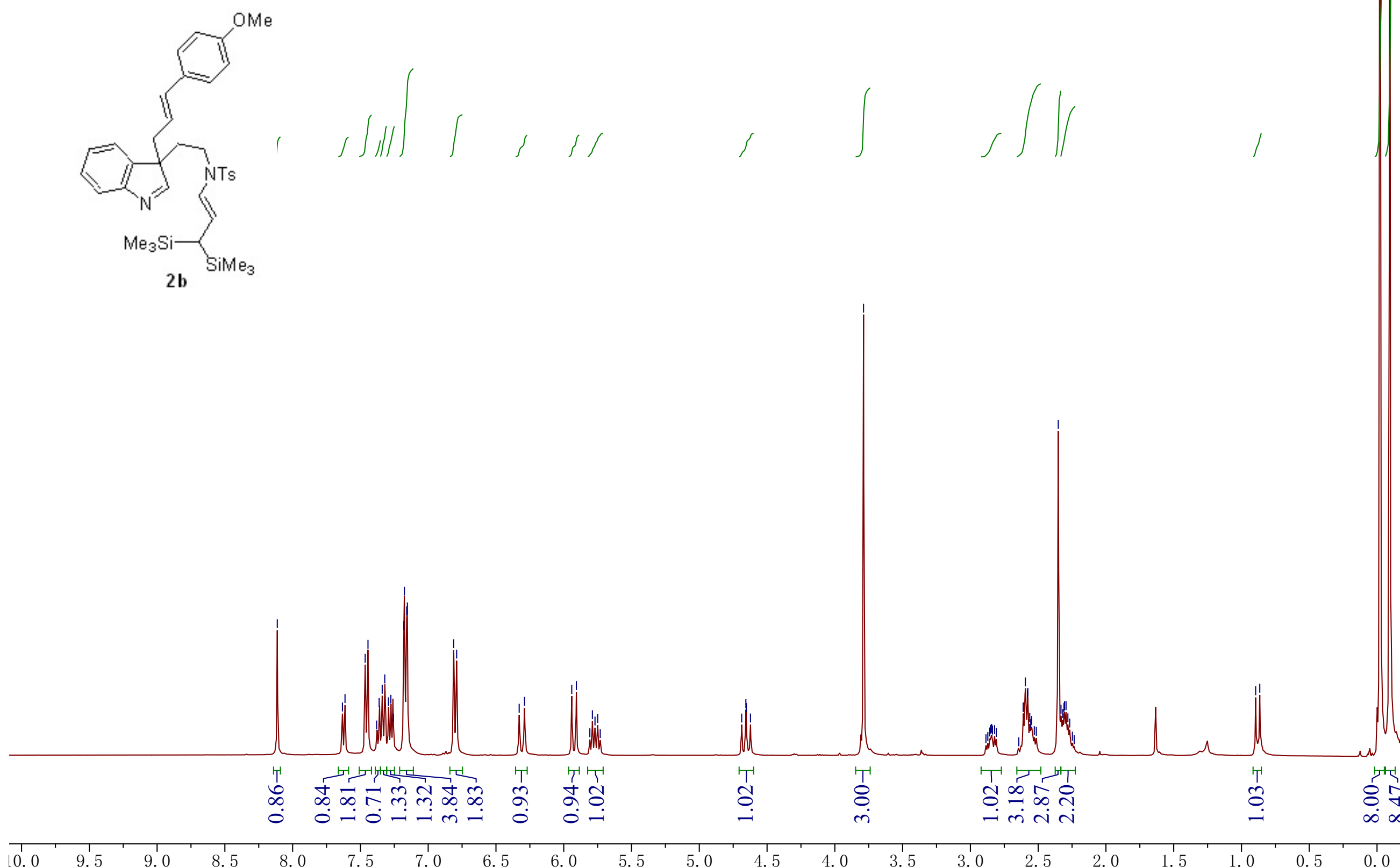

9.0

0

5

. 0.5 

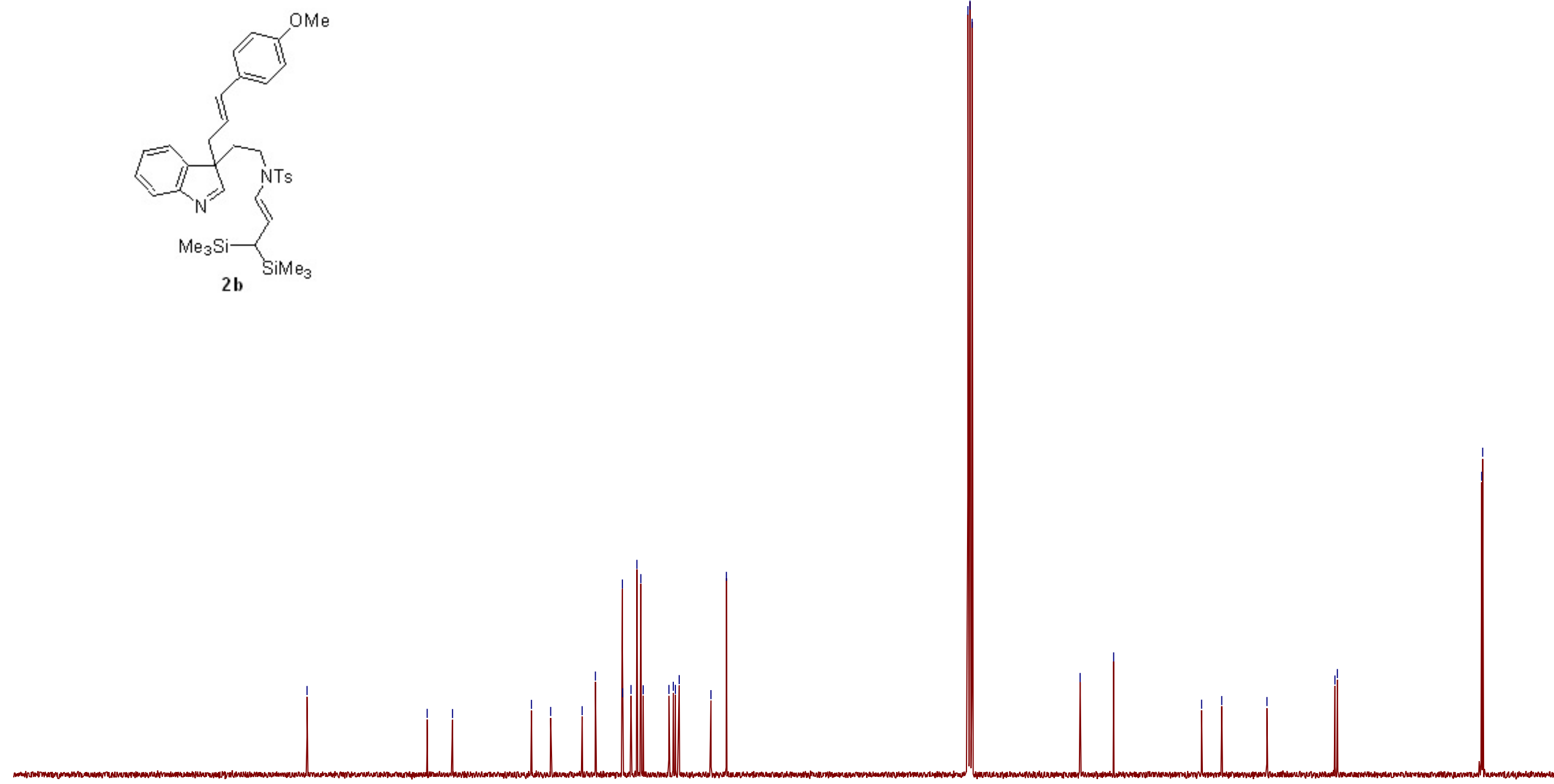


\section{CY-6-56-B3_H1_CDCl3_2019-12-3.40.fid - $400 \mathrm{MHz}$}

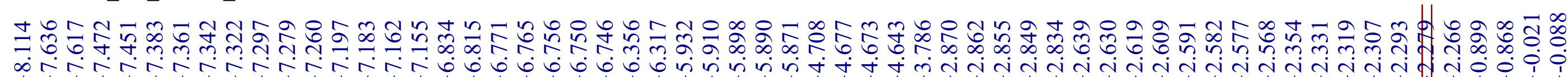
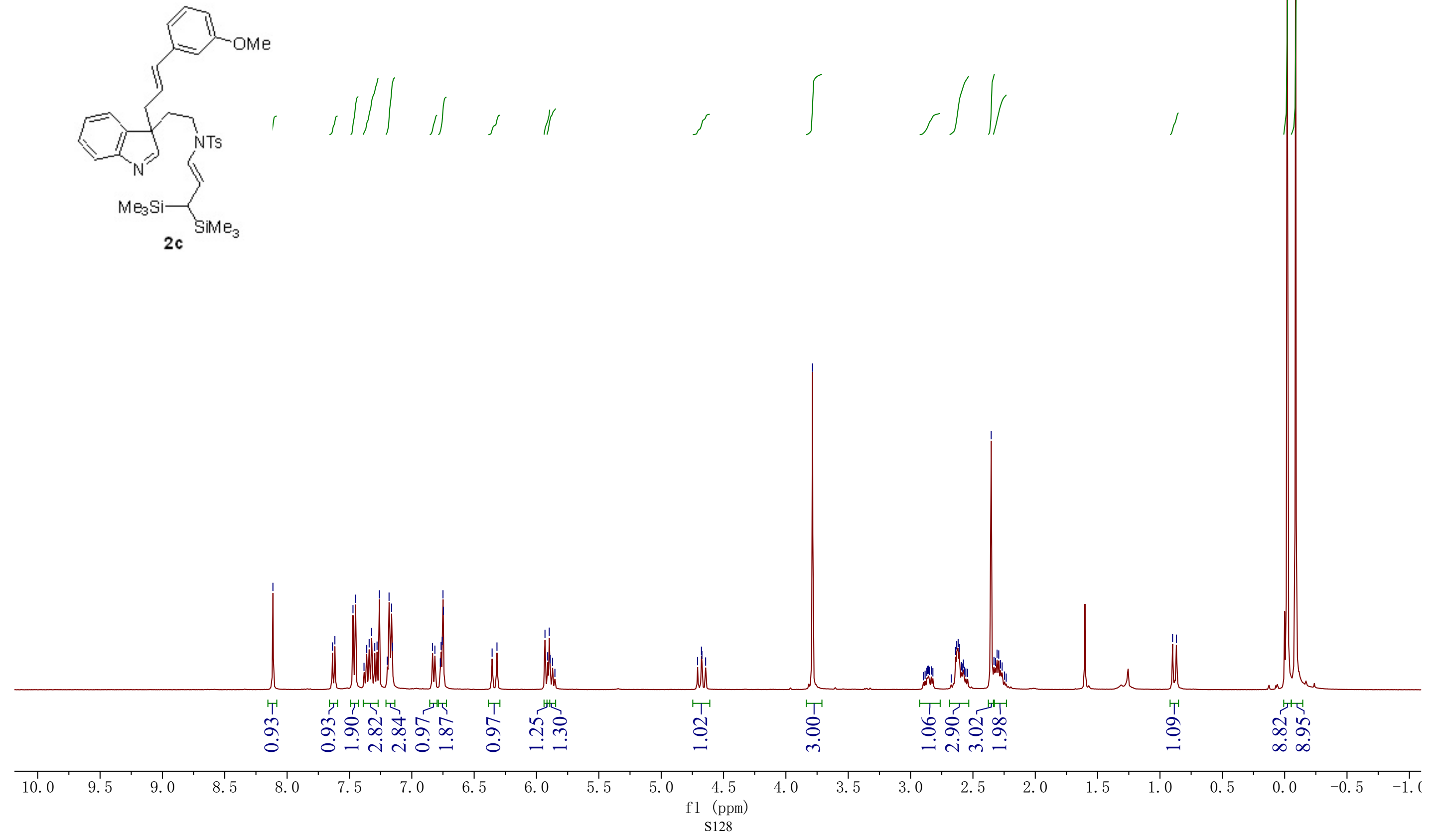

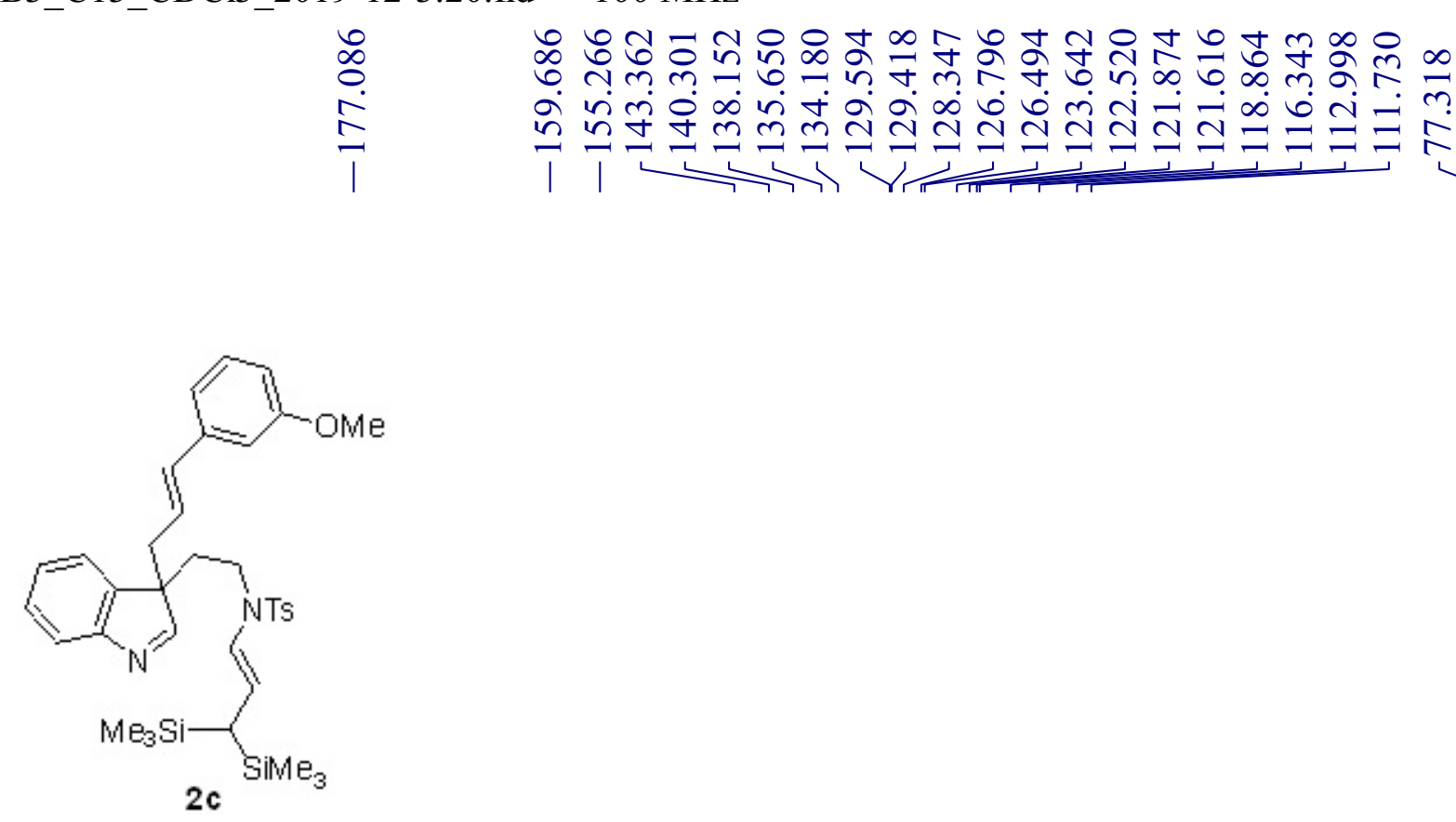

$\stackrel{+}{\lessgtr} \infty$

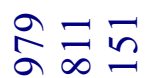

$\infty \vec{y}$

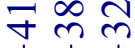

ฟัज

ํํำ
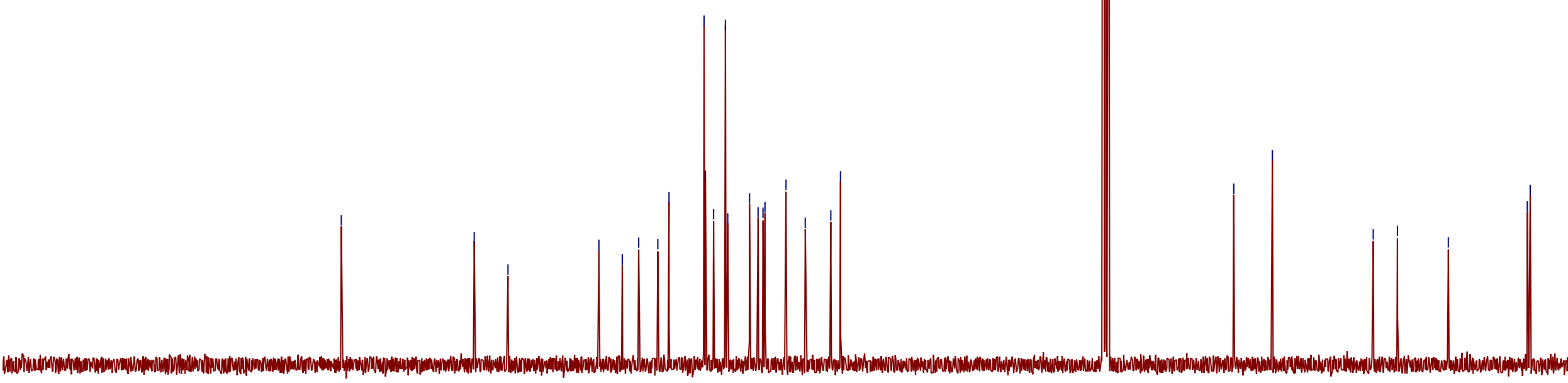

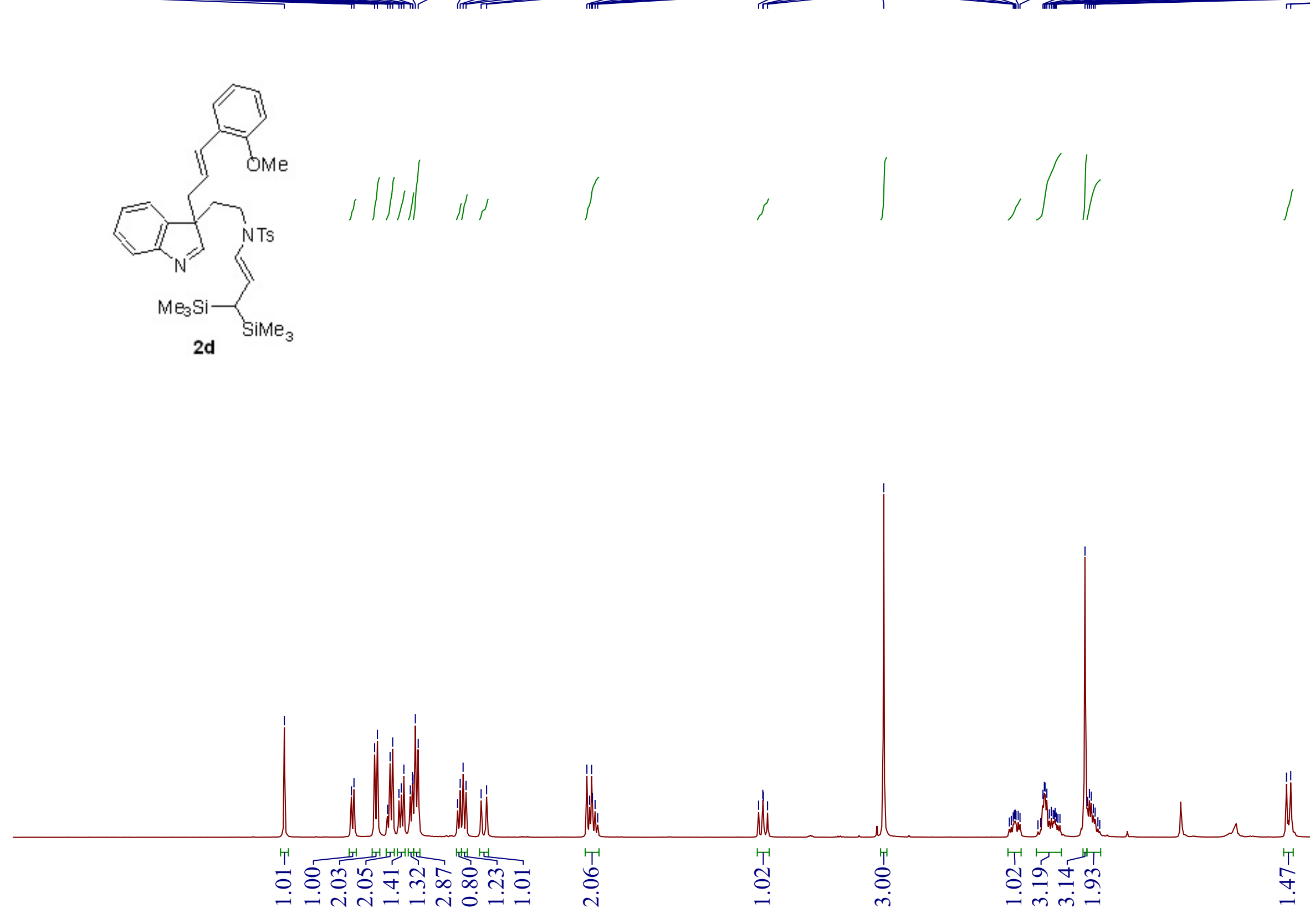

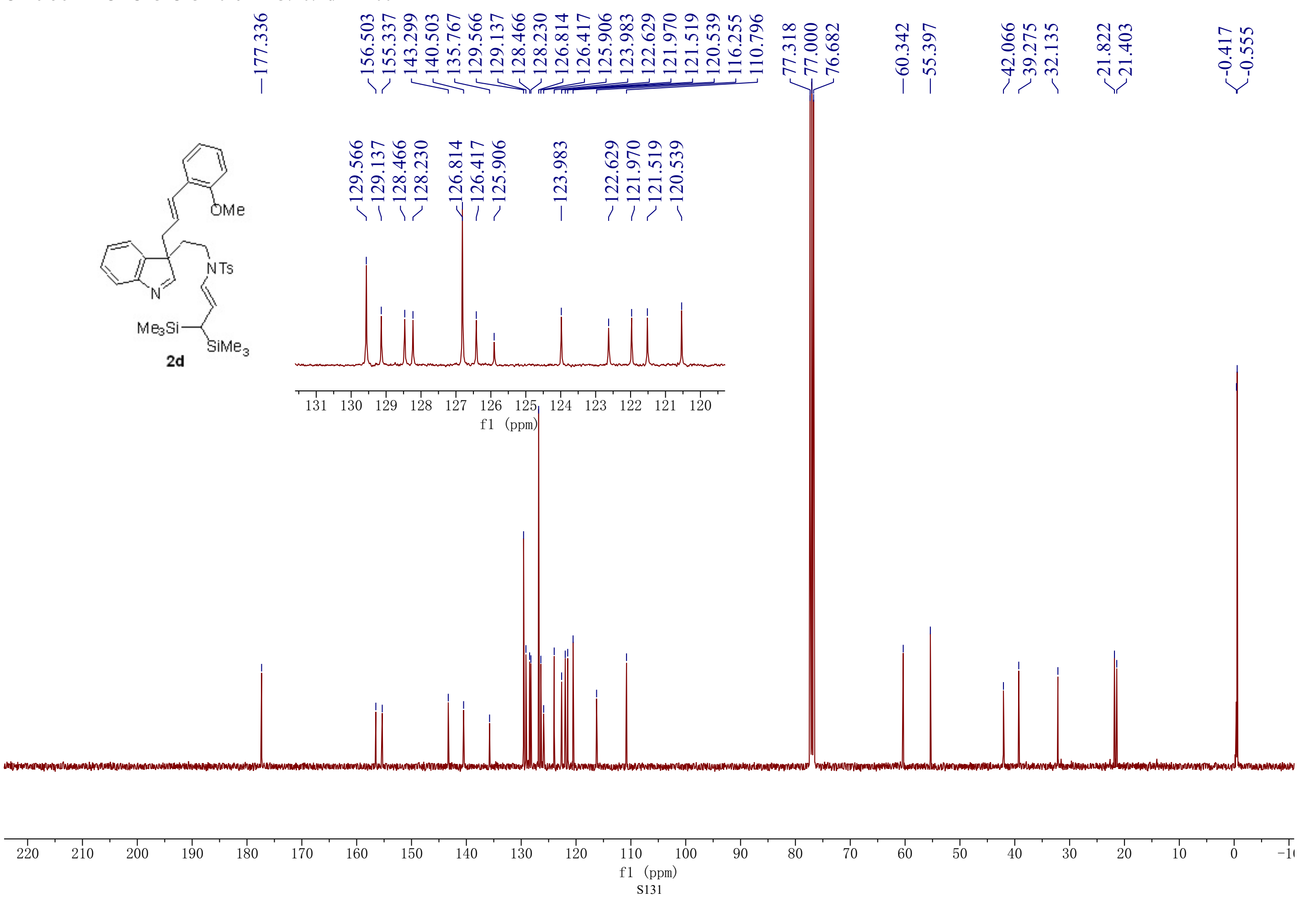

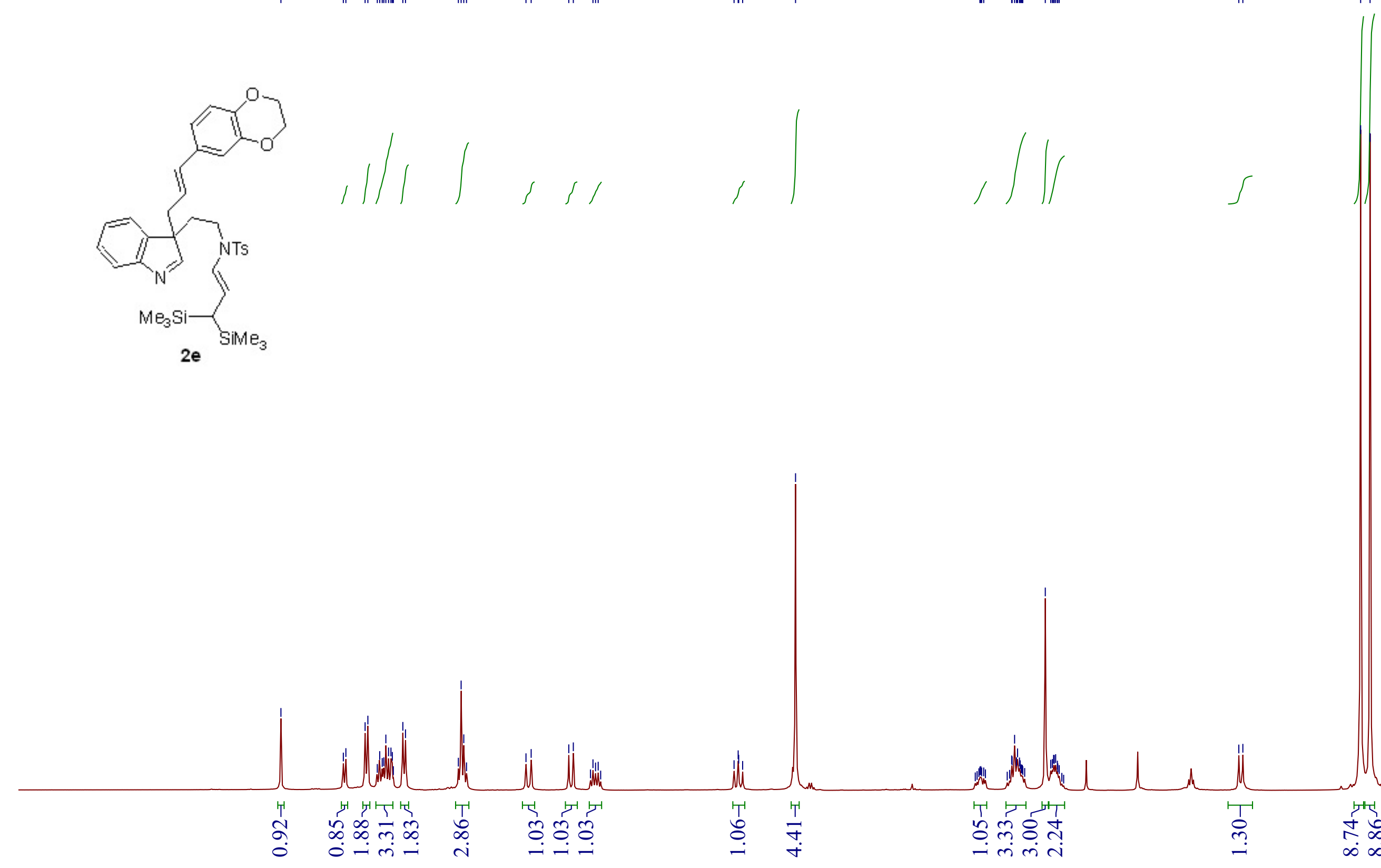


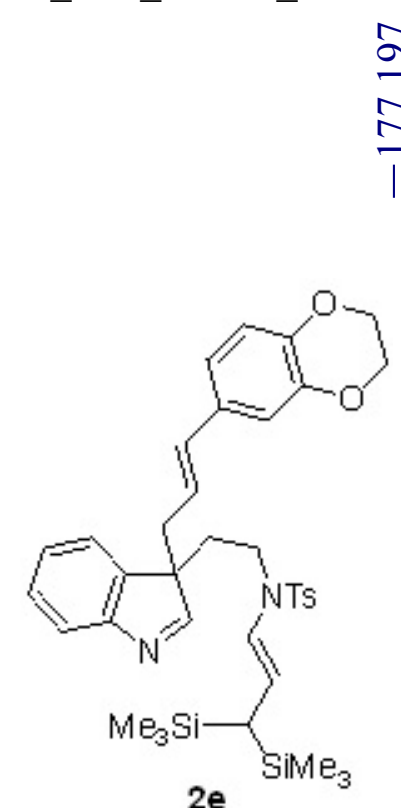

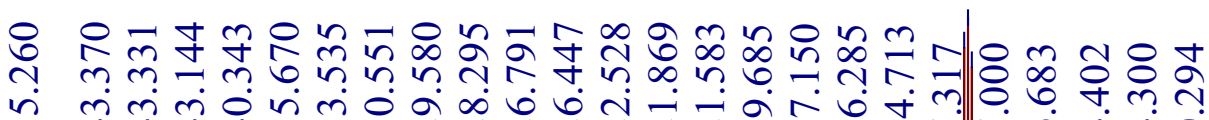

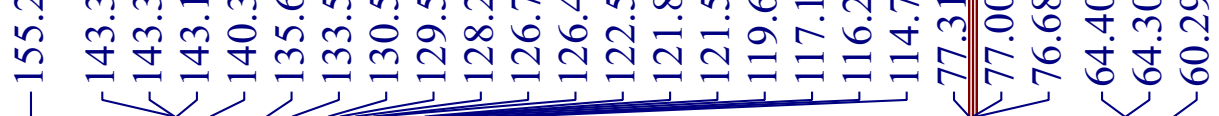

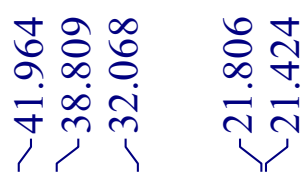

ì

$2 e$

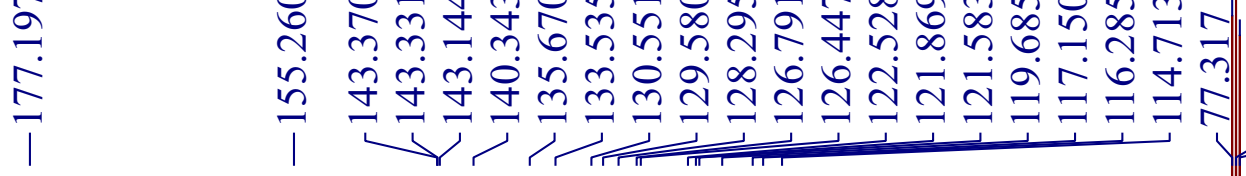



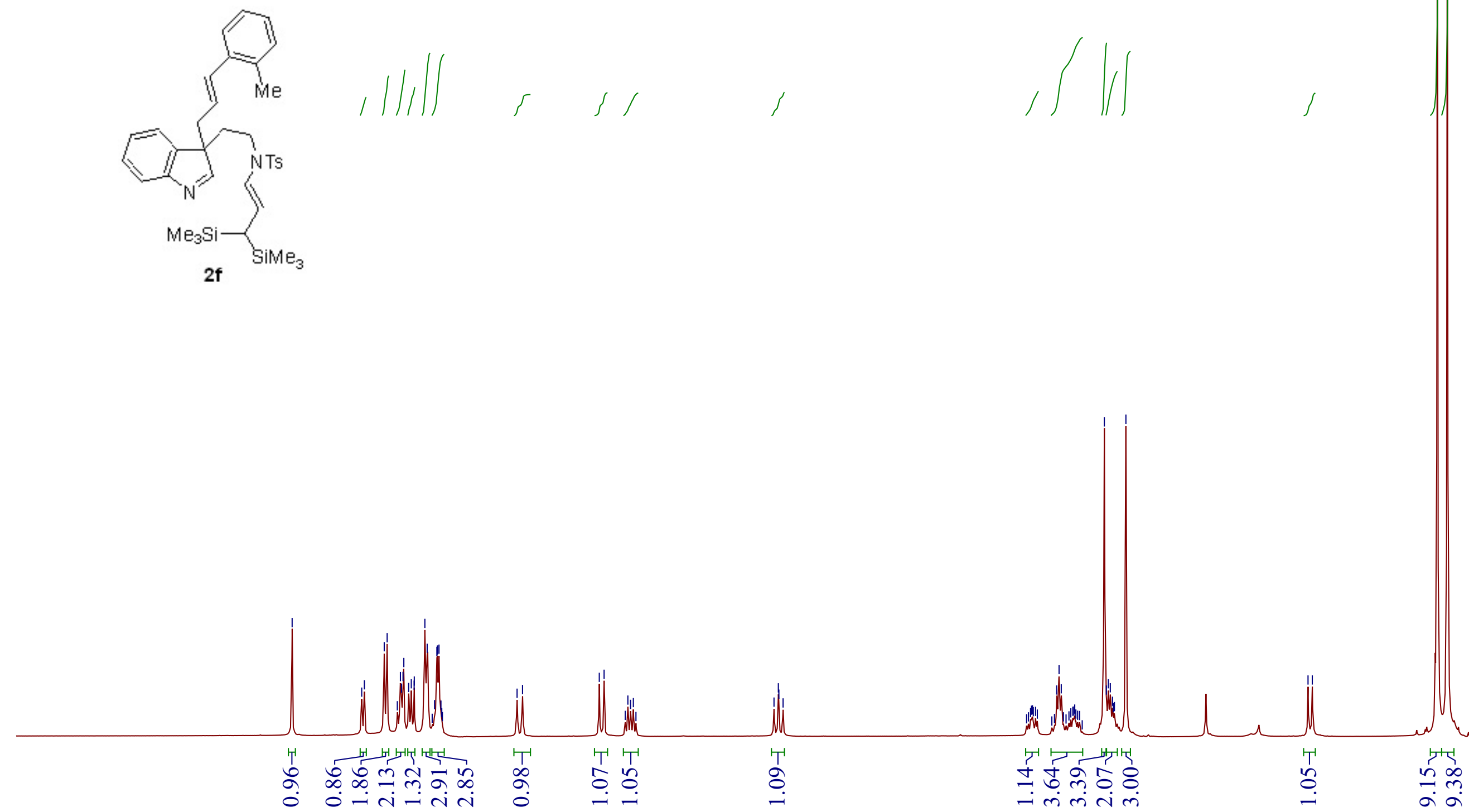


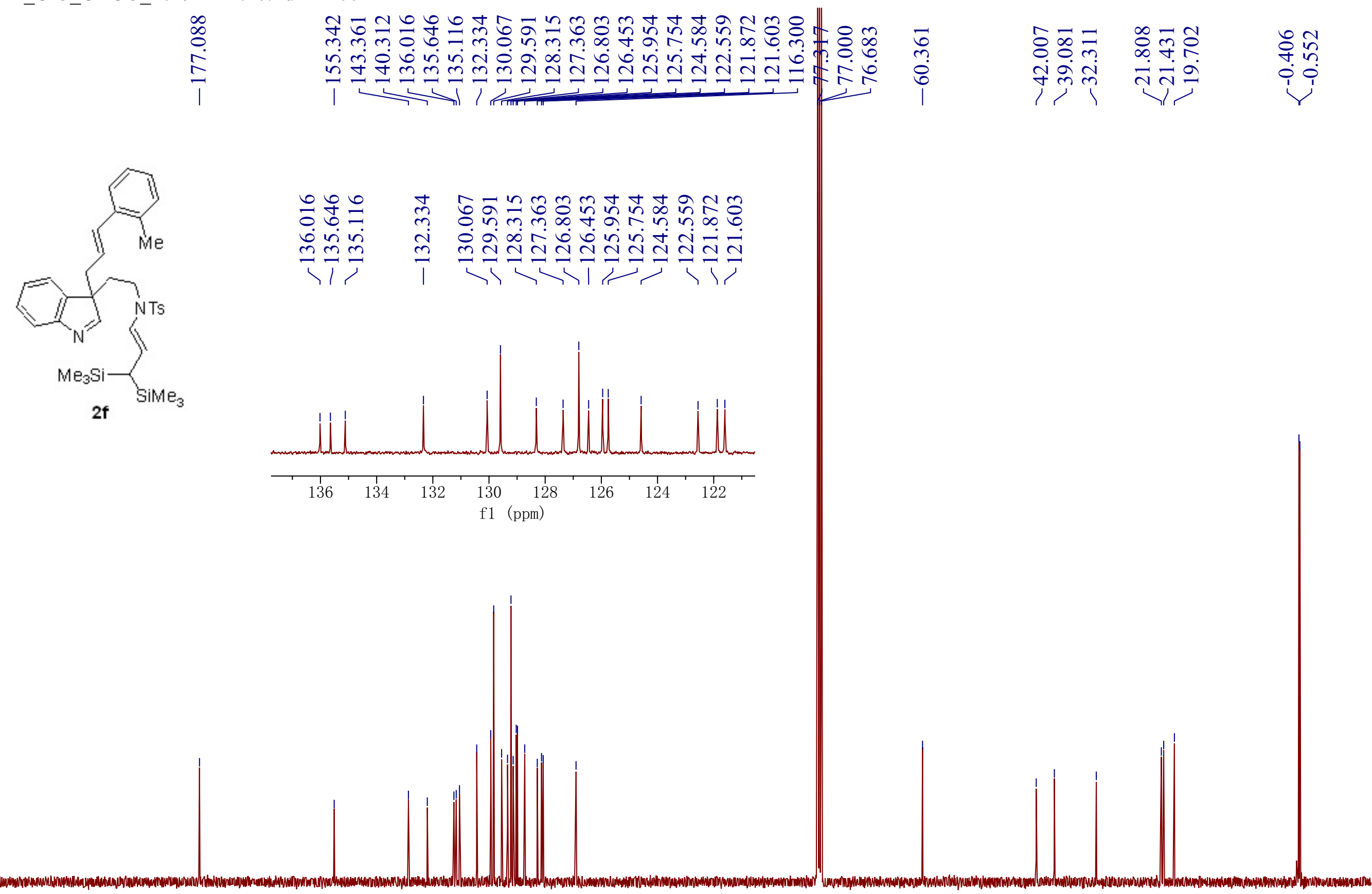



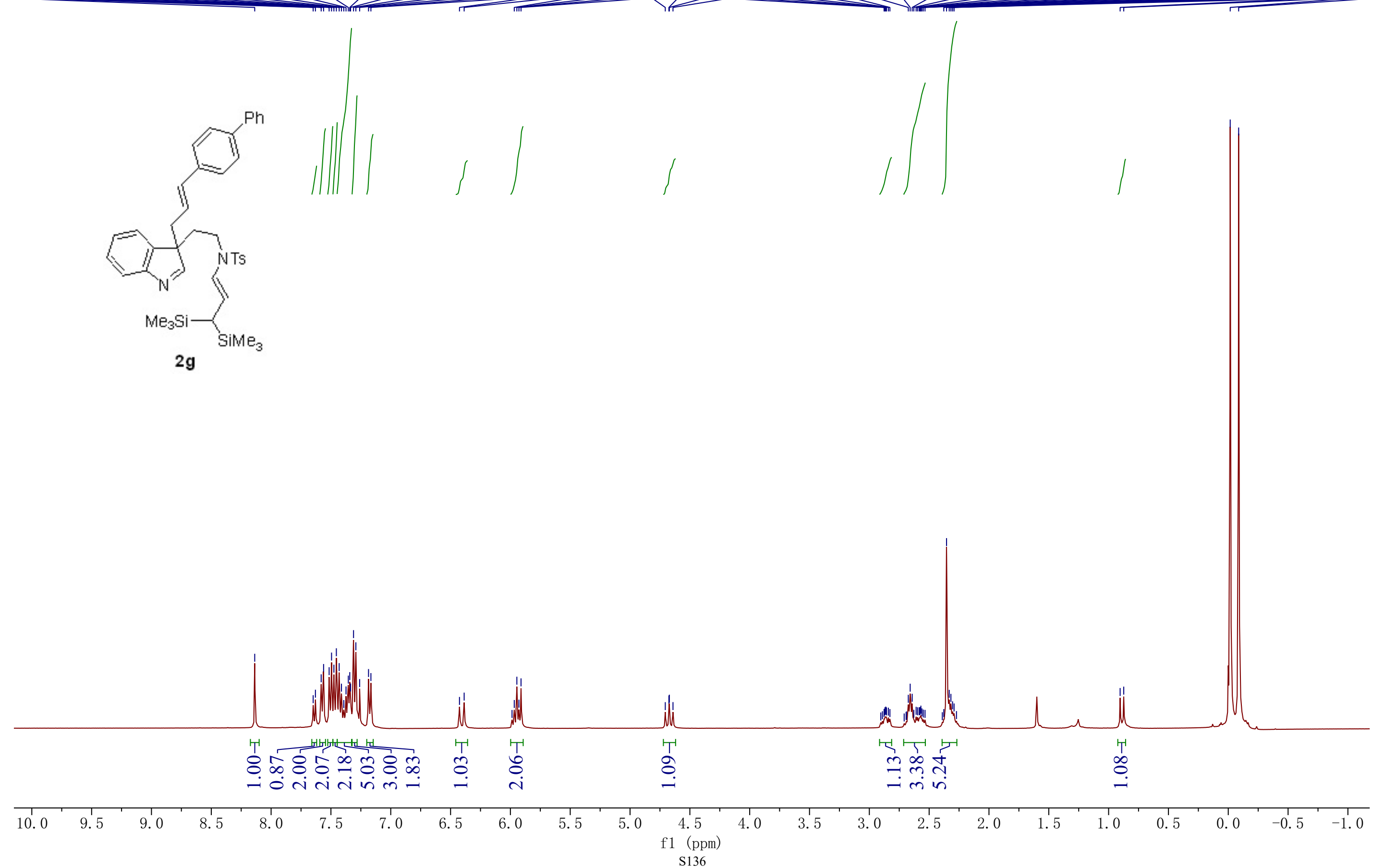


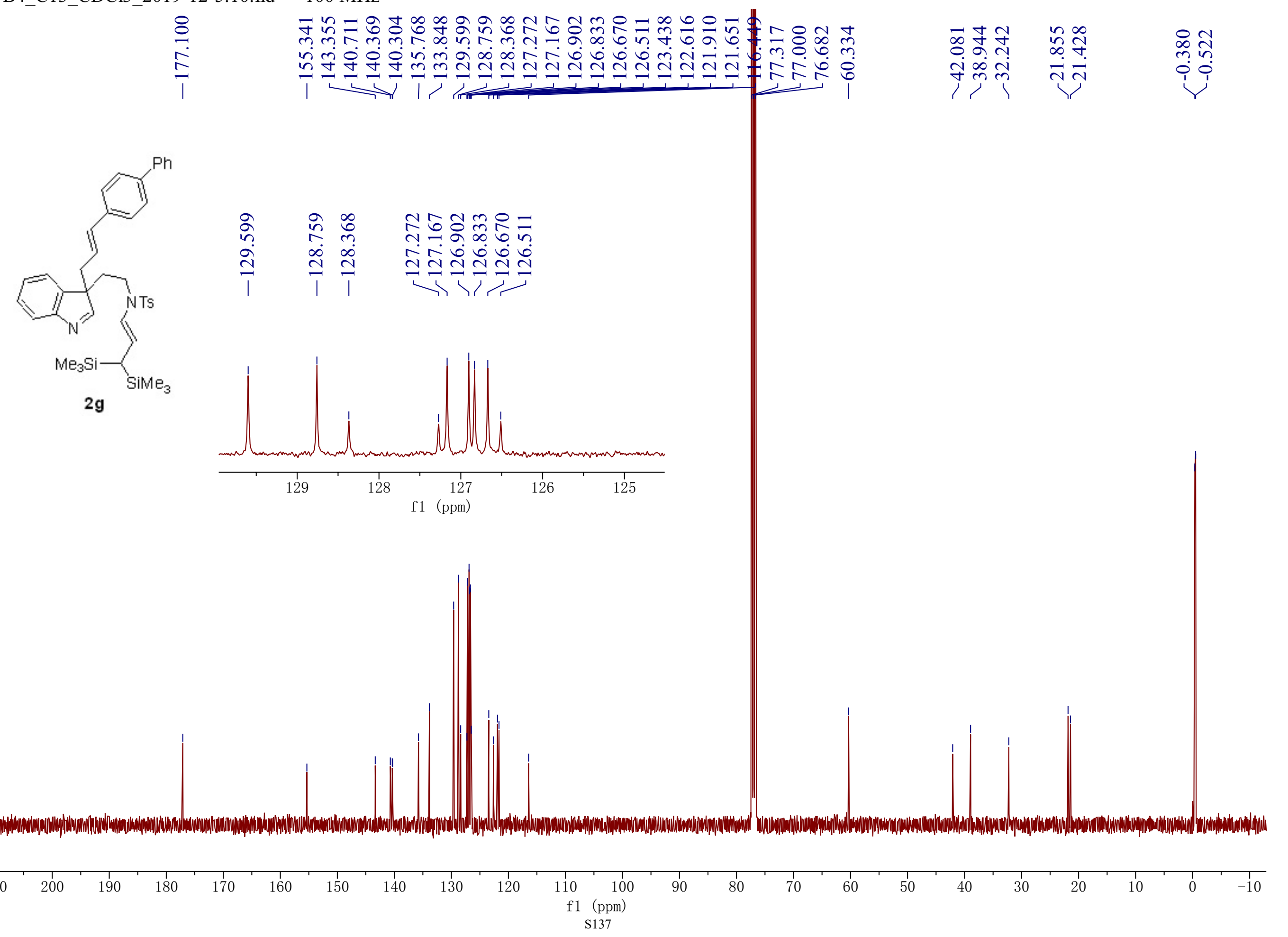



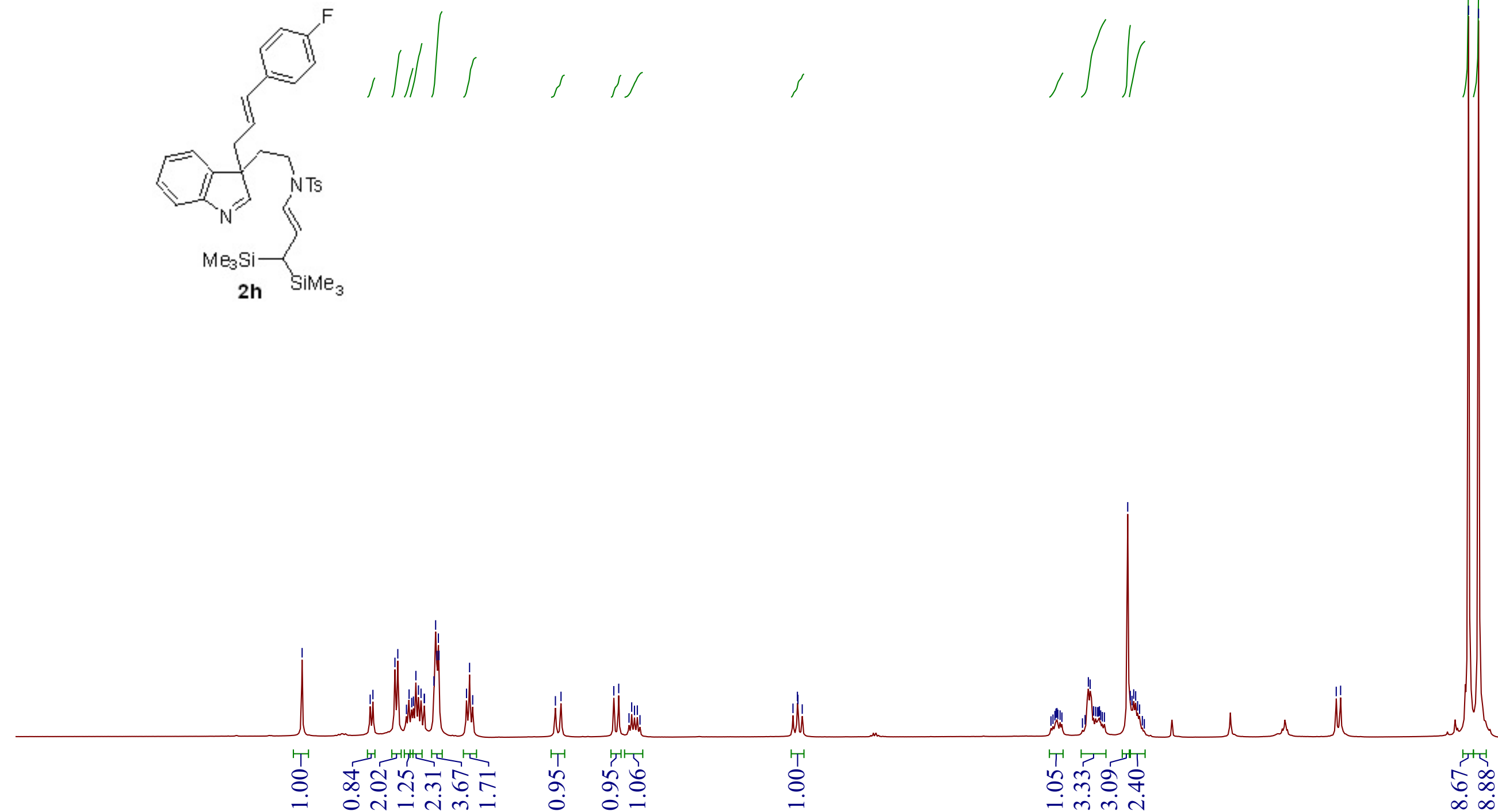

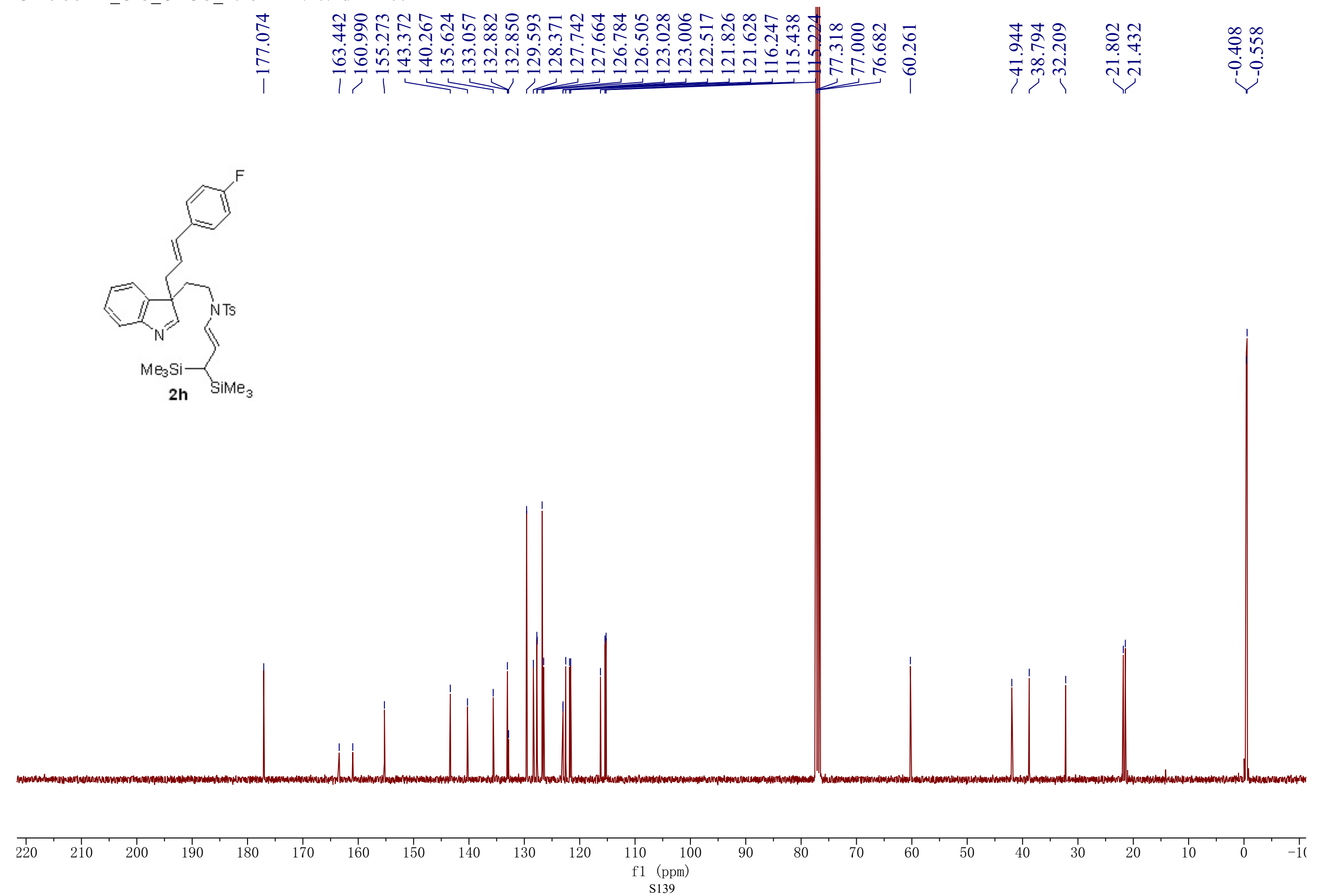

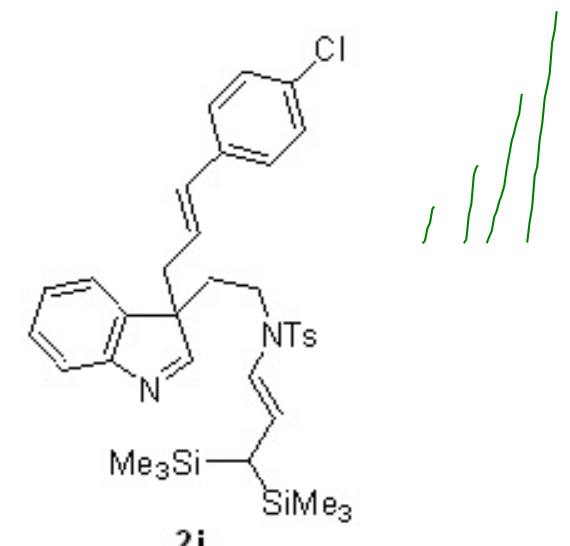

rim
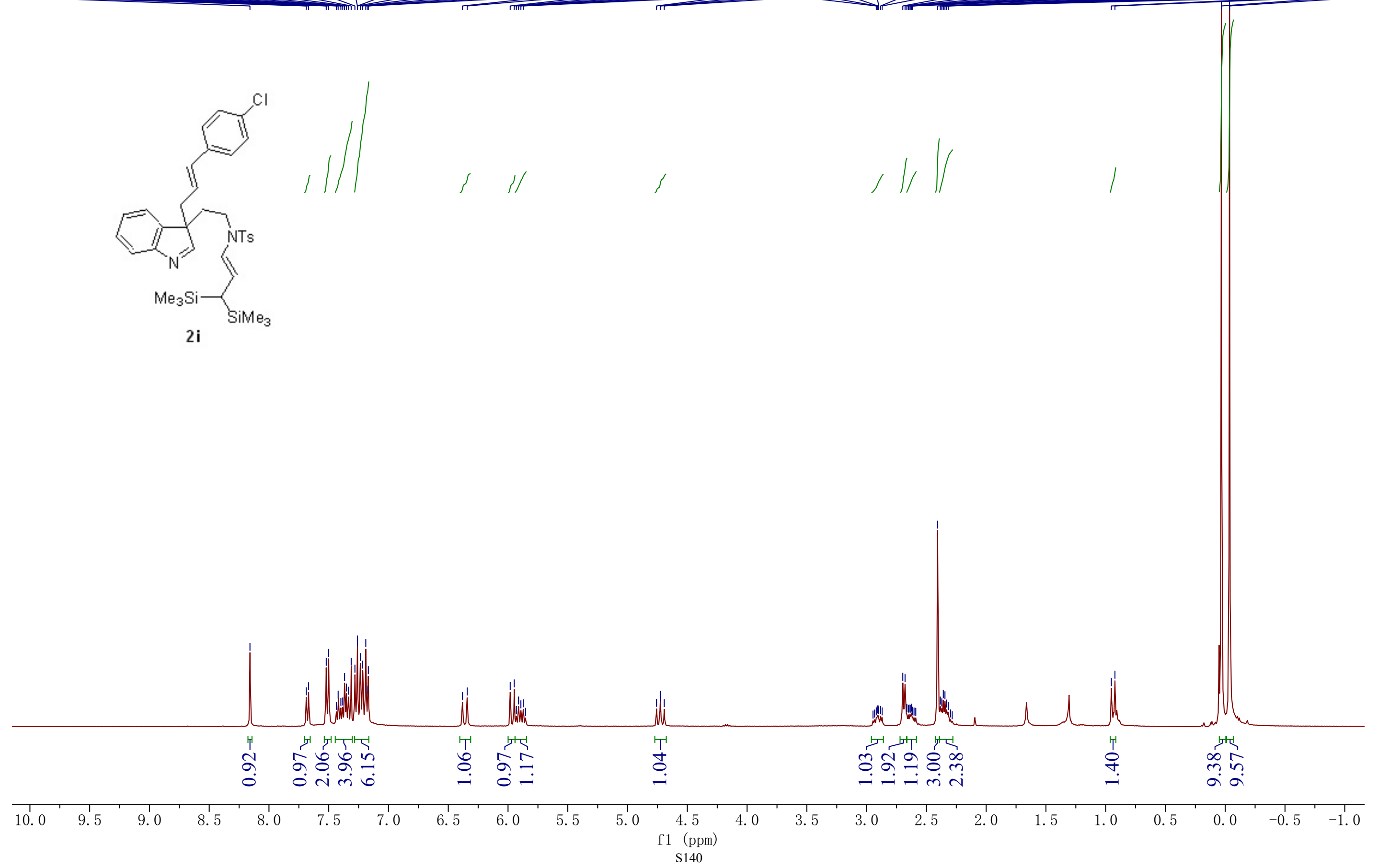


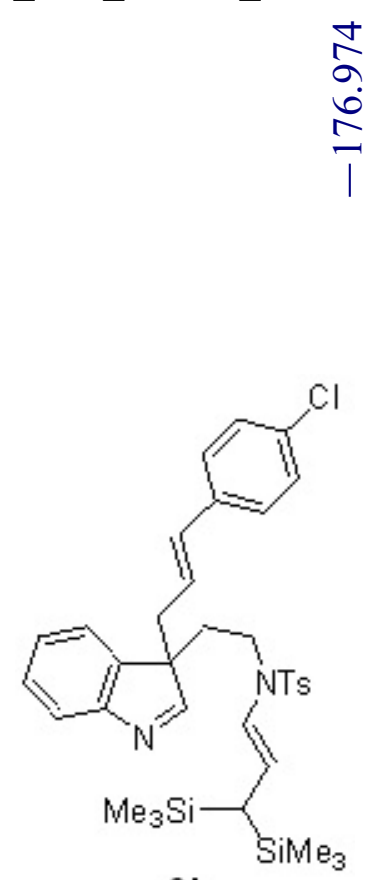

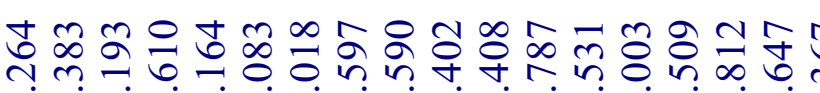

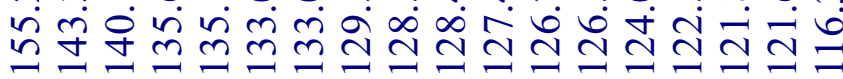

$2 \mathbf{i}$

$$
\begin{aligned}
& \text { 응 } \quad \infty \frac{\infty}{0} \\
& \text { लnं min }
\end{aligned}
$$

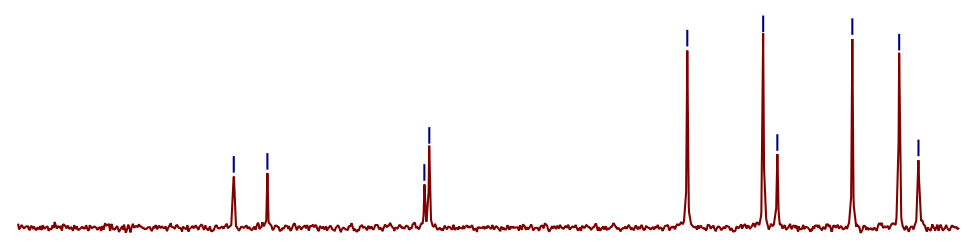

$\begin{array}{lllllllllllllllll}138 & 137 & 136 & 135 & 134 & 133 & 132 & 131 & 130 & 129 & 128 & 127 & 126\end{array}$ f1 (ppm)

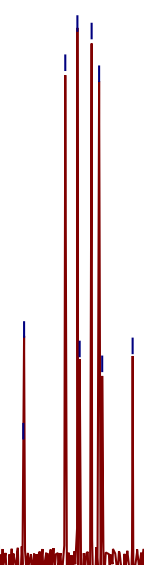



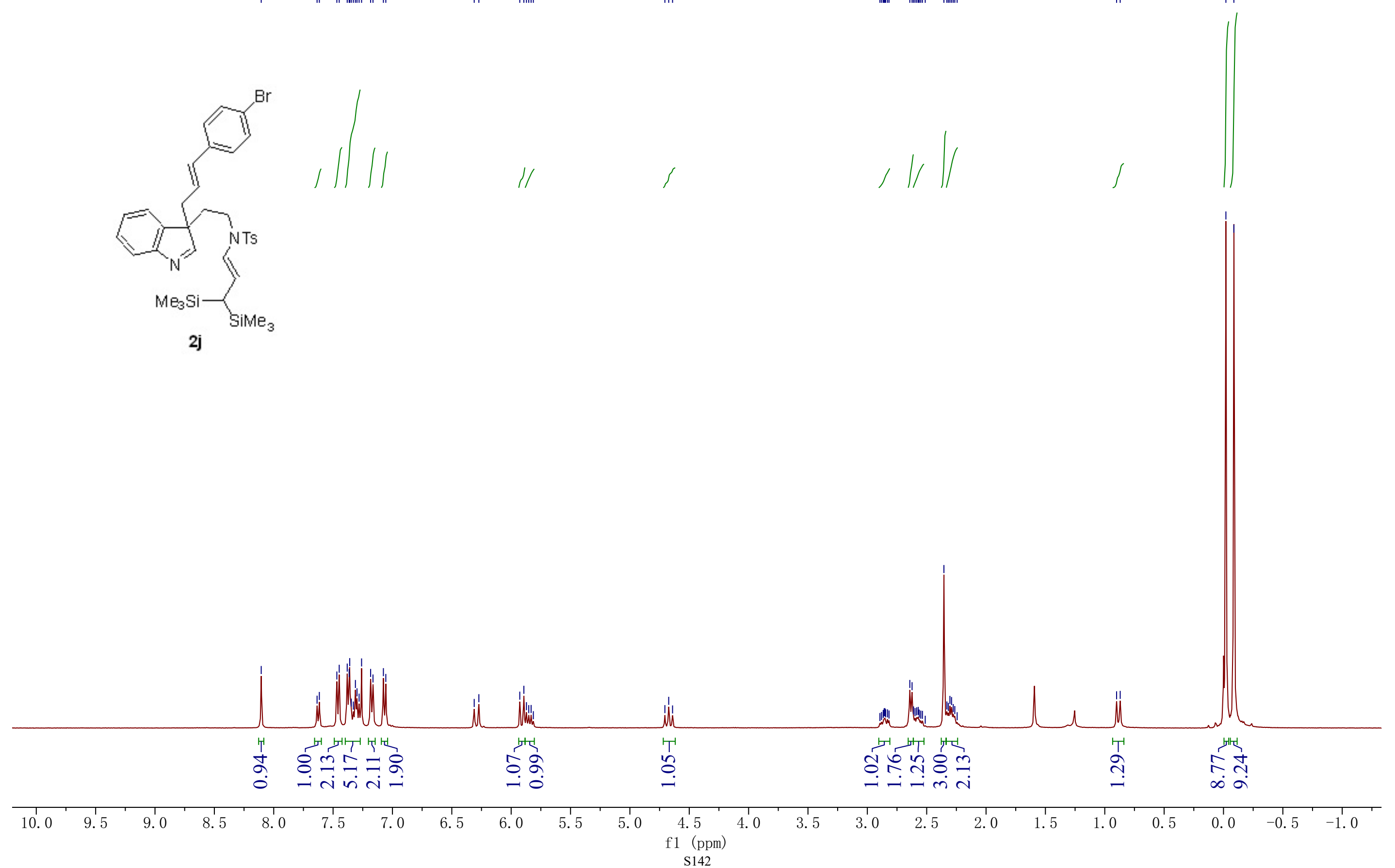


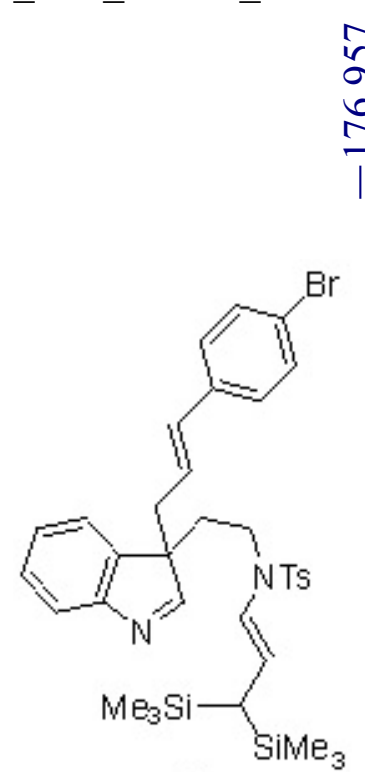

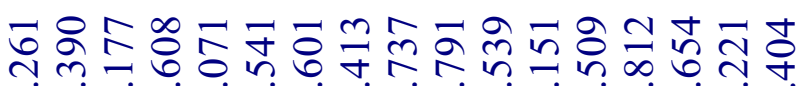

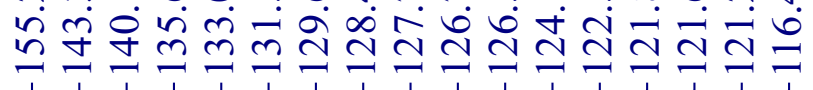
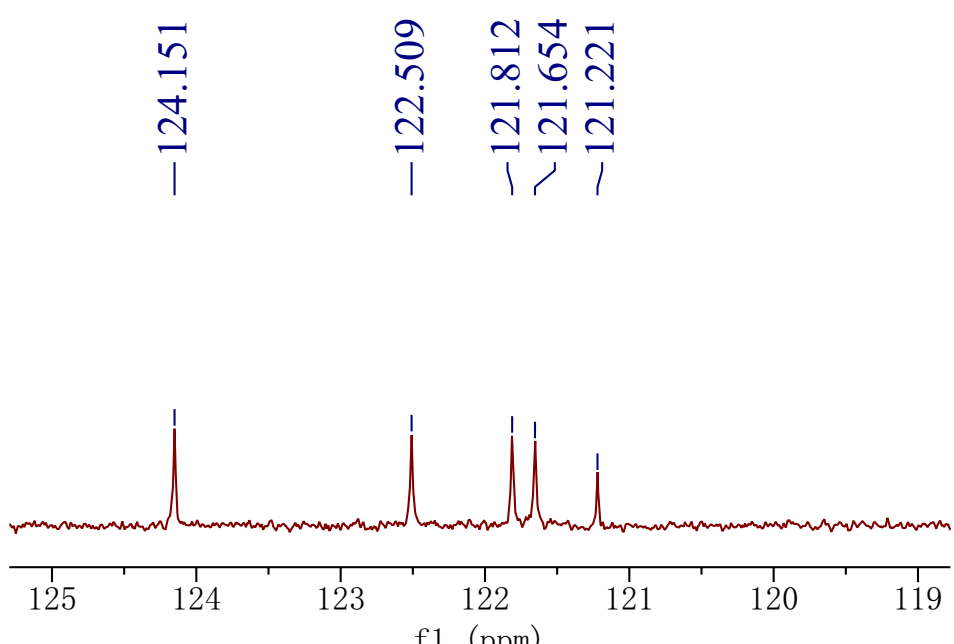

f1 (ppm) 

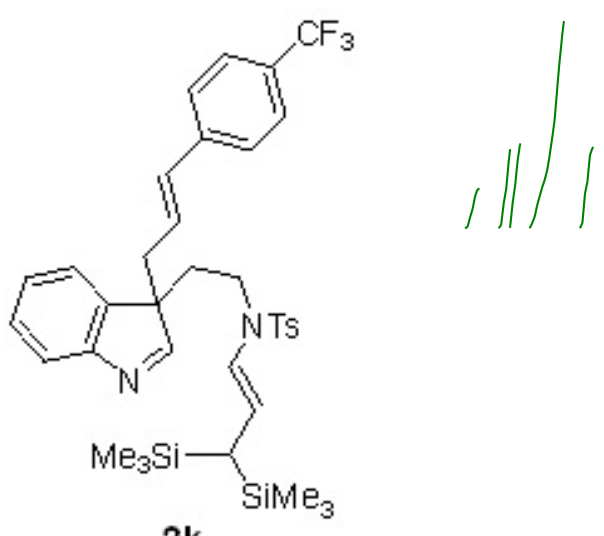

2k

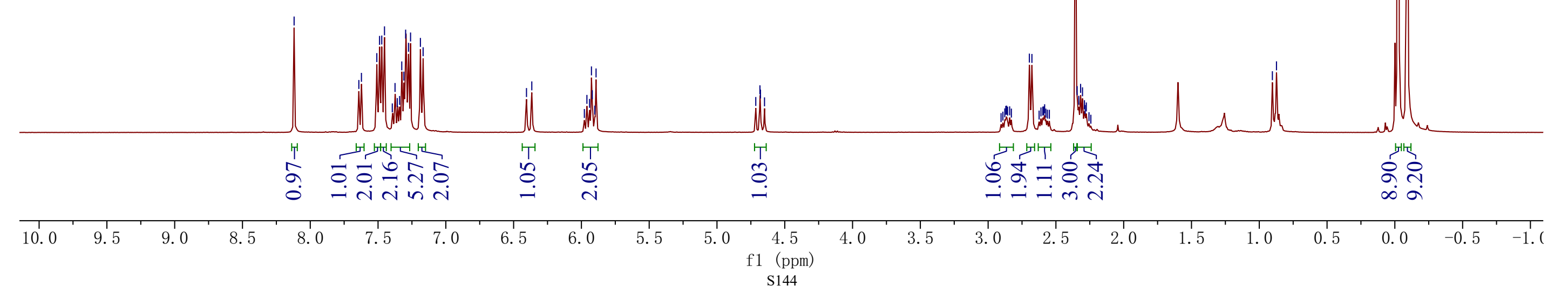




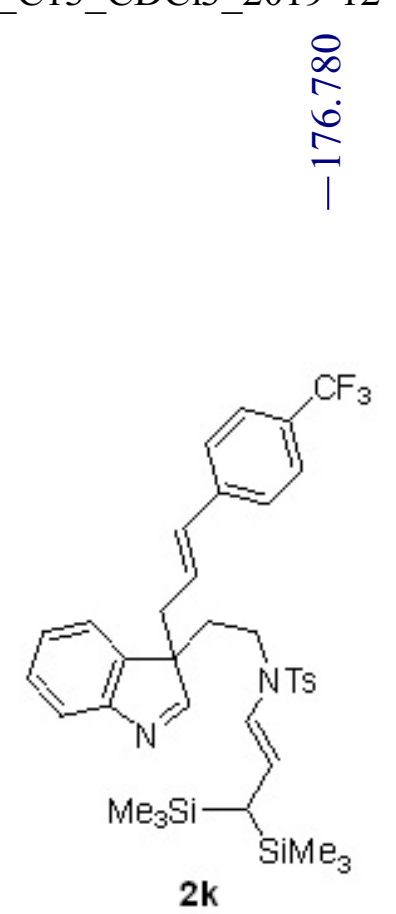

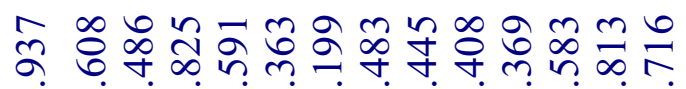

๙

1 展

$2 k$

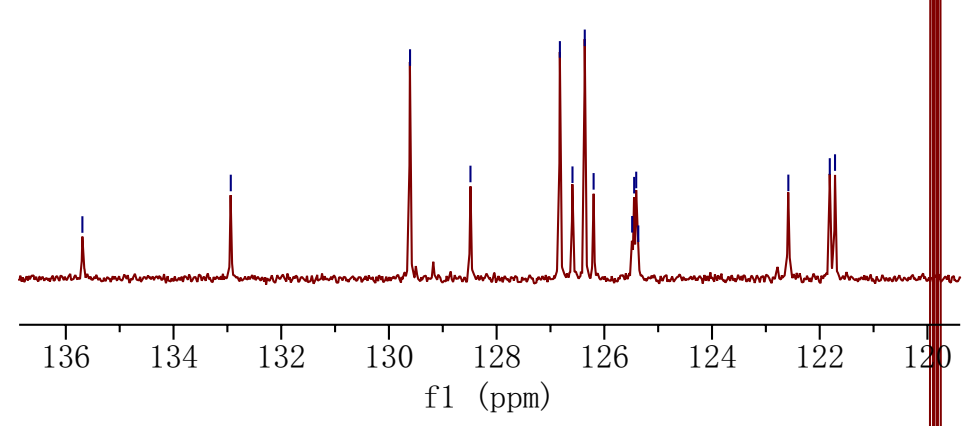



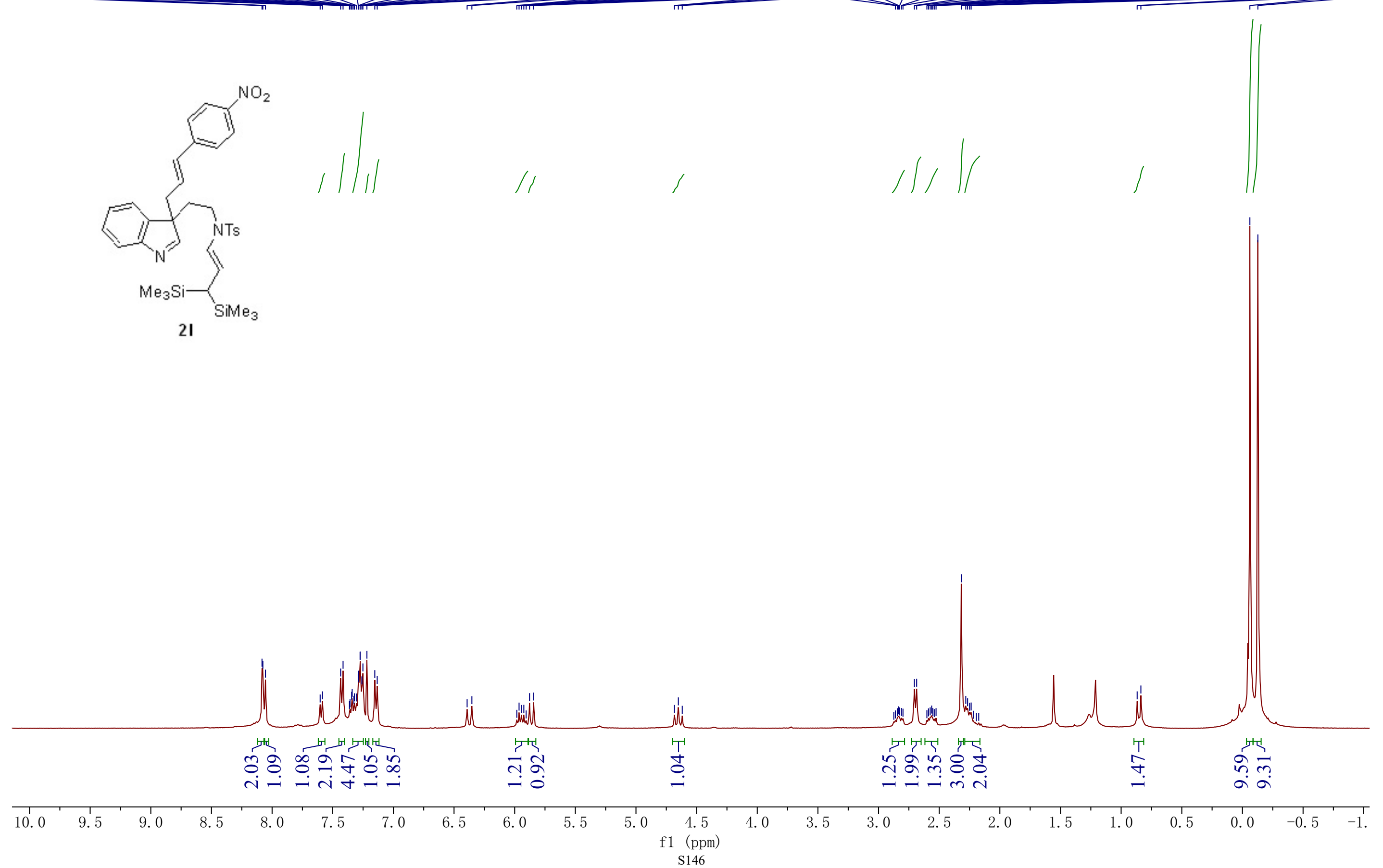


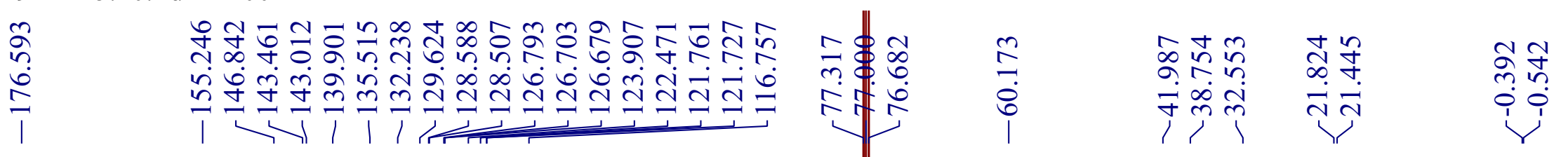

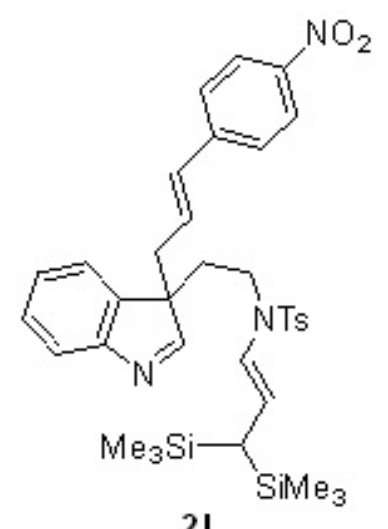

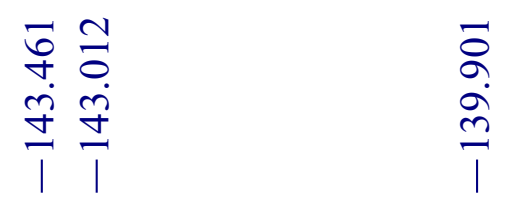

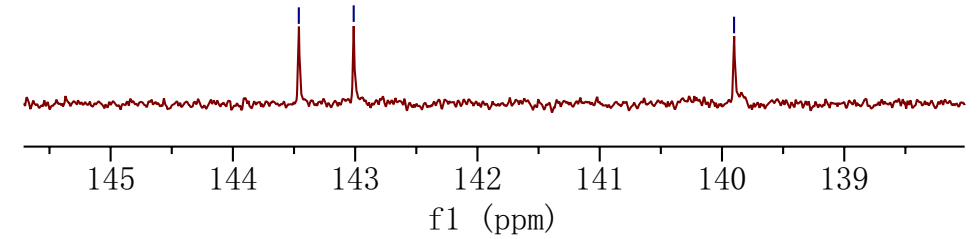



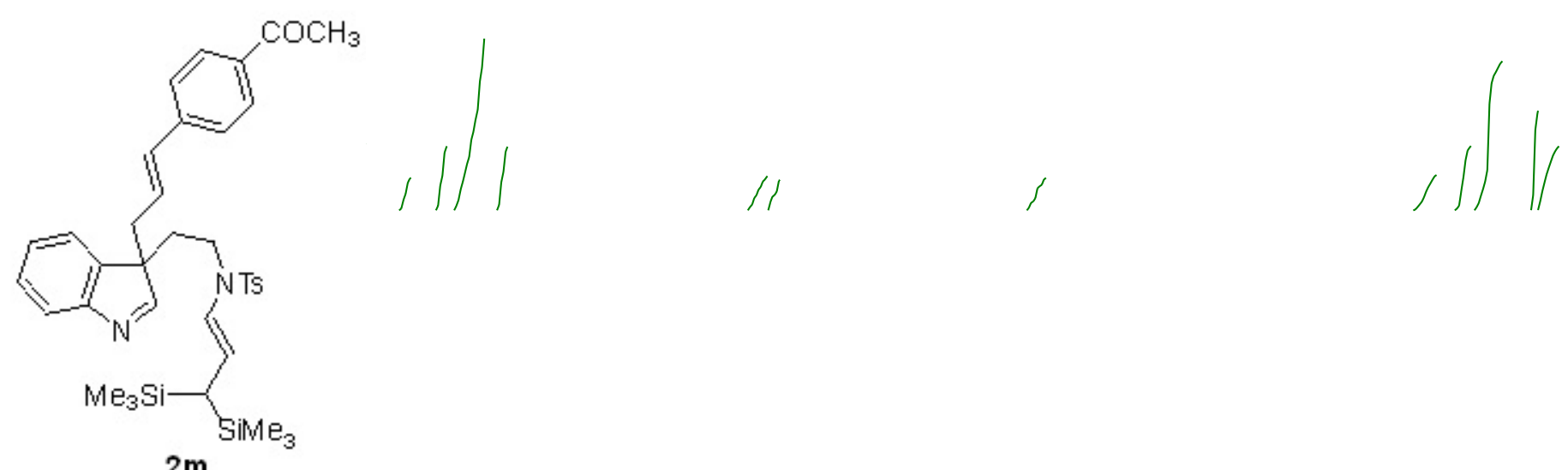

$2 \mathrm{~m}$

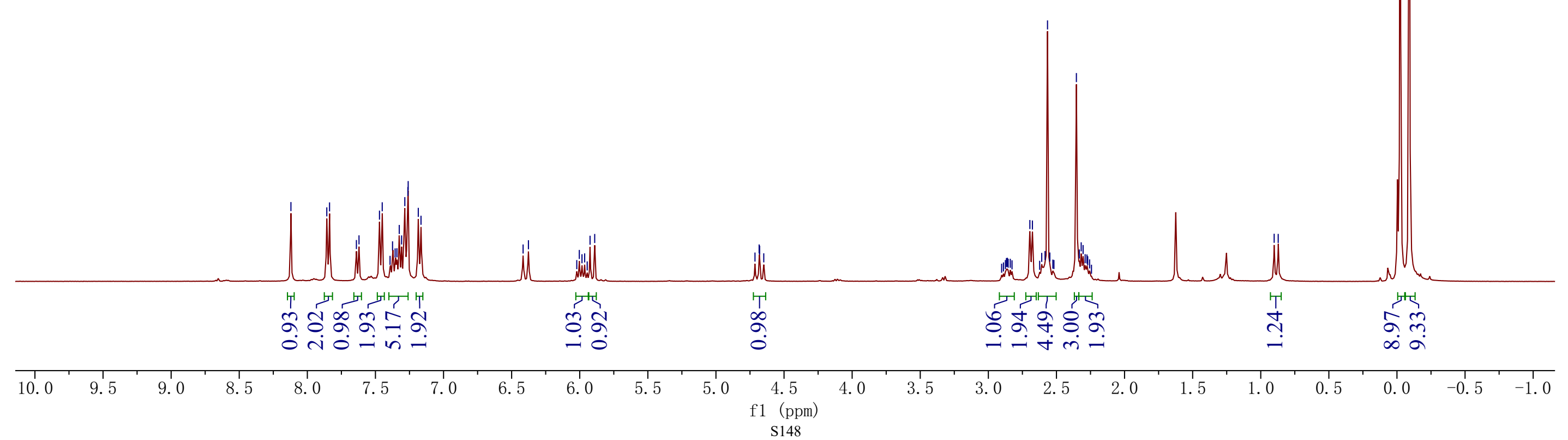




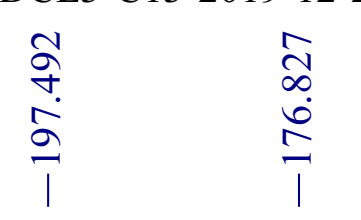

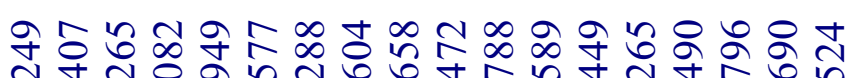

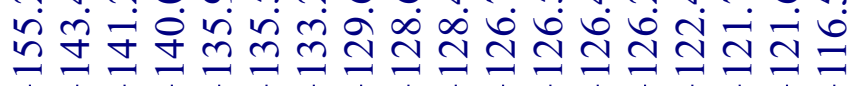
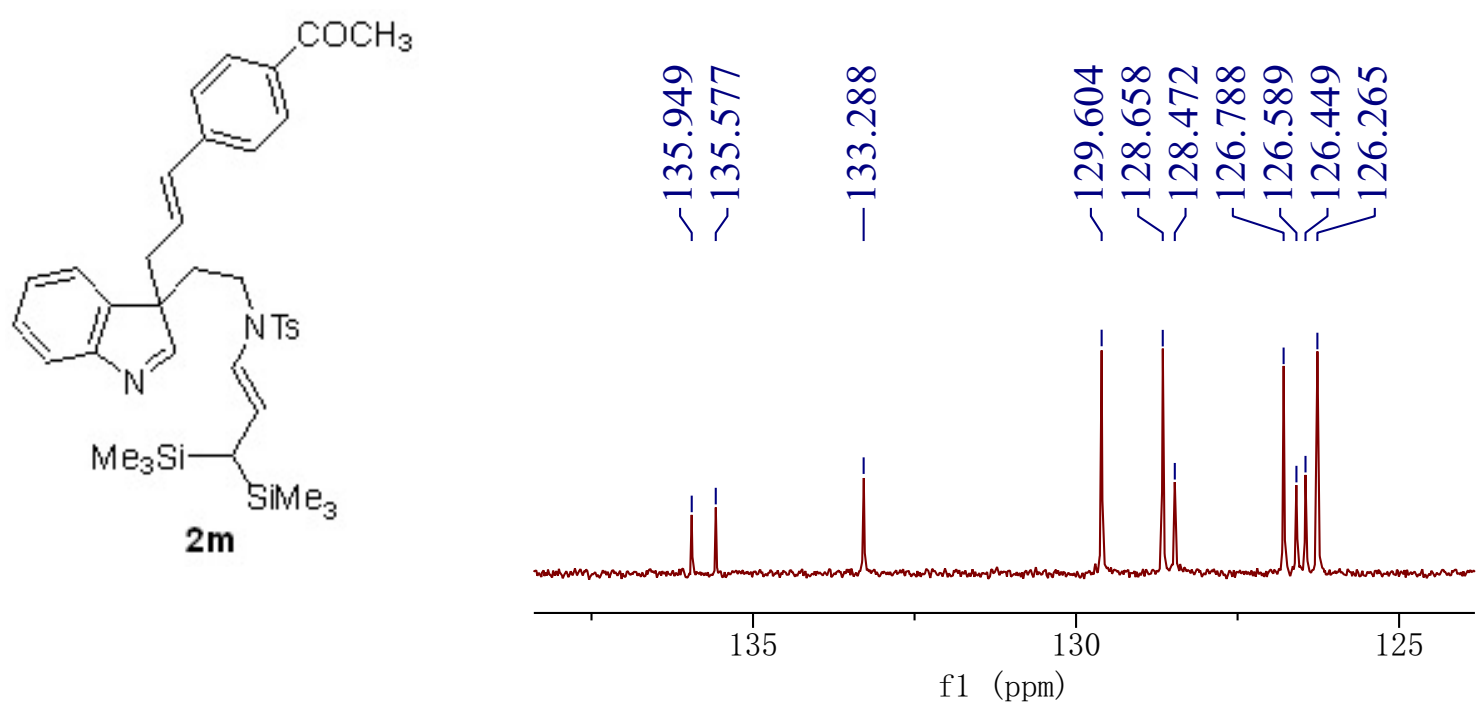

$2 \mathrm{~m}$

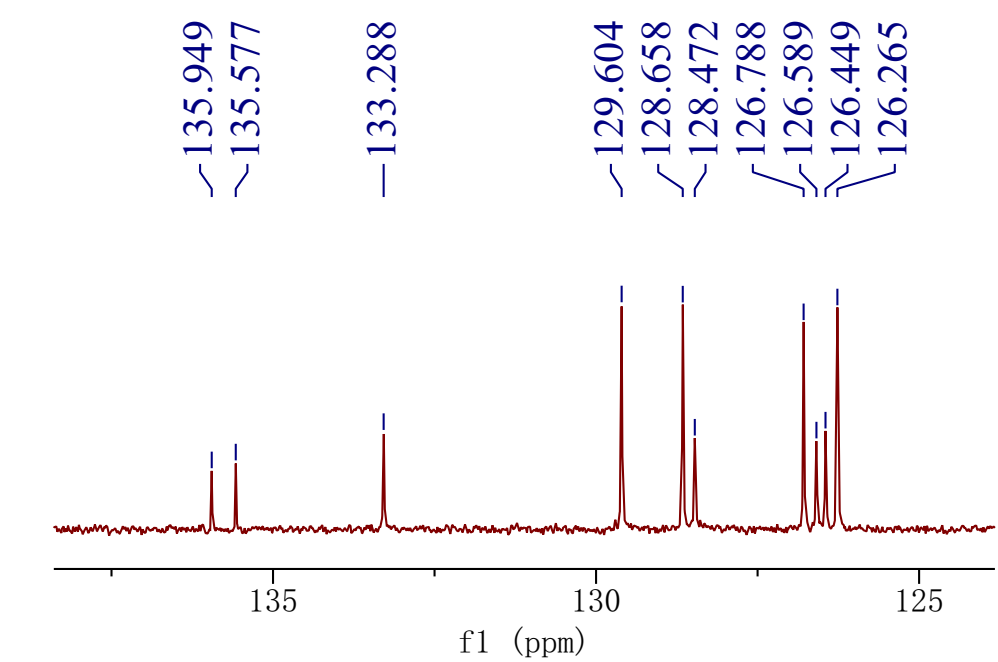




\section{CY-6-86-B2_H1_CDCl3_2019-12-25.20.fid - $400 \mathrm{MHz}$}

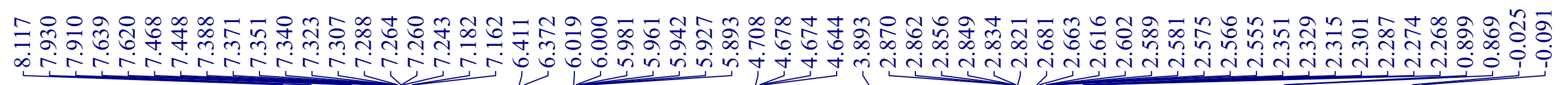
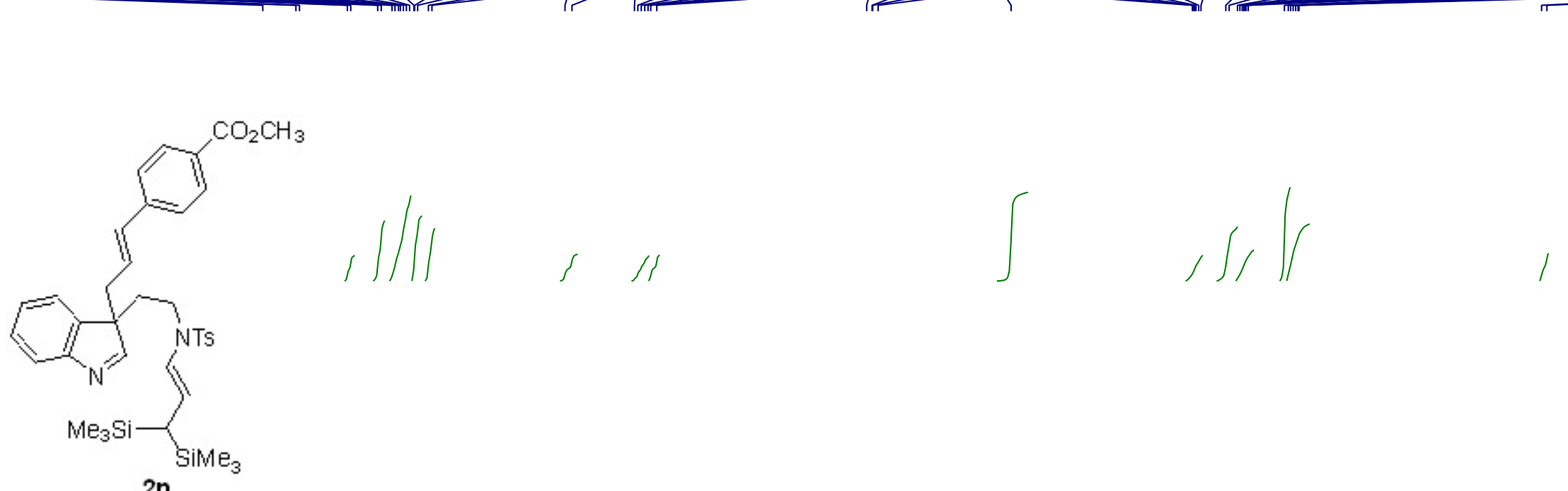

2n

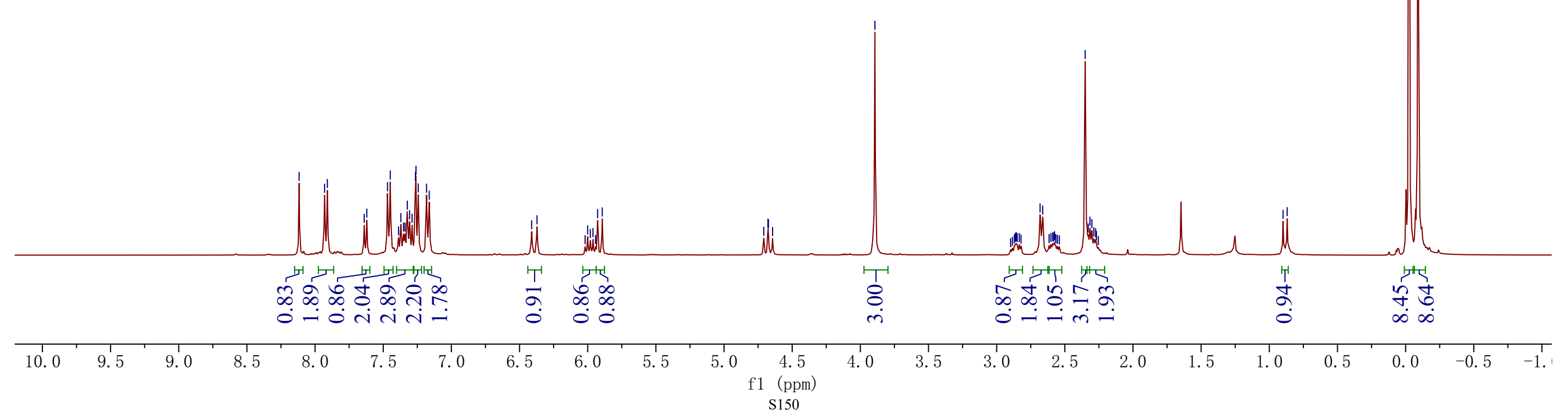



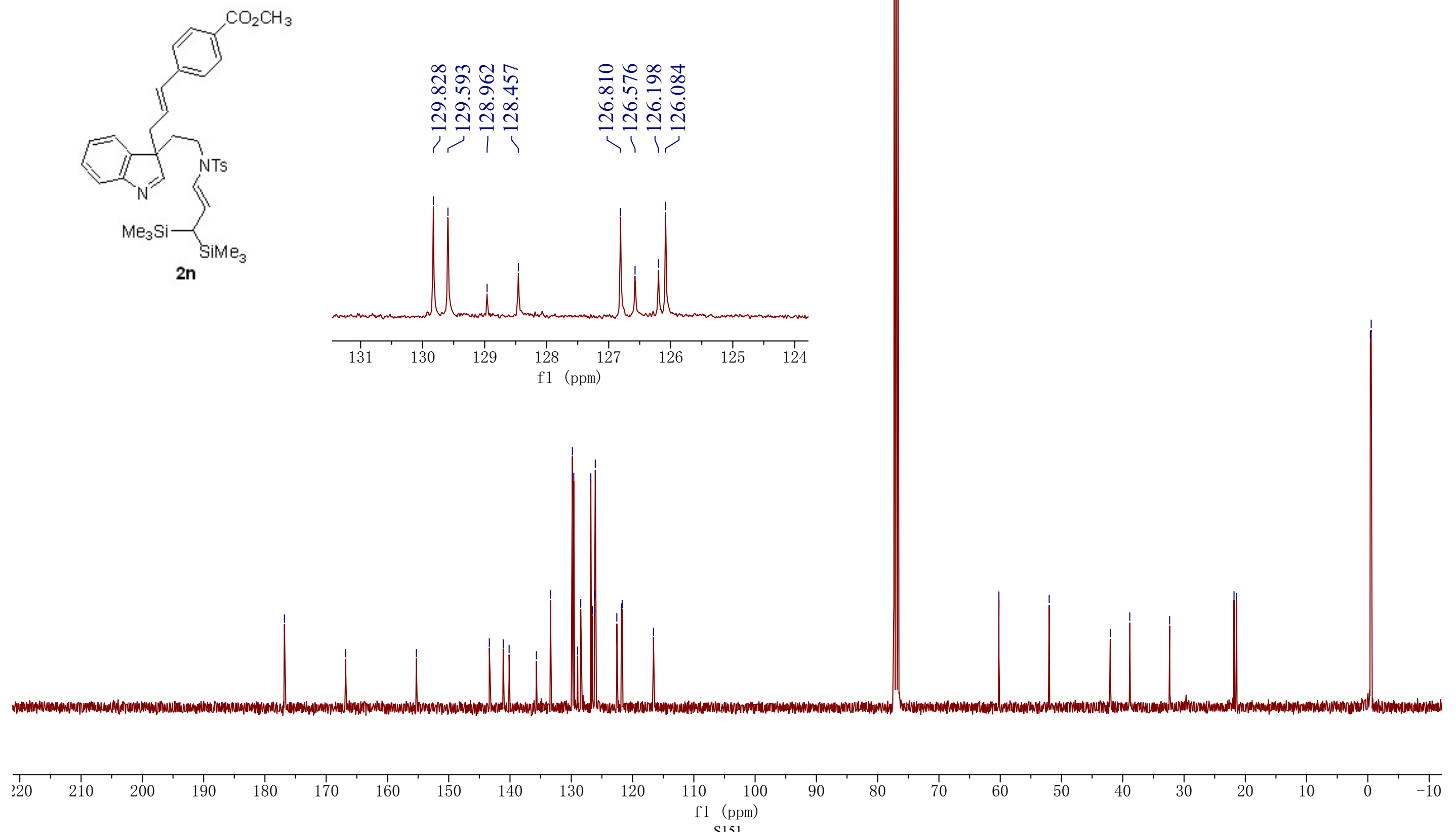

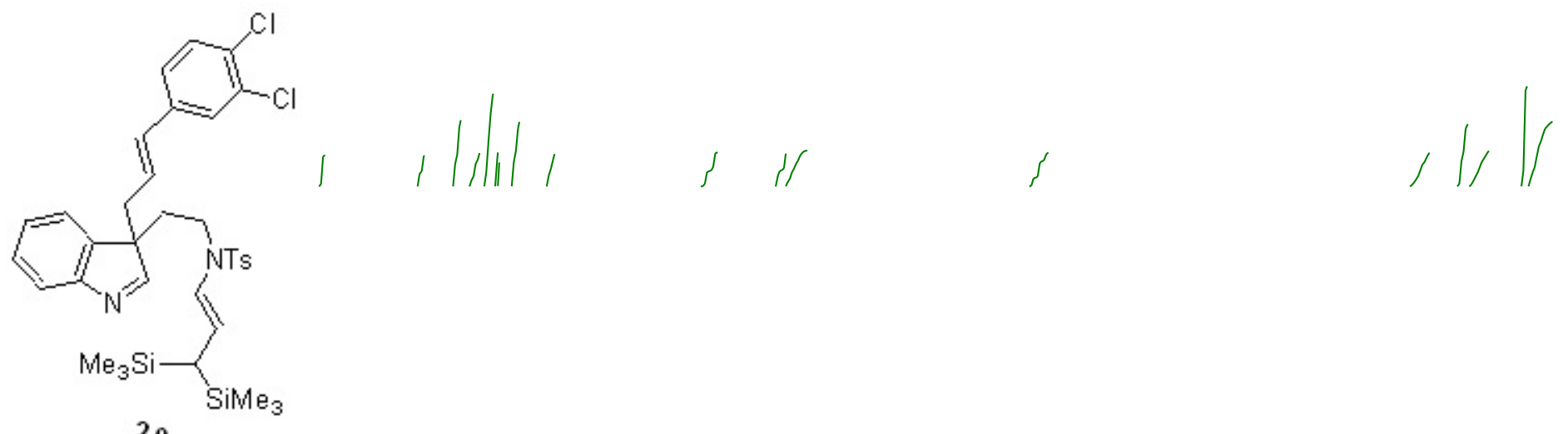

20

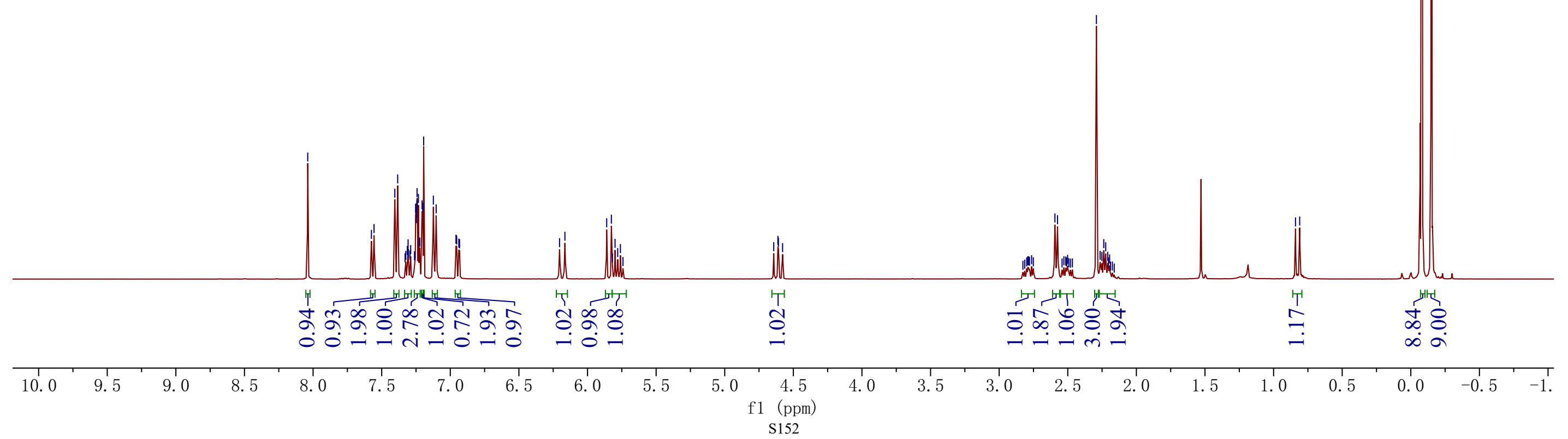



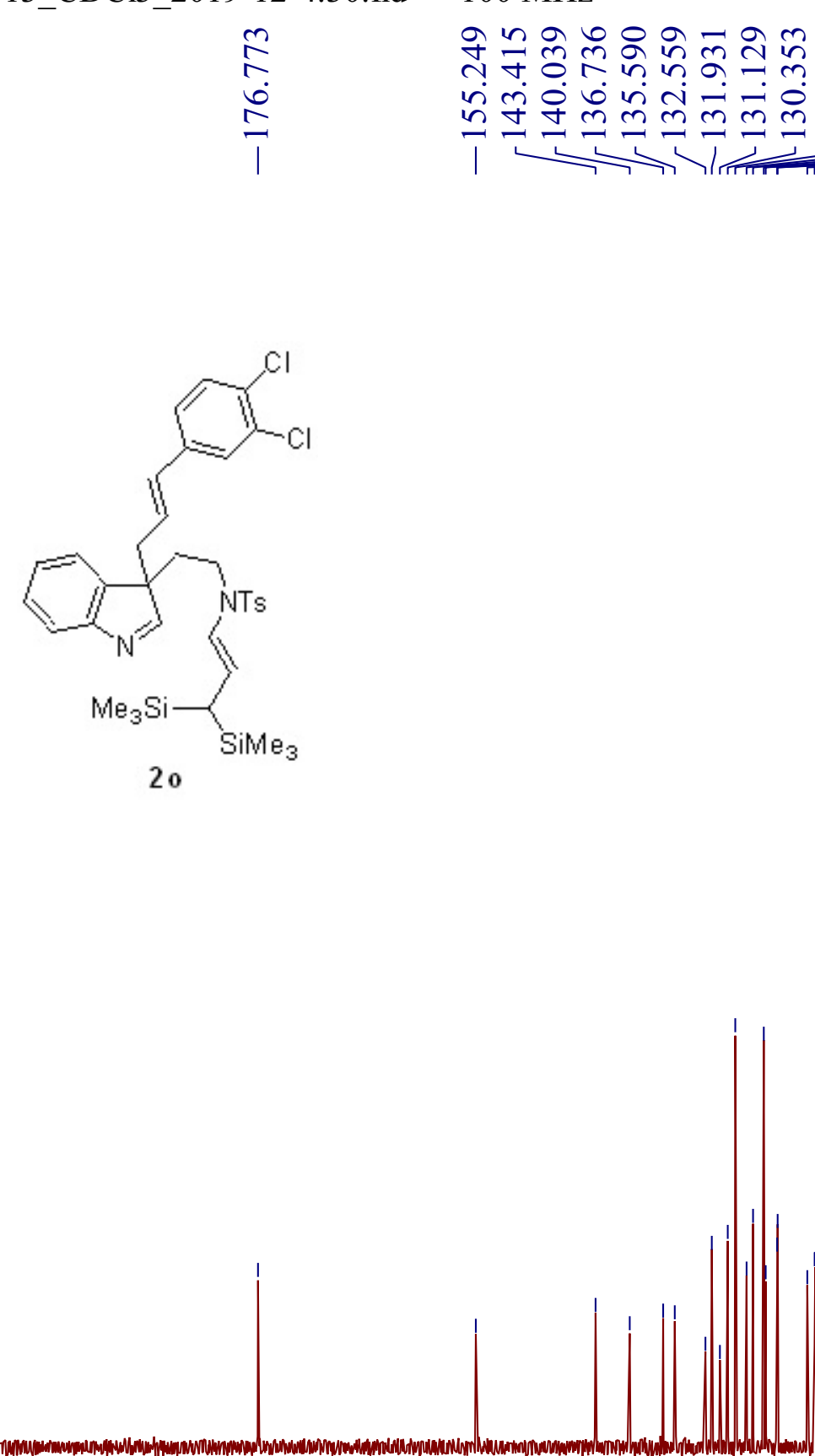

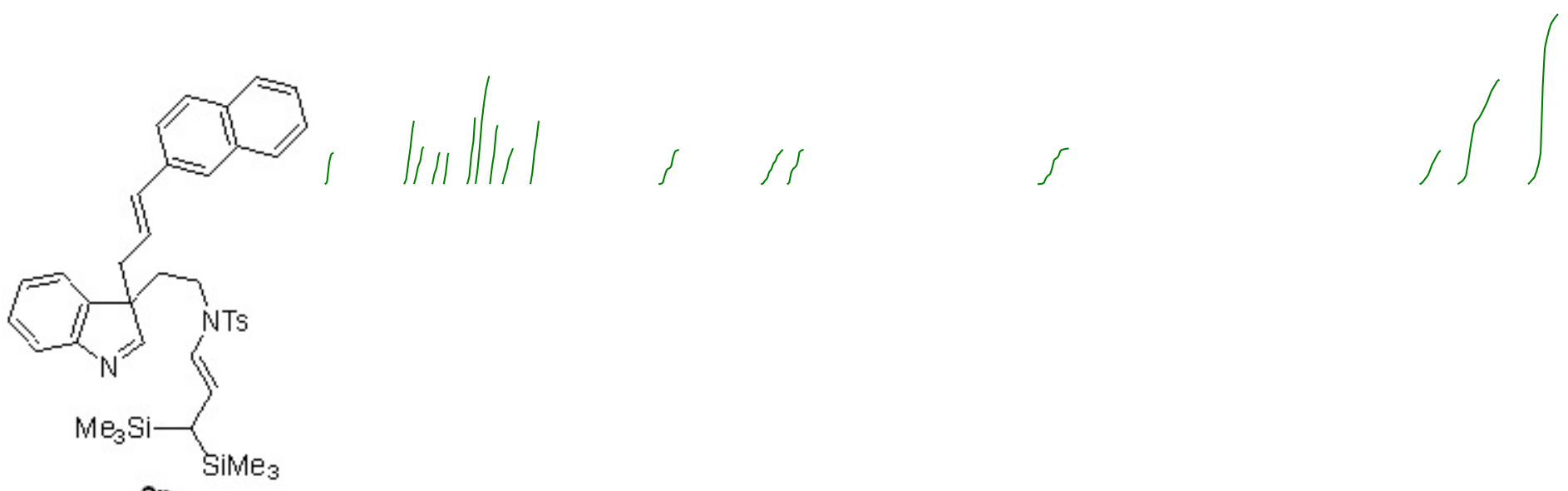

$2 p$

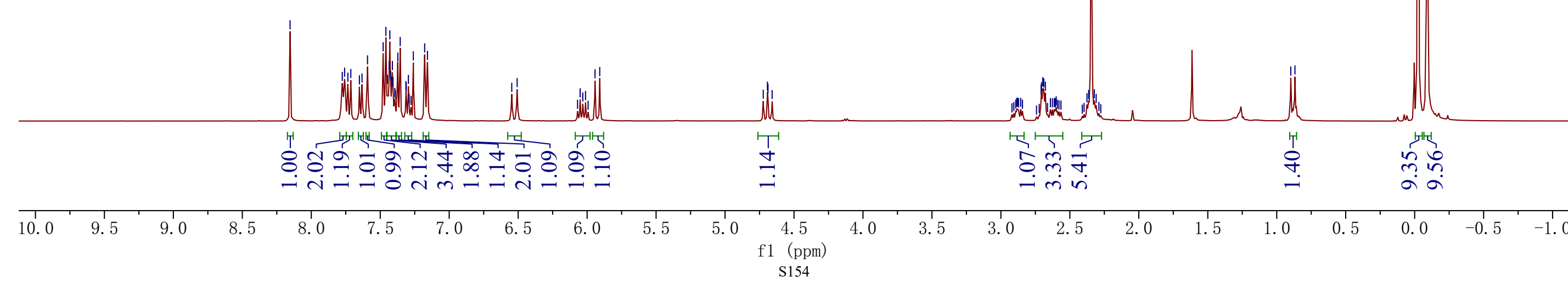




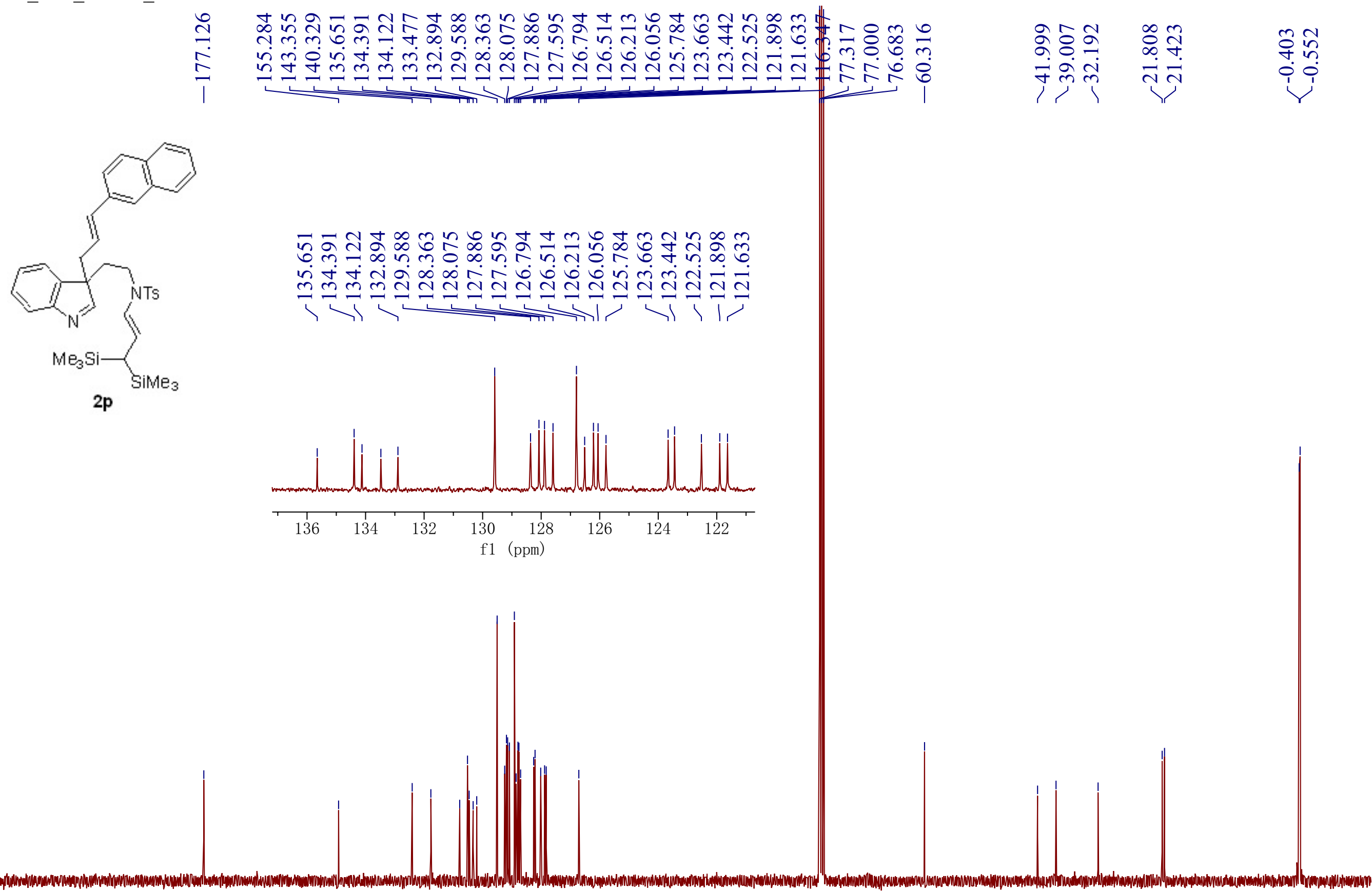



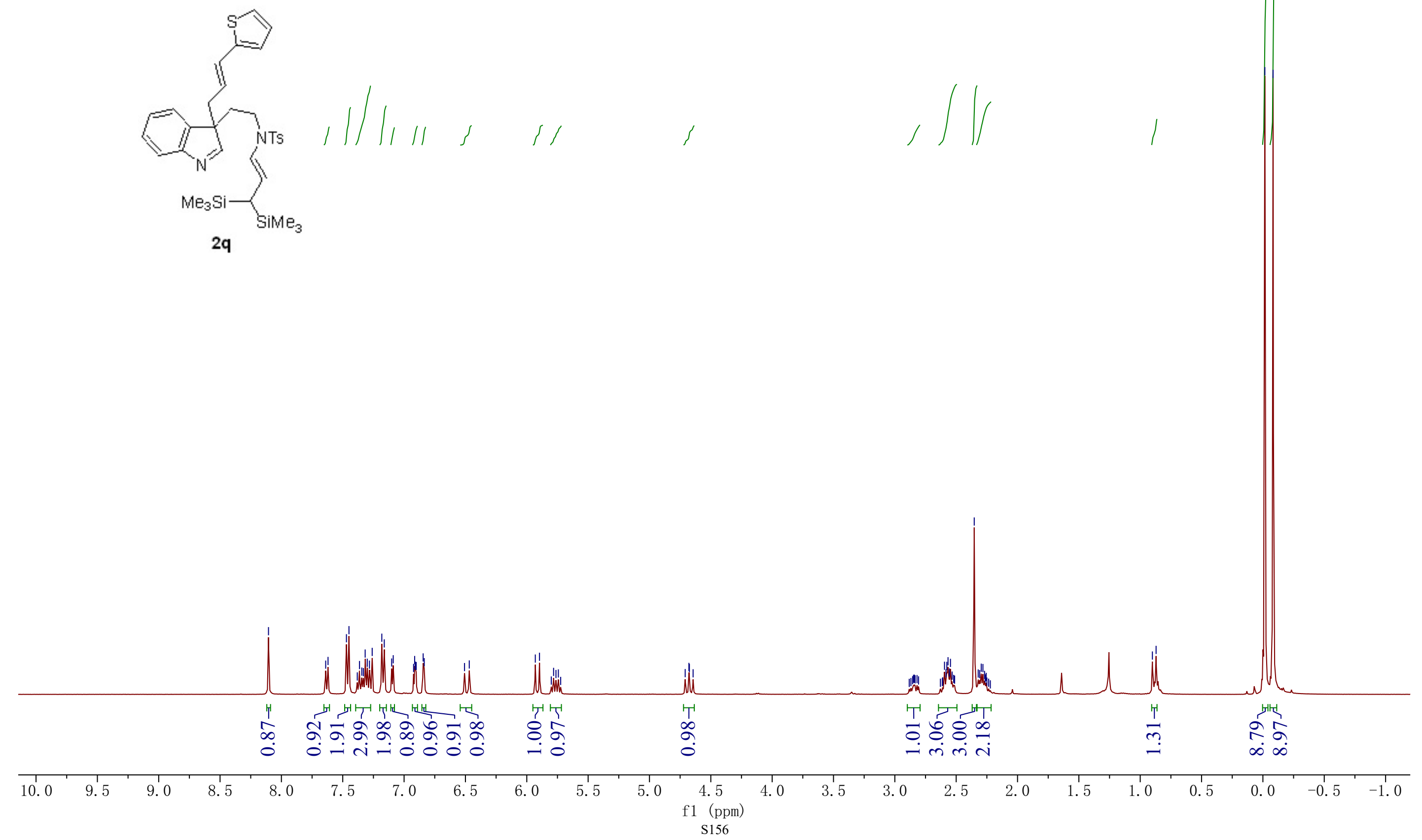


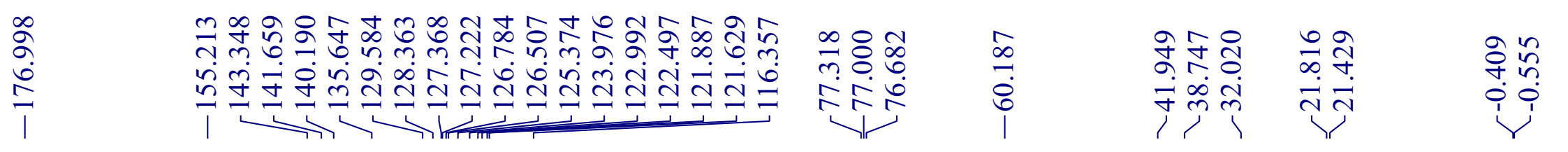
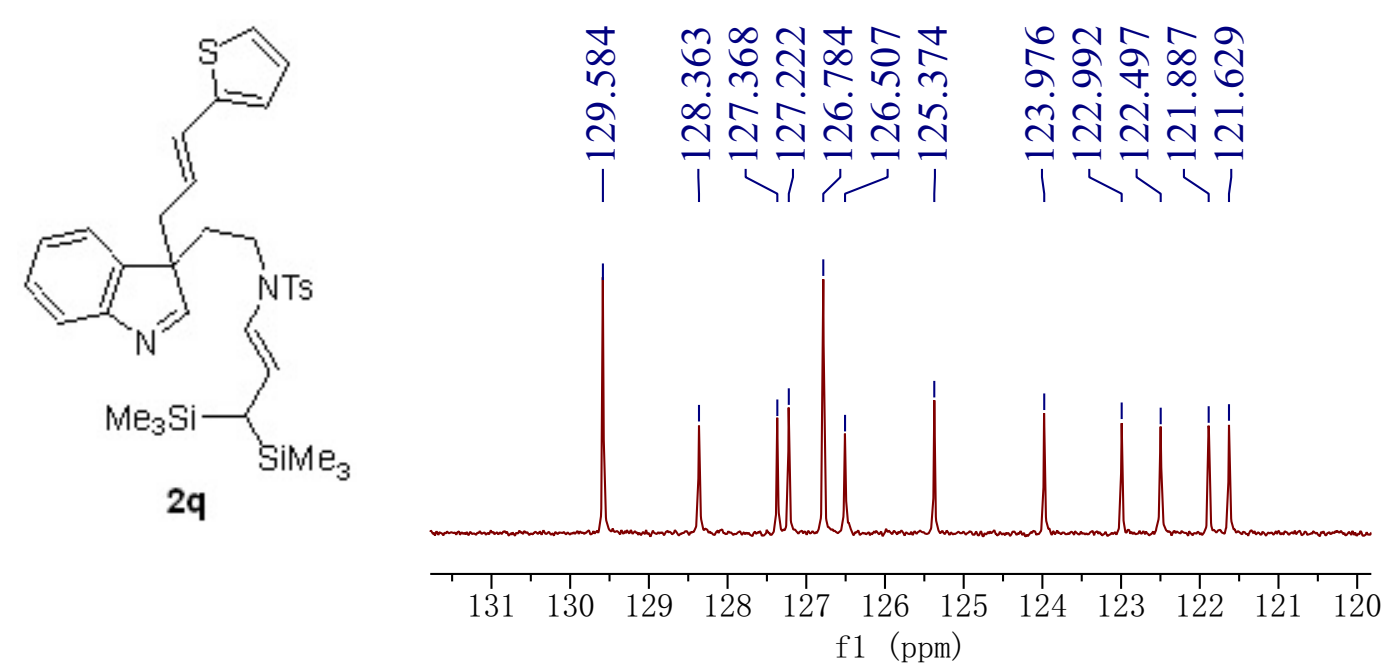

$2 q$

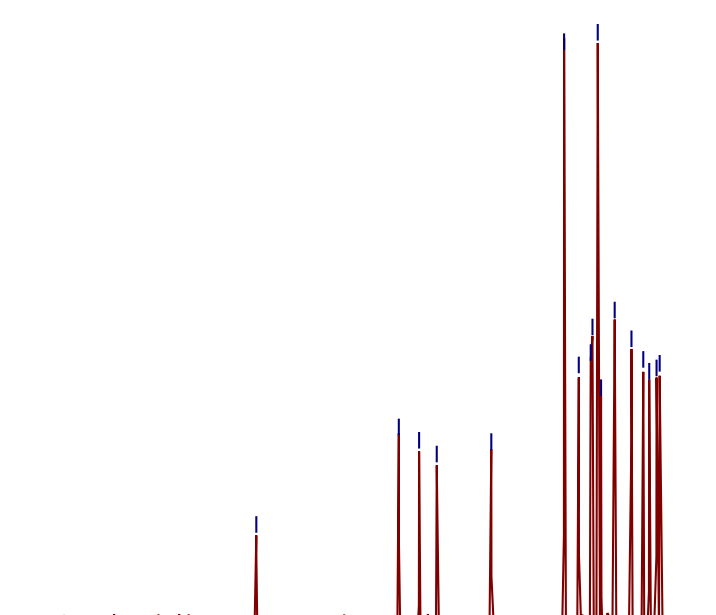



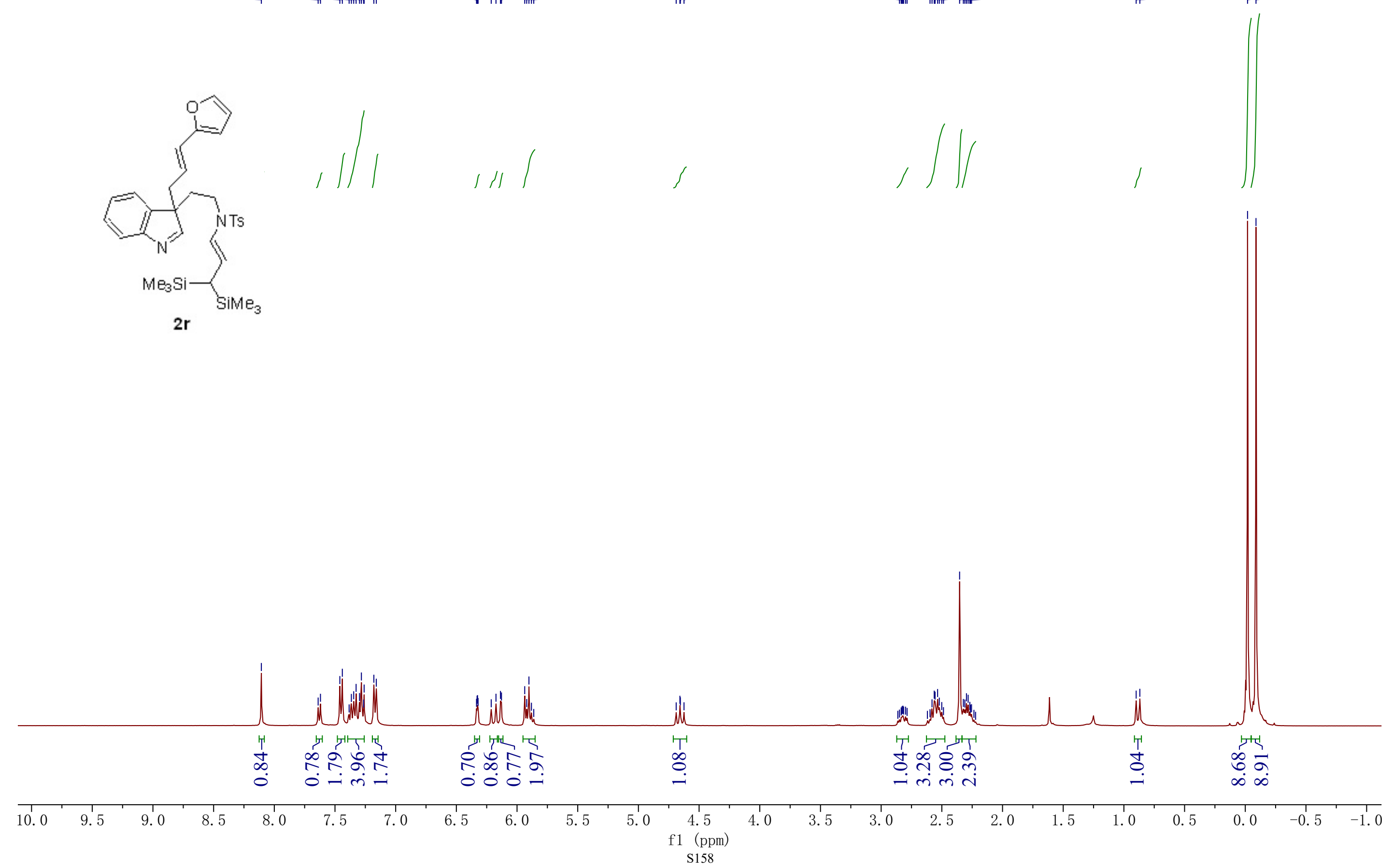


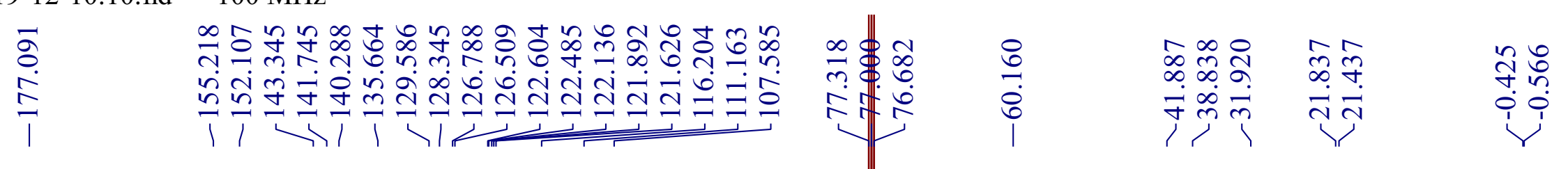
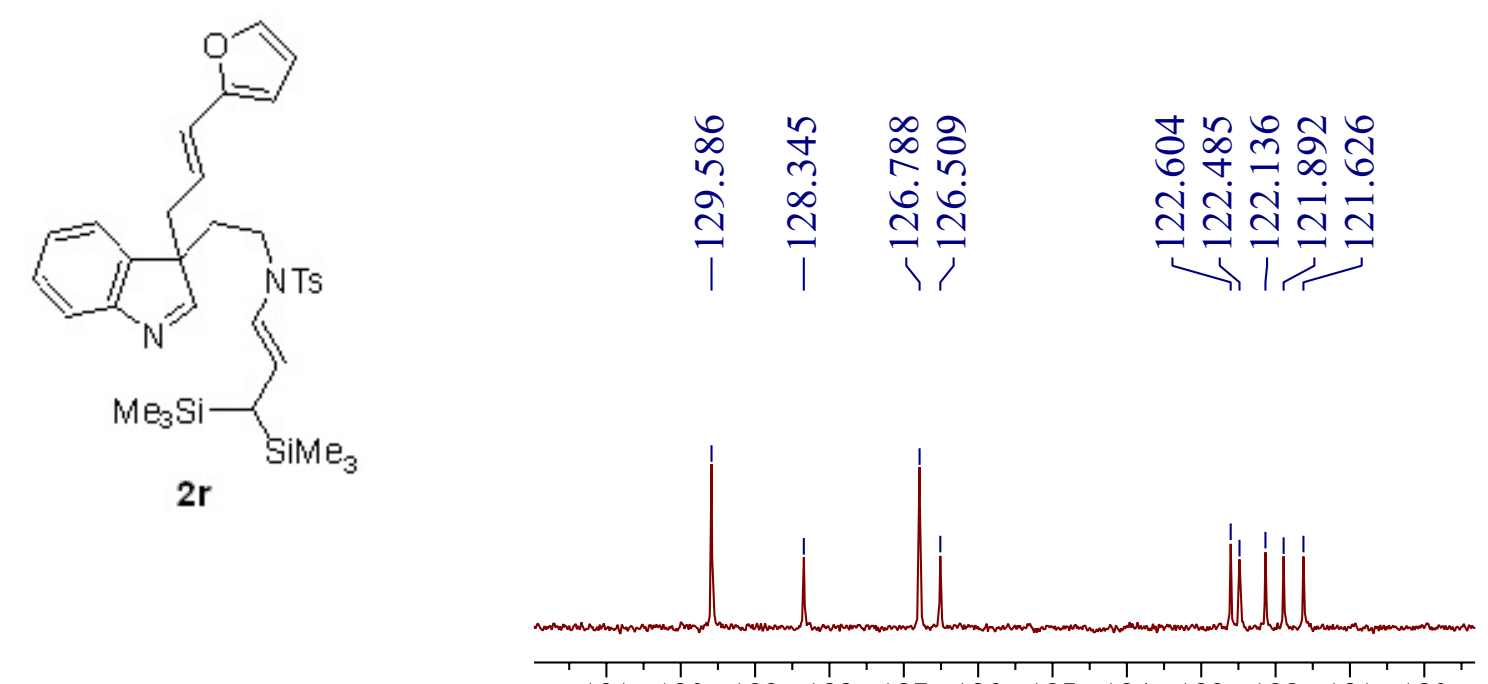

$\begin{array}{llllllllllll}131 & 130 & 129 & 128 & 127 & 126 & 125 & 124 & 123 & 122 & 121 & 120\end{array}$ f1 (ppm)

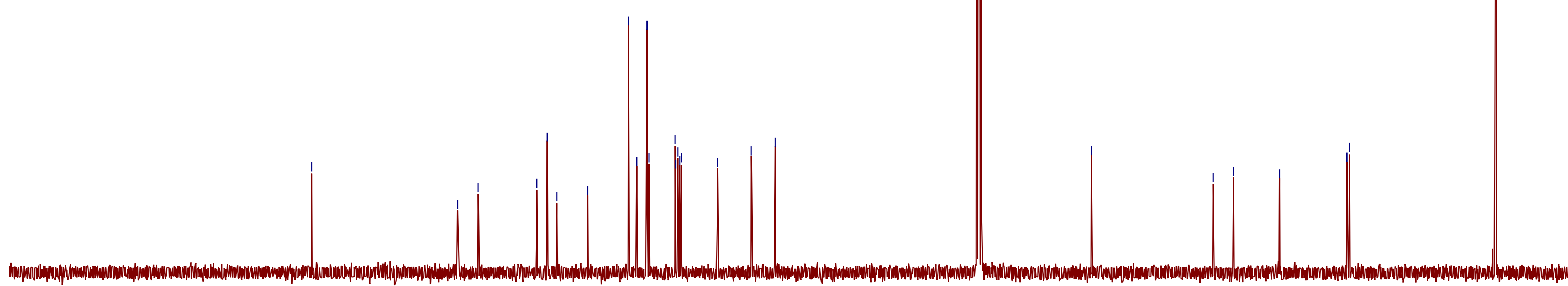




\section{CY-6-86-B3_H1_CDCl3_2019-12-25.30.fid - $400 \mathrm{MHz}$}

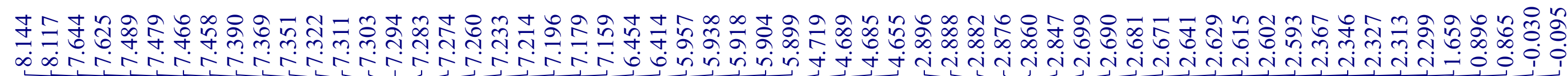
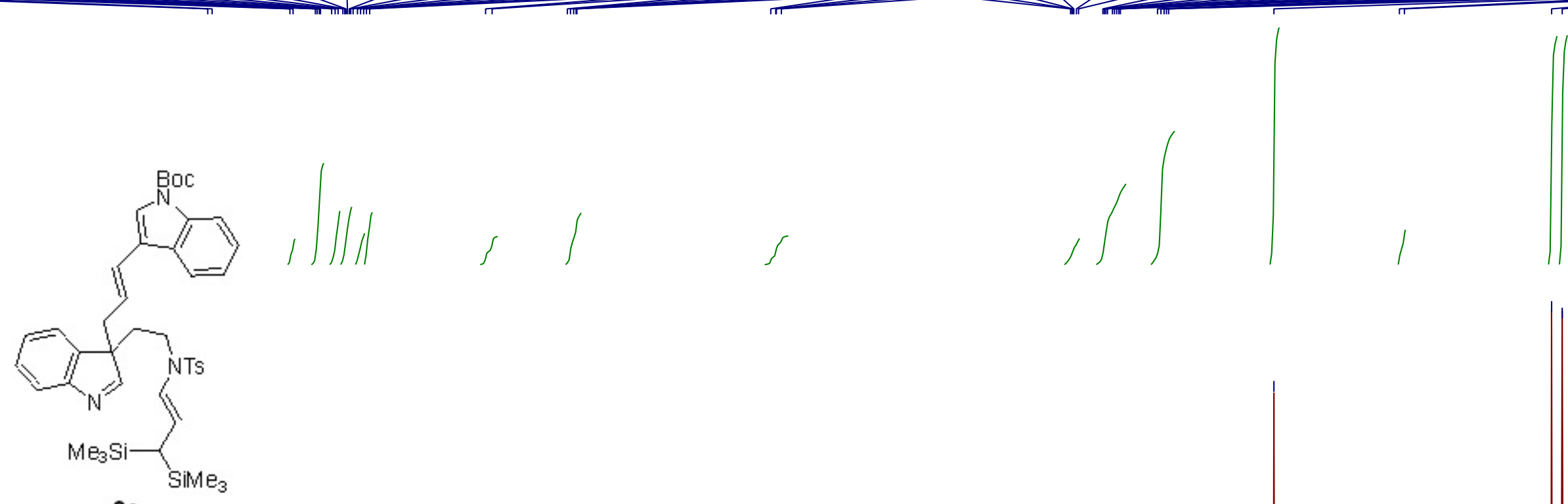

$2 s$

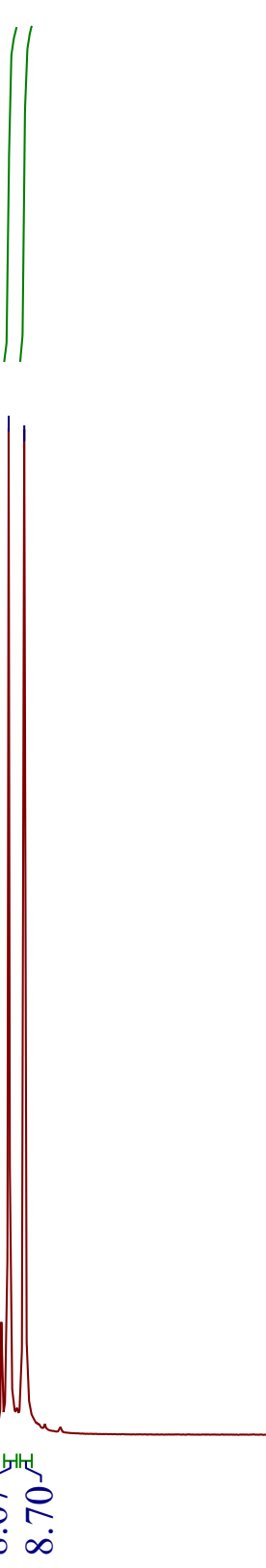

$\begin{array}{llll}10.0 & 9.5 & 9.0 & 8.5\end{array}$

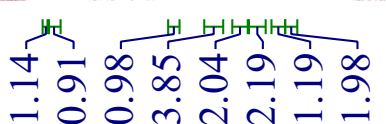

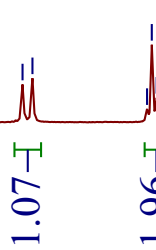

綟

(n)

管

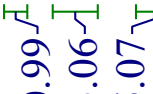

○!

$\stackrel{1}{\text { กิ }}$

6웅

8. 0

6.5

$6.0 \quad 5.5$

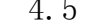

4. 0 3.

2. 0 1. 1.5

$1.0 \quad 0.5$

0.0 


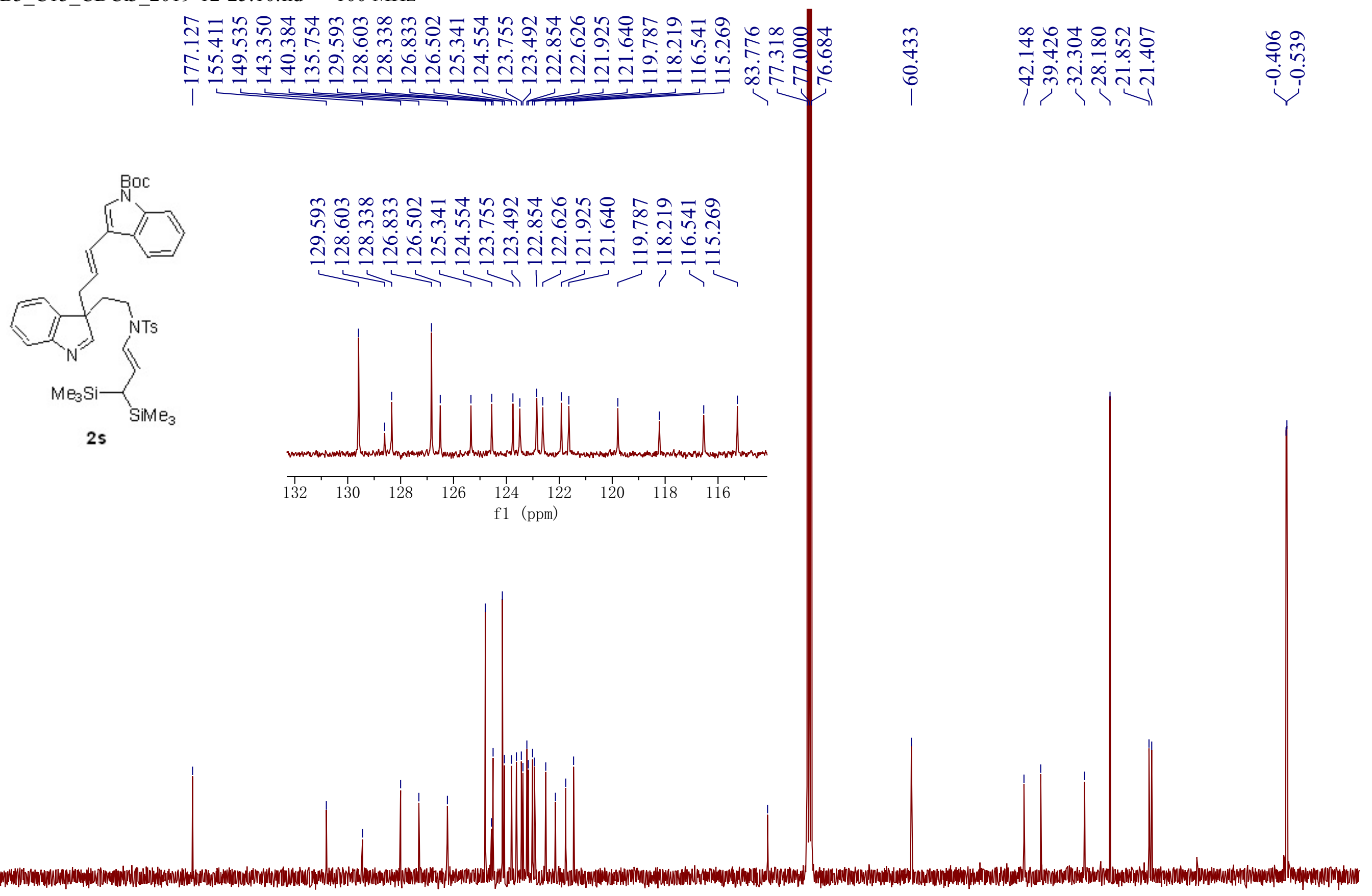



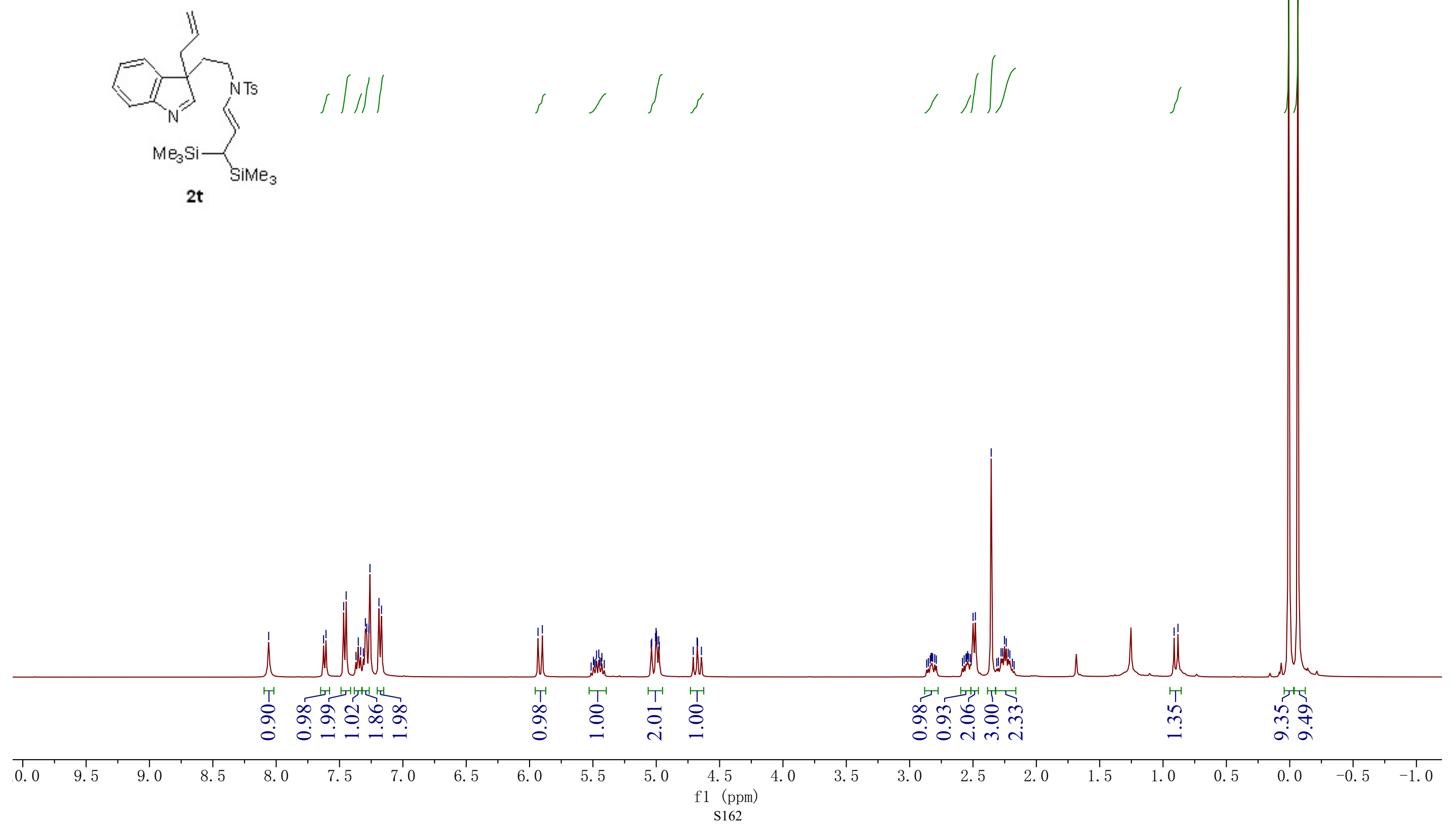

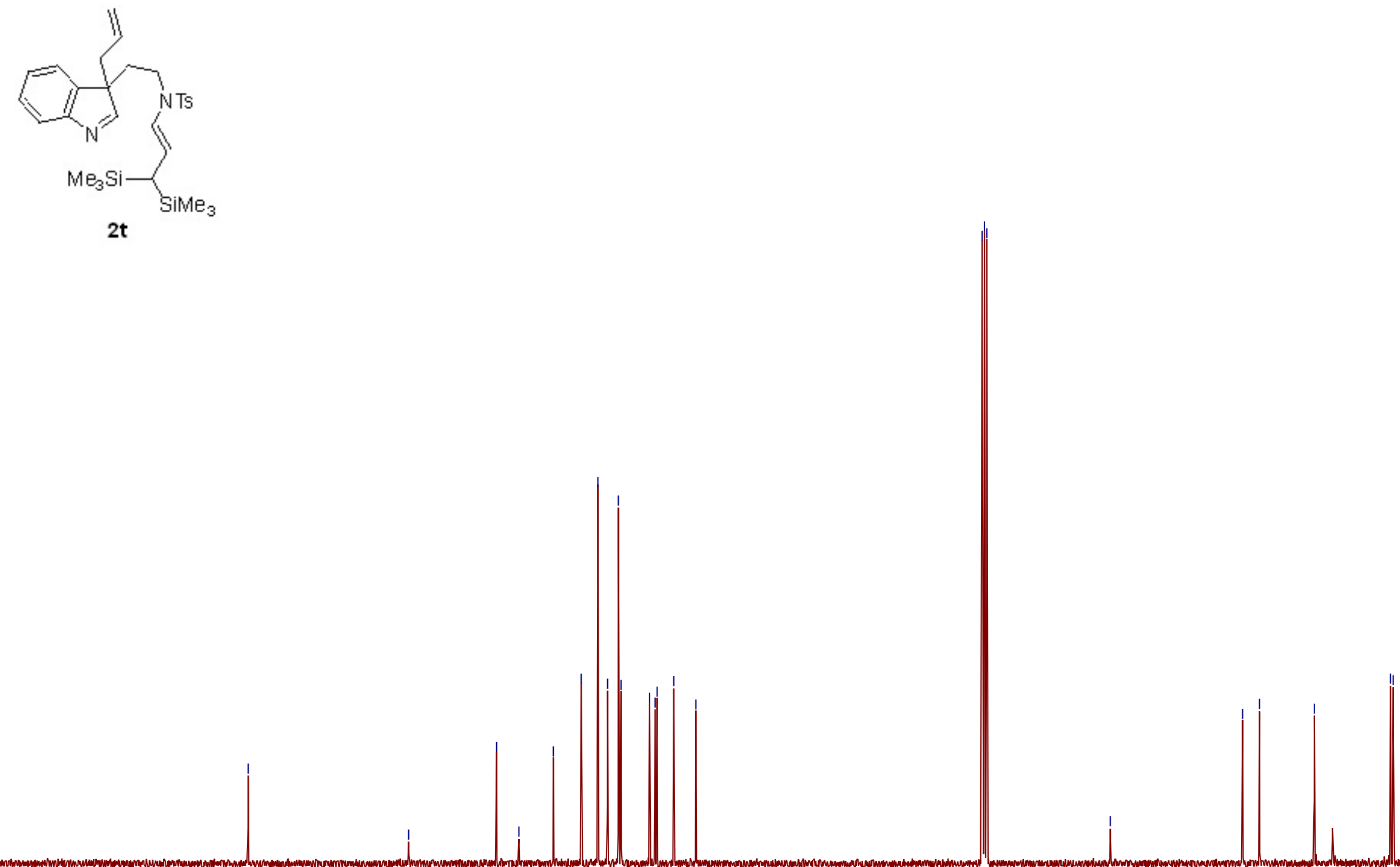


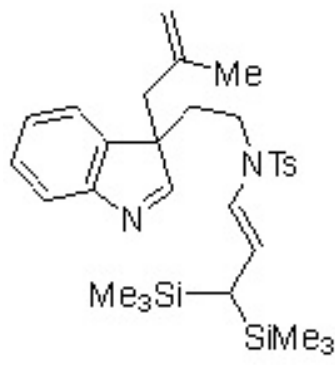

2u
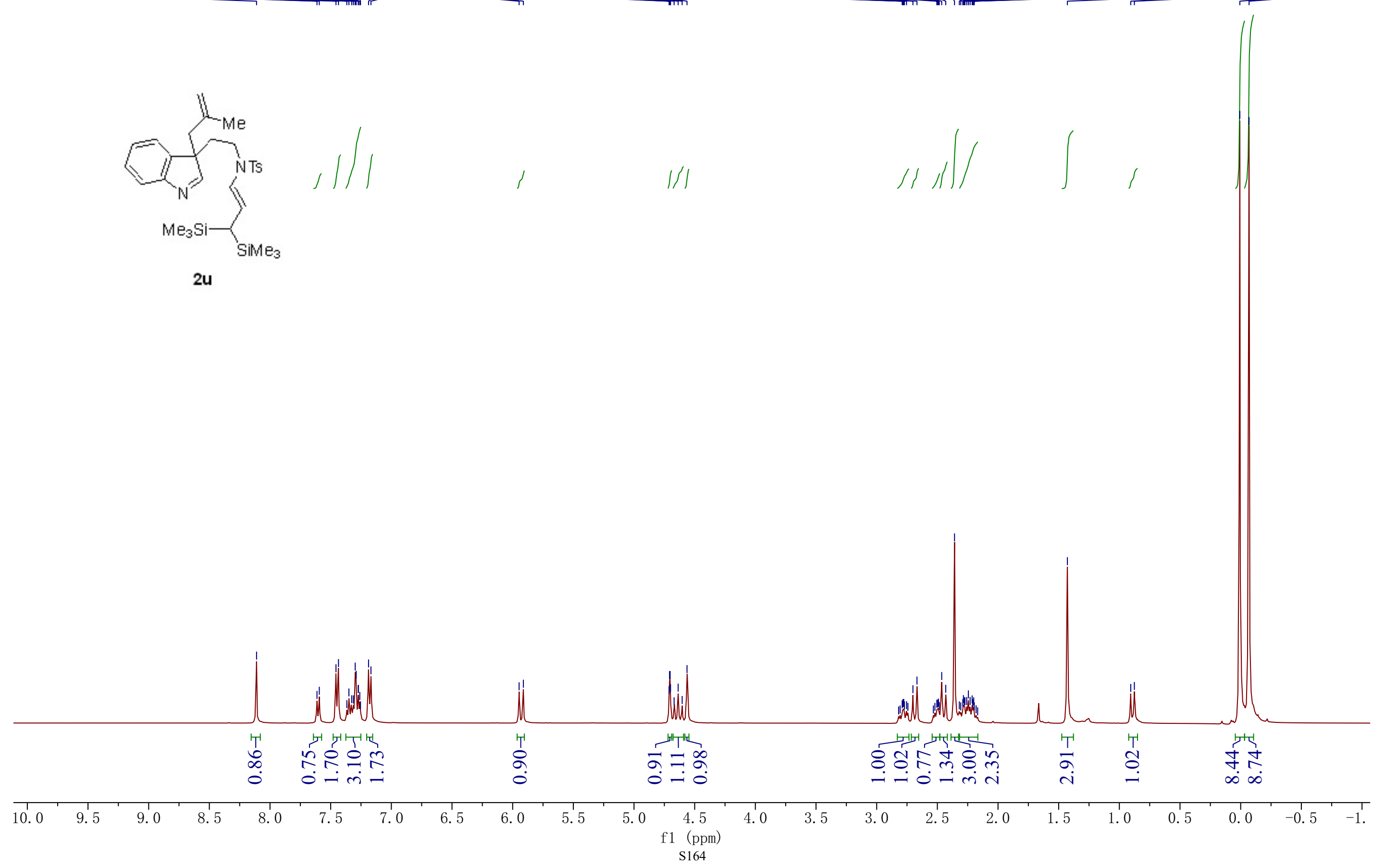


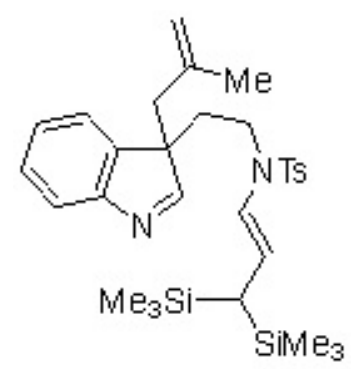

$2 \mathrm{u}$

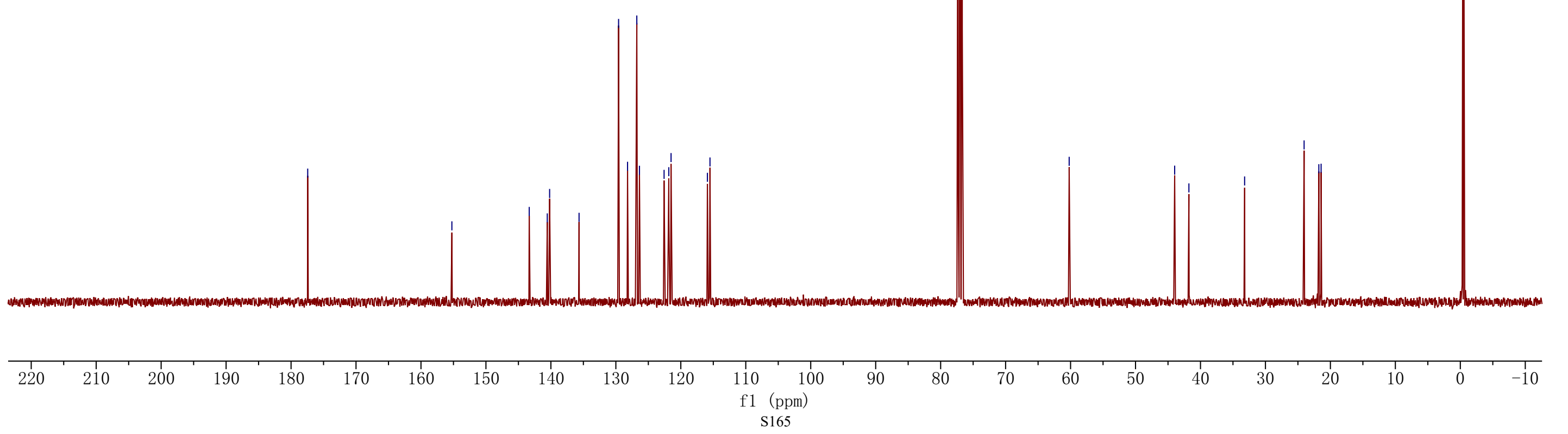



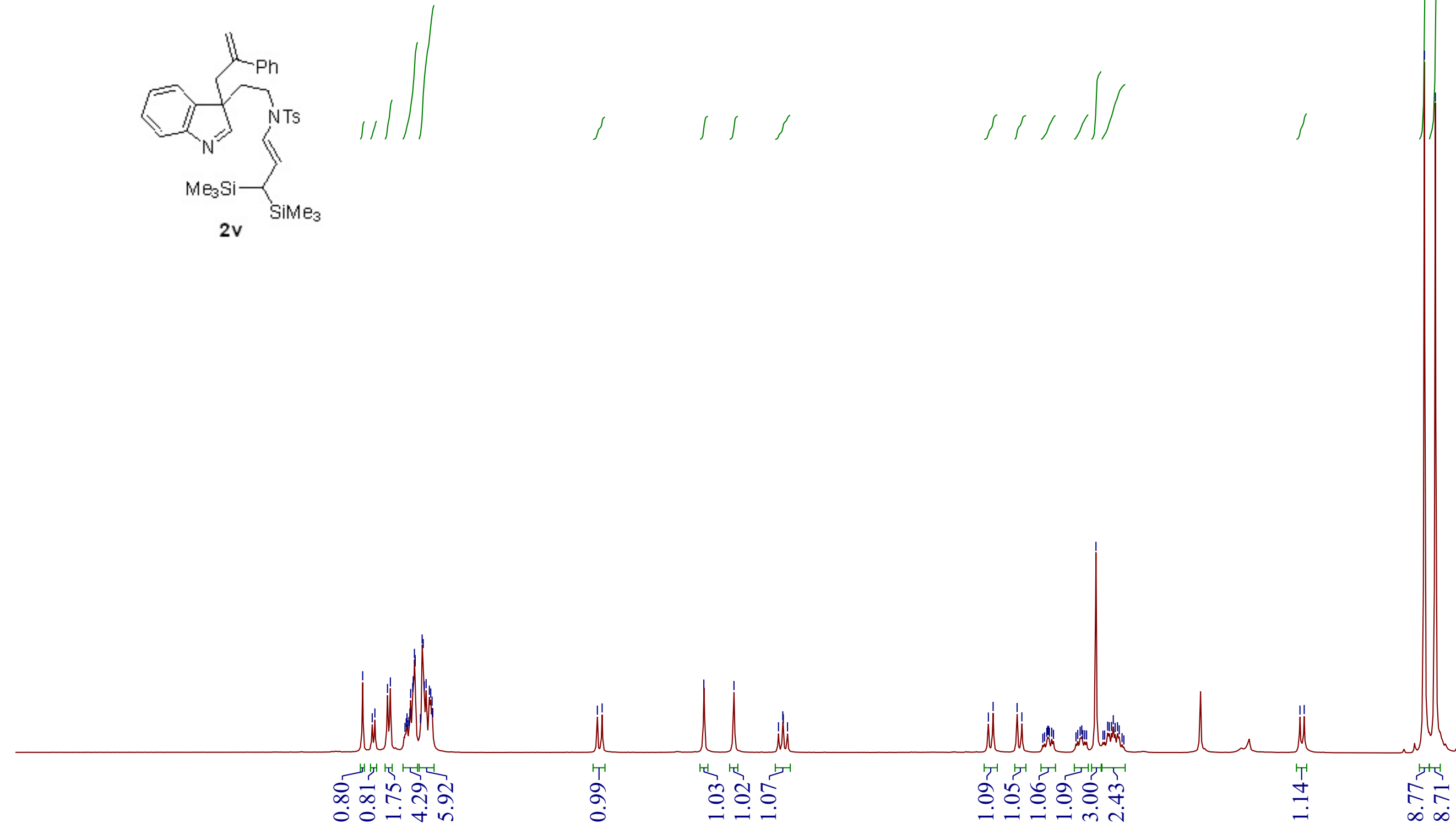

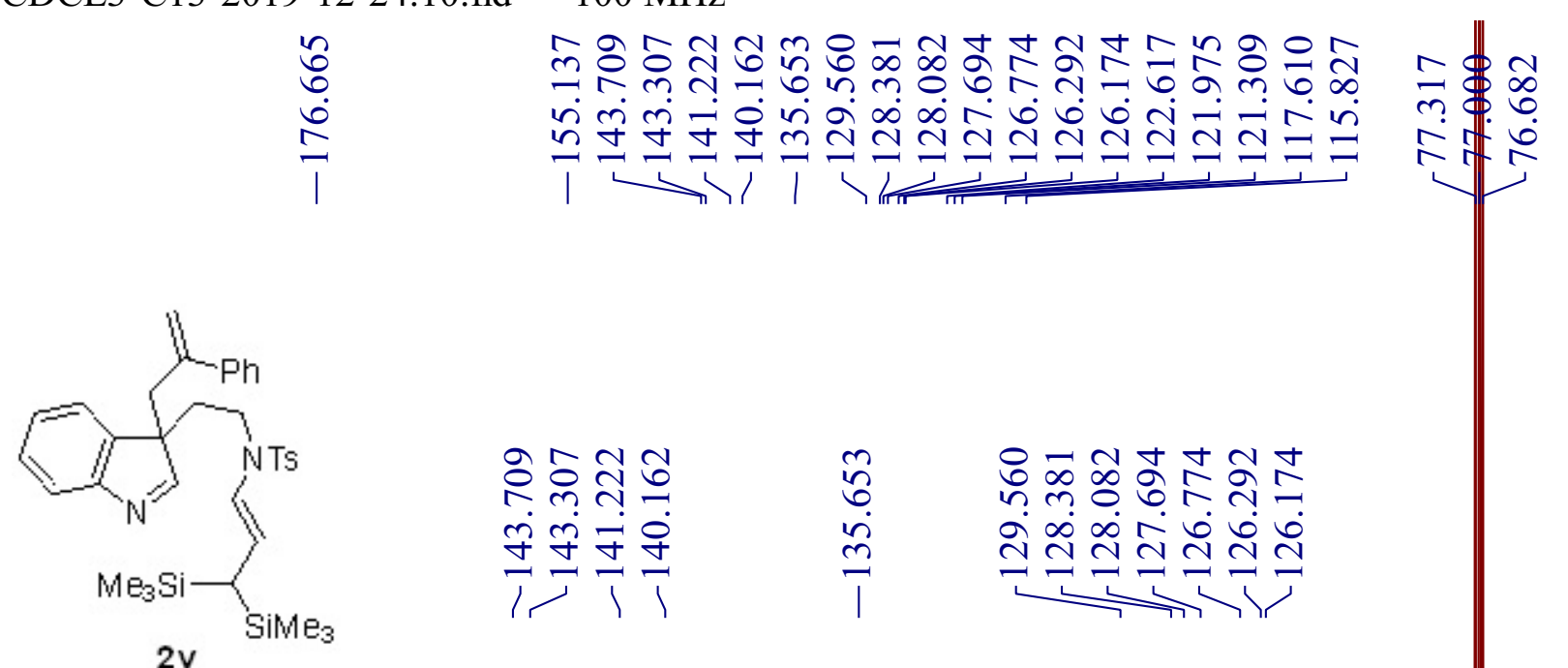

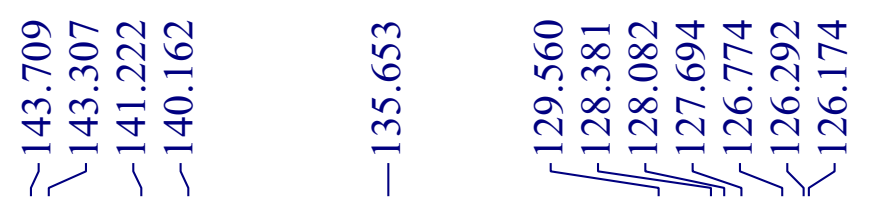
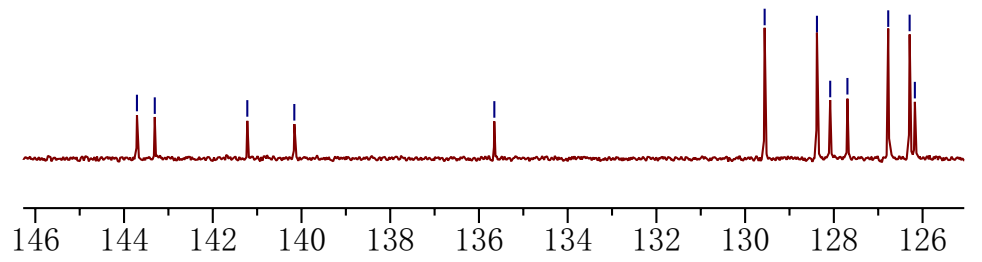

f1 (ppm)

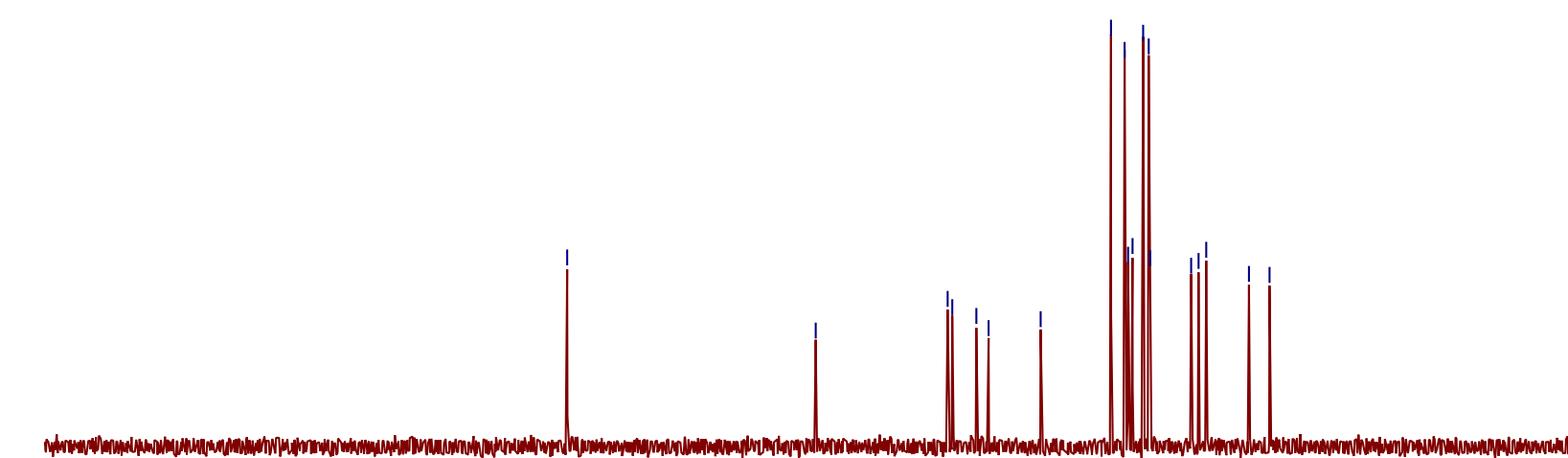



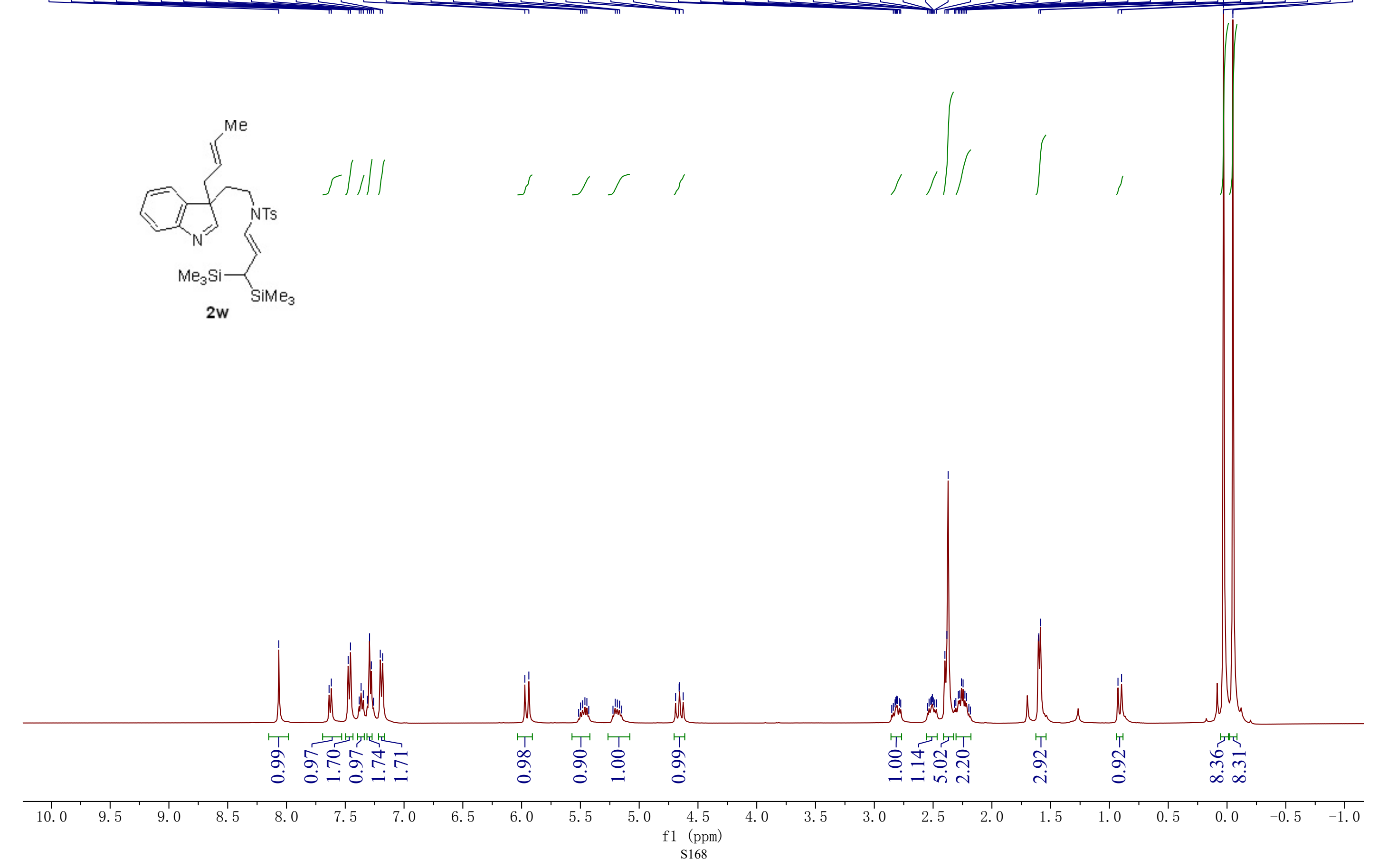


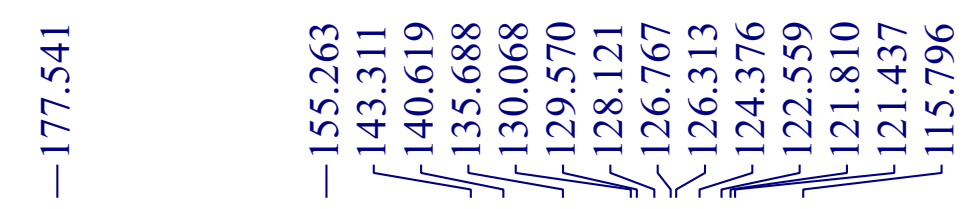

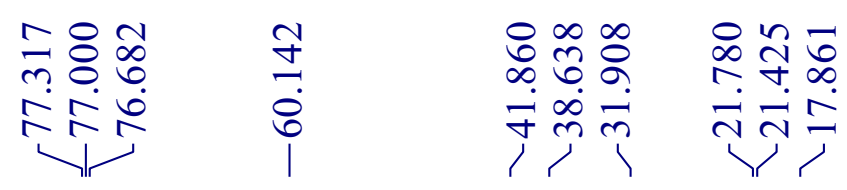

نn

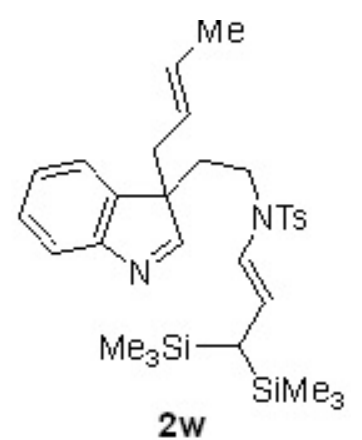

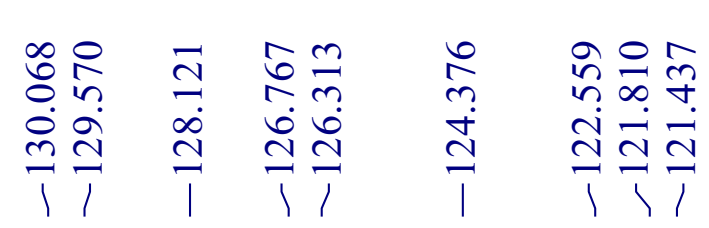

$2 w$

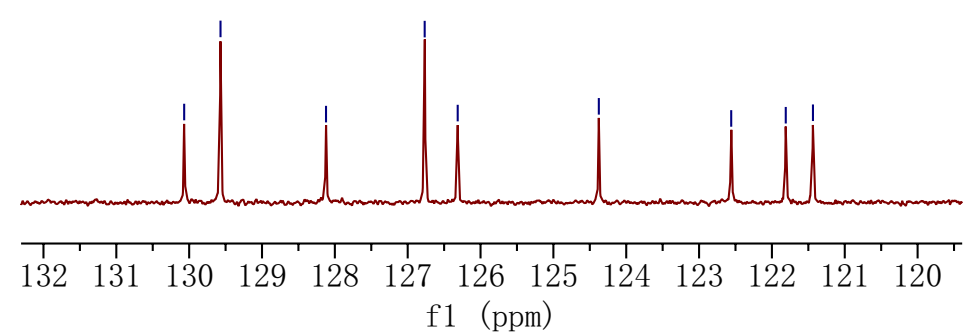

f1 (ppm)
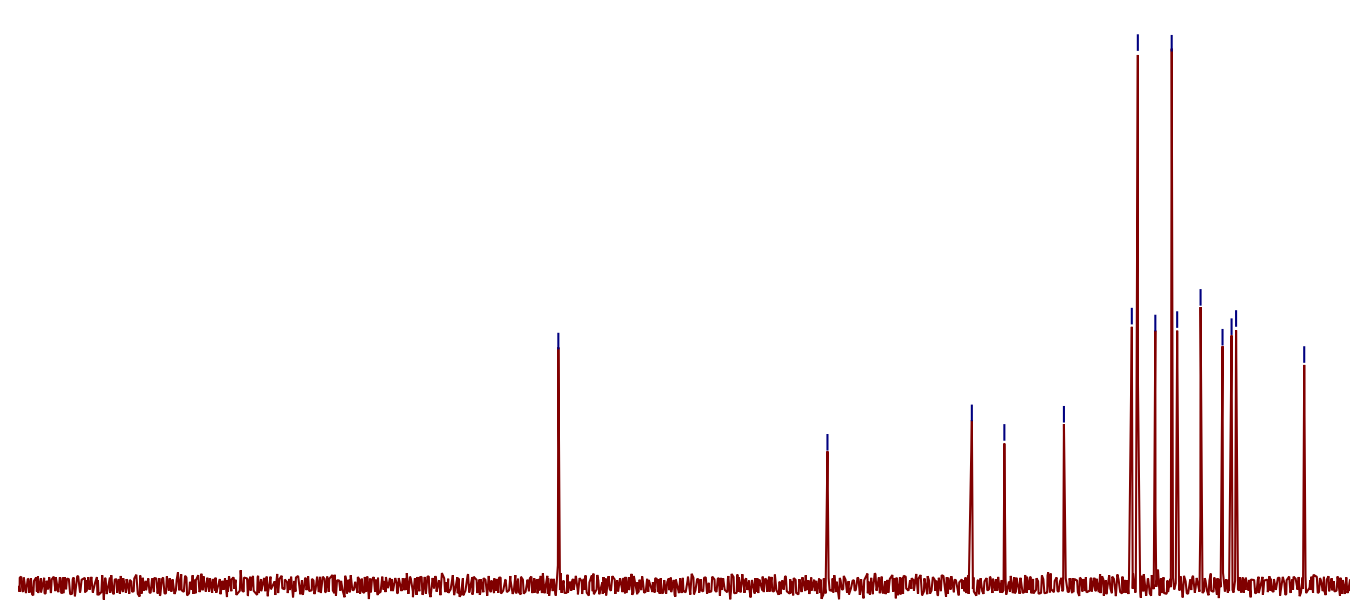

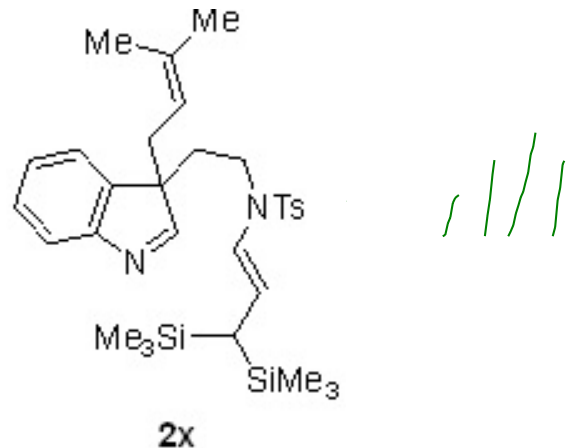

$2 x$

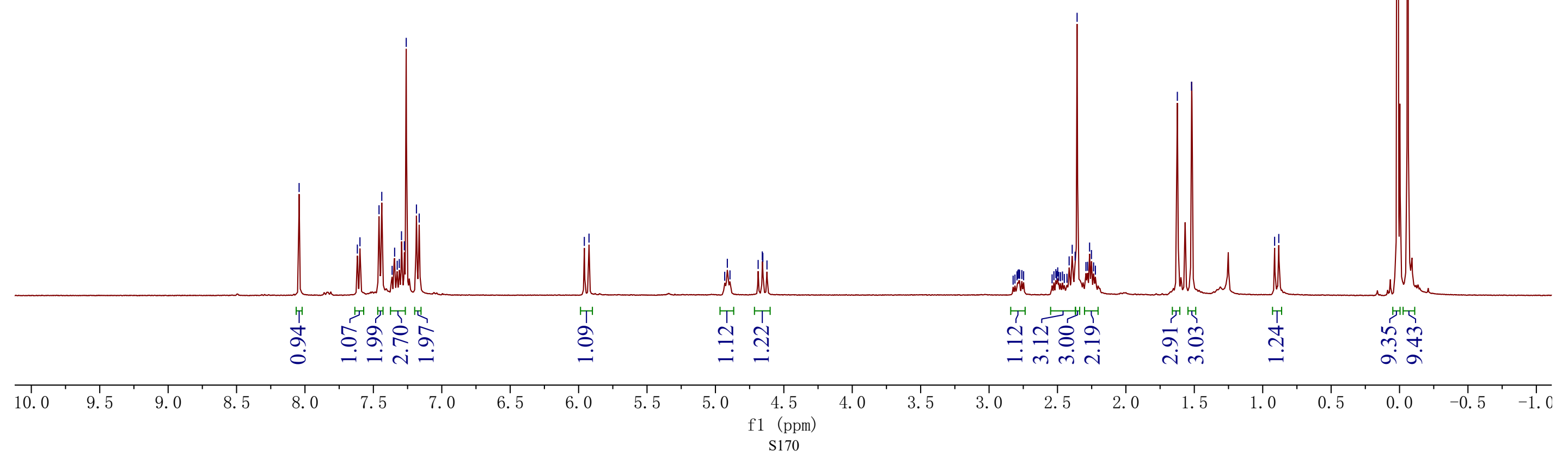



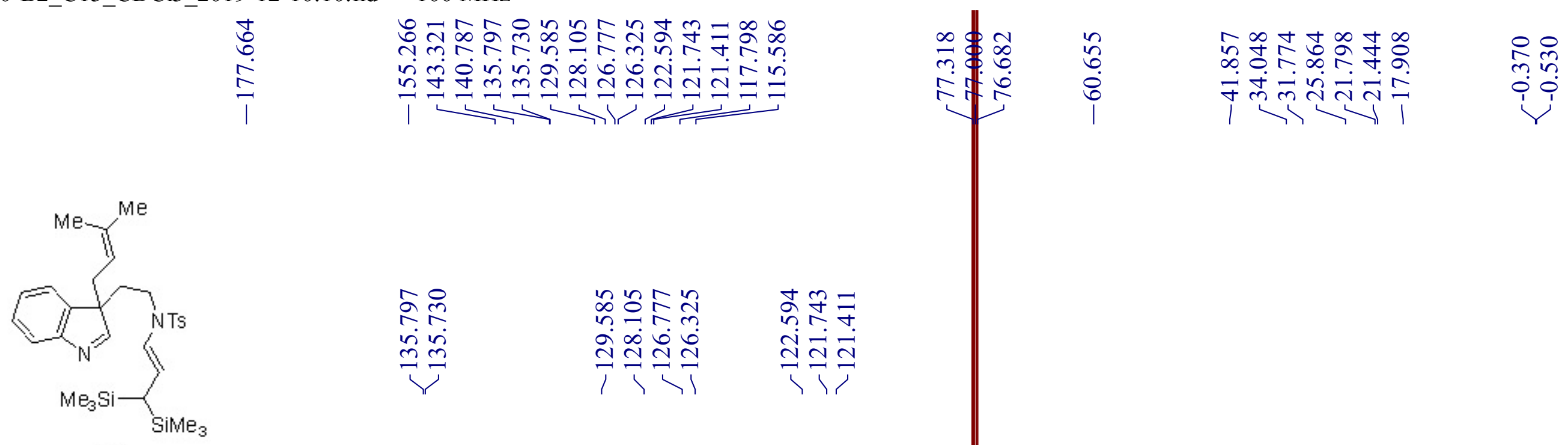

$2 x$
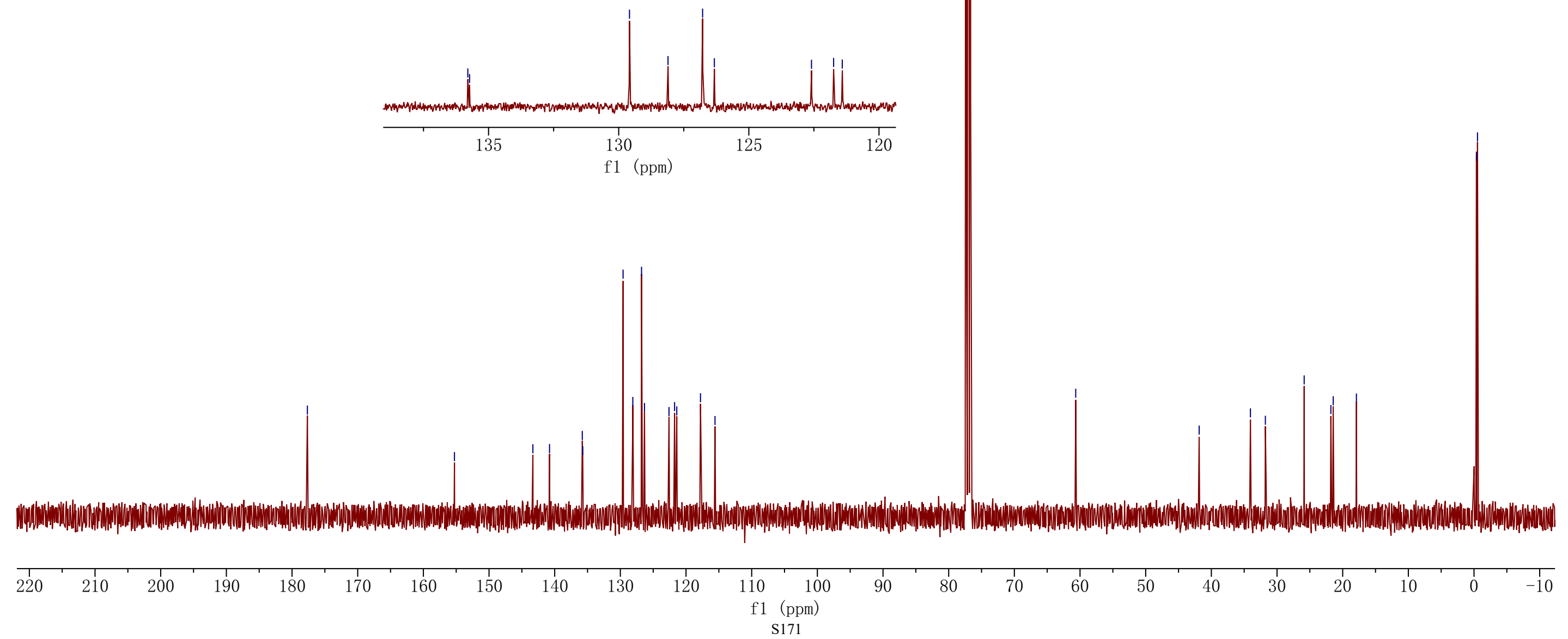

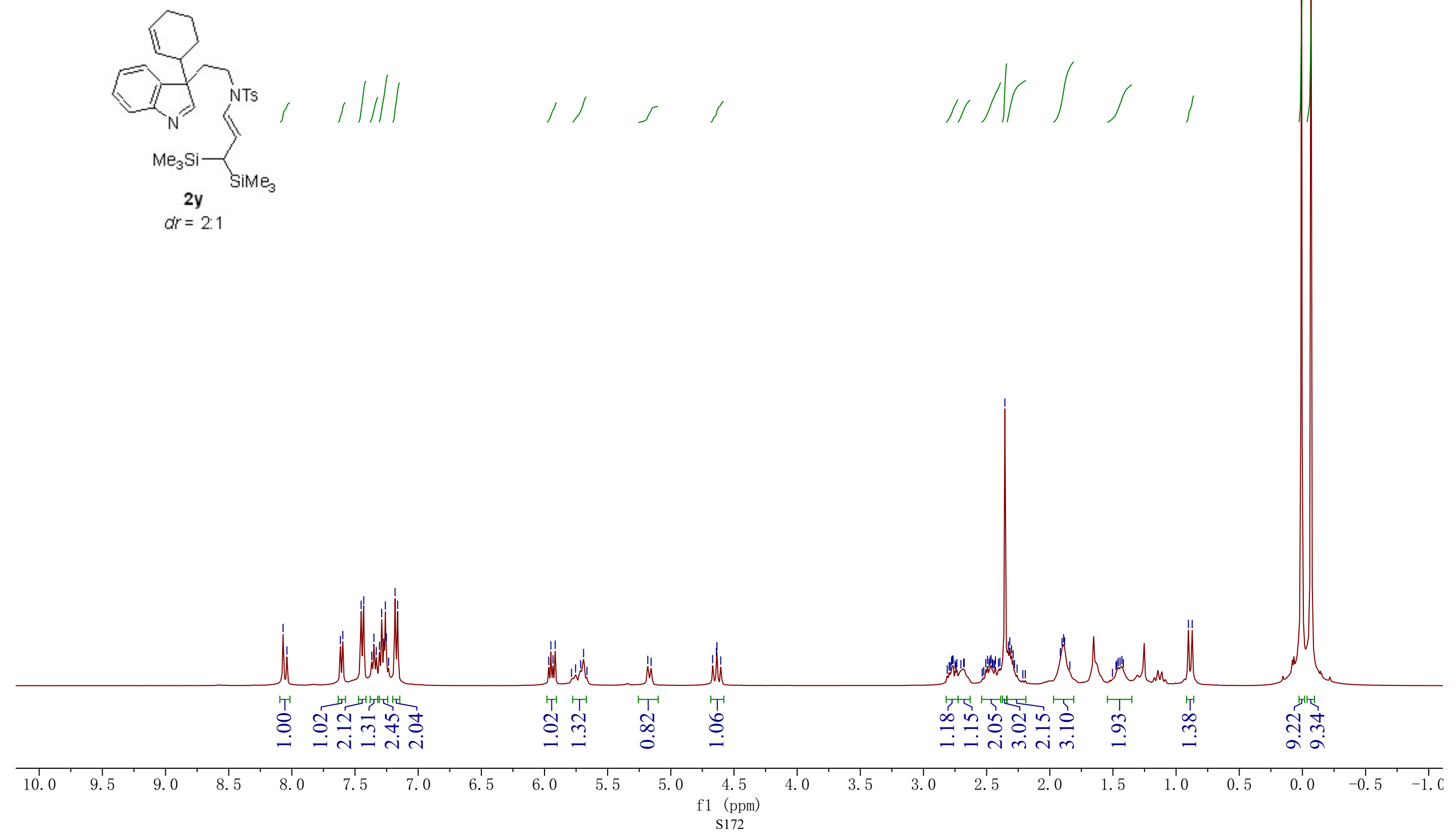


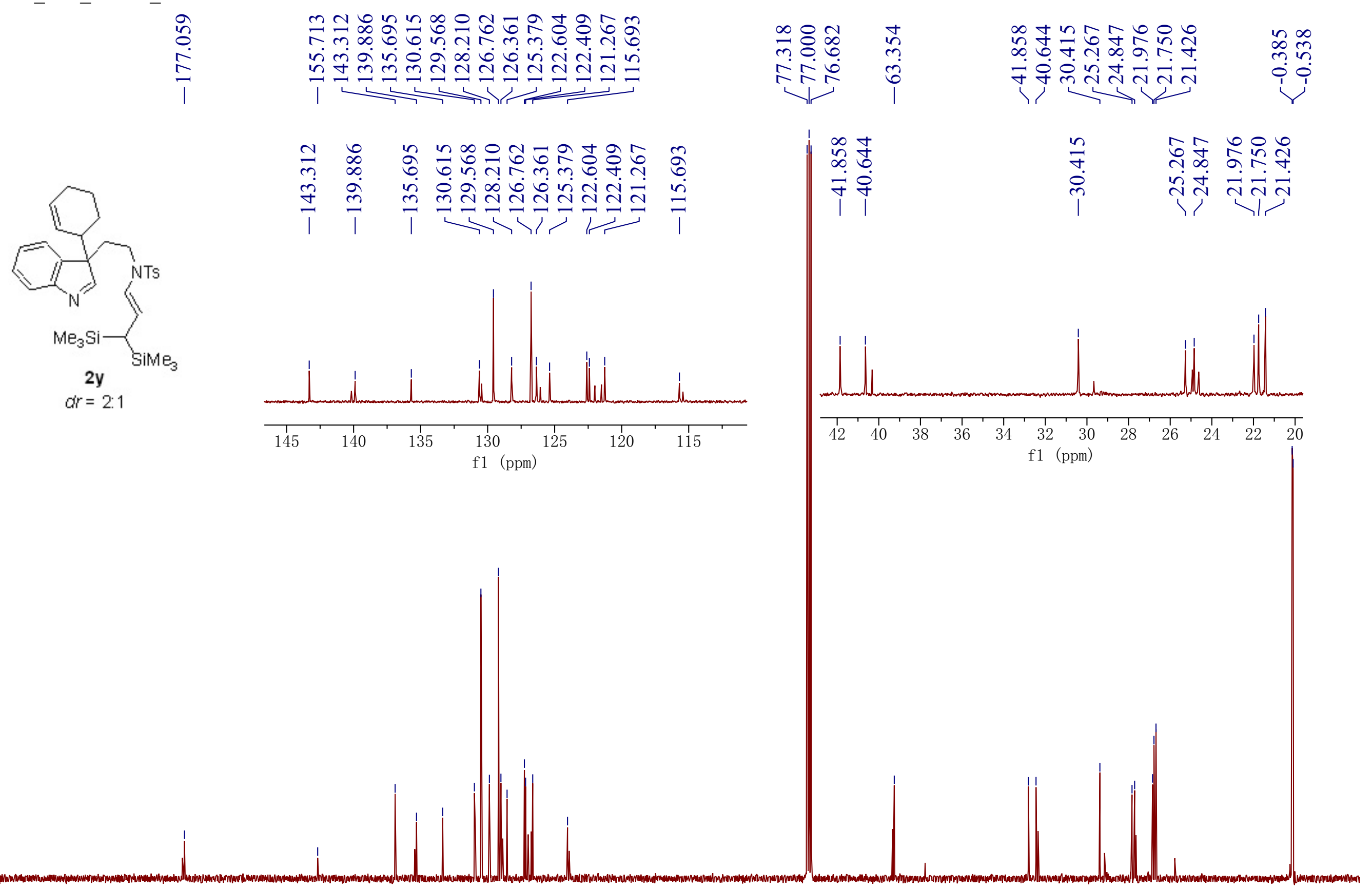



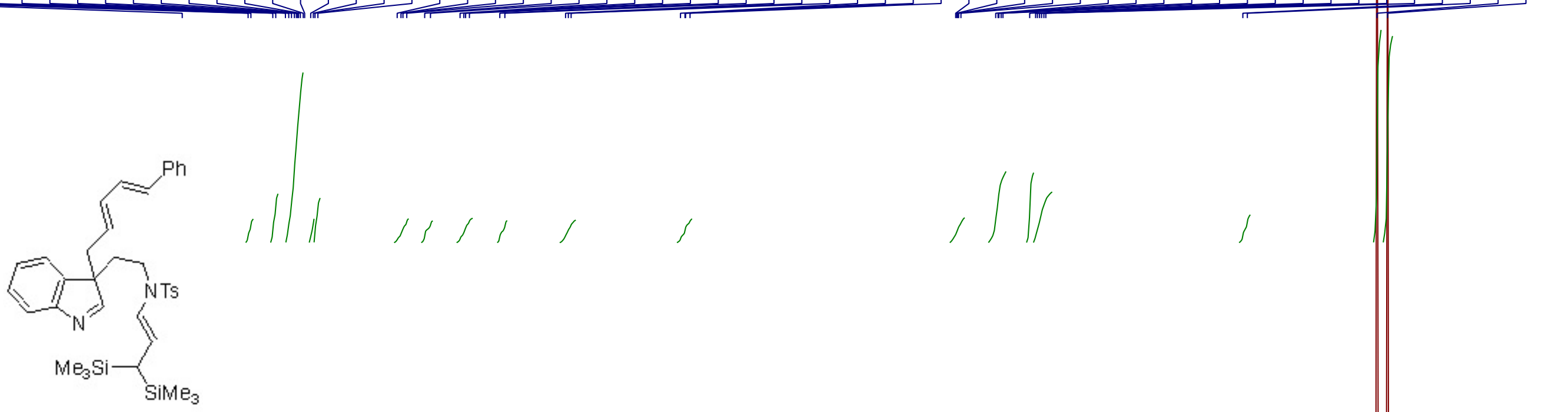

$2 z$
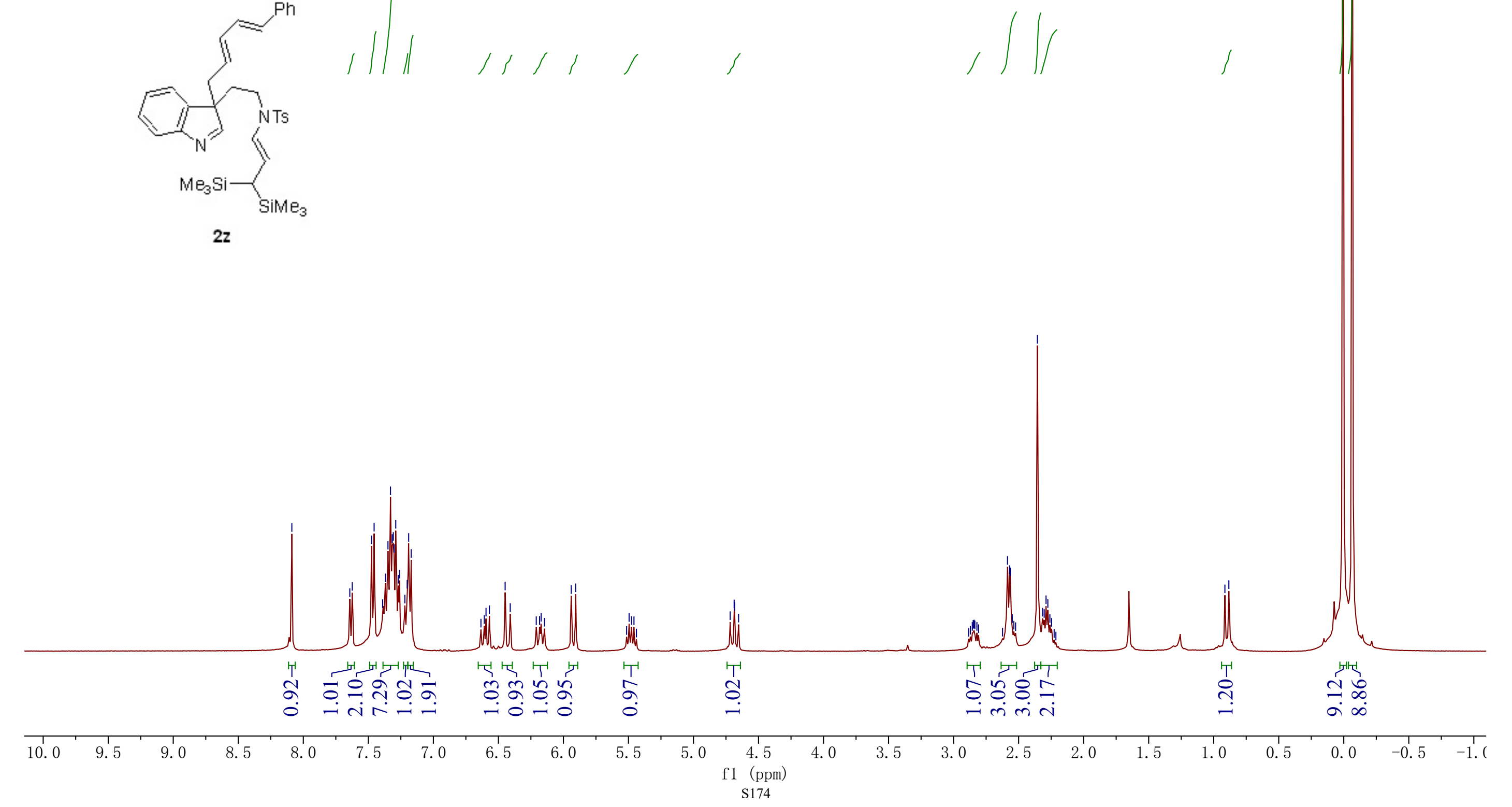


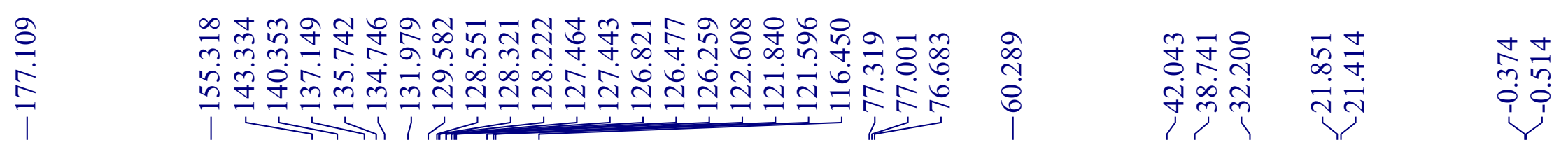

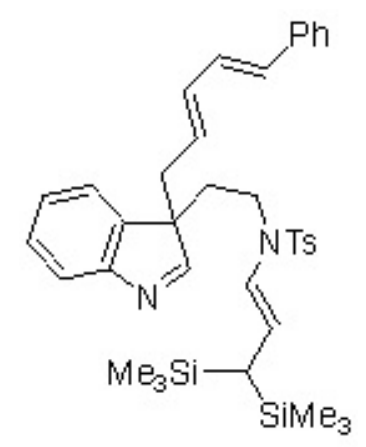

$2 z$
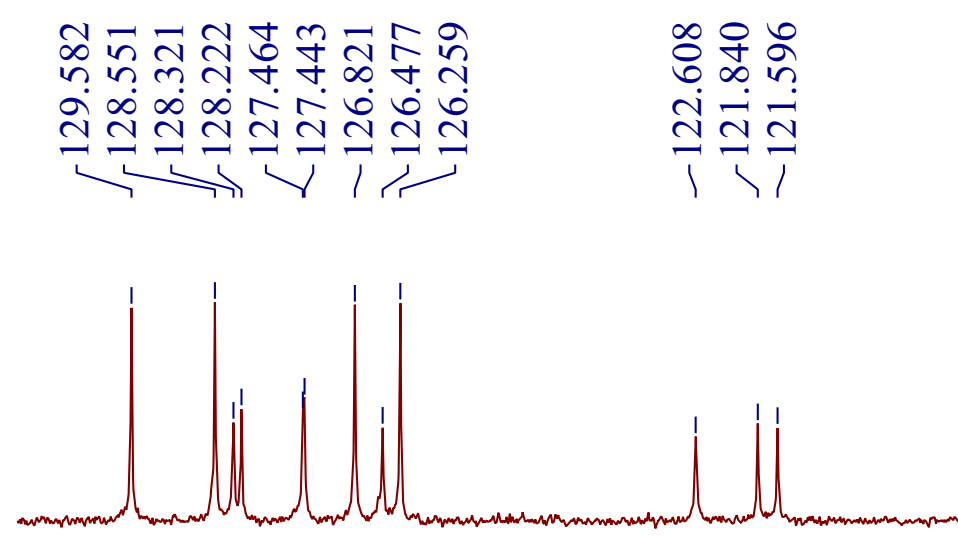

$\begin{array}{llllllllllll}130 & 129 & 128 & 127 & 126 & 125 & 124 & 123 & 122 & 121 & 120\end{array}$

f1 (ppm)
○ \

$\sqrt{2} \sqrt{1}$

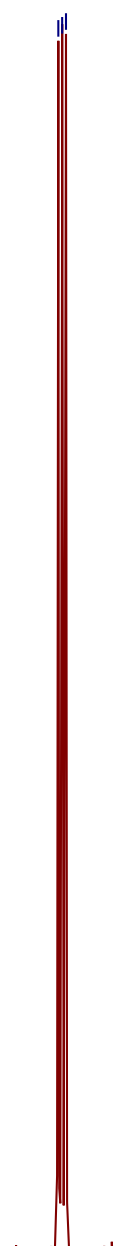

\begin{tabular}{|c|c|c|c|c|c|c|c|c|c|c|c|c|c|c|c|c|c|c|c|c|c|c|}
\hline $\begin{array}{r}1 \\
220\end{array}$ & 210 & 200 & $\begin{array}{l}19 \\
190\end{array}$ & 180 & 170 & 160 & 150 & 140 & 130 & 120 & $\begin{array}{c}110 \quad 100 \\
\mathrm{f} 1 \quad \text { (ppm) } \\
\text { S175 }\end{array}$ & $\begin{array}{l}1 \\
90\end{array}$ & 80 & $\begin{array}{l}1 \\
70\end{array}$ & 60 & 50 & 40 & 30 & 20 & 10 & 0 & $-1 c$ \\
\hline
\end{tabular}



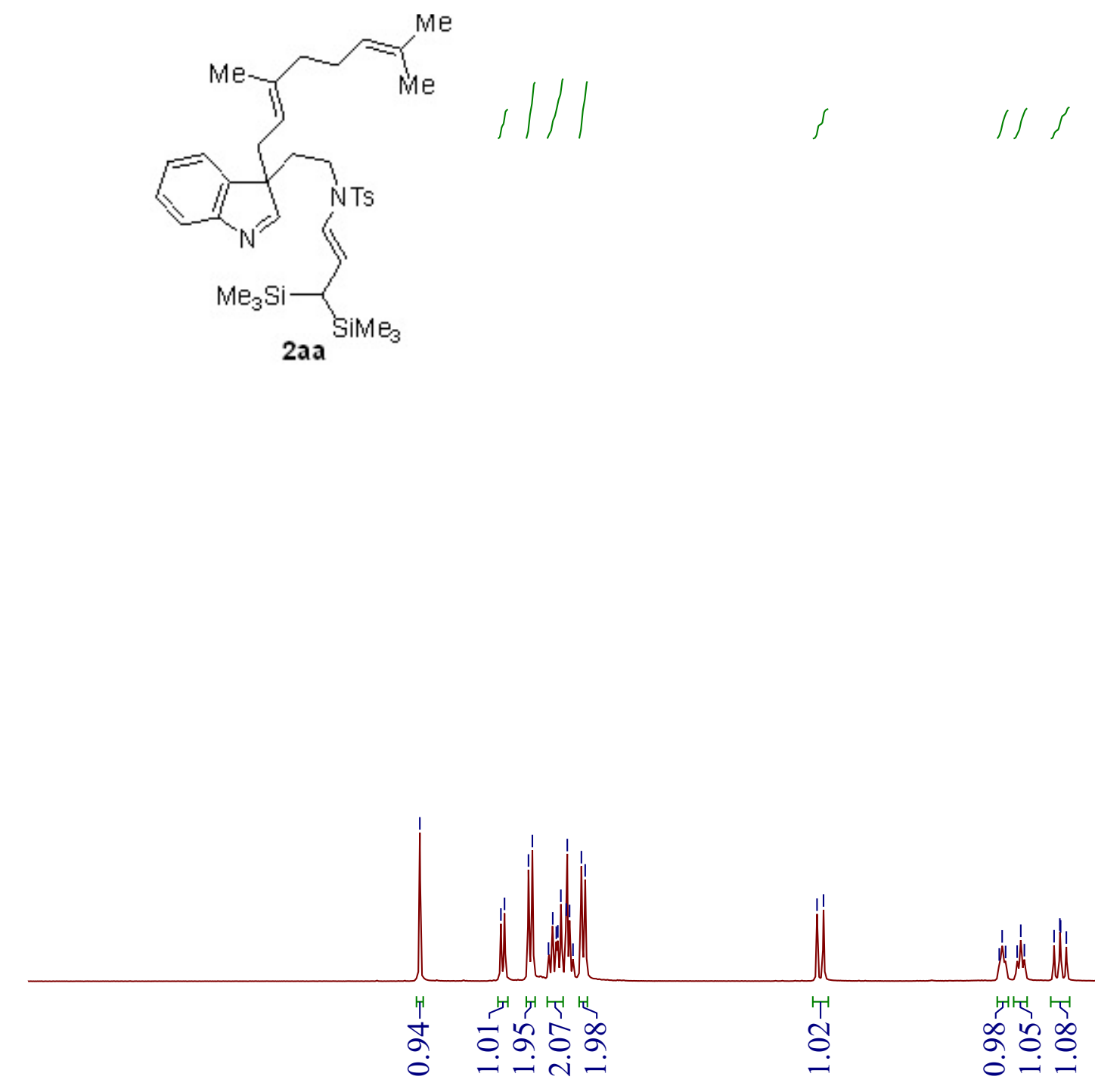

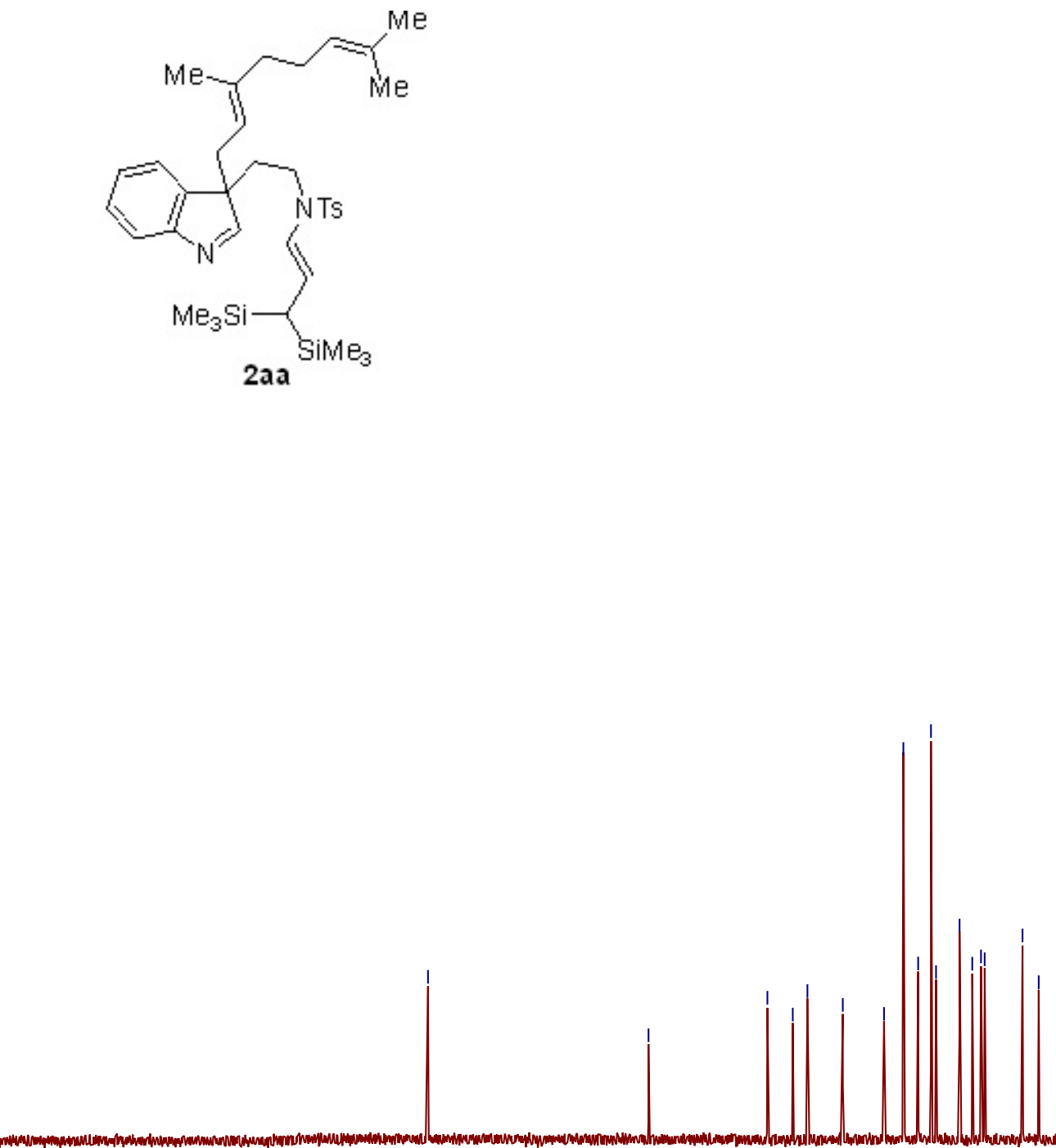


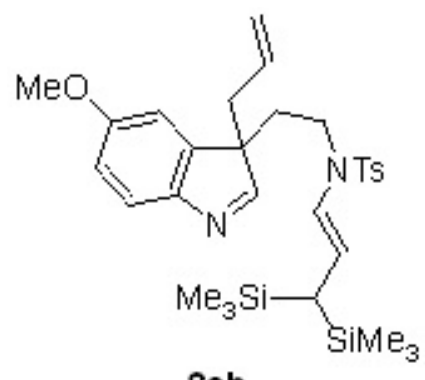

2ab

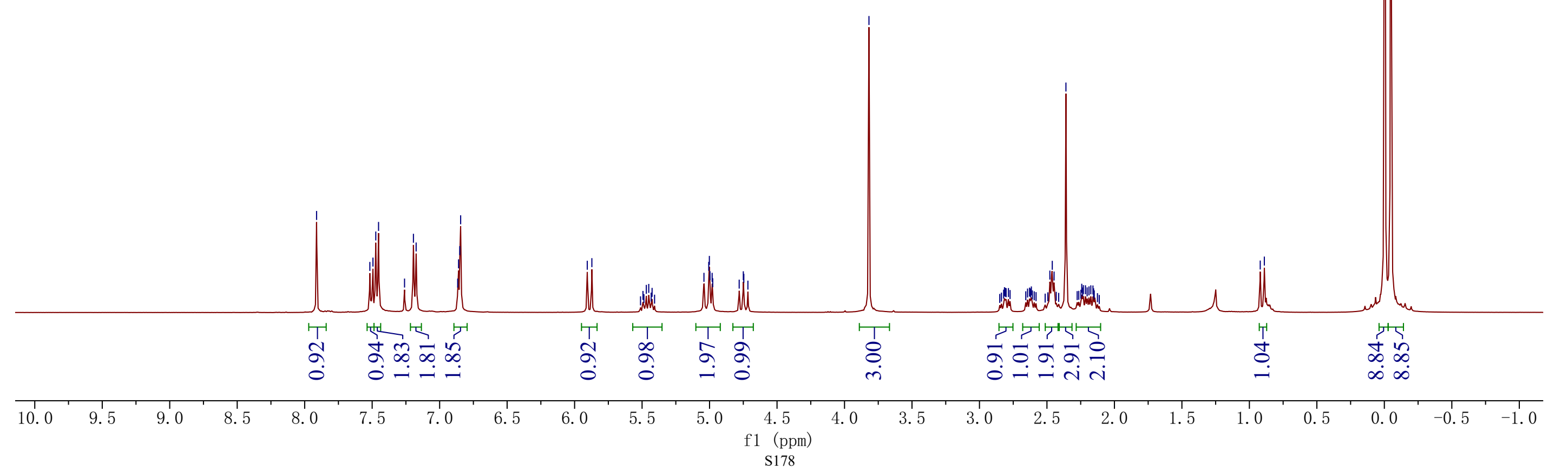


CY-7-6_C13_CDCl3_2020-5-4.30.fid - $100 \mathrm{MHz}$

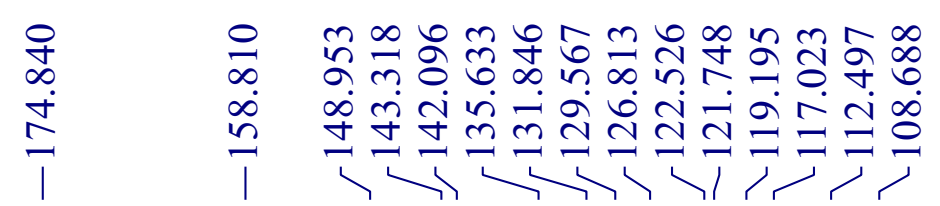

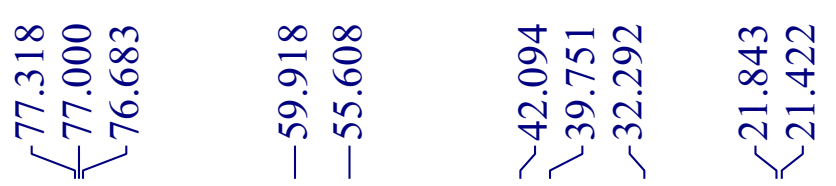

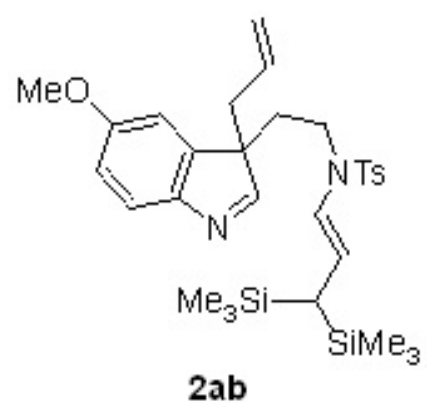

$2 a b$

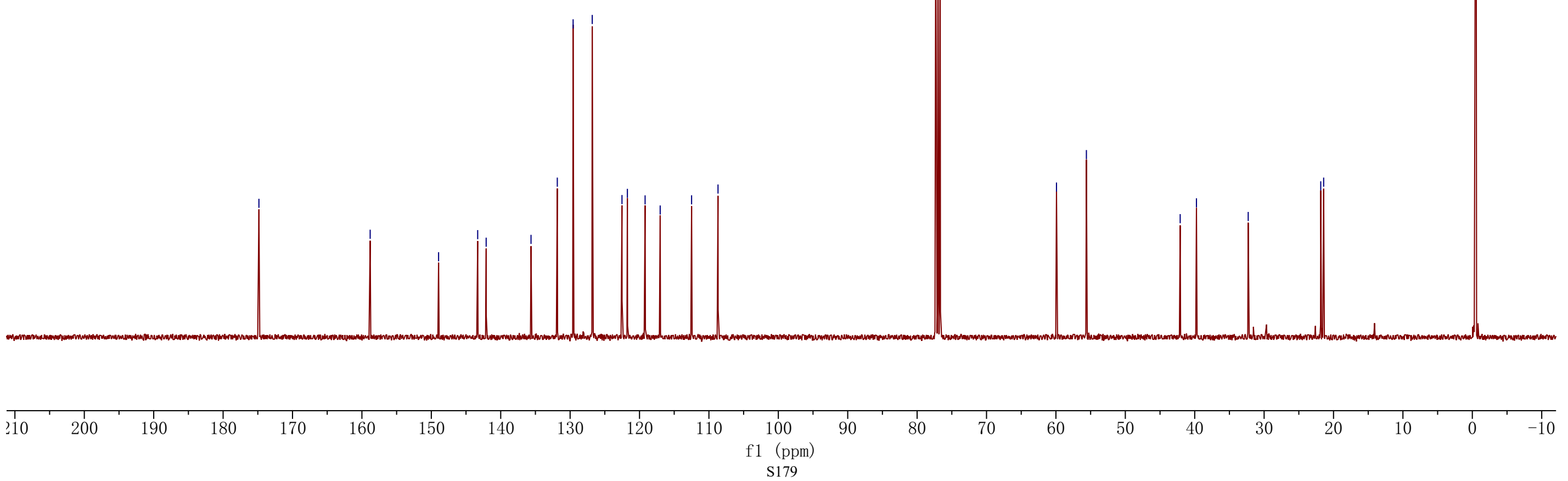



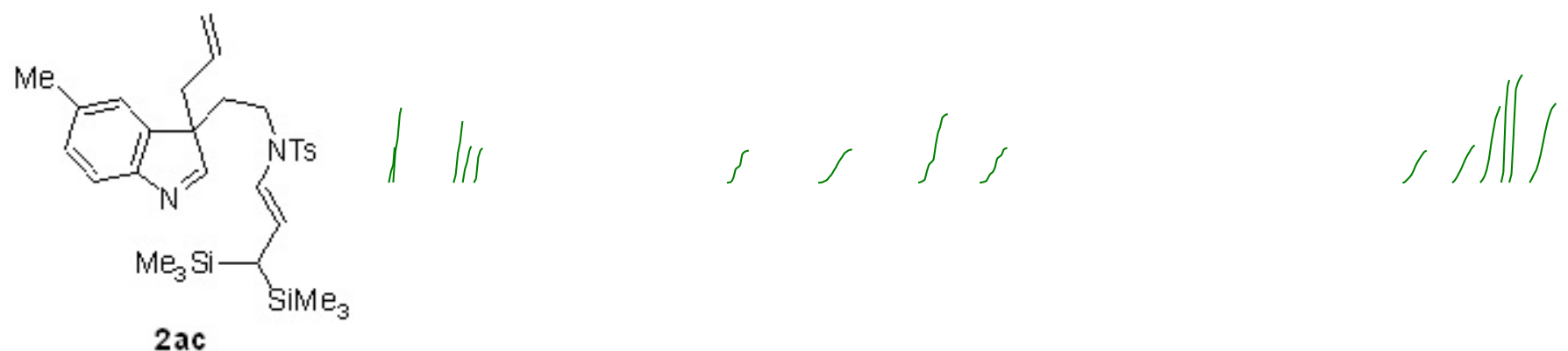

$2 \mathrm{ac}$

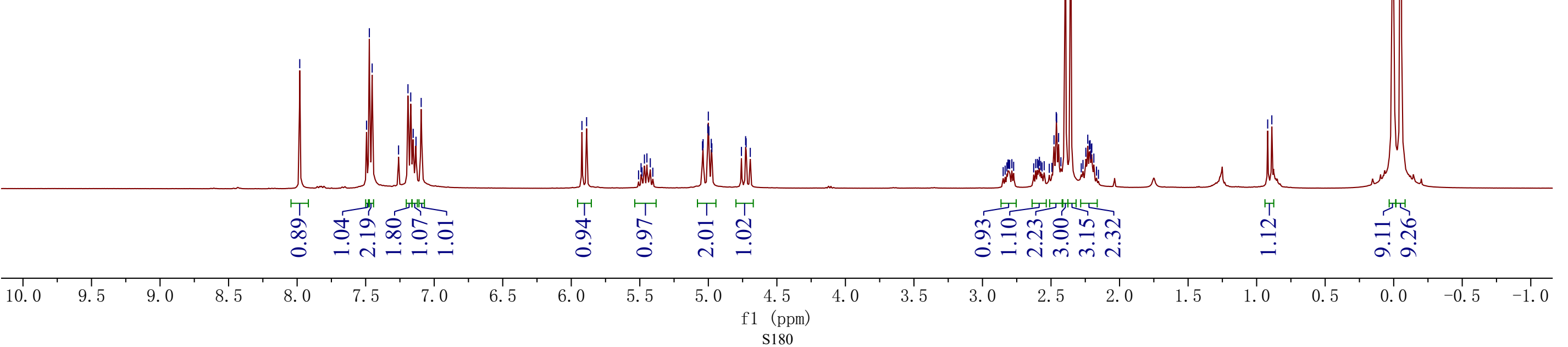




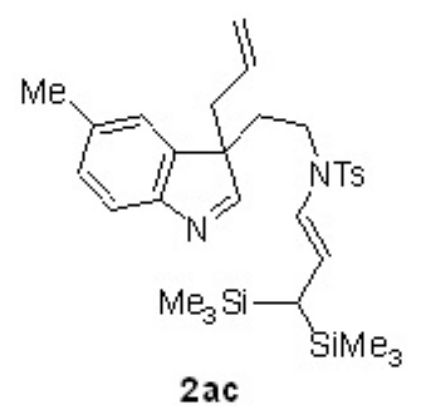

$2 a c$

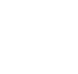

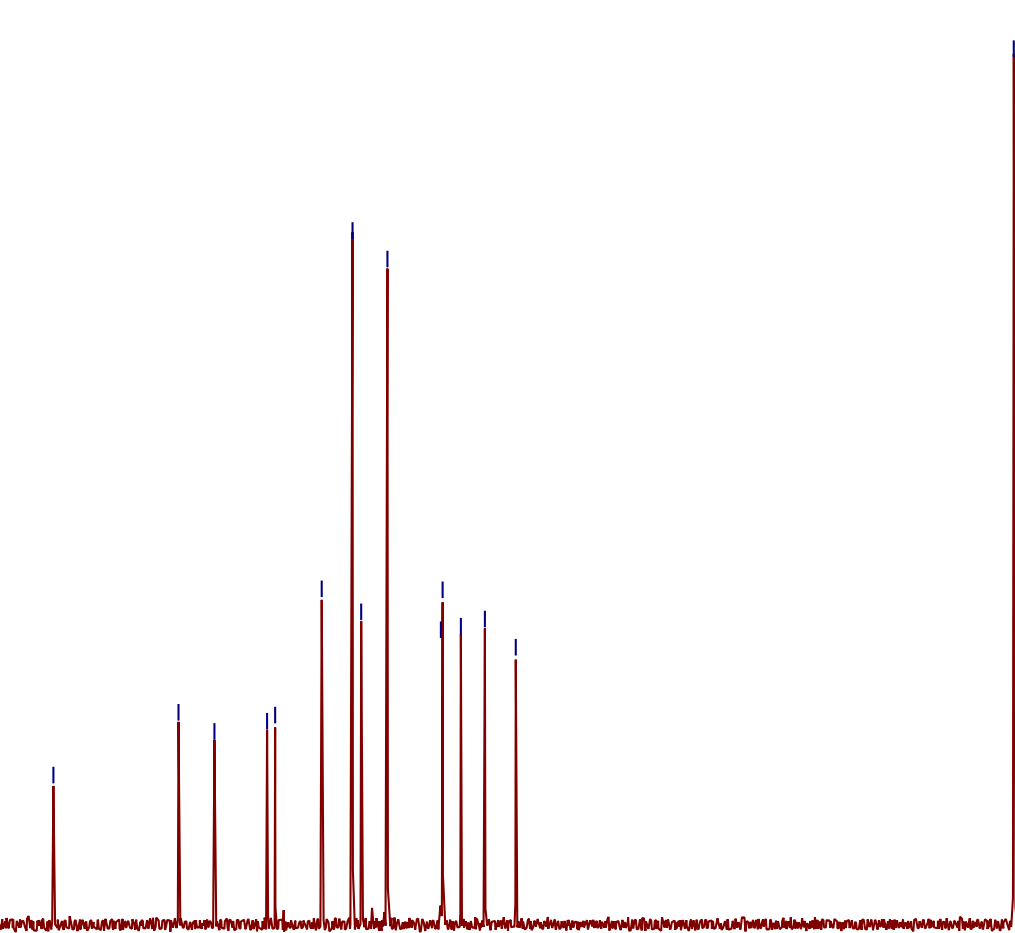



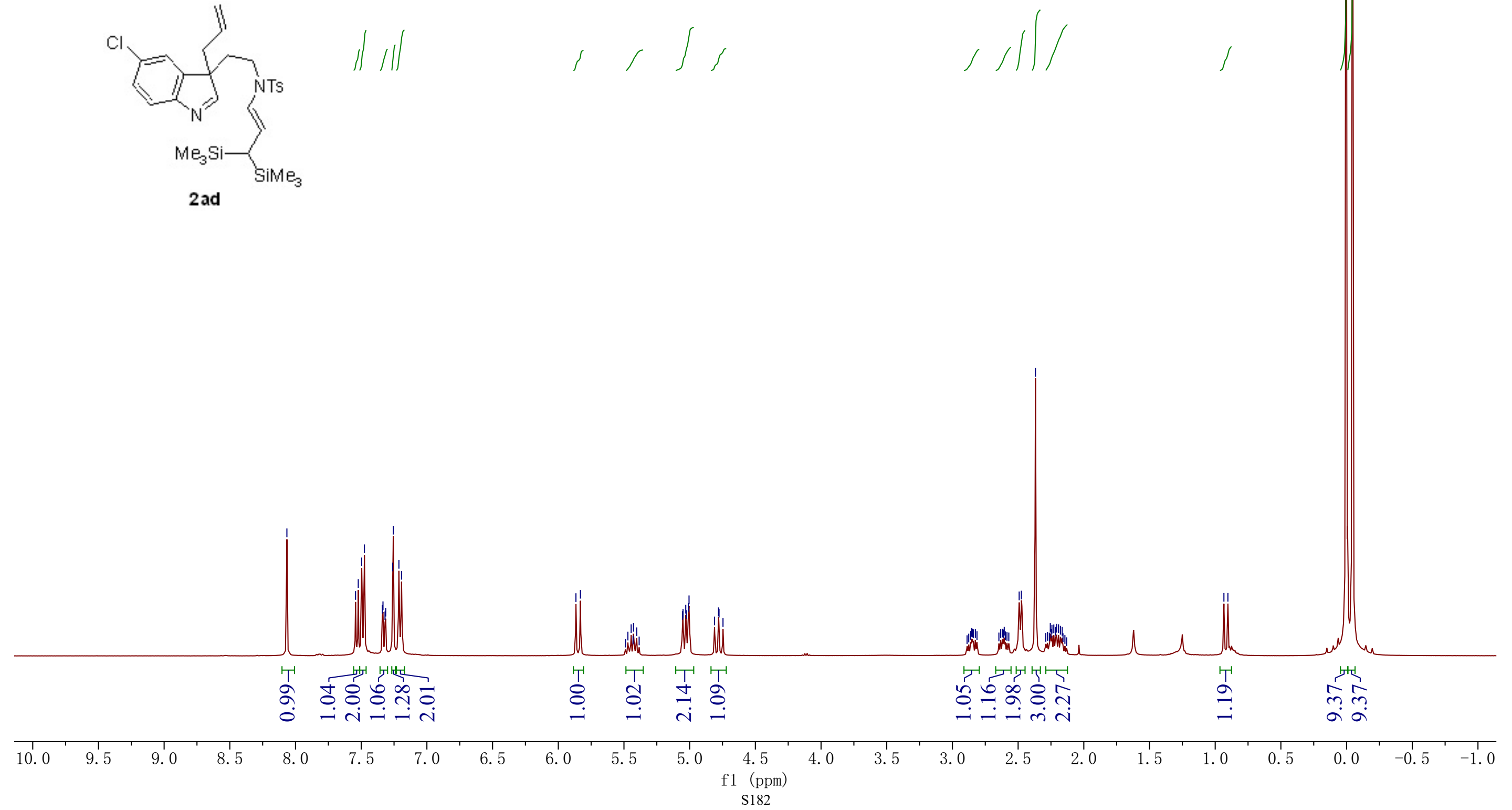


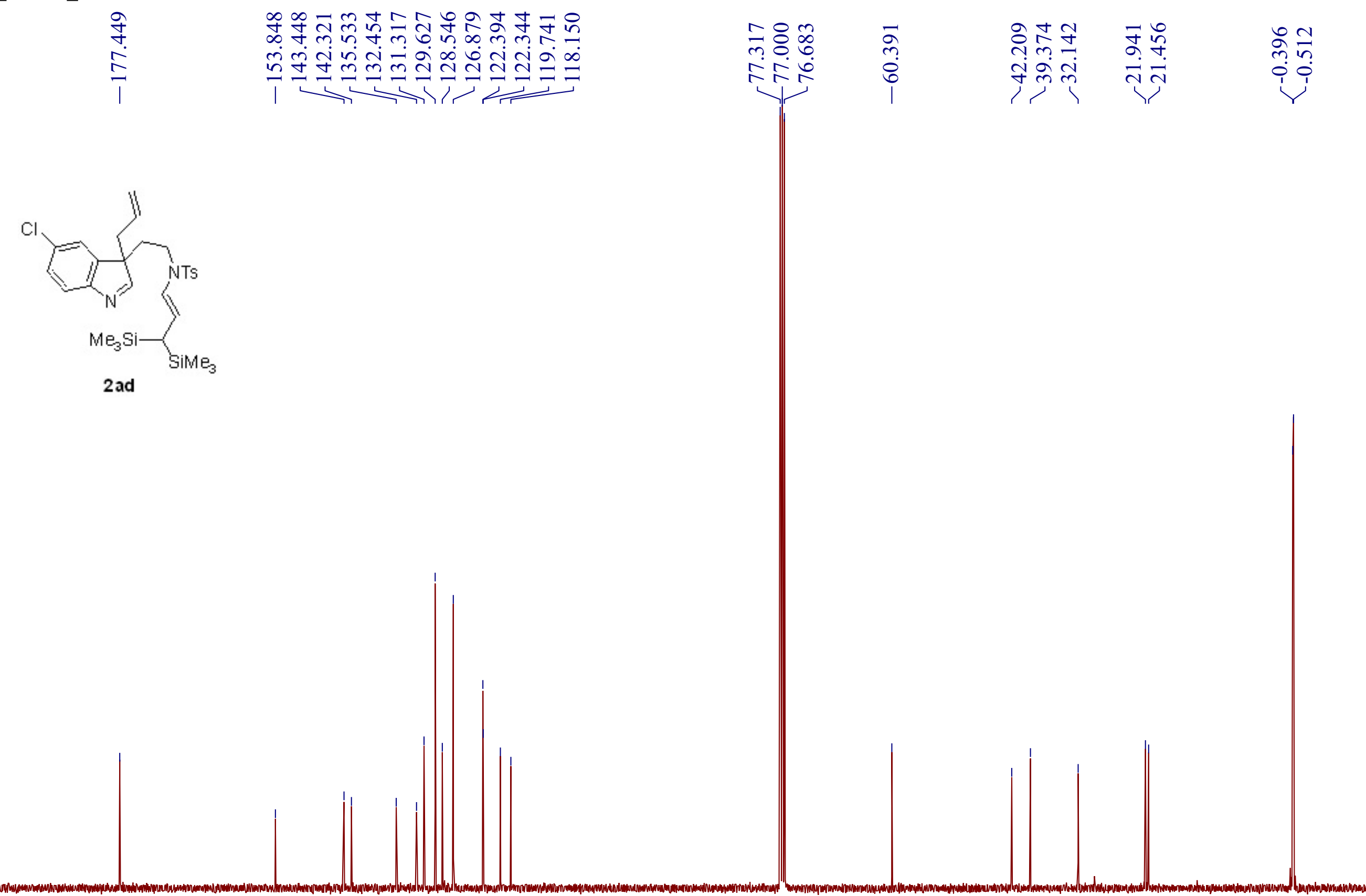



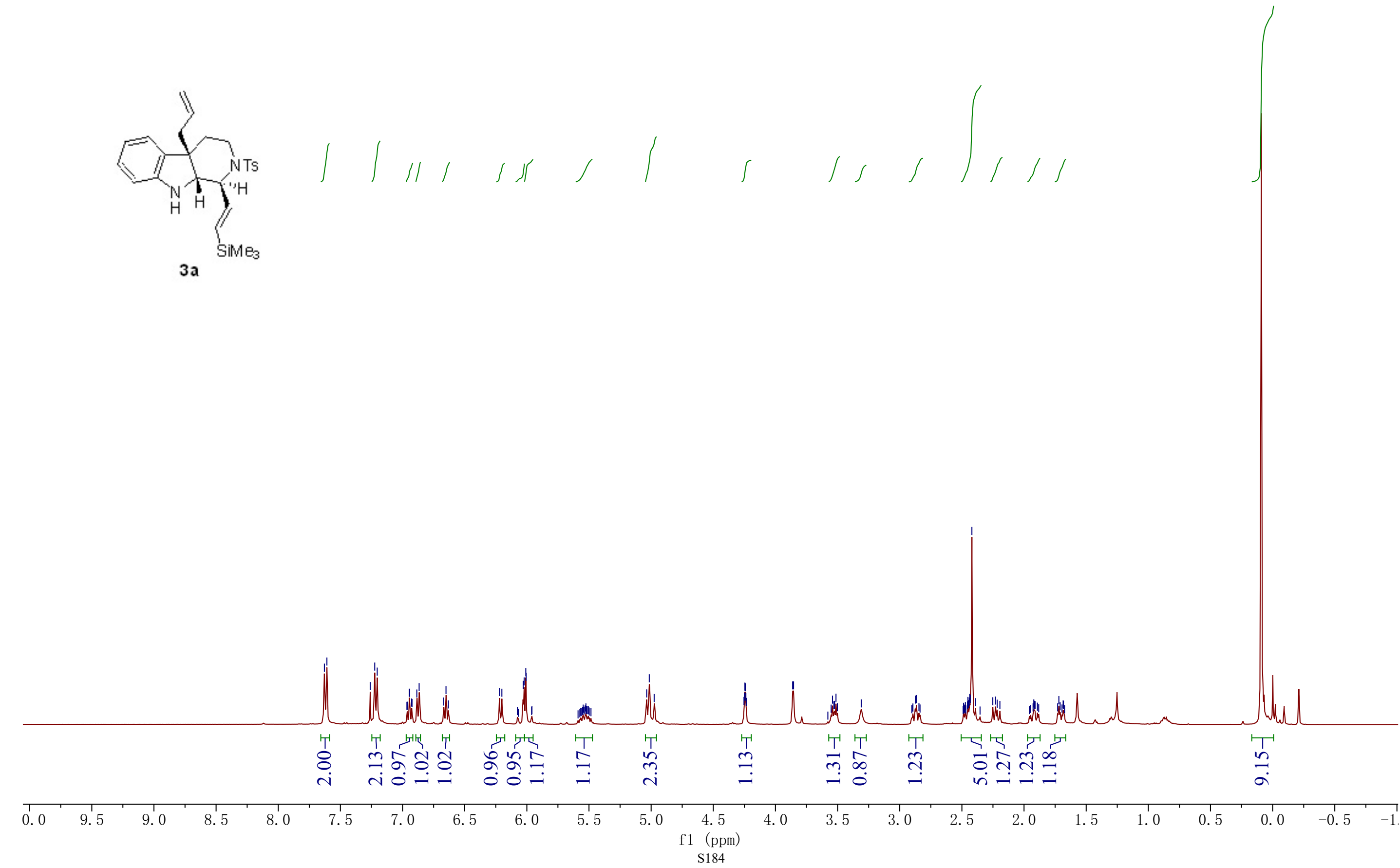


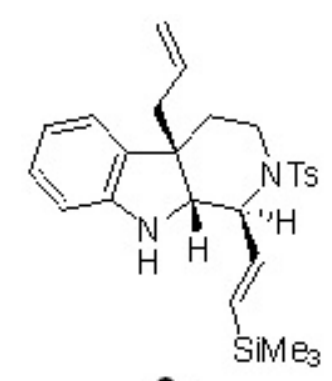

$3 a$

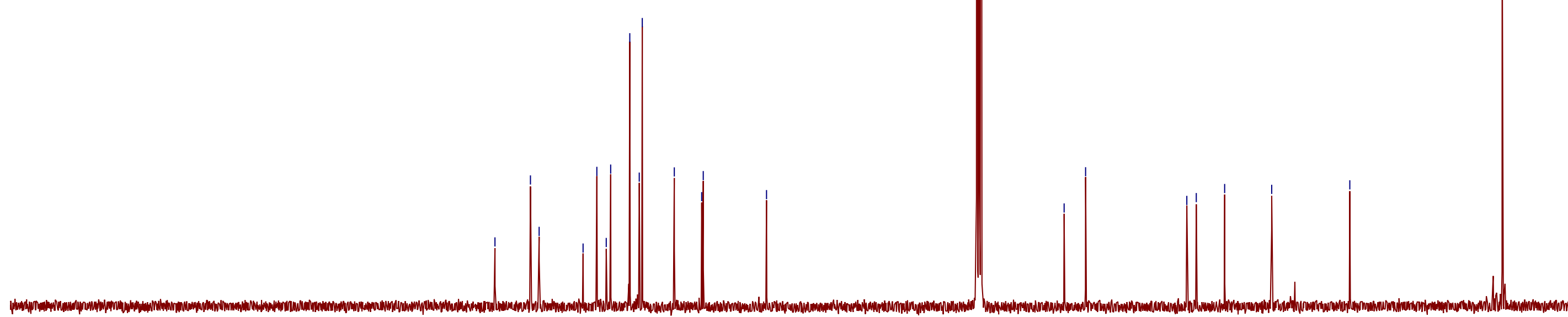


CY-6-50-2_DEPT135_CDCl3_2019-11-28.13.fid - $100 \mathrm{MHz}$

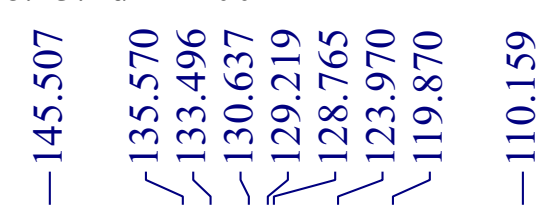

$\infty \frac{n}{n}$

ติ ชิ

5
0

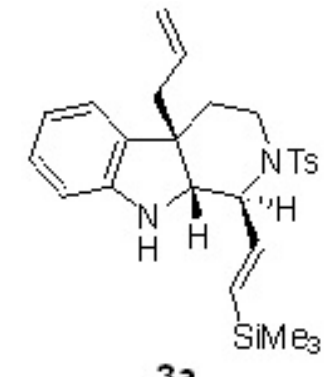

$3 a$

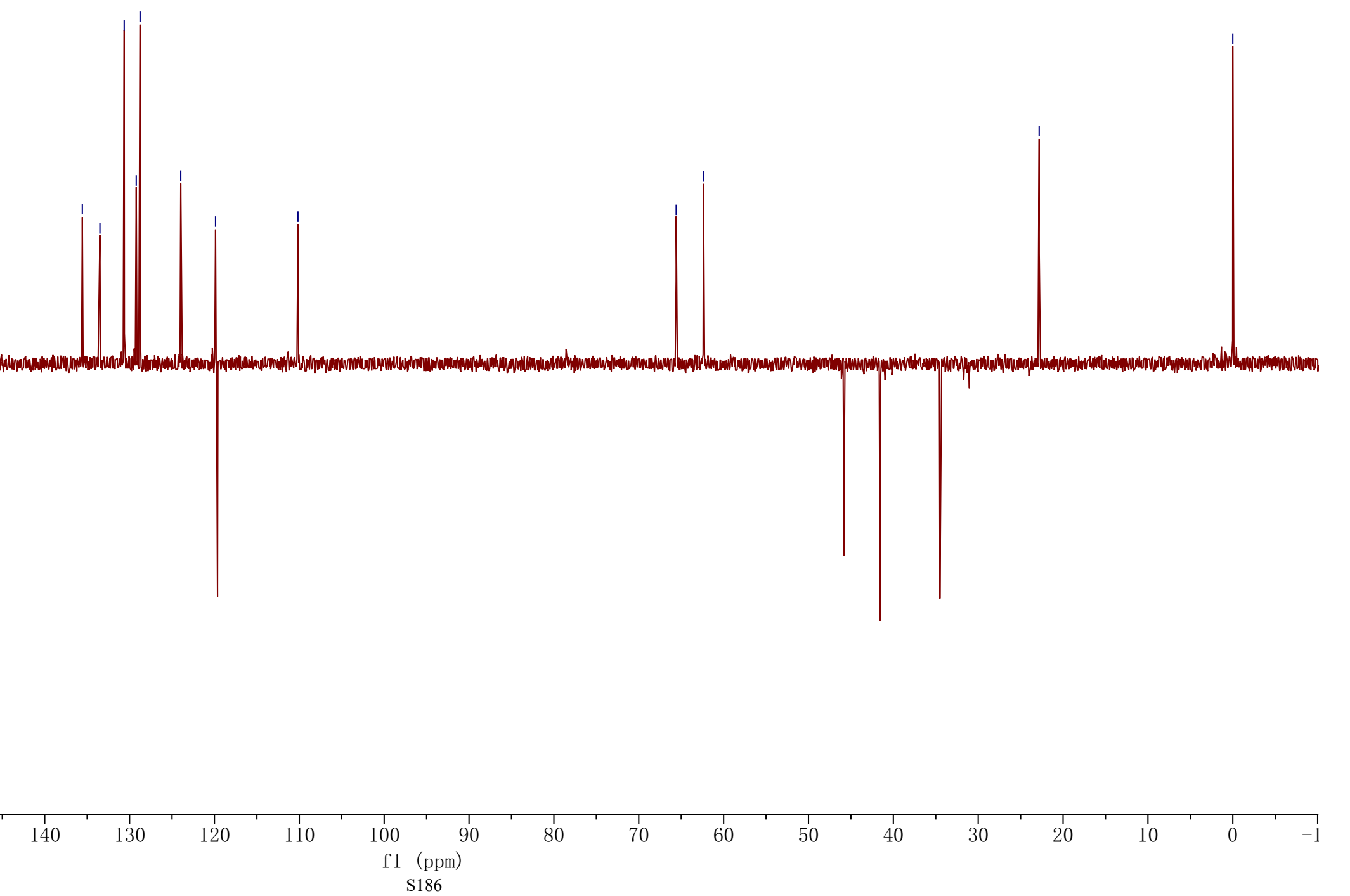




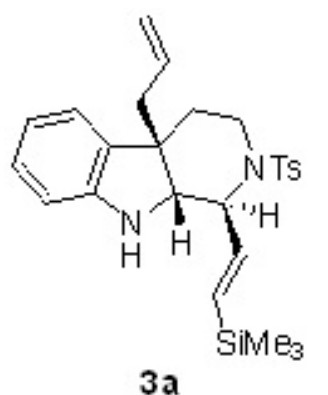

CY-6-50-2_2D_CDCl3_2019-11-28.11.ser - H1-H1 COSY $400 \mathrm{MHz}$

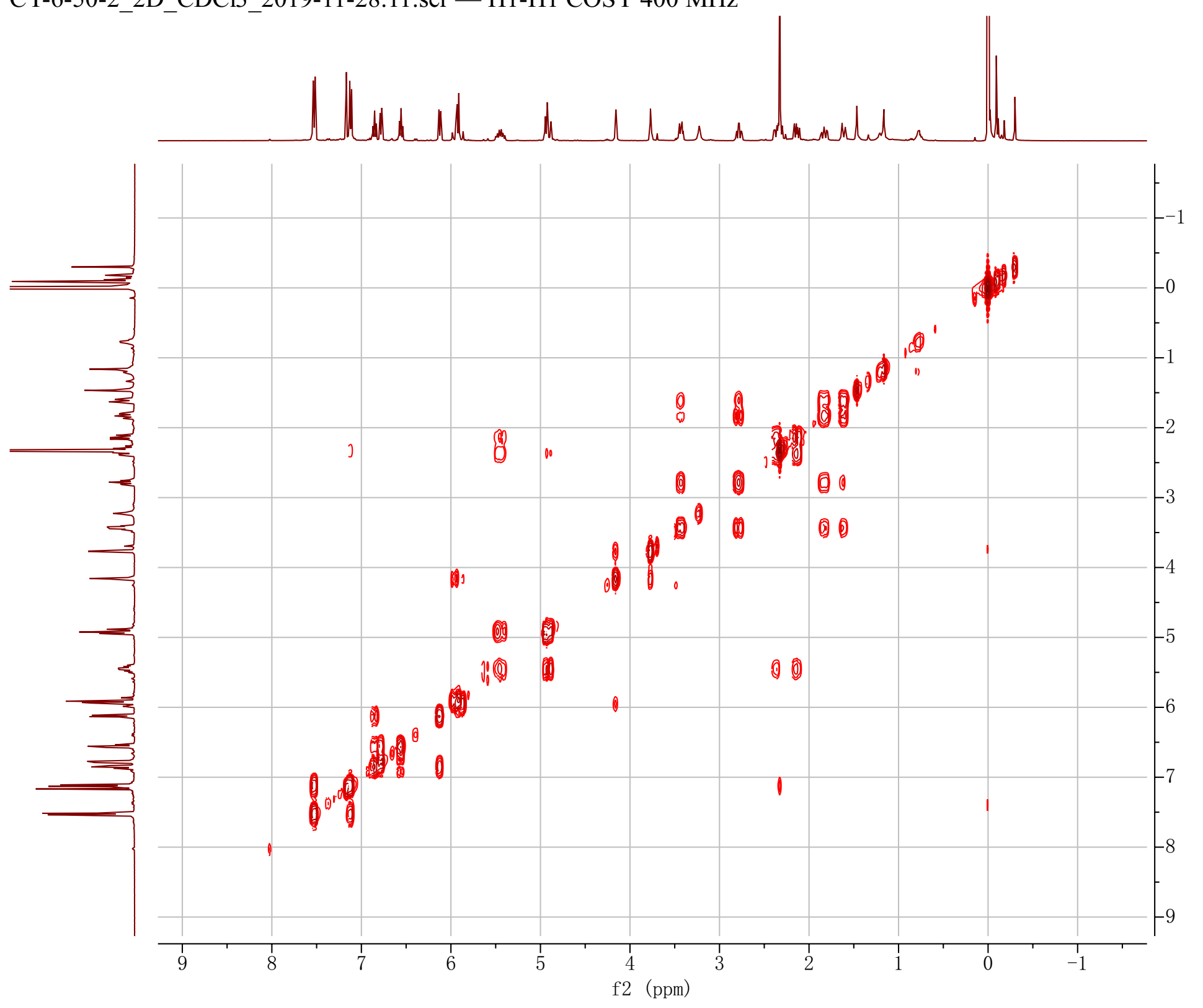




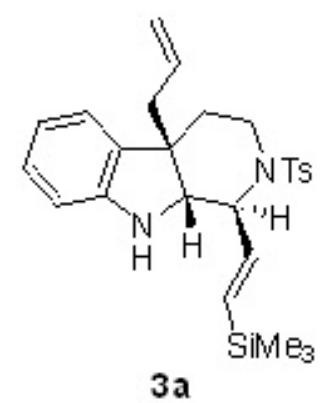

CY-6-50-2_2D_CDCl3_2019-11-28.12.ser - H1-C13 HSQC $400 \mathrm{MHz}$

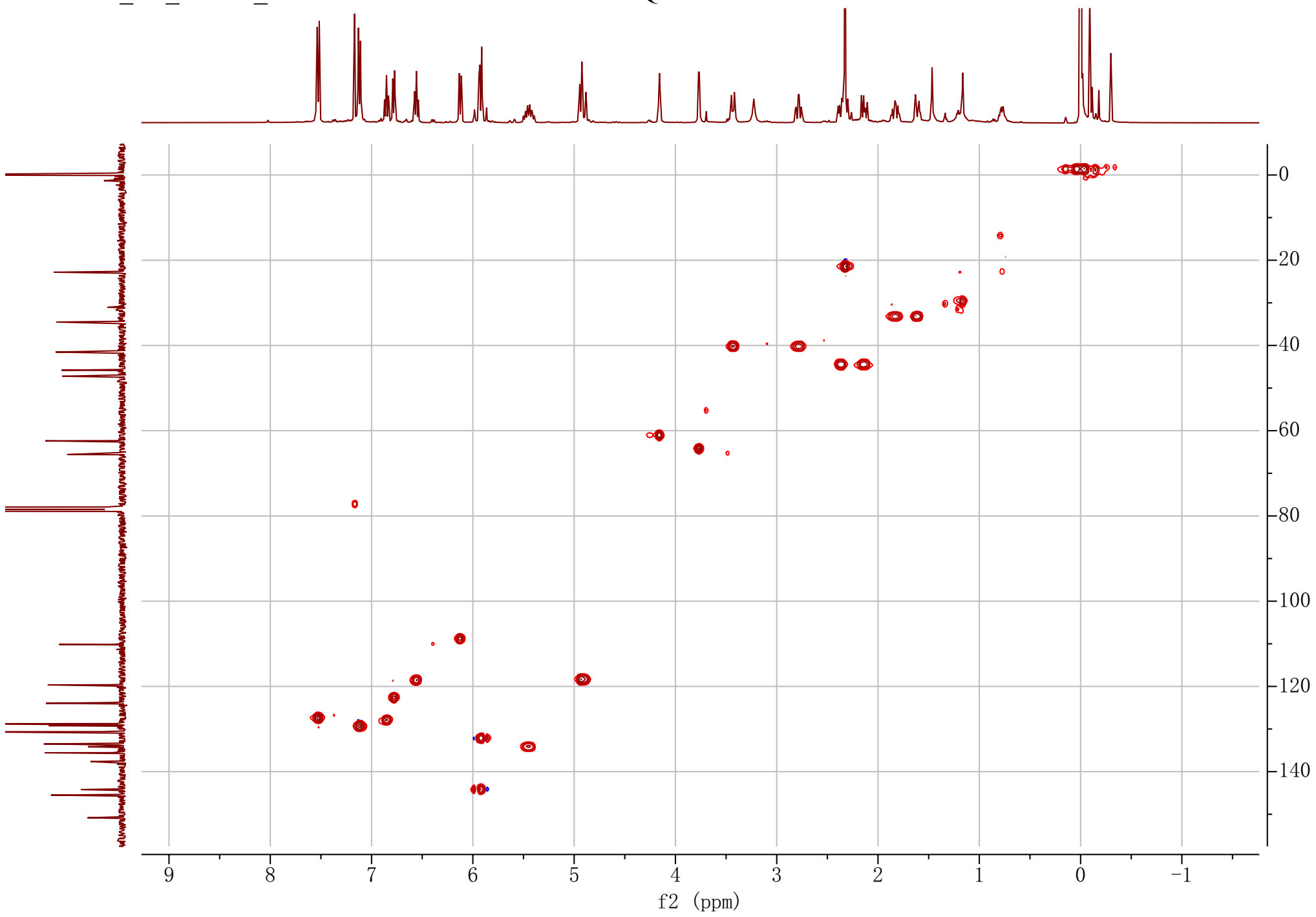



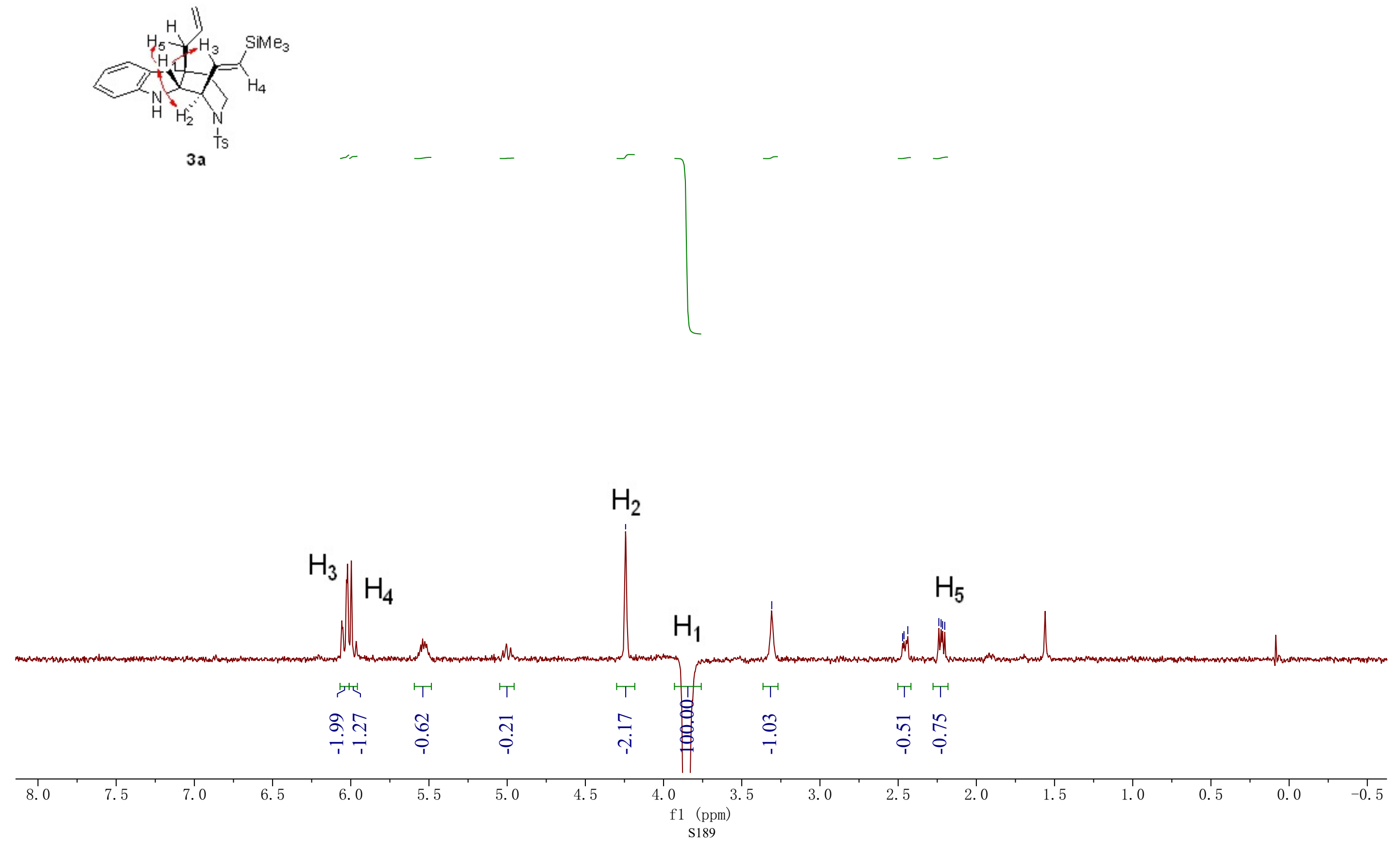
CY-6-50-2-CDCL3-NOESY1D4.25-2019-12-2 - $600 \mathrm{MHz}$

ํํ유.

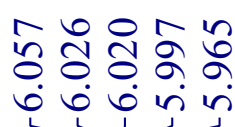

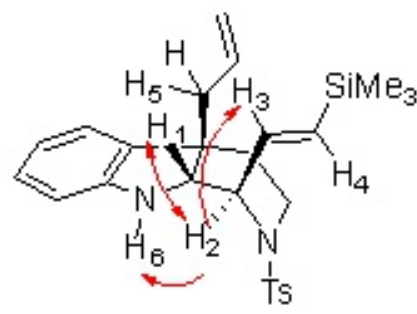

$3 a$

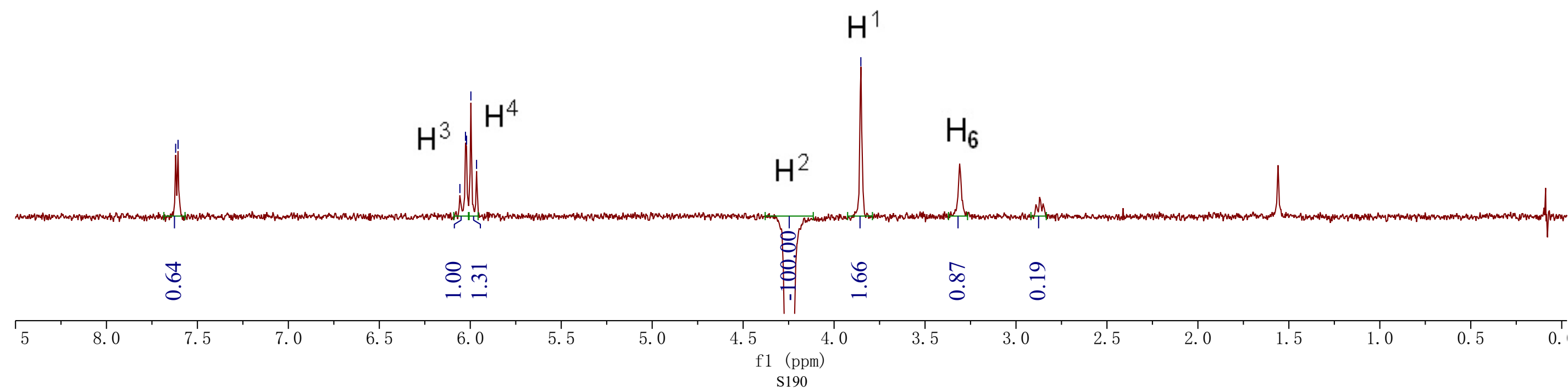



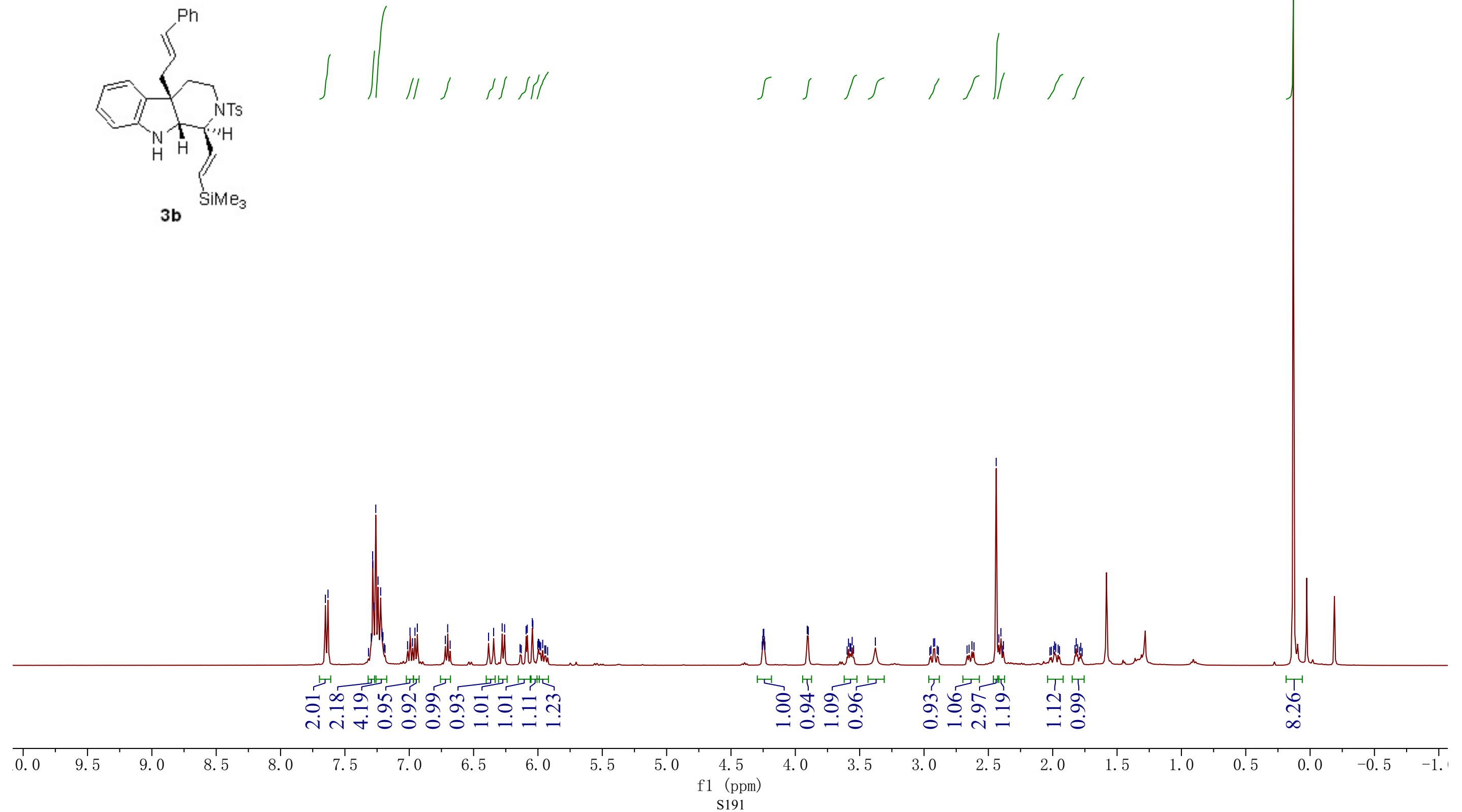

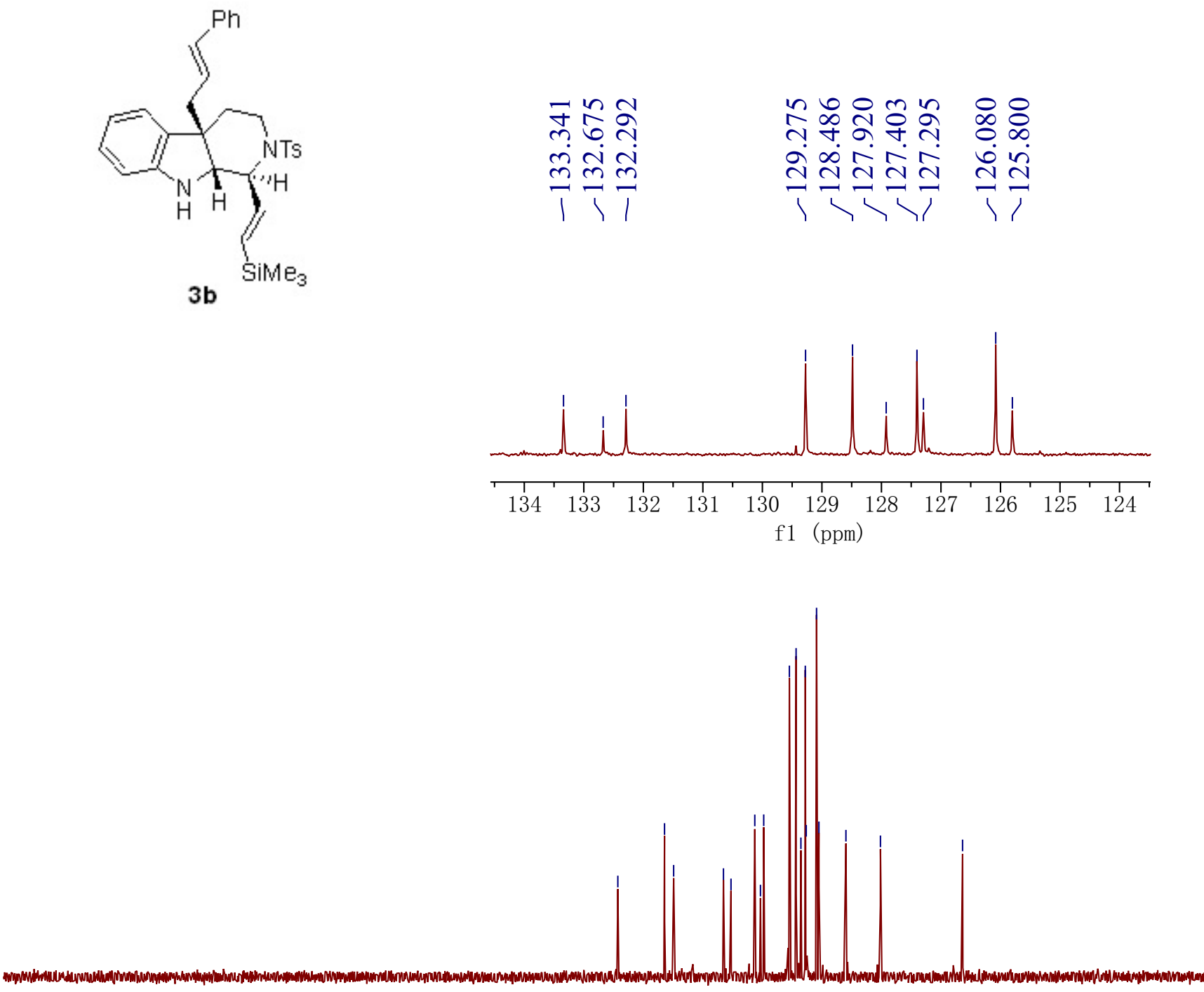

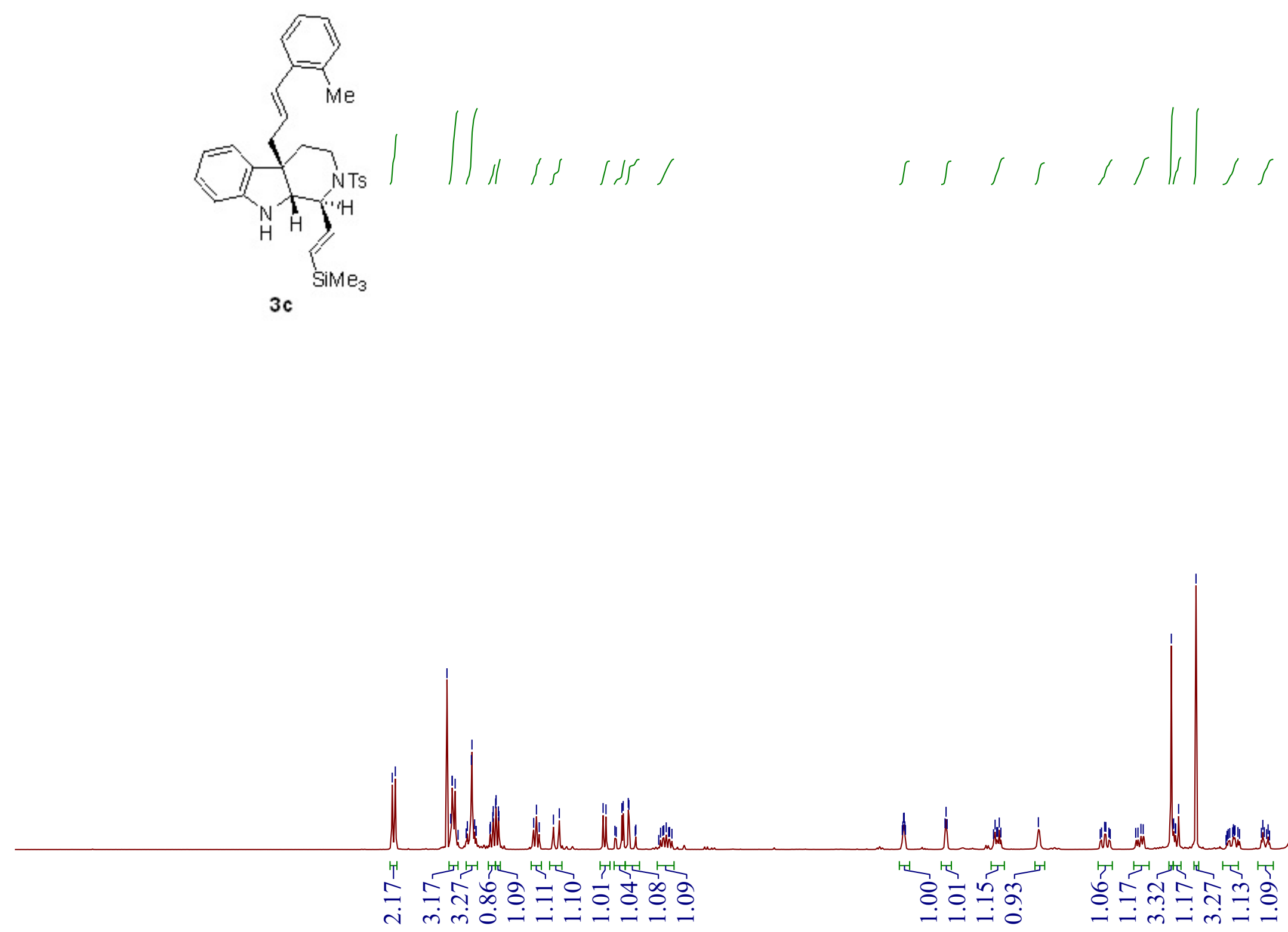

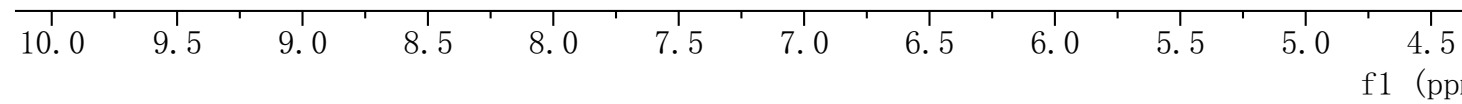



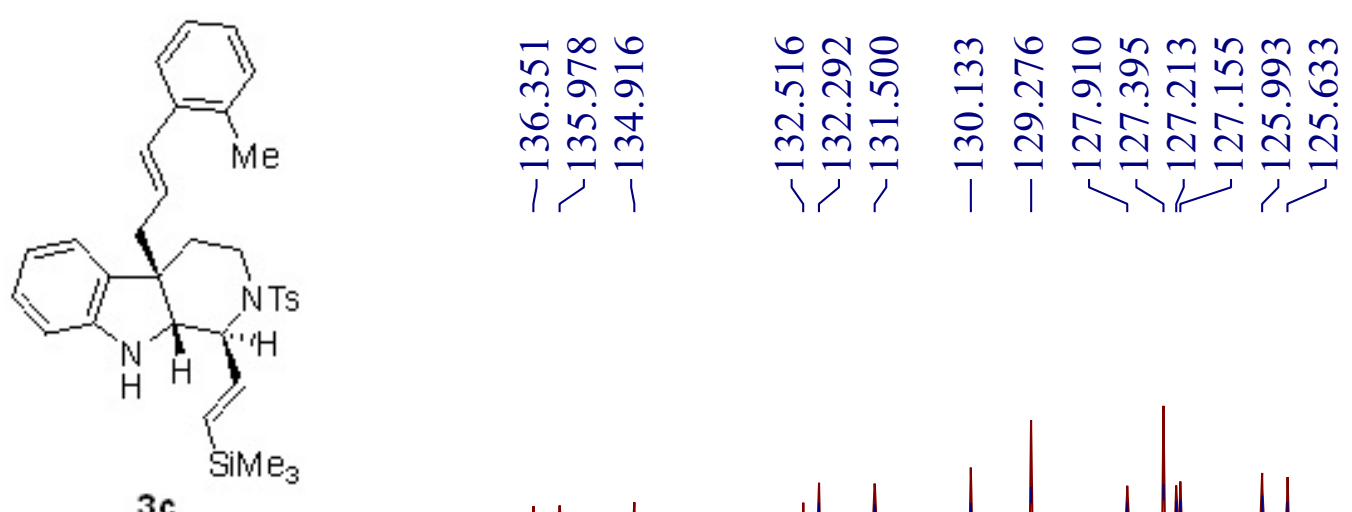

3c

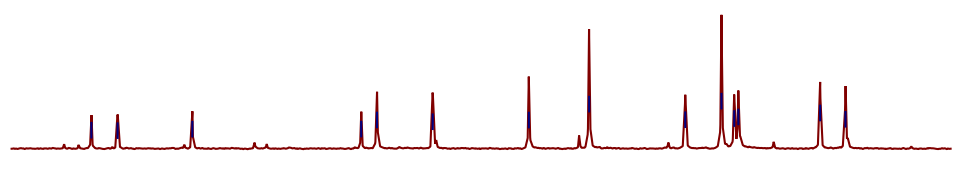

$\begin{array}{lllllllllllll}137 & 136 & 135 & 134 & 133 & 132 & 131 & 130 & 129 & 128 & 127 & 126 & 125\end{array}$ f1 (ppm)

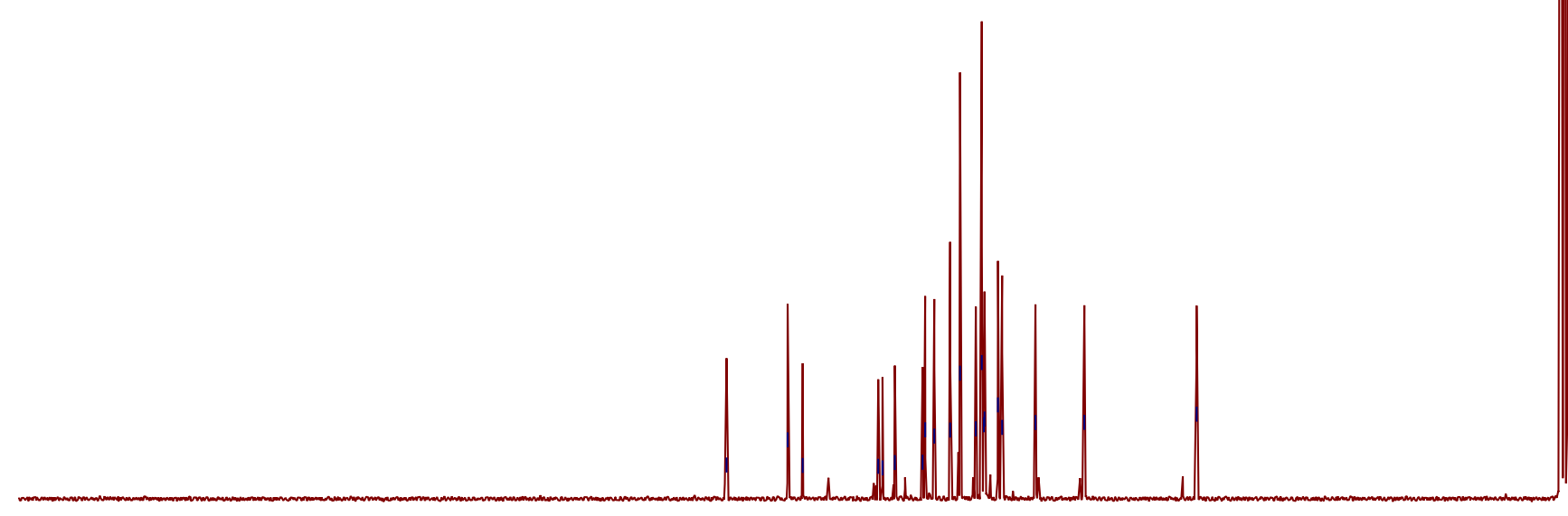

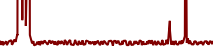

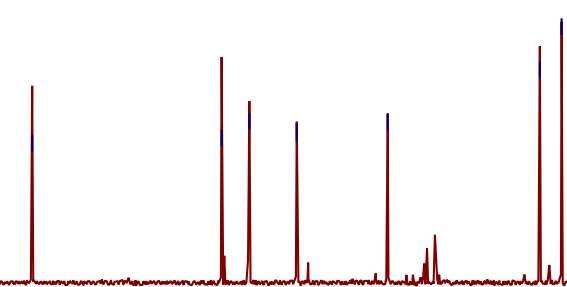

80

70

60

50 

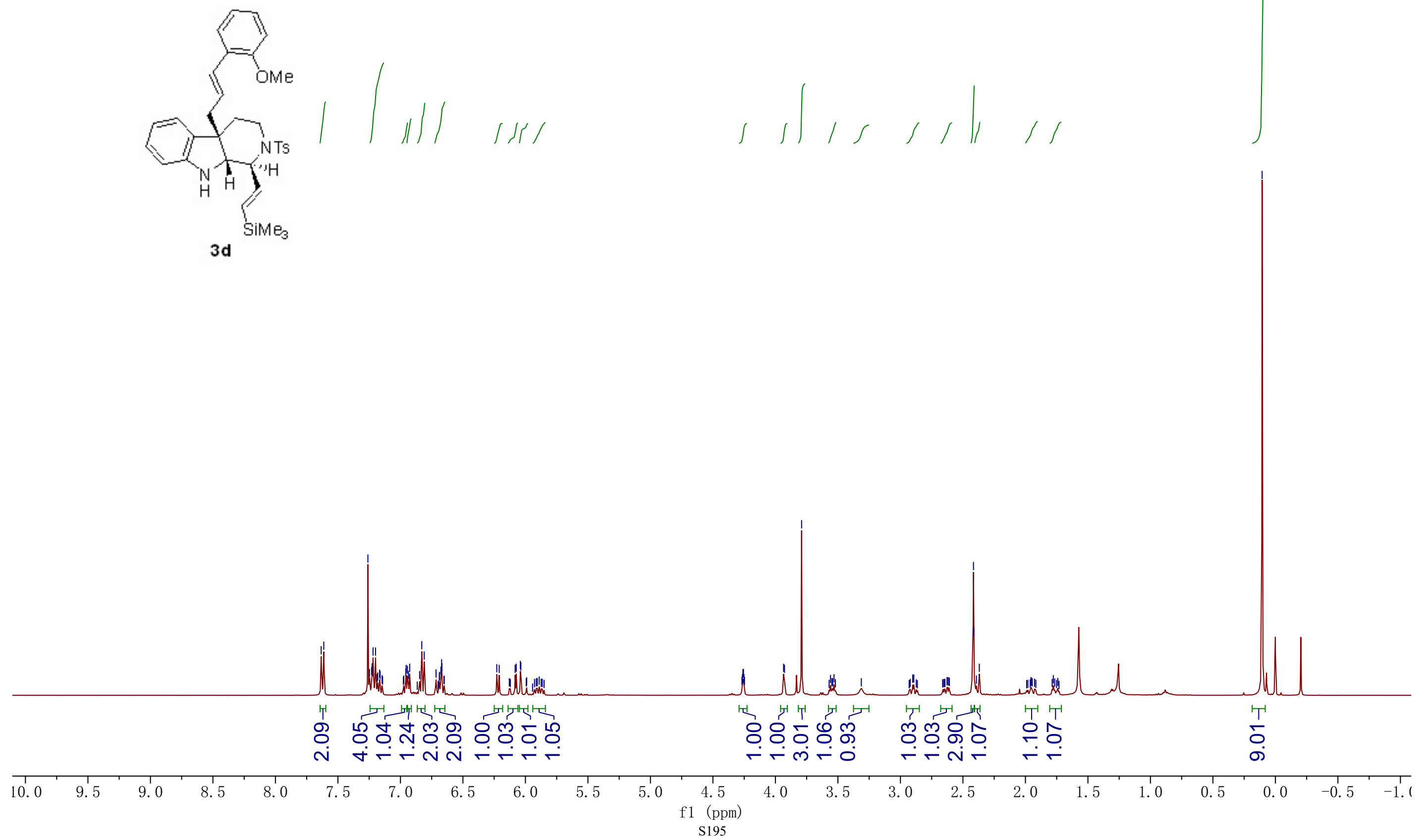


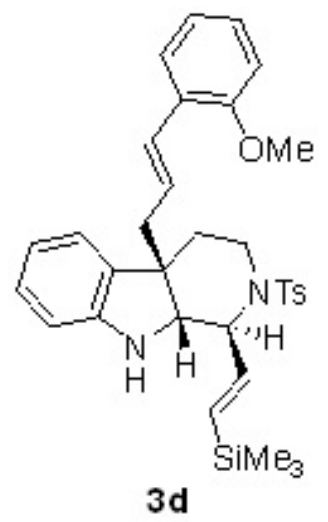

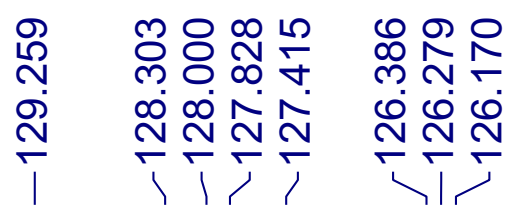
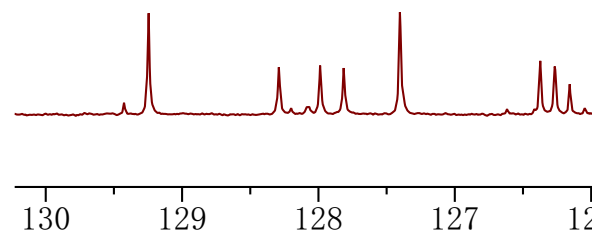

129

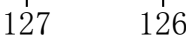

125

124
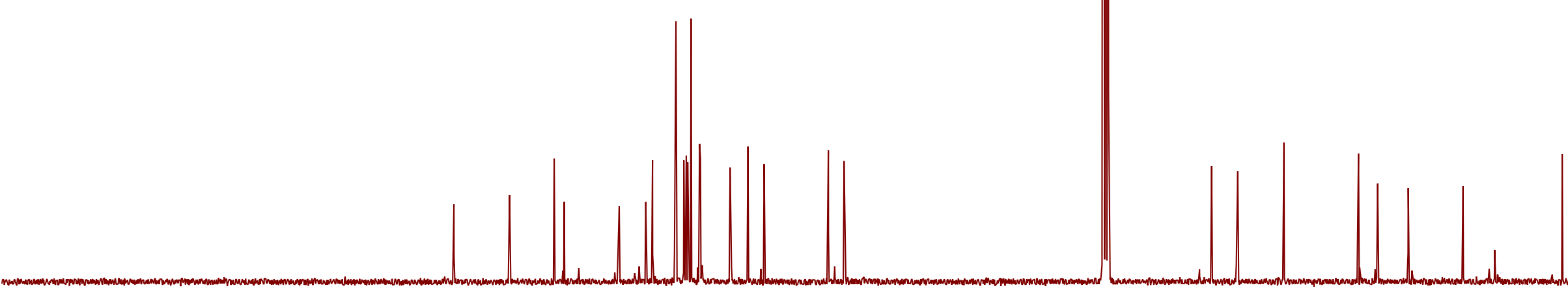

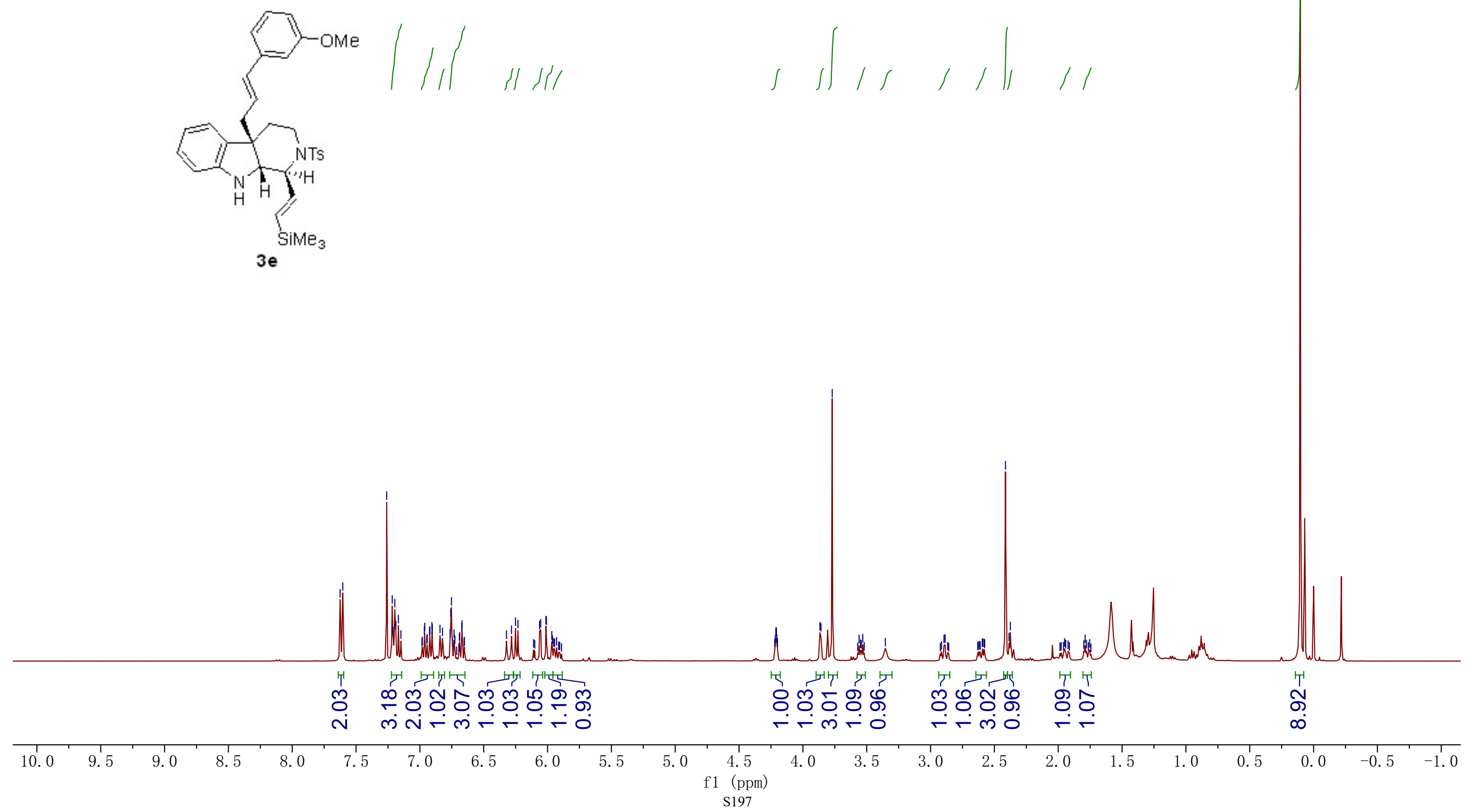


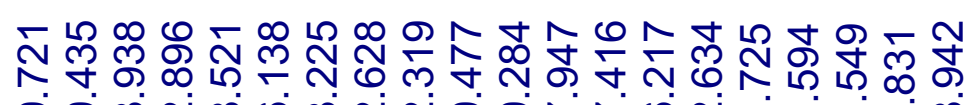

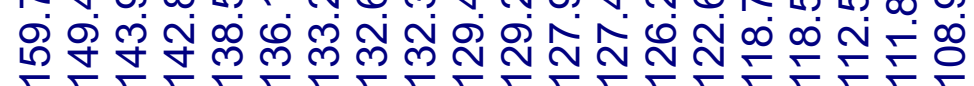

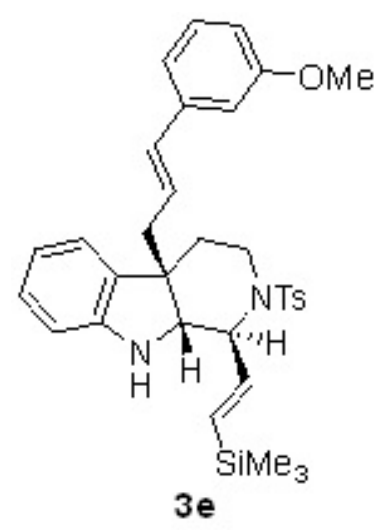

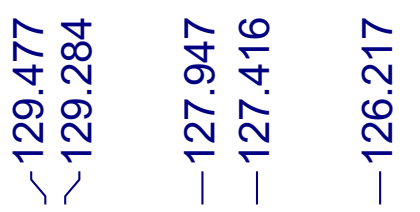

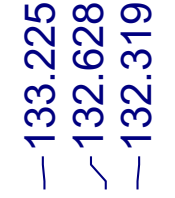

ָั
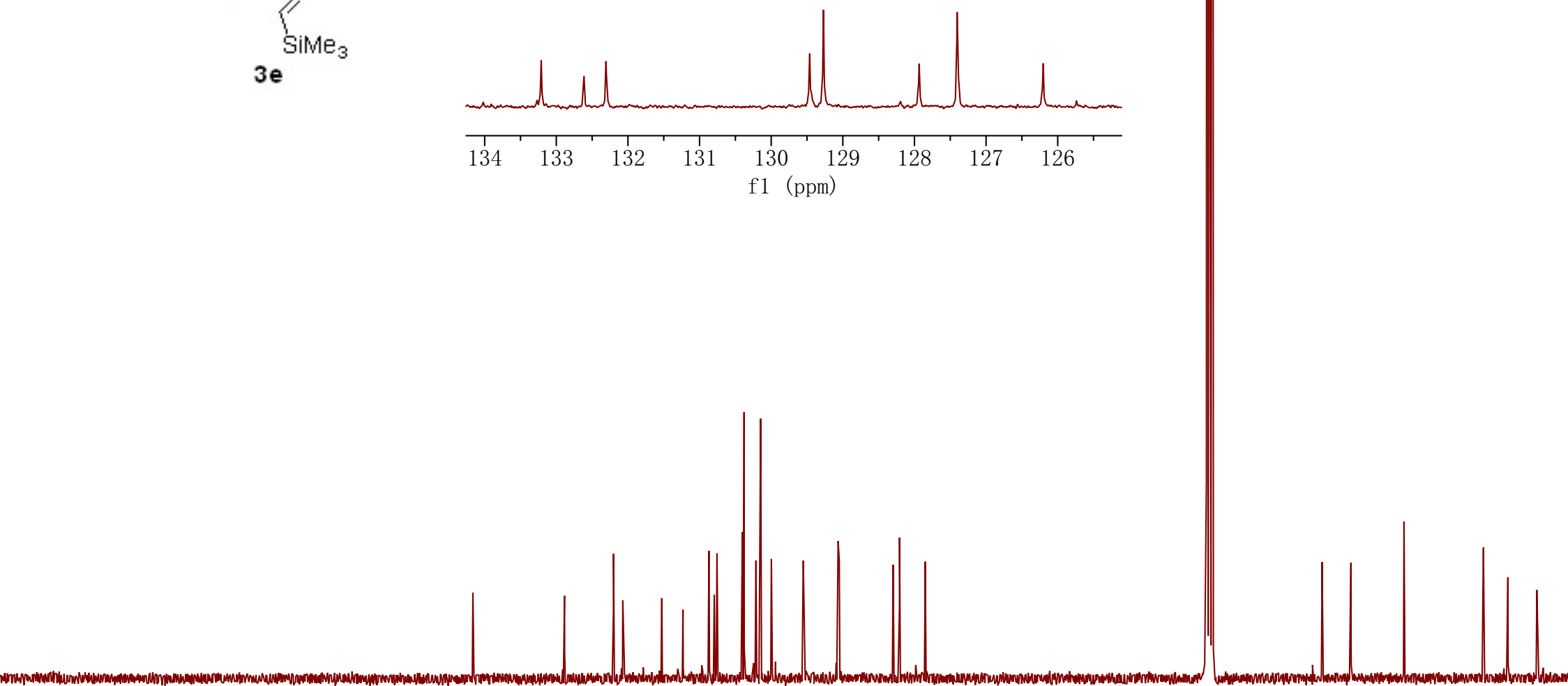

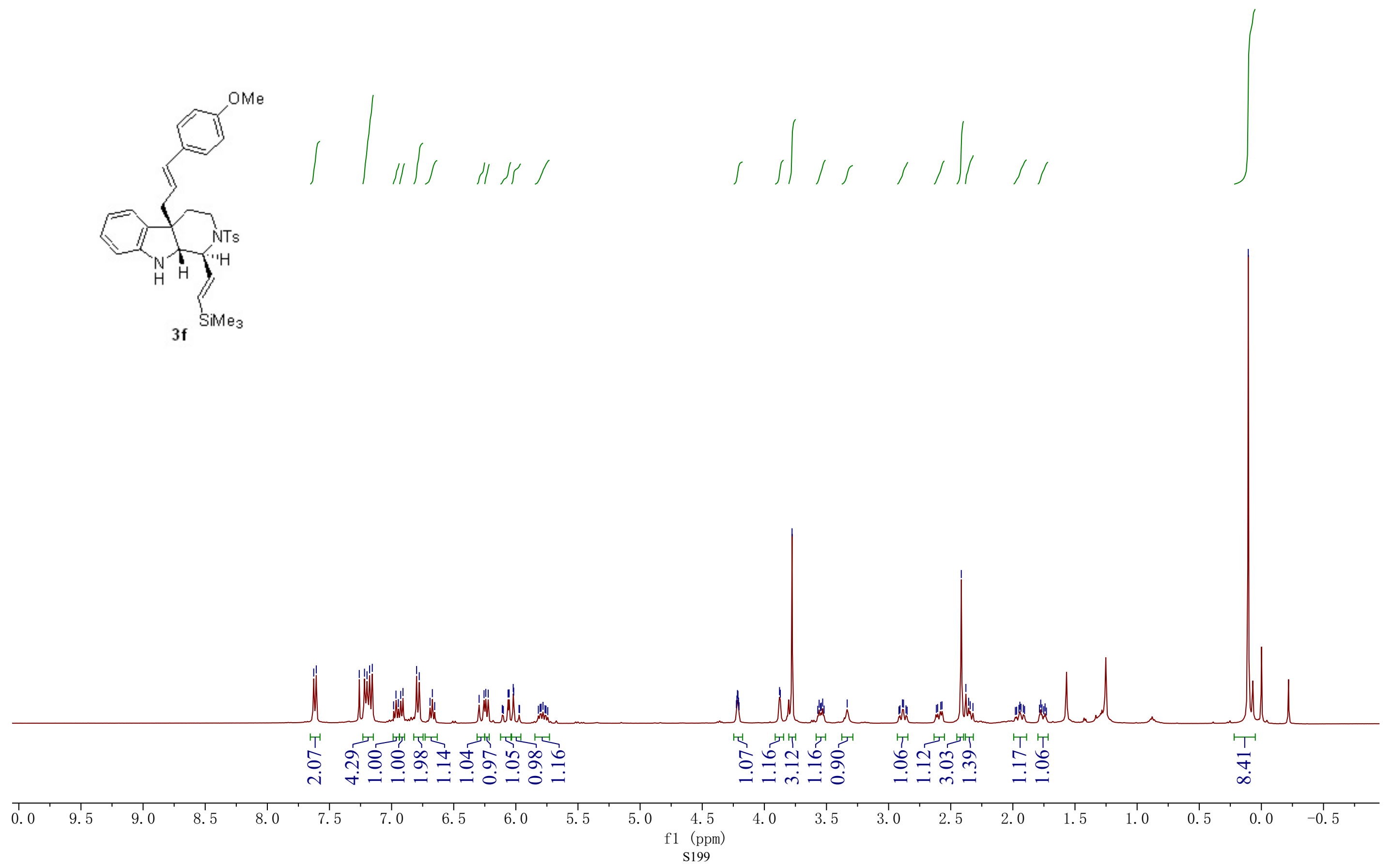


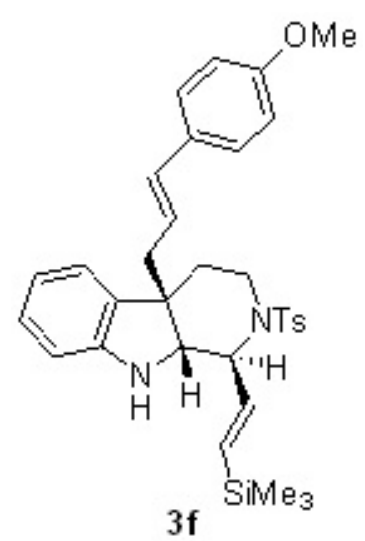

$$
\begin{aligned}
& \text { 항ํำ } \\
& \text { กั่ กี่ }
\end{aligned}
$$
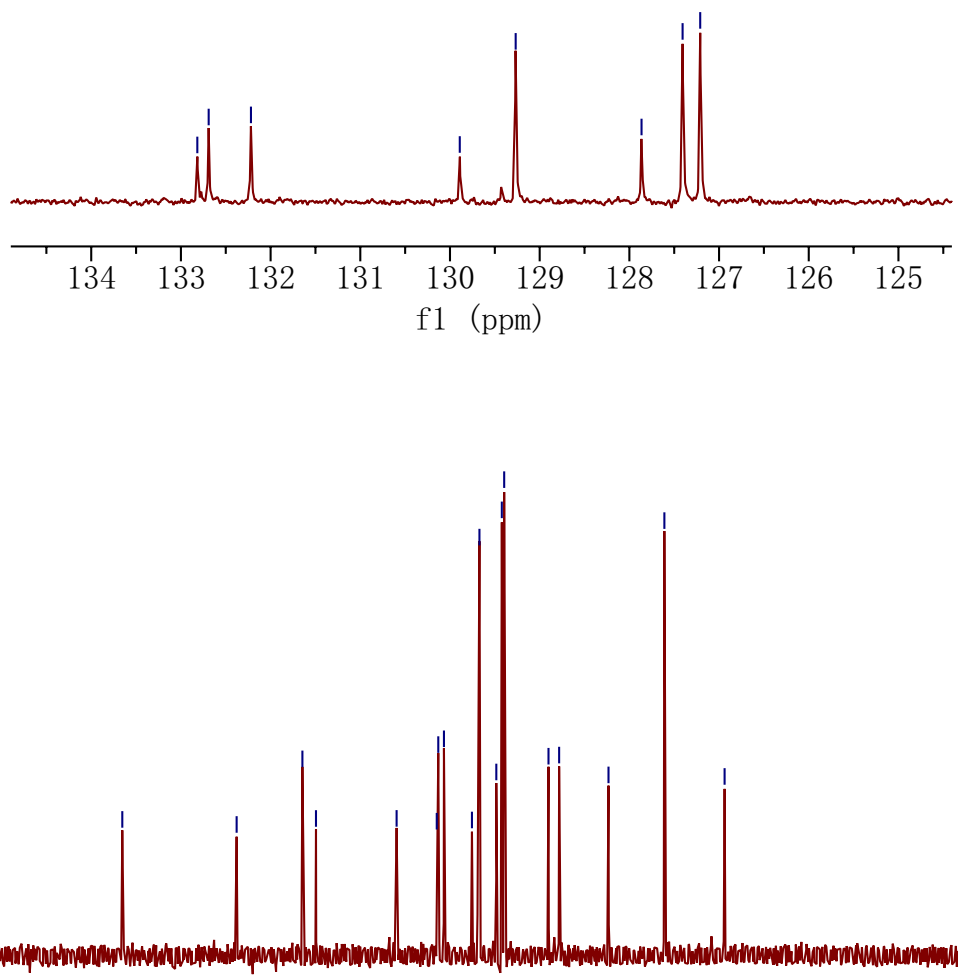


\section{SXY-4-31-CDCL3-H1-2020-10-7.80.fid - $400 \mathrm{MHz}$}

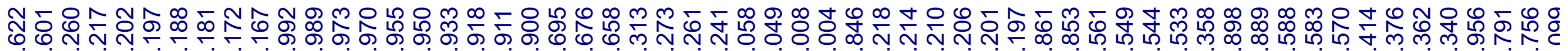

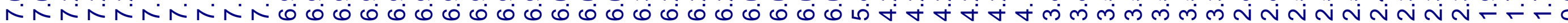
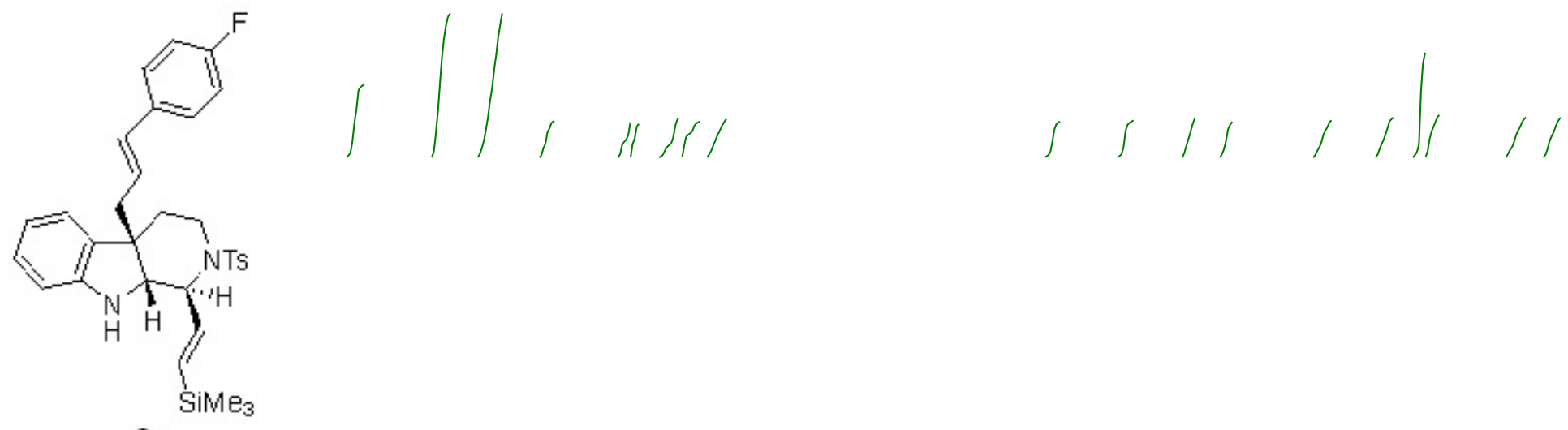

$3 \mathrm{~g}$

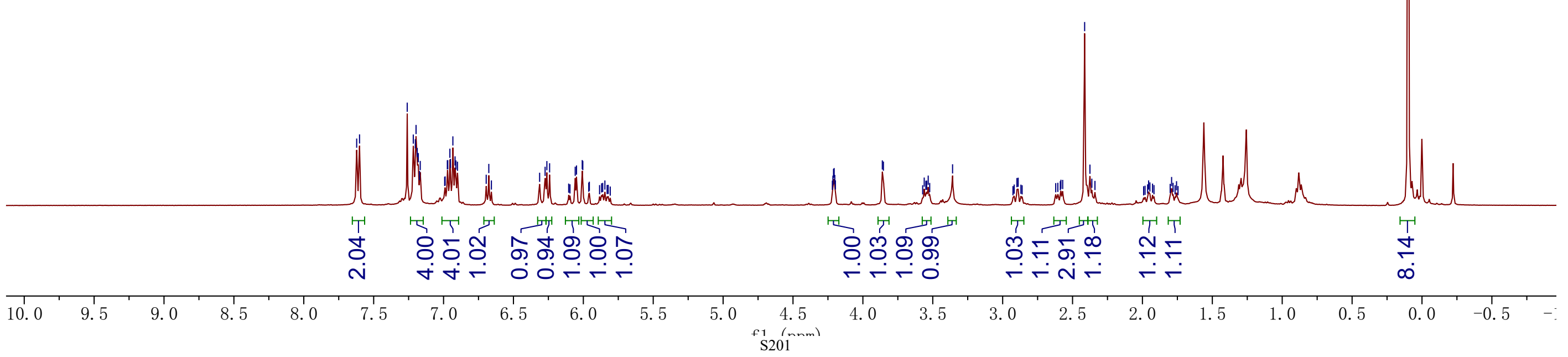




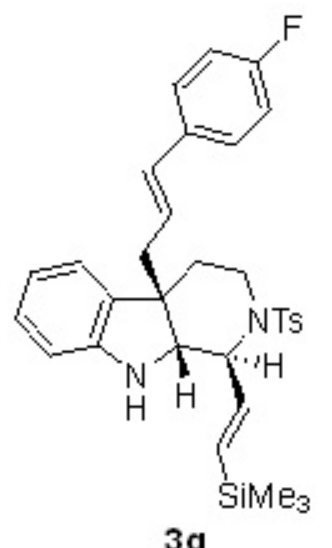

$3 g$

\begin{tabular}{|c|}
\hline 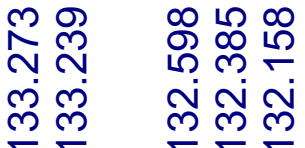 \\
\hline 11 \\
\hline
\end{tabular}

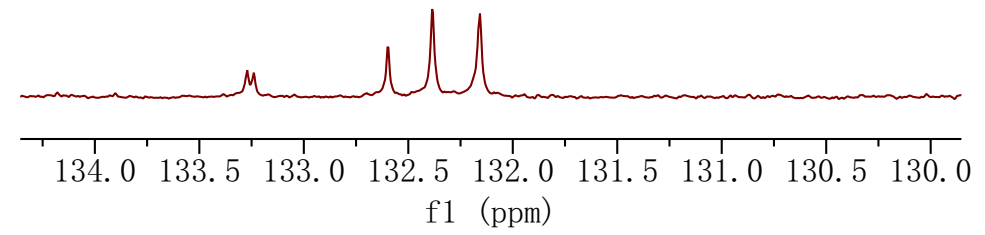

f1 (ppm)

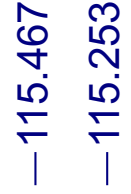

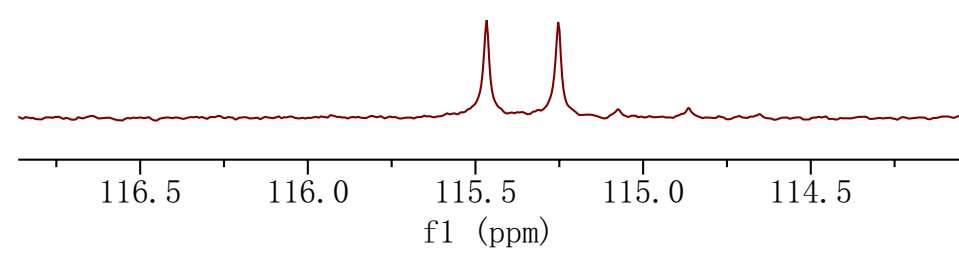

T10 

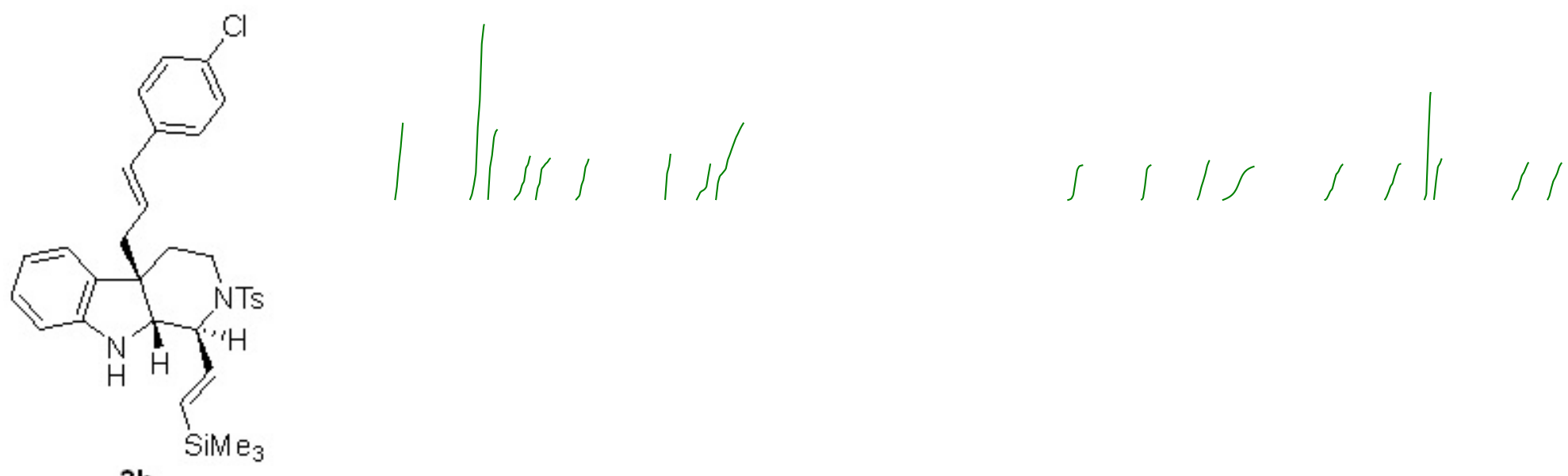

$3 \mathrm{~h}$

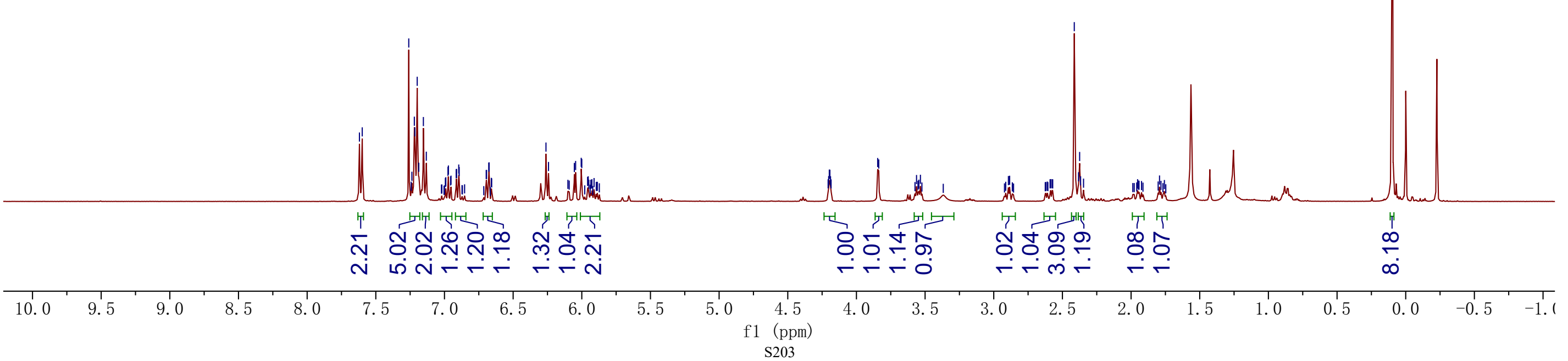




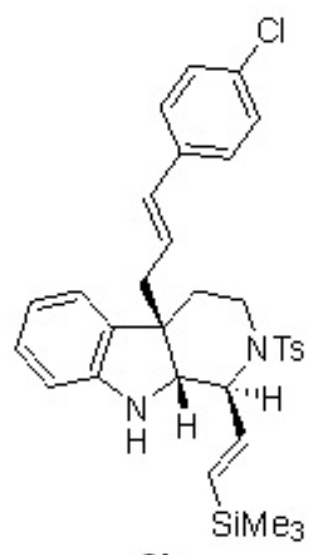

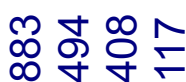
กิ่ กิ กิ

$3 \mathrm{~h}$

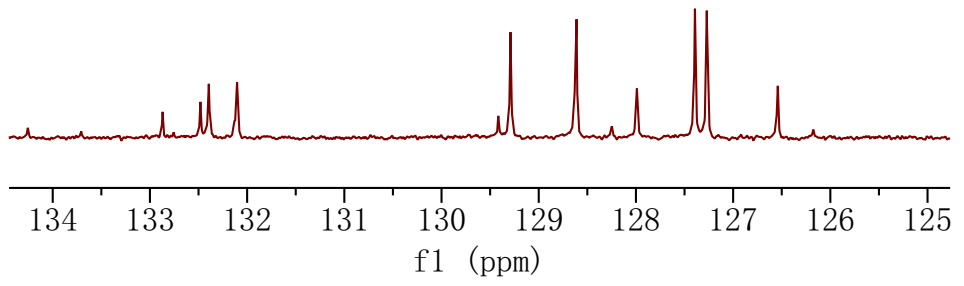



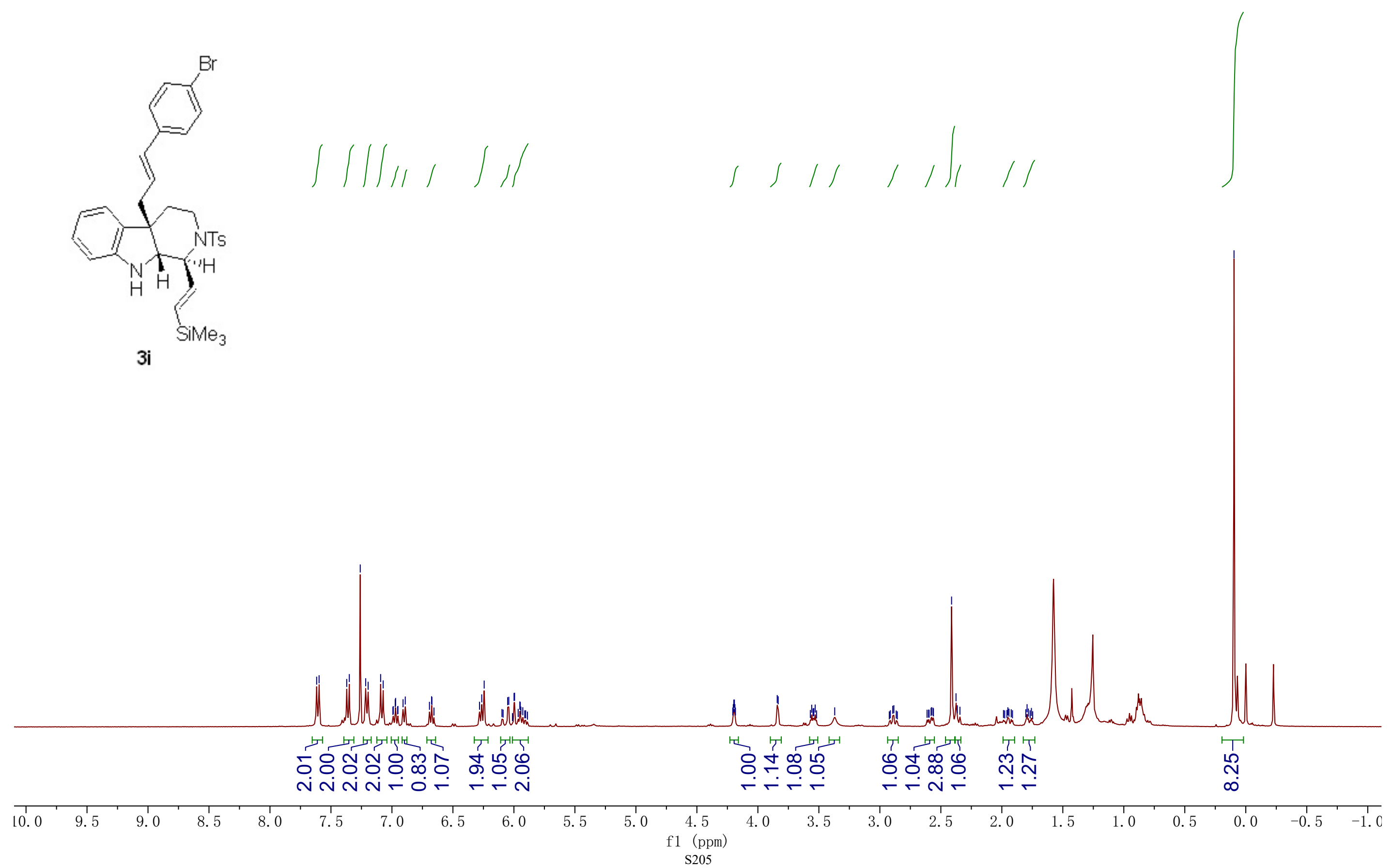

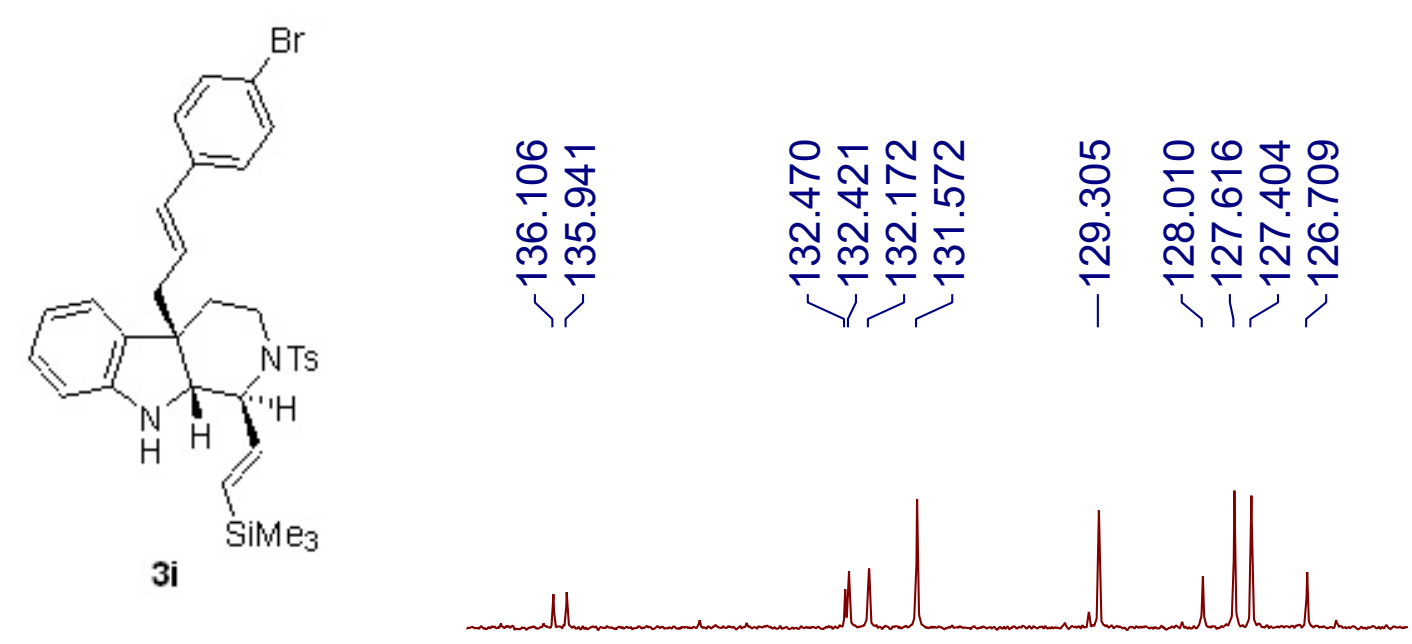

\begin{tabular}{lllllllllllll}
\hline 137 & 136 & 135 & 134 & 133 & 132 & 131 & 130 & 129 & 128 & 127 & 126
\end{tabular} f1 (ppm)
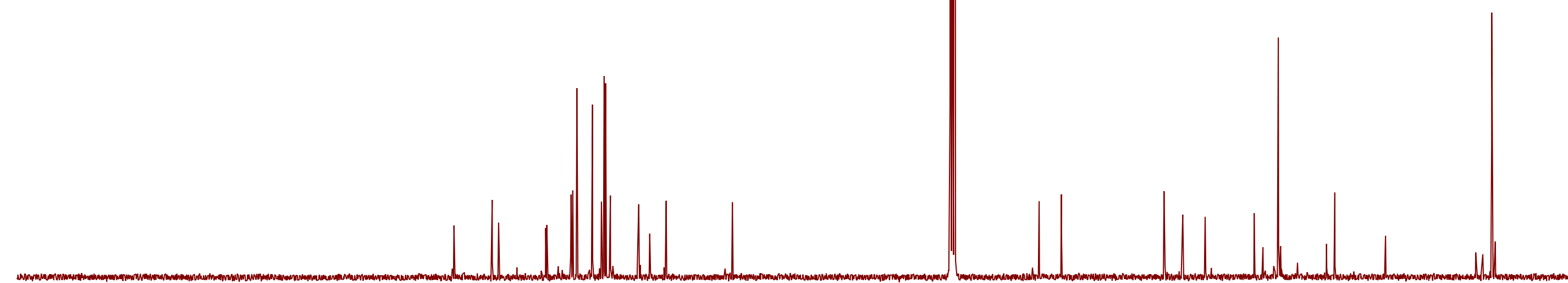

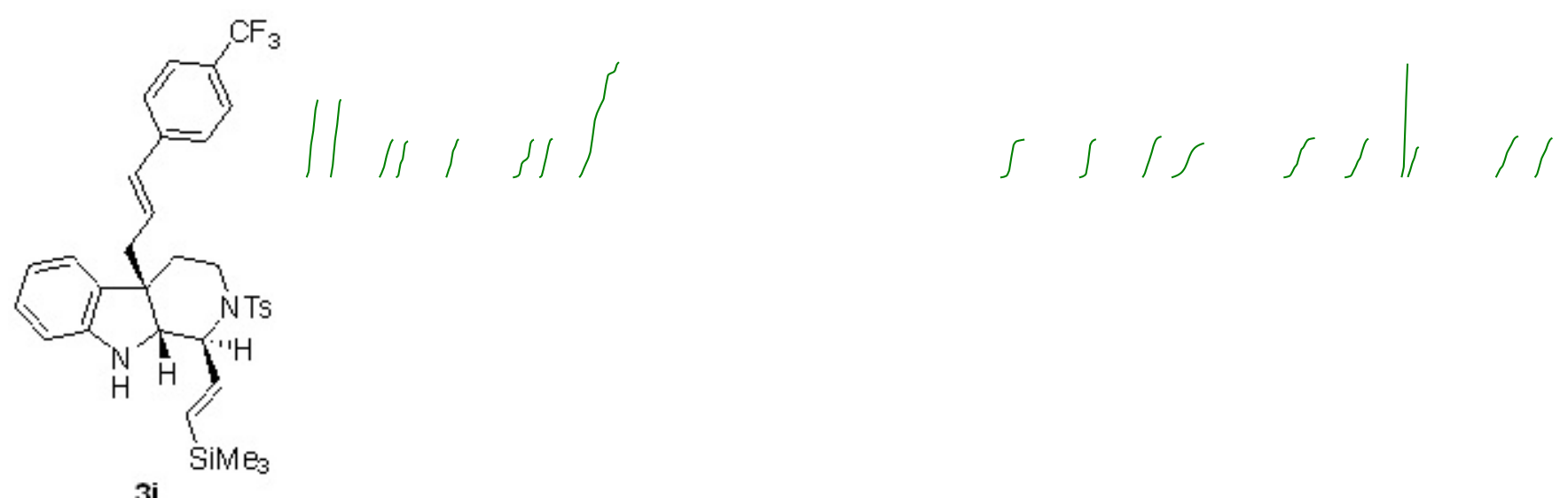

3 j

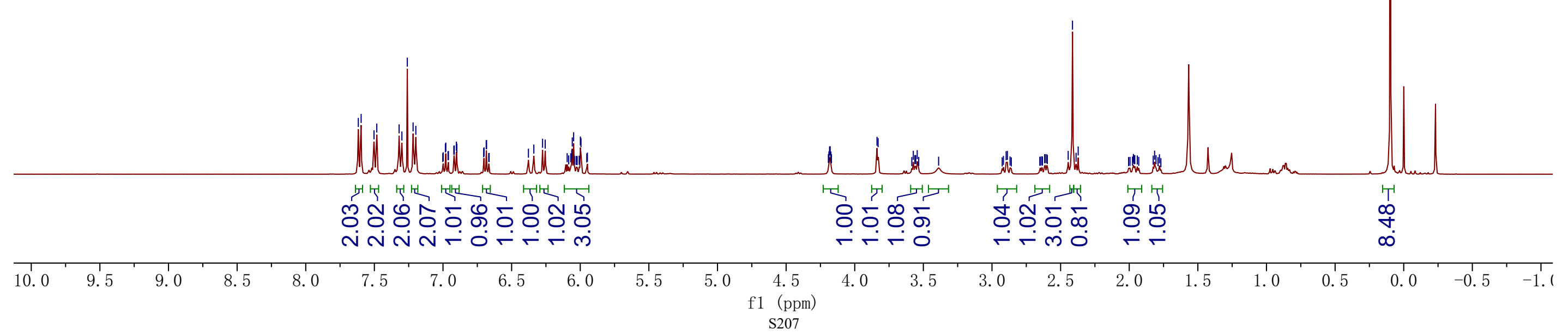




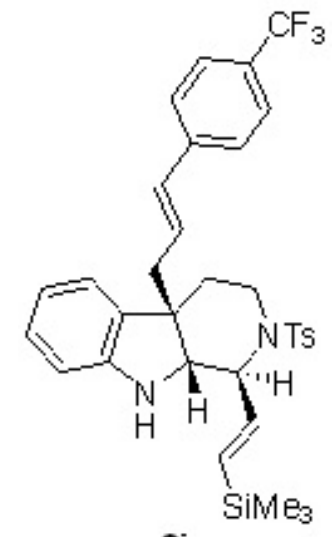

3j

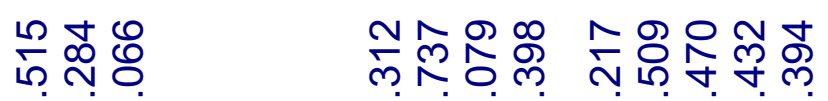

กิ่ กิ่

m $\dddot{m}$

$\stackrel{\sim}{\sim} \stackrel{\sim}{N} \stackrel{\sim}{N} \stackrel{\sim}{N} \stackrel{\sim}{N}$
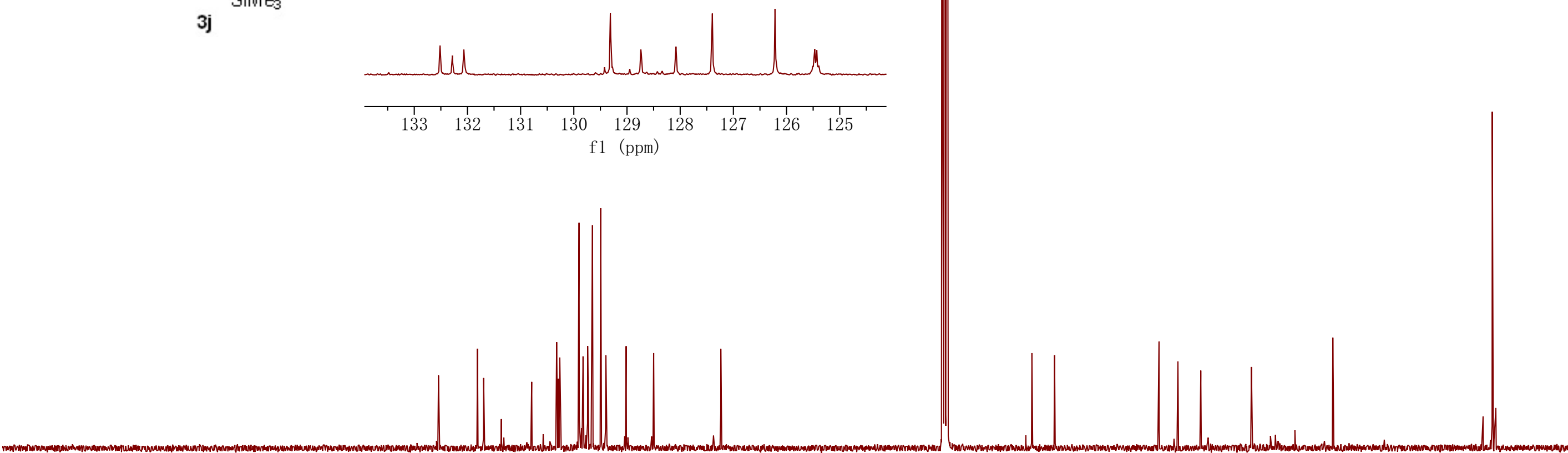

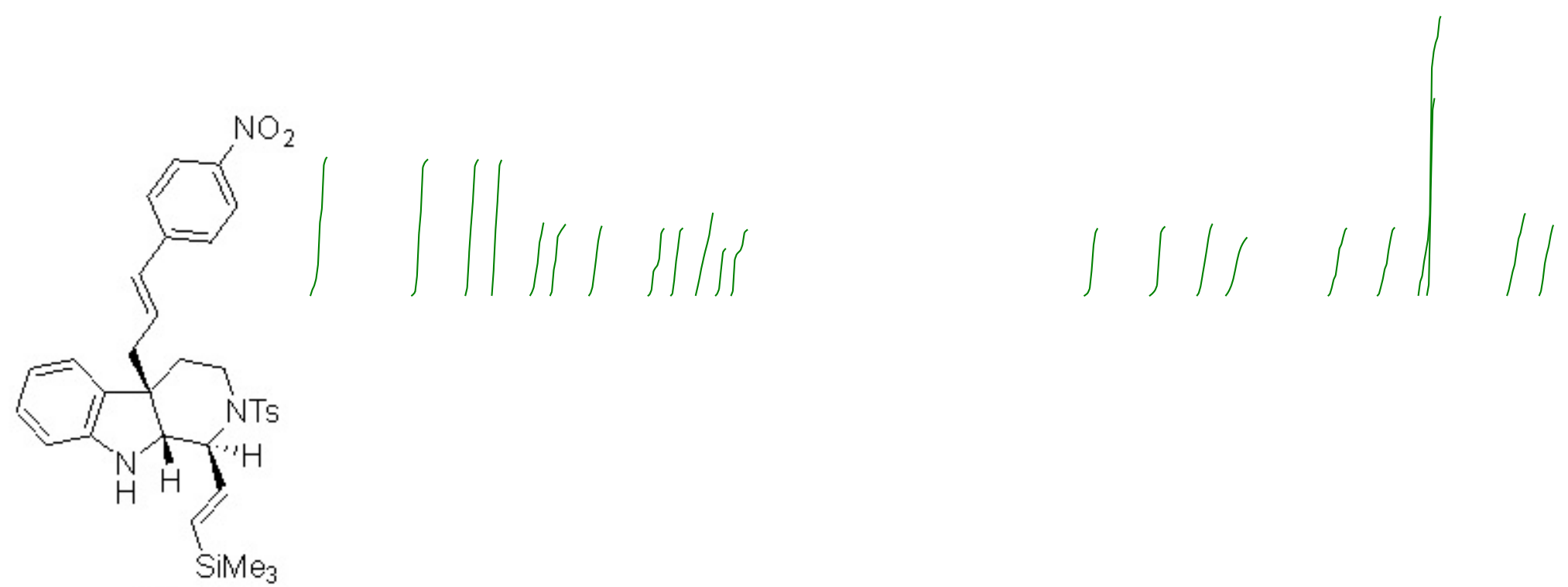

$3 \mathrm{k}$

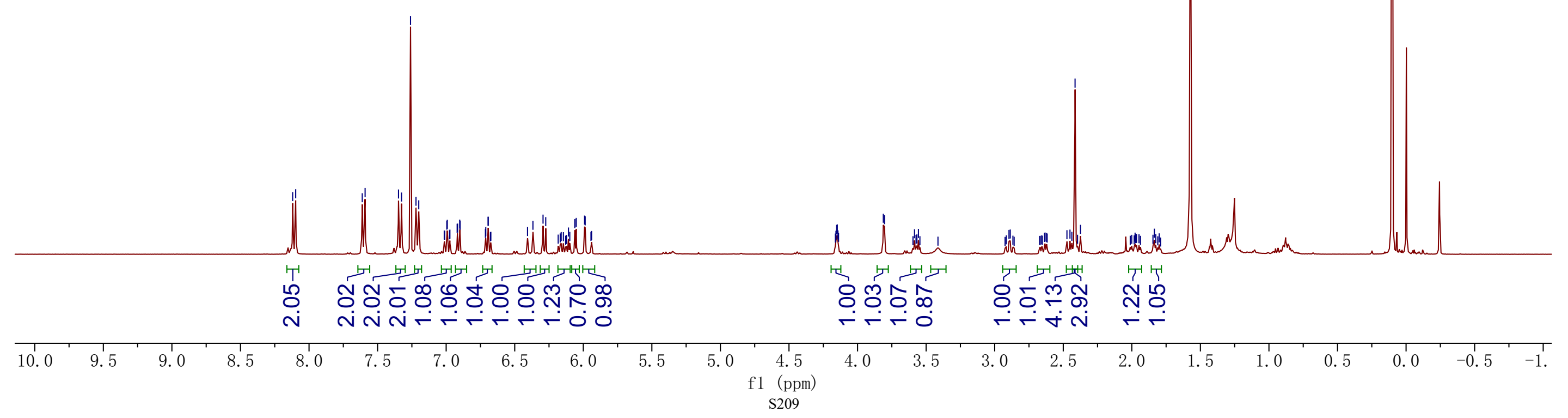




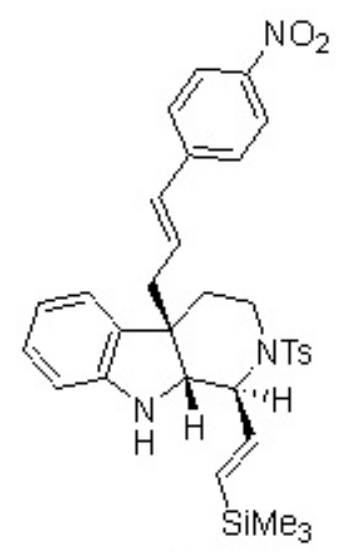

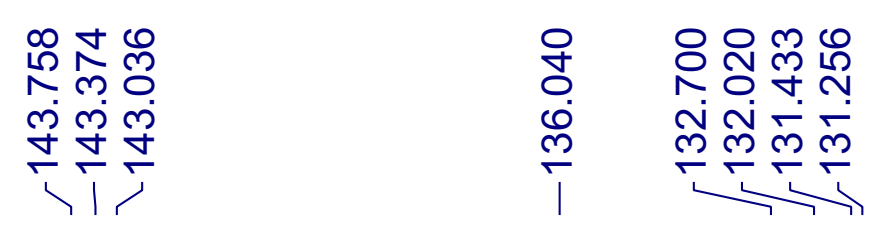

3k

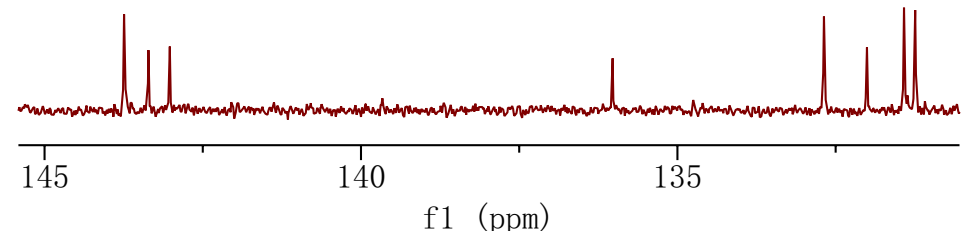

f1 (ppm) 200 

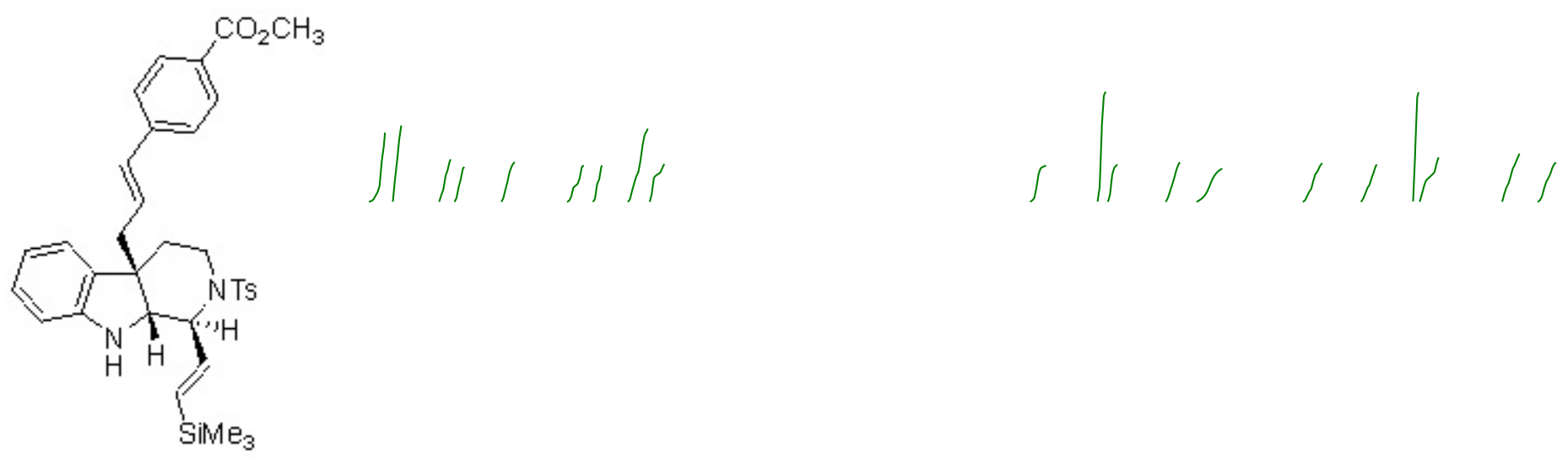

31

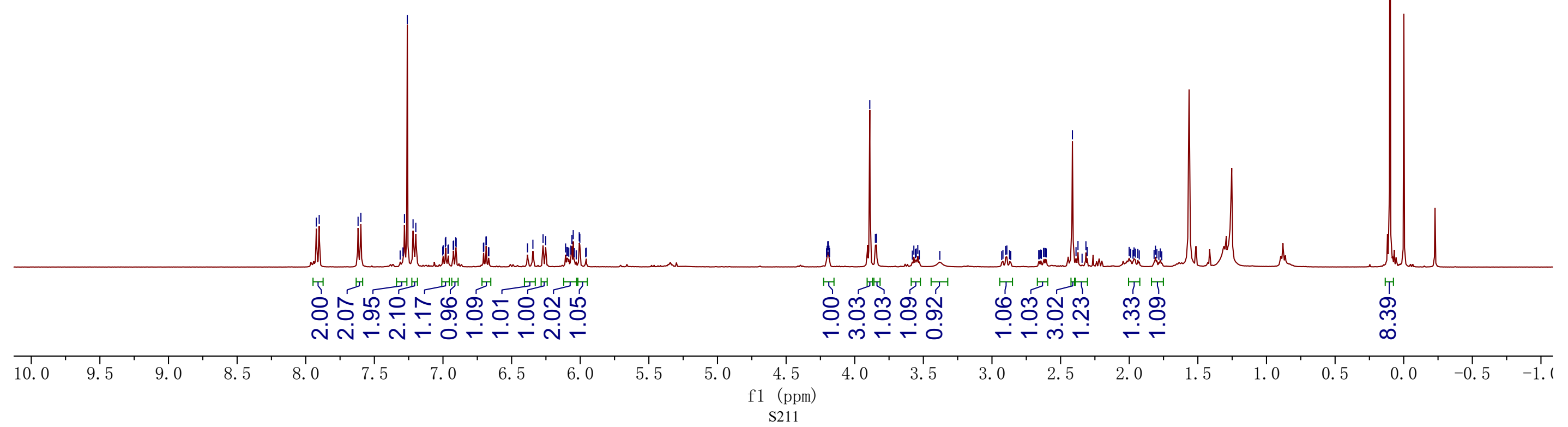



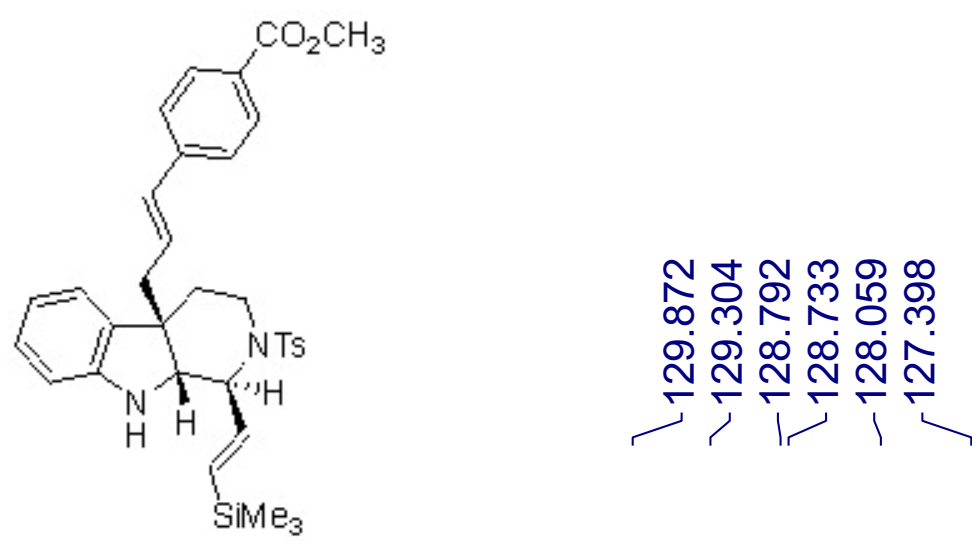

31
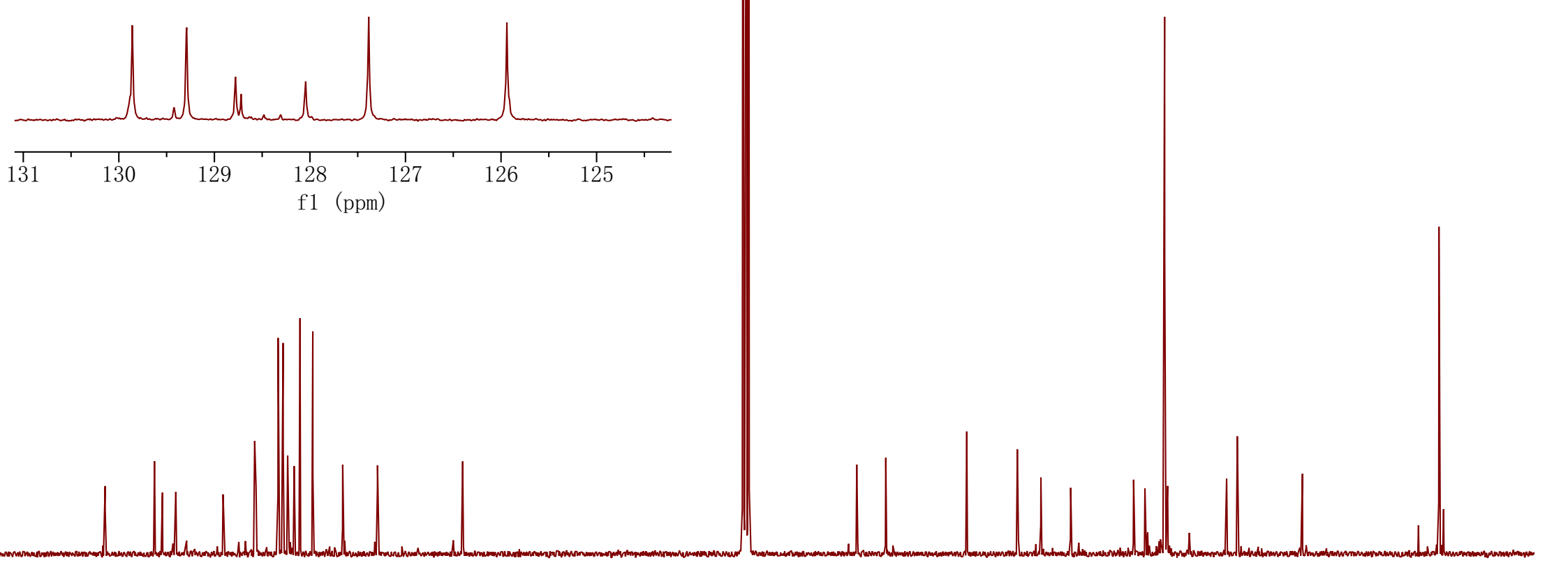

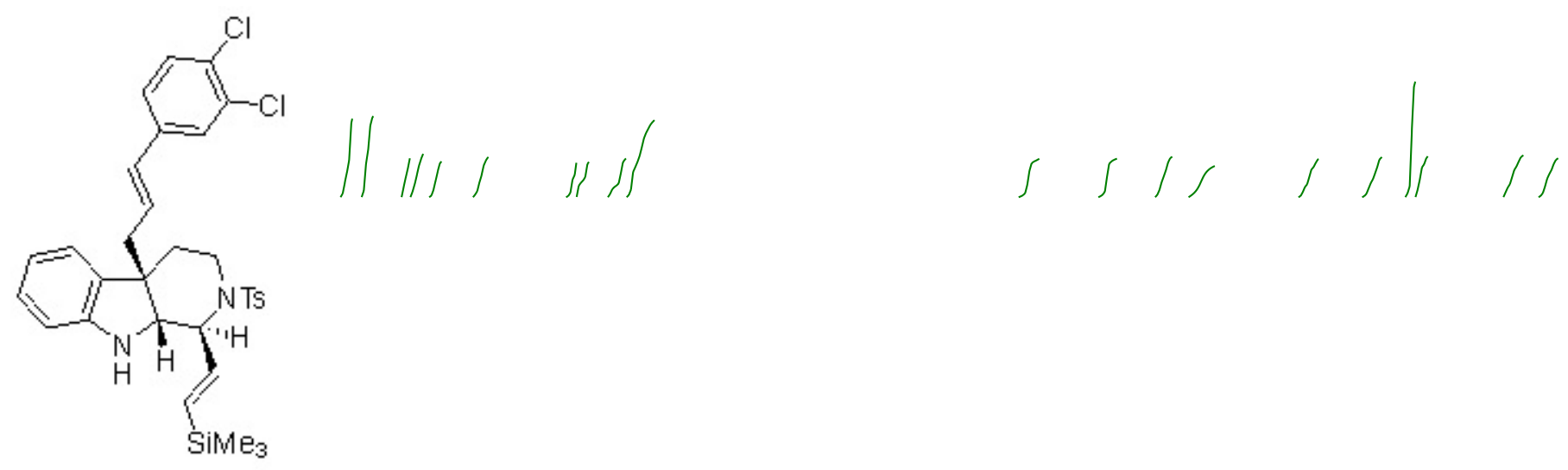

$3 m$

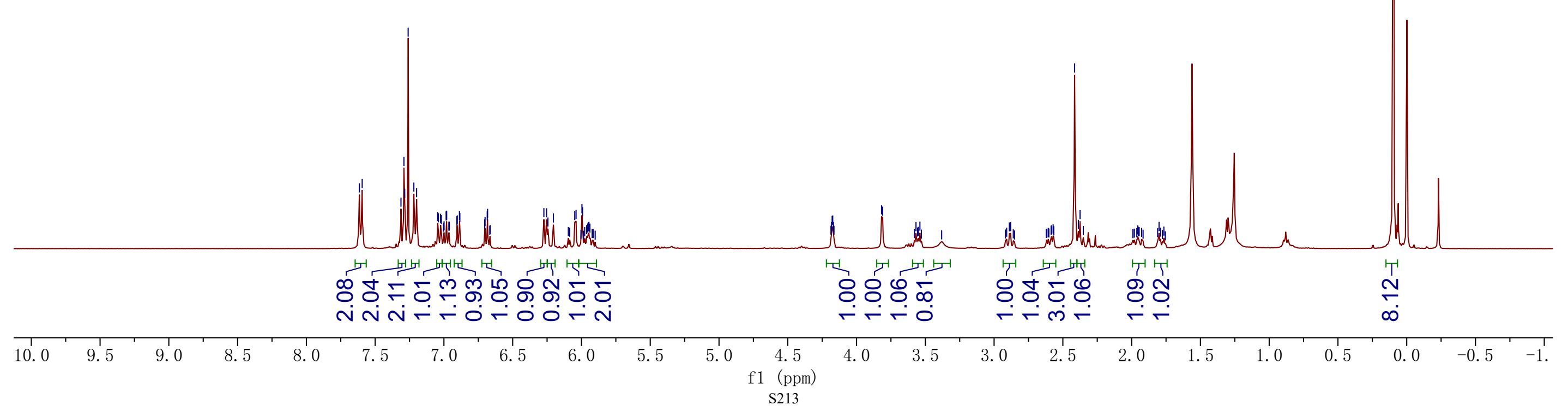




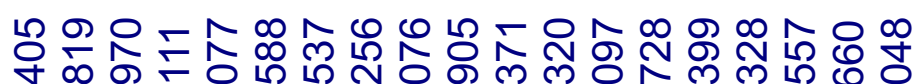

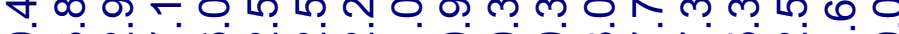

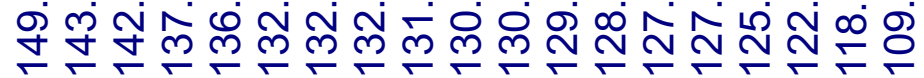

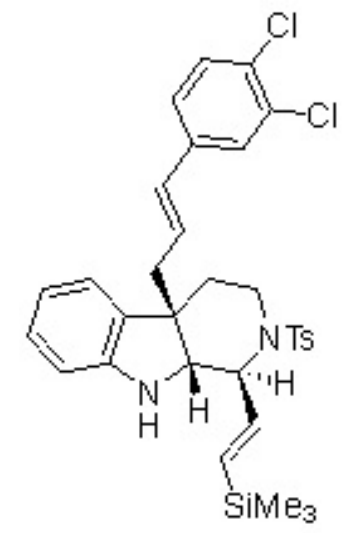

$3 m$
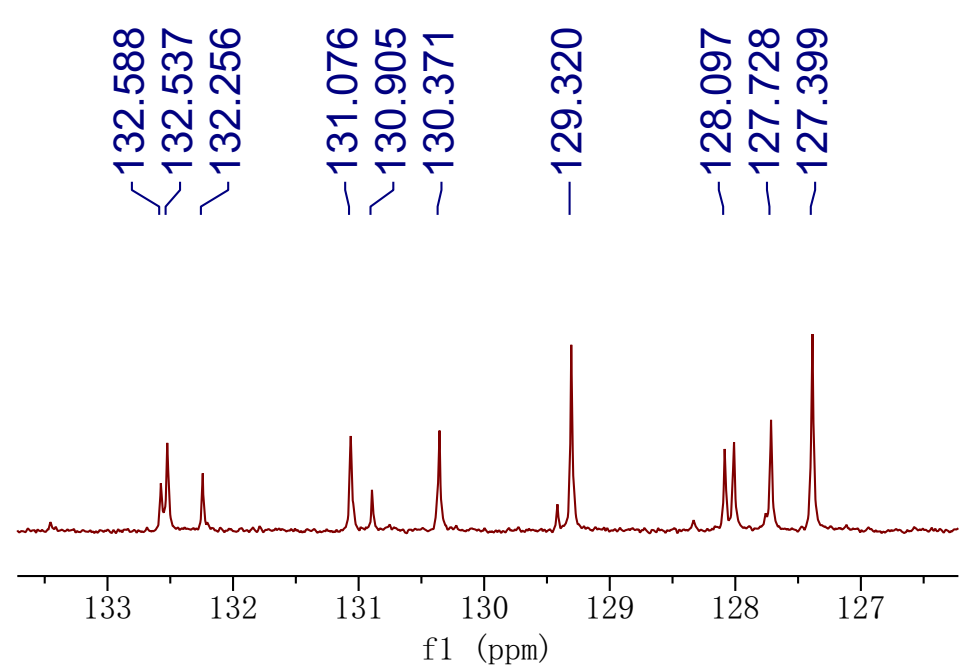


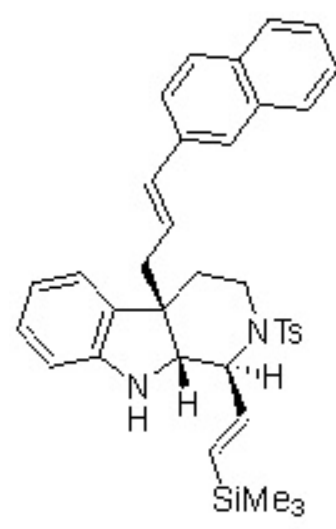

3n

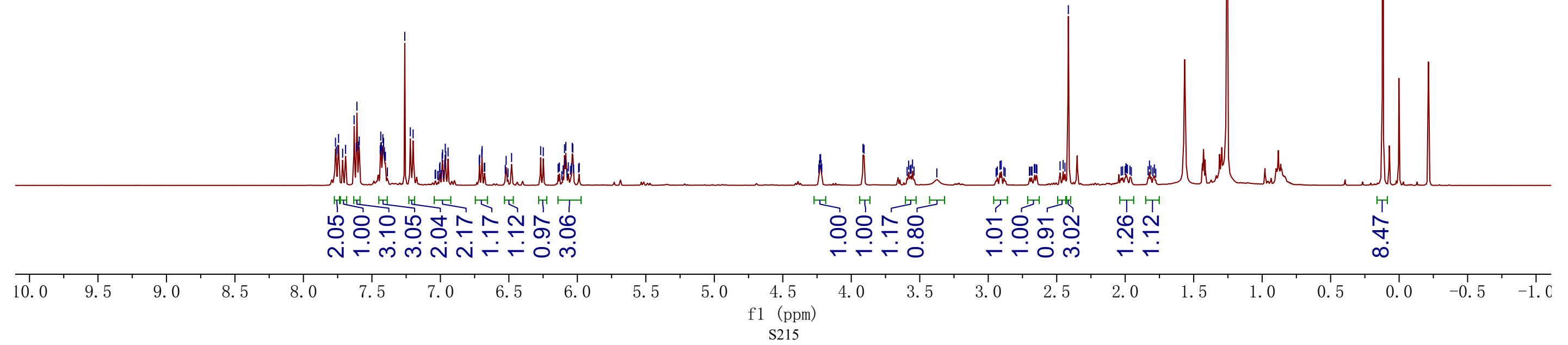




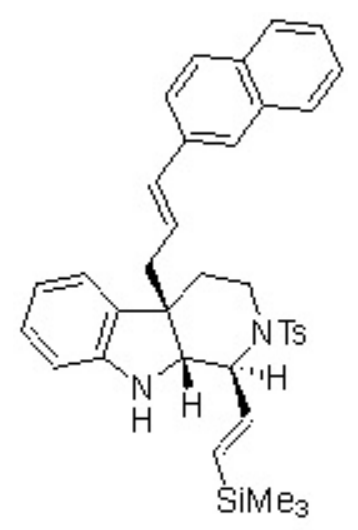

3n
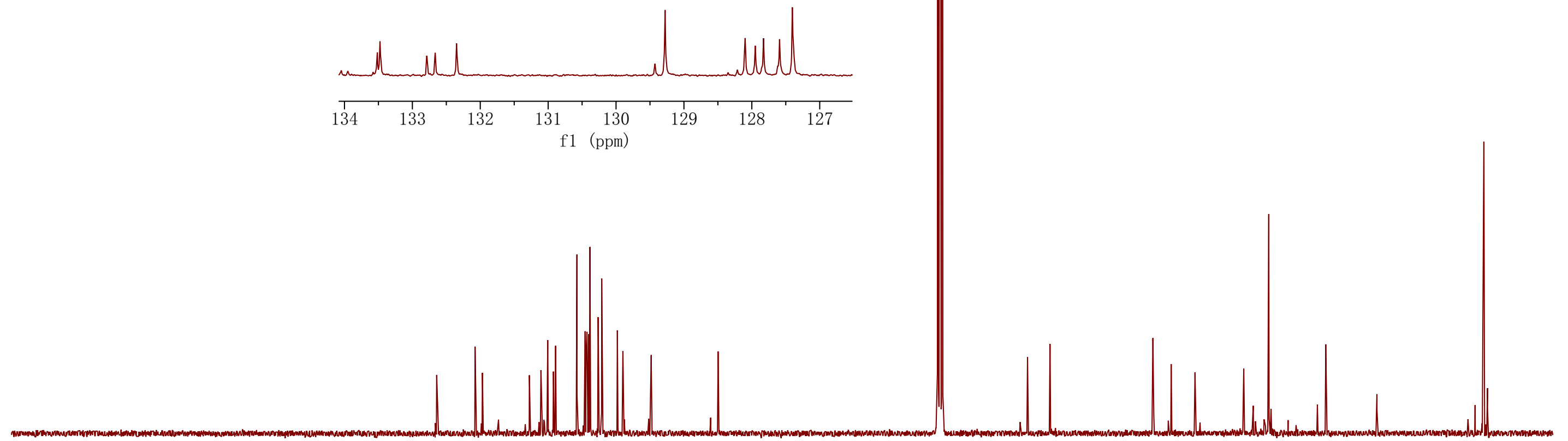

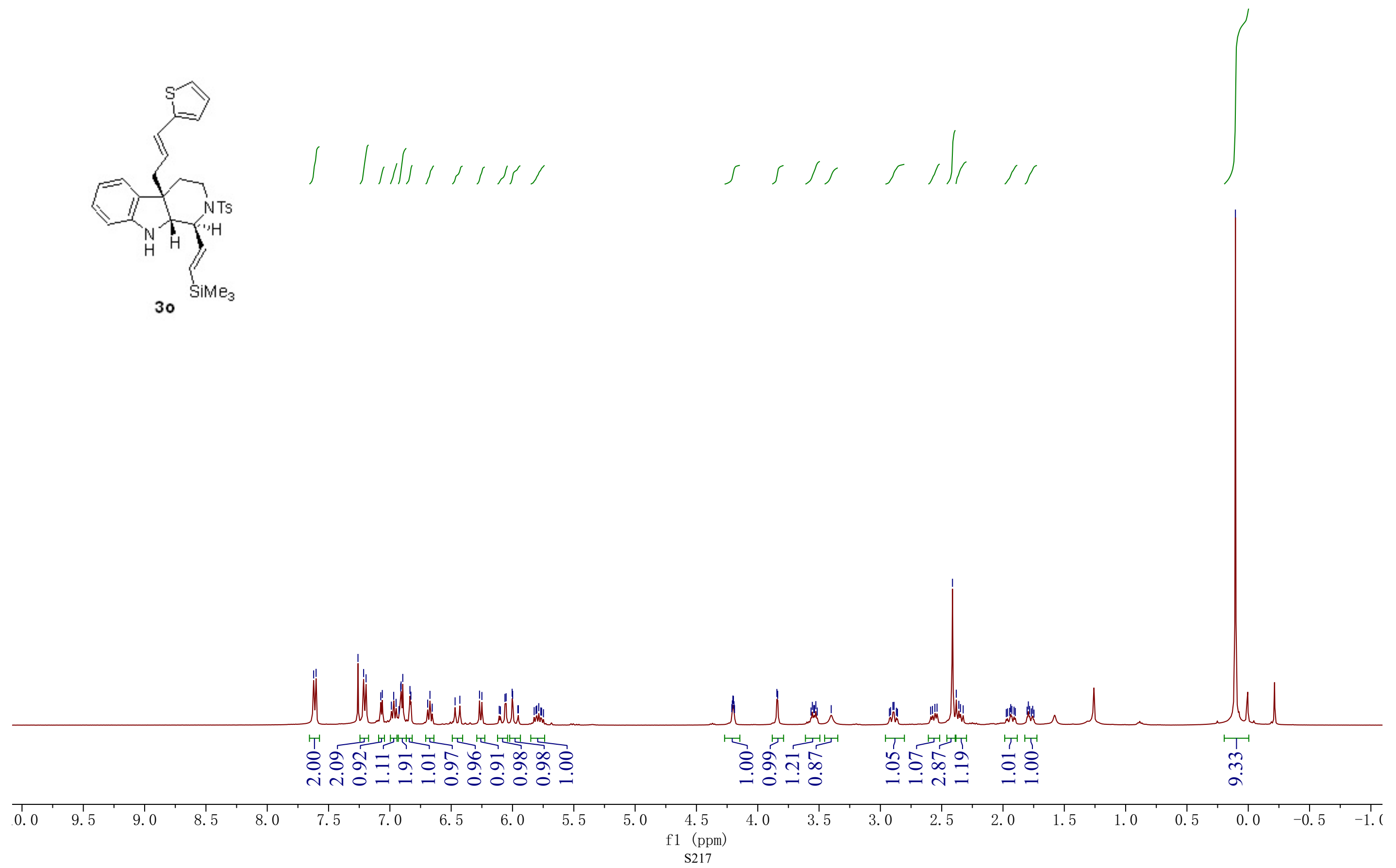

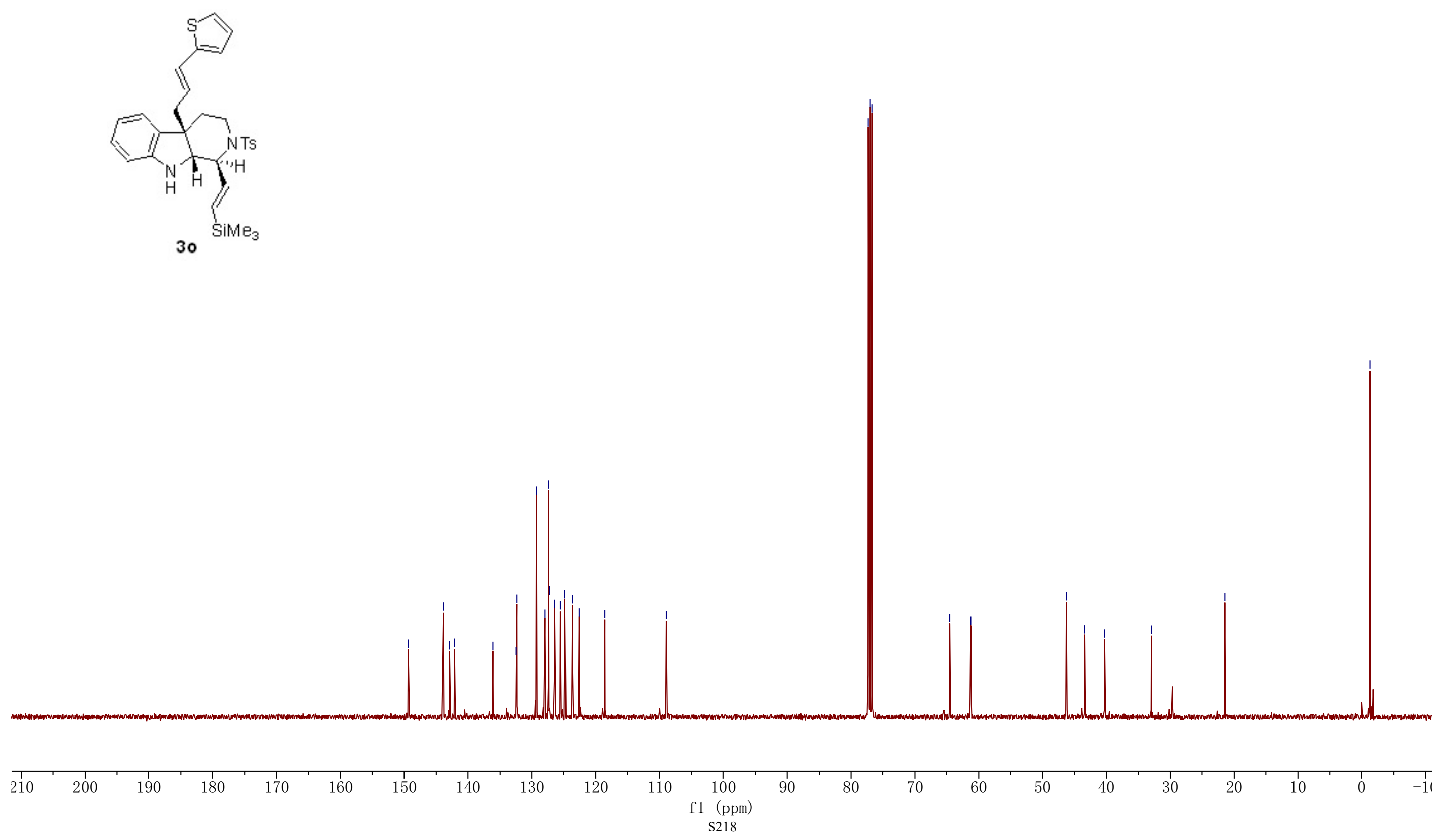

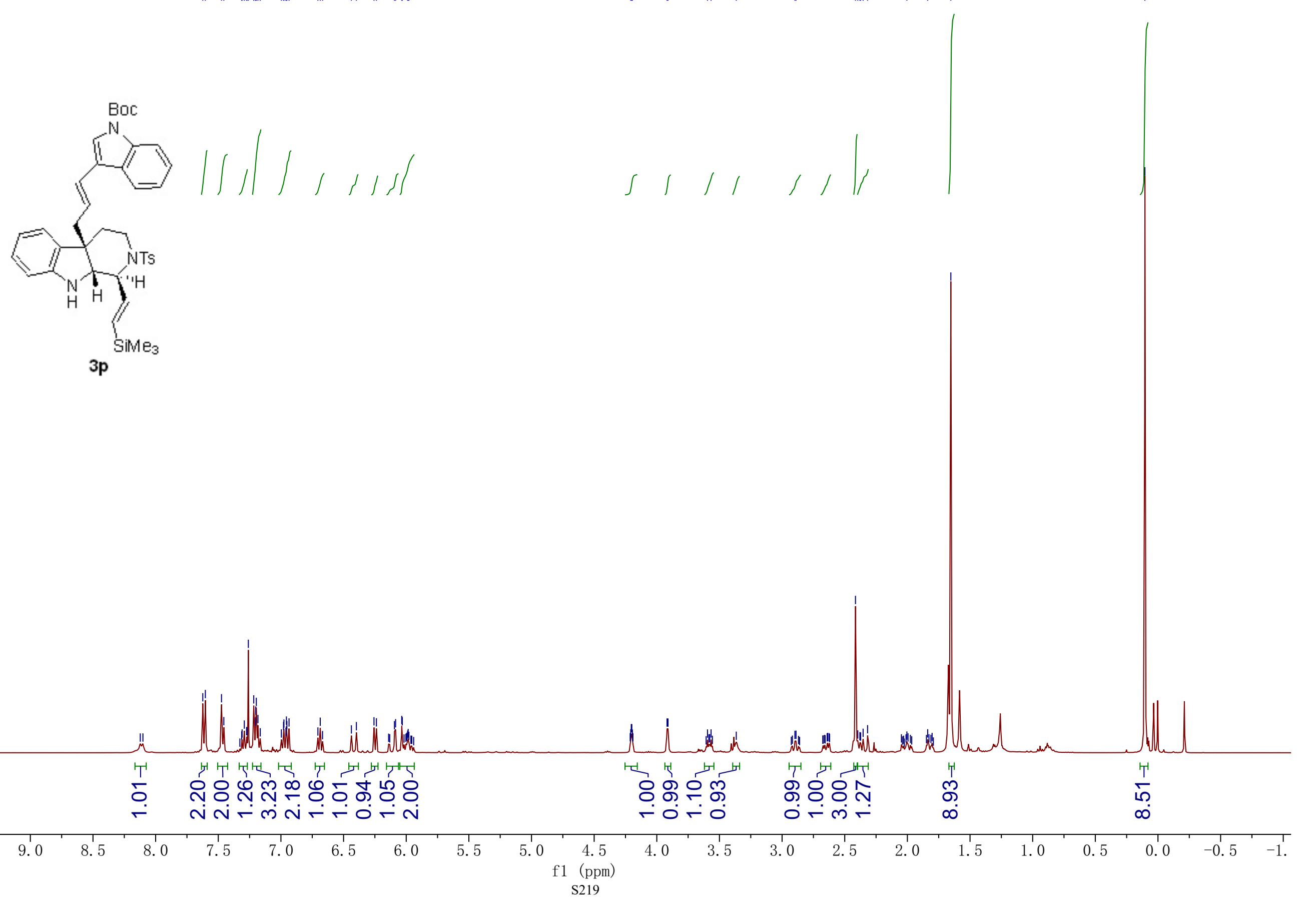

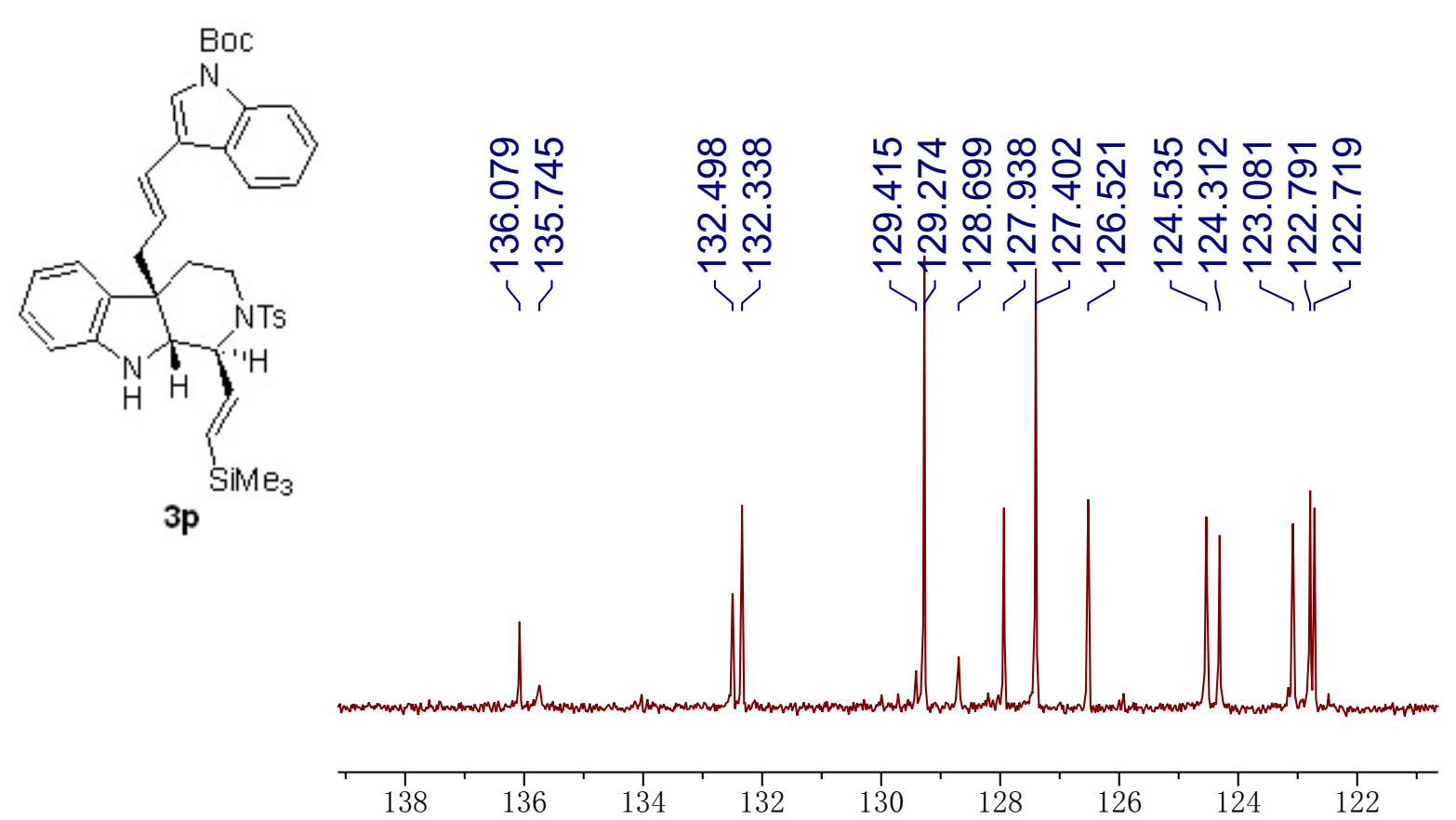

1 ( ppm)

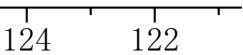

(1)

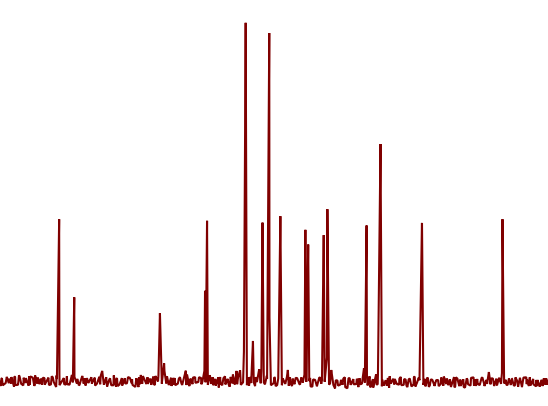



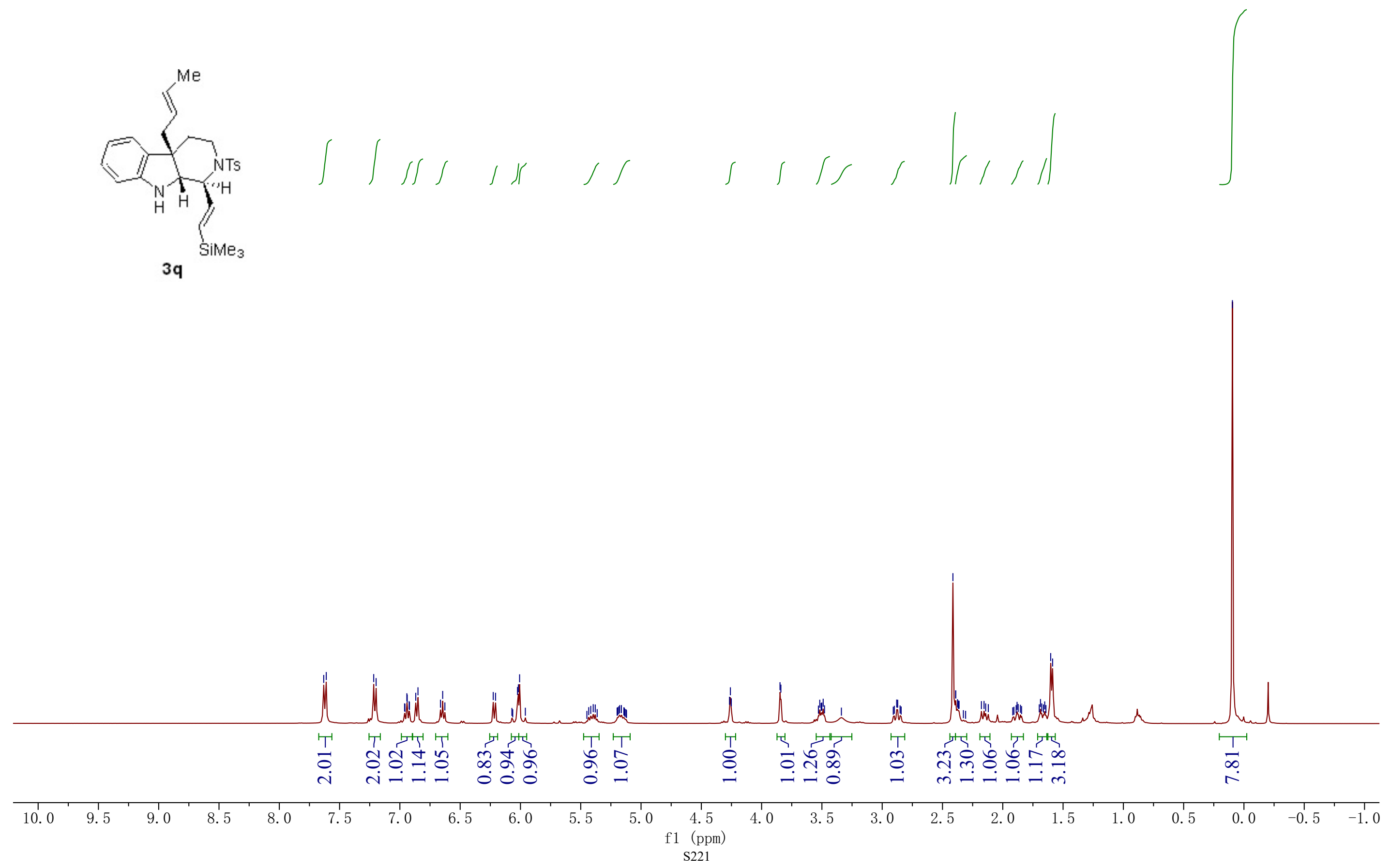


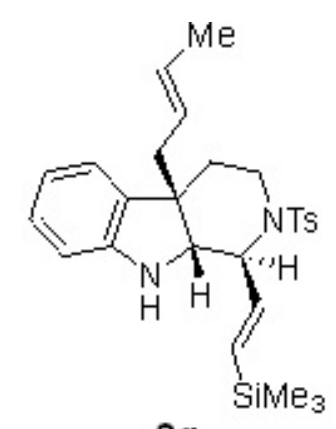

$3 q$

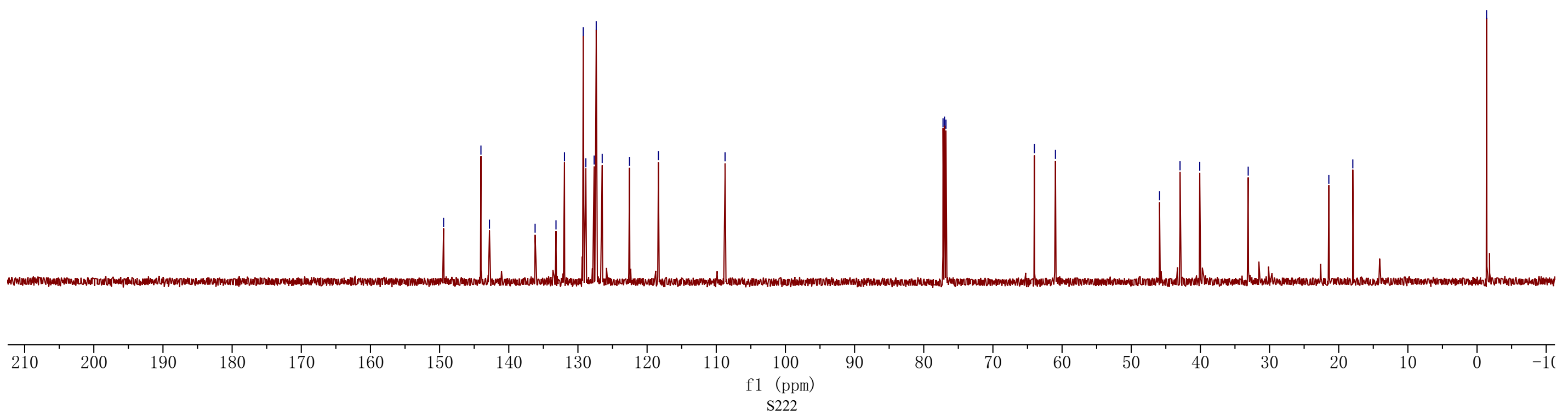



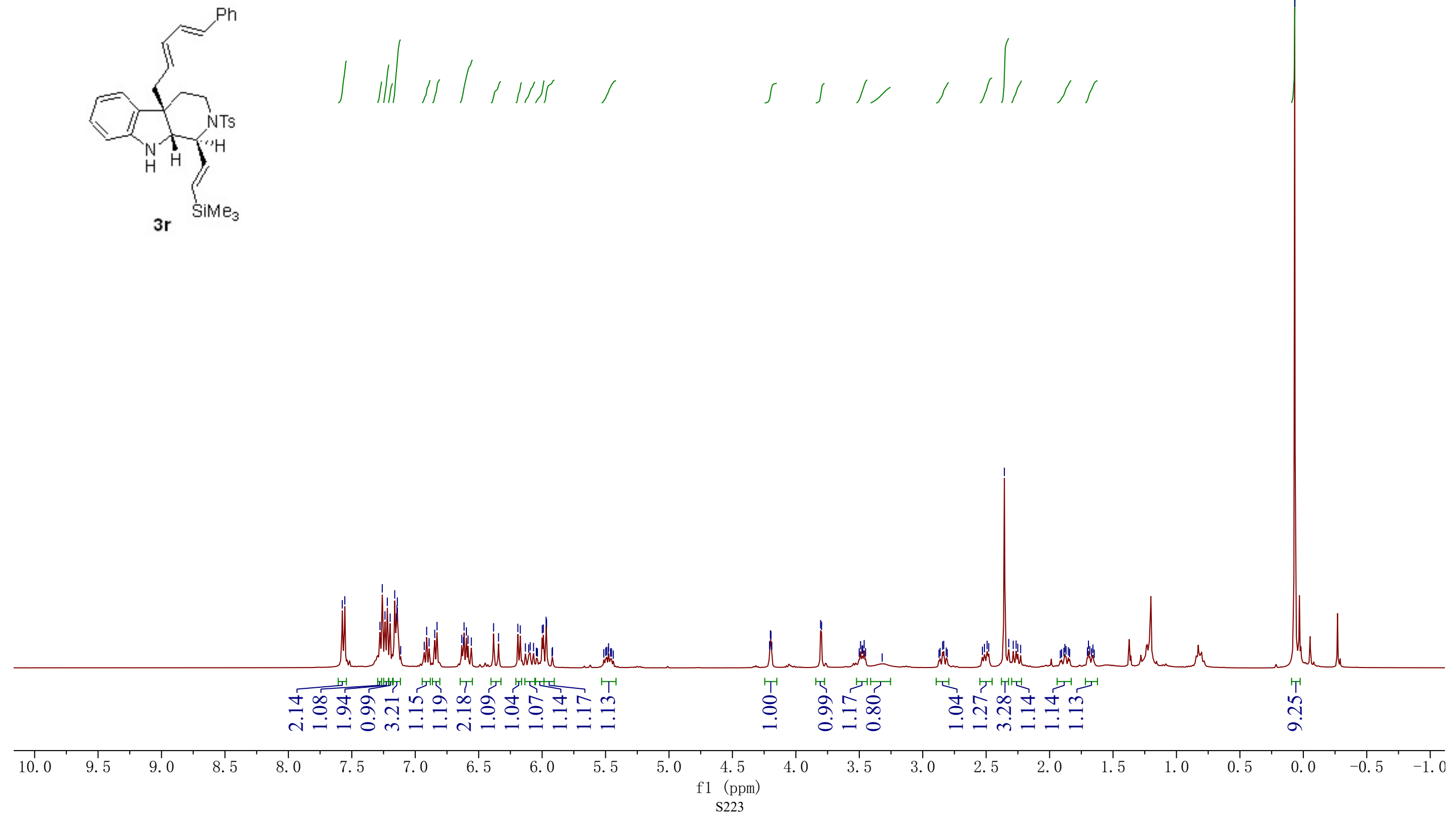

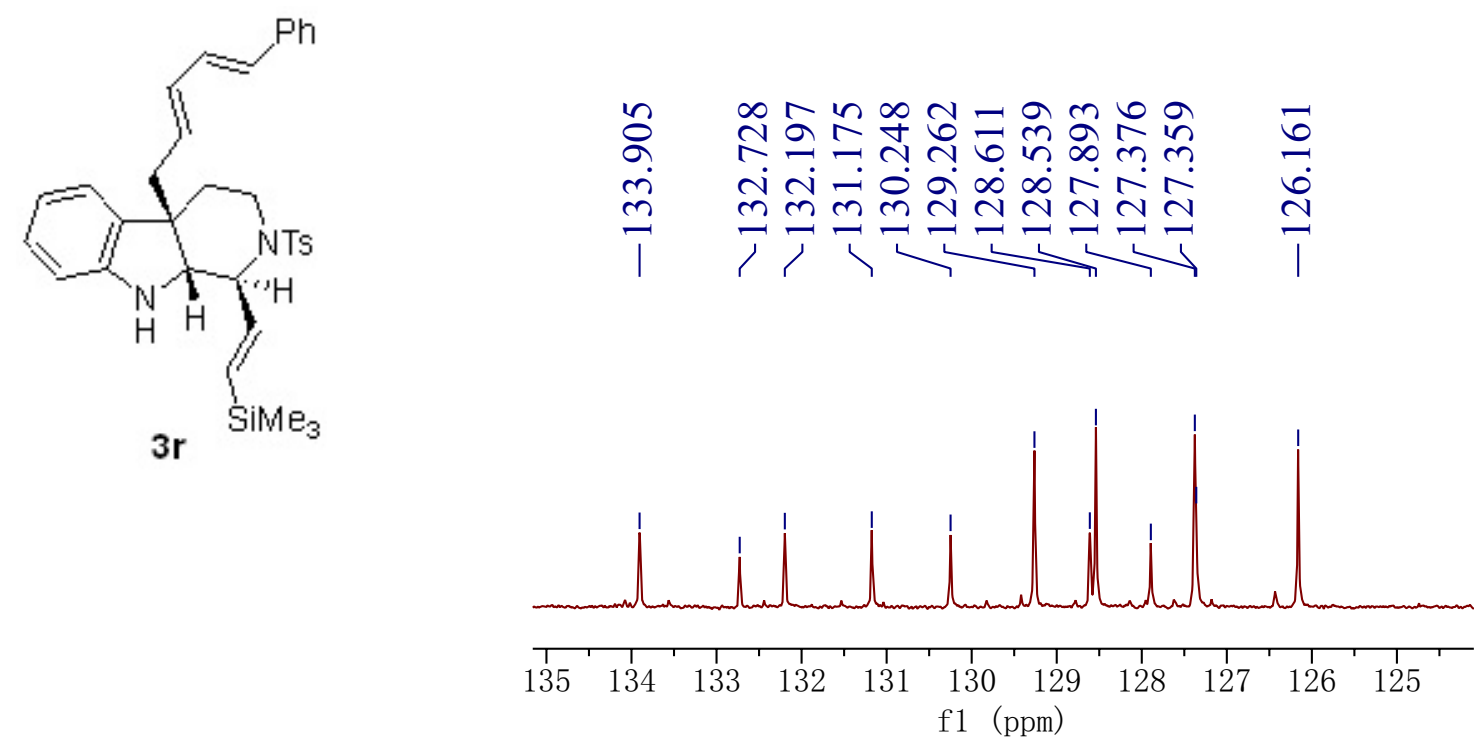

f1 (ppm)
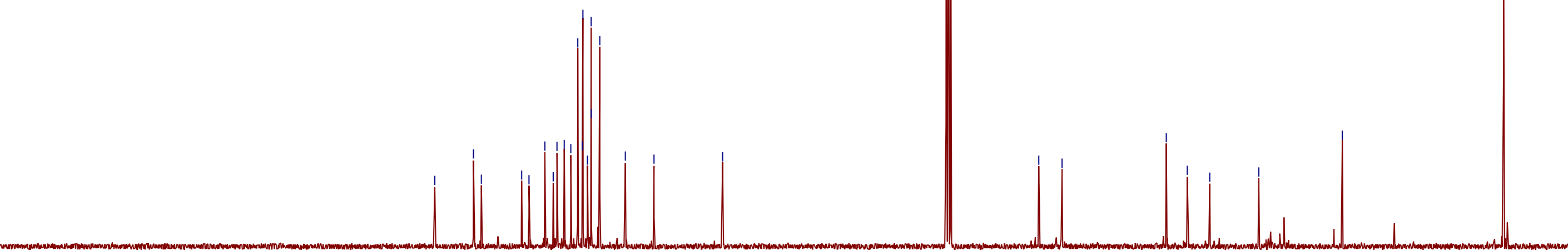

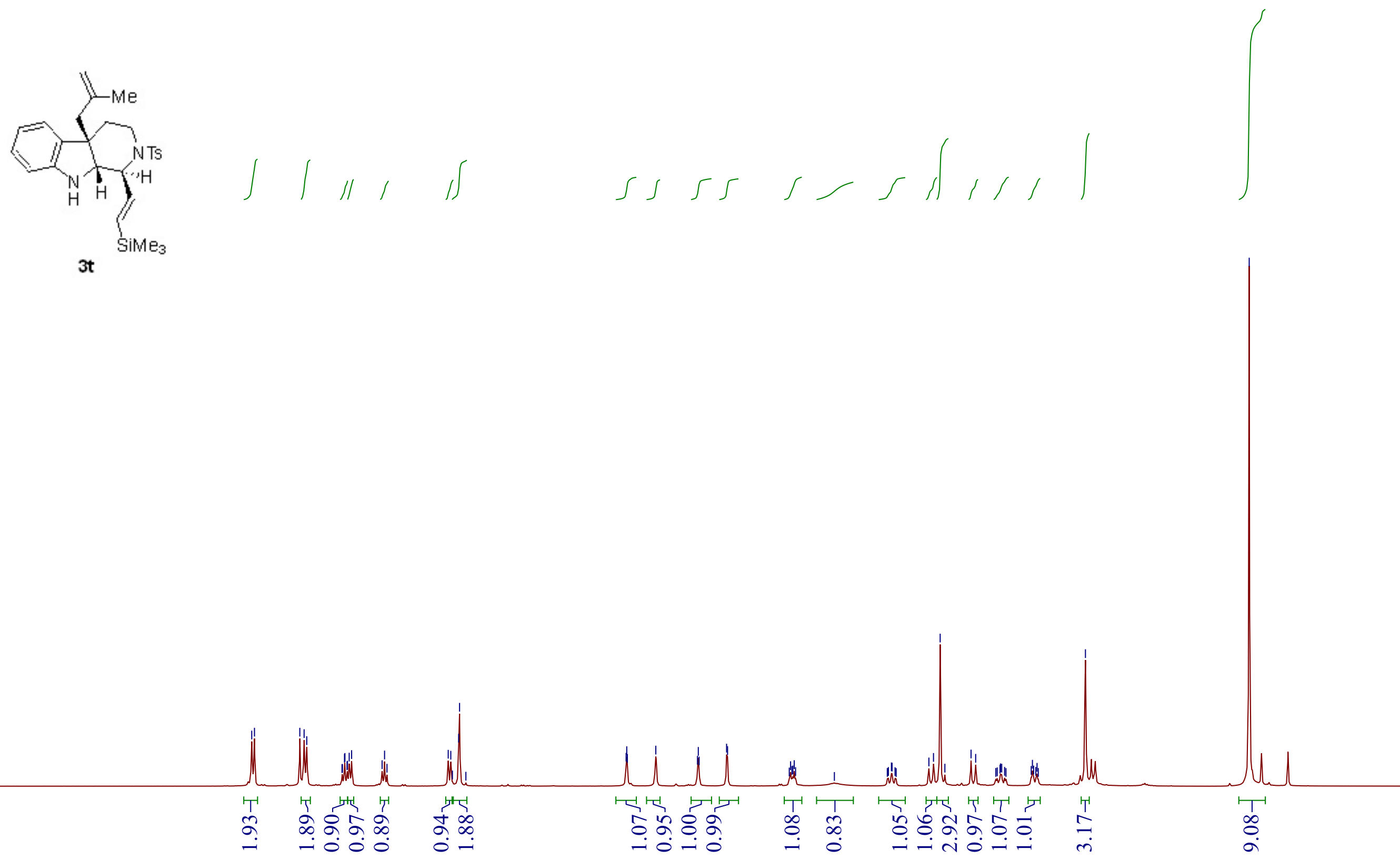


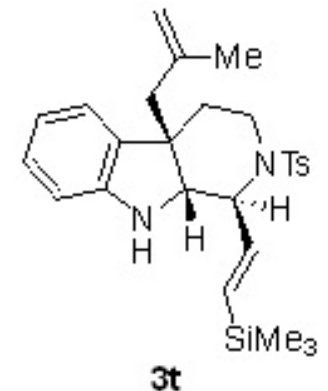

$3 t$

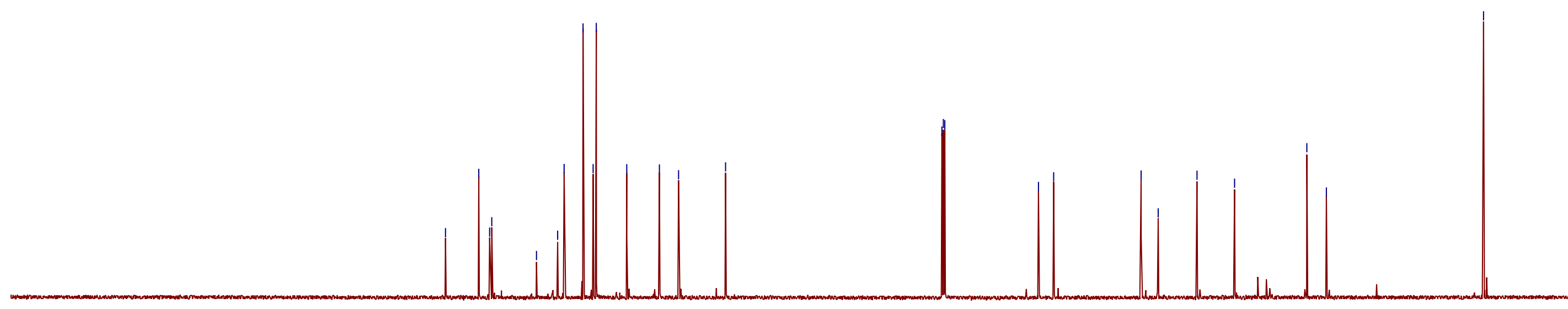



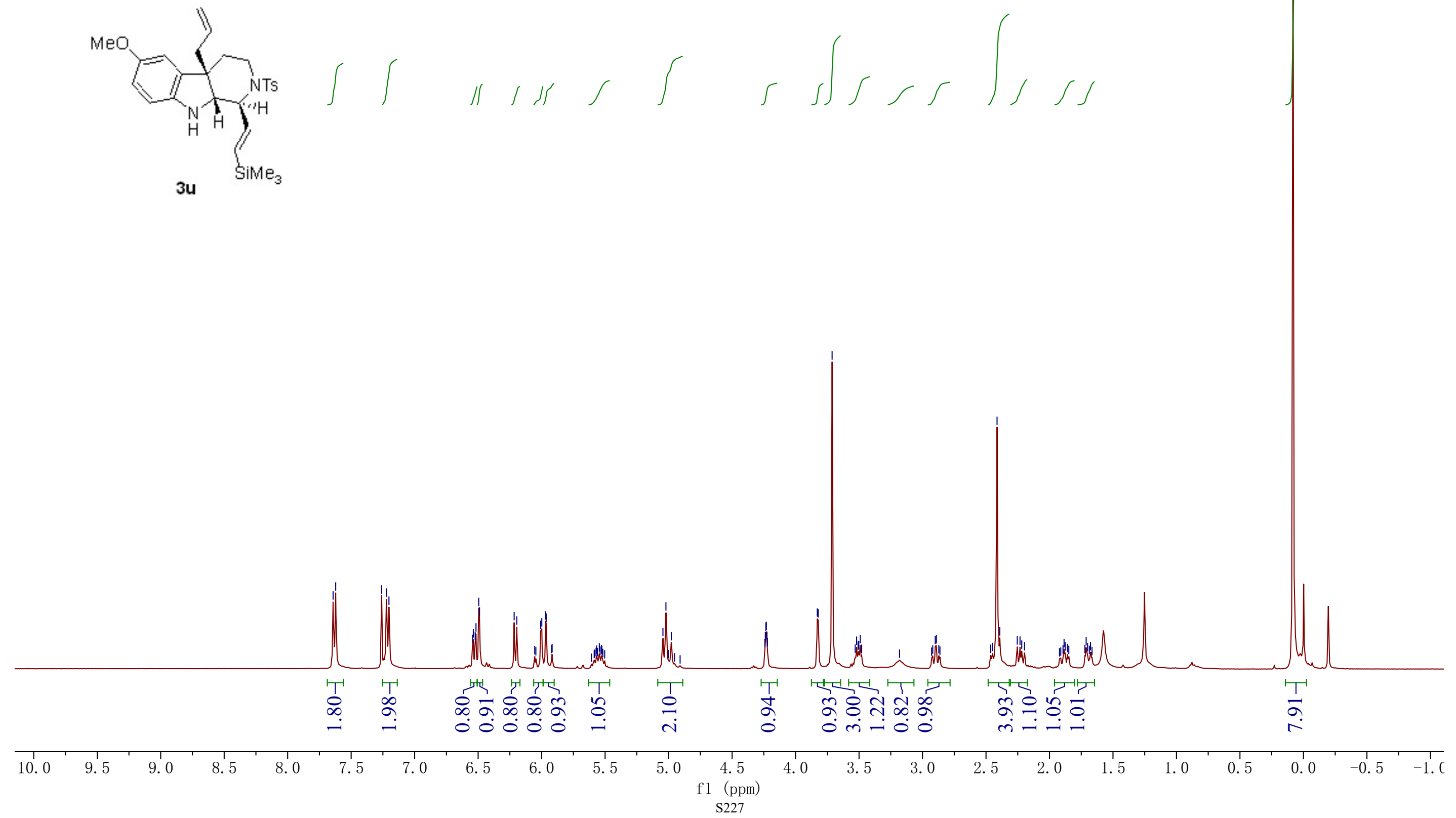


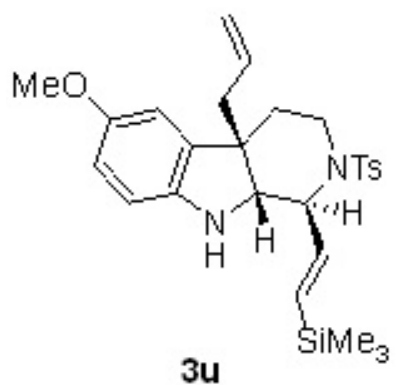

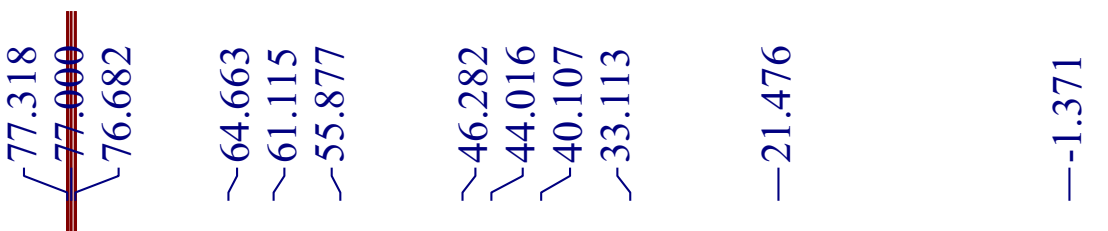



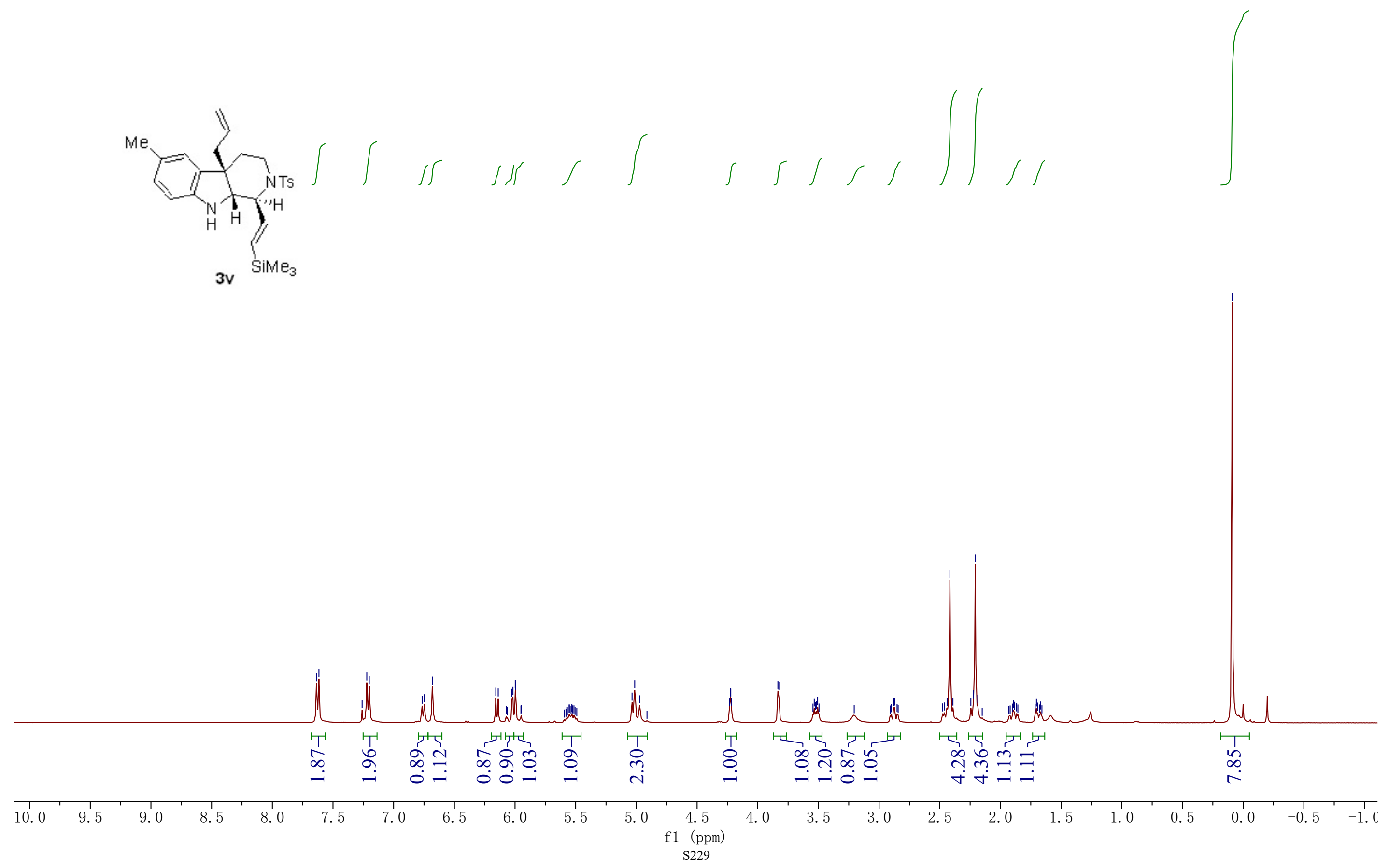

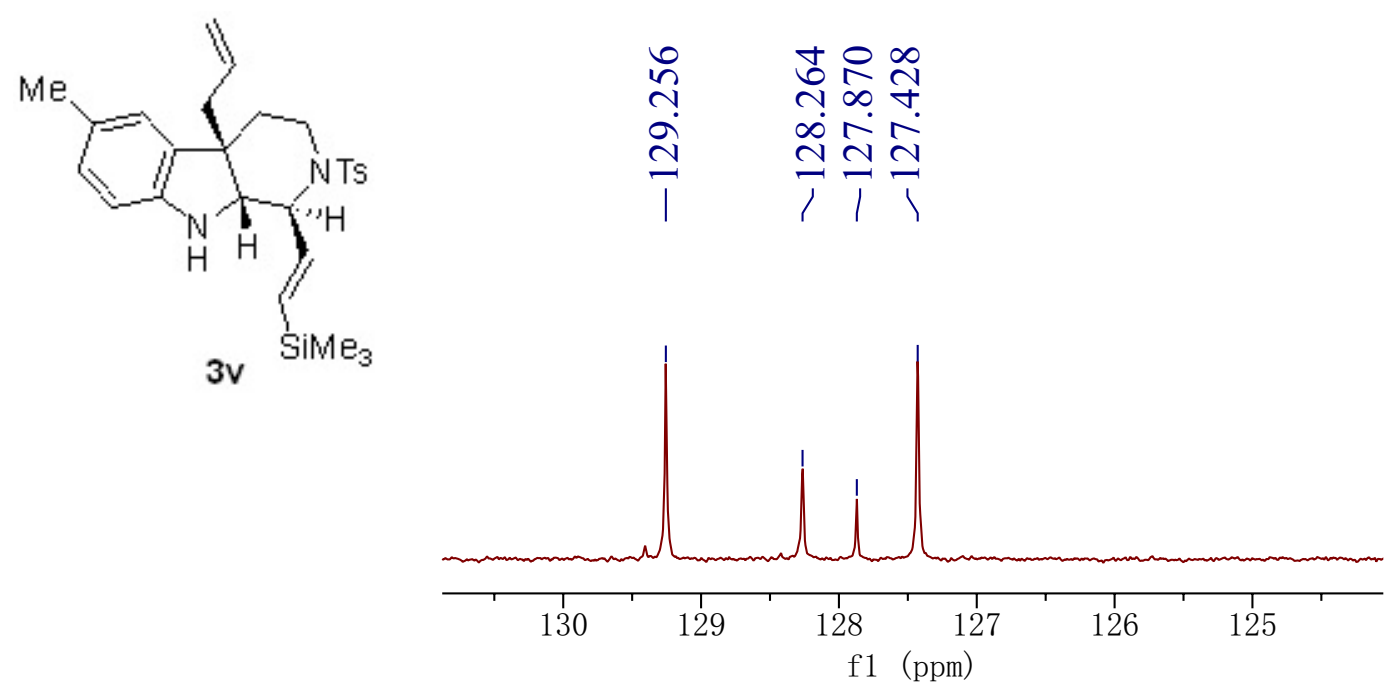

3

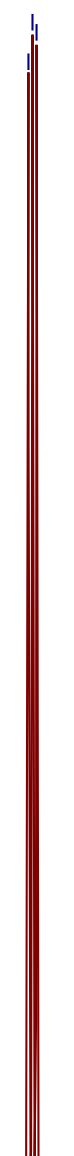




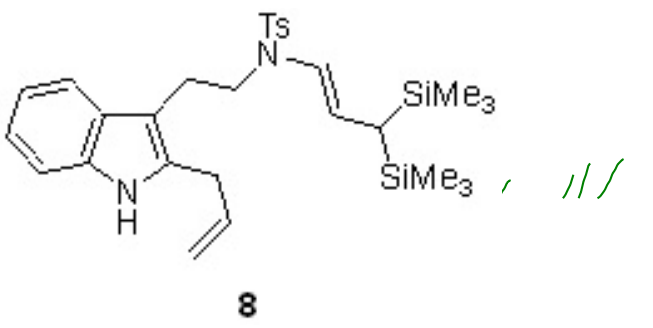

minn

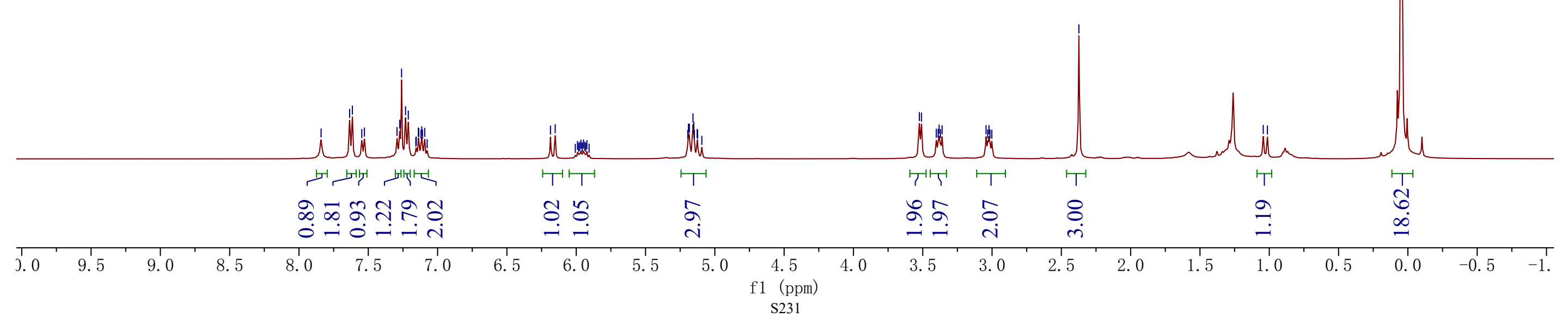



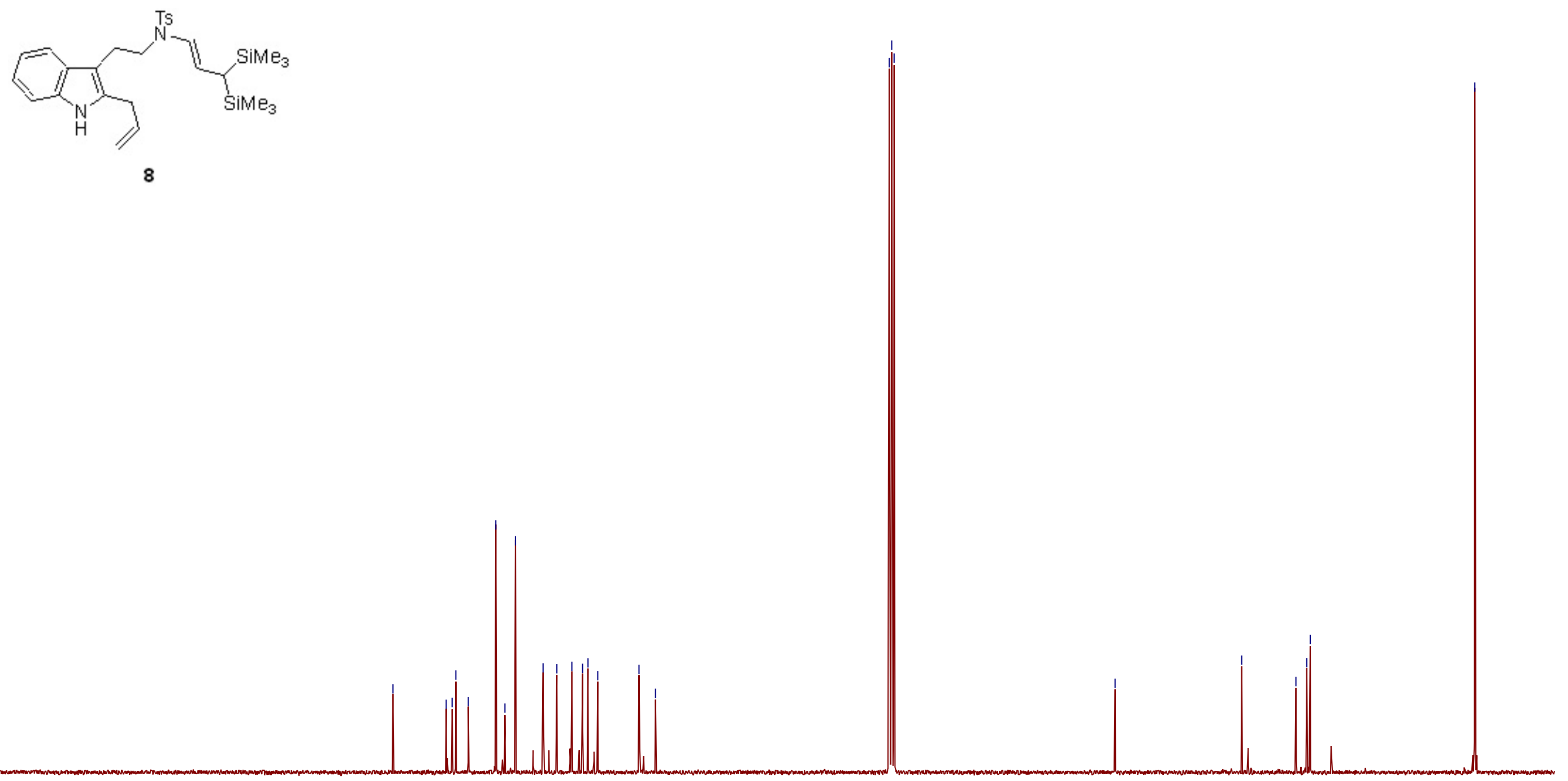

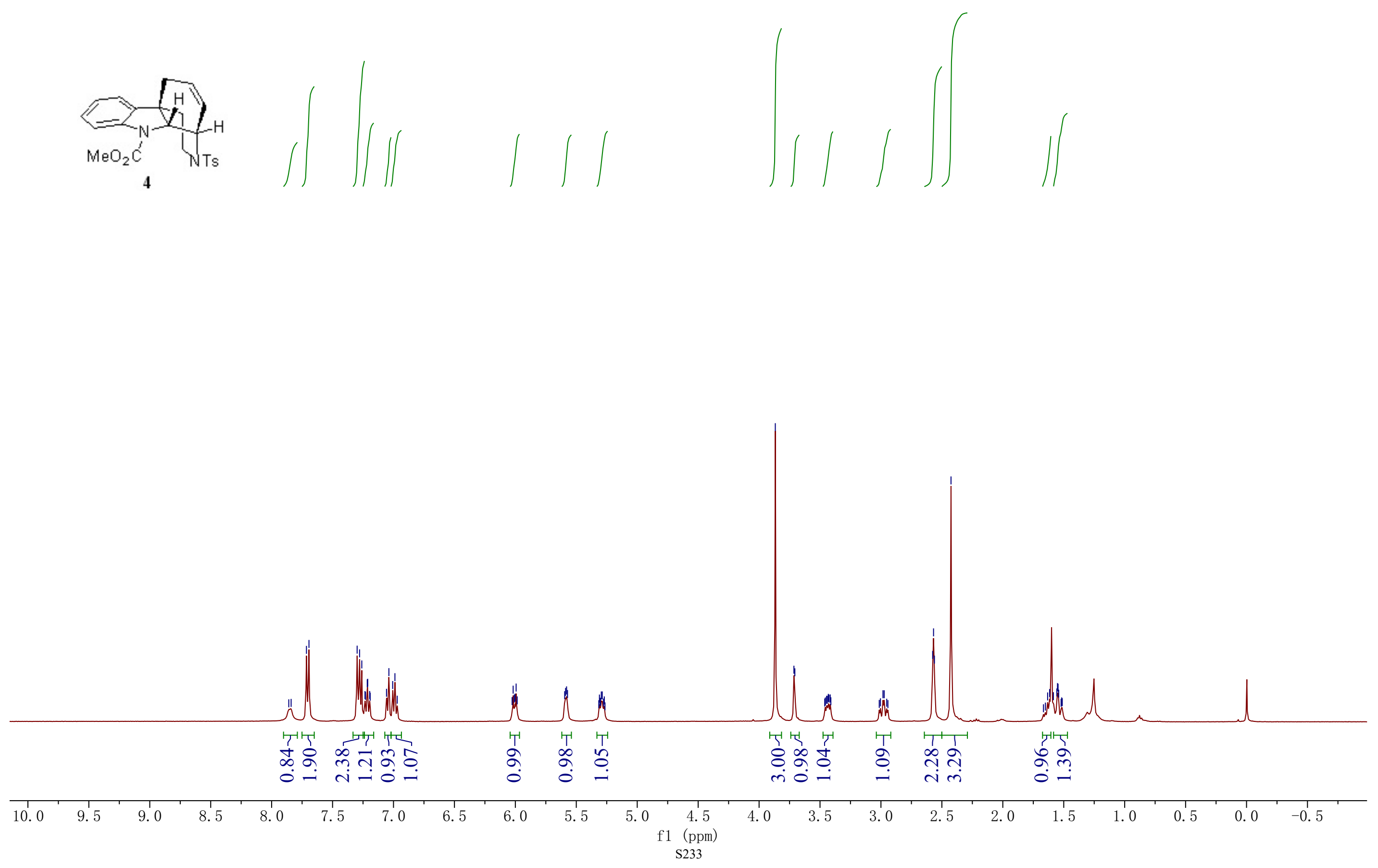


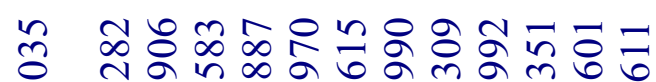

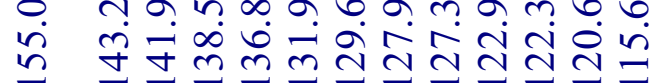

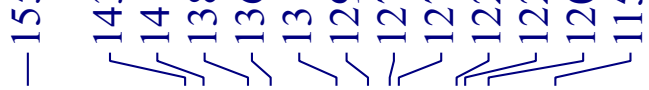

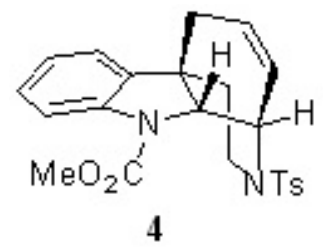

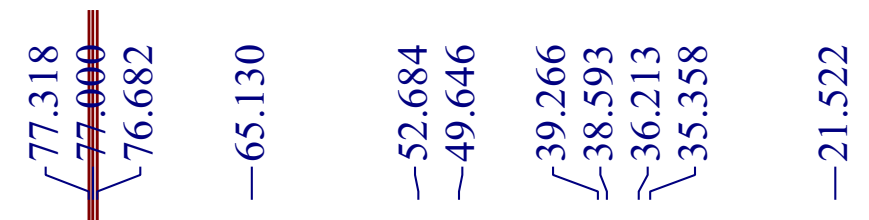

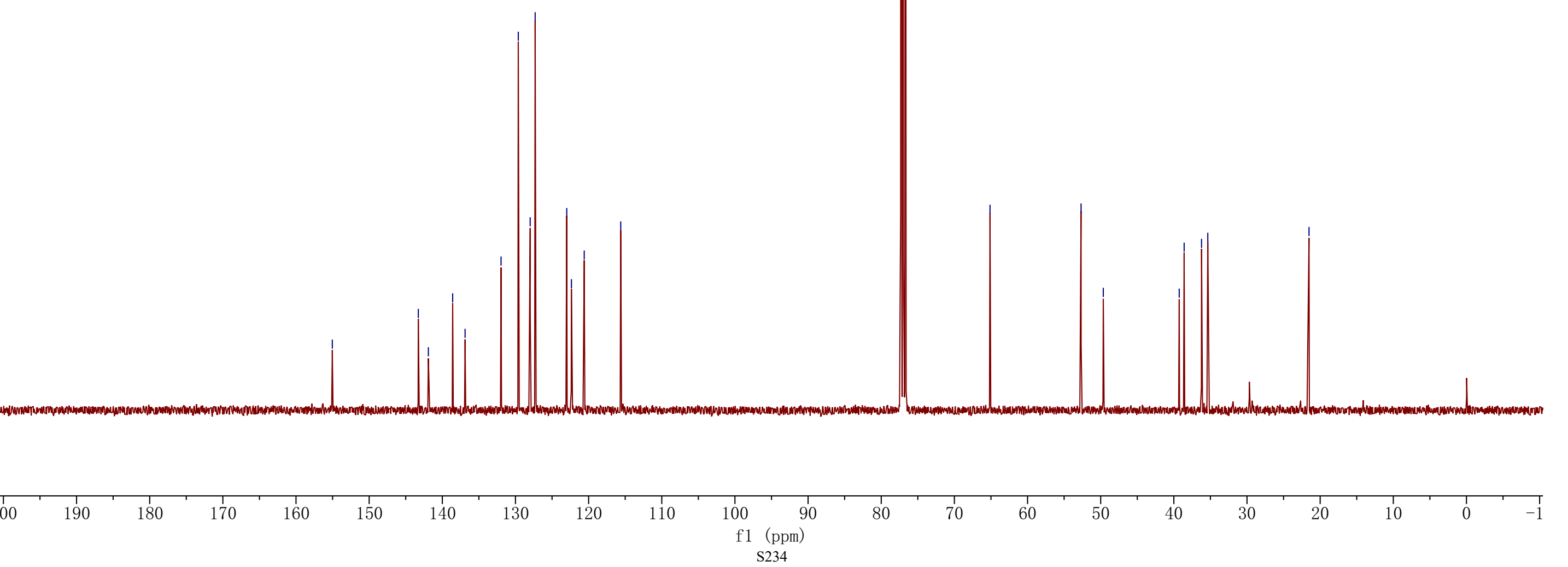



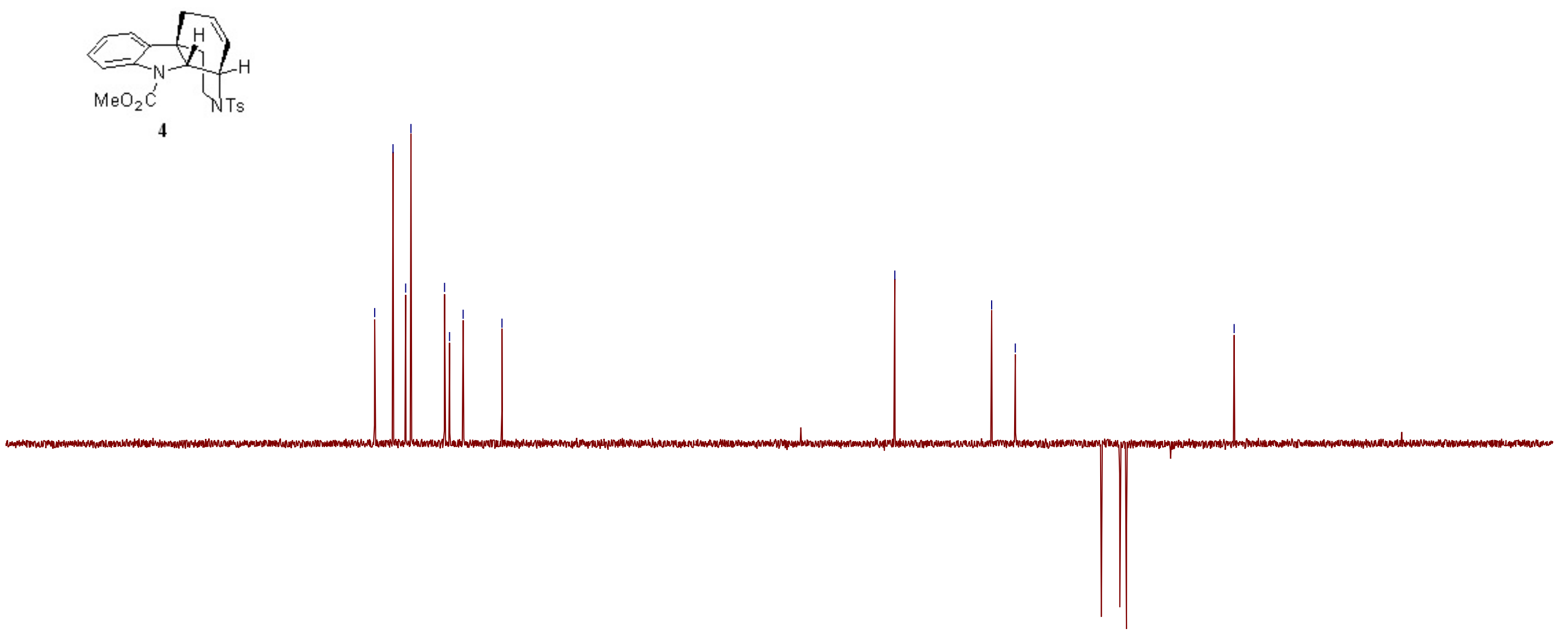

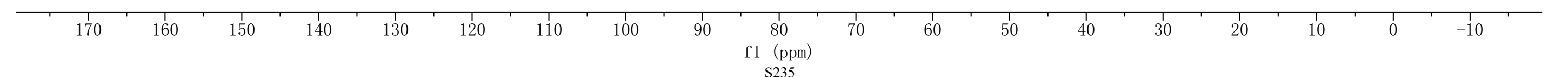




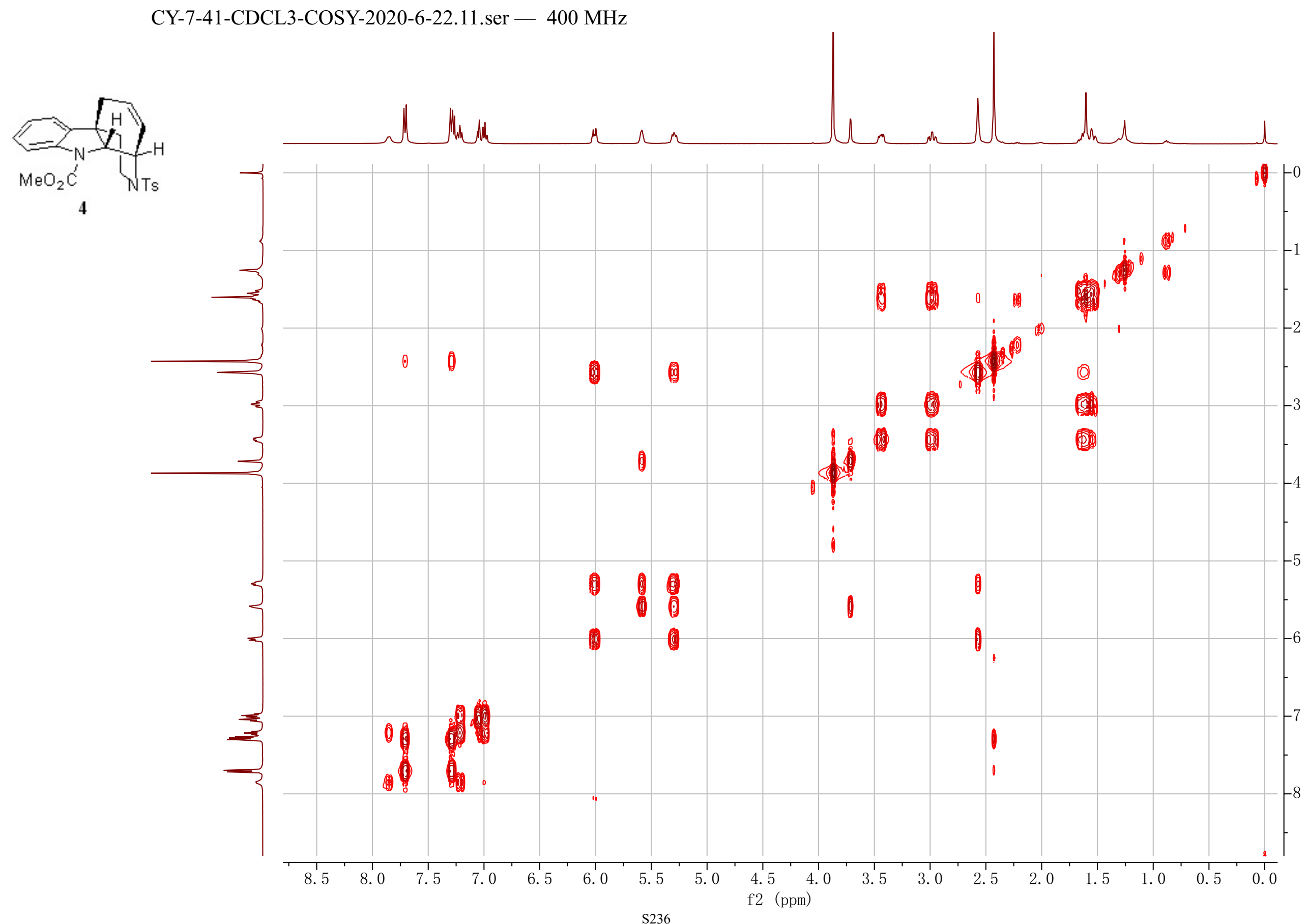




\section{CY-7-41-CDCL3-HSQC-2020-6-22.10.ser - $400 \mathrm{MHz}$}
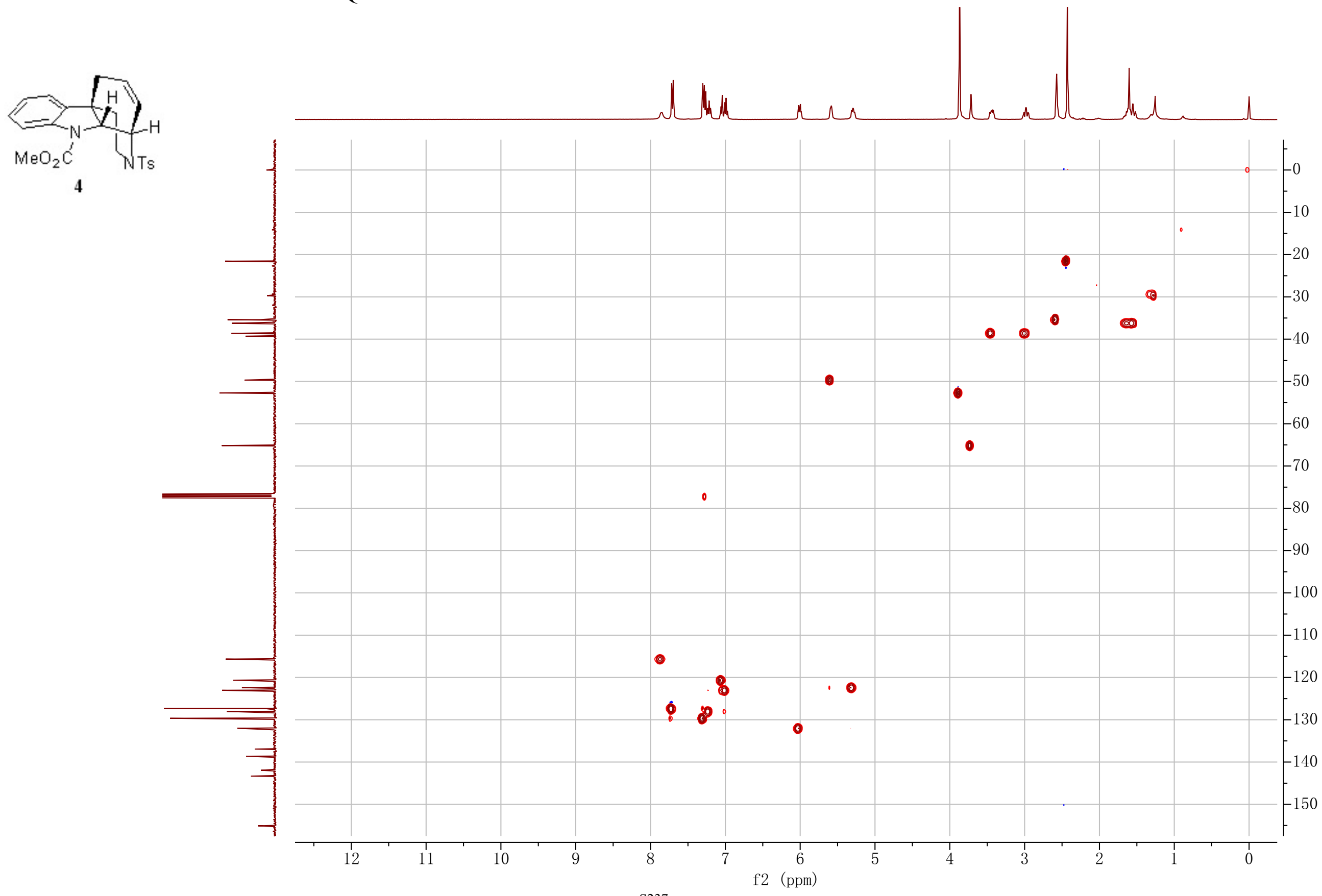


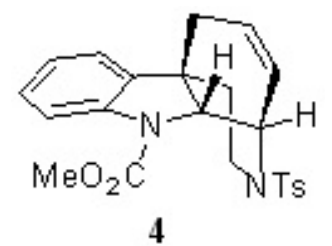

$\mathrm{H}_{3}$

$\mathrm{H}_{1}$
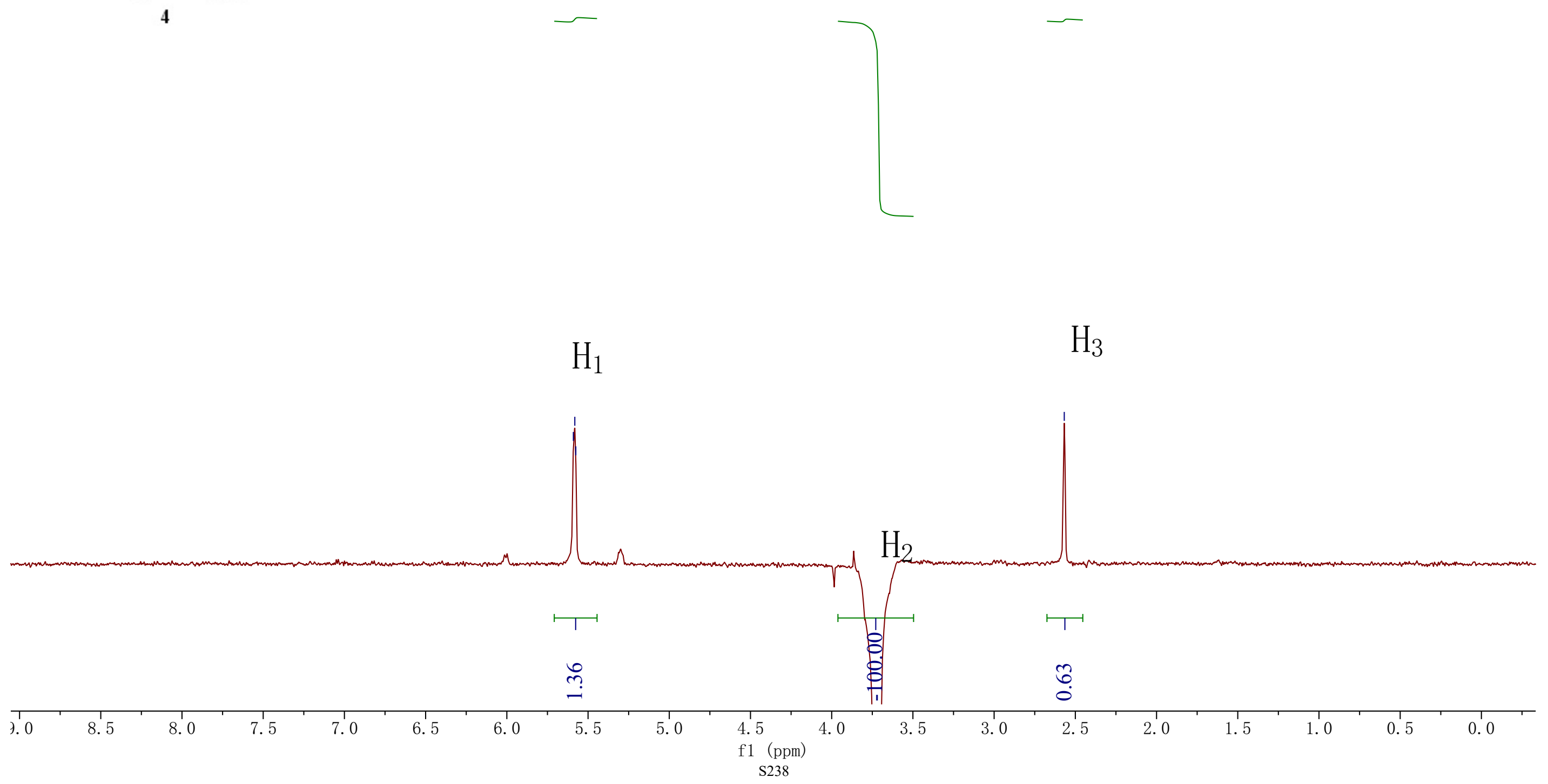

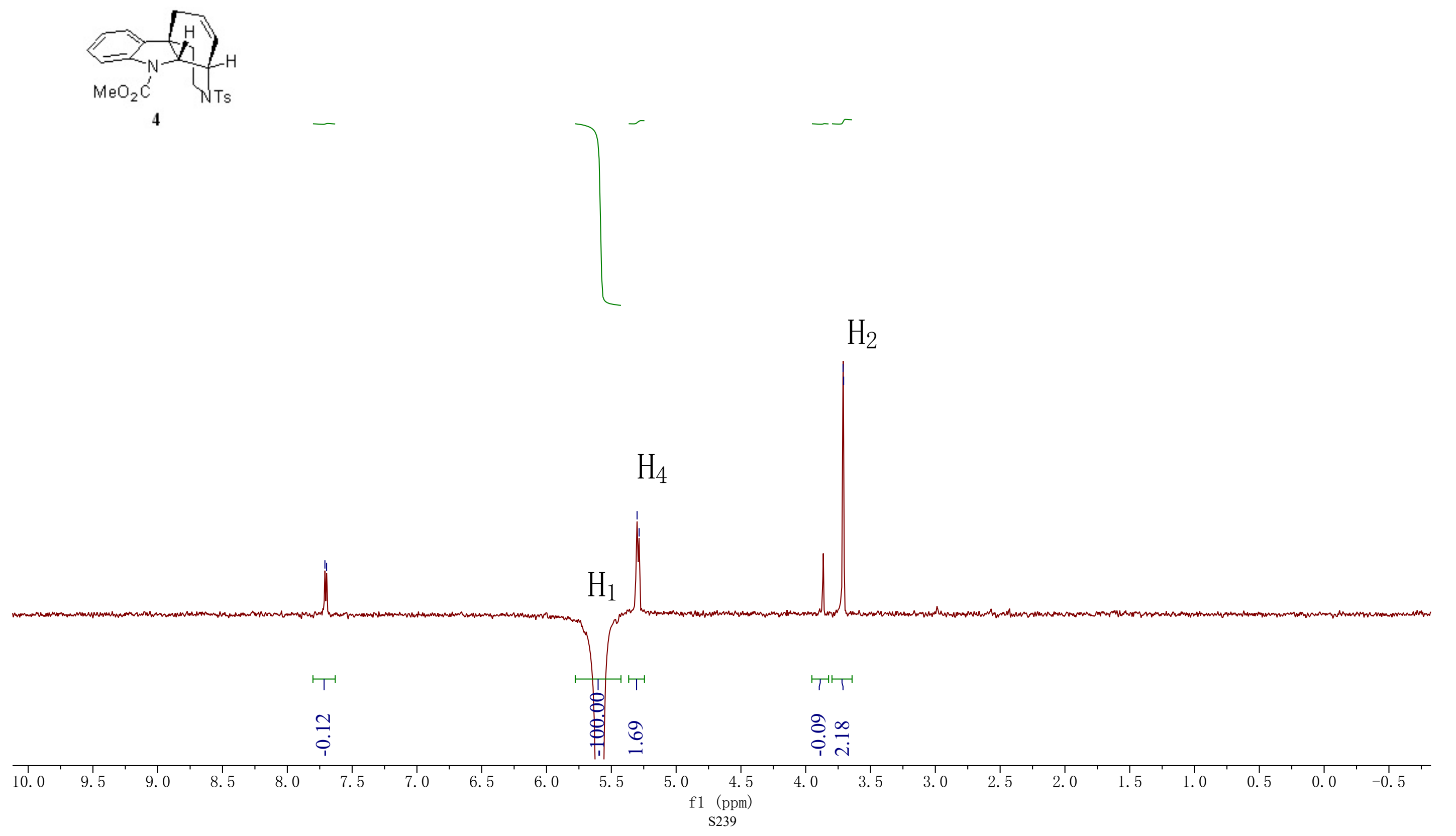